



\title{
Electronic Structure and Reactivity of
} Bioinspired Organometallic Iron

Complexes Relevant to Small Molecule

\section{Activation}

\author{
Dissertation \\ zur Erlangung des mathematisch-naturwissenschaftlichen Doktorgrades \\ „Doctor rerum naturalium“ \\ der Georg-August-Universität Göttingen \\ im Promotionsprogramm BioMetals \\ der Georg-August University School of Science (GAUSS)
}

vorgelegt von

Claudia Gisela Kupper M.Sc.

aus Gera 



\section{Betreuungsausschuss}

\section{Prof. Dr. F. Meyer}

Institut für Anorganische Chemie, Georg-August-Universität Göttingen

\section{Prof. Dr. I. Feußner}

Abteilung Biochemie der Pflanze, Albrecht-von-Haller-Institut der Pflanzenwissenschaften Universität Göttingen

Prof. Dr. E. Nordlander

Lunds Universitet, Schweden

\section{$\underline{\text { Mitglieder der Prüfungskommission }}$}

\section{Referent: Prof. Dr. F. Meyer}

Institut für Anorganische Chemie, Georg-August-Universität Göttingen

\section{Korreferent: Prof. Dr. I. Feußner}

Abteilung Biochemie der Pflanze, Albrecht-von-Haller-Institut der Pflanzenwissenschaften Universität Göttingen

Weitere Mitglieder der Prüfungskommision:

Prof. Dr. L. Ackermann (Institut für Organische und Biomolekulare Chemie, GeorgAugust-Universität Göttingen)

Jun.-Prof. Dr. S. Demir (Institut für Anorganische Chemie, Georg-August-Universität Göttingen)

Prof. Dr. S. Schneider (Institut für Anorganische Chemie, Georg-August-Universität Göttingen)

Prof. Dr. I. Siewert (Institut für Anorganische Chemie, Georg-August-Universität Göttingen) 



\section{Table of Contents}

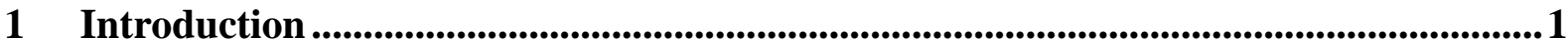

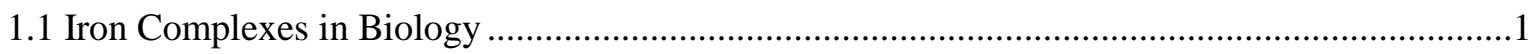

1.1.1 Oxoiron Chemistry in Nature and High-Valent Intermediates ...........................................2

1.1.2 Iron Enzymes Related to the Biogeochemical Nitrogen Cycle ............................................

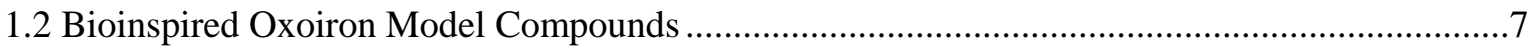

1.2.1 Oxoiron(IV) Model Systems and their Typical Ligand Scaffolds ......................................

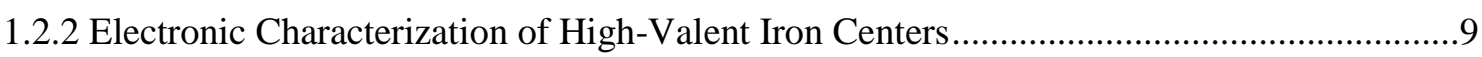

1.2.3 Oxidation and Oxygenation Reactivity of Oxoiron(IV) Species .....................................10

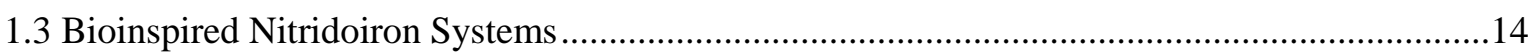

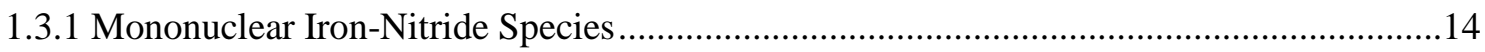

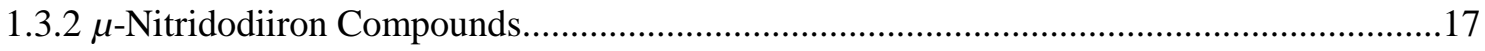

1.4 Synthetic Iron-Complexes with Nitrogen Oxides .....................................................................19

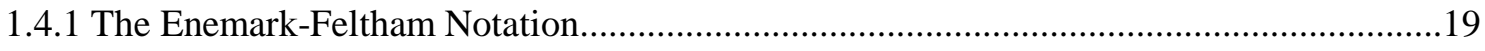

1.4.2 Model Compounds and Their Main Characteristics .........................................................19

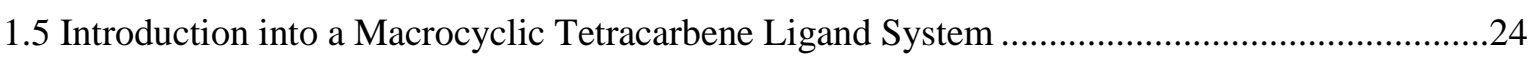

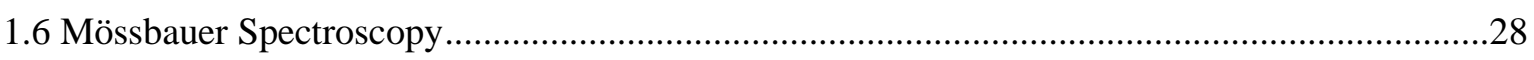

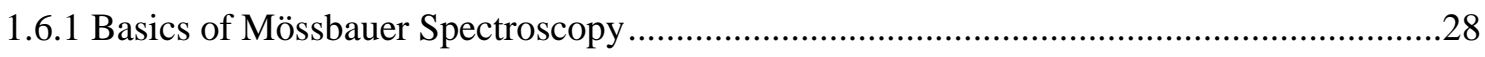

1.6.2 Hyperfine Interactions in Mössbauer Spectroscopy .......................................................29

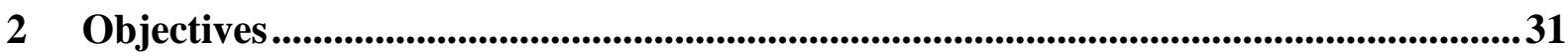

3 Characterization of a Carbene Coordinated Oxoiron(IV) Complex..........................33

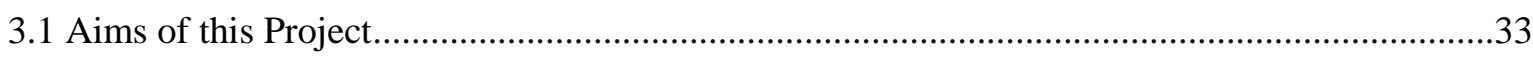

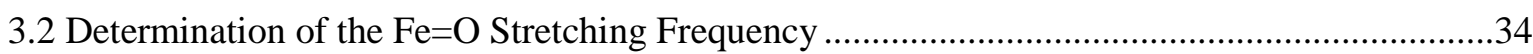

3.3 Characterization of the Electronic Structure of $\mathbf{2}$ by MCD .........................................................

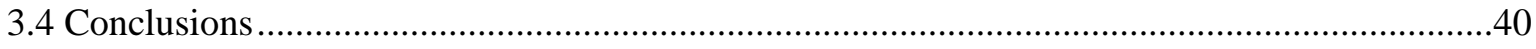

4 C-H Bond Activation by an Organometallic Oxoiron(IV) Complex ........................... 41

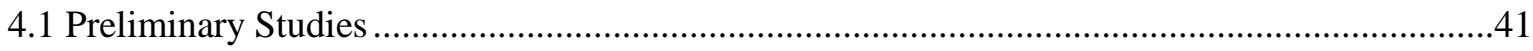

4.2 Reaction of Oxoiron(IV) Complex 2 Toward 1,4-Cyclohexadiene ............................................43

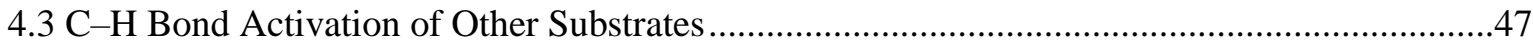

4.4 Characterization of the HAA Mechanism by Labeling Experiments and EPR Spectroscopy ....51

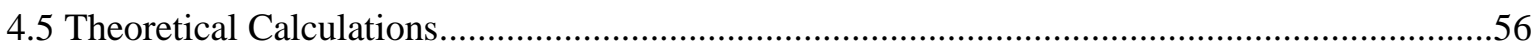

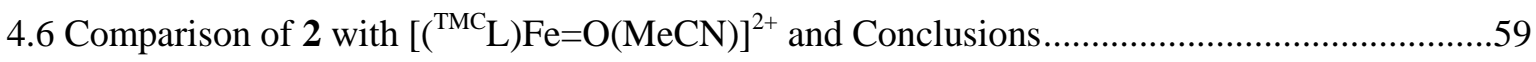

5 Unprecedented Reactivity of a $\mu$-Oxodiiron(III) Species ........................................61

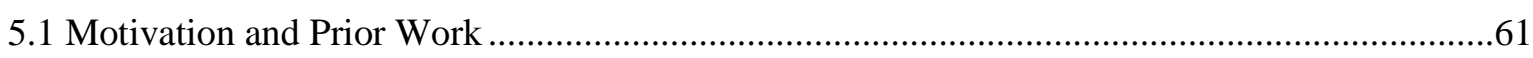

5.2 Oxygen Atom Transfer Reactivity Toward Trimethylphosphine ............................................62 


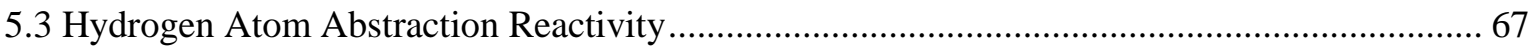

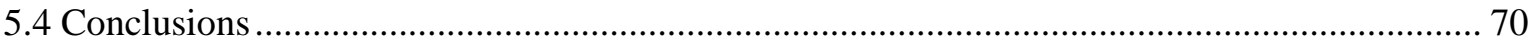

6 Conversion of NHC Coordinated Iron Precursors with Azides ................................... 71

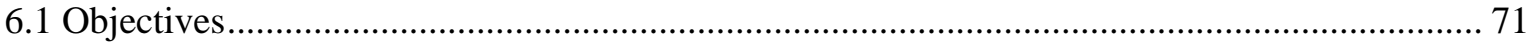

6.2 Bis-Azido Complexes of Organometallic Iron(II) and Iron(III) Centers .................................. 72

6.2.1 Characterization of a Bis-Azidoiron(II) Complex (5) _..................................................... 72

6.2.2 Oxidation of an Organometallic Iron(II) Complex ....................................................... 74

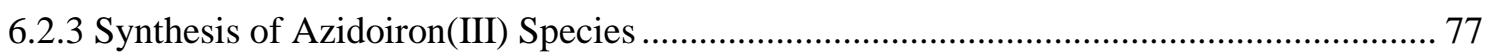

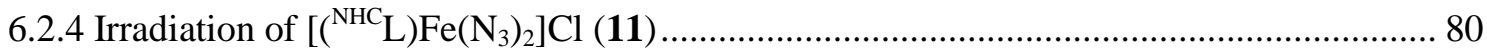

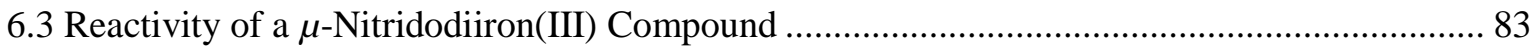

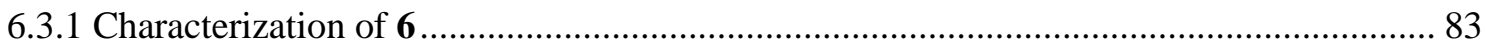

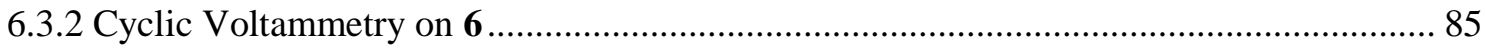

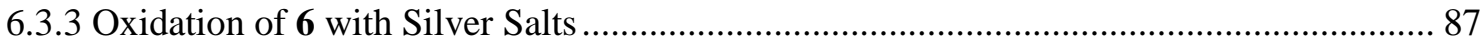

6.3.4 Conversion of 6 with Nitrosonium Tetrafluoroborate....................................................... 94

7 Formation of Organometallic Iron(V) Species............................................................. 99

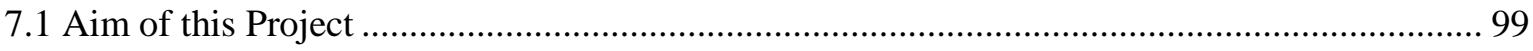

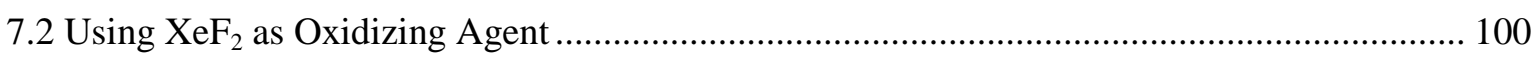

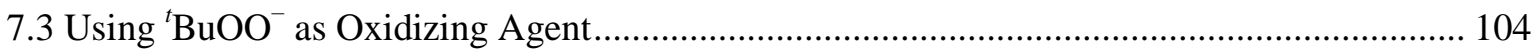

8 Macrocyclic Iron NHC Complexes with Nitric Oxides ......................................... 111

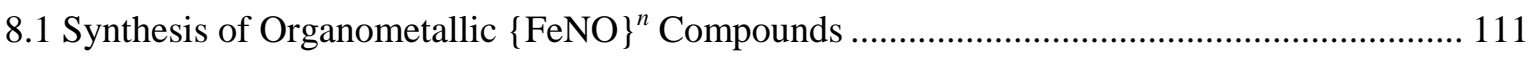

8.2 Characterization of a Complete Series of $\{\mathrm{FeNO}\}^{6},\{\mathrm{FeNO}\}^{7}$ and $\{\mathrm{FeNO}\}^{8}$......................... 116

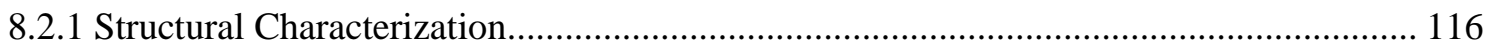

8.2.2 Magnetic Properties of the Newly Synthesized FeNO Compounds ................................. 119

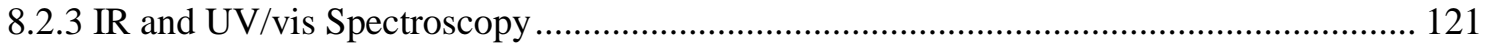

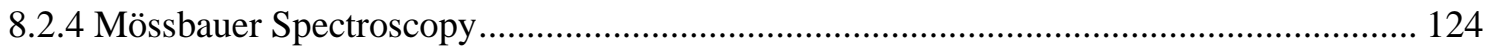

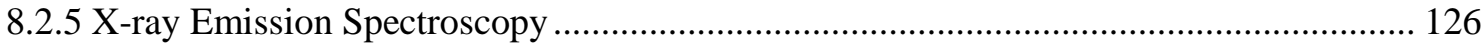

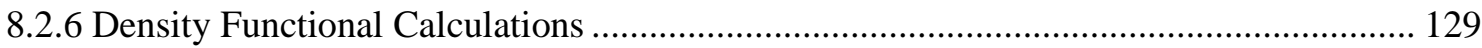

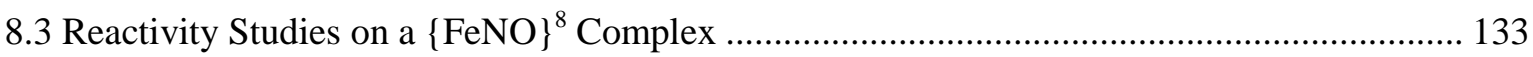

9 Development of New Hybride Ligand Systems ........................................................... 135

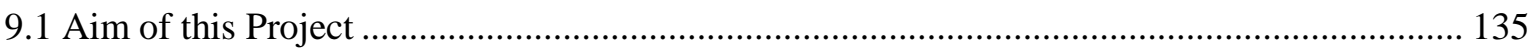

9.2 A ‘Carbeneporphyrinoid' Macrocycle for the Generation of Metal Complexes....................... 136

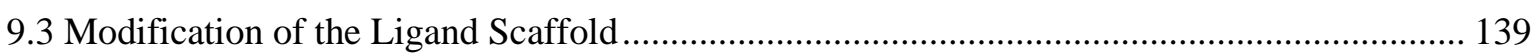

10 Summary

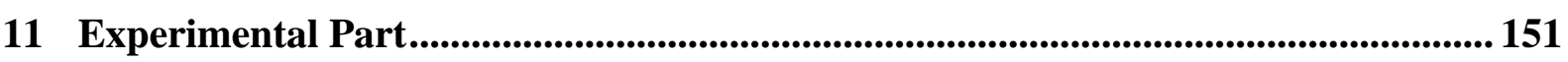

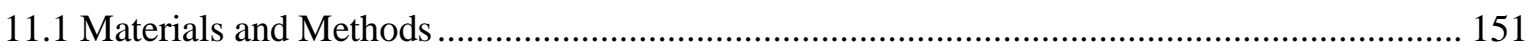

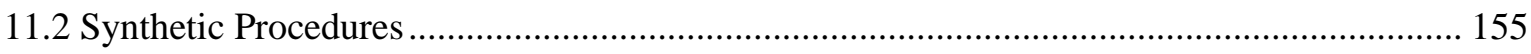

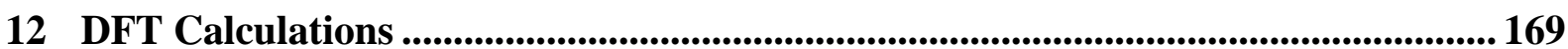


13 Crystallography ................................................................................................................ 185

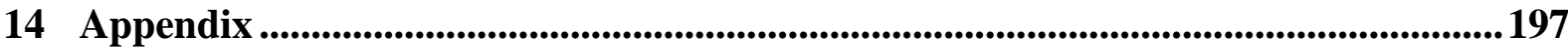

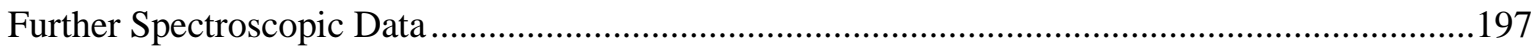

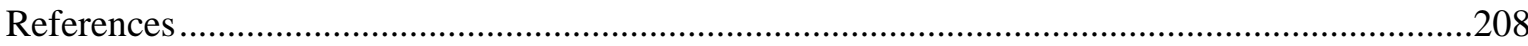

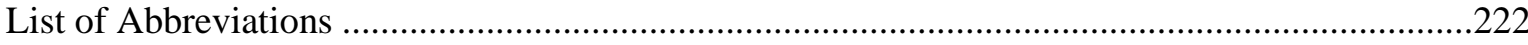

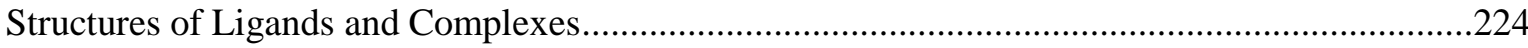

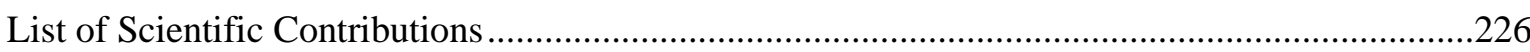





\section{Introduction}

\subsection{Iron Complexes in Biology}

Behind oxygen, silicon, and aluminum, iron is the fourth most abundant element in earth's crust and occurs in a range of minerals in its most common and stable oxidation states two and three. ${ }^{[1,2]}$ Moreover, it plays a central role in living cells where it contributes to numerous cellular processes. These include, for example, electron transfer processes such as those involving Fe-S-clusters, thanks to the accessibility of the oxidation states two and three. ${ }^{[3]}$ Iron is also present in a protein of primary importance for life, hemoglobin, which is responsible for the red color of blood. ${ }^{[4,5]}$ Hemoglobin is able to bind dioxygen; this allows to circumvent the low solubility of $\mathrm{O}_{2}$ in blood and, thus, transport to biological tissues. Even more interesting, biological systems also involve iron intermediates in unusual oxidation states. For example, iron(I) species are postulated during the reversible reduction of protons to dihydrogen by iron-only hydrogenases. ${ }^{[6]}$ More electrondeficient iron(IV) or iron(V) centers can instead occur during enzymatic oxidation processes. One example for this behavior is represented by heme iron(II) centers, which can activate dioxygen leading to high-valent oxoiron species. In biological systems, these $\mathrm{Fe}=\mathrm{O}$ intermediates functionalize unactivated $\mathrm{C}-\mathrm{H}$ bonds with high efficiency and stereospecificity. ${ }^{[5]}$ Therefore, a detailed understanding of the intermediate oxoiron(IV) and -(V) species is of high interest. So far, three different coordination modes of $\mathrm{Fe}=\mathrm{O}$ species have been found in nature that will be introduced in section 1.1.1. ${ }^{[7]}$

Besides $\mathrm{O}_{2}$, iron containing enzymes are involved in the activation and transfer of another molecule of primary importance for life: $\mathrm{N}_{2}$. In similarity to the involvement of $\mathrm{Fe}=\mathrm{O}$ compounds in oxygen atom transfer processes, high-valent nitridoiron(V) species are the key intermediates in nitrogen atom transfer reactions (1.1.2) ${ }^{[8]}$ An outstanding enzyme in this regard is nitrogenase with its FeMo cofactor that performs dinitrogen activation; the initial process in the biogeochemical nitrogen cycle. ${ }^{[9,10]}$ One mechanistic scenario for the reduction of dinitrogen to ammonia includes both a low-valent iron(I) and a high-valent iron(IV) species giving a perfect example for the use of a wide range of iron oxidation states in biological systems. The denitrification step in the biogeochemical nitrogen cycle involves other iron-nitrogen species, iron nitrosyl and -nitroxyl compounds. Such Fe-NO species also play an important role in mammals, for example as activator for muscle relaxation as further discussed in 1.1.2. ${ }^{[11]}$ Besides the activation of $\mathrm{O}_{2}, \mathrm{~N}_{2}$ and $\mathrm{NO}$, iron complexes play many more important roles in biology. Still, due to their relevance for the work developed during this thesis, only these topics will be discussed in detail within the next sections. 


\subsubsection{Oxoiron Chemistry in Nature and High-Valent Intermediates}

As mentioned above, iron centers play an important role in dioxygen chemistry in a variety of living creatures. Typically, iron(II) centers in oxygenase enzymes activate dioxygen giving initial iron(III) superoxo species. Subsequent one-electron reduction by co-substrates as well as protonation can lead to peroxo and hydroperoxo compounds which finally undergo hetero- and homolytic cleavage leading to $\mathrm{Fe}^{\mathrm{IV}}=\mathrm{O}$ and $\mathrm{Fe}^{\mathrm{V}}=\mathrm{O}$ species. ${ }^{[7]}$ These high-valent oxoiron intermediates are highly reactive toward electrophilic attack and hydrogen atom abstraction (HAA) due to both unpaired spin density on the oxygen atom and electron-deficient iron centers. ${ }^{[12]}$ Consequently, detection or isolation of these species in biological systems is rather challenging, yet huge success in understanding enzymatic mechanisms has been achieved during the last decades of persistent research. ${ }^{[13]}$

As already mentioned within the first paragraph of this work, heme iron species such as hemoglobin play an important role in the activation of dioxygen for substrate oxidation and oxygenation. The protoporphyrin IX ligand system can perfectly stabilize high-valent iron intermediates. The most popular example, Compound I (Cpd-I), occurs in the catalytic activation of dioxygen by cytochrome P450 oxygenases (Scheme 1). ${ }^{[14,15]}$ The iron(II) center (a) of this enzyme reacts with $\mathrm{O}_{2}$ giving (su)peroxo and hydroperoxo intermediates (b-d). Heterolytic cleavage of the $\mathrm{O}-\mathrm{O}$ bond in $\mathbf{d}$ subsequently forms the cysteine coordinated oxoiron(IV) porphyrine radical Cpd-I as the active species in substrate oxidation. Consequently, both the iron center and the non-innocent ligand scaffold in $\mathbf{d}$ undergo one-electron oxidation upon formation of Cpd-I while the equally possible formation of an iron(V) center is not observed. Similar highvalent heme oxoiron species are also found in catalase and peroxidase enzymes. ${ }^{[7]}$

A second coordination geometry found in high-valent oxoiron active sites is represented by mononuclear non-heme iron centers coordinated by a '2-His-1-carboxylate' facial triad. One famous example of this are the Rieske dioxygenases which are proposed to catalyze the dihydroxylation of unsaturated $\mathrm{C}-\mathrm{C}$ bonds via a $\mathrm{HO}-\mathrm{Fe}^{\mathrm{V}}=\mathrm{O}$ intermediate. ${ }^{[16]}$ The catalytic cycle of another mononuclear non-heme iron system, taurine $\alpha$-ketoglutarate dioxygenase, is depicted in Scheme 1. The crystal structure of the active species TauD-J could be fully characterized in 2003 and represents the first unambiguously identified enzymatic non-heme oxoiron(IV) center. ${ }^{[17,18]}$ TauD- $J$ is formed after homolytic cleavage of the $\mathrm{O}-\mathrm{O}$ bond in dioxygen. In this case, a pathway involving ligand oxidation similar to the one observed in heme systems is not possible due to the redox-innocent non-heme ligand scaffold, but an $\alpha$-ketogluterate $(\alpha-K G)$ co-substrate is used instead for providing two electrons. ${ }^{[12]}$ 

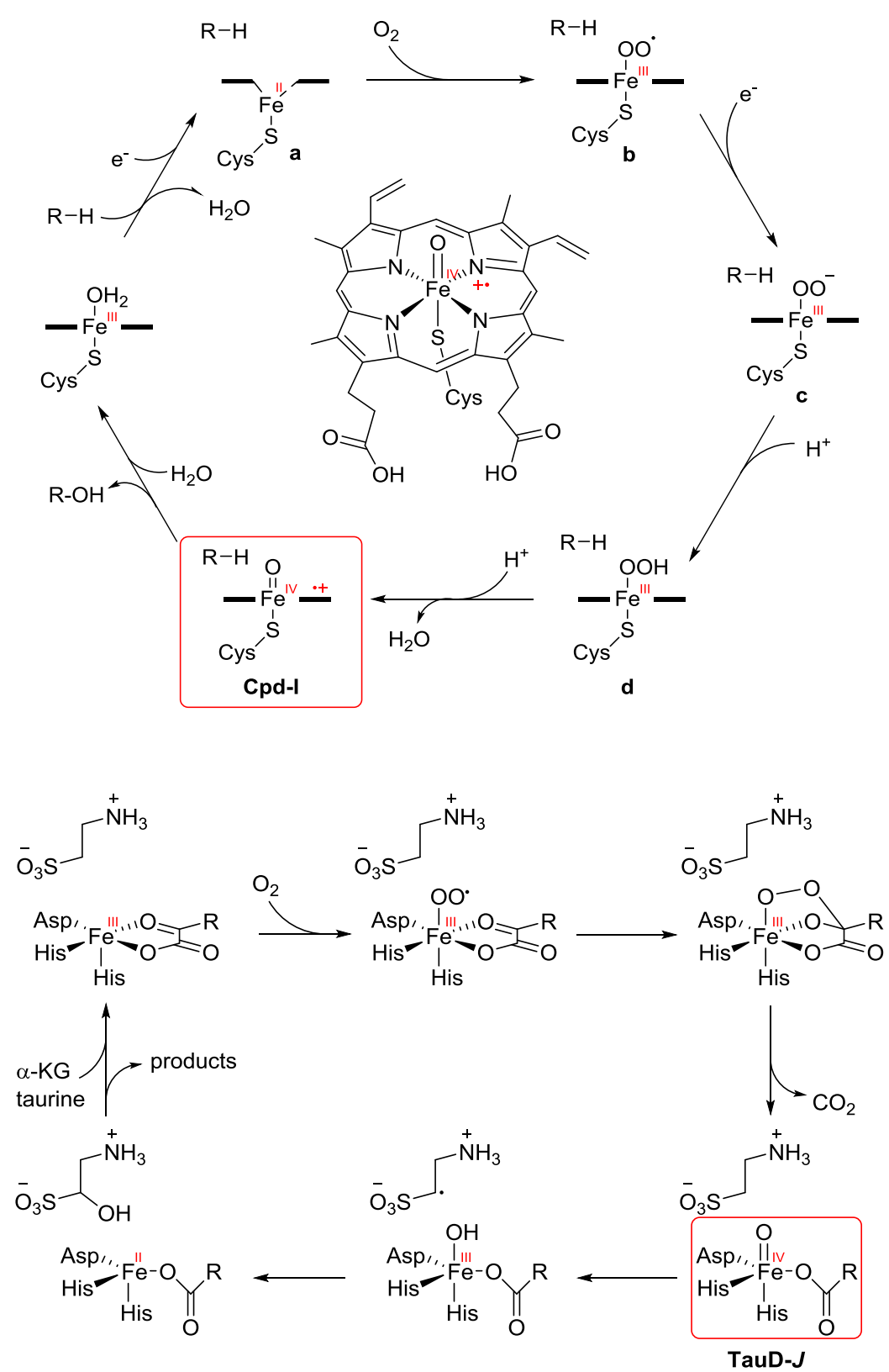

Scheme 1. Proposed catalytic cycles of cytochrome P450 oxygenase (top) and taurine $\alpha$-KG dioxygenase (bottom). ${ }^{[13]}$

Finally, also dinuclear non-heme active centers are found in the enzymatic activation of $\mathrm{C}-\mathrm{H}$ bonds. Typically, the iron atoms are coordinated by two histidine and four carboxylate groups. ${ }^{[7]}$ The most famous example is intermediate $\mathrm{Q}$ of the soluble methane monooxygenase (sMMO) whose exact structure has been under study since the 1990s. ${ }^{[19,20]}$ This enzyme catalyzes the oxidation of methane to methanol which is of high interest for possible industrial applications. Banerjee et al. have recently confirmed that $\mathrm{Q}$ contains a bis- $\mu$-oxo-diiron(IV) center with both bridging oxygen atoms originating from $\mathrm{O}_{2}$. Other diiron active sites are found in toluene monooxygenase, fatty acid desaturases and ribonucleotide reductase. ${ }^{[18]}$ 


\subsubsection{Iron Enzymes Related to the Biogeochemical Nitrogen Cycle}

For a long time, nitrogen oxide species $\left(\mathrm{NO}_{\mathrm{x}}\right)$ have been mainly known for their toxicity and their involvement in air pollution. ${ }^{[21-23]}$ An important member of this family, nitric oxide ( $\mathrm{NO}$ ), can be either highly toxic due to its radical character but also serve as important secondary messenger molecule at nanomolar concentrations. It plays crucial roles in mammalian physiology such as blood pressure control, neurotransmission, immune response, tissue damage, and carcinogenesis. ${ }^{[22-}$ ${ }^{25]}$ Its interaction with metallobiosites, in particular with heme and non-heme iron centers, is of high relevance in this respect. The most important receptor for 'NO in mammals is the soluble gyanylate cyclase sGC. ${ }^{[11,26]}$ This enzyme contains an iron(II) heme active site coordinated by an additional axial histidine. Upon reaction with nitric oxide, it forms a five coordinated heme iron nitrosyl complex, thus the axial histidine becomes disconnected. This initiates a cascade of enzymatic conversions that finally activate Protein kinase $\mathrm{G}$ which mediates the dilation of blood vessels, also called vasodilation.

The sGC activity is strongly connected to nitric oxide synthase (NOS) enzymes, responsible for 'NO synthesis in mammals. In this process, ${ }^{\mathrm{NO}}$ is released upon hydroxylation and oxidation of L-arginin, catalyzed by cytochrome P450 enzymes. ${ }^{[27]}$ In bacteria, nitrosyl compounds are intermediates in the denitrification of nitrate to dinitrogen as part of the biogeochemical nitrogen cycle (Scheme 2). ${ }^{[22,23,28,29]}$

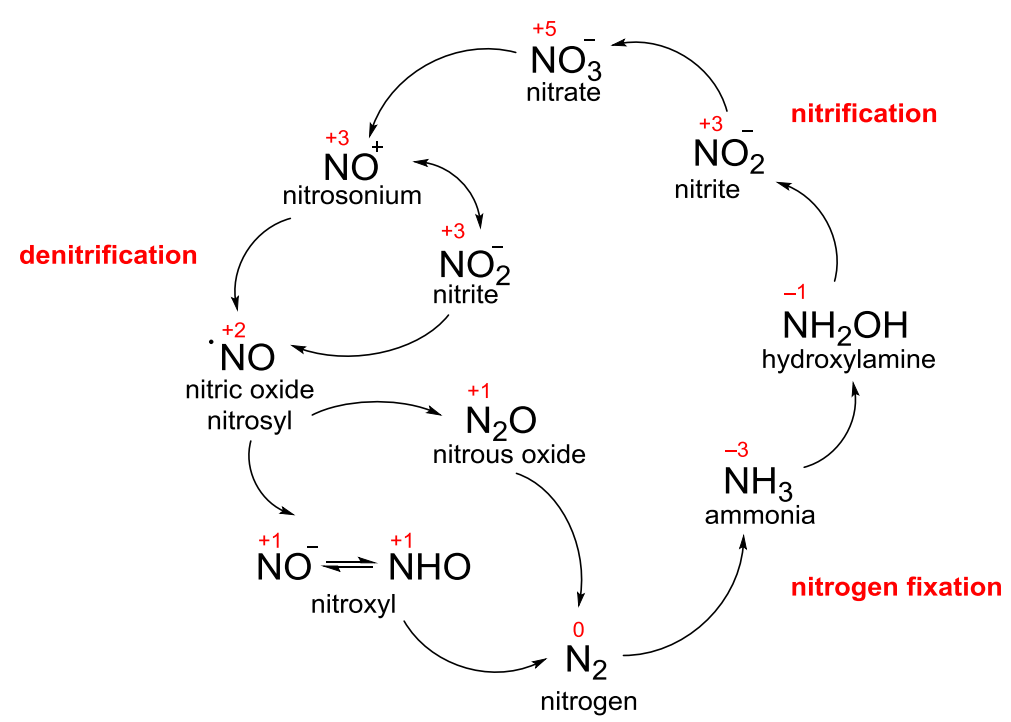

Scheme 2. The biogeochemical nitrogen cycle. ${ }^{[28]}$

The stepwise reduction processes within the bacterial and fungal dissimilatory denitrification pathways are performed by metalloenzymes, including iron-containing nitric oxide reductases such as rNORs and FNORs. ${ }^{[30]}$ Proposed key intermediates during nitric oxide reduction are iron nitrosyl and nitroxyl complexes of both heme and non-heme types. ${ }^{[22,23,31]}$ Ferric nitrosyl species that are 
able to transport and release 'NO are formed upon nitrite reduction mediated by heme $c d_{1}$ NIRs, as well. ${ }^{[32]}$ One of the enzymes that catalyze the further reduction of 'NO to the less toxic nitrous oxide $\left(\mathrm{N}_{2} \mathrm{O}\right)$ is NorBC. ${ }^{[1,33]}$ Present in bacteria, it contains a heme iron center (NorC) close to a non-heme center (NorB). How exactly this dinuclear system mediates the conversion of two "NO molecules into $\mathrm{N}_{2} \mathrm{O}$ and water is still under debate. According to the proposed trans-type mechanism, both iron centers initially form mono nitrosyl iron species with the NO ligands close to each other in the enzyme pocket. Afterwards, the nitrosyls presumably are coupled via a radical or dipolar mechanism and a $\mu$-oxodiiron(III) center is formed upon $\mathrm{N}_{2} \mathrm{O}$ release. Another $\mathrm{NO}$ reducing enzyme is the fungal P450nor which forms a six-coordinated ferric nitrosyl as the first intermediate. ${ }^{[34]}$ In the following steps, this species is doubly reduced to give a ferrous nitroxyl compound. The exact nature of this intermediate has not been clarified yet, thus it remains unclear if this species is protonated. Furthermore, the spin state at the iron center is not clarified yet. ${ }^{[35]}$ Finally, also flavodiiron proteins can work as NO reducing enzymes besides their oxygen activation reactivity. ${ }^{[36,37]} \mathrm{A}$ mechanistic insight for this class of enzymes is still lacking, however it is assumed that two mononitrosyl units are formed in the non-heme diiron active site during the first step. In a second step, reduced iron nitroxyl species might be formed and initiate formation and release of $\mathrm{N}_{2} \mathrm{O}$.

Summarizing, in many of these processes conversions between nitrosonium $\mathrm{NO}^{+}$, nitrosyl ${ }^{\circ} \mathrm{NO}$, and nitroxyl $\mathrm{NO}^{-}$play an important role. When connected to an iron center, this redox equilibrium directly corresponds to addition and substraction of electrons to an (FeNO) moiety. This idea is connected to a central part of this work that is discussed in chapter 8 .

Besides being a component of the $\mathrm{NO}_{\mathrm{x}}$ molecules described so far, nitrogen is also, together with carbon and hydrogen, one of the most important components in biomolecules and is essential for all living organisms. ${ }^{[2]}$ However, most species are able to assimilate it as ammonia or nitrate only while activation of atmospheric dinitrogen is a prerogative of a few bacteria. ${ }^{[38-40]}$ This dinitrogen fixation process is another important step within the biogeochemical nitrogen cycle (Scheme 2). The reduction of dinitrogen to ammonia constitutes an equally fascinating biocatalytic transformation compared to $\mathrm{O}_{2}$ reduction. ${ }^{[41]}$ The industrial analogue is found in the energy consuming and expensive Haber-Bosch process that needs high pressure and temperature. The biological nitrogen-fixation process is catalyzed by the microbial enzyme nitrogenase. ${ }^{[10,42]}$ The Mo-dependent nitrogenase enzyme catalyzes the conversion of $\mathrm{N}_{2}$ into $\mathrm{NH}_{3}$ at moderate temperatures and with high efficiency, in contrast to the Haber-Bosch conditions. The active site (MoFe protein) consists of a multimetallic iron-moybdenum cofactor (FeMo-co, Scheme 3) and an auxiliary ' $\mathrm{P}$ ' cluster assumed to mediate electron transfer. An additional [4Fe-4S] clustercontaining protein called dinitrogenase reductase provides the electrons for $\mathrm{MoFe}$ reduction. ${ }^{[10]}$ Despite the exact structure of the FeMo cofactor was solved in $2011,{ }^{[9]}$ a daunting number of 
mechanistic questions behind dinitrogen activation remain unanswered. In fact, it is still unclear which metal binds $\mathrm{N}_{2}$ initially; both iron and molybdenum are in fact available. For the former, iron(IV) intermediates have been proposed, while the latter is a highly redox active species with most common oxidation states ranging from + III to + VI. One popular scenario is the Chatt cycle ${ }^{[43,44]}$ shown in the iron-mediated distal pathway in Scheme $3 .{ }^{[45]}$ In this case, the mechanism would involve a high-valent nitridoiron species, similar to surface bound nitridoiron intermediates suggested during the Haber-Bosch process. ${ }^{[46]}$

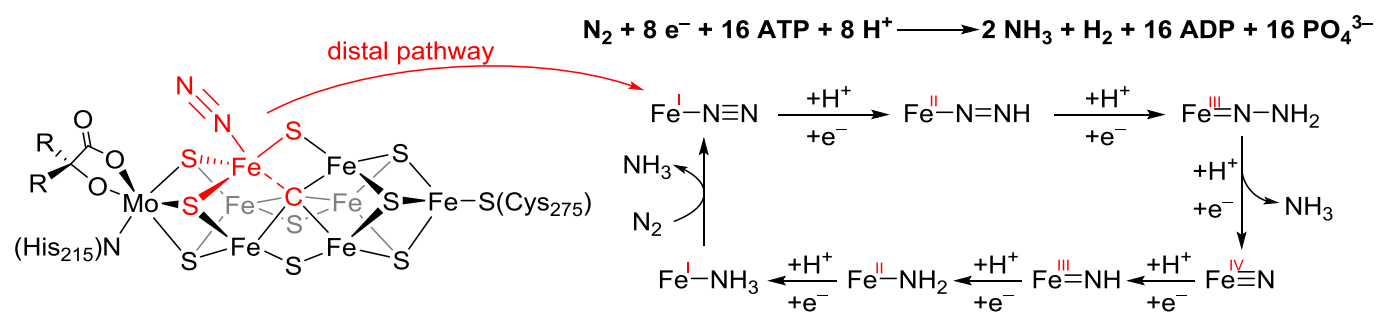

Scheme 3. FeMo cofactor and the proposed distal pathway of dinitrogen activation at a single iron site in the dinitrogenase enzyme. ${ }^{[45]}$

High-valent nitrido- $(\mathrm{Fe} \equiv \mathrm{N})$ or imidoiron $(\mathrm{Fe}=\mathrm{NR})$ species are also proposed as intermediates in nitrogen atom and nitrogen group transfer reactions performed by cytochrome $\mathrm{P} 450$ enzymes. ${ }^{[47]} \mathrm{In}$ analogy to oxoiron species, they are assumed to be crucial for activation and nitrogenation of $\mathrm{C}-\mathrm{H}$ bonds. Even though many model compounds for such species have been synthesized so far (see 1.3), direct evidence of the involvement of these compounds in biology is still missing. 


\subsection{Bioinspired Oxoiron Model Compounds}

The described enzymes, together with many more fascinating natural systems, perform biocatalytic transformations that are, in many cases, still quite difficult to reproduce for synthetic chemistry. In terms of selectivity and efficiency, natural processes work better than similar industrial ones mainly by using non-toxic and abundant metal catalysts as well as by benefitting from low activation barriers. To reproduce these natural processes, a better insight into the molecular and electronic structures of the active species is needed. Unfortunately, studies on the biological systems are very difficult due to the high reactivity and instability of the intermediates formed during the catalytic cycles. To overcome the difficulties in studying the enzyme's activities in biological environment, synthetic bioinorganic chemists isolate compounds to use them as functional models. These model compounds help to understand fundamental aspects such as likely mechanisms, critical interactions, and properties of active intermediates. In the same way, model compound studies allow for the use of non-biological conditions, such as low temperatures or water-free environments, which can facilitate the detection and isolation of unstable intermediates. A better understanding of the enzymatic processes will help in setting the basis for the development of new synthetic or catalytic routes, which may result in the improvement of current industrial processes. ${ }^{[11]}$

\subsubsection{Oxoiron(IV) Model Systems and their Typical Ligand Scaffolds}

The first heme oxoiron(IV) system could be synthesized in 1981 with the use of a mesotetramesityl porphinato anion (TMP) ligand system. Groves et al. oxidized [(TMP) $\left.\mathrm{Fe}^{\mathrm{III}}(\mathrm{Cl})\right]$ with meta-chloroperbenzoic (mCPBA) acid at $-78{ }^{\circ} \mathrm{C}$ yielding the corresponding $\mathrm{Fe}^{\mathrm{IV}}=\mathrm{O}$ species with an additional porphyrine radical similar to Cpd-I (Figure $1 \mathbf{A}) \cdot{ }^{[48,49]}$ During the following years, this complex has been characterized using a wide range of spectroscopic methods and initial reactivity studies have been performed as well. The electronic structure has been described as a low spin $S=1 \mathrm{Fe}^{\mathrm{IV}}=\mathrm{O}$ moiety ferromagnetically coupled with the $S=1 / 2$ porphyrin radical leading to a total spin of $S_{\mathrm{T}}=3 / 2$, which turned out to be the common electronic structure for heme systems. Since then, a number of heme oxoiron(IV) models with varying electron density on the iron center have been presented and their oxidizing power has been compared. ${ }^{[49,50]}$

The investigation of d-d transitions with the help of UV/vis spectroscopy is an important method in characterizing metal complexes. Unfortunately, the organic porphyrin ligand scaffold in heme systems shows intense optical features itself, such as the strong Soret band around $400 \mathrm{~nm}$ and Q 
bands around $600 \mathrm{~nm}$ that dominate the UV/vis spectra of heme $\mathrm{Fe}^{\mathrm{IV}}=\mathrm{O}$ species. ${ }^{[51]}$ To overcome this problem, it has been necessary to design and isolate non-heme model systems.

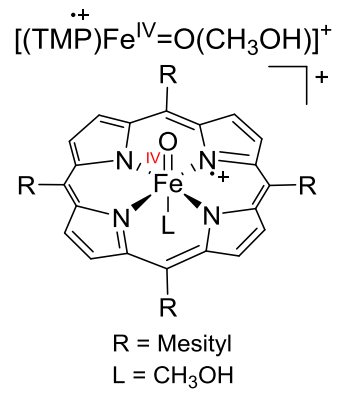

A

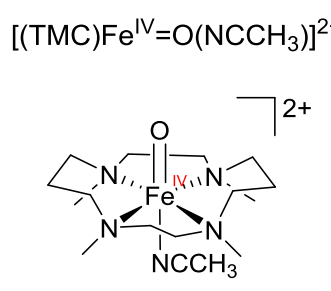

B

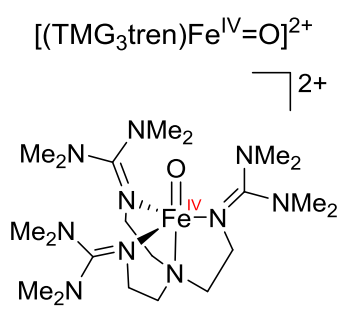

C
$\left[(\mathrm{TAML}) \mathrm{Fe}^{\mathrm{V}}=\mathrm{O}^{-}\right.$

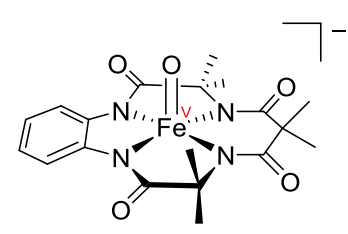

D

Figure 1. High-valent oxoiron complexes supported by tetradentate nitrogen donor systems. ${ }^{[48,49,52-56]}$

Wieghardt et al. synthesized the first non-heme oxoiron(IV) system by ozonolysis of $\left[(\mathrm{cy}-\mathrm{ac}) \mathrm{Fe}^{\mathrm{III}}\left(\mathrm{CF}_{3} \mathrm{SO}_{3}\right)\right]^{+}$at $-80{ }^{\circ} \mathrm{C}(\mathrm{cy}-\mathrm{ac}=$ cyclam-acetate $) .{ }^{[57]}$ This report in 2000 represents the starting point of a successful period for the synthesis and characterization of high-valent non-heme model compounds. Soon after this, Rohde and co-workers presented the first crystal structure of a mononuclear $S=1$ oxoiron(IV) complex (Figure $1 \mathbf{B}$ ). ${ }^{[53]}$ The use of a tetramethylcyclam (TMC) system instead of cy-ac helped to increase the stability of the high-valent species and allowed for its isolation at room temperature (RT). The iron(II) starting material was oxidized with the oxygen atom donor iodosylbenzene in order to obtain the desired $\mathrm{Fe}^{\mathrm{IV}}=\mathrm{O}$ compound. This synthetic strategy still represents a common way to generate oxoiron(IV) complexes. Since then, numerous model complexes have been published, most of them presenting multichelating $N$-donors and, more importantly, a low spin state $S=1 .^{[52,58-60]}$ This is in contrast to the high spin state $S=2$ found for the biological non-heme systems TauD-J, prolyl hydroxylase, or halogenase CytC3. The first crystal structure of a synthetic non-heme $S=2$ system was published as recently as 2010 and used the sterically demanding trigonal ligand scaffold $\left(\mathrm{TMG}_{3}\right.$ tren $=1,1,1-\operatorname{tris}\left\{2-\left[N^{2}-(1,1,3,3-\right.\right.$ tetramethylguanidino)]ethyl \}amine) shown in Figure 1 C. ${ }^{[54,55]}$

In the field of mononuclear high-valent oxoiron models, the synthesis of an iron(V) system had been also quite challenging. Since heme-based systems mainly stabilize an oxoiron(IV) $\pi$-cation radical unit instead for an iron(V) atom, the use of non-heme models with their redox-innocent ligand systems had been necessary. The first synthetic $\mathrm{Fe}^{\mathrm{V}}=\mathrm{O}$ species was prepared by Münck, Collins, et al. in 2007, oxidizing [(TAML) $\left.\mathrm{Fe}^{\mathrm{III}}\left(\mathrm{H}_{2} \mathrm{O}\right)\right]^{-}$with an excess mCPBA at $-60{ }^{\circ} \mathrm{C}(\mathrm{TAML}=$ tetraamido macrocyclic ligand, Figure 1 D). ${ }^{[56]}$ The resulting species was identified to be a low spin $S=1 / 2$ system with a very low isomer shift $\left(\delta=-0.42 \mathrm{~mm} \mathrm{~s}^{-1}\right)$ in its Mössbauer spectrum and a short $\mathrm{Fe}=\mathrm{O}$ bond based on EXAFS. X-ray diffraction studies were prevented by the low stability of the compound and, thus, by the inability to isolate single crystals. Unexpectedly, it turned out to be a rather weak oxidant, able to activate the weak $\mathrm{C}-\mathrm{H}$ bond in dihydroanthracene only. The first 
direct detection of a synthetic $\mathrm{HO}-\mathrm{Fe}^{\mathrm{V}}=\mathrm{O}$ intermediate as it is proposed to exist in Rieske dioxygenase was presented by Costas et al. in 2011. ${ }^{[61]}$ The use of a ${ }^{\mathrm{Me}, \mathrm{H}}$ Pytacn ligand (1-(2'pyridylmethyl)-4,7-dimethyl-1,4,7-triazacyclo-nonane) coordinating the central iron allowed for cis-dihydroxylation of $\mathrm{C}=\mathrm{C}$ double bonds and detection of the $\mathrm{HO}-\mathrm{Fe}^{\mathrm{V}}=\mathrm{O}$ intermediate by variable-temperature mass spectrometry.

The synthesis of model compounds as described so far has enabled a better insight in the behavior of oxoiron species; in particular regarding the influence of spin state, oxidation state, and electron richness of the iron centers on the overall reactivity of the complexes. Answers to these questions on the basis of current knowledge are given in the following sections.

\subsubsection{Electronic Characterization of High-Valent Iron Centers}

The majority of $\mathrm{d}^{4}$ oxoiron(IV) model compounds synthesized so far has fourfold symmetry octahedral or square pyramidal - and a low spin $S=1$ state. Based on model compound studies, high spin $S=2$ species seem to be more favorable in threefold symmetry (Figure 1, C) while fourfold symmetry induces low spin $S=1$ character. The d-orbital splittings for both cases are shown in Figure $2 .{ }^{[7]}$ In case of $\mathrm{C}_{4 \mathrm{v}}$ geometry, the classical $1+2+1+1 \mathrm{~d}$-orbital energy scheme is found. The energy difference between the low lying $d_{x y}$ orbital and the LUMO $\left(d_{x^{2}-y^{2}}\right)$ is higher than the energy required for spin pairing, thus a $S=1$ triplet state is energetically favored. In contrast to this, in $C_{3 v}$ symmetry the $d_{x^{2}-y^{2}}$ remains degenerate with the $d_{x y}$ orbital and a quintet state becomes more stable. Summarizing, the geometry of the ligand system shows a distinct influence on the spin state of the $\mathrm{Fe}=\mathrm{O}$ moiety.

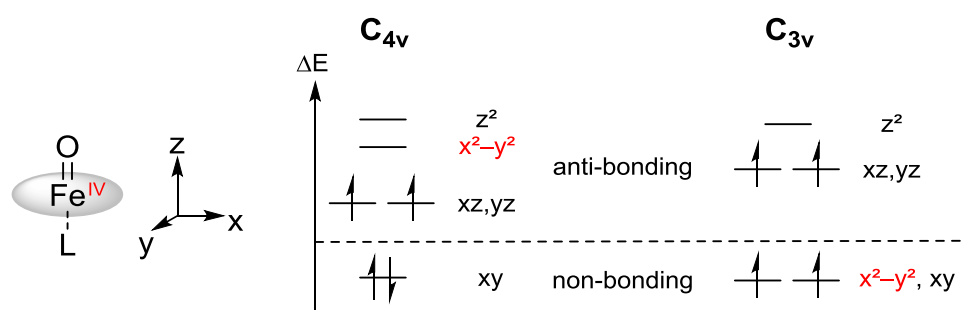

Figure 2. Tetragonal and trigonal ligand field splitting of high-valent oxoiron(IV) complexes; the figure was adapted from Hohenberger et al..$^{[7]}$

Electronic ground states of bioinorganic compounds in general are described using the interplay of different methods. Of particular importance for this work are electron paramagnetic resonance spectroscopy (EPR), superconducting quantum interference device (SQUID) measurements, and Mössbauer spectroscopy. The latter method is introduced further in subchapter 1.6. Additionally, 
resonance Raman, IR, and UV/vis spectroscopy are useful techniques for the characterization of model compounds, as will be shown within this work.

\subsubsection{Oxidation and Oxygenation Reactivity of Oxoiron(IV) Species}

As anticipated, high-valent oxoiron species have been described as reactive intermediates in $\mathrm{C}-\mathrm{H}$ bond activation. More concretely, they are able to perform aromatic and aliphatic hydroxylation processes, alkylaromatic oxidation, as well as alcohol oxidation on different types of $\mathrm{C}-\mathrm{H}$ bonds. ${ }^{[58]}$ Excellent substrates for mechanistic studies of these reactions are $9 H$-xanthene, 9,10-dihydroanthracene (DHA), 1,4-cyclohexadiene (CHD) and 9H-fluorene since they have weak $\mathrm{C}-\mathrm{H}$ bonds which can be easily activated. The key reaction step in $\mathrm{C}-\mathrm{H}$ bond activation is the hydrogen atom abstraction (HAA) usually characterized by large kinetic isotop effects. Mechanistically, two different pathways can follow on the HAA step, depending on heme and nonheme systems. In the mechanistic scenario for heme $\mathrm{Fe}^{\mathrm{IV}}=\mathrm{O}$ species, HAA is followed by an oxygen rebound of the organic radical back to the hydroxoiron(III) intermediate. Finally, the radical-rebound process leads to formation of the alcoholic product and an iron(II) species in ratio 1:1 (Scheme 4).

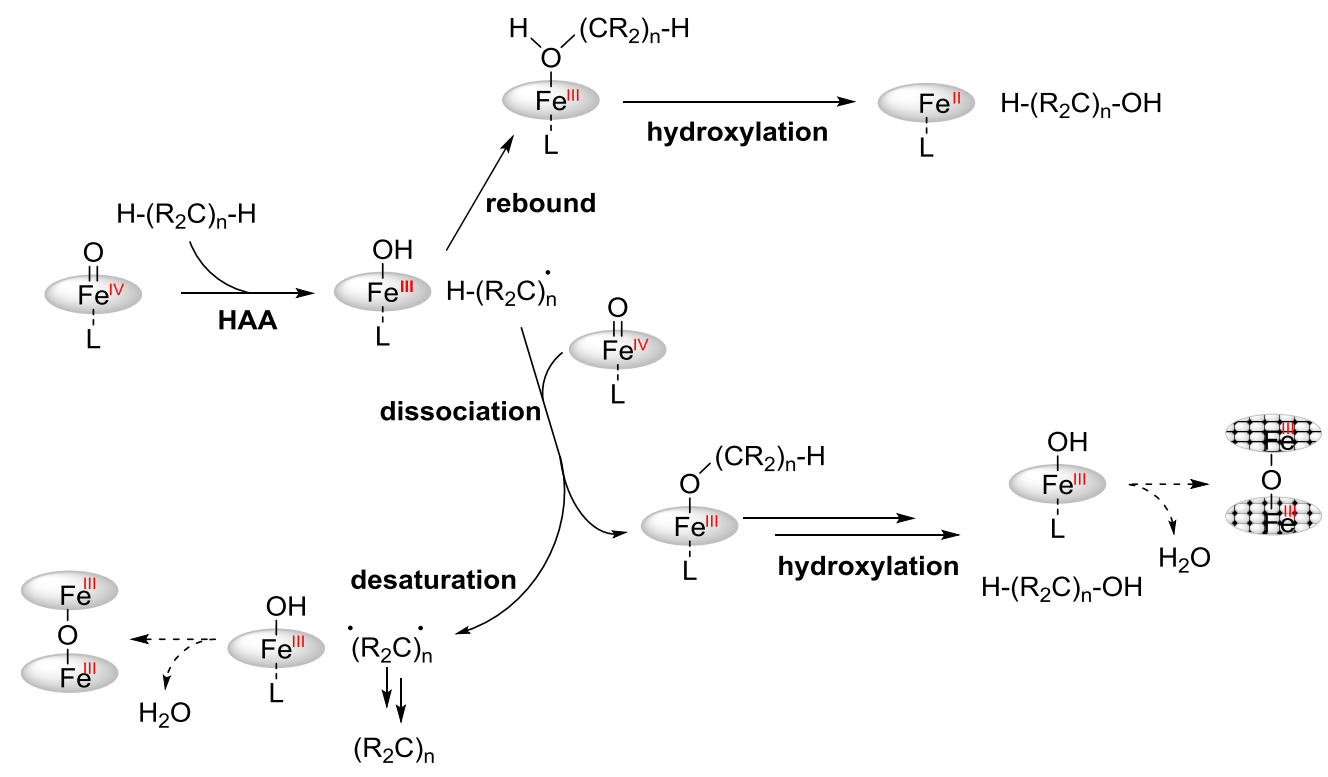

Scheme 4. Selected $\mathrm{C}-\mathrm{H}$ bond activation pathways by oxoiron(IV) species. ${ }^{[62,63]}$

In case of most non-heme models, a different pathway has been observed since dissociation of the organic radical after HAA is energetically more favorable than rebound. ${ }^{[62,63]}$ It has been observed that the initial substrate radical reacts further with a second $\mathrm{Fe}^{\mathrm{IV}}=\mathrm{O}$ species resulting in a substrate:catalyst product ratio of 1:2 and iron(III) species as product. ${ }^{[64]}$ The initially generated hydroxoiron(III) species is usually not the final product due to its high reactivity and low stability. 
The detection and characterization of $\mathrm{Fe}-\mathrm{OH}$ intermediates as well as their decomposition processes are still under investigation. A common scenario is the dimerization of two $\mathrm{Fe}-\mathrm{OH}$ species to a $\mu$-oxodiiron(III) compound as shown in Scheme 4.

The most striking observation within the field of $\mathrm{C}-\mathrm{H}$ bond activation is the hydroxylation of unactivated alkanes such as cyclohexane, first reported in 2004 by Nam, Que and co-workers. ${ }^{[65]}$ The driving force for $\mathrm{C}-\mathrm{H}$ bond activation processes is controlled by the redox potential and basicity of the iron center and can therefore be modulated by varying the ligand system. ${ }^{[66]}$

Furthermore, oxoiron(IV) species are intermediates in N-dealkylation processes, alkene epoxidation as well as oxygen atom transfer (OAT) reactions. OAT processes on sulfur and phosphorus compounds can be described as shown in Scheme 5. In a two electron process, atoms from small molecules such as $\mathrm{O}_{2}$ or $\mathrm{N}_{2}$ are transferred to larger substrates via high-valent metal intermediates. ${ }^{[67]}$

$$
\stackrel{n+}{M}=\mathrm{O}+\mathrm{E} \longrightarrow \mathrm{M}^{(\mathrm{n}-2)+}+\mathrm{O}=\mathrm{E}
$$

Scheme 5. Oxygen atom transfer processes by oxometal species. ${ }^{[67]}$

The rate of OAT can often be rationalized in terms of thermodynamic driving forces. ${ }^{[68]}$ A linear relationship between the $\mathrm{Fe}^{\mathrm{IV}} / \mathrm{Fe}^{\mathrm{III}}$ redox potential and the rates of OAT has been reported in studies on thioanisole. ${ }^{[69]}$ Mechanistically, two distinct pathways are conceivable. The process can either take place via a direct oxygen transfer (DOT) or via a stepwise electron transfer / oxygen atom transfer process $(\mathrm{ET} / \mathrm{OAT}) .{ }^{[58]}$

Electron- ${ }^{[70]}$ and hydride-transfer reactions, oxygen exchange with water and intermolecular oxygen atom transfer by non-heme oxoiron(IV) models are also of current interest. ${ }^{[58]}$ Oxygen exchange with $\mathrm{H}_{2}{ }^{18} \mathrm{O}$ is used for the generation of $\mathrm{Fe}={ }^{18} \mathrm{O}$ species for Raman studies, for example. The determined rates are slow and excess of water is needed. ${ }^{[71]}$ Recent studies by the groups of Costas and Que have shown that oxygen atom exchange occurs - depending on the supporting ligands -via reversible association of water or via a subsequent rate determining proton-transfer step. ${ }^{[72]}$ Intermolecular OAT from one $\mathrm{LFe}^{\mathrm{IV}}=\mathrm{O}$ species to $\mathrm{L}^{\prime} \mathrm{Fe}^{\mathrm{II}}$ via $\mu$-oxoiron(III) dimers is used for comparison of their oxidizing power. ${ }^{[73,74]}$

Many factors are known to have an influence on the reactivity of oxoiron(IV) species. For example, the role of the spin state in non-heme iron systems is currently under debate. DFT calculations have predicted a higher reactivity of species in the $S=2$ quintet state compared to $S=1$ triplet compounds. ${ }^{[75]}$ The characteristics of the $\mathrm{Fe}=\mathrm{O}$ bond should be similar in both spin states since the z-orbitals are not affected by the change in spin state (compare Figure 2), but the activation barrier for oxidation reactions is supposed to be smaller for $S=2$ species. Up to now, no experimental 
proof has been found for this assumption. The best known high spin $\mathrm{Fe}^{\mathrm{IV}}=\mathrm{O}$ system $\left(\left[\left(\mathrm{TMG}_{3} \text { tren }\right) \mathrm{Fe}=\mathrm{O}\right]^{2+}\right.$, Figure $\left.1 \mathrm{C}\right)$ shows reactivity similar to $S=1$ models due to its steric hindrance. ${ }^{[55,76]}$ The so far most reactive $S=2$ complex $\left[(\mathrm{TQA}) \mathrm{Fe}^{\mathrm{IV}}(\mathrm{O})(\mathrm{NCMe})\right]^{2+}(\mathrm{TQA}=$ tris(quinolin-2-ylmethyl)amine, Figure $3 \mathbf{E}$ ) is only slightly more reactive than the most effective low spin counterpart $\left[\left(\mathrm{Me}_{3} \mathrm{NTB}\right) \mathrm{Fe}^{\mathrm{IV}}(\mathrm{O})\right]^{2+}\left(\mathrm{Me}_{3} \mathrm{NTB}=\operatorname{tris}((1-m e t h y l-1 \mathrm{H}-\right.$ benzo[d]imidazole-2yl)methyl)amine, Figure $3 \mathbf{F}) \cdot{ }^{[77-79]}$ A so called 'two state reactivity' model is used to explain the high reactivity of the $S=1$ species. ${ }^{[80]}$ As observed for many other non-heme oxoiron(IV) models as well, the triplet ground state of compound $\mathbf{F}$ is close in energy with the quintet excited state. Initiated by the substrate and while progressing toward the transition state, the spin state changes and the reaction occurs via the quintet energy surface with significantly lower activation barriers. In the case of Nam's $\mathrm{Me}_{3} \mathrm{NTB}$ system, the conversion from $S=1$ to $S=2$ is exothermic and the activation barrier for HAA of CHD was calculated to be only $0.3 \mathrm{kcal} \mathrm{mol}^{-1}$. As said, the influence of the spin state in oxoiron(IV) species is not fully understood yet and further investigations are needed. In fact, the model systems with $S=2$ spin state cannot be used to proof the proposed higher reactivity of quintet states. At the same time, the low spin species can easily access two spin states ( $S=1$ and $S=2)$ and are unfit to act as 'triplet-only' models.

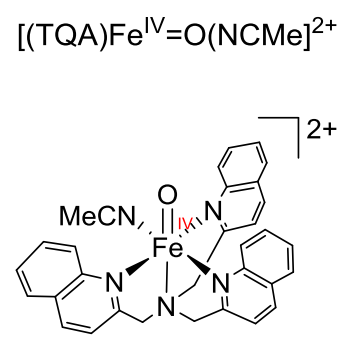

E

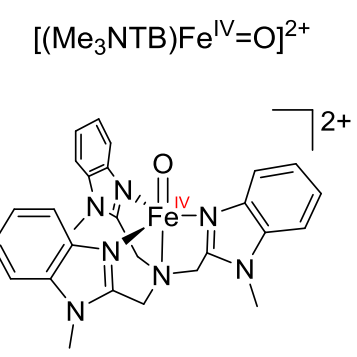

$\mathbf{F}$

Figure 3. Schematic drawing of the so far most reactive $S=2$ oxoiron(IV) complex $\mathbf{E}$ and the most reactive $S=1$ example $\mathbf{F} .^{[77-79]}$

As expected, not only the spin state of $\mathrm{Fe}=\mathrm{O}$ systems but also the oxidation state itself determines the reactivity of oxometal compounds. An outstanding experimental study in this context has been performed by Collins et al. ${ }^{[81]}$ Comparing the OAT toward thioanisole, they found the TAML coordinated oxoiron(V) species to be more reactive than the corresponding oxoiron(IV) by four orders of magnitude (compare Figure 1 D). A DFT study on this topic was performed by Neese and co-workers. ${ }^{[82]}$ According to their results, the activation barrier for $\mathrm{C}-\mathrm{H}$ bond oxidation decreases with higher oxidation state of the iron center in agreement with an increase of electrophilicity.

Another factor with a huge influence on the reactivity of oxoiron(IV) species is the ligand system. Starting with $\left[\left(\mathrm{TMP}^{+\cdot}\right) \mathrm{Fe}^{\mathrm{IV}}=\mathrm{O}\left(\mathrm{CH}_{3} \mathrm{OH}\right)\right]^{+}$, numerous different heme model compounds bearing electron-rich or -deficient porphyrines have been synthesized and their reactivity has been compared. ${ }^{[13,49,50]}$ These experiments have revealed that the oxidant power decreases with 
increasing electron-richness of the porphyrine. Additionally, by studying the influence of the axial ligand, it has been shown that an increase of its electron donating properties corresponds to higher rates in OAT and HAA (Figure 4).
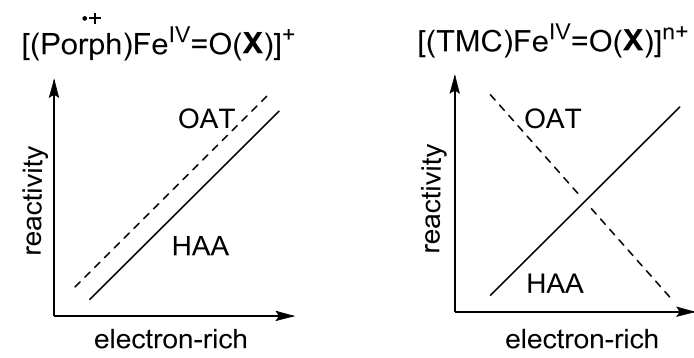

Figure 4. Schematic drawings comparing the axial ligand effects in oxygen atom transfer (OAT) and hydrogen atom abstraction (HAA) by heme (left) and non-heme (right) high-valent oxoiron(IV) models.

The axial ligand effect in non-heme models has been studied initially on $\mathrm{Fe}^{\mathrm{IV}}=\mathrm{O}$ species with the TMC ligand system and turned out to be more complicated. ${ }^{[83]}$ As shown in Figure 4, the rate of HAA behaves similar to the heme-models while the rate of OAT decreases with more electron donating axial ligands. According to the theoretical background, a more electron-rich iron center corresponds to a lower real oxidation state and thus to a less reactive metal center. Therefore, only OAT processes in non-heme models show the expected behavior. The counter intuitive behavior observed for heme models and HAA of non-heme systems has been explained using the 'two state reactivity' model. Theoretical studies have shown that a more electron donating axial ligand reduces the energy gap between triplet and quintet states and thus the overall activation barrier decreases. Furthermore, Shaik and co-workers observed that only calculations that include tunneling agree with the observed antielectrophilic trend, giving support for the "tunneling control" hypothesis by Schreiner et al. ${ }^{[84-86]}$

Other studies dealing with ligand effects have concentrated on the topology of linear tetradentate N4 ligands ${ }^{[87]}$ or the ring size of a TMC. ${ }^{[88]}$ Hitomi et al. have shown that electron-donating groups in the backbone of the ligand sets do not only influence the reactivity but also the selectivity of oxygenation reactions. ${ }^{\left[{ }^{89]}\right.}$ Moreover, a recent theoretical analysis by Li and co-workers points out that equatorial ligands tune the reactivity of high-valent oxoiron species more than axial ones. ${ }^{[0]}$ Consequently, even though numerous high-valent oxoiron models have been reported so far, synthetic chemists are still asked to invent novel ligand systems on the way to mimicking nature's efficient and selective catalysts. 


\subsection{Bioinspired Nitridoiron Systems}

While direct evidence for the existence of high-valent nitridoiron compounds in biological systems is still lacking, the work on synthetic compounds has been very successful during the last two decades. ${ }^{[7]}$ Compared to an oxo ligand, the nitrido one has a smaller effective nuclear charge and p-orbitals that energetically match better with the $d_{x z, y z}$ orbitals of iron. This allows for a better $\pi$-donating ability and stabilization of higher oxidation states of the iron center. Consequently, the synthesis of nitridoiron species in the oxidation states $+\mathrm{V}$ and $+\mathrm{VI}$ has been successful as will be described in the following section. Interestingly, higher oxidation states seem to be preferred also in dinuclear systems, as shown in 1.3.2. Several mixed-valent $\mathrm{Fe}^{\mathrm{III}}-\mathrm{N}-\mathrm{Fe}^{\mathrm{IV}}$ complexes as well as $\mathrm{Fe}^{\mathrm{IV}}-\mathrm{N}-\mathrm{Fe}^{\mathrm{IV}}$ species have been reported while no $\mu$-nitridodiiron(III) species is known so far. This is in huge contrast to the quite common $\mu$-oxodiiron(III) compounds in oxygen chemistry.

\subsubsection{Mononuclear Iron-Nitride Species}

In 1989, Wagner and Nakamoto reported the synthesis of the first nitridoiron(V) species. ${ }^{[1]}$ They irradiated $\left[(\mathrm{TPP}) \mathrm{Fe}^{\mathrm{III}}\left(\mathrm{N}_{3}\right)\right]$ with a laser at $30 \mathrm{~K}$ and detected the matrix-stabilized $\mathrm{Fe}^{\mathrm{V}} \equiv \mathrm{N}$ compound via resonance Raman spectroscopy (TPP = tetraphenylporphyrin). However, higher temperatures led to decomposition and formation of the corresponding $\mu$-nitrido-diiron species. Laser irradiation of azido compounds as shown in Scheme 6 is still one of the general procedures to generate nitridoiron species.

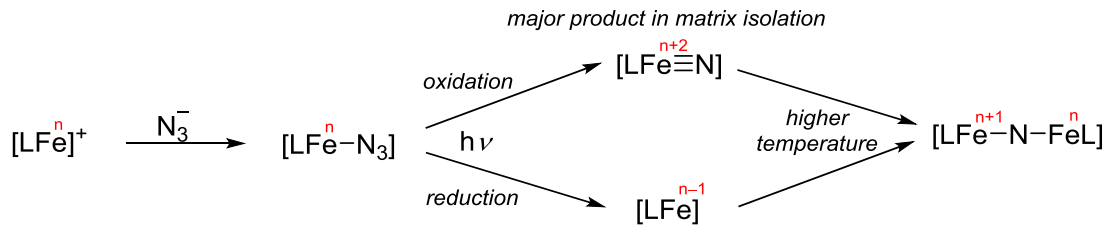

Scheme 6. Common synthetic route to obtain high-valent iron-nitrido species. ${ }^{[7]}$

The same strategy was used by Wieghardt et al. ten years later irradiating trans- and cis-diazido iron(III)cyclam. ${ }^{[92]}$ Depending on the starting material, photoreduction upon homolytic cleavage of the $\mathrm{Fe}-\mathrm{N}_{3}$-bond or photooxidation upon heterolytic $\mathrm{N}-\mathrm{N}$-bond cleavage followed by $\mathrm{N}_{2}$-release was preferred. Within their work, the group also presented Mössbauer data for the $\left[\mathrm{N}_{3}-(\text { cyclam }) \mathrm{Fe}^{\mathrm{V}} \equiv \mathrm{N}\right]^{+}$species. The isomer shift $\left(\delta=-0.04 \mathrm{~mm} \mathrm{~s}^{-1}\right)$ is similar to comparable oxoiron(IV) species and therefore higher than expected for an iron(V) center. This high IS has been explained by enhanced covalency of the $\mathrm{Fe} \equiv \mathrm{N}$-bond compared to a $\mathrm{Fe}=\mathrm{O}-$ bond. In the following years, the group has further characterized the $\mathrm{Fe}^{\mathrm{V}} \equiv \mathrm{N}$ species as $S=1 / 2$ and presented a similar cyclam-acetato coordinated system. ${ }^{[8,57,93]}$ Additionally, they examined in which way the laser- 
initiated bond cleavage can be modulated. With $\left[\mathrm{N}_{3}-(\text { cyclam }) \mathrm{Fe}^{\mathrm{III}}-\mathrm{N}_{3}\right]^{+}$, it was shown that irradiation at $266 \mathrm{~nm}$ leads to formation of the unstable $\left[\mathrm{N}_{3}-(\text { cyclam }) \mathrm{Fe}^{\mathrm{V}} \equiv \mathrm{N}\right]^{+}$species, while irradiation with a 355 or $532 \mathrm{~nm}$ laser leads to $\mathrm{Fe}-\mathrm{N}_{3}$ bond cleavage. ${ }^{[94]}$ However, with the cyclamacetato coordinated mono azido system an opposing trend was observed. Irradiation with a $266 \mathrm{~nm}$ laser leads to photoreduction while irradiation at $450 \mathrm{~nm}$ initiates the desired photooxidation. ${ }^{[5]}$ For the latter system it has been concluded that irradiation into the ligand-to-metal charge transfer (LMCT) band around $450 \mathrm{~nm}$ induces $\mathrm{N}-\mathrm{N}$ bond cleavage and formation of the $\mathrm{Fe}^{\mathrm{V}} \equiv \mathrm{N}$ species. Typically, azido complexes show a LMCT band in this area that gives them a characteristic reddish color. However, irradiation into this transition seems not to be a generally valid method and further studies are necessary to understand the photochemistry of azide compounds.

The use of a more sterically hindered $\mathrm{Me}_{3} \mathrm{cy}$-ac ligand allowed Wieghardt and co-workers to isolate the first nitridoiron(VI) compound (Figure 5 A) in 2006. ${ }^{[2]}$ They initially oxidized the iron(III)azido complex and irradiated, in a second step, the obtained $\mathrm{Fe}^{\mathrm{IV}}-\mathrm{N}_{3}$ species in frozen matrix with a laser, receiving the $\mathrm{Fe}^{\mathrm{VI}} \equiv \mathrm{N}$ compound with a low IS of $\delta=-0.29 \mathrm{~mm} \mathrm{~s}^{-1}$. The oxidation state $+\mathrm{VI}$ is still considered to be the highest accessible for iron and, before the cited work, has been known only for $\mathrm{FeO}_{4}{ }^{2-}$.

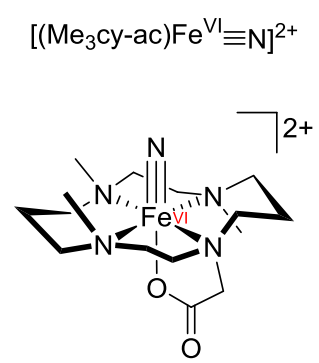

A
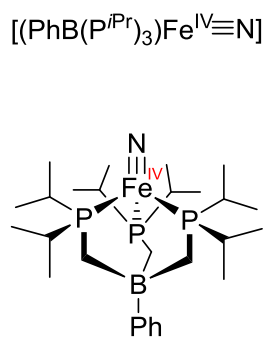

B

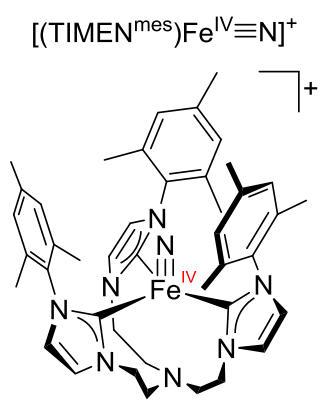

C
$\left[\left(\mathrm{PhB}(\mathrm{tBu}-\mathrm{Im})_{3}\right) \mathrm{Fe}^{\mathrm{V}} \equiv \mathrm{N}\right]^{+}$

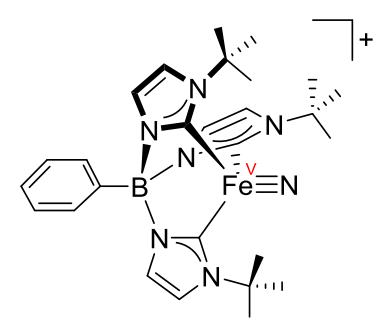

D

Figure 5. Selected characterized high-valent nitridoiron species. ${ }^{[2,41,95,96]}$

In 2004, Peters et al. succeeded in the formation of the first room temperature stable nitridoiron(IV) model compound. ${ }^{[41]}$ In contrast to the ligands with fourfold symmetry employed till then, the use of a tridentate ligand with threefold symmetry (phenyl-tris(diisopropylphosphino)borate, $\mathrm{PhBP}_{3}^{i \mathrm{Pr}}{ }_{3}^{-}$) increased the stability of the coordinated $\mathrm{Fe}^{\mathrm{IV}} \equiv \mathrm{N}$ moiety drastically (Figure 5 B). Additionally, a different strategy for generation of the high-valent species was employed. Instead for azide irradiation, lithium 2,3,5,6-dibenzo-7-azabicyclo[2.2.1]hepta-2,5diene (dbabh) was used as $\mathrm{N}$-atom transfer reagent. Interestingly, treatment of the tetrahedral $\mathrm{Fe}^{\mathrm{IV}} \equiv \mathrm{N}$ species with a weak acid under reducing conditions led to formation of ammonia in similarity to the Chatt mechanism proposed for nitrogenase. ${ }^{[43,44]}$ Upon crystallization, Peters and co-workers observed dimerization of the complex leading to an $\mathrm{Fe}^{\mathrm{I}}-\mathrm{N}-\mathrm{Fe}^{\mathrm{I}}$ compound. This 
fascinating 6-electron reduction ( $2 \mathrm{Fe}^{\mathrm{IV}} \equiv \mathrm{N} \rightarrow \mathrm{Fe}^{\mathrm{I}}-\mathrm{N}-\mathrm{Fe}^{\mathrm{I}}$ ) emphasizes the special characteristics of the electron rich phosphinoborate ligand system which is able to stabilize both high and low oxidation states of iron.

The use of a bulkier but still electron rich tris-carbene system (TIMEN ${ }^{\text {mes }}$ ) enabled for the first $\mathrm{X}$-ray crystallographic characterization of a $\mathrm{Fe}^{\mathrm{IV}} \equiv \mathrm{N}$ species (Figure 5 C). ${ }^{\left[{ }^{[5]}\right]}$ This trigonalpyramidal compound is air and moisture stable at room temperature and does not show any dimerization because of the sterically hindered ligand system. In the same year, Smith and coworkers also isolated a nitridoiron(IV) species coordinated by a tris-carbene borate ligand scaffold $\left(\mathrm{PhB}(t \mathrm{Bu}-\mathrm{Im})_{3}\right)$ starting from the corresponding azido precursor. ${ }^{[97]}$ This compound could be further oxidized yielding the first fully characterized $\mathrm{Fe}^{\mathrm{V}} \equiv \mathrm{N}$ system (Figure 5 D). ${ }^{\left[{ }^{[6]}\right.}$ In an aqueous and reductive medium it releases almost quantitatively ammonia concomitant with formation of the iron(II) species.

To explain the fascinating higher stability of nitridoiron species with a threefold symmetry with respect to the fourfold ones, the ligand field splitting must be considered (Figure 6). ${ }^{[7,46]}$ Compared to oxoiron species, the $\mathrm{d}_{\mathrm{xz}, \mathrm{yz}}$ orbitals are much higher in energy, due to a stronger $\pi$-interaction with nitrido ligands than with the oxo ones as already mentioned before. This leads to a significantly larger gap between non-bonding and anti-bonding orbitals in octahedral and square planar systems and therefore destabilization of iron(IV) or -(V) species. Instead, iron(VI) compounds are, based on this model, stable. Experimental proof can be found in the successful isolation of the first non-heme iron(VI) species by Wieghardt et al. as described above. ${ }^{[2]}$ In case of threefold systems, a different situation is found. The $\mathrm{d}_{\mathrm{x}^{2}-\mathrm{y}^{2}}$ orbital is on a similar energy level as the $\mathrm{d}_{\mathrm{xy}}$ orbital and up to four electrons can be accommodated in these relatively low-lying orbitals. Consequently, threefold nitridoiron species with the oxidation states IV and $\mathrm{V}$ are stable as shown by the recent success in their isolation. Due to the large non-bonding/anti-bonding gap in both coordination geometries, all species are anticipated to be low spin.

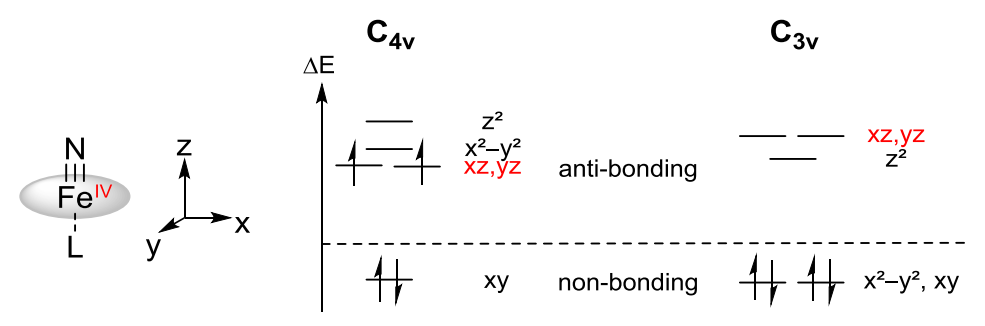

Figure 6. Tetragonal and trigonal ligand field splitting of high-valent nitridoiron(IV) complexes; the figure was adapted from Hohenberger et al. ${ }^{[7]}$

The stability of tetrahedral nitridoiron systems has allowed to study their reactivity. ${ }^{[98]}$ In contrast to its oxidized $\mathrm{Fe}^{\mathrm{V}}$ homologue, $\left[\left(\mathrm{PhB}(t \mathrm{Bu}-\mathrm{Im})_{3}\right) \mathrm{Fe}^{\mathrm{IV}} \equiv \mathrm{N}\right]$ does not react with protons but treatment 
with TEMPO-H leads to formation of ammonia, as found for $\left[\left(\mathrm{PhBP}^{i \mathrm{Pr}}{ }_{3}\right) \mathrm{Fe}^{\mathrm{IV}} \equiv \mathrm{N}\right] .^{[99]}$ More interesting, Smith et al. observed complete nitrogen atom transfer by treatment of the $\mathrm{Fe}^{\mathrm{IV}} \equiv \mathrm{N}$ compound with tert-butylisonitrile. ${ }^{[100]}$ This three electron process is of high interest as analog to the well understood oxygen atom transfer by high-valent oxoiron species. However, in this field further studies are aimed at obtaining a better understanding of the reactivity of nitridoiron compounds.

\subsection{2 $\mu$-Nitridodiiron Compounds}

Dinuclear iron complexes with a bridging nitrido unit have already been known for decades and their iron centers are typically characterized by relatively high oxidation states. ${ }^{[46]}$ An early example is represented by the heme system [(TPP) $\mathrm{Fe}^{3.5}-\mathrm{N}-\mathrm{Fe}^{3.5}$ (TPP)] reported in $1976 .^{[101]}$ This compound shows a linear and symmetric $\mathrm{Fe}-\mathrm{N}-\mathrm{Fe}$ moiety, which is indicative of a fully delocalized unpaired electron. Valence-tautomerism is assumed to be prevalent in mixed-valent $\mu$-nitrido systems due to a strong electronic coupling of the metal centers. A theoretical study on the structure of this complex has been conducted by Que and co-workers, who concluded that the linear $\mathrm{Fe}-\mathrm{N}-\mathrm{Fe}$ fashion enables a highly covalent $\pi$-bonding interaction leading to the observed delocalized electronic structure. ${ }^{[102]}$ More than two decades later also the corresponding one electron oxidized TPP coordinated $\mu$-nitridodiiron(IV) species could be fully characterized and compared to the mixed-valent analog. ${ }^{[103]}$

As described within the previous section, Wieghardt and co-workers studied cis and transcoordinated cyclam diazidoiron species and isolated a high-valent $\mathrm{Fe}^{\mathrm{V}} \equiv \mathrm{N}$ compound in frozen matrix, while irradiation in cold solution $\left(-35^{\circ} \mathrm{C}\right)$ led to formation of $\mathrm{Fe}^{\mathrm{III}}-\mathrm{N}-\mathrm{Fe}^{\mathrm{IV}}$ systems. ${ }^{[2]}$ Moreover, they again observed a strong antiferromagnetic coupling between the iron centers, but found localized valencies, as indicated by two distinct Mössbauer doublets. The same observation has been made for a mixed-valent $\mathrm{Fe}^{\mathrm{III}}-\mathrm{N}-\mathrm{Fe}^{\mathrm{IV}}$ compound coordinated by 1,4,7-triazacyclononane (TACN) and tetrachlorocatecholate ligands, while the corresponding diiron(IV) system showed diamagnetic behaviour. ${ }^{[104]}$

The comparison of $\mu$-nitridodiiron models with their $\mu$-oxo analogues is of current interest since it provides insight into their structural, electronic, and catalytic properties. A comparative study on isoelectronic dinuclear octapropylporphyrazine systems with bridging oxo, nitrido and carbido ligands was presented by Sorokin and co-workers, mainly focusing on structural influences. ${ }^{[105]}$

Besides these high-valent compounds, also a low-valent $\mu$-nitridodiiron(II) species coordinated by the $\mathrm{PhBP}_{3}$ ligand as well as the corresponding $\mathrm{Fe}^{\mathrm{III}}-\mathrm{N}-\mathrm{Fe}^{\mathrm{II}}$ system have been reported. ${ }^{[106]}$ Both 
compounds show pseudotetrahedrally coordinated iron centers and bent $\mathrm{Fe}-\mathrm{N}-\mathrm{Fe}$ moieties $\left(\sim 140^{\circ}\right)$, and are able to activate dihydrogen leading to diiron $\mu-\mathrm{NH}-\mu-\mathrm{H}$ compounds. Upon treatment with hydrochloric acid, the diiron(II) diamond core releases ammonia in close analogy to the findings made with mononuclear systems.

Focusing again on high-valent $\mu$-nitridodiiron models, they also provide fascinating applications. ${ }^{[105]}$ For example, an outstanding work was performed by Sorokin and co-workers on the activation of methane. In 2008, they found that treatment of a phthalocyanine coordinated $\mathrm{Fe}^{\mathrm{III}}-\mathrm{N}-\mathrm{Fe}^{\mathrm{IV}}$ complex forms a putative $\mathrm{O}=\mathrm{Fe}^{\mathrm{V}}-\mathrm{N}-\mathrm{Fe}^{\mathrm{IV}}$ intermediate upon reaction with $\mathrm{H}_{2} \mathrm{O}_{2} \cdot{ }^{[107]}$ This species is then active in the catalytic oxidation of methane to methanol, formaldehyde, and formic acid. In a similar study published in 2012, they obtained an N-bridged high-valent oxodiiron(IV) porphyrin radical species upon reaction of the mixed-valent $\mathrm{Fe}^{\mathrm{III}}-\mathrm{N}-\mathrm{Fe}^{\mathrm{IV}}$ TPP system with $m \mathrm{CPBA}$ at $-90{ }^{\circ} \mathrm{C} .{ }^{[108]}$ This species could be characterized via UV/vis, EPR, MB and mass spectrometry and showed OAT toward methane yielding methanol. Furthermore, $\mu$-nitridodiiron systems have been shown to be active catalysts in the peroxide mediated oxidation of benzene ${ }^{[109]}$ and alkylaromatic compounds ${ }^{[110]}$ as well as in the formation of $\mathrm{C}-\mathrm{C}$ bonds. ${ }^{[111]}$ The activation of the strong $\mathrm{C}-\mathrm{F}$ bond is of importance for organic synthesis and $\mu$-nitridodiiron compounds turned out to be active toward deflourination. ${ }^{[112]}$ Consequently, this class of compounds offers a huge variety of applications providing motivation for synthetic chemists to develop new model systems and investigate their reactivity. 


\subsection{Synthetic Iron-Complexes with Nitrogen Oxides}

\subsubsection{The Enemark-Feltham Notation}

Before describing the characteristics of iron complexes coordinated by NO, which is the topic of this section, it is necessary to introduce the Enemark-Feltham notation. NO is a redox non-innocent ligand that forms highly covalent bonds with metal ions. This aggravates the assignment of oxidation states for the metal center and the NO ligand. In fact, many electronic structures are typically accessible for M-NO species and the exact positions of the metal-d and NO- $\pi^{*}$ electrons remain ambiguous. To overcome these difficulties, the FeNO moiety in its entirety is classified according to the Enemark-Feltham notation. ${ }^{[13]}$ As summarized below, the number of $\pi^{*}$ electrons of the NO ligand is added to the number of d-electrons of the metal center and the total number of electrons is then denoted as $n$ in $\{\mathrm{FeNO}\}^{n}$. In case of mononuclear mononitrosyl species, $\{\mathrm{FeNO}\}^{6-8}$ compounds are known, which will be introduced in the following sections.

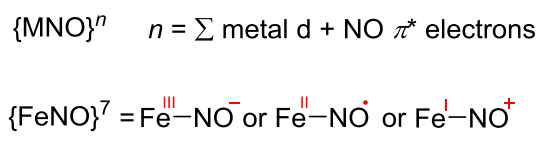

\subsubsection{Model Compounds and Their Main Characteristics}

As briefly introduced in 1.1.2, iron nitrosyl compounds and their redox interconversions play important roles in nature. In many cases, exact mechanisms and fundamentals of the biological NO-chemistry are still not fully understood. Consequently, synthetic chemists are asked to characterize model compounds to provide a better understanding of the versatile chemistry of iron nitrosyls.

\section{Heme Model Compounds}

Many five coordinated (5C) porphyrin ferrous nitrosyl compounds $\{\mathrm{FeNO}\}^{7}$ have been synthesized with the aim of mimicking the biologically important heme nitrosyls. ${ }^{[28,114,115]}$ Due to the high affinity of an iron(II) center for the nitric oxide radical, they easily form $\{\mathrm{FeNO}\}^{7}$ compounds of high stability. Heme $\{\mathrm{FeNO}\}^{7}$ complexes are characterized by short Fe-NO bonds $(1.73 \AA)$, bent $\mathrm{Fe}-\mathrm{N}-\mathrm{O}$ moieties $\left(140-145^{\circ}\right)$, and stretching frequencies at around $\tilde{v}=1670-1700 \mathrm{~cm}^{-1}$ and $\tilde{v}=520-540 \mathrm{~cm}^{-1}$ for $\mathrm{N}-\mathrm{O}$ and $\mathrm{Fe}-\mathrm{NO}$, respectively. Their spin state has been characterized as low spin $S=1 / 2$. The SOMO in these species originates from strong $\sigma$-donation of the singly occupied $\mathrm{NO}\left(\pi^{*}\right)$ orbital into the $\mathrm{Fe}\left(\mathrm{d}_{\mathrm{z}^{2}}\right)$ orbital and is typically largely NO-based. ${ }^{[1]}$ This interaction is 
optimized in bent $\mathrm{Fe}-\mathrm{N}-\mathrm{O}$ moieties; however, the energetic benefit of the bending is quite small and easily overcome by steric effects. ${ }^{[116,117]}$ Due to the covalency of the Fe-NO bond, the unpaired electron is largely delocalized resulting in a noticeable $\mathrm{Fe}^{\mathrm{I}}-\mathrm{NO}^{+}$character of heme $\{\mathrm{FeNO}\}^{7}$ systems. Additionally, the NO ligand works as medium-strong $\pi$-acceptor.

Six-coordinate model compounds with additional trans $\mathrm{N}$-donor ligands show weaker $\mathrm{Fe}-\mathrm{NO}$ and $\mathrm{N}-\mathrm{O}$ bonds according to IR-stretches and bond lengths. ${ }^{[1]}$ This direct correlation between the two bonds is consistent with a weaker $\sigma$-donation from the NO fragment to the iron center, resulting in a higher $\mathrm{Fe}^{\mathrm{II}}-\mathrm{NO}$ character. In general, the axial ligands in model compounds show only small binding constants in line with the biological behavior observed in sGC (compare section 1.1.2). ${ }^{[11,26]}$

The one-electron oxidation of $5 \mathrm{C}$ or $6 \mathrm{C}$ heme $\{\mathrm{FeNO}\}^{7}$ species is in many cases reversible and leads to the corresponding $\{\mathrm{FeNO}\}^{6}$ compounds. ${ }^{[115]}$ The redox process is largely ligand centered and leads to a $\mathrm{Fe}^{\mathrm{II}}-\mathrm{NO}^{+}$character with only a weak $\sigma$-interaction and a strong $\pi$-backbonding of the iron $\mathrm{d}_{\mathrm{xz}}$ and $\mathrm{d}_{\mathrm{yz}}$ orbitals into the NO- $\pi^{*}$ orbital. ${ }^{[11]}$ This interaction is maximized in linear $\mathrm{Fe}-\mathrm{N}-\mathrm{O}$ moieties as found in structures determined by X-ray crystallography. The strong $\pi$-backbonding is also confirmed by NO stretching frequencies of about $\tilde{v} \sim 1900 \mathrm{~cm}^{-1}$, much lower than the one known for free $\mathrm{NO}^{+}\left(\tilde{v}=2390 \mathrm{~cm}^{-1}\right)$.

In contrast to the well characterized $\{\mathrm{FeNO}\}^{6}$ models, isolation of the reduced $\{\mathrm{FeNO}\}^{8}$ species has been more difficult due to their high reactivity and instability. ${ }^{[22,23]}$ Pioneering spectroelectrochemical studies by Kadish et al. showed a reversible 1-electron reduction of porphyrin-based $\{\mathrm{FeNO}\}^{7}$ complexes, ${ }^{[18]}$ and Ryan et al. performed first studies to investigate the spectroscopic signatures of the $\{\mathrm{FeNO}\}^{8}$ species and their reactivity toward acids and bases. ${ }^{[119,120]}$ The first $\{\mathrm{FeNO}\}^{8}$ porphyrin complexes could be isolated in 2010 by Doctorovich et al. even though they were not able to obtain crystals suitable for X-ray crystallography. ${ }^{[121]}$ However, with the help of DFT studies, these investigations already allowed a general understanding of the $\left\{\mathrm{FeNO}^{7}{ }^{7}\right.$ reduction process. ${ }^{[122]}$ The theoretical picture of the electronic differences upon reduction of low spin species is shown in Scheme 7, left. Upon reduction, a second electron is added into the NO-based SOMO of the $\{\mathrm{FeNO}\}^{7}$ species resulting in a diamagnetic low spin $\{\mathrm{FeNO}\}^{8}$ compound. The reduction process can be described as ligand based in the same way as the oxidation described above, and gives the compounds a $\mathrm{Fe}^{\mathrm{II}}-\mathrm{NO}^{-}$character. 


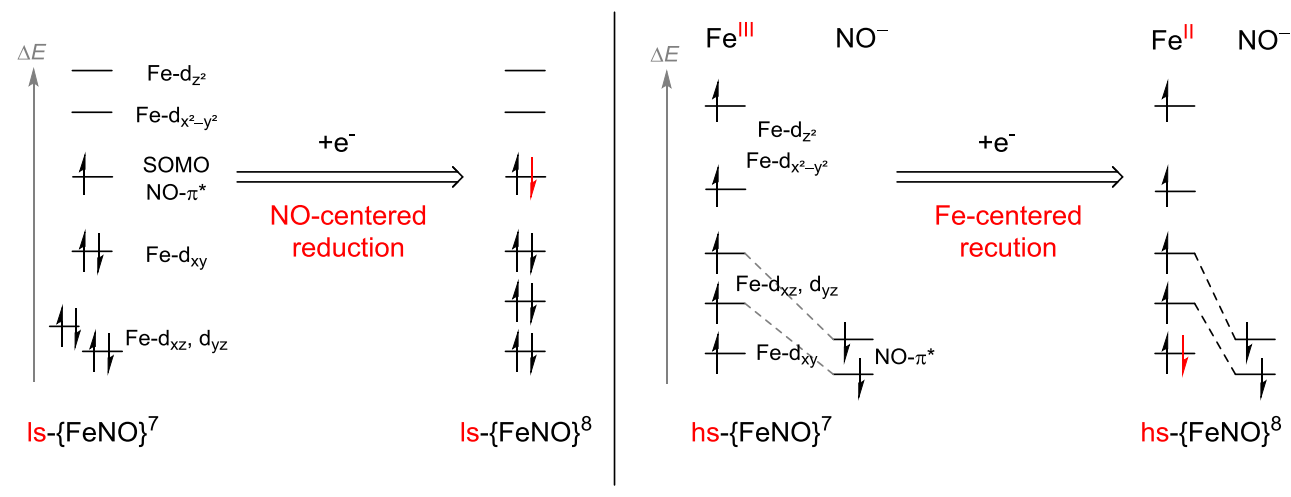

Scheme 7. Illustration of the electronic changes upon reduction for low spin (left) and high spin (right) $\{\mathrm{FeNO}\}$ systems. ${ }^{[31,122,123]}$

Since the nitroxyl ligand shows a stronger trans-effect than the nitrosyl, $6 \mathrm{C}$ species in $\{\mathrm{FeNO}\}^{8}$ compounds become even less stable. Moreover, the nitroxyl group is quite basic and is easily protonated, even if its instability usually leads to disproportionation accompanied by $\mathrm{H}_{2}$ release. ${ }^{[119,121]}$ Disproportionation can be however prevented by using bulky ligands, as shown by Lehnert and co-workers, who recently presented characterization and reactivity data for a ferrous bis-picket fence porphyrin coordinated $\{\mathrm{FeNO}\}^{8}$ species including its protonation to $\{\mathrm{FeNHO}\}^{8}$ and suppressed disproportionation. ${ }^{[124]}$ On the way to more stable heme $\{\mathrm{FeNO}\}^{8}$ systems, porphyrins with electron-withdrawing substituents are helpful, as shown by Doctorovich and coworkers using octabromo-[tetrakis-(pentafluorophenyl)]porphyrin $\left(\mathrm{TFPPBr}_{8}\right) \cdot{ }^{[121]}$ Finally, in 2015 $\mathrm{Hu}$ and $\mathrm{Li}$ presented the first X-ray crystallographic structure determination of a $\{\mathrm{FeNO}\}^{8}$ system coordinated by this TFPPBr 8 ligand. ${ }^{[125]}$ Comparison of the $\{\mathrm{FeNO}\}^{7}$ and $\{\mathrm{FeNO}\}^{8}$ species showed drastic changes upon reduction, in line with former theoretical and experimental studies. ${ }^{[121,124]}$ The $\mathrm{Fe}-\mathrm{N}-\mathrm{O}$ angle decreases from $149^{\circ}$ to $122^{\circ}$ while the $\tilde{v}(\mathrm{~N}-\mathrm{O})$ stretch also decreases from 1718 to $1540 \mathrm{~cm}^{-1}$. ${ }^{[125]}$

\section{Non-heme Model Compounds}

In addition to studies performed on heme systems, also many non-heme model compounds have been presented. ${ }^{[28,116]}$ The majority of these $\{\mathrm{FeNO}\}^{7}$ systems have been reported with $S=3 / 2$ ground states while few examples showing $S=1 / 2$ ground states have a coordination environment and an electronic structure largely mimicking the ones of heme systems. ${ }^{[126]}$ Interestingly, a couple of early examples showed temperature or pressure dependent spin-crossover ${ }^{[127-129]}$ In the case of a $[\mathrm{Fe}($ salen$) \mathrm{NO}]$ system $\quad($ salen $=N, N$ '-ethylenebis(salicylimine $)$ ), both spin states could be characterized and showed marked differences such as increased bending of the $\mathrm{Fe}-\mathrm{N}-\mathrm{O}$ moiety in the low spin state. ${ }^{[128,130]}$ 
According to studies performed by Solomon and co-workers, the ground state of high spin $\{\mathrm{FeNO}\}^{7}$ species with a total spin of $S_{\mathrm{T}}=3 / 2$ was characterized as an $\mathrm{Fe}^{\mathrm{III}} S=5 / 2$ species antiferromagnetically coupled with an $S=1 \mathrm{NO}^{-} \cdot{ }^{[117]}$ High spin $\left(S_{\mathrm{T}}=3 / 2\right)\{\mathrm{FeNO}\}^{7}$ species show slightly longer Fe-NO bonds (1.73-1.78 $\AA$ ) compared to their low spin $\left(S_{\mathrm{T}}=1 / 2\right)$ analogues and a wide range of possible $\mathrm{Fe}-\mathrm{N}-\mathrm{O}$ angles $\left(146-178^{\circ}\right) .{ }^{[86]}$ However, the large majority of non-heme $\{\mathrm{FeNO}\}^{7}$ species has bent $\mathrm{Fe}-\mathrm{N}-\mathrm{O}$ moieties and only few exceptions with quasi-linear units stand out, either 5C with $S=1 / 2$ or pseudotetrahedral (4C) with $S=3 / 2$ ground states. ${ }^{[131-133]}$ In most of these examples the linearity of the Fe-N-O unit largely depends on steric effects, ${ }^{[116,134,135]}$ but also the electronic structure plays a significant role. Detailed DFT studies by Conradie and Ghosh have identified metal $\mathrm{d}_{z} / \mathrm{p}_{z}$ mixing, which is enhanced upon pyramidalization in square-pyramidal $\{\mathrm{FeNO}\}^{7}$ complexes, as one key factor in the linearity of the $\mathrm{Fe}-\mathrm{N}-\mathrm{O}$ unit because it minimizes repulsion between the $\mathrm{Fe}\left(\mathrm{d}_{\mathrm{z}^{2}}\right)$ orbital and the $\sigma$ lone pair of NO${ }^{[136,137]}$ The $\tilde{v}(\mathrm{~N}-\mathrm{O})$ stretching frequencies of non-heme $\{\mathrm{FeNO}\}^{7}$ models are typically in the range from 1700 to $1800 \mathrm{~cm}^{-1}$ and are therefore higher than the ones for low spin species. ${ }^{[16]}$

Typically, non-heme $\{\mathrm{FeNO}\}^{7}$ models show quasi-reversible to irreversible reductions in cyclic voltammetry experiments. Consequently, only few reports on non-heme $\{\mathrm{FeNO}\}^{8}$ compounds can be found in the literature. A pioneering work was presented in 2000 by Wieghardt and coworkers. ${ }^{[138]}$ They performed electrochemical reduction and oxidation of a cyclam coordinated $\{\mathrm{FeNO}\}^{7}$ complex with an axial chlorido ligand. The starting material, initially described as intermediate spin iron $(S=3 / 2)$ antiferromagnetically coupled with an $\mathrm{NO}^{-}(S=1)$, showed reversible reduction and oxidation processes leading to the corresponding $\{\mathrm{FeNO}\}^{8}$ and $\{\mathrm{FeNO}\}^{6}$ compounds. However, isolation of the reduced species failed because of the weakly coordinated axial chlorido ligand, but was successful after replacing it with a pentacoordinated cyclam-ac ligand instead. ${ }^{[123]}$ The higher stability of the latter system allowed for isolation and characterization of the first series of $\{\mathrm{FeNO}\}^{6-8}$ in solid material while crystal structures were determined for the $\{\mathrm{FeNO}\}^{6}$ and $\{\mathrm{FeNO}\}^{7}$ species only. The series showed stretching frequencies of $\tilde{v}(\mathrm{~N}-\mathrm{O})=1903,1607$, and $1271 \mathrm{~cm}^{-1}$. The huge differences between $\tilde{v}(\mathrm{~N}-\mathrm{O})$ in the three species has been explained by almost entirely ligand centered redox processes, as supported by DFT calculations (Scheme 7, left). In all FeNO moieties within this series, iron(II) low spin centers $(S=1 / 2)$ are found, coordinated by $\mathrm{NO}^{+}, \mathrm{NO}^{-}$, and $\mathrm{NO}^{-}$along the series. These new data on the cyclam-ac coordinated iron nitrosyl complexes are in contrast to the electronic characterization of the cyclam coordinated chlorido $\{\mathrm{FeNO}\}^{7}$ species, described as $\left\{\mathrm{Fe}_{S=3 / 2} \mathrm{NO}_{S=1}^{-}\right\}$four years earlier. ${ }^{[138]}$ This represents well the difficulties in making correct assignements of oxidation states in these $\{\mathrm{FeNO}\}$ complexes. Finally, a recent study by Harrop et al. used a heme-like $4 \mathrm{~N}$-donor ligand for stabilization of the $\{\mathrm{FeNO}\}^{7}$ and $\{\mathrm{FeNO}\}^{8}$ species, but the corresponding oxidized 
species is missing in this report. ${ }^{[139]}$ The reduction was again assigned to occur largely ligand centered, with a low spin iron(II) center in both complexes.

Successful reduction of high spin $\{\mathrm{FeNO}\}^{7}$ compounds has been reported even more seldom. The high spin reduced species are usually highly reactive and show, for example, fast disproportionation and formation of dinitrosyl iron species $\left\{\mathrm{Fe}(\mathrm{NO})_{2}\right\}^{10} \cdot{ }^{[140]}$ Only in 2013, Lehnert and co-workers could synthesize a $\{\mathrm{FeNO}\}^{8}$ species sterically protected by a $\mathrm{TMG}_{3}$ tren ligand scaffold (compare Figure 1 C). ${ }^{[31,122]}$ Its electronic state was described as a high spin $\mathrm{Fe}^{\mathrm{II}}$ center coupled to $\mathrm{NO}^{-}$resulting in $S_{\mathrm{T}}=1$ (Scheme 7, right). Therefore, in this case the reduction occurs metal centered and at a more positive potential. In a comparative study of heme versus non-heme iron-nitroxyl compounds, it was reported that metal centered reduction is a general feature of nonheme high spin species while low spin complexes show ligand centered reduction. ${ }^{[122]}$ Within this work, it will be shown that this rule is not true for non-heme tetracarbene coordinated $\{\mathrm{FeNO}\}^{n}$ species.

Similar to the low spin analogues, the $\{\mathrm{FeNO}\}^{8} \mathrm{TMG}_{3}$ tren system can be protonated with weak acids leading to a putative $\{\mathrm{FeNHO}\}^{8}$ species. ${ }^{[31]}$ However, this compound was not stable at room temperature and could not be isolated. This behavior raised the question if a metal centered reduction, as it was assigned for this system, does indeed promote protonation at the NO moiety or if it does favor protonation at the iron center instead. The system presented in this work directly draws on this question as will be discussed in chapter 8 . 


\subsection{Introduction into a Macrocyclic Tetracarbene Ligand System}

As presented in section 1.3.1, the breakthrough compounds in the chemistry of high-valent nitridoiron complexes have been synthesized with the use of scorpionato-type N-heterocyclic carbene (NHC) ligand scaffolds. The TIMEN ${ }^{\text {mes[95] }}$ and $\mathrm{PhBIm}_{3}{ }^{[96,97]}$ systems (Figure 5 C and D) show perfectly well the special characteristics and advantages of carbene ligands. Their strong $\sigma$-donor ability enables for the stabilization of metal centers in high oxidation states, but also low oxidation states, in particular iron(I) and iron(0), are known to be stable with carbene donors. ${ }^{[141,142]}$ Thus, the possible combination of NHC ligands with iron centers in a range of oxidation states provides the opportunity to study unique structures, bonding situations, and reactivities. ${ }^{[143]}$

The most prominent example of the fascinating properties and possibilities provided by NHC ligands is represented by the Grubbs catalyst. ${ }^{[144]}$ Exchange of a phosphine with a NHC ligand improved its stability and, even more important, its activity toward olefin metathesis. NHCs show huge similarity to phosphine ligands but they are typically more basic and show a smaller tendency toward dissociation. ${ }^{[145]}$ The $\mathrm{M}-\mathrm{C}$ bonds are mainly of $\sigma$-bonding character, while only very little contribution from $\pi$-backbonding is observed, resulting in longer $\mathrm{M}-\mathrm{C}$ bonds compared to Fischerand Schrock-carbenes. ${ }^{[146]}$

A crucial aspect in coordination chemistry is the control and fine tuning of electronic and steric properties of the ligand sphere. ${ }^{[142]}$ For this purpose, NHCs are well suited and numerous iron NHC compounds with interesting properties and catalytic applications have been synthesized. ${ }^{[143]}$ Rather new is the use of NHC ligands in mimicking biological systems such as Fe-S-clusters or dinitrosyl compounds. As presented in the previous sections, most of the reported and structurally authenticated $\mathrm{Fe}-\mathrm{NO}$ and $\mathrm{Fe}=\mathrm{O}$ species are supported by $\mathrm{N}-$, O-, or $\mathrm{S}$-donor ligands, likely because of the biological relevance of such a ligand environment. Apart from the unique $\left[\mathrm{Fe}(\mathrm{CN})_{4}(\mathrm{NO})\right]^{2-}$ compound, organometallic $\mathrm{Fe}-\mathrm{NO}$ complexes featuring $\mathrm{Fe}-\mathrm{C}$ bonds are rare and were, before starting with this work, limited to few $\mathrm{N}$-confused porphyrin systems with a $\left\{\mathrm{N}_{3} \mathrm{C}\right\}$ donor set. $^{[147,148]}$

In 2010, Jenkins et al. described the synthesis of tetraimidazolium macrocycles with two methylene and two ethylene bridges as well as protons or phenyl groups in the imidazole backbones. ${ }^{[149]}$ Conversion of these tetraanionic ligand scaffolds with platinum salts allowed for the isolation of tetracarbene coordinated metal complexes. In the following year, they reported the catalytic activity of a corresponding iron complex (Scheme $8 \mathbf{A}$ ) in aziridination. ${ }^{[150]}$ Within this publication the authors have proposed that the catalytically active species is an imidoiron(IV) complex (Scheme 8, middle) and evidence for this proposal was reported in 2014 by isolation of the tetrazene complex B. ${ }^{[151]}$ These studies are of high interest since they illustrate the possibility to stabilize high 
oxidation states on an iron center coordinated by such a tetracarbene macrocycle. While the group of Jenkins was successful in the isolation of further metal complexes ( $\mathrm{Ag}, \mathrm{Pd}, \mathrm{Ni}, \mathrm{Co}, \mathrm{Ru}, \mathrm{Cr}$ ) supported by the tetracarbene system with phenyl groups in the backbone, ${ }^{[152]}$ they did not describe the synthesis of further complexes without these phenyl groups in the carbene-backbone. In 2013, Meyer et al. reported the isolation of the less sterically hindered iron(II) analog 1 (Scheme 9) using the basic iron(II) precursor $\left\{\mathrm{Fe}\left[\mathrm{N}\left(\mathrm{SiMe}_{3}\right)_{2}\right]_{2}\right\}_{2}{ }^{[153]}$

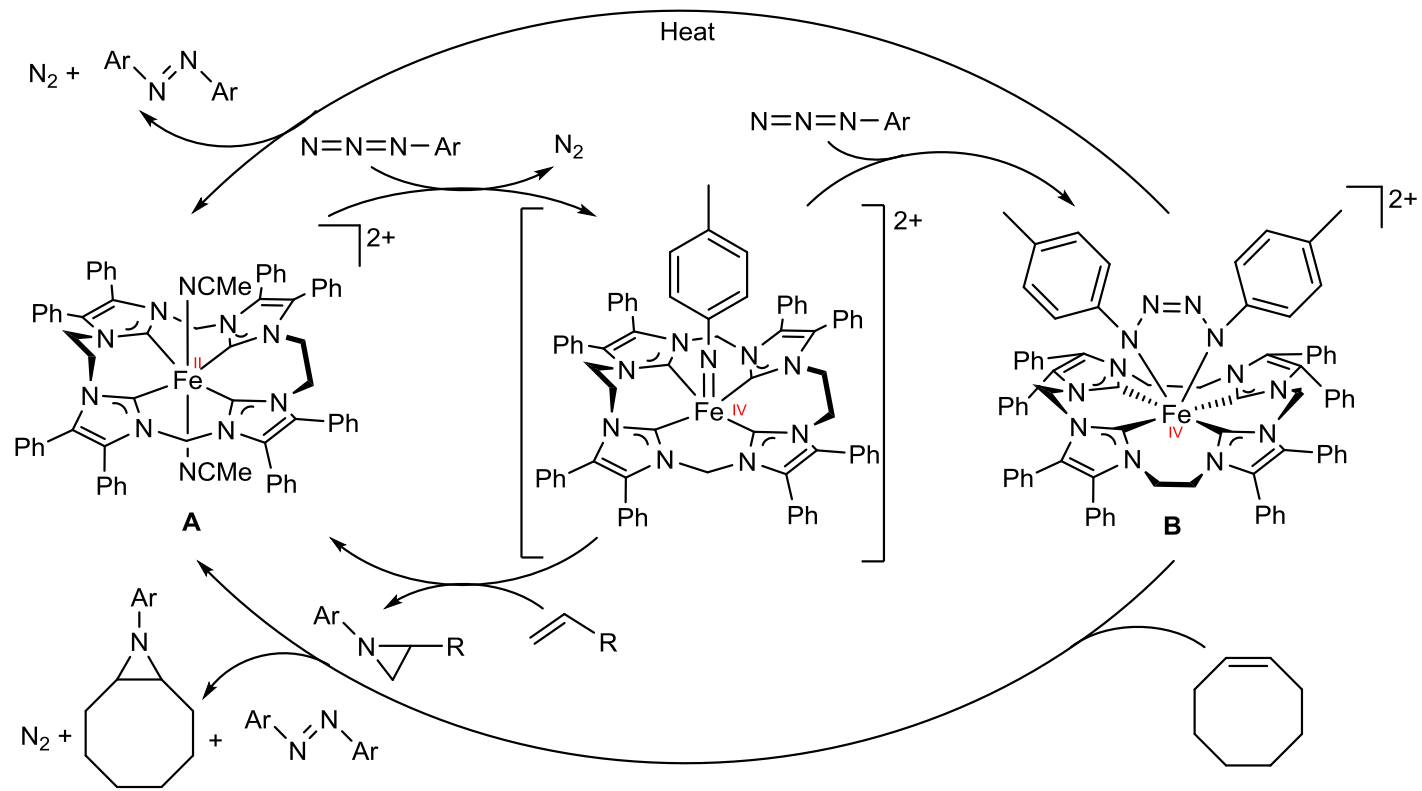

Scheme 8. Reaction cycle of aziridination showing the proposed imide intermediate (center) and the isolated tetrazene complex $\mathbf{B}$ (right) starting from the tetracarbene coordinated iron(II) complex $\mathbf{A}$ (left). ${ }^{[151]}$

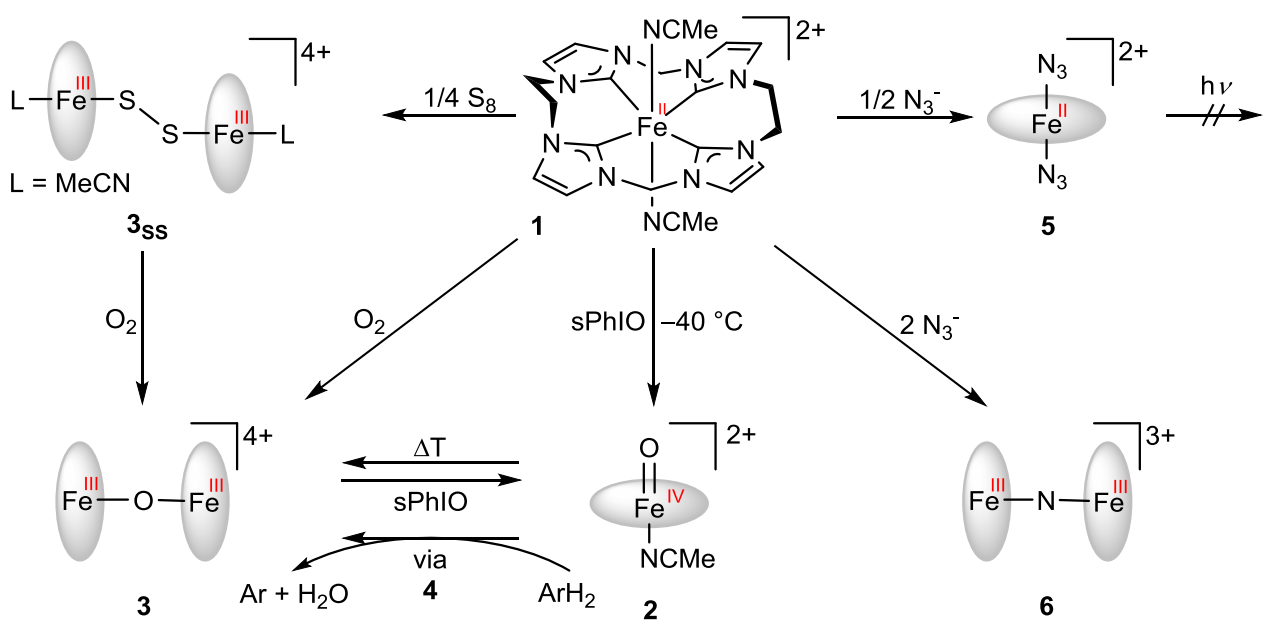

Scheme 9. Preliminiary work on the conversion of the tetracarbene coordinated iron(II) compound $\mathbf{1}$ by Steffen Meyer ${ }^{[154]}$ and Iris Klawitter. ${ }^{[144]}$ The grey ellipse around the iron nucleus represents the tetracarbene macrocyclic ligand scaffold $\left({ }^{\mathrm{NHC}} \mathrm{L}\right)$.

Starting from this iron(II) tetracarbene complex 1, Meyer et al. were also successful in the isolation of the first example of an organometallic oxoiron(IV) moiety (Scheme 9, 2) coordinated by the popular $N$-heterocyclic carbene scaffold. ${ }^{[153]}$ Compared to typical nitrogen donor systems, this 
macrocyclic carbene ligand $\left({ }^{\mathrm{NHC}} \mathrm{L}\right)$ induces quite special characteristics. For example, the strong $\sigma$-donor ability of the equatorial carbenes leads to an oblate charge distribution around the iron core resulting in large Mössbauer quadrupole splittings. Besides Mössbauer spectroscopy, the oxoiron(IV) species $\mathbf{2}$ could be intensively characterized by an interplay of different methods, in particular X-ray crystallography, UV/vis, XAS, as well as SQUID measurements. However, the electronic state was not fully clarified and a characterization of the $\mathrm{Fe}=\mathrm{O}$ bond strength apart from the bond length has not been reported. Additionally, first reactivity studies were performed but no kinetic parameters could be obtained. Within this work, the answers to these remaining questions will be presented in chapters 3 and 4 .

Prior to this work, the iron(II) compound $\mathbf{1}$ has also allowed the isolation of $\mu$-oxodiiron(III) species 3 and a trans-1,2 end-on disulfide-bridged iron dimer $\mathbf{3}_{\text {SS }}$ (Scheme 9). ${ }^{[153,156]}$ Iris Klawitter studied the reactivity of $\mathbf{1}$ versus azide compounds and sythesized complexes $\mathbf{5}$ and $\mathbf{6}^{[155]}$ The $\mu$-nitrido compound $\mathbf{6}$ has been the first example of an $\mathrm{Fe}^{\mathrm{III}}-\mathrm{N}-\mathrm{Fe}^{\mathrm{III}}$ system and highlights the ability of the carbene ligand in making model systems with iron in unusual oxidation states available.

In parallel to this work, several modifications of the carbene ligand scaffolds have been reported. For example, the methyl bridges could be replaced by $\mathrm{B}\left(\mathrm{Me}_{3}\right)_{2}$ groups leading to dianionic macrocyclic carbene systems. ${ }^{[157,158]}$ While a bigger macrocyclic system with propylene bridges was already been known before ${ }^{[159]}$ also a smaller system with exclusively methylene linkers could be established recently (Scheme 10 C) ${ }^{[160]}$ Kühn and co-workers were furthermore able to isolate the bis(carbonyl) complex $\mathbf{D}$ as well as the mononitrosyl $\{\mathrm{FeNO}\}^{7}$ complex $\mathbf{E}$ coordinated by this smaller tetracarbene system. The latter will be of further importance in chapter 8 . Moreover, they explored the oxidation behavior of $\mathbf{C}$ and observed significant differences to the system studied in this work. ${ }^{[161]}$ For example, their analogous $\mu$-oxodiiron(III) system has a bent $\mathrm{Fe}-\mathrm{O}-\mathrm{Fe}$ moiety $\left(163^{\circ}\right)$ in contrast to the linear oxo bridge in 3 .

Having these tetracarbene donor-systems with their unusual properties compared to other nonheme models in hand, it is also of interest to generate macrocyclic hybrid ligand systems with both carbene and nitrogen donor atoms. A combination of classical coordination chemistry with organometallic systems is expected to lead to intermediate properties compared to nitrogen-only or carbene-only coordinated models. Characterization of such compounds can provide a better understanding of the specific influences of both donor systems. For example, Iris Klawitter worked on a mixed pyridine-carbene ligand scaffold and its iron complexes (Scheme 10 F), but many other combinations are clearly possible. ${ }^{[155,162]}$ A part of this work will also deal with this topic (chapter 9). 


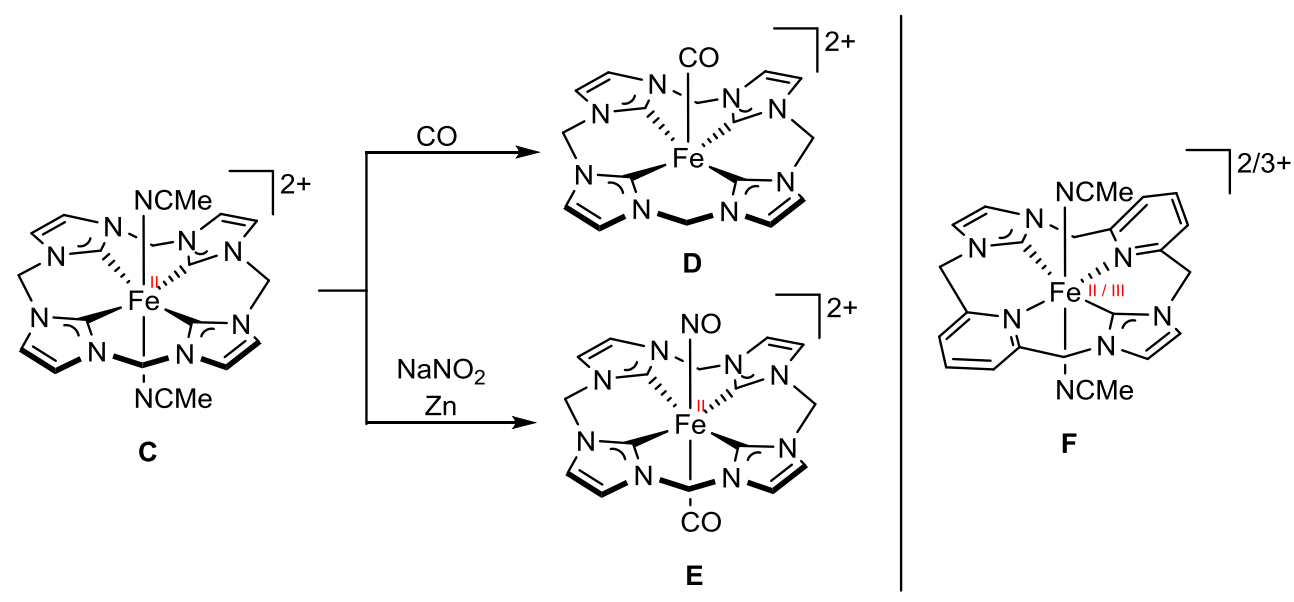

Scheme 10. Synthesis of complexes $\mathbf{D}$ and $\mathbf{E}$ according to Kühn et al. (right) and the macrocyclic pyridinecarbene coordinated iron(II) and (III) complexes F synthesized by Meyer and co-workers (left). ${ }^{[162]}$ 


\subsection{Mössbauer Spectroscopy}

Since the complexes investigated in this work were intensively analyzed with the help of Mössbauer spectroscopy (MB), a brief introduction into this technique should be given in this section. Mössbauer spectroscopy was founded in 1958 by the German physicist R. L. Mößbauer, who observed the effect of recoilless emission and absorption of nuclear $\gamma$-radiation. ${ }^{[163,164]}$ The fundamentals of this spectroscopy are discussed below.

\subsubsection{Basics of Mössbauer Spectroscopy}

A free atom in an excited nuclear state emits photons of certain energy upon transformation into a different state, for example the ground state. This process is accompanied by recoil with the recoil energy $E_{\mathrm{R}}$.

$$
E_{R}=\frac{E_{\gamma}^{2}}{2 m c^{2}}
$$

If a $\gamma$-quantum gets absorbed by a second atom, both atoms show recoil in the opposed direction. Therefore, the corresponding emitted energy $E_{\mathrm{S}}(\mathrm{S}=$ source $)$ is drastically decreased upon absorption and cannot excite the absorbing atom into a suitable excited state anymore. However, resonant core- $\gamma$-absorption becomes possible when the emitting atom is incorporated into a lattice. Coming back to formula (i), incorporation of a single atom into a lattice corresponds to a huge increase in the mass and therefore smaller recoil energy. In case of such recoilless emission, the so called 'Mössbauer effect', $E_{\mathrm{S}}$ is monochromatic. To use the Mössbauer effect for spectroscopy, $E_{\mathrm{S}}$ needs to be modulated according to the core energy level $E_{\mathrm{A}}$ of the absorbing nucleus. Taking advantage of the Doppler effect, this is achieved by moving the source in a certain velocity $\left(v / \mathrm{mm} \mathrm{s}^{-1}\right)$ toward and away from the absorber. This allows for an exact assignment of the absorbers' ground state in relation to the source. The observed differences can be put in relation to the local chemical and electronic environment of the absorbing atom.

The nucleus most commonly used for MB studies is ${ }^{57} \mathrm{Fe}$. The appropriate source for measurements on iron is ${ }^{57}$ Co. As shown in Scheme 11, this radioactive nucleus transforms with a half-life of 272 days into a ${ }^{57} \mathrm{Fe}$ excited state with a nuclear spin of $I=5 / 2$ which further decays to a second excited state with $I=3 / 2$ (Mössbauer niveau). From this level it passes into the $I=1 / 2$ ground state by emission of a $14.4 \mathrm{keV} \gamma$-quantum. This energy then excites the absorbing nucleus. 


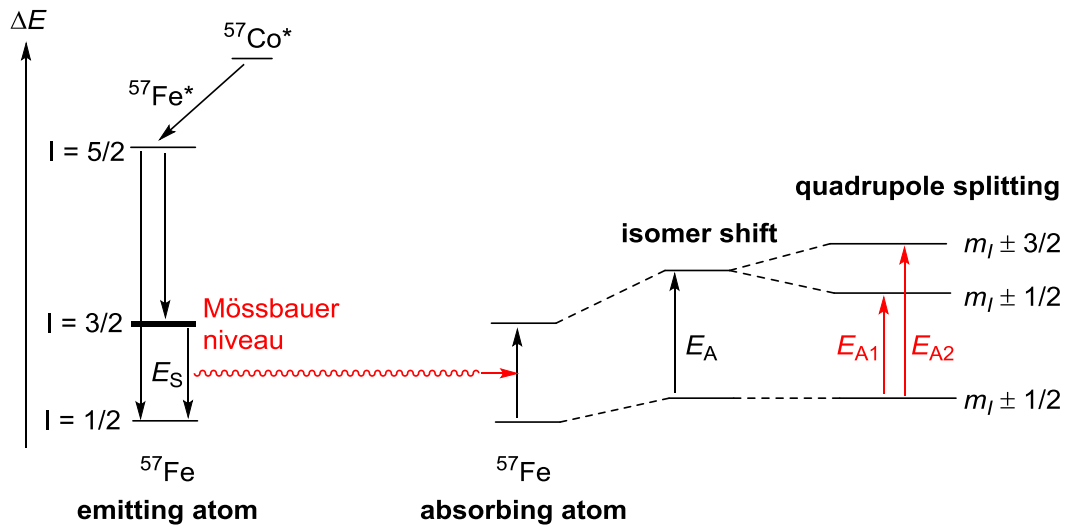

Scheme 11. Illustration of the principles behind Mössbauer spectroscopy.

Since the nuclear properties are influenced by surrounding electrons, the MB measurement provides valuable chemical information about valence and oxidation state, symmetry, and magnetic behavior. There are three types of hyperfine interactions.

\subsubsection{Hyperfine Interactions in Mössbauer Spectroscopy}

The energy shift of the Mössbauer absorption line $E_{\mathrm{A}}$ compared to the emitted $14.4 \mathrm{keV}\left(E_{\mathrm{S}}\right)$ is called isomer shift (IS, $\delta=E_{\mathrm{A}}-E_{\mathrm{S}}$ ). ${ }^{[163]}$ It is based on electrostatic interaction of the nucleus with its surrounding electrons and therefore relies on the electron density around the iron center. The electrons in s-orbitals with non-zero electron density at the nucleus are directly connected to the IS due to the electric monopole interaction. In particular, higher s-electron density leads to lower $\delta$-values. However, p-, d- and f-electrons show an indirect effect on the IS since they shield s-electrons and therefore lower the s-electron density at the nucleus. Consequently, higher p- and d-electron density leads to higher shielding, reduced s-electron density and finally a higher IS. Due to this effects, the IS largely depends on the oxidation state, the spin state, and bonding interactions around the iron center. In typical cases, higher iron oxidation states (lower d-electron density) show less shielding of the s-electrons and therefore a lower IS. However, this negative correlation is mainly valid for high spin compounds or high oxidation states. ${ }^{[163,165]}$ In low spin compounds with lower oxidation states, other characteristics such as $\sigma$ - and $\pi$-interactions correlate with changes in the bond length and have been shown to play a significant role. In particular, a higher d-electron density can be balanced out by increase of iron backbonding into the ligand orbitals leading to negligible changes in the IS. Such effects have been reported for a number of low-valent dinitrosyl low spin complexes ${ }^{[166]}$ as well as for iron(IV)alkylidines with unusually high IS. ${ }^{[167]}$ The latter case represents a perfect example for the influence of bond lengths on $\delta$. For a series of $\mathrm{Fe}-\mathrm{N}_{2}{ }^{+}, \mathrm{Fe}-\mathrm{N}_{2}$ and $\mathrm{Fe}-\mathrm{N}_{2}{ }^{-}$complexes reported by Peters et al., even a reverse positive correlation of the IS with 
the iron oxidation state has been reported. ${ }^{[165,168]}$ Consequently, the isomer shift parameter alone does not allow for an unambiguous assignment of oxidation states as will be of relevance within this work as well.

Another feature of $\mathrm{MB}$ spectra is the quadrupole splitting ( $\mathrm{QS}$ or $\left.\Delta E_{\mathrm{Q}}\right)$. Caused by electric quadrupole interactions, the nucleus shows an electric quadrupole moment. This interacts with the electric field gradient (EFG) and, for $I=3 / 2$, leads to splitting into two twofold degenerated nucleus states with $m_{I} \pm 1 / 2$ and $m_{I} \pm 3 / 2$ (iron case, Scheme 11). Therefore, the absorbing atom gets excited into two different states and two absorption lines are observed. The energy difference of the two absorption lines - the so called quadrupole splitting - relies on two effects. First, a nonsymmetric arrangement of the ligands leads to large QS. Second, a non-cubical distribution of the nucleus' valence electrons also increases the QS. For example, the tetracarbene coordinated iron complexes reported by Meyer et al. typically show very large QS parameters. ${ }^{[153,156]}$ The strong equatorial $\sigma$-donor system leads to an oblate and therefore non-cubic charge distribution around the nucleus. More examples in this context will be given within this thesis.

A third type of hyperfine interactions in MB spectroscopy is the magnetic dipole interaction. Within an intrinsic or external magnetic field, the nuclear spins prefer particular orientations. The $m_{I}$ states of ground and excited state split further into their magnetic sublevels. This so called nuclear Zeeman effect results in a spectrum with up to six absorption lines according to the selection rules for nuclear transitions $\left(\Delta m_{I}=0, \pm 1\right)$. In case of an intrinsic magnetic field as induced by paramagnetic compounds, paramagnetic relaxation is of high relevance. If the relaxation time is slower than the Mössbauer time scale $\left(10^{-7} \mathrm{~s}\right)$, a magnetic splitting of the Mössbauer doublets is observed while no effect is noticed when the relaxation time is faster. In case of similar time scales, an unsymmetric broadening of the lines is induced.

A typical experiment is performed at $80 \mathrm{~K}$ on solid material or frozen solution to increase the intensity of recoilless emission. It should be noted that the briefly introduced parameters can show temperature-dependence; however, this effect will not be discussed in detail herein. 


\section{Objectives}

The main course of this work deals with the preparation and characterization of novel tetracarbene coordinated iron compounds as models for biorelevant intermediates. As already demonstrated by Steffen Meyer and Iris Klawitter, the organometallic ligand scaffold ${ }^{\mathrm{NHC}} \mathrm{L}$ shows distinct influences on the electronic and structural properties of the iron center when compared to nitrogencoordinated model compounds predominantly found in literature. ${ }^{[153-155]}$ Of particular interest in this regard are the $\mathrm{Fe}=\mathrm{O}$ stretching frequency and the ligand field splitting of oxoiron(IV) complex 2. Furthermore, it was planned to study the reactivity of $\mathbf{2}$ in $\mathrm{C}-\mathrm{H}$ bond activation and to compare the obtained kinetic parameters with data reported for $\mathrm{N}$-coordinated high-valent oxoiron systems. These comprehensive studies on the properties of $\mathbf{2}$ and its corresponding reactivity are expected to improve the current understanding regarding the factors that control the reactivity of $\mathrm{Fe}^{\mathrm{IV}}=\mathrm{O}$ compounds. In contrast to literature described $\mu$-oxodiiron(III) compounds that are typically the dead end of oxidation chemistry, the carbene-coordinated $\left[\left({ }^{\mathrm{NHC}} \mathrm{L}\right) \mathrm{Fe}{ }^{\mathrm{III}}-\mathrm{O}-\mathrm{Fe}^{\mathrm{III}}\left({ }^{\mathrm{NHC}} \mathrm{L}\right)\right]^{4+}$ species 3 is reactive toward substrates with small oxidizing potentials and weak $\mathrm{C}-\mathrm{H}$ bonds. As supported by scrambling experiments by Iris Klawitter, this so far unprecedented reactivity has been attributed to a disproportionation of $\mathbf{3}$ into $\left[\left({ }^{\mathrm{NHC}} \mathrm{L}\right) \mathrm{Fe}^{\mathrm{II}}(\mathrm{MeCN})_{2}\right]^{2+}$ (1) and $\left[\left({ }^{\mathrm{NHC}} \mathrm{L}\right) \mathrm{Fe}^{\mathrm{IV}} \mathrm{O}(\mathrm{MeCN})\right]^{2+}$ (2) in solution. Within this work, it was planned to study the reactivity of $\mathbf{3}$ in order to obtain insight into the proposed disproportionation equilibrium. As depicted in Scheme 12, the results of these projects are described in chapters 3-5.

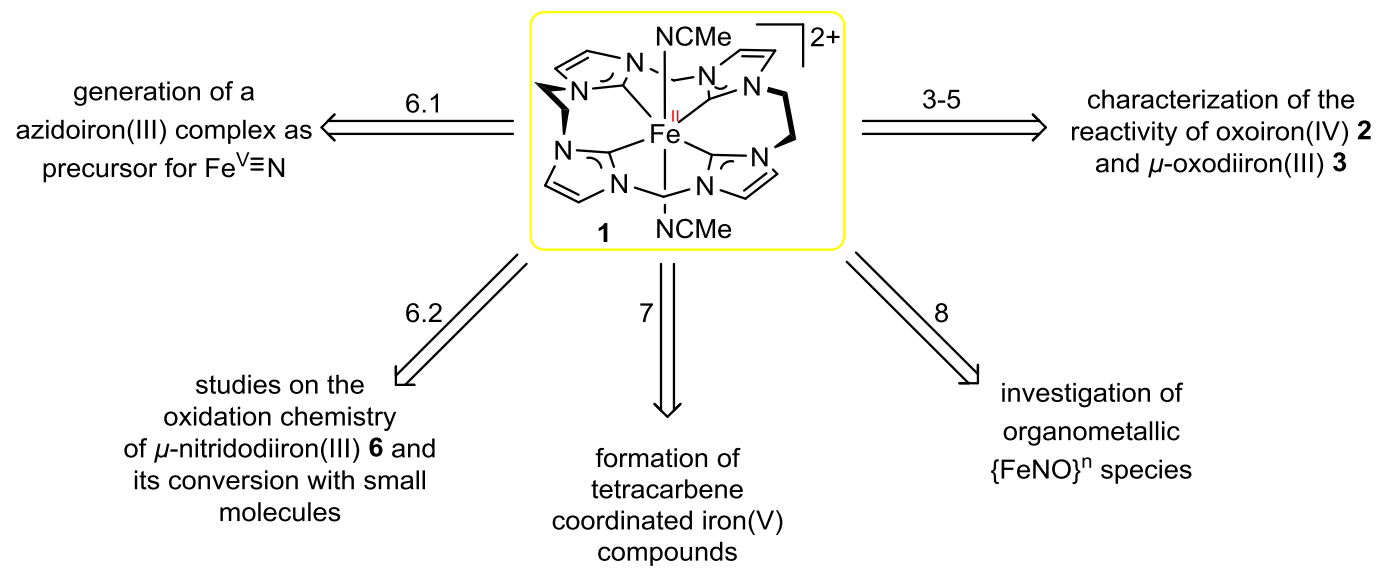

Scheme 12. Goals of this work dealing with tetracarbene-coordinated iron complexes, the numbers above the arrows correspond to the chapters.

Inspired by the work on bis-azido iron complexes by Wieghardt et al., ${ }^{[8,57,92]}$ the generation of azidoiron(III) complexes as precursors for corresponding nitridoiron(V) species was another goal of this work. It was planned to study two strategies: first, oxidation of the bis-azidoiron(II) complex 5 and second, conversion of a suitable iron(III) precursor with azide salts. Once having the clean 
bis-azidoiron(III) compound in hand, it was aimed at searching for suitable irradiation conditions on the way to $\mathrm{Fe}^{\mathrm{V}} \equiv \mathrm{N}$ systems.

As described in chapter 1 , conversion of 1 with 0.5 eq. azide salt leads to formation of an unprecedented $\mu$-nitridodiiron(III) species (6) while literature described $\mu$-nitrido systems are typically in the mixed-valent iron(III)-iron(IV) or high-valent diiron(IV) oxidation state. Thus, full characterization of $\mathbf{6}$ as well as studies on its redox chemistry and reactivity were desired. The results of these azide projects are summarized in chapter 6 .

Motivated by the high stability and fascinating features of oxoiron(IV) complex 2, it was of high interest to generate iron compounds in even higher oxidation states. While common strategies have already been excluded by Iris Klawitter, the strong oxidizing and fluorination reagent xenon difluoride was used for studies herein. Furthermore, a second promising strategy was adapted by Que et al. who reported that the attack of deprotonated tert-butylperoxide at the axial MeCN ligand can result in formation of an iron(V) compound. ${ }^{[169]}$

Studies on the reactivity of the tetracarbene coordinated iron(II) complex $\mathbf{1}$ toward nitric oxide are described in chapter 8 . The high affinity of iron(II) centers toward the $\mathrm{NO}$ radical is expected to allow for the generation of the corresponding $\{\mathrm{FeNO}\}^{7}$ species. Characterization of the obtained product in comparison with literature reported heme and non-heme models was pursued. Moreover, its redox properties should be examined, hoping for possible oxidation and reduction of the $\{\mathrm{FeNO}\}^{7}$ moiety to its corresponding $\{\mathrm{FeNO}\}^{6}$ and $\{\mathrm{FeNO}\}^{8}$ analogs. As presented in section 1.4, no complete series of fully characterized $\{\mathrm{FeNO}\}^{6-8}$ compounds is known so far, providing motivation for detailed investigation of the tetracarbene-coordinated systems.

Finally, it was planned to synthesize new hybride ligand systems for the generation of iron centers coordinated by both nitrogen and carbene donors. These model compounds are expected to show intermediate properties when compared to nitrogen-only and carbene-only coordinated iron complexes, thus allowing for a better understanding of the effects of the NHC ligands on the properties and reactivity of the corresponding iron complexes. 


\section{Characterization of a Carbene Coordinated Oxoiron(IV) Complex}

\subsection{Aims of this Project}

Organometallic oxoiron(IV) model complex 2 (Scheme 13) has already been characterized intensively by Steffen Meyer. ${ }^{[153]}$ Still, some questions had been left unanswered and present a starting point for this work. Parts of this chapter have been adapted from a recent publication. ${ }^{[170]}$

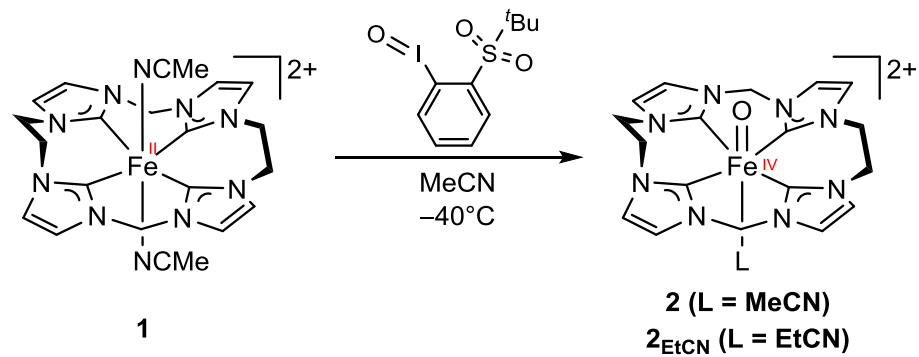

Scheme 13. Synthetic procedure to oxoiron(IV) complex $2^{[153]}$

Steffen Meyer was able to determine the structure of $\mathbf{2}_{\mathrm{EtCN}}$ by X-ray crystallography and reported a $\mathrm{Fe}-\mathrm{O}$ bond length of $1.661 \AA$ A. Compared to other structurally characterized model systems, this bond length is within the expected range but no direct conclusions about the bonding interaction could be drawn from this finding because of two reasons: First, the Fe-O moieties of $S=1$ (1.639$1.667 \AA)^{[53,171,172]}$ and $S=2(1.661 \AA, 1.680 \AA)^{[54,76]}$ systems do not show drastic differences in general. Second, comparison with the reported models is aggravated by the fact that the carbene donors show completely different properties than the typical N/O donor systems. Therefore, a characterization of the $\mathrm{Fe}=\mathrm{O}$ bond strength via $\mathrm{Raman}$ or IR spectroscopy was aimed at. Typically, the stretching frequencies of oxoiron moieties are determined with the help of ${ }^{18} \mathrm{O}$-labeling experiments and show values of about $810-850 \mathrm{~cm}^{-1} \cdot{ }^{[7,52]}$ Prior to this work, a labeling with ${ }^{18} \mathrm{O}$ had not been successful and Raman studies on non-labeled material did not allow for an unambiguous assignment of the $\mathrm{Fe}=\mathrm{O}$ stretch.

With the help of a SQUID measurement, the spin state of the tetracarbene oxoiron(IV) complex 2 could be clearly determined as low spin $S=1 .{ }^{[153]}$ Additionally, an anisotropy value $\left(|D|=16.8 \mathrm{~cm}^{-1}\right)$ smaller than for other $\mathrm{Fe}^{\mathrm{IV}}=\mathrm{O}$ species was observed, indicative of a large separation of the $S=1$ ground state and the $S=2$ excited state. ${ }^{[52]}$ This finding was explained by destabilization of the $d_{x^{2}-y^{2}}$ orbital due to the strong equatorial donor system. However, the exact electronic structure of $\mathbf{2}$ remained unclear and was the focus of a collaboration project with Shengfa Ye at the MPI-CEC in Mülheim. 


\subsection{Determination of the $\mathrm{Fe}=\mathrm{O}$ Stretching Frequency}

In terms of ${ }^{18} \mathrm{O}$-labeling of oxoiron(IV) species, two methods are available. It is possible to start directly from the labeled artificial oxidant, in this case soluble ${ }^{18} \mathrm{O}$-iodosylbenzene (ArI $\left.\mathrm{I}^{18} \mathrm{O}\right)$. Additionally, $\mathrm{Fe}=\mathrm{O}$ complexes have been shown to undergo oxygen atom exchange with water, which can be used for labeling as well. ${ }^{[58,71]}$ Both methods have been employed within this work.

The synthesis of soluble iodosylbenzene was adapted from Protasiewicz et al. ${ }^{[173]}$ During the last step, the iodanediyl intermediate is treated with aquaeous metalhydroxide solution in order to give the iodosyl-group. Consequently, treatment with $\mathrm{M}^{18} \mathrm{OH}$ in ${ }^{18} \mathrm{O}$-water should lead to formation of the labeled iodosylbenzene. The basic mixture was prepared by addition of labeled water to sodium hydride under inert atmosphere. In analogy to the 'normal' synthesis, the product was obtained as a slightly yellow solid after crystallization. Synthesis of $\mathbf{2}$ according to Meyer et al. ${ }^{[153]}$ was successful with both labeled and non-labeled ArIO and crystals suitable for resonance Raman (rRaman) and AT-IR spectroscopy could be obtained. However, neither method showed characteristic shifts within the desired region and the $\mathrm{Fe}=\mathrm{O}$ stretching frequency could not be assigned.

Oxygen exchange on 2 with ${ }^{18} \mathrm{O}$-water was studied using a cryo mass spectrometer in the group of Miquel Costas at the University of Girona, Spain. According to high resolution mass spectra (HR-MS, Figure 7), a ${ }^{16} \mathrm{O}:{ }^{18} \mathrm{O}$ ratio of $1: 1$ was achieved about 90 min after addition of 250 eq. of $\mathrm{H}_{2}{ }^{18} \mathrm{O}$ to an ice-cooled solution of in situ generated 2 . Within $5 \mathrm{~h}$, the amount of $\left[\left({ }^{\mathrm{NHC}} \mathrm{L}\right) \mathrm{Fe}={ }^{18} \mathrm{O}\right](\mathrm{OTf}){ }^{+}$increased up to a ratio of 1:2 while only little decomposition was observed. Consequently, oxygen exchange with ${ }^{18} \mathrm{O}$-water turned out to be a suitable method for generating ${ }^{18} \mathrm{O}-2$ and therefore was also used in bigger scale reactions. Even though small amounts of crystals suitable for rRaman studies and IR measurements in solution could be obtained, no unambiguous assignment of the $\mathrm{Fe}=\mathrm{O}$ stretch was possible. Presumably, the intensity of the $\mathrm{Fe}=\mathrm{O}$ stretch in both techniques is too weak to allow a distinct detection of shifts upon labeling. 


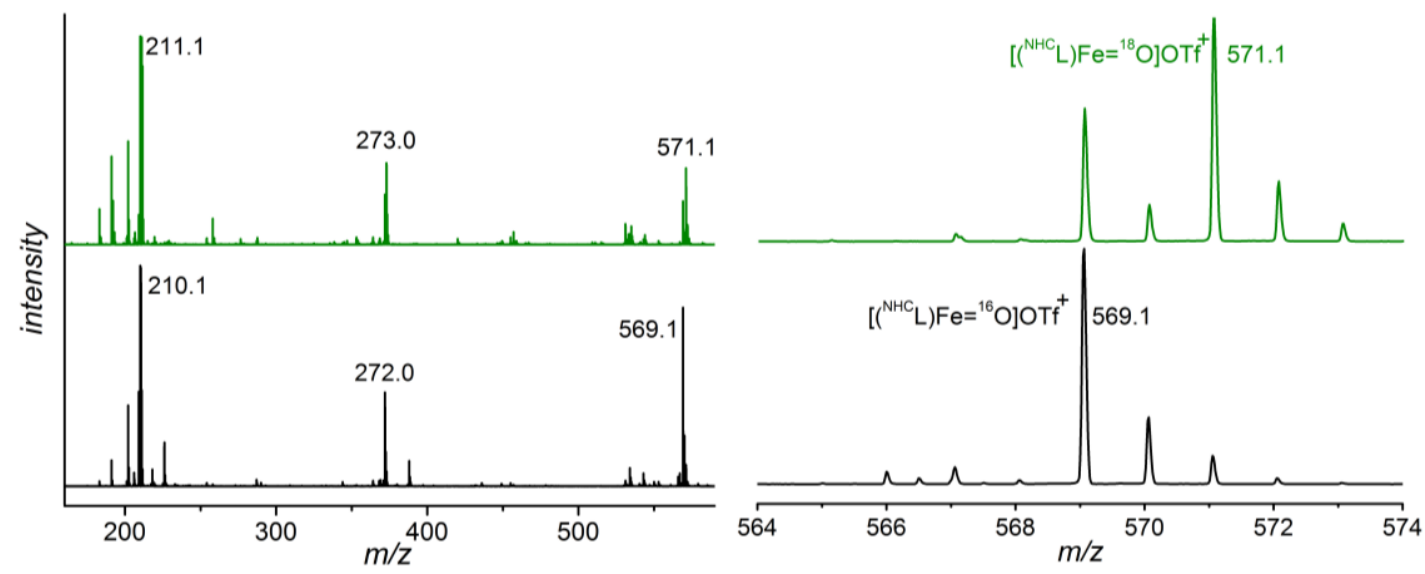

Figure 7. ESI-HR-MS spectrum of oxygen exchange in 2 with $\mathrm{H}_{2}{ }^{18} \mathrm{O}$. The non-labeled starting material is depicted in black (bottom), the reaction mixture after $5 \mathrm{~h}$ with significantly increased amount of $\mathrm{Fe}={ }^{18} \mathrm{O}$ in green (top). Signals in the black spectrum are assigned as follows: $210.1\left[\left({ }^{\mathrm{NHC}} \mathrm{L}\right) \mathrm{Fe}={ }^{16} \mathrm{O}\right]^{2+}, 272.0$ $\left.\left[\left({ }^{\mathrm{NHC}} \mathrm{L}\right) \mathrm{Fe}={ }^{16} \mathrm{O}(\mathrm{IAr})\right]^{2+}, 569.1\left[\left({ }^{\mathrm{NHC}} \mathrm{L}\right) \mathrm{Fe}={ }^{16} \mathrm{O}\right](\mathrm{OTf})\right)^{+}$. The signal at $m / z=569.1$ is shown in higher resolution on the right side. The shifts to the corresponding signals in the green spectrum verify successful ${ }^{18} \mathrm{O}$-labeling.

Finally, the infrared spectra of labeled and non-labeled $\left[\left({ }^{\mathrm{NHC}} \mathrm{L}\right) \mathrm{Fe}=\mathrm{O}(\mathrm{NCMe})\right]^{2+}$ have been obtained by helium tagging infrared photodissociation (IRPD) spectroscopy in the group of Jana Roithová in Prague. ${ }^{[170,174]}$ Samples of 2 suitable for IRPD measurements have been prepared as described in the experimental part. The labeled species in particular was again generated by oxygen exchange between the oxoiron complex and $\mathrm{H}_{2}{ }^{18} \mathrm{O}$ in $\mathrm{MeCN}$.

The group of Jana Roithová provides helium tagging IRPD spectroscopy, a unique technique to determine the IR spectrum of isolated ions. Therein, the complex ions are generated by electrospray ionization, mass-selected and transferred to an ion trap. Since this trap is operating at $3 \mathrm{~K}$, the internal energy of the ions is very low and they form adducts with helium atoms. Subsequently, absorbed photons of an IR beam increase the internal energy of the helium complexes and initiate their dissociation. The eliminated helium atoms are analyzed by mass spectrometry and the energy of the absorbed IR photon can be determined from the energy of the helium atom. This method turned out to be a suitable technique for determination of the $\mathrm{Fe}=\mathrm{O}$ stretching frequency in $\mathbf{2}$. As shown in Figure 8, a signal at $\tilde{v}=832 \pm 3 \mathrm{~cm}^{-1}$ for the non-labeled compound is found to shift to $\tilde{v}=799 \pm 3 \mathrm{~cm}^{-1}$ for the $\mathrm{Fe}={ }^{18} \mathrm{O}$ complex. This shift of $\Delta \tilde{v}=33 \mathrm{~cm}^{-1}$ is in the expected range for a diatomic oxoiron vibration. The $\mathrm{Fe}=\mathrm{O}$ stretching frequency of $\mathbf{2}$ is perfectly in line with those reported for other non-heme models $\left(814-854 \mathrm{~cm}^{-1}\right) .{ }^{[52]}$ 


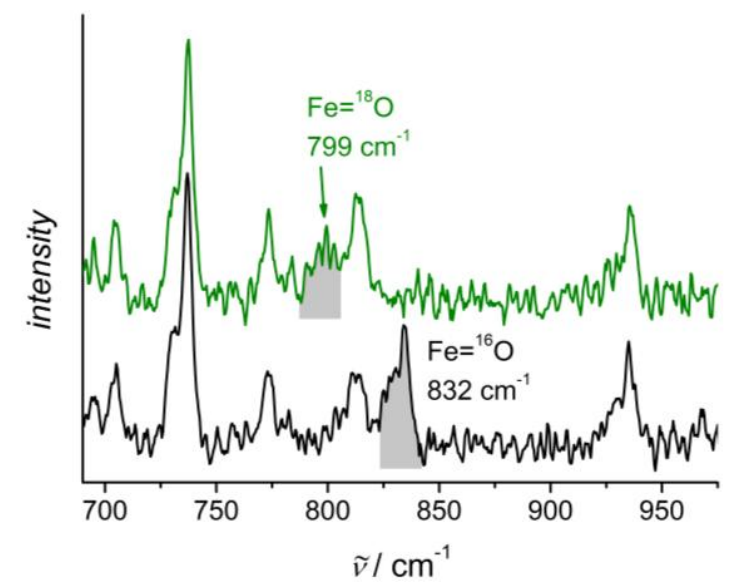

Figure 8. Helium tagging IRPD spectrum of $\left[\left({ }^{\mathrm{NHC}} \mathrm{L}\right) \mathrm{Fe}^{\mathrm{IV}}={ }^{16} \mathrm{O}(\mathrm{NCMe})\right]^{2+}$ (black) and its ${ }^{18} \mathrm{O}$-labeled isotopomer (green). ${ }^{[170]}$

In summary, the $\mathrm{Fe}=\mathrm{O}$ stretching frequency could be determined by sophisticated spectroscopic methods and provides valuable insights into properties of $\mathbf{2}$. The found stretching frequency and shift upon ${ }^{18} \mathrm{O}$-labeling indicate that the $\mathrm{Fe}=\mathrm{O}$ moiety is only barely affected by the strong $\sigma$-donating carbene ligand in comparison with typical N-donor models. ${ }^{[170]}$ 


\subsection{Characterization of the Electronic Structure of 2 by MCD}

The electronic structure of $\mathbf{2}$ was characterized in detail using the interplay of UV/vis/nIR spectroscopy, magnetic circular dichroism (MCD) and density functional theory (DFT). Since the simulation, theoretical interpretation of the data as well as the final assignment of the observed transitions was mainly performed by the collaborator Shengfa Ye, the results will just be presented briefly herein. A detailed description is given in a recent joint publication. ${ }^{[170]}$

The spectral characteristics of 2 in the UV/vis and nIR region have been already reported by Steffen Meyer. ${ }^{[153,154]}$ For comparison with MCD spectra, a new UV/vis/nIR spectrum in high concentration $(4 \mathrm{mM})$ was recorded at RT. The spectrum is dominated by a broad band around $\tilde{v}=26000 \mathrm{~cm}^{-1}\left(\lambda_{\max }=400 \mathrm{~nm}, \varepsilon=1100 \mathrm{M}^{-1} \mathrm{~cm}^{-1}\right)$ that is only partly shown in Figure 9 due to its high intensity. Additionally, two weak transitions in the near IR region at $\tilde{v}=16500 \mathrm{~cm}^{-1}$ $\left(\lambda_{\max }=600 \mathrm{~nm}, \varepsilon=160 \mathrm{M}^{-1} \mathrm{~cm}^{-1}\right)$ and $\tilde{v}=12000 \mathrm{~cm}^{-1}\left(\lambda_{\max }=830 \mathrm{~nm}, \varepsilon=30 \mathrm{M}^{-1} \mathrm{~cm}^{-1}\right)$ are observed. These bands give rise to rich MCD spectra and allowed to characterize the electronic structure of $2 .^{[170]}$

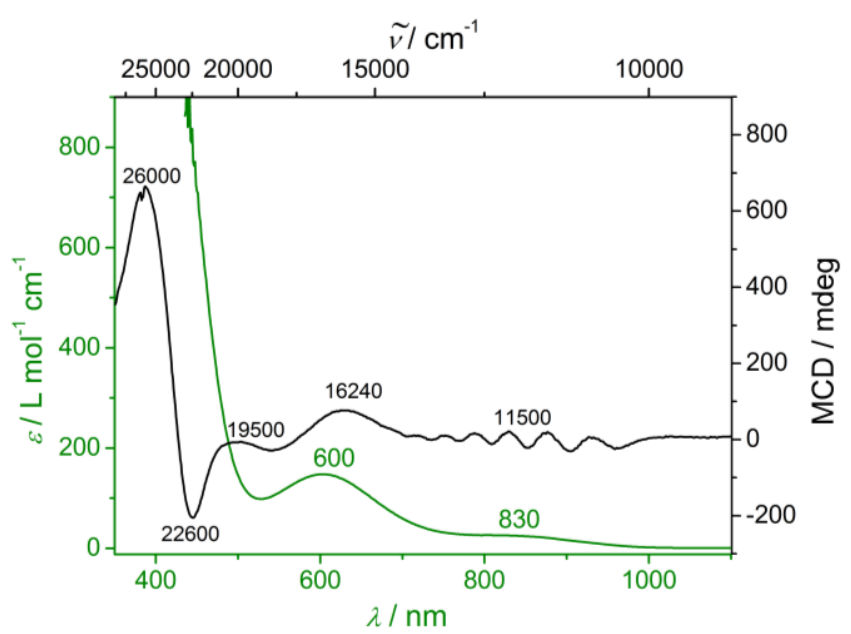

Figure 9. RT absorption spectrum (MeCN, $4 \mathrm{mM}$, green) and $\mathrm{MCD}$ spectrum (PrCN, $1.63 \mathrm{mM}, 20 \mathrm{~K}, 10 \mathrm{~T}$, black) of 2 .

In MCD spectroscopy, the sample is placed in a strong magnetic field and irradiated with circularly polarized light. Since this light is oriented parallel to the magnetic field, the sample absorbs left and right circularly polarized light in a different manner. In case of one-electron excitations, the sign of the MCD signal is determined by the symmetry of the involved orbitals. One major advantage of MCD in comparison to other absorption techniques is the possibility to detect transitions of weak intensity such as d-d transitions. The intensity of the MCD signal is a function of wavelength, temperature and magnetic field. In the experiments presented herein, the wavelength was varied between $\tilde{v}=5000$ and $30000 \mathrm{~cm}^{-1}$ while a temperature of $2-80 \mathrm{~K}$ and a field intensity of $10 \mathrm{~T}$ were 
used. The observed spectra are described by so called $A, B$ and $C$ term transitions. For compound 2 , the spectra are dominated by the $C$-term whose intensity arises from spin-orbit coupling (SOC). This SOC can occur between ground state and excited state or between the two excited states. Some of the observed transitions are assigned as pseudo $A$-term signals resulting from overlapping $C$-term transitions. Simulation of the $C$-term intensity according to the selection rules for MCD transitions and equation (ii) allows for an assignment of the observed transitions. ${ }^{[170,175]}$

$$
\frac{\Delta \varepsilon}{E}=\frac{\gamma}{4 \pi S} \int_{0}^{\pi} \int_{0}^{2 \pi} \sum_{i} N_{i}\left(l_{x}\left\langle\hat{S}_{x}\right\rangle_{i} M_{y z}^{\mathrm{eff}}+l_{y}\left\langle\hat{S}_{y}\right\rangle_{i} M_{z x}^{\mathrm{eff}}+l_{z}\left\langle\hat{S}_{z}\right\rangle_{i} M_{x y}^{\mathrm{eff}}\right) \sin \theta d \theta
$$

Here, $\frac{\Delta \varepsilon}{E}$ is the MCD intensity, $\gamma$ is a collection of constants, $S$ is the total spin of the ground state, $N_{i}$ is the Boltzmann population of the $i^{\text {th }}$ magnetic sublevel of the electronic ground state, $l_{x, y, z}$ are the directional cosine values of the angles between the magnetic field and the molecular coordinate system, and $\left\langle\hat{S}_{x, y, z}\right\rangle_{i}$ are the expectation values of the $\mathrm{x}, \mathrm{y}, \mathrm{z}$ component of the spin operator $\hat{S}$ over the $i^{\text {th }}$ magnetic eigenstate, respectively. The $M_{v w}^{\text {eff }}$ factors $(\mathrm{v}, \mathrm{w}=\mathrm{x}, \mathrm{y}, \mathrm{z})$ are effective transition dipole moment products that are fit parameters, but also can be theoretically calculated. ${ }^{[170,175]}$

The most prominent positive bands in the experimental MCD spectra of 2 are found at $\tilde{v}=26000$, 19500 and $16240 \mathrm{~cm}^{-1}$ while a negative signal is observed at $\tilde{v}=22600 \mathrm{~cm}^{-1}$ (Figure 9). Additionally, a vibrational progression appears at $\tilde{v}=11500 \mathrm{~cm}^{-1}$. All the observed bands in the absorption and MCD spectra could be simulated by 8 different $d$-d transitions. Two of these simulated bands with maxima at $\tilde{v}=16240 \mathrm{~cm}^{-1}$ and $19570 \mathrm{~cm}^{-1}$ arise from two-electron excitations and shall not be of further interest herein. Assignment of the remaining six bands according to the nature of their $\mathrm{d}-\mathrm{d}$ transition is shown in Table $1 .^{[170,175]}$

Table 1. Experimental and calculated excitation energies of key d-d transitions along with the assignments for complexes $\left[\left({ }^{\mathrm{NHC}} \mathrm{L}\right) \mathrm{Fe}=\mathrm{O}(\mathrm{NCMe})\right]^{2+} \mathbf{2}$ and $[(\mathrm{SR}-\mathrm{TPA}) \mathrm{Fe}=\mathrm{O}(\mathrm{NCMe})]^{+} \cdot{ }^{[170,176]}$

\begin{tabular}{|c|c|c|c|c|}
\hline \multirow[t]{2}{*}{ Transition } & \multicolumn{2}{|c|}{$\left[\left({ }^{\mathrm{NHC}} \mathrm{L}\right) \mathrm{Fe}=\mathrm{O}(\mathrm{NCMe})\right]^{2+} 2$} & \multicolumn{2}{|c|}{$[(\mathrm{SR}-\mathrm{TPA}) \mathrm{Fe}=\mathrm{O}(\mathrm{NCMe})]^{2+}$} \\
\hline & Exp. $\left(\mathrm{cm}^{-1}\right)$ & Calc. $\left(\mathrm{cm}^{-1}\right)$ & Exp. $\left(\mathrm{cm}^{-1}\right)$ & Calc. $\left(\mathrm{cm}^{-1}\right)$ \\
\hline$d_{x y} \rightarrow d_{x z, y z}$ & 12410,12930 & 13100,13200 & - & 13320,13400 \\
\hline$d_{x z, y z} \rightarrow d_{z^{2}}$ & 15700,18220 & 18820,18860 & 18200 & 17960,18050 \\
\hline$d_{x z, y z} \rightarrow d_{x^{2}-y^{2}}$ & 22660,25920 & 25010,25160 & 13700,15220 & 11840,12060 \\
\hline
\end{tabular}

The low-energy vibronic progression at $\tilde{v}=11500 \mathrm{~cm}^{-1}$ in MCD and $\tilde{v}=12000 \mathrm{~cm}^{-1}$ in the $\mathrm{nIR}$ is assigned to a transition of mainly $d_{x y} \rightarrow d_{x z, y z}$ character (Scheme 14). Its fine structure is attributed to a $\mathrm{Fe}=\mathrm{O}$ stretching mode and allows for determination of $\tilde{v}_{\mathrm{Fe}=\mathrm{O}}$ in the excited state $\left(616 \pm 15 \mathrm{~cm}^{-1}\right)$ based on the average line spacing. A similar vibronic progression $\left(610 \mathrm{~cm}^{-1}\right)$ in the nonbonding $\rightarrow \pi^{*}$ excited state was also observed for the TMC coordinated oxoiron(IV) system 
(Figure $1 \mathbf{B}$ ). ${ }^{[177]}$ Since this progression directly correlates with the $\pi$-bonding amount of the $\mathrm{Fe}=\mathrm{O}$ bond, it can be concluded that the bond characters of both systems are very similar despite their different ligand scaffolds. Furthermore, the well characterized $[(\mathrm{SR}-\mathrm{TPA}) \mathrm{Fe}=\mathrm{O}(\mathrm{NCMe})]^{2+}$ system (SR-TPA $=$ tris(3,5-dimethyl-4-methoxypyridyl-2-methyl)amine) shows a $d_{x y} \rightarrow d_{x z, y z}$ transition in a similar area indicative of a comparable energy difference between these orbitals (Table 1). ${ }^{[170,176]}$

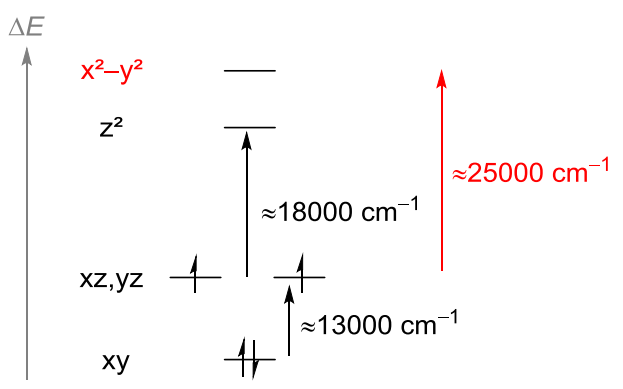

Scheme 14. Assignment of the d-d-transitions observed for oxoiron(IV) complex 2.

The nIR band at $\tilde{v}=16500 \mathrm{~cm}^{-1}$, found at $\tilde{v}=16240 \mathrm{~cm}^{-1}$ in MCD simulation arises from a $\mathrm{d}_{\mathrm{xz}, \mathrm{yz}} \rightarrow \mathrm{d}_{\mathrm{z}^{2}}$ transition with a maximum at $\tilde{v}=15700 \mathrm{~cm}^{-1}$. A second transition of this nature shows a maximum at $\tilde{v}=18220 \mathrm{~cm}^{-1}$. Both energies - corresponding to the energetic separation of the involved orbitals - agree well with data reported for the pyridyl-amine coordinated oxoiron(IV) species. ${ }^{[176]}$ The broad band at $\tilde{v}=26000 \mathrm{~cm}^{-1}$ in UV/vis and MCD was assigned to a $\mathrm{d}_{\mathrm{xz}, \mathrm{yz}} \rightarrow \mathrm{d}_{\mathrm{x}^{2}-\mathrm{y}^{2}}$ transition (Scheme 14, red arrow). It corresponds to the positive MCD signal at $\tilde{v}=26000 \mathrm{~cm}^{-1}$ and the negative band at $\tilde{v}=22600 \mathrm{~cm}^{-1}$. The experimentally and theoretically determined energy for this transition is much higher than what was found for $[(\mathrm{SR}-\mathrm{TPA}) \mathrm{Fe}=\mathrm{O}(\mathrm{NCMe})]^{2+}$ (Table 1). ${ }^{[176]}$ Even more interestingly, the energetic gap between the $d_{x z, y z}$ orbitals and $d_{x^{2}-y^{2}}$ is even higher than $\Delta E\left(\mathrm{~d}_{\mathrm{xz}, \mathrm{yz}}-\mathrm{d}_{\mathrm{z}^{2}}\right)$. Thus, the $\mathrm{d}_{\mathrm{x}^{2}-\mathrm{y}^{2}}$ orbital in $\mathbf{2}$ is not the lowest unoccupied orbital as it typically is observed in oxoiron(IV) models. While the $\mathrm{d}_{\mathrm{x}^{2}-\mathrm{y}^{2}}$ orbital is strongly destabilized due to the strong $\sigma$-donating ligand system, the $\mathrm{d}_{\mathrm{z}^{2}}$ orbital remains largely unaffected and is found at a similar energy compared to the $\mathrm{d}_{\mathrm{z}^{2}}$ in $[(\mathrm{SR}-\mathrm{TPA}) \mathrm{Fe}=\mathrm{O}(\mathrm{NCMe})]^{+}$. In conclusion, an increased HOMO-LUMO gap in $\mathbf{2}$ is determined experimentally in agreement with the conclusions made in the initial publication by Meyer et al. ${ }^{[153]}$ Additional DFT studies indicate that the resulting triplet ground state in $\mathbf{2}$ is stabilized by $18.7 \mathrm{kcal} \mathrm{mol}^{-1}$ with respect to the quintet state. ${ }^{[170]}$ 


\subsection{Conclusions}

Summed up, with the help of the collaborators Jana Roithová and Shengfa Ye, the tetracarbene coordinated oxoiron(IV) compound 2 could be characterized further and open questions about its electronic structure could be answered. The $\mathrm{Fe}=\mathrm{O}$ stretching frequency could be determined in the ground $\left(\tilde{v}=832 \pm 3 \mathrm{~cm}^{-1}\right)$ and the excited state $\left(\tilde{v}=616 \pm 15 \mathrm{~cm}^{-1}\right)$ and comparison with N/O coordinated systems showed that the $(\mathrm{FeO})$ core in 2 features similar properties. A combined $\mathrm{UV} / \mathrm{vis} / \mathrm{MCD}$ theory approach yielded valuable insights into the electronic structure of 2 . These studies revealed that the ligand field splitting of $\mathbf{2}$ differs significantly from that of related models featuring $\mathrm{N}$-donors. In $\mathbf{2}$, the $\mathrm{d}_{\mathrm{x}^{2} \mathrm{y}^{2}}$ orbital is higher in energy than the $\mathrm{d}_{\mathrm{z}^{2}}$ orbital, resulting in an increased triplet-quintet gap. This may have implications for reactivity which will be explored in the following chapter. 


\section{C-H Bond Activation by an Organometallic Oxoiron(IV) Complex}

\subsection{Preliminary Studies}

Within this chapter, the kinetics of $\mathrm{Fe}^{\mathrm{IV}}=\mathrm{O}$ complex 2 in oxidation reactions are presented. Major parts of this project were studied during a research stay in the group of Miquel Costas in Girona, Spain. The results are based on preliminary studies by Steffen Meyer and Iris Klawitter. ${ }^{[153-155]}$ Steffen Meyer had already followed the reaction of $\mathbf{2}$ toward various substrates. He attempted to perform oxygen atom transfer (OAT) reactions, but could not detect any OAT toward typical substrates such as thioanisole or styrene, in huge contrast to reports on other $\mathrm{Fe}^{\mathrm{IV}}=\mathrm{O}$ species described in the literature (see chapter 1.2). This observation has been reproducibly confirmed within this work. However, Steffen Meyer observed that 2 shows hydrogen atom abstraction (HAA) reactivity toward substrates with weak C-H bonds as illustrated in Scheme 15. ${ }^{[153,154]}$

a) Formation and decomposition of $\mathbf{2}$ :
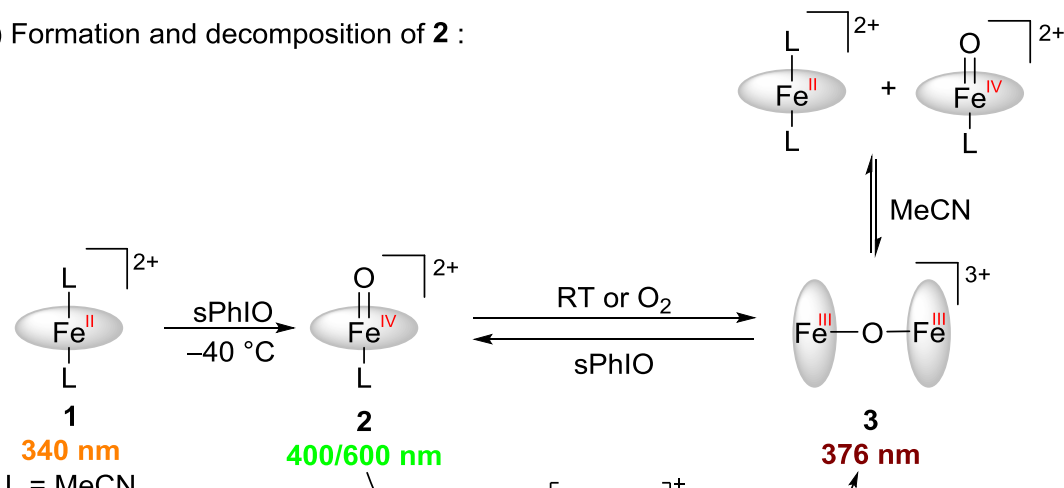

2

$\mathrm{L}=\mathrm{MeCN}$

$400 / 600 \mathrm{~nm}$

b) $\mathrm{C}^{-} \mathrm{H}$ activation:
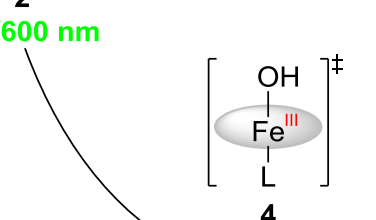

3

$376 \mathrm{~nm}$

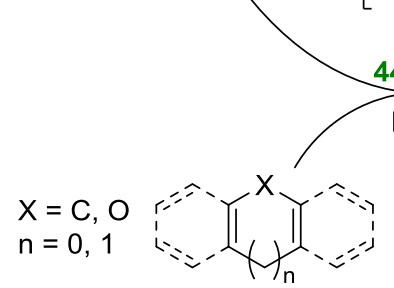

4

$\mathrm{MeCN}$

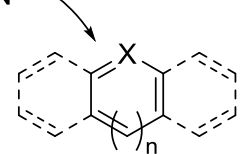

Scheme 15. Formation, decomposition and reactivity of $\mathrm{Fe}^{\mathrm{IV}}=\mathrm{O} 2$ including the characteristic absorbances in $\mathrm{UV} / \mathrm{vis}$ for iron complexes 1-4.

The addition of 1,4-cyclohexadiene (CHD) and 9,10-dihydroanthracene (DHA) led to a fast reaction and formation of $\mu$-oxodiiron(III) complex 3 . $^{[153,154]}$ Further on, Iris Klawitter observed first indications on at least two different processes occurring during reaction with CHD. After addition of substrate to in situ generated $\mathbf{2}$, she observed the formation of an intermediate species with an absorption in UV/vis at $\lambda_{\max }=448 \mathrm{~nm}$, which was suggested to be hydroxoiron complex 4 resulting from initial $\mathrm{H}$-atom abstraction. Subsequently, this species decomposes giving the final 
mixture of products, whose exact composition depends on the amount of substrate used. Due to the high complexity of the process of $\mathrm{C}-\mathrm{H}$ activation as monitored by $\mathrm{UV} / \mathrm{vis}$, kinetic parameters for the reaction of $\mathbf{2}$ with weak $\mathrm{C}-\mathrm{H}$ bonds could not be determined prior to this work.

As proven by scrambling experiments, the $\mu$-oxoiron(III) complex $\mathbf{3}$ is unstable in solution and shows disproportionation into the iron(II) species $\mathbf{1}$ and oxoiron(IV) compound 2. Accordingly, $\mathbf{3}$ gives signals of rather low intensity in mass spectrometry while the signals corresponding to $\mathbf{1}$ and $\mathbf{2}$ are of higher intensity, due to cleavage of the relatively weak $\mathrm{Fe}-\mathrm{O}-\mathrm{Fe}$ bond upon ionization. A similar splitting of a $\mathrm{Fe}^{\mathrm{IV}}-\mathrm{O}-\mathrm{Fe}^{\mathrm{IV}}$ system during mass spectrometry measurements was reported by Collins et al. ${ }^{[178]}$ Via this disproportionation equilibrium, $\mathbf{3}$ can also act as reactive species. While this chapter focuses on the reactivity of $\mathrm{Fe}^{\mathrm{IV}}=\mathrm{O}$ compound 2 , studies on the reactivity of $\mathrm{Fe}^{\mathrm{III}}-\mathrm{O}-\mathrm{Fe}^{\mathrm{III}}$ complex 3 will be presented in chapter 5 .

The results of this chapter recently have been published online and parts of this work have been adapted from this publication with permission from ACS. ${ }^{[179]}$ 


\subsection{Reaction of Oxoiron(IV) Complex 2 Toward 1,4-Cyclohexadiene}

Initial studies on $\mathrm{C}-\mathrm{H}$ abstraction activity have been performed using $\mathrm{CHD}$ as substrate. Compared to the work by Meyer and Klawitter, a higher concentration of 2 was chosen (1 mM rather than $0.15 \mathrm{mM})$. In literature, this is the predominant concentration used in kinetic studies on high-valent oxoiron complexes and allows following small changes in absorbance more easily. All reactions were perfomed at $-40{ }^{\circ} \mathrm{C}$ since this low temperature helps in avoiding the self-decay of $\mathrm{Fe}^{\mathrm{IV}}=\mathrm{O} 2$ to $\mu$-oxo species 3. $^{[179]}$

The changes observed in UV/vis spectroscopy upon addition of 50 eq. of CHD are shown in Figure 10. The initial spectrum of 2 contains two bands at $\lambda_{\max }=400$ and $600 \mathrm{~nm}$. While the reaction proceeds, the features observed in the UV/vis region start changing. The two characteristic bands increase in intensity and shift to $\lambda_{\max }=367$ and $558 \mathrm{~nm}$ and a new band at $\lambda_{\max }=448 \mathrm{~nm}$ appears (left, first process). About 100 seconds after addition of substrate, the bands at $\lambda_{\max }=448$ and $558 \mathrm{~nm}$ reach their maximum absorbance and start decreasing whereas the band at $\lambda_{\max }=367 \mathrm{~nm}$ and a new band at $\lambda_{\max }=675 \mathrm{~nm}$ arise (right, second process). ${ }^{[179]}$
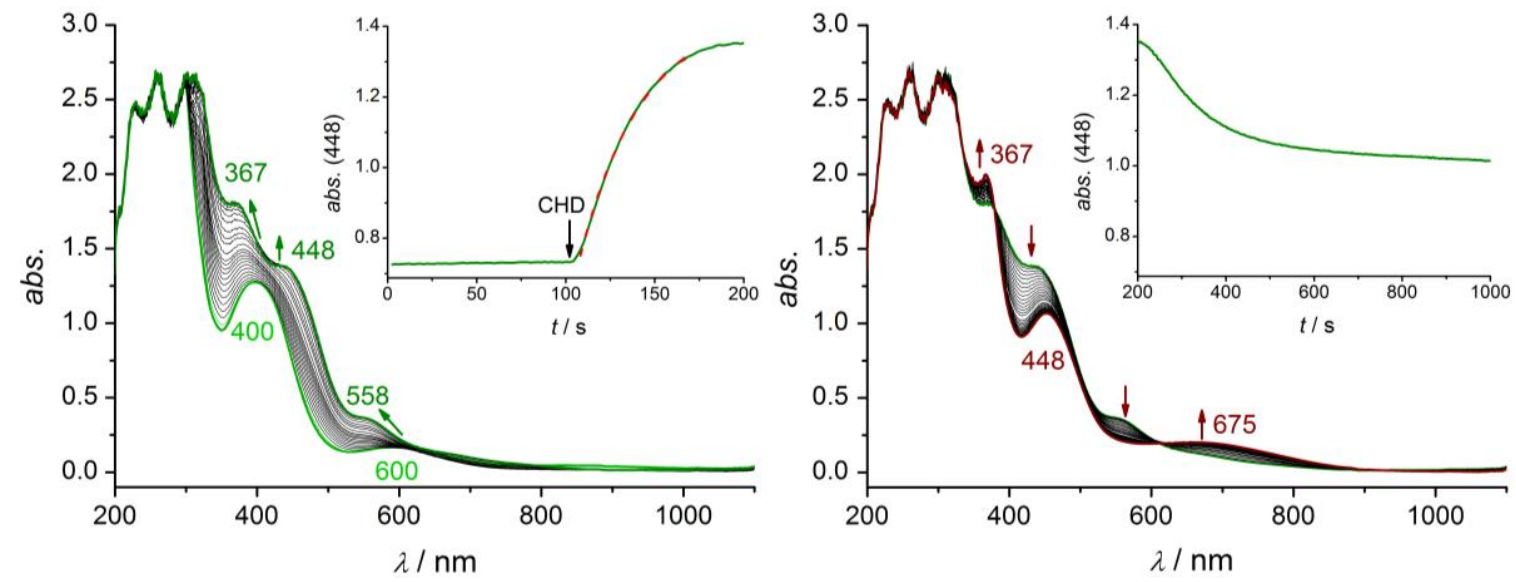

Figure 10. UV/vis spectral changes observed upon reaction of 2 with 50 equivalents $\mathrm{CHD}$ at $-40{ }^{\circ} \mathrm{C}$ in $\mathrm{MeCN}$ during the first (left) and second process (right). Insets: Kinetic trace at $448 \mathrm{~nm}$ for this transformation and corresponding exponential fit (red dashed line). Addition of substrate is indicated by an arrow. Reprinted with permission from ACS. ${ }^{[179]}$

With all these spectral changes occurring, analysis to obtain kinetic data is rather challenging. The common strategy in literature consists in following the decay of the active species upon addition of different amounts of certain substrates. This is not a suitable approach in this case since the decay species (i.e. $\mu$-oxodiiron(III) 3) has UV/vis features in the same area. However, the band at $\lambda_{\max }=448 \mathrm{~nm}$ shows a marked change in absorbance during both processes which is consistent for different concentrations of oxoiron(IV) 2 and different substrates. Its increase in intensity likely corresponds to the formation of $\mathrm{Fe}^{\mathrm{III}}-\mathrm{OH} 4$, which results from $\mathrm{H}$-atom abstraction from the 
substrate. It is further proposed that formation of $\mathbf{4}$ is proportional to a decreasing amount of $\mathrm{Fe}^{\mathrm{IV}}=\mathrm{O} 2$. Therefore, the observed $k_{\mathrm{obs}}$ for generation of $\mathbf{4}$ (a negative value when fitted to a single exponential) can be considered as the $k_{\mathrm{obs}}$ (positive) for the decrease of species $2 .^{[179]}$

The behavior of the absorbance at $\lambda_{\max }=367 \mathrm{~nm}$, which corresponds to $\mu$-oxodiiron $\mathbf{3}$, changes with varying excess of substrate since more iron(II) complex (absorbance at $\lambda_{\max }=340 \mathrm{~nm}$ ) is formed. Therefore, it is not a suitable absorbance to be followed. Following the band at $\lambda_{\max }=448 \mathrm{~nm}$ instead, the traces can be fitted to a single exponential and compared for different concentrations of substrate while maintaining a constant concentration of $2(1 \mathrm{mM})$. A plot of the observed rate constant $k_{\mathrm{obs}} v s$. concentration of CHD gives a good linear fit with a slope of 4.76 $10^{-4} \mathrm{M}^{-1} \mathrm{~s}^{-1}$ (Figure 11, left). Since the concentration is given in $\mathrm{mM}$, this value corresponds to a $k_{2}$ of $0.48 \mathrm{M}^{-1} \mathrm{~s}^{-1}$ for the reaction of 2 with CHD. The second order rate constant $k_{2}$ needs to be adjusted according to the reaction stoichiometry. Based on the number of abstractable weak $\mathrm{C}-\mathrm{H}$ bonds of the substrate (four in the case of CHD) this correction gives a $k_{2}$ ' of $0.12 \mathrm{M}^{-1} \mathrm{~s}^{-1}$. [179]
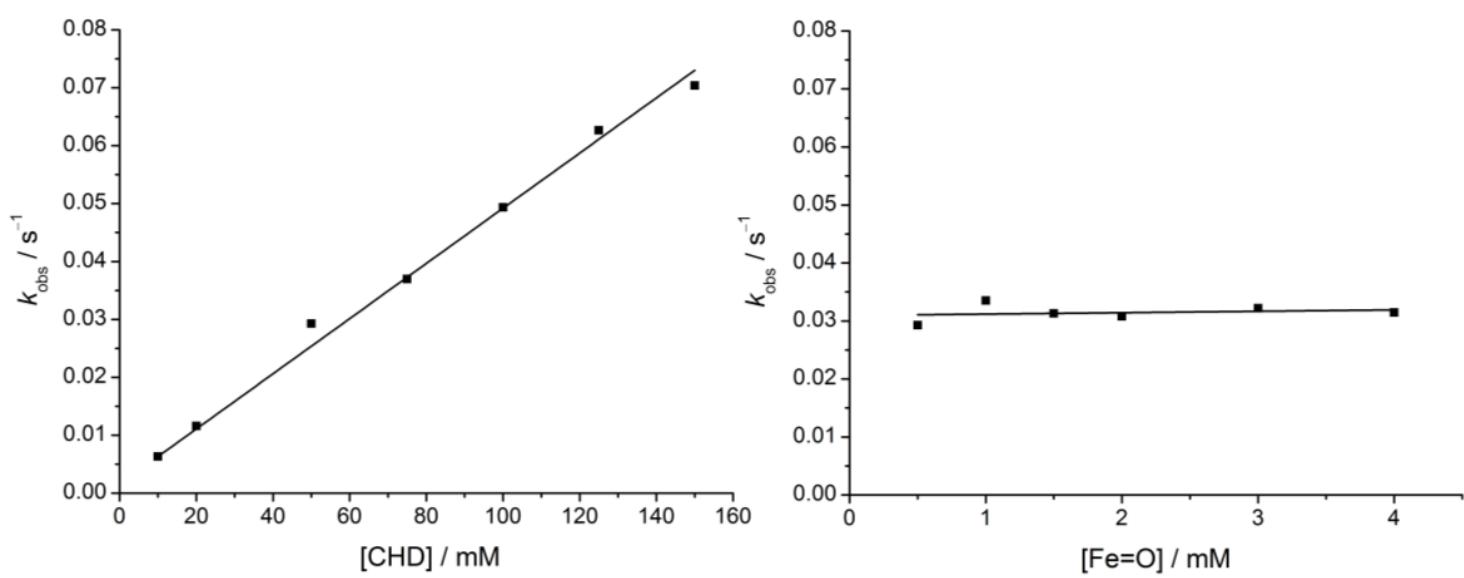

Figure 11. Left: Plot of the observed reaction rates $\left(k_{\text {obs }}\right)$ of $2(1 \mathrm{mM}) v s$. concentration of CHD at $-40{ }^{\circ} \mathrm{C}$ in MeCN. Right: Plot of the observed reaction rates $\left(k_{\mathrm{obs}}\right)$ with $[\mathrm{CHD}]=60 \mathrm{mM} v s$. concentration of 2 . $^{[179]}$

Furthermore, experiments varying the concentration of 2 while keeping the concentration of CHD constant $(60 \mathrm{mM})$, were performed. The observed $k_{\text {obs }}$ values were found to be constant in all experiments in agreement with $k_{\mathrm{obs}}=k_{2}$ [CHD] (Figure 11, right). This observation suggests that the process follows pseudo first order kinetics and that the reaction is a bimolecular process. ${ }^{[179]}$

As demonstrated in chapter 1.2.3, proton abstraction by an oxoiron(IV) species is expected to result in formation of a hydroxoiron(III) species. In order to gain evidence for the formation of $\mathrm{Fe}^{\mathrm{III}}-\mathrm{OH}$ 4, cryospray mass spectrometry experiments were performed. 2 was generated in situ at $-40{ }^{\circ} \mathrm{C}$ in $\mathrm{MeCN}$ and its initial spectrum was recorded. CHD (100 eq.) was added and the evolution of the spectrum was followed over time (Figure 12, left). The initial spectrum of $\mathbf{2}$ consists of two main peaks at $m / z=210.1$ and 569.1 (di- and mono-cationic species); only the mono-cationic signal is 
shown in Figure 12. Upon substrate addition, a significant increase of the peaks at $\mathrm{m} / \mathrm{z}=210.6$ and 570.1, which correspond to protonated and simultaneously reduced complex $\mathbf{4}$, is observed. At the same time, increase of a signal at $m / z=202.1$ is observed that is attributed to the decomposition of $\mu$-oxoiron(III) species 3 in MS. As mentioned above, $\mathbf{3}$ is highly unstable under harsh ionization conditions and breaks into the iron(II) species 1 and oxoiron(IV) 2. This fragmentation also explains the high intensity of the signal at $\mathrm{m} / z=569.1$ after reaction time of $120 \mathrm{~s}^{[179]}$
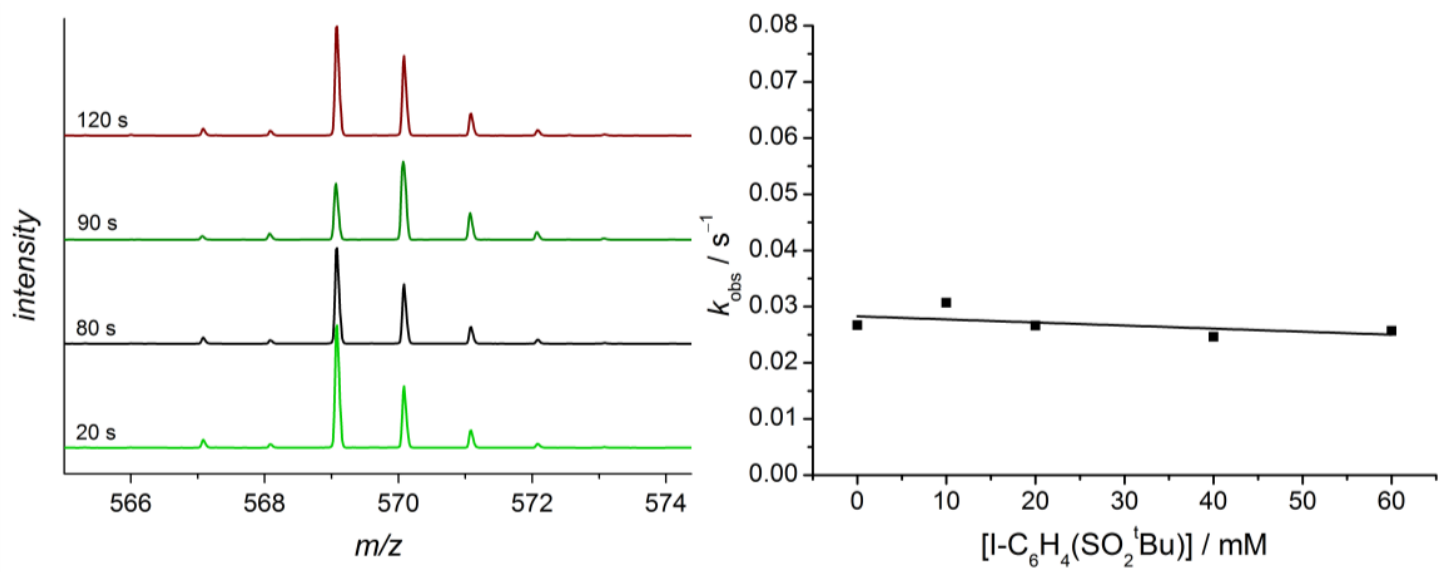

Figure 12. Left: Part of the cryospray mass spectrum of $2(0.25 \mathrm{mM})$ during the reaction with CHD $(25 \mathrm{mM})$. The signal at $m / z=569.1$ is assigned to $\left[\left({ }^{\mathrm{NHC}} \mathrm{L}\right) \mathrm{Fe}^{\mathrm{IV}} \mathrm{O}(\mathrm{OTf})\right]^{+}$and at 570.1 to $\left.\left[{ }^{\mathrm{NHC}} \mathrm{L}\right) \mathrm{Fe}^{\mathrm{III}} \mathrm{OH}(\mathrm{OTf})\right]^{+}$. Right: Plot of the observed reaction rates $\left(k_{\mathrm{obs}}\right)$ of $\mathbf{2}(1 \mathrm{mM}) v s$. concentration of I-Ar with [CHD] each $20 \mathrm{mM} \cdot{ }^{[179]}$

Interestingly, a peak at $m / z=372.0$ could be assigned to $\mathrm{Fe}^{\mathrm{IV}}=\mathrm{O} 2$ with additional coordination of the iodoarene derivative $\mathrm{I}_{-} \mathrm{C}_{6} \mathrm{H}_{4}-\left(\mathrm{SO}_{2}{ }^{\mathrm{t}} \mathrm{Bu}\right)$, which results from the reaction between soluble iodosylbenzene and 1. In 2014, Latour and Nam have described the formation of [(TMC)Fe ${ }^{\text {III }-O-I A r] ~(U V / v i s ~ a b s o r b a n c e ~} \lambda_{\max }=640 \mathrm{~nm}$ ) which decays very fast to form the corresponding $\mathrm{Fe}^{\mathrm{IV}}=\mathrm{O}$ species $\left(\lambda_{\max }=740 \mathrm{~nm}\right) \cdot{ }^{[180]}$ However, a similar existence of two different compounds was not observed in case of the tetracarbene system reported herein. Both the in situ and the crystalline $\mathrm{Fe}^{\mathrm{IV}}=\mathrm{O} 2$ show characteristic UV/vis absorbances at $\lambda_{\max }=400$ and $600 \mathrm{~nm}$. Moreover, the rate of reaction of $\mathbf{2}$ toward CHD was only very slightly affected by addition of high excess of I-Ar (Figure 12, right). If there was an equilibrium involving the coordination of this I-Ar species, the addition of a large excess would be expected to have a significant effect on the observed rates. All together, these results lead to the suggestion that the observed coordination only occurs due to effects in the cryospray mass spectrometer and can be neglected in the description of the reaction of 2 with CHD. ESI-MS studies carried out in Göttingen at harsher ionization conditions did not show formation of such an adduct while at the mass spectrometer in Prague the coordination of up to four I-Ar species was observed. The latter finding gives evidence for an interaction of the iodoarene derivative with the four carbene backbones. ${ }^{[179]}$ 
A suitable experimental approach for determining thermodynamic parameters is the Eyring plot. ${ }^{[181]}$ Therefore, the conversion of 2 with 20 eq. CHD was performed at varying temperature and the determined $k_{2}$ values were plotted according to equation (iii).

$$
\ln \frac{k}{T}=\frac{-\Delta H^{\ddagger}}{R} \cdot \frac{1}{T}+\ln \frac{k_{B}}{h}+\frac{\Delta S^{\ddagger}}{R}
$$

As expected, the reaction proceeds faster with rising temperature. The observed $k_{\mathrm{obs}}$ values correspond to $k_{2}=k_{\mathrm{obs}} / 20$ and $\ln \left(k_{2} / T\right)$ was plotted $v s$. 1/T. As it is shown in Figure 13, the data led to a very nice linear fit which allows to derive values for $\Delta H^{\star}=35.6 \mathrm{~kJ} \mathrm{~mol}^{-1}$ and $\Delta S^{\ddagger}=-96.0 \mathrm{~J} \mathrm{~K}^{-1} \mathrm{~mol}^{-1}$. The corresponding thermodynamic parameters in $\mathrm{kcal} \mathrm{mol}^{-1}$ are given in Table 2. ${ }^{[179]}$

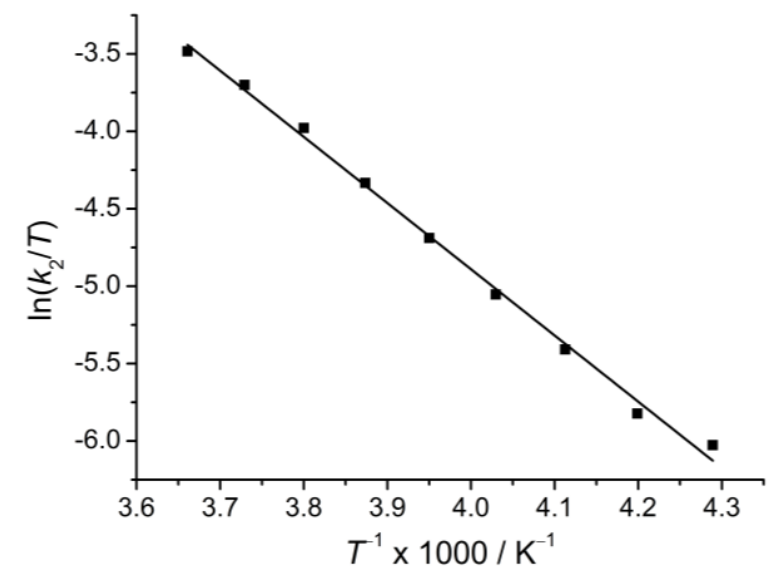

Figure 13. Eyring plot for the reaction of $2(1 \mathrm{mM})$ with CHD $(20 \mathrm{mM})$ at various temperatures $\left(-40-0{ }^{\circ} \mathrm{C}\right)$. Reprinted with permission of ACS. ${ }^{[179]}$

Table 2. Thermodynamic parameters for the reaction of 2 with CHD and DHA. ${ }^{[179]}$

\begin{tabular}{ccc}
\hline & {$\left[\left({ }^{\mathrm{NHC}} \mathbf{L}\right) \mathbf{F e O}\right]^{2+}$ with CHD } & {$\left[\left({ }^{\mathrm{NHC}} \mathbf{L}\right) \mathbf{F e O}\right]^{2+}$ with DHA } \\
\hline $\boldsymbol{\Delta} \boldsymbol{H}^{\ddagger} / \mathrm{kcal} \mathrm{mol}^{-1}$ & $8.5(2)$ & $8.5(2)$ \\
$\boldsymbol{\Delta} \boldsymbol{S}^{\ddagger} / \mathrm{cal} \mathrm{K}^{-1} \mathbf{~ m o l}^{-1}$ & $-22.9(8)$ & $-22.4(7)$ \\
$\boldsymbol{\Delta} \boldsymbol{G}_{\mathbf{}_{\mathbf{0}{ }^{\circ} \mathrm{C}} / \mathrm{kcal} \mathrm{mol}^{-1}}$ & $15.2(9)$ & $15.1(8)$ \\
\hline
\end{tabular}

In conclusion, the reaction of oxoiron(IV) complex 2 with CHD was studied in detail and its kinetic and thermodynamic parameters were determined. First evidence for the formation of a hydroxoiron(III) intermediate $\mathbf{4}$ was found, detailed studies on the formation of this species are presented in section 4.4. The weakest $\mathrm{C}-\mathrm{H}$ bond in $\mathrm{CHD}$ has a bond dissociation energy (BDE) of $78 \mathrm{kcal} \mathrm{mol}^{-1}$. Within the next section, the investigation of HAA on substrates with slightly higher and lower BDEs is presented. ${ }^{[179]}$ 


\subsection{C-H Bond Activation of Other Substrates}

The process of $\mathrm{C}-\mathrm{H}$ bond activation using 9,10-dihydroanthracene $\left(\mathrm{DHA}, \mathrm{BDE}=77 \mathrm{kcal} \mathrm{mol}^{-1}\right)$ as a substrate was followed in analogy to experiments carried out with CHD (Figure 14, left). Since the $\mathrm{C}-\mathrm{H}$ bond dissociation energy of the weakest $\mathrm{C}-\mathrm{H}$ bond of DHA is smaller compared to $\mathrm{CHD}$ $\left(78 \mathrm{kcal} \mathrm{mol}^{-1}\right)$, the reaction was slightly faster. In contrast to CHD, DHA is a solid at RT and only sparsely soluble in MeCN and had to be dissolved in DCM before addition. Because of this experimental issues, only up to 50 eq. of DHA could be added to the reaction mixture. Higher amounts of DHA led to precipitation inside the cuvette. Besides this limitation, the observed $k_{\mathrm{obs}}$ values gave a good linear fit with a slope of $7.57 \cdot 10^{-4} \mathrm{M}^{-1} \mathrm{~s}^{-1}$ corresponding to $k_{2}{ }^{\prime}=0.19 \mathrm{M}^{-1} \mathrm{~s}^{-1}$. [179]
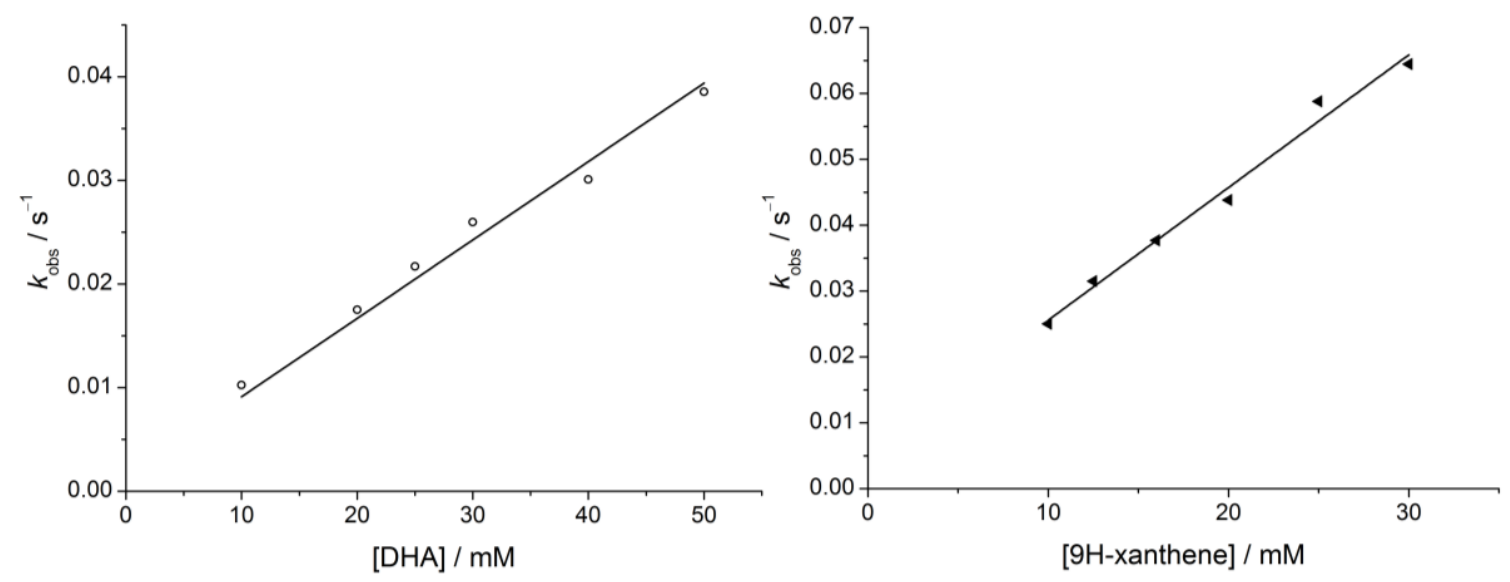

Figure 14. Plot of the observed reaction rates $k_{\mathrm{obs}}$ of $2(1 \mathrm{mM}) v s$. concentration of DHA (left) and $9 H$-xanthene (right) at $-40{ }^{\circ} \mathrm{C}$ in $\mathrm{MeCN} .{ }^{[179]}$

The reaction of 2 toward excess of DHA was also followed at $-20{ }^{\circ} \mathrm{C}\left(k_{2}{ }^{\prime}=0.72 \mathrm{M}^{-1} \mathrm{~s}^{-1}\right)$ and $0{ }^{\circ} \mathrm{C}$ $\left(k_{2}{ }^{\prime}=2.8 \mathrm{M}^{-1} \mathrm{~s}^{-1}\right.$, Appendix Figure 1). The obtained $k_{2}$ values were plotted according to the Eyring equation (iii) and show a good linear correlation. The resulting activation parameters (Table 2) are very similar compared to the values determined for $\mathrm{CHD}$, in good agreement with the similar bond dissociation energy for the weakest $\mathrm{C}-\mathrm{H}$ bond in both substrates. ${ }^{[179]}$

Additionally, the reaction with DHA was used to determine the yield in $\mathrm{C}-\mathrm{H}$ activation using GC-FID methods. Therefore, the reaction of crystalline material of $\mathrm{Fe}^{\mathrm{IV}}=\mathrm{O} 2$ with 10 eq. of DHA was performed two times and stopped by filtration through silica after 3 min and $1 \mathrm{~h}$. According to GC-FID, the only observed product was anthracene suggesting a mechanism without oxygen rebound (compare chapter 1 , Scheme 4 ) ${ }^{[64]}$ Consequently, $\mathrm{Fe}^{\mathrm{IV}}=\mathrm{O}$ complex 2 is acting as a $1 \mathrm{e}^{-}$ oxidant only and two high-valent iron centers are required to form one anthracene molecule. According to this finding, the maximum yield (100\%) of the reaction corresponds to the formation of 0.5 eq. anthracene with respect to $\mathrm{Fe}^{\mathrm{IV}}=\mathrm{O} 2$ ( 1 eq.). Based on GC-FID, yields of $64 \%$ (0.32 eq.) after $3 \mathrm{~min}$ and of $74 \%(0.37 \mathrm{eq}$.$) after 1 \mathrm{~h}$ were observed. The obtained data allowed for 
comparison of the yield after the initial process, which takes about 3 min with 10 eq. of DHA, with the yield after $60 \mathrm{~min}$. After $3 \mathrm{~min}$, the maximum intensity of the absorbance at $\lambda_{\max }=448 \mathrm{~nm}$ was reached. As described before, this likely corresponds to the formation of $\mathrm{Fe}^{\mathrm{III}}-\mathrm{OH}$ complex 4 and therefore a significant yield of anthracene is already expected at this point. In line with this assumption, the major amount (87\%) of the final yield was already formed after 3 min reaction time. ${ }^{[179]}$

Furthermore, the process of HAA was also studied using $9 H$-xanthene - a substrate with a markedly weaker $\mathrm{C}-\mathrm{H}$ bond dissociation energy $\left(75.5 \mathrm{kcal} \mathrm{mol}^{-1}\right)$ compared to CHD. Consequently, the reaction was much faster and the initial process only took about 20 seconds. Therefore, the concentration of substrate could not be increased to more than 30 eq. As shown in Figure 14 (right), a good linear correlation of the $k_{\text {obs }}$ values $v s$. concentration of xanthene was observed. A $k_{2}$ ' of $1.1 \mathrm{M}^{-1} \mathrm{~s}^{-1}$ was obtained, which is approximately one order of magnitude higher than for CHD. ${ }^{[179]}$

Finally, various equivalents of $9 H$-fluorene with a significantly stronger $\mathrm{C}-\mathrm{H}$ bond $\left(\mathrm{BDE}=80 \mathrm{kcal} \mathrm{mol}^{-1}\right)$ were added to in situ generated $\mathrm{Fe}^{\mathrm{IV}}=\mathrm{O} 2$ and the formation of the band at $\lambda_{\max }=448 \mathrm{~nm}$ was followed over time. As expected, this reaction was much slower compared to the other substrates. Additionally, using more than 80 eq. of substrate led to precipitation as described for DHA and xanthene. Still, the plot of $k_{\mathrm{obs}} v s$. concentration of fluorene as shown in Figure 15 (left) gave a good linear fit with a very small $k_{2}$ value of $6.44 \cdot 10^{-3} \mathrm{M}^{-1} \mathrm{~s}^{-1}$ and $k_{2}$ ' of $3.22 \cdot 10^{-3} \mathrm{M}^{-1} \mathrm{~s}^{-1}$ which is about two orders of magnitude smaller compared to CHD. The differences in the reaction rates among the four substrates are illustrated in Figure 15 (right). Especially the fast reaction with xanthene and the very slow conversion in case of fluorene stand out. With 2,3-dimethylbutane $\left(\mathrm{BDE}=84 \mathrm{kcal} \mathrm{mol}^{-1}\right)$ no reaction could be observed. ${ }^{[179]}$
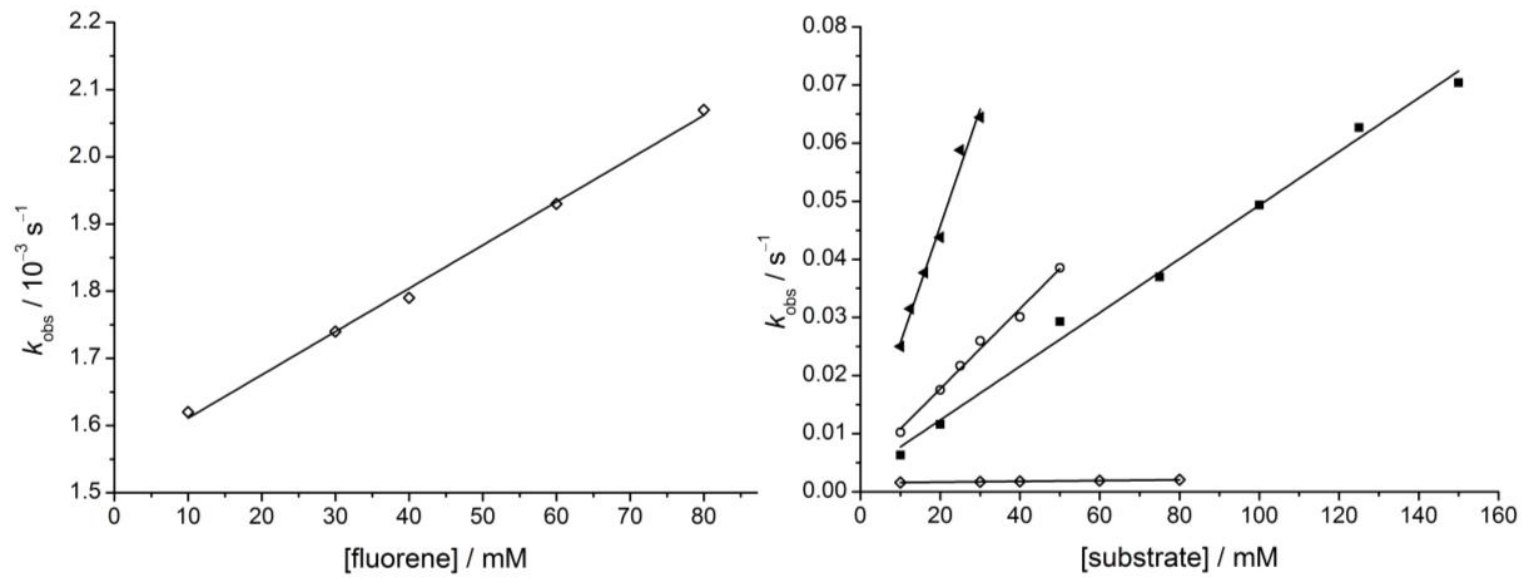

Figure 15. Left: Plot of the observed reaction rates $\left(k_{\text {obs }}\right)$ of $2(1 \mathrm{mM}) v s$. concentration of fluorene at $-40{ }^{\circ} \mathrm{C}$ in MeCN. Right: Observed rates for the reaction of $\mathrm{Fe}^{\mathrm{IV}}=\mathrm{O} 2$ with various substrates ( 4 xanthene, $\circ \mathrm{DHA}$, $\mathrm{CHD}, \diamond$ fluorene). Reprinted with permission of ACS. ${ }^{[179]}$ 
Table 3. Kinetic properties of $\mathrm{Fe}^{\mathrm{IV}}=\mathrm{O}$ complex 2 in $\mathrm{C}-\mathrm{H}$-bond activation of various substrates. ${ }^{[179]}$

\begin{tabular}{|c|c|c|c|c|}
\hline & BDE $\left(\mathrm{kcal} \mathrm{mol}^{-\mathbf{1}}\right)$ & $k_{2}\left(\mathrm{M}^{-1} \mathrm{~s}^{-1}\right)$ & $k_{2}^{\prime}\left(\mathrm{M}^{-1} \mathrm{~s}^{-1}\right)$ & $\log \left(k_{2}^{\prime}\right)$ \\
\hline Xanthene & 75.5 & 2.2 & 1.1 & 0.041 \\
\hline DHA & 77 & 0.76 & 0.19 & -0.723 \\
\hline 1,4-CHD & 78 & 0.47 & 0.12 & -0.924 \\
\hline Fluorene & 80 & 0.0064 & 0.0032 & -1.49 \\
\hline 2,3-Dimethylbutane & 84 & & no reaction & \\
\hline
\end{tabular}

The obtained kinetic parameters are summarized in Table 3. For all substrates, $\log \left(k_{2}^{\prime}\right)$ was plotted $v s$. the bond dissociation energy of the weakest $\mathrm{C}-\mathrm{H}$ bond (Figure 16). The correlation gives a good linear fit with a slope of -0.39 , which resembles the value obtained for previously reported oxoiron(IV) complexes. ${ }^{[65,70,83,182]}$ The observed sensitivity of the reaction rate to the BDE of the substrate is indicative of $\mathrm{H}$-atom abstraction as the first step of the process of $\mathrm{C}-\mathrm{H}$-bond activation by $2 .^{[179]}$

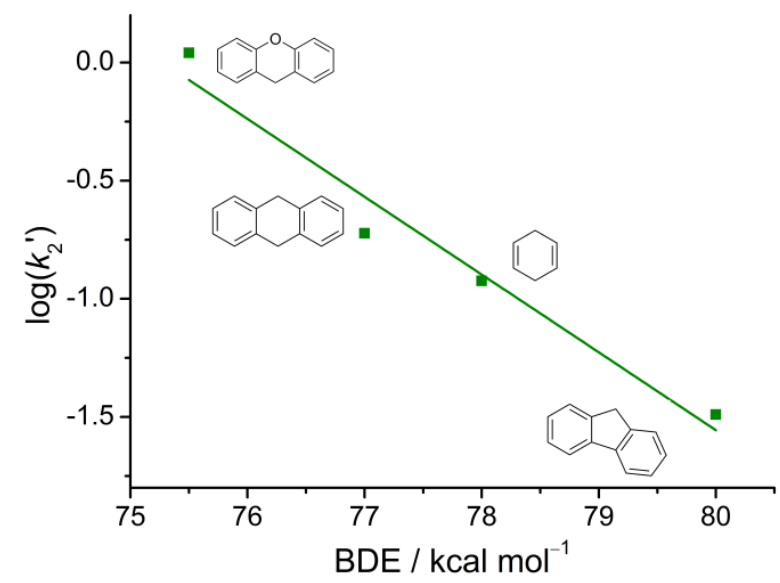

Figure 16. Plot of $\log \left(k_{2}{ }^{\circ}\right)$ against the $\mathrm{C}-\mathrm{H}$ BDE of different substrates for 2. Reprinted with permission of ACS. ${ }^{[179]}$

In summary, the kinetics of the reaction of $\mathbf{2}$ toward five different substrates has been analyzed. Several indications point to a stepwise mechanism with HAA as the first step. First, the changes in UV/vis absorbance clearly indicate a two-step process. In particular, the kinetic trace of the absorbance at $\lambda_{\max }=448 \mathrm{~nm}$ shows the most drastic changes upon substrate activation since it increases during the first and decreases during the second process. In a similar period of time, a signal likely corresponding to the $\mathrm{Fe}^{\mathrm{III}}-\mathrm{OH}$ species $\mathbf{4}$ is detected in mass spectrometry. In agreement with mechanisms reported in the literature, it was concluded that the first step consists of $\mathrm{H}$-atom abstraction by oxoiron(IV) 2 and formation of the corresponding hydroxoiron(III) complex 4. Consequently, product formation should be largely accomplished at this point which was verified experimentally by GC-FID analysis. ${ }^{[179]}$ 
Further evidence for a HAA mechanism might be provided by labeling experiments with deuterated substrates. According to reports in literature, ${ }^{[63,88]}$ detection of highly reactive intermediates such as $\mathbf{4}$ is possible with EPR spectroscopy. The results of these experiments are presented in the following section. ${ }^{[179]}$ 


\subsection{Characterization of the HAA Mechanism by Labeling Experiments and EPR Spectroscopy}

To gain further insight into the mechanism of HAA, deuterated dihydroanthracene was used to study the kinetic isotope effect (KIE) at three different temperatures (Figure 17, left). As summarized in Table 4, the reaction with DHA- $d_{4}$ is much slower than with DHA in all cases; comparative plots are also given in Appendix Figure 2. Especially at $-40{ }^{\circ} \mathrm{C}$, a remarkably high KIE was observed while the deperature dependence shows the expected decrease of the KIE with increasing temperature. Similarly high KIEs at low temperatures have been reported for taurine $\alpha$-KG dioxygenase (TauD) and highly reactive non-heme iron compounds that are able to oxidize ethylbenzole whereas particulate methane monooxygenase (pMMO) and most of the cyclamcoordinated iron complexes show lower KIEs (10-20). ${ }^{[65,83,183,184]}$ However, all these unusually high values suggest non-classical hydrogen tunneling effects ${ }^{[185]}$ and are consistent with hydrogen atom abstraction as the rate determine step. Furthermore, recent theoretical work by Mandal and Shaik described high KIEs as indication for the reaction to proceed via a $S=1$ reactive spin state rather than $S=2 .{ }^{[186]}$ This is in perfect agreement with the observed large triplet-quintet separation in 2 and the expected single-state mechanism along the triplet surface. Additionally, they observed that very high KIEs as experimentally observed for $\mathbf{2}$ correspond to rather thermoneutral reactions that allow for tunneling on the entire energy profile. ${ }^{[179]}$
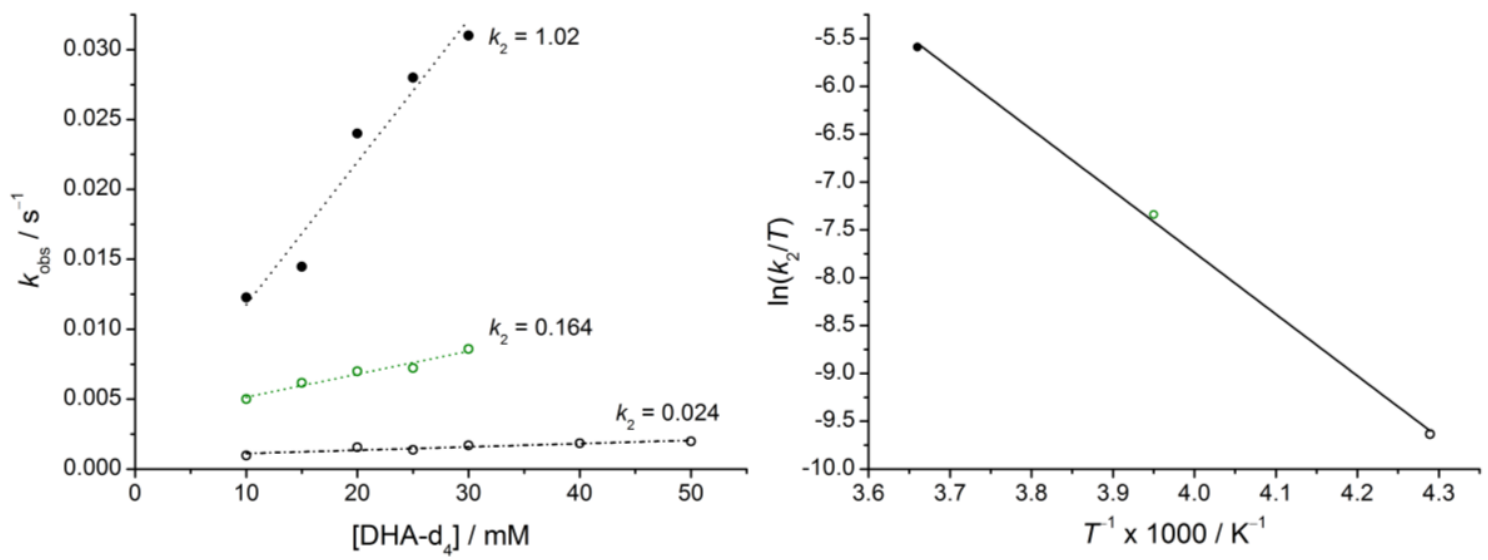

Figure 17. Plot of the observed reaction rates $k_{\mathrm{obs}}$ of $2(1 \mathrm{mM}) v s$. concentration of DHA- $\mathrm{d}_{4}$ at $-40{ }^{\circ} \mathrm{C}(\mathrm{\circ})$, $-20{ }^{\circ} \mathrm{C}(\circ)$ and $0{ }^{\circ} \mathrm{C}(\bullet)$ in $\mathrm{MeCN}$ (left) and the resulting Eyring plot (right). The $k_{2}$ values are given in $\mathrm{M}^{-1} \mathrm{~s}^{-1}$. Reprinted with permission of ACS. ${ }^{[179]}$

The determined $k_{2}$ values for the three different temperatures were also plotted according to the Eyring equation (iv) showing a very good linear correlation (Figure 17, right). The observed slope of the linear fit corresponds to an enthalpie of activation of $\Delta H^{*}=13.0 \pm 0.5 \mathrm{kcal} \mathrm{mol}^{-1}$ and the $y$-intercept gives rise to an entropie of activation of $\Delta S^{\ddagger}=-11.6 \pm 2.0 \mathrm{cal} \mathrm{K}^{-1} \mathrm{~mol}^{-1}$. Thus, the 
enthalpie of activation for the reaction of $\mathbf{2}$ toward a $\mathrm{C}-\mathrm{D}$ bond is significantly higher compared to activation of a $\mathrm{C}-\mathrm{H}$ bond in agreement with the higher $\mathrm{BDE}$ of the former. ${ }^{[179]}$

Table 4. Kinetic properties of $\mathrm{Fe}^{\mathrm{IV}}=\mathrm{O}$ complex 2 in $\mathrm{C}-\mathrm{H}-$ bond activation toward DHA and DHA- $\mathrm{d}_{4}$ at different temperatures and the corresponding KIEs. ${ }^{[179]}$

\begin{tabular}{|c|c|c|c|}
\hline & \multicolumn{2}{|c|}{$k_{2}\left(M^{-1} s^{-1}\right)$} & \multirow{2}{*}{$\begin{array}{l}\text { KIE } \\
k_{\mathrm{H}} / k_{\mathrm{D}}\end{array}$} \\
\hline & DHA & DHA-d ${ }_{4}$ & \\
\hline$-40{ }^{\circ} \mathrm{C}$ & 0.76 & $0.024(5)^{\mathrm{a}}$ & $32 \pm 8^{a}$ \\
\hline$-20{ }^{\circ} \mathrm{C}$ & 2.9 & 0.16 & 18 \\
\hline $\mathbf{0}^{\circ} \mathbf{C}$ & 11.3 & 1.0 & 11 \\
\hline
\end{tabular}

a: The error of these values was derived from repetitive experiments.

During a research stay at the MPI-CEC, the reaction was studied by EPR spectroscopy. Therefore, oxoiron(IV) 2 was generated in situ from iron(II) precursor $\mathbf{1}$ by addition of iodosylbenzene in a glove box and subsequently transferred into an EPR tube. In analogy to the UV/vis experiments, the $\mathrm{MeCN}$ solution was cooled to $-40{ }^{\circ} \mathrm{C}$ and an excess of CHD was added (10 eq.). The tube was closed tightly and frozen in liquid nitrogen after $\sim 2 \mathrm{~min}$. At this point, the solution had already changed its color from bright green to brownish corresponding to the changes observed in UV/vis. An EPR spectrum of the frozen sample was measured at $\sim 10 \mathrm{~K}$ and is shown in Figure 18. Simulation of the spectrum revealed the presence of three different species, all low spin $S=1 / 2$ in nature. The main species (58\%) shows $g$-values $2.33,2.14,1.94$ while the characteristics of a second rhombic species $(26 \%)$ are only slightly different $(g=2.30,2.17,1.95)$. Additionally, an axial signal with $g_{1}=2.05$ and $g_{2}=2.01$ is found at low intensity $(16 \%)$. Even though this sharp signal is reminiscent of an organic radical, its line-width is still indicative of a heavy atom contribution. All three signals could correspond to a hydroxoiron(III) species but an exact assignment appears to be rather challenging. To gain further insight, additional experiments were performed.

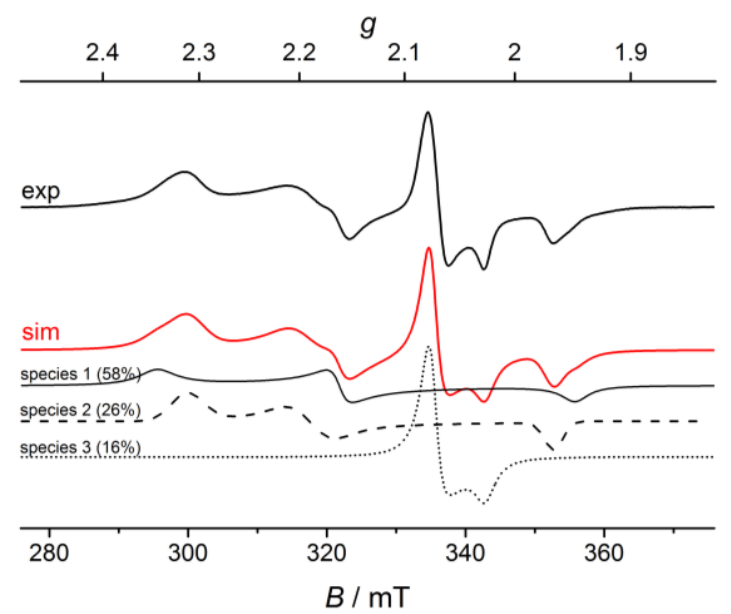

Figure 18. X-band EPR spectrum obtained upon reaction of in situ generated $2(1 \mathrm{mM})$ with 10 eq. of CHD $(\mathrm{MeCN}, 10 \mathrm{~K})$. 
A more concentrated sample of $2(3 \mathrm{mM})$ was used to determine the spin concentration by comparison with a copper standard. The reaction, frozen after about 2 min, showed a spin concentration of $\sim 0.5 \mathrm{mM}$. Furthermore, a time-dependent measurement was performed. Therefore, the reaction mixture with in situ generated 2 was frozen directly after addition of CHD (5 eq.) and an EPR spectrum at $\mathrm{t} \sim 10 \mathrm{~s}$ was recorded. A signal of rather small intensity was observed consisting of the described three sub-species. However, in this sample the intensity of the axial signal was found to be higher compared to the rhombic species. Still, repeated unfreezing of the sample in a cooling bath $\left(-40{ }^{\circ} \mathrm{C}\right)$ for about $30 \mathrm{~s}$ and subsequent EPR measurements in refrozen solution nicely showed a simultaneous increase and decrease in intensity of all signals with a maximum reached at $\sim 350 \mathrm{~s}$. Taking into account the differences in substrate concentration, this result correlates well with the observation made in UV/vis and Cryo-MS experiments described in section 4.2 (100 eq. CHD, maximum at $\sim 100 \mathrm{~s}$ ). The results are in good agreement with the proposed formation of the EPR-active hydroxoiron(III) species 4 during the first process which subsequently decomposes to the EPR-silent compounds $\mu$-oxoiron(III) $\mathbf{3}$ and iron(II) $\mathbf{1}$.

Looking for a simplified spectroscopic signature, the EPR studies were repeated with crystalline material of $\mathrm{Fe}^{\mathrm{IV}}=\mathrm{O} 2$. However, as depicted in Figure 19, left, the spectrum again consists of three subspecies. After $\sim 2 \min$ as well as $\sim 4$ min reaction time, an axial $S=1 / 2$ signal that resembles the $g$-values described for the reaction with in situ generated $\mathbf{2}$ is observed in very high intensity (78\%). Furthermore, a very small signal for a rhombic $S=1 / 2$ species is found, giving values similar to the two sub-species in the initial experiment $(g=2.32,2.15,1.95 ; 8 \%)$. Finally, a new axial signal typical for an $S=3 / 2$ compound with $g=4.00,4.00,2.00$ appears (14\%).
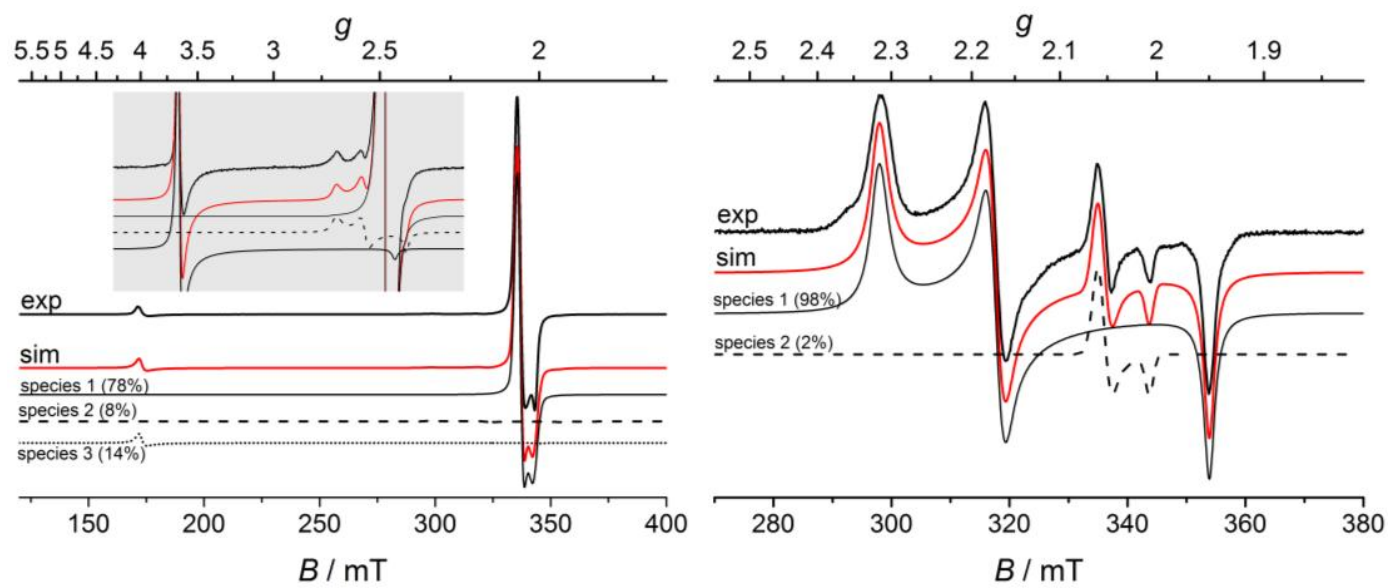

Figure 19. EPR spectra of the conversion of crystalline $2(3 \mathrm{mM})$ with 50 eq. CHD (left) and after addition of $\mathrm{I}-\mathrm{C}_{6} \mathrm{H}_{4}-\left(\mathrm{SO}_{2}{ }^{\mathrm{t}} \mathrm{Bu}\right)$ (6 eq., right) to this solution. The inset shaded in grey represents an enlargement of the spectral characteristics.

To gain further insight into the differences between reactions of in situ generated and crystalline material of 2,6 eq. of the iodoarene $\mathrm{I}_{-} \mathrm{C}_{6} \mathrm{H}_{4}-\left(\mathrm{SO}_{2}{ }^{\mathrm{t}} \mathrm{Bu}\right)$ were added to the latter yellowish sample 
leading to an immediate color change to brown and drastic changes in the EPR spectrum (Figure 19 , right). The $S=3 / 2$ signal vanishes while the rhombic $S=1 / 2$ signal increases in intensity. It accounts for $98 \%$ of the spin in the resulting sample; the remaining $2 \%$ correspond to the sharp axial $S=1 / 2$ signal. Consequently, excess of I-Ar seems to initiate conversion of the two axial species to the rhombic $S=1 / 2$ compound.

Based on these findings, assumptions about the nature of the observed species can be made. Considering the ligand field of tetragonal oxoiron species (Figure 20), an $S=3 / 2$ iron(III) system would correspond to a $\left(d_{\mathrm{xy}}\right)^{2}\left(d_{\mathrm{xz}} / d_{\mathrm{yz}}\right)^{2}\left(d_{\mathrm{z}^{2}}\right)^{1}\left(d_{\mathrm{x}^{2}-\mathrm{y}^{2}}\right)^{0}$ electronic configuration. To stabilize such a species, the $d_{z^{2}}$ orbital would have to be lowered in energy, for example through release of the axial solvent molecule. Consequently, the observed intermediate spin compound is expected to be the five-coordinated compound $\left[\left({ }^{\mathrm{NHC}} \mathrm{L}\right) \mathrm{Fe}{ }^{\mathrm{III}}-\mathrm{OH}\right](\mathrm{OTf})_{2}$ 4a. In contrast to this, the rhombic $S=1 / 2$ signal likely corresponds to a six-coordinated species. Since this species is largely favored in presence of excess I-Ar, axial coordination of the iodoarene may result in formation of the low spin six-coordintated species $\left[\left({ }^{\mathrm{NHC}} \mathrm{L}\right) \mathrm{Fe}{ }^{\mathrm{III}}-\mathrm{OH}(\mathrm{I}-\mathrm{Ar})\right](\mathrm{OTf})_{2}$ 4b. However, this seems to be in disagreement with the results described in section 4.2 that pointed toward coordination of I-Ar at the carbene backbone rather than axial coordination with no direct influence on the reactivity of 2 . Finally, the axial $S=1 / 2$ signal is found both in reactions starting from crystalline and in situ generated material. It is expected to be six-coordinated as well. Both axial coordination of a solvent molecule or a triflate counter anion are conceivable $(\mathbf{4 c})$. The latter case is supported by EPR studies on $\left[\left({ }^{\mathrm{NHC}} \mathrm{L}\right) \mathrm{Fe}^{\mathrm{III}} \mathrm{Cl}\right](\mathrm{OTf})_{2}(S=3 / 2)$. Upon addition of excess sodium triflate, a sharp signal $(g=2.05,2.05,2.01)$ similar to the axial signal observed herein appears in the broad spectrum of the five-coordinated chlorido compound (further details see chapter 6).

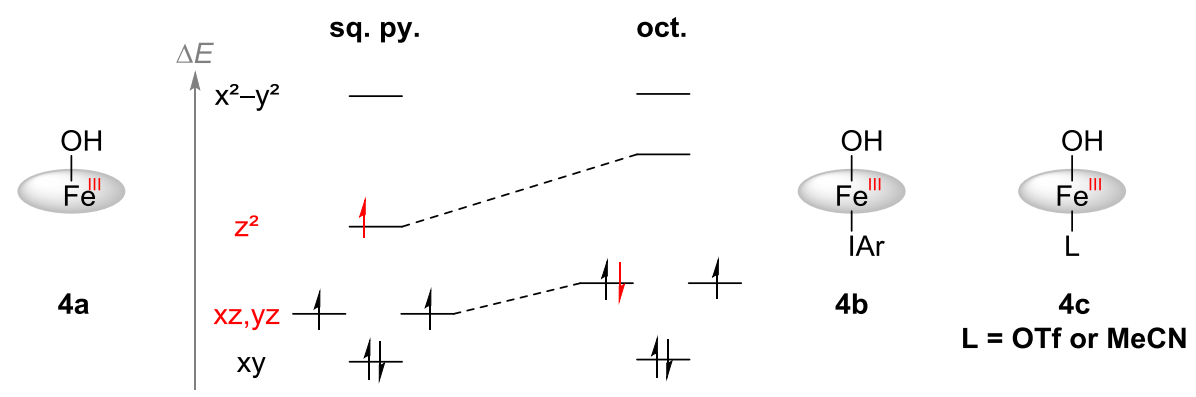

Figure 20. Ligand field splittings for $S=3 / 2$ (left) and $S=1 / 2$ (right) iron(III) species and the corresponding proposed hydroxoiron(III) compounds $\mathbf{4 a - 4 c}$.

Another possibility is the formation of a dinuclear iron(III)-iron(IV) species. The typical mechanistic scenario for decomposition of $\mathrm{Fe}^{\mathrm{III}}-\mathrm{OH}$ intermediates described in the literature consists in the dimerization of two $\mathrm{Fe}^{\mathrm{III}}-\mathrm{OH}$ species yielding the EPR-silent $\mathrm{Fe}^{\mathrm{III}}-\mathrm{O}-\mathrm{Fe}^{\mathrm{III}}$ complex and water (compare section 1.2.3). Presumably, the $\mathrm{Fe}^{\mathrm{III}}-\mathrm{OH}$ species could also react with the $\mathrm{Fe}^{\mathrm{IV}}=\mathrm{O}$ starting material and give an EPR-active $\mathrm{L}-\mathrm{Fe}^{\mathrm{IV}}-\mathrm{O}(\mathrm{H})-\mathrm{Fe}^{\mathrm{III}}-\mathrm{O}(\mathrm{H})$ compound. Thus, 
studies on $\mu$-oxodiiron(III) $\mathbf{3}$ examining this possibility in detail were performed. However, electrochemical oxidation of an $\mathrm{MeCN}$ solution of $\mathbf{3}$ in presence of hydroxide (1 eq. of $\left.\left[\mathrm{BnMe}_{3} \mathrm{~N}\right] \mathrm{OH}\right)$ did not lead to a detectable signal in the EPR spectrum.

Motivated by the observed differences between the reactions of in situ generated $\mathbf{2}$ and crystalline $\mathbf{2}$ with CHD, further UV/vis experiments were performed. Addition of excess I-Ar to both solutions of the in situ prepared $\mathrm{Fe}^{\mathrm{IV}}=\mathrm{O} 2$ and crystalline material led to similar results as described for the 'normal' kinetics reported in 4.2. However, since crystalline material already partly decomposes when dissolved in $\mathrm{MeCN}$, the observed UV/vis spectra are not as clean as for the in situ prepared reactions. When using the crystalline material only, the observed increase of the $448 \mathrm{~nm}$ trace during the first reaction process is significantly smaller. This is in agreement to the different colors observed for the rhombic $S=1 / 2$ species from in situ prepared 2 and the axial signal observed in measurements on crystalline starting material. Consequently, the band at $448 \mathrm{~nm}$ likely corresponds to the rhombic $S=1 / 2$ signals while the axial signals cannot be assigned to a prominent transition in the $\mathrm{UV} /$ vis region.

Thus, iodoarene, as it is present in the in situ generated reaction mixture, shows a distinct influence on the nature of the EPR active intermediates. However, while it seems to change the pathway of the reaction, the rate of the initial HAA remains unaffected by excess of I-Ar. 


\subsection{Theoretical Calculations}

To gain further insight into the reactivity of $\left[\left({ }^{\mathrm{NHC}} \mathrm{L}\right) \mathrm{Fe}^{\mathrm{IV}}=\mathrm{O}\right]^{2+}$ complex 2, DFT calculations were performed in cooperation with the MPI-CEC. As described in chapter 3, 2 possesses a triplet $(S=1)$ ground state which is energetically well separated from the quintet $(S=2)$ excited state by $18.7 \mathrm{kcal} \mathrm{mol}^{-1}$. Due to the extremely strong donating ability of the carbene ligand, the $d_{\mathrm{x}^{2} \mathrm{y}^{2}}$ orbital was calculated to be highest in energy; the $d_{z^{2}}$ orbital represents the LUMO of the ground state. Thus, the electron configurations of the lowest energy triplet and quintet states are $\left(d_{\mathrm{xy}}\right)^{2}\left(d_{\mathrm{xz}} / d_{\mathrm{yz}}\right)^{2}\left(d_{\mathrm{z}^{2}}\right)^{0}\left(d_{\mathrm{x}^{2}-\mathrm{y}^{2}}\right)^{0}$ and $\left(d_{\mathrm{xy}}\right)^{1}\left(d_{\mathrm{xz}} / d_{\mathrm{yz}}\right)^{2}\left(d_{\mathrm{z}^{2}}\right)^{1}\left(d_{\mathrm{x}^{2}-\mathrm{y}^{2}}\right)^{0}$, respectively. Moreover, calculations predict a huge difference in the $\mathrm{Fe}-\mathrm{O}$ bond length of $\mathbf{2}$ when going from triplet to quintet state. The optimized geometries of ${ }^{3} \mathbf{2}$ and ${ }^{5} \mathbf{2}$ demonstrate that the $\mathrm{Fe}-\mathrm{O}$ bond length elongates from 1.651 to 1.733 A due to occupation of the antibonding $\mathrm{d}_{\mathrm{z}^{2}}$ orbital. ${ }^{[179]}$

HAA processes for $\mathbf{2}$ were investigated on the triplet as well as quintet potential energy surfaces and are presented in Figure 21 (CHD) and Appendix Figure 3 (DHA). Furthermore, it was taken into account that $\mathbf{2}$ - as observed in the crystal structure - could still have an axial solvent molecule $(\mathbf{2}$, left $)$ or could react as five coordinate species $\left(\mathbf{2}^{\prime}\right.$, middle $) .{ }^{[179]}$
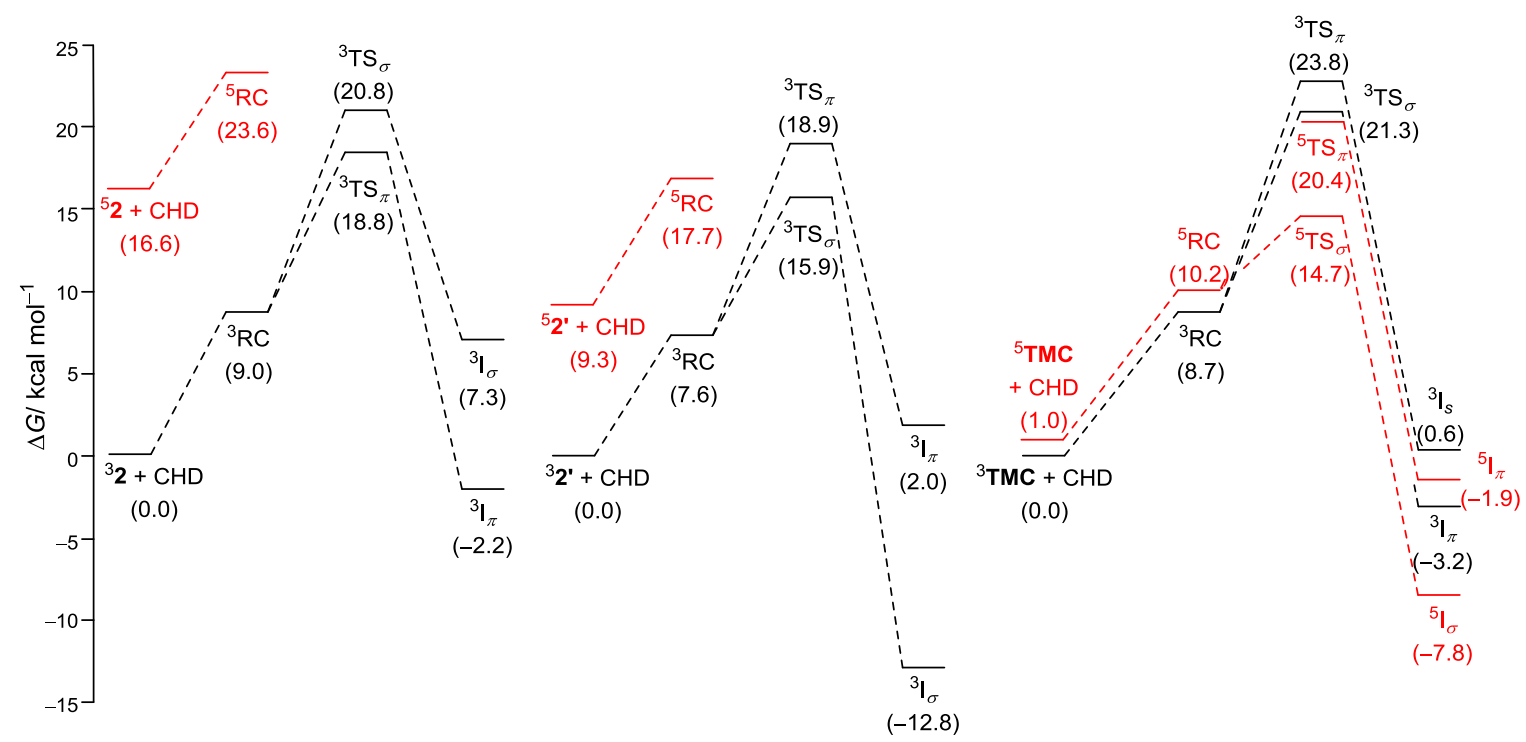

Figure 21. Reaction free energy profile for the first $\mathrm{H}$-atom abstraction of CHD by $\mathbf{2}$ (left), 2' (middle) and $\left.\left[{ }^{\mathrm{TMC}} \mathrm{L}\right) \mathrm{Fe}^{\mathrm{IV}}=\mathrm{O}(\mathrm{MeCN})\right]^{2+}($ TMC , right $) .{ }^{[179]}$

In a tetragonal $\mathrm{Fe}^{\mathrm{IV}}=\mathrm{O}$ geometry, the key frontier molecular orbitals likely to be involved in HAA are the singly occupied $\pi^{*} \mathrm{~d}_{\mathrm{xz}, \mathrm{yz}}$ and the typically unoccupied $\sigma^{*} \mathrm{~d}_{\mathrm{z}^{2}}$ orbitals (compare inset Scheme 16). ${ }^{[187]}$ In quintet ${ }^{5} \boldsymbol{2}$, the $d_{\mathrm{z}^{2}}$ (SOMO) is already populated with a spin-up electron and population with a second spin-up electron via the ${ }^{5} \sigma$-pathway is not possible. Therefore, only a spin-down electron can be transferred to the iron center with the ${ }^{5} \pi$-route being more favorable. Consequently, all attempts to locate the ${ }^{5} \sigma$ transition state converged to the corresponding ${ }^{5} \pi$ transition state. ${ }^{[179]}$ 


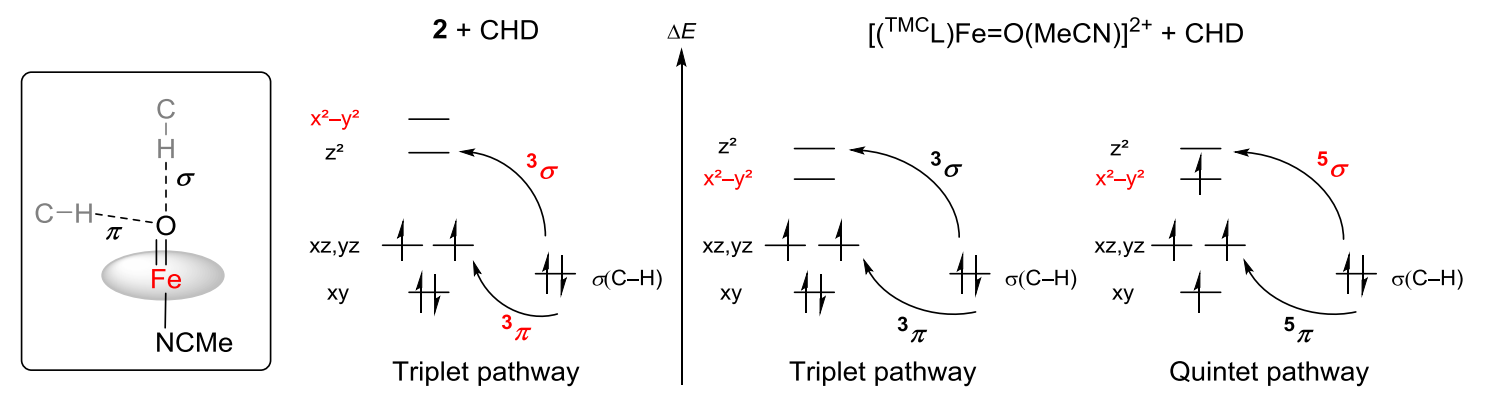

Scheme 16. Electron transfer diagram for the possible HAA pathways of $\mathbf{2}$ (left) and $\left[\left({ }^{\mathrm{TMC}} \mathrm{L}\right) \mathrm{Fe}^{\mathrm{IV}}=\mathrm{O}(\mathrm{MeCN})\right]^{2+}$ (right). The red color highlights the most favorable pathways as well as the differences in the energy of the $\mathrm{d}_{\mathrm{x}^{2}-\mathrm{y}^{2}}$ orbital. The inset on the left side gives a simplified comparison of $\sigma$ and $\pi$ pathway. ${ }^{[179]}$

Among all $\mathrm{H}$-atom abstraction pathways in $\mathbf{2}$ and 2', the triplet $\pi$ and $\sigma$ trajectories are the most efficient ones. On the Gibbs free energy scale, they show almost equal activation barriers of about $19 \mathrm{kcal} \mathrm{mol}^{-1}$ (2) and $16 \mathrm{kcal} \mathrm{mol}^{-1}\left(\mathbf{2}^{\prime}\right)$. Interestingly, the corresponding triplet transition states $\left({ }^{3} \mathbf{T S}_{\pi}\right.$ and $\left.{ }^{3} \mathbf{T S}_{\sigma}\right)$ are $\sim 3 \mathrm{kcal} \mathrm{mol}^{-1}$ lower in free energy than the quintet reaction complex ${ }^{5} \mathbf{R C}$, indicating that two-state-reactivity can be safely ruled out in the process of $\mathrm{C}-\mathrm{H}$ bond oxidation in 2 and 2'. Hence, the relative activity of the H-atom abstraction by 2 follows the descending order $\mathbf{3} \boldsymbol{\pi} \approx \mathbf{3} \boldsymbol{\sigma}>\mathbf{5} \boldsymbol{\pi}$. Scheme 16, left, shows the preferred triplet pathway for $\mathbf{2}$ toward CHD. In the case of the $\mathbf{3} \boldsymbol{\pi}$ pathway, only a small energetic difference between starting material $\left({ }^{3} \mathbf{2}+\mathrm{CHD}\right)$ and ${ }^{3} \mathbf{I}_{\pi}$ was calculated. ${ }^{[179]}$ This result is consistent with the conclusion by Mandal and Shaik that large KIEs correspond to rather thermoneutral reactions. ${ }^{[186]}$

Explanation for the similar activation energy calculated for ${ }^{3} \sigma$ - and ${ }^{3} \pi$-pathway is found in the energetic ordering of the orbitals. In case of $\mathbf{2}$, addition of one spin-up electron from the electron donating orbital $\left(\sigma_{\mathrm{C}-\mathrm{H}}\right)$ into the low-lying $\mathrm{Fe}-\mathrm{d}_{\mathrm{z}^{2}}$ orbital is of comparable energy as addition of a spin-down electron into $d_{x z, y z}$. However, upon occupation of the $d_{z^{2}}$ orbital, the trans axial $\mathrm{Fe}-\mathrm{N}_{\mathrm{ax}}$ distance (3.491 $\AA$ ) in the transition state ${ }^{3} \mathbf{T} \mathbf{S}_{\sigma}$ is significantly elongated, which leads to dissociation of $\mathrm{MeCN}$ from the iron center. A similar situation was found for the quintet reactant complex in which the Fe- $d_{z^{2}}$ orbital is already populated with one spin-up electron in the beginning. The preferred five-fold coordination is also represented by the slightly lower activation energy of 2 ' compared to 2. Additionally, the nature of the most favorable intermediate ' $\mathbf{I}$ ' differs for the reactions of $\mathbf{2}$ and 2'. While a slightly exergonic process leads to ${ }^{3} \mathbf{I}_{\boldsymbol{\pi}}$ in case of 6-coordinated species $\mathbf{2}$, a ${ }^{3} \mathbf{I}_{\sigma}$ intermediate of very low energy is found for the five-coordinated compound $\mathbf{2}$ '. This is in agreement with results obtained by EPR experiments as described in 4.4. Therein, the formation of intermediate ${ }^{3} \mathbf{I}_{\sigma}$ with an iron $S=3 / 2$ center coupled to a substrate radical via the ${ }^{3} \sigma$-pathway was only observed in the absence of I-Ar and is associated to a five-coordinated species. ${ }^{[179]}$ 
The calculated activation parameters for the $\mathrm{H}$-atom abstraction of CHD are in good agreement with the experimental results (Table 2). Notably, the $\mathrm{C}-\mathrm{H}$ bond activation of $\mathrm{CHD}$ and DHA features similar barriers of $\sim 19 \mathrm{kcal} \mathrm{mol}^{-1}$, which is in line with the fact that they possess similar $\mathrm{C}-\mathrm{H}$ bond dissociation energies (78 and $77 \mathrm{kcal} \mathrm{mol}^{-1}$, respectively). ${ }^{[179]}$

As already illustrated in Figure 21 and Scheme 16, the reactivity of 2 was compared with the well studied complex $\left[\left({ }^{\mathrm{TMC}} \mathrm{L}\right) \mathrm{Fe}^{\mathrm{IV}}=\mathrm{O}(\mathrm{MeCN})\right]^{2+}$ and significant differences have been found, which will be discussed in the following chapter. 


\subsection{Comparison of 2 with $\left[\left({ }^{\mathrm{TMC}} \mathrm{L}\right) \mathrm{Fe}=\mathrm{O}(\mathrm{MeCN})\right]^{2+}$ and Conclusions}

The experimentally obtained bi-molecular reaction rates allowed for a direct comparison with the values for $\left[\left({ }^{\mathrm{TMC}} \mathrm{L}\right) \mathrm{Fe}^{\mathrm{IV}}=\mathrm{O}(\mathrm{MeCN})\right]^{2+}$ reported in literature. ${ }^{[88]}$ The rates depicted in Table 5 clearly indicate that $\mathbf{2}$ shows higher HAA reactivity compared to the TMC-coordinated model by about two to three orders of magnitude depending on the substrates. Comparison with other literature known $\mathrm{Fe}^{\mathrm{IV}} \mathrm{O}$ compounds classifies $\left[\left({ }^{\mathrm{TMC}} \mathrm{L}\right) \mathrm{Fe}^{\mathrm{IV}}=\mathrm{O}(\mathrm{MeCN})\right]^{2+}$ to be of rather weak and $\mathbf{2}$ of average reactivity. ${ }^{[187]}$ The so far most reactive oxoiron(IV) complexes do not only show reaction rates up to four orders of magnitude higher than $\mathbf{2}$ but are also able to activate substrates with stronger $\mathrm{C}-\mathrm{H}$ bonds such as cyclohexane $\left(\mathrm{BDE}=99 \mathrm{kcal} \mathrm{mol}^{-1}\right) .{ }^{[185]}$ As described in the introduction, numerous reports on factors governing the oxidation and oxygenation reactivity of oxoiron(IV) compounds show crucial effects of the supporting ligands. ${ }^{[58]}$ Even small changes such as different axial ligands or varied chelate ring sizes can significantly increase or decrease the reaction rate. Considering this, it is not unexpected that the tetracarbene macrocycle leads to different reactivity in $\mathrm{C}-\mathrm{H}$ bond activation compared to the TMC system. Still, further insight on a theoretical level was required for a more thorough understanding of the reasons for the observed differences. ${ }^{[179]}$

Table 5. Comparison of the kinetic parameters of 2 and $\left[\left({ }^{\mathrm{TMC}} \mathrm{L}\right) \mathrm{Fe}^{\mathrm{IV}}=\mathrm{O}(\mathrm{MeCN})\right]^{[88]}$ at $-40{ }^{\circ} \mathrm{C} .{ }^{[179]}$

\begin{tabular}{ccc}
\hline & {$\left[\left({ }^{\mathrm{NHC}} \mathbf{L}\right) \mathbf{F e}^{\mathrm{IV}}=\mathbf{O}(\mathbf{M e C N})\right]^{2+}(2)$} & {$\left[\left({ }^{\mathrm{TMC}} \mathbf{L}\right) \mathbf{F e}^{\mathrm{IV}}=\mathbf{O}(\mathbf{M e C N})\right]^{2+}$} \\
\hline $\boldsymbol{k}_{\mathbf{2}}$ (Xanthene) & 2.2 & 0.066 \\
$\boldsymbol{k}_{\mathbf{2}}$ (DHA) & 0.76 & 0.0025 \\
$\boldsymbol{k}_{\mathbf{2}}$ (CHD) & 0.47 & $6.4 \cdot 10^{-4}$ \\
$\boldsymbol{k}_{\mathbf{2}}$ (Fluorene) & 0.0064 & $3.2 \cdot 10^{-5}$ \\
\hline
\end{tabular}

The calculations by Bhaskar Mondal and Shengfa Ye support the previous observation that the TMC complex has a triplet $(S=1)$ ground state and a quintet $(S=2)$ excited state which is close in energy $\left(2.7 \mathrm{kcal} \mathrm{mol}^{-1}\right)$. Unlike in $\mathbf{2}$, the $d_{\mathrm{z}^{2}}$ orbital of the TMC coordinated species was calculated to be highest in energy followed by the $d_{\mathrm{x}^{2}-\mathrm{y}^{2}}$ orbital similar to what was found for the SR-TPA coordinated complex studied in chapter 3 . Hence, the electronic configurations of the triplet and quintet states are $\left(d_{\mathrm{xy}}\right)^{2}\left(d_{\mathrm{xz}} / d_{\mathrm{yz}}\right)^{2}\left(d_{\mathrm{x}^{2}-\mathrm{y}^{2}}\right)^{0}\left(d_{\mathrm{z}^{2}}\right)^{0}$ and $\left(d_{\mathrm{xy}}\right)^{1}\left(d_{\mathrm{xz}} / d_{\mathrm{yz}}\right)^{2}\left(d_{\mathrm{x}^{2}-\mathrm{y}^{2}}\right)^{1}\left(d_{\mathrm{z}^{2}}\right)^{0}$, respectively. As a consequence, the $\mathrm{Fe}=\mathrm{O}$ bond is unaffected when going from the triplet to the quintet state while slightly elongated $\mathrm{Fe}-\mathrm{N}$ bonds in the equatorial plane $\left(d_{\mathrm{x}^{2}-\mathrm{y}^{2}}\right)$ are found. ${ }^{[179]}$

As shown in Figure 21 (right), among the four alternatives the quintet $\sigma$ route is calculated to have the lowest activation barrier $\left({ }^{5} \mathbf{T S}_{\boldsymbol{\sigma}}\right)$ in $\mathrm{H}$-atom abstraction reactivity. Furthermore, the ${ }^{\mathbf{5}} \boldsymbol{\sigma}$ route possesses the highest exergonicity to form intermediate ${ }^{5} \mathbf{I}_{\sigma}$. The reactivity of the first H-atom 
abstraction on CHD by $\left[\left({ }^{\mathrm{TMC}} \mathrm{L}\right) \mathrm{Fe}^{\mathrm{IV}}=\mathrm{O}(\mathrm{MeCN})\right]^{2+}$ decreases in the order ${ }^{5} \boldsymbol{\sigma}>{ }^{5} \boldsymbol{\pi} \approx^{\mathbf{3}} \boldsymbol{\sigma}>{ }^{\mathbf{3}} \boldsymbol{\pi}$. Despite the process via $^{3} \mathbf{I}_{\sigma}$, all reaction channels are exergonic. ${ }^{[179]}$

Consequently, the TMC coordinated oxoiron(IV) system follows the well-established reaction pathway via ${ }^{5} \mathbf{T S}_{\sigma}$ (Scheme 16, right) and represents a typical example for 'two-state reactivity'. Still, based on DFT calculations, a similar efficiency of the tetracarbene- and the TMC coordinated system in HAA is expected. However, the observed experimental differences of about 2 orders of magnitude correspond to only 2-4 $\mathrm{kcal} \mathrm{mol}^{-1}$. Given the well-accepted error range of DFT, an unambiguous prediction of reaction rates is not possible. ${ }^{[179]}$

It can be concluded that $\mathbf{2}$ serves as the first isolated 'single-state reactivity' model that reacts along the triplet surface only. Still, it shows intermediate efficiency in HAA compared to literature reported model complexes. In particular, it exceeds the $\left[\left({ }^{\mathrm{TMC}} \mathrm{L}\right) \mathrm{Fe}^{\mathrm{IV}}=\mathrm{O}(\mathrm{MeCN})\right]^{2+}$ system that reacts via the quintet surface. Consequently, the simplified assumption that higher reactivity can be ascribed to quintet pathways when compared to triplet routes must be reconsidered. These findings show the importance of spin state in oxoiron(IV) systems but also the limitations of rationalizing higher reactivity based on spin state only. Furthermore, the study presented herein elucidates the complexity of the interplay of different factors that determine the reactivity of high-valent $\mathrm{Fe}=\mathrm{O}$ complexes. ${ }^{[179]}$

Right before the completion of this work, Cantú Reinhard and de Visser published a similar theoretical study on the reactivity of an oxoiron(IV) system coordinated by a smaller tetracarbene macrocycle with methylene bridges only (compare chapter 1 , Scheme 10 ). ${ }^{[188]}$ Within their calculations, they mainly focused on the reactivity difference between the five-coordinated $\mathrm{Fe}=\mathrm{O}$ carbene complex and its six-coordinated $\mathrm{MeCN}-\mathrm{Fe}=\mathrm{O}$ analog in comparison with the naturally prevalent $[(\mathrm{Porph}) \mathrm{Fe}=\mathrm{O}(\mathrm{SH})]$ system. Their results on the ground state of the comparable carbenecoordinated iron(IV) $=\mathrm{O}$ systems agree well with the calculations presented in this work. In particular, they also find a triplet ground state energetically separated from its quinted excitet state and the same orbital order when compared to $\mathbf{2}$. While expmerimental studies on $\mathbf{2}$ did not show any OAT reactivity toward styrene, propene, and ethylbenzene, Cantú Reinhard and de Visser report relatively small activation barriers for the putative 5C system (13-16 kcal mol $\left.{ }^{-1}\right)$. Significantly larger barriers have been calculated for the 6C complex (21-23 kcal mol $\left.{ }^{-1}\right)$. Furthermore, they also describe the ${ }^{3} \boldsymbol{\sigma}$ pathway as the most favorable possibility among the initial $\mathrm{C}-\mathrm{H}$ bond activation processes on propene and ethylbenzene and conclude that the quinted surface cannot take a role in catalysis. Finally, they summarize that the calculated $5 \mathrm{C}$ system in the gas phase and in apolar solvents can keep pace with P450 CpdI models while coordination of a polar solvent $(\mathrm{MeCN})$ largely reduces the activity due to a rise in energy of the iron $\left(\mathrm{d}_{\mathrm{z}^{2}}\right)$ orbital. ${ }^{[179]}$ 


\section{Unprecedented Reactivity of a $\mu$-Oxodiiron(III) Species}

\subsection{Motivation and Prior Work}

Since the 1960s, autoxidation processes of heme and non-heme iron(II) systems have been studied at low temperature as well as room temperature. ${ }^{[189,190]}$ Upon reaction with oxygen, different intermediates have been observed; namely mono- or diiron peroxo species and high-valent oxoiron(IV) compounds. At RT, coupling of these reactive species leads to stable $\mu$-oxodiiron(III) species as the final reaction product. ${ }^{[191,192]}$ In agreement with this, Steffen Meyer reported on the formation of $\left[\left\{\left({ }^{\mathrm{NHC}} \mathrm{L}\right) \mathrm{Fe}\right\}_{2} \mathrm{O}\right](\mathrm{OTf})_{3} 3$ at RT while isolation of high-valent $\left[\left({ }^{\mathrm{NHC}} \mathrm{L}\right) \mathrm{Fe}=\mathrm{O}\right](\mathrm{OTf})_{2}$ species $\mathbf{2}$ was possible only at low temperature. ${ }^{[153]}$ As shown in the previous chapter, $\mathbf{2}$ is able to activate $\mathrm{C}-\mathrm{H}$ bonds with moderate efficiency. In contrast to oxoiron(IV) species, the oxidizing potential of the $\mathrm{Fe}^{\mathrm{III}}-\mathrm{O}-\mathrm{Fe}^{\mathrm{III}}$ unit is typically too low for oxidation reactions such as $\mathrm{C}-\mathrm{H}$ activation or oxygen atom transfer. ${ }^{[189,190]}$ However, due to their high stability in solution as well as under aerobic conditions they are frequently used for oxygenation reactions but additional oxidants are necessary in all cases. ${ }^{[193-198]}$ Oxidants such as hydrogen peroxide, peroxy acids or dioxygen generate reactive species such as (hydro)peroxo compounds. The $\mathrm{Fe}^{\mathrm{III}}-\mathrm{O}-\mathrm{Fe}^{\mathrm{III}}$ species themselves have shown catalytic activity for conversions such as transesterification or acylation only. ${ }^{[199]}$

As already mentioned in chapter 4 , the reaction of $\mathbf{2}$ with various substrates did not stop at formation of $\mathrm{Fe}^{\mathrm{III}}-\mathrm{O}-\mathrm{Fe}^{\mathrm{III}}$ species $\mathbf{3}$ but instead slow conversion leading to the final iron(II) product 1 was observed. Based on this initial observation, Iris Klawitter performed scrambling experiments, which indicated disproportionation of $\mathbf{3}$ in solution (compare chapter 4, Scheme 15). ${ }^{[155]}$ She concluded that $\mathbf{3}$ disproportionates in $\mathrm{MeCN}$ into $\mathbf{1}$ and 2; the latter one then acts as active species in the observed reactivity. These initial observations call for a detailed investigation of the reactivity of $\mathbf{3}$ in comparison to oxoiron(IV) species $\mathbf{2}$, including an accurate determination of the equilibrium constant for the disproportionation. 


\subsection{Oxygen Atom Transfer Reactivity Toward Trimethylphosphine}

As mentioned in the previous chapter, the oxoiron(IV) compound $\mathbf{2}$ showed no reactivity in oxygen atom transfer (OAT) toward thioanisole or triphenylphosphine. ${ }^{[154]}$ Only when using trimethylphosphine $\left(\mathrm{PMe}_{3}\right)$ as substrate, an oxygenation process significantly faster than self-decay of $\mathbf{2}$ was observed and could be followed by UV/vis spectroscopy (Figure 22, left).
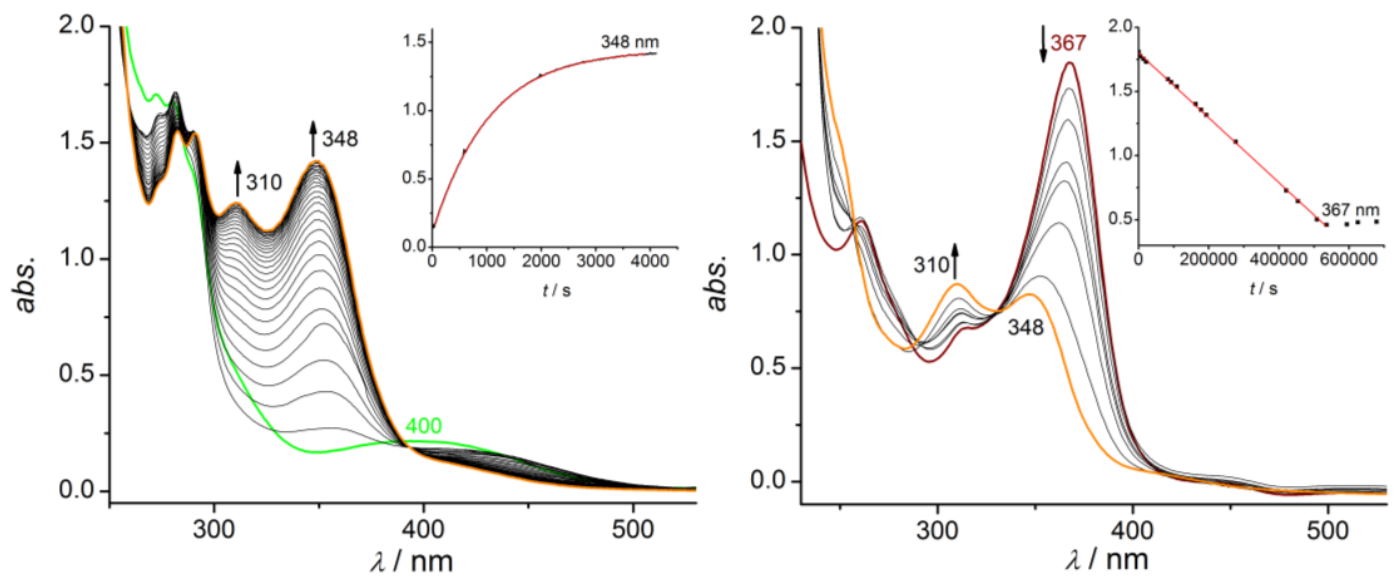

Figure 22. Spectral changes observed in OAT processes of 2 (left, $0.2 \mathrm{mM}, 100$ eq. $\mathrm{PMe}_{3}$ ) and 3 (right, $0.1 \mathrm{mM}, 400$ eq. $\mathrm{PMe}_{3}$ ) upon reaction with $\mathrm{PMe}_{3}$ at $0{ }^{\circ} \mathrm{C}$ in $\mathrm{MeCN}$. In both reactions, iron(II) bisphosphine complex 7 (orange) with its characteristic bands at $\lambda_{\max }=310$ and $348 \mathrm{~nm}$ is formed. The insets show the kinetic traces for the characteristic wavelengths $\lambda_{\max }=348$ (2) and $367 \mathrm{~nm}$ (3) that were used to determine $k_{\text {obs }}$ values.

Starting from the characteristic broad band of 2 at $\lambda_{\max }=400 \mathrm{~nm}$, addition of large excess of $\mathrm{PMe}_{3}$ led to formation of two new bands at $\lambda_{\max }=310$ and $348 \mathrm{~nm}$. Two-electron OAT processes of oxoiron(IV) species typically yield the corresponding iron(II) species as the final product. ${ }^{[200,201]}$ However, the characteristics of the final reaction mixture do not match with the UV/vis transition at $\lambda_{\max }=340 \mathrm{~nm}$ reported for $\left[\left({ }^{\mathrm{NHC}} \mathrm{L}\right) \mathrm{Fe}(\mathrm{MeCN})_{2}\right]^{2+} \mathbf{1}^{[153,154]}$ Therefore, ESI-MS measurements of the reaction mixture were performed and signals at $\mathrm{m} / \mathrm{z}=278.1$ and 705.1 were detected that have been assigned to the bis-(phosphino)iron(II) compound $7 \quad\left(\left[{ }^{\mathrm{NHC}} \mathrm{L}\right) \mathrm{Fe}\left(\mathrm{PMe}_{3}\right)_{2}\right]^{2+}$ and $\left[\left({ }^{\mathrm{NHC}} \mathrm{L}\right) \mathrm{Fe}\left(\mathrm{PMe}_{3}\right)_{2}\right](\mathrm{OTf})^{+}$, respectively). Coordination of excess phosphine to an iron(II) species has been reported for many different systems before. For example, Balch et al. already reported a similar finding in $1980 .{ }^{[202]}$ Still, the reaction was performed in larger scale and $\left[\left({ }^{\mathrm{NHC}} \mathrm{L}\right) \mathrm{Fe}\left(\mathrm{PMe}_{3}\right)_{2}\right](\mathrm{OTf})_{2}$ was crystallized by diffusion of $\mathrm{Et}_{2} \mathrm{O}$ into the $\mathrm{MeCN}$ reaction mixture at RT. As shown in Figure 2, the axial MeCN molecules in compound $\mathbf{1}$ are substituted by $\mathrm{PMe}_{3}$ in $\mathbf{7}$. This leads to significant structural changes compared to 1. 7 crystallizes in the monoclinic space group $P 2_{1} / \mathrm{n}$ instead of $P \overline{1}$ and, even more interesting, the iron sits on a crystallo-graphically imposed inversion center. The local symmetry around the iron center is $\mathrm{D}_{2 \mathrm{~d}}$. The $\mathrm{Fe}-\mathrm{C}$ bonds of the trans carbene donors are of exactly the same length (2.0206 and 2.0231 $\mathrm{A})$ and longer compared to 
1 (average $2.00 \AA$ ). Additionally, the Fe-P bonds are also identical and much longer than the corresponding $\mathrm{Fe}-\mathrm{NCMe}$ bonds in $\mathbf{1}$. Moreover, 7 shows accurate angles of $180^{\circ}$ as well as almost exact $90^{\circ}$ around the iron center resulting in a much more symmetric structure in $\mathbf{7}$ compared to the one found for $\mathbf{1}$ and other macrocyclic tetracarbene iron compounds presented before or as part of this work. ${ }^{[22,153,156]}$ Evidently, OAT from oxoiron(IV) 2 toward $\mathrm{PMe}_{3}$ yields the corresponding iron(II) species coordinated by excess of phosphine. Further proof for a single step OAT process ${ }^{[0]}$ was found by EPR spectroscopy. A frozen solution of the reaction mixture gave no signal, ruling out the occurrence of a paramagnetic iron(III) intermediate.
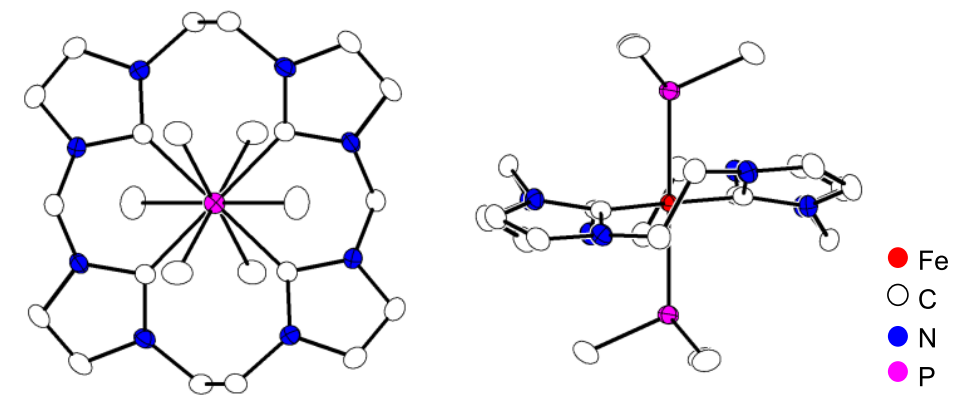

Figure 23. Molecular structure of the cation of 7 along the P-Fe-P axis (top view, left) and in the side view (right) (50\% probability thermal ellipsoids); anions and hydrogen atoms are omitted for clarity, important bond lengths and angles are given in chapter 13 .

With these findings in hand, detailed studies on the reactivity of $\mu$-oxo compound 3 toward $\mathrm{PMe}_{3}$ were performed. 3 shows a characteristic transition at $\lambda_{\max }=367 \mathrm{~nm}$ in the UV/vis region and addition of $\mathrm{PMe}_{3}$ led to slow decrease of this band and formation of the same final spectrum as it was found for the reaction of oxoiron(IV) 2 with $\mathrm{PMe}_{3}$ (Figure 22). As already described by Steffen Meyer, ${ }^{[153]} \mathbf{3}$ shows very strong antiferromagnetic coupling between the two iron centers leading to a silent X-band EPR spectrum and sharp signals in NMR spectra typical for diamagnetic species. Consequently, the conversion of $\mathbf{1}$ to $\mathbf{4}$ was also followed via ${ }^{1} \mathrm{H},{ }^{13} \mathrm{C}$ and ${ }^{31} \mathrm{P}-\mathrm{NMR}$ spectroscopy. The latter helped in particular to distinguish between free $\mathrm{PMe}_{3}(-62.1 \mathrm{ppm})$, coordinated $\mathrm{Fe}-\mathrm{PMe}_{3}(22.7 \mathrm{ppm})$ and oxidized $\mathrm{O}=\mathrm{PMe}_{3}(35.5 \mathrm{ppm})$. The obtained spectroscopic details are given in the experimental part. The main difference between the OAT process of $\mathbf{2}$ and $\mathbf{3}$ is the time needed for full conversion. While 2 oxidizes excess of $\mathrm{PMe}_{3}$ at $0{ }^{\circ} \mathrm{C}$ within a couple of hours, this process is much slower when starting from $\mathbf{3}$ and takes several weeks.

As shown in Scheme 17, the reaction of $\mathbf{3}$ with $\mathrm{PMe}_{3}$ occurs via a pre-equilibrium in which $\mathbf{3}$ disproportionates into $\mathbf{2}$ and $\mathbf{1}$. In the second step, both species react with the phosphine in a fast process yielding 7. However, the intermediates are not detectable within this process and further proof for the proposed equilibrium was desired. As already mentioned in 4.1, Iris Klawitter performed a scrambling experiment starting with the $\mu$-oxo compound $\mathbf{3}$ described herein and a similar one with methyl groups in the carbene backbones. While no scrambled species was found in 
a fresh mixture of the two $\mu$-oxo complexes, ESI-MS studies revealed slow formation of the scrambled $\mu$-oxo compound with different macrocyclic ligands coordinating each one of the iron centers. ${ }^{[155]}$ Moreover, the huge difference in reaction time when starting from $\mathbf{3}$ or $\mathbf{2}$ is indicative of an equilibrium lying mainly on the side of the $\mu$-oxo compound. Consequently, only small amounts of $\mathbf{2}$ and $\mathbf{1}$ would be formed and are expected to react immediately with the huge excess of substrate, in line with them not being detectable. Furthermore, comparison with literature shows that a similar but light induced disproportionation of $\mu$-oxoiron(III) compounds has already been observed before several times and has been reviewed. ${ }^{[189,190]}$ Moreover, Vanka, Gupta and coworkers recently presented reactivity studies on a $\mathrm{Fe}^{\mathrm{IV}}-\mathrm{O}-\mathrm{Fe}^{\mathrm{IV}}$ compound that shows a similar disproportionation into the corresponding $\mathrm{Fe}^{\mathrm{V}}=\mathrm{O}$ and $\mathrm{Fe}^{\mathrm{IIII}}$ species. ${ }^{[203]}$

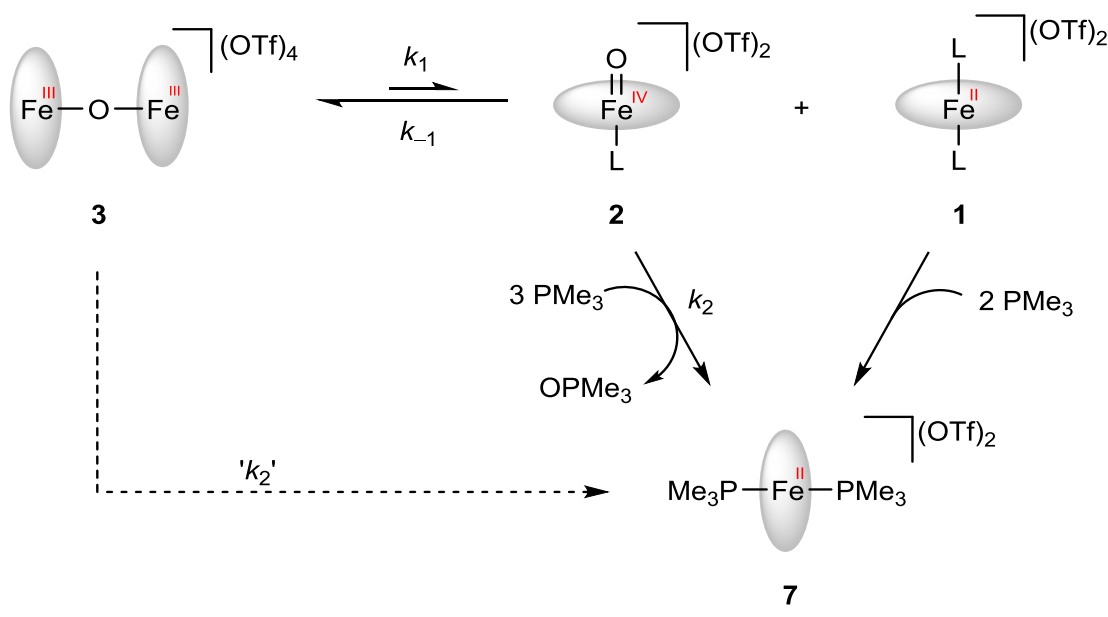

Scheme 17. Reaction scheme of the OAT process to $\mathrm{PMe}_{3}$ by $\mu$-oxodiiron(III) compound $\mathbf{3}(\mathrm{L}=\mathrm{MeCN})$.

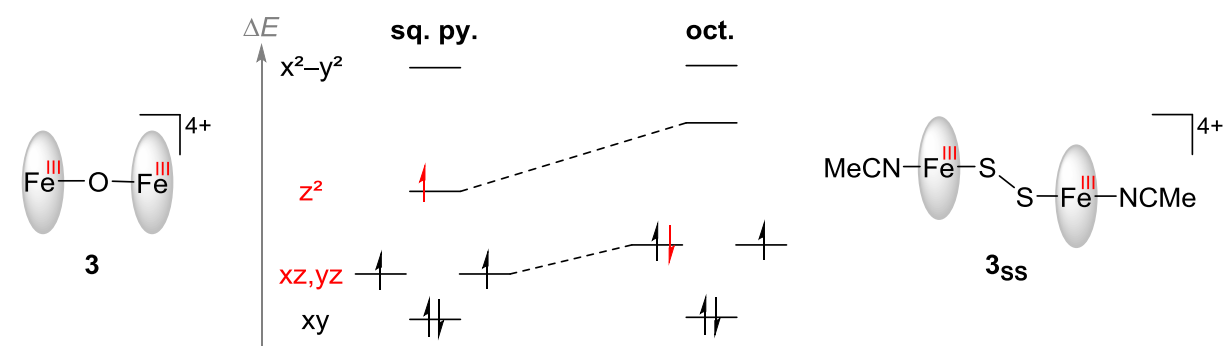

Figure 24. Comparison of the ligand field splittings in $\mathbf{3}$ and $\mathbf{3}_{\mathrm{ss}}$.

Explanation for the observed disproportionation is found in the electronic state of $\mathbf{3}$. As calculated by Oliver Krahe, ${ }^{[156]}$ the five coordinated iron centers in $\mathbf{3}$ are in the intermediate local spin state $(S=3 / 2)$ in contrast to the initially proposed low spin states. ${ }^{[153]}$ Thus, the $\mathrm{d}_{\mathrm{Z}^{2}}$ orbital is singly occupied and a five-fold coordination without a donating $\mathrm{MeCN}$ ligand in the axial position is favored over six-fold coordination (Figure 24). For comparison, the metal ions in the trans-1,2 endon disulfide-bridged iron(III) dimer $\mathbf{3}_{\mathrm{SS}}$ are coordinated octahedrally with additional MeCN ligands 
trans to the disulfide bridge, in agreement with their $S=1 / 2$ spin states. Consequently, it is likely that coordination of $\mathrm{MeCN}$ or $\mathrm{PMe}_{3}$ in solution induces cleavage of the $\mathrm{Fe}-\mathrm{O}-\mathrm{Fe}$ bond in 3 .

To allow for a direct comparison of both oxygenation processes, kinetic parameters for the reaction of 2 and 3 with $\mathrm{PMe}_{3}$ have been determined. Therefore, reactions of each complex solution (2: $0.2 \mathrm{mM}, 3: 0.1 \mathrm{mM}$ in $\mathrm{MeCN}$ ) with various amounts of the substrate were carried out and the characteristic time trace in UV/vis $(2: 346 \mathrm{~nm}, 3: 367 \mathrm{~nm})$ was fitted to a single exponential in case of $\mathbf{2}$ and linear in case of $\mathbf{3}$. The obtained $k_{\mathrm{obs}}$ values were plotted $v s$. the corresponding substrate concentration and showed a good linear correlation (Figure 25). The $k_{2}$ values of $\mathbf{2}$ and $\mathbf{3}$ differ by three orders of magnitude. It should be noted that the ' $k_{2}$ ' value for $\mathbf{3}$ represents the overall reaction rate consisting of the equilibrium constant $K_{\text {eq }}=k_{1} / k_{-1}$ as well as the rate constant $k_{2}$ for the reaction of oxoiron(IV) 2 with $\mathrm{PMe}_{3}$.
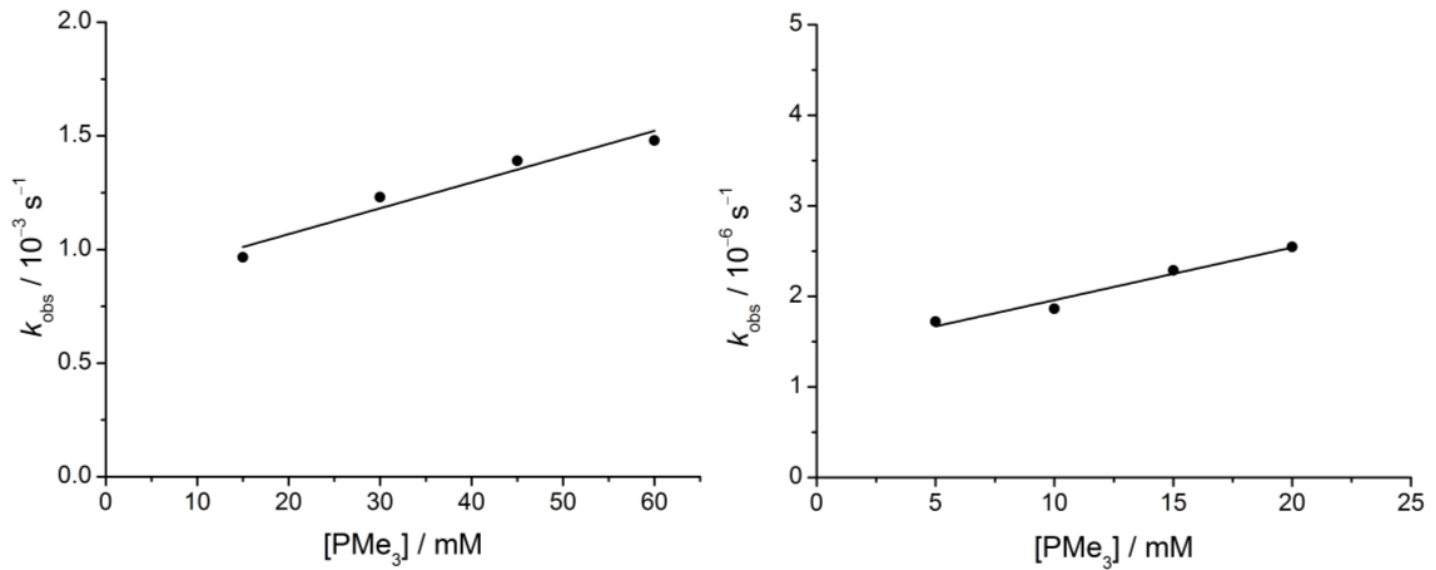

Figure 25. Kinetic data obtained for $\left.\left[{ }^{\mathrm{NHC}} \mathrm{L}\right) \mathrm{Fe}=\mathrm{O}(\mathrm{MeCN})\right](\mathrm{OTf})_{2} \mathbf{2}$ (left) and $\left[\left\{\left({ }^{\mathrm{NHC}} \mathrm{L}\right) \mathrm{Fe}\right\}_{2} \mathrm{O}\right](\mathrm{OTf})_{3} 3$ (right) with $\mathrm{PMe}_{3}$ at $0{ }^{\circ} \mathrm{C}$ in $\mathrm{MeCN}$.

In case of 2 , a $k_{2}$ value of $1.1 \cdot 10^{-2} \mathrm{~s}^{-1} \mathrm{M}^{-1}$ at $0{ }^{\circ} \mathrm{C}$ was determined, which is small compared to the $k_{2}$ values found for $\mathrm{C}-\mathrm{H}$ activation reactions by 2 at $-40{ }^{\circ} \mathrm{C}$ in agreement with the longer reaction time. The ' $k_{2}$ ' value for $\mathbf{3}\left(6.0 \cdot 10^{-5} \mathrm{~s}^{-1} \mathrm{M}^{-1}\right)$ is much smaller than the one obtained for 2 which indicates that the substrate concentration has only little influence on the reaction rate but ' $k_{2}$ ' rather depends on the equilibrium constant of the pre-equilibrium of $\mathbf{3}$ into $\mathbf{1}$ and $\mathbf{2}$, thus the disproportionation is the rate determining step. Moreover, the linear decrease of the absorbance at $\lambda_{\max }=367 \mathrm{~nm}$ for the reaction of $\mathbf{3}$ with $\mathrm{PMe}_{3}$ is indicative of a reaction of zero order. Since $\mathbf{1}$, once formed via the disproportionation of $\mathbf{3}$, reacts immediately with $\mathrm{PMe}_{3}$ giving $\mathbf{7}$, its concentration is always close to zero and thus the equilibrium is not effected by any changes in the ratio of 1:2 during the reaction and the concentration of $\mathbf{2}$ is constant during the whole process. Thus, it is included in the observed rate constant resulting in a linear behavior of $k_{\mathrm{obs}}$.

A suitable way to manipulate chemical equilibria is the subsequent addition of one of the involved species to shift the equilibrium to the other side. In this case, addition of the iron(II) species $\mathbf{1}$ is 
most promising since it is not an active species for neither OAT nor $\mathrm{C}-\mathrm{H}$ reactions. A similar strategy has already been used by Vanka and Gupta in their study on an active $\mathrm{Fe}^{\mathrm{IV}}-\mathrm{O}-\mathrm{Fe}^{\mathrm{IV}}$ compound. ${ }^{[203]}$ However, this method is not appropriate for OAT of $\mathbf{2}$ and $\mathbf{3}$ toward $\mathrm{PMe}_{3}$. As stated above, additional amounts of the iron(II) compound 1 would directly undergo $\mathrm{MeCN} \rightarrow \mathrm{PMe}_{3}$ ligand exchange and thus will be removed from the equilibrium. Consequently, a more suitable system was needed. Thus, the reactivity of $\mathbf{2}$ and $\mathbf{3}$ versus dihydroanthracene (DHA) was studied in a similar comparative way. 


\subsection{Hydrogen Atom Abstraction Reactivity}

As presented in section 4.3, the reaction of the oxoiron(IV) species 2 with DHA was studied in detail via UV/vis spectroscopy. No oxygenation but instead selective oxidation of DHA to anthracene was observed and a $k_{2}$ value of $0.76 \mathrm{~mol}^{-1} \mathrm{~s}^{-1}$ at $-40{ }^{\circ} \mathrm{C}$ was determined. With the help of activation parameters derived from DFT calculations and Eyring analysis (chapter 4), this value can be transferred to a $k_{2}$ of $\sim 35 \mathrm{~mol}^{-1} \mathrm{~s}^{-1}$ at RT. Having the kinetic data for 2 already available, the reaction of $\mathbf{3}$ with various excess of DHA was studied (Scheme 18).

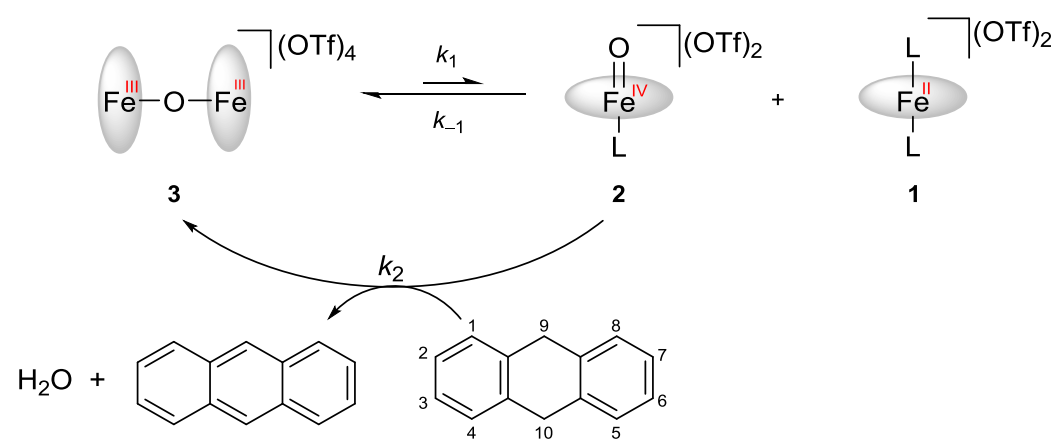

Scheme 18. Reaction scheme of the $\mathrm{C}-\mathrm{H}$ activation process toward dihydroanthracene by $\mu$-oxodiiron(III) 3 .

Initial experiments were followed via UV/vis measurements in analogy to the studies described in 5.2, but absorption spectroscopy turned out to be no suitable method in this case. The observed bands for starting material $3(367 \mathrm{~nm})$, anthracene $(338,356,376 \mathrm{~nm})$, and product $1(340 \mathrm{~nm})$ overlapped and no consistent data could be obtained. Instead, the reaction was followed by ${ }^{1} \mathrm{H}-\mathrm{NMR}$ spectroscopy which gave reliable and consistent data. Under fully inert conditions, $\mathbf{1}$ and DHA selectively convert to $\mathbf{3}$ and anthracene, giving literature reported NMR spectra. ${ }^{[153]}$ Compared to CHD - a substrate well suited for HAA reactivity studies due to its high solubility DHA was preferred for these studies due to the characteristic ${ }^{1} \mathrm{H}-\mathrm{NMR}$ signals for the protons at positions 9 and 10 .

In contrast to UV/vis experiments, a higher concentration of $\mathbf{3}(5 \mathrm{mM})$ was needed for the NMR studies and only a slight excess of DHA could be used (10, 15, and 20 eq.). Under these conditions, the formation of anthracene was basically independent of the substrate concentration; all reactions showed comparable $k_{\mathrm{obs}}$ values. This finding is indicative of this disproportionation being substrateindependent and thus, the disproportionation appears to be a general characteristic of compound $\mathbf{3}$ in MeCN solution. ${ }^{[155]}$ Compared to the OAT process toward $\mathrm{PMe}_{3}$, the reaction in this case gets slower throughout the HAA process since the iron(II) product $\mathbf{1}$ accumulates in the solution and thus shifts the equilibrium even more to the starting material 3. 
Possible effects on this pre-equilibrium were studied by addition of various amounts of iron(II) complex 1 (1.6, 3.2 and 6.4 eq.) to the reaction mixture. Exemplary ${ }^{1} \mathrm{H}-\mathrm{NMR}$ spectra for the reaction of 3 with DHA ( $~ 7$ eq.) after addition of 1.6 eq. 1 are shown in Figure 26. Formation of anthracene and iron(II) complex 1 was followed using the peaks at $8.50 \mathrm{ppm}$ (black box, $\int 2 \mathrm{H}$ ) and $6.03 \mathrm{ppm}$ (orange box, $\int 4 \mathrm{H}$ ), respectively. Decrease of $\mu$-oxodiiron(III) 3 was followed using the doublet at $6.29 \mathrm{ppm}$ (brown box, $\sqrt{2} \mathrm{H}$ ). The corresponding time traces are shown in the right plot.
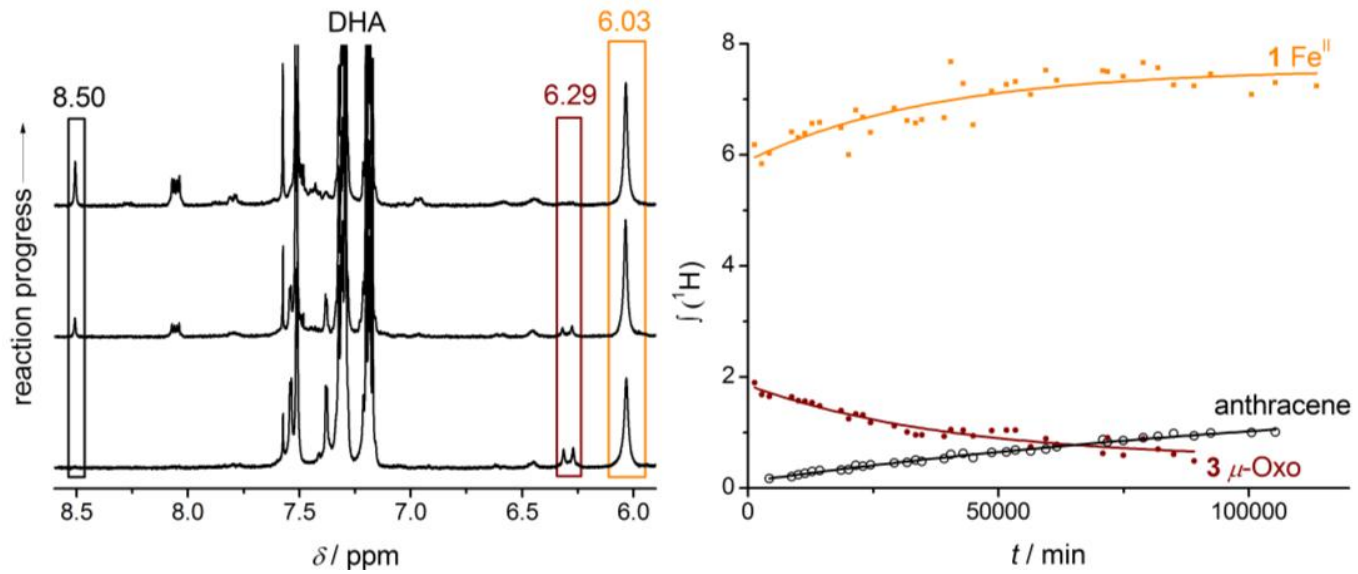

Figure 26. Left: ${ }^{1} \mathrm{H}-\mathrm{NMR}$ spectra of the reaction mixture of the $\mu$-oxo compound 3 with DHA and additional 1.6 eq. of iron(II) 2 at beginning ( $t=1 \mathrm{~d}$, bottom), mid reaction time ( $t=30 \mathrm{~d}$, middle) and end of the reaction ( $t=75 \mathrm{~d}$, top). Right: Kinetic traces for the development of the signals at $8.50 \mathrm{ppm}$ (anthracene), $6.29 \mathrm{ppm}$ ( $\mu$-oxo 3 ), $6.03 \mathrm{ppm}\left(\mathrm{Fe}^{\mathrm{II}} \mathbf{1}\right)$.
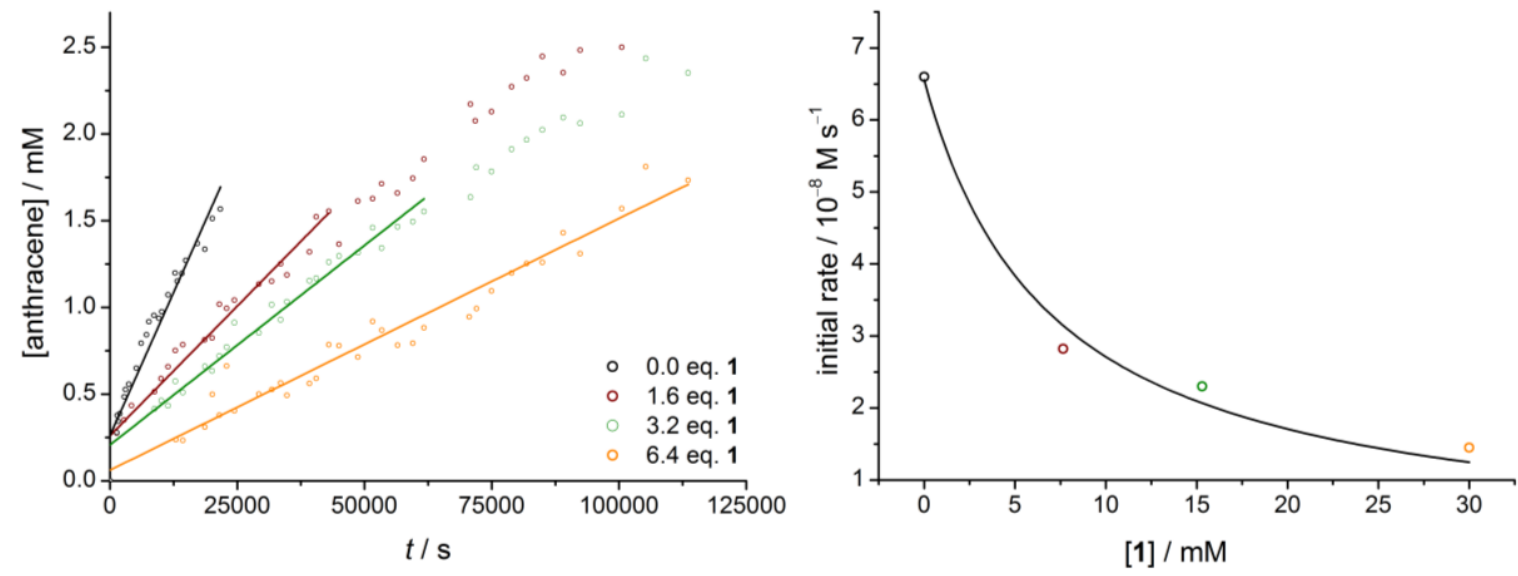

Figure 27. Left: Formation of anthracene during the reaction of $\mathbf{3}(5 \mathrm{mM})$ with DHA $(35 \mathrm{mM})$ and different amounts of 1 (black: 0 eq., brown: 1.6 eq., green: 3.2 eq., orange: 6.4 eq.) at RT determined by ${ }^{1} \mathrm{H}-\mathrm{NMR}$ spectroscopy. The initial increase in [anthracene] was fitted to a linear curve with the slope representing the initial rate. Right: Plot of the determined initial rate values for the reaction of $\mathbf{3}$ with DHA versus concentration of $\mathbf{3}$. The data points were fitted using equation (iv).

One oxoiron(IV) molecule is able to abstract one proton from DHA. Thus, two high-valent species are needed to form one anthracene molecule and the maximum yield for these reactions is 0.5 eq. anthracene with respect to 1 eq. $\mu$-oxo compound 3. In the NMR spectrum, formation of protons 9 and 10 of anthracene at $8.50 \mathrm{ppm}$ was followed and consequently an integral of 1 represents $100 \%$ 
conversion. In case of the reaction with 1.6 eq. iron(II), the process was followed until the end, indicating full conversion in agreement with the clean reaction observed in the ${ }^{1} \mathrm{H}-\mathrm{NMR}$ spectra with no side products being detected. Due to the very long reaction time ( $>2$ months), only formation of anthracene up to a minimum concentration of $1.5 \mathrm{mM}$ was followed for all four reactions with varying iron(II) concentration, as shown in Figure 27, left.

Significant decrease of the initial reaction rate was observed for increasing amounts of $\mathbf{1}$, clearly in line with inhibition of the disproportion of $\mu$-oxodiiron(III) 3. For each of the four experiments presented in Figure 27, the reaction progress until formation of 0.6 eq. anthracene $(1.5 \mathrm{mM})$ was fitted with a linear curve and initial rates were determined. A plot of these initial rates $v s$. $\left[\mathrm{Fe}^{\mathrm{II}}\right]_{0}$ clearly shows the decrease in reaction rate with increasing amounts of $\mathbf{1}$. The obtained data points were fitted according to equation (iv) which allowed for determination of an equilibrium constant for the disproportionation equilibrium of $3^{[203]}$ The error of $2.5 \cdot 10^{-8} \mathrm{~mol} \mathrm{l}^{-1}$ was roughly estimated by test fits with slightly changed values for $k_{2} \sim 35 \pm 12 \mathrm{~mol}^{-1} \mathrm{~s}^{-1}$.

$$
\begin{aligned}
& \text { rate }=\frac{k_{1} k_{2}[3][D H A]}{k_{-1}[1]+k_{2}[D H A]} \\
& K_{e q}=\frac{k_{1}}{k_{-1}}=\frac{1.3 \cdot 10^{-5} \frac{1}{s}}{174 \frac{l}{\mathrm{mols}}}=(7.5 \pm 2.5) \cdot 10^{-8} \frac{\mathrm{mol}}{\mathrm{l}}
\end{aligned}
$$

The very small equilibrium constant is within the expected region and in line with the impossibility to detect $\mathbf{1}$ or $\mathbf{2}$ as intermediates during the reaction process. Additionally, it agrees with measurements of $\mathbf{3}$ in solution (eg. NMR, MB) that cannot be used to detect the presence of $\mathbf{1}$ or $\mathbf{2}$. Compared to the $K_{\text {eq }}$ determined by Vanka and Gupta for their $\mu$-oxodiiron(IV) system $\left(K_{\mathrm{eq}}=9.7 \cdot 10^{-7} \mathrm{~mol} \mathrm{l}^{-1}\right)$, the result for $\mathbf{3}$ is found in a similar range. According to equation (vi), this value can be transferred to a Gibbs free energy of about $40 \mathrm{~kJ} \mathrm{~mol}^{-1}\left(9.6 \mathrm{kcal} \mathrm{mol}^{-1}\right)$ for the disproportionation of $\mathbf{3}$ at $\mathrm{RT}\left(20^{\circ} \mathrm{C}\right)$.

$$
\Delta G=-R T \ln K
$$

Thus, deep insight into the disproportionation equilibrium of a $\mu$-oxodiiron(III) species that allows for unprecedented reactivity in OAT and HAA is achieved. 


\subsection{Conclusions}

Solutions of organometallic $\mu$-oxodiiron(III) compound 3 in $\mathrm{MeCN}$ show a disproportionation equilibrium into the corresponding oxoiron(IV) species 2 and iron(II) complex 1 . No previous example of such a disproportionation of a $\mathrm{Fe}^{\mathrm{III}}-\mathrm{O}-\mathrm{Fe}^{\mathrm{III}}$ species that leads to a reactive high-valent iron compound without addition of further oxidants could be found in literature. This fascinating observation can be attributed to the special characteristics of the equatorial tetracarbene ligand system. Due to the ligand's strong $\sigma$-donor ability, the iron $\mathrm{d}_{\mathrm{x}^{2}-\mathrm{y}^{2}}$ orbital lies highest in energy. Thus, the $\mathrm{d}_{\mathrm{z}^{2}}$ orbital is singly occupied in the $S=3 / 2$ ground state of the iron centers in $\mathbf{3}$ and square pyramidal coordination is favored over octahedral coordination. Coordination of a sixth ligand in solution, i.e. $\mathrm{MeCN}$ or $\mathrm{PMe}_{3}$, likely induces cleavage of the $\mathrm{Fe}-\mathrm{O}-\mathrm{Fe}$ bond and formation of the reactive compound 2 .

The resulting reactivity of $\mathbf{3}$ in OAT and HAA processes was studied kinetically and, in case of oxidation of DHA to anthracene, the equilibrium constant for the disproportionation of $\mathbf{3}$ could be determined. In agreement with the observed slow reaction, $K_{\text {eq }}$ is very small, $(7.5 \pm 2.5) \cdot 10^{-8} \mathrm{~mol} \mathrm{l}^{-1}$.

In case of OAT processes toward $\mathrm{PMe}_{3}$, a bis-(phosphino)iron(II) complex is formed that is not part of the equilibrium anymore. Consequently, the presence of phosphine acts as an additional driving force for the disproportionation of $\mathbf{3}$ in a similar way as pyridine was used in a study by Ercolani et al. ${ }^{[204]}$ Additionally, the UV/vis experiments with $\mathrm{PMe}_{3}$ were performed at much lower concentration $(0.1 \mathrm{mM})$ than the NMR experiments with DHA as substrate $(5 \mathrm{mM})$. Since the disproportionation is likely solvent induced, the concentration is also expected to have an influence on $K_{\text {eq }}$. Thus, $K_{\text {eq }}$ for the reaction of $\mathbf{3}$ with $\mathrm{PMe}_{3}$ can be assumed to be larger compared to what was found for reaction with DHA but no definite values could be obtained. 


\section{Conversion of NHC Coordinated Iron Precursors with Azides}

\subsection{Objectives}

Azidoiron(II) and -iron(III) compounds are important precursors to their corresponding high-valent nitridoiron(IV) and -iron(V) species. ${ }^{[7]}$ As previously mentioned in chapter 1.3, irradiation of azido complexes can, depending on the conditions, initiate release of dinitrogen with concomitant formation of the desired nitridoiron moiety. These nitridoiron compounds are model systems for intermediates proposed in enzymatic processes related to the biogeochemical nitrogen cycle, in particular during the activation of dinitrogen by the enzyme nitrogenase ${ }^{[45]}$ Furthermore, they offer a range of applications, such as the release of ammonia upon protonation ${ }^{[41]}$ and nitrogen atom transfer. ${ }^{[100]}$

Preliminary work on this project was done by Iris Klawitter, ${ }^{[155]}$ where she was able to generate the bis-azidoiron(II) compound $\mathbf{5}$ upon conversion of the tetracarbene coordinated iron(II) species $\mathbf{1}$ with excess $\left[{ }^{n} \mathrm{Bu}_{4} \mathrm{~N}\right] \mathrm{N}_{3}$ in DMF (Scheme 9). Due to its neutral character, the green product is only partially soluble in common solvents preventing full characterization at that point. Within this work, crystallization of $\left[\left({ }^{\mathrm{NHC}} \mathrm{L}\right) \mathrm{Fe}\left(\mathrm{N}_{3}\right)_{2}\right](\mathbf{5})$ was pursued, in order to obtain further evidence for its successful isolation. Although no crystalline material was obtained by Iris Klawitter, laser irradiation experiments were performed on the crude product of $\mathbf{5}$ at various temperatures and no tendency for formation of a high-valent nitrodoiron(IV) was observed. However, in contrast to its stability upon irradiation, $\mathbf{5}$ immediately changes color from green to red in air, indicative of a high tendency for oxidation. This provides motivation for the generation of the corresponding bis-azidoiron(III) compound, described in section 6.2. Upon release of $\mathrm{N}_{2}$, a nitridoiron(V) compound is expected to form.

Highly unstable nitridoiron intermediates, isolated in frozen matrix, have been shown to generate stable $\mu$-nitridodiiron systems in solution (compare 1.3.2). Several compounds in $\mathrm{Fe}^{\mathrm{III}}-\mathrm{N}-\mathrm{Fe}^{\mathrm{IV}}$ or $\mathrm{Fe}^{\mathrm{IV}}-\mathrm{N}-\mathrm{Fe}^{\mathrm{IV}}$ oxidation states have been reported in the literature while compound $\mathbf{6}$, first reported by Iris Klawitter, is the first example of a $\mu$-nitridodiiron(III) species. ${ }^{[155]}$ Further characterization of 6 as well as elucidation of its oxidation chemistry and reactivity was pursued. The results of this project are presented in section 6.3. 


\subsection{Bis-Azido Complexes of Organometallic Iron(II) and Iron(III) Centers}

\subsubsection{Characterization of a Bis-Azidoiron(II) Complex (5)}

As reported by Iris Klawitter, the conversion of $\left[\left({ }^{\mathrm{NHC}} \mathrm{L}\right) \mathrm{Fe}(\mathrm{MeCN})_{2}\right](\mathrm{OTf})_{2} \mathbf{1}$ with an excess of the azide salt $\left[{ }^{n} \mathrm{Bu}_{4} \mathrm{~N}\right] \mathrm{N}_{3}$ leads to the formation of a partially soluble green precipitate. ${ }^{[155]}$ The IR spectrum of solid material of 5 shows a prominent resonance at $\tilde{v}=2022 \mathrm{~cm}^{-1}$, which is shifted by $\Delta \tilde{v}=25 \mathrm{~cm}^{-1}$ compared to the vibration of the free $\mathrm{N}_{3}{ }^{-}$anion. This value is typical for the antisymmetric $\mathrm{N}_{3}^{-}$stretching vibration in octahedral ferrous complexes with trans azido ligands $\left(2014-2051 \mathrm{~cm}^{-1}\right) \cdot{ }^{[57,162,205,206]}$ Furthermore, the MB spectrum of the crude product 5 shows a quadrupole doublet with an isomer shift of $\delta=0.32 \mathrm{~mm} \mathrm{~s}^{-1}$ and a quadrupole splitting of $\Delta E_{\mathrm{Q}}=2.39 \mathrm{~mm} \mathrm{~s}^{-1}$, which is indicative of an iron(II) species, similar to the spectrum recorded for the precursor compound $\mathbf{1}\left(\delta=0.23 \mathrm{~mm} \mathrm{~s}^{-1}, \Delta E_{\mathrm{Q}}=2.10 \mathrm{~mm} \mathrm{~s}^{-1}\right) .{ }^{[153,155]}$

Within this work, 5 was synthesized according to the described procedure. ${ }^{[155]}$ To remove impurities, such as remaining $\left[{ }^{n} \mathrm{Bu}_{4} \mathrm{~N}\right] \mathrm{N}_{3}$, the green compound was further washed with unpolar $\mathrm{Et}_{2} \mathrm{O}$ and THF as well as $\mathrm{MeOH}$, yielding pure $\left[\left({ }^{\mathrm{NHC}} \mathrm{L}\right) \mathrm{Fe}\left(\mathrm{N}_{3}\right)_{2}\right]$ as confirmed by elemental analysis. The slight solubility of $\mathbf{5}$ in MeCN provides a means for crystallization. Slow diffusion of $\mathrm{Et}_{2} \mathrm{O}$ into a slightly purple $\mathrm{MeCN}$ solution of the crude product at RT led to formation of two different types of crystals: a green main species and small amounts of a red compound. Both structures were determined by single $\mathrm{X}$-ray crystallography. The green species represents the neutral $\left[\left({ }^{\mathrm{NHC}} \mathrm{L}\right) \mathrm{Fe}\left(\mathrm{N}_{3}\right)_{2}\right]$ compound 5 (Figure 28, left), while the red crystals correspond to $\left[\left({ }^{\mathrm{NHC}} \mathrm{L}\right) \mathrm{Fe}\left(\mathrm{N}_{3}\right)(\mathrm{MeCN})\right] \mathrm{N}_{3}\left(\mathbf{5}_{\mathrm{MeCN}}\right.$, Figure 28, right).
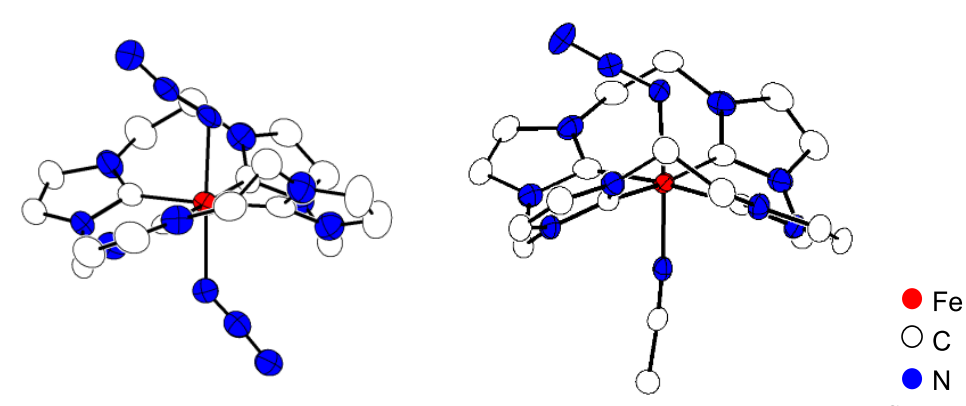

Figure 28. ORTEP plots of the molecular structures of the compounds $\left[\left({ }^{\mathrm{NHC}} \mathrm{L}\right) \mathrm{Fe}\left(\mathrm{N}_{3}\right)_{2}\right] \mathbf{5}$ (left) and $\left.\left[{ }^{\mathrm{NHC}} \mathrm{L}\right) \mathrm{Fe}\left(\mathrm{N}_{3}\right)(\mathrm{MeCN})\right]^{+} \mathbf{5}_{\mathrm{MeCN}}$ (right) (50\% probability thermal ellipsoids); hydrogen atoms and anions $\left(\mathrm{N}_{3}{ }^{-}\right.$ in the case of $\mathbf{5}_{\mathrm{MeCN}}$ ) are omitted for clarity, important bond lengths and angles are tabulated in Table 6.

Compounds $\mathbf{5}$ and $\mathbf{5}_{\mathrm{MeCN}}$ crystallized in the monoclinic space group $P 2_{1} / n$ while the starting material 1 crystallized in the triclinic $P \overline{1}$ space group. In the case of $\mathbf{5}$, the ligands show rotational disorder, with the coordinating iron atoms located in the center of the virtual rotation axis. So far, it was not possible to obtain crystals without such a disorder. Relevant bond lengths and angles are 
tabulated in Table 6 . In both systems, the azide ligand(s) are nearly linear $\left(\mathbf{5}: 167^{\circ} / 169^{\circ}, \mathbf{5}_{\mathrm{MeCN}}\right.$ : $\left.178^{\circ}\right)$ and are bound to the iron ion in a bent $\mathrm{Fe}-\mathrm{N}-\mathrm{N}$ fashion $\left(\mathbf{5}: 119^{\circ} / 136^{\circ}, \mathbf{5}_{\mathrm{MeCN}}: 120^{\circ}\right)$ resulting in an almost linear axial coordination of the iron center $\left(5: 174^{\circ}, \mathbf{5}_{\mathrm{MeCN}}: 176^{\circ}\right)$.

Table 6. Structural parameters, bond lengths $[\AA]$ and angles $\left[{ }^{\circ}\right]$ for the complexes of relevance in section 6.2.

\begin{tabular}{|c|c|c|c|c|c|c|c|}
\hline & $\mathbf{1}^{[153]}$ & $5^{*}$ & $\mathbf{5}_{\mathrm{MeCN}}$ & 9 & $8^{*}$ & 10 & 11 \\
\hline Space group & $P \overline{\mathbf{1}}$ & $P 2_{1} / \mathrm{n}$ & $P 2_{1} / \mathrm{n}$ & $C 2 / \mathrm{c}$ & $P \overline{\mathbf{1}}$ & $P \overline{\mathbf{1}}$ & $P \overline{\mathbf{1}}$ \\
\hline coordination & OC-6 & OC-6 & OC-6 & OC-6 & SPY -5 & OC-6 & OC-6 \\
\hline av. $d\left(F e-C^{\mathrm{NHC}}\right)[\AA]$ & 2.00 & 1.99 & 1.99 & 2.02 & 2.01 & 2.00 & 2.01 \\
\hline $\mathbf{d}\left(\mathbf{F e}-\mathbf{N}_{3}\right)[\AA]$ & - & 2.11 & 2.06 & - & - & 1.94 & 1.98 \\
\hline d(Fe-NCMe) $[\AA ̊]$ & 1.92 & - & 1.92 & 1.93 & - & - & - \\
\hline $\mathbf{d}(\mathbf{F e}-\mathbf{C l})[\AA ̊ A]$ & - & - & - & - & 2.27 & 2.34 & - \\
\hline $\mathbf{d}\left(\mathbf{F e N}-\mathbf{N}_{2}\right)[\AA]$ & - & 1.15 & 1.20 & - & - & 1.21 & 1.16 \\
\hline $\mathbf{d}\left(\mathrm{FeN}_{2}-\mathbf{N}\right)[\AA]$ & - & 1.17 & 1.17 & - & - & 1.16 & 1.16 \\
\hline$<\left(\mathbf{L}_{\mathbf{1}}-\mathbf{F e}-\mathbf{L}_{2}\right)\left[{ }^{\circ}\right]$ & 180 & 174 & 176 & 177 & - & 177 & 178 \\
\hline$<\left(\mathbf{F e}-\mathbf{N}-\mathbf{N}_{2}\right)\left[{ }^{\circ}\right]$ & - & $119 / 136$ & 120 & - & - & 122 & 121 \\
\hline$<(\mathbf{N}-\mathbf{N}-\mathbf{N})\left[^{\circ}\right]$ & - & $167 / 169$ & 178 & - & - & 175 & 176 \\
\hline
\end{tabular}

* values are given for $(\mathrm{A})$.

Crystallization of both $\mathbf{5}$ and $\mathbf{5}_{\mathrm{MeCN}}$ from the same $\mathrm{MeCN}$ solution indicates the existence of a $\mathrm{MeCN} /$ azide ligand exchange equilibrium. To further investigate the proposed equilibrium, $\left[{ }^{n} \mathrm{Bu}_{4} \mathrm{~N}\right] \mathrm{N}_{3}$ was added in portions of 1 eq. to a MeCN solution of the iron(II) precursor 1 (Figure 29, left). Starting with $\left.\left[{ }^{\mathrm{NHC}} \mathrm{L}\right) \mathrm{Fe}(\mathrm{MeCN})_{2}\right]^{2+} \mathbf{1}$, stepwise exchange of the $\mathrm{MeCN}$ ligands is expected, affording the intermediate $\left[\left({ }^{\mathrm{NHC}} \mathrm{L}\right) \mathrm{Fe}(\mathrm{MeCN})\left(\mathrm{N}_{3}\right)\right]^{+}$species $\mathbf{5}_{\mathrm{MeCN}}$ as well as the final compound $\left[\left({ }^{\mathrm{NHC}} \mathrm{L}\right) \mathrm{Fe}\left(\mathrm{N}_{3}\right)\right] \mathbf{5}$. However, based on the UV/vis, only a decrease of the characteristic band of $\mathbf{1}$ at $\lambda_{\max }=339 \mathrm{~nm}$ and an increase of two new bands at $\lambda_{\max }=372$ and $545 \mathrm{~nm}$ without any intermediate was observed, resulting in the final spectrum after addition of 16 eq. of $\left[{ }^{n} \mathrm{Bu}_{4} \mathrm{~N}\right] \mathrm{N}_{3}$. The corresponding reaction mixture showed an intense pink color, in clear difference to the slightly purple solution when solving 5 in $\mathrm{MeCN}$. For comparison, 5 was dissolved in $\mathrm{MeCN}$ and $\mathrm{MeOH}$ in a glove box and an UV/vis absorption spectrum was recorded (Figure 29, right). The spectrum of the green $\mathrm{MeOH}$ solution revealed maxima at $\lambda_{\max }=265,364$ and $438 \mathrm{~nm}$ while transitions at $\lambda_{\max }=339,374$ and $523 \mathrm{~nm}$ were observed in the pink MeCN solution. Based on literature, ${ }^{[153]}$ the band at $\lambda_{\max }=339 \mathrm{~nm}$ in the latter spectrum can be assigned to bis-acetronitrile coordinated iron(II) (1) that is likely part of the dissociation/association equilibrium in the MeCN solution. The maximum at $\lambda_{\max }=374 \mathrm{~nm}$ is consistent with the maximum observed during the titration experiment, while the third band at $\lambda_{\max }=523 \mathrm{~nm}$ is shifted compared to $\lambda_{\max }=545 \mathrm{~nm}$. Both transitions were assigned to $\mathrm{N}_{3} \rightarrow$ Fe LMCT transitions, while the maximum at $\lambda_{\max }=374 \mathrm{~nm}$ 
likely corresponds to a carbene $\rightarrow \mathrm{Fe}^{\mathrm{II}}$ LMCT transition. The band at $\lambda_{\max }=545 \mathrm{~nm}$ (Figure 29, left) was assigned to a $\mathrm{N}_{3} \rightarrow \mathrm{Fe}^{\mathrm{III}}$ transition based on the studies described in 6.2.3 while the weaker band at $\lambda_{\max }=523 \mathrm{~nm}$ represents a $\mathrm{N}_{3} \rightarrow \mathrm{Fe}^{\mathrm{II}}$ transition. In conclusion, the iron center in 1 gets partially oxidized during titration with $\left[{ }^{n} \mathrm{Bu}_{4} \mathrm{~N}\right] \mathrm{N}_{3}$, although it was attempted to work under strictly inert conditions.
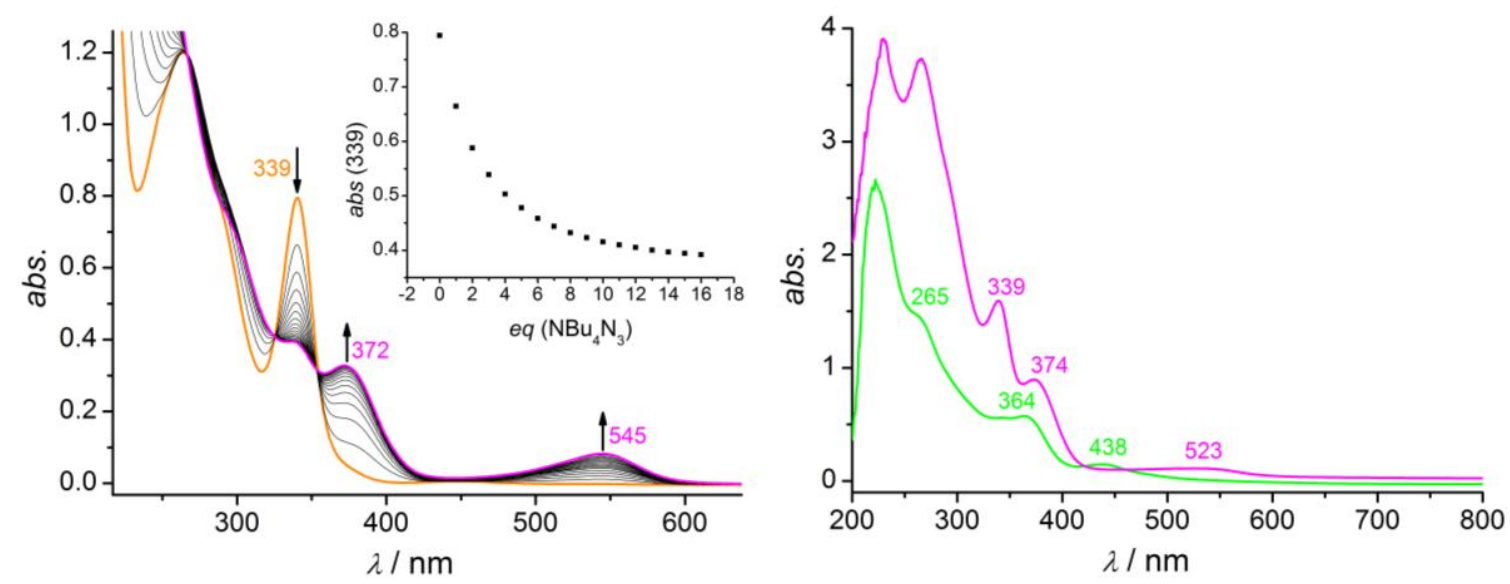

Figure 29. Stepwise addition of $\left[{ }^{n} \mathrm{Bu}_{4} \mathrm{~N}\right] \mathrm{N}_{3}$ to a MeCN solution of $\mathbf{1}(0.1 \mathrm{mM}$, orange followed by UV/vis. Further analysis revealed that a mixture of $\mathbf{5}_{\mathrm{MeCN}}$ and the oxidized species $\mathbf{1 1}$ was formed. Right: UV/vis spectrum of $\mathbf{5}$ in $\mathrm{MeCN}$ (magenta) and $\mathrm{MeOH}$ (green).

The oxidation process of $\mathbf{5}$ was further studied via MB spectroscopy. Upon addition of three different oxidants (dry dioxygen, the radical cation thianthrenyl tetrafluoroborate, and ferrocenium tetrafluoroborate) to a suspension of $\mathbf{5}$ in $\mathrm{MeCN}$, an intense dark pink solution was obtained. However, MB, IR and UV/vis studies revealed no formation of a clean species but rather a mixture of different compounds in all three cases. Consequently, it was necessary to generate the corresponding bis-azidoiron(III) compound via a different route. Specifically, the iron(II) precursor 1 was first oxidized to an iron(III) species that was subsequently reacted with $\left[{ }^{n} \mathrm{Bu}_{4} \mathrm{~N}\right] \mathrm{N}_{3}$, as described in the next sections.

\subsubsection{Oxidation of an Organometallic Iron(II) Complex}

Motivated by a reversible oxidation in the cyclic voltammogram of $\mathbf{1}$, Iris Klawitter oxidized $\left[\left({ }^{\mathrm{NHC}} \mathrm{L}\right) \mathrm{Fe}(\mathrm{MeCN})_{2}\right](\mathrm{OTf})_{2}$ with Magic Blue $\left(\left[\mathrm{N}\left(\mathrm{C}_{6} \mathrm{H}_{4}-4-\mathrm{Br}_{3}\right] \mathrm{SbCl}_{6}\left(\mathrm{E}_{1 / 2}=1.3 \mathrm{~V}\right.\right.\right.$ in $\left.\mathrm{MeCN}^{[207]}\right) .{ }^{[155]}$ In agreement with electrochemical oxidation experiments, treatment of 1 with Magic Blue resulted in a purple reaction mixture whereas crystallization upon slow diffusion of $\mathrm{Et}_{2} \mathrm{O}$ into the $\mathrm{MeCN}$ solution led to the formation of orange crystals of $\left[\left({ }^{\mathrm{NHC}} \mathrm{L}\right) \mathrm{FeCl}\right](\mathrm{OTf})_{2}(\mathbf{8})$ in good yields $(75 \%)$. The molecular structure of $\mathbf{8}$ is shown in Figure 30 (right) and pertinent structural parameters are given in Table 6. The UV/vis spectrum of the obtained crystals shows distinct differences 
compared to the initially generated purple solution, indicating that $\mathbf{8}$ does not correspond to the in situ generated material. It was proposed that an initial bis-acetonitrile coordinated iron(III) species 9 is formed, that abstracts a chloride from the $\mathrm{SbCl}_{6}{ }^{-}$counter ion upon crystallization. ${ }^{[155]}$

When this reaction was repeated as part of this work, slight modification of the crystallization conditions allowed for the isolation of purple crystals suitable for single crystal X-ray crystallography. In particular, fast diffusion of $\mathrm{Et}_{2} \mathrm{O}$ into a more concentrated $\mathrm{MeCN}$ solution of the in situ oxidized purple compound as well as crystallization at $-35{ }^{\circ} \mathrm{C}$ led to formation of single crystals of $\left.\left[{ }^{\mathrm{NHC}} \mathrm{L}\right) \mathrm{Fe}(\mathrm{MeCN})_{2}\right]\left(\mathrm{SbCl}_{6}\right)_{3}(\mathbf{9})$. As shown in Figure 30 (left), the iron centers in 9 are found in the expected octahedral coordination environment with two $\mathrm{MeCN}$ ligands in the axial positions. Compound 9 crystallizes in the monoclinic $C 2 / \mathrm{c}$ space group and shows a similar coordination mode as its iron(II) analog (Table 6). However, the average $\mathrm{Fe}-\mathrm{C}$ and $\mathrm{Fe}-\mathrm{NCMe}$ bonds in $\mathbf{9}$ are slightly longer compared to $\mathbf{1}$, in agreement with weaker back bonding ability of the oxidized iron center.
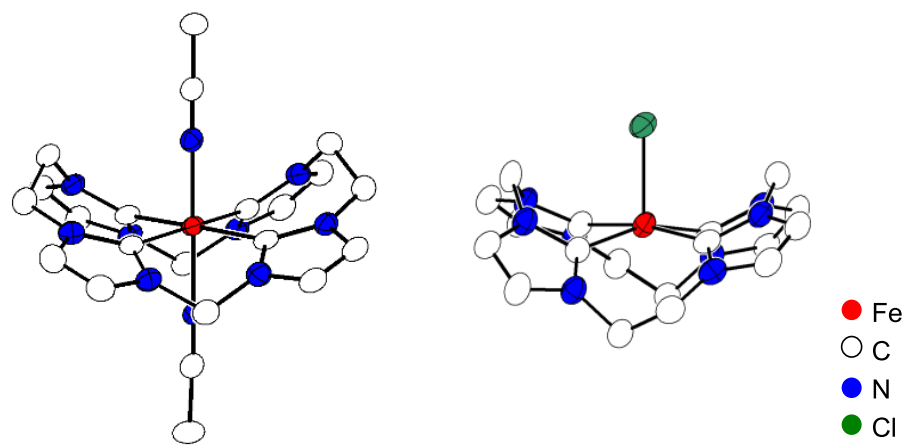

Figure 30. ORTEP plots of the molecular structures of the cations $\left.\left[{ }^{\mathrm{NHC}} \mathrm{L}\right) \mathrm{Fe}(\mathrm{MeCN})_{2}\right]^{3+} \mathbf{9}$ (left) and $\left[\left({ }^{\mathrm{NHC}} \mathrm{L}\right) \mathrm{FeCl}\right]^{2+} \mathbf{8}^{[155]}$ (right) (50\% probability thermal ellipsoids); hydrogen atoms and anions are omitted for clarity, important bond lengths and angles are tabulated in Table 6.

MB spectroscopy on 8 was previously performed by Iris Klawitter $\left(\delta=0.11 \mathrm{~mm} \mathrm{~s}^{-1}\right.$ and $\Delta E_{\mathrm{Q}}=4.52 \mathrm{~mm} \mathrm{~s}^{-1}$ ) along with SQUID measurements, which determined the $5 \mathrm{C}$ species' spin state to be $S=3 / 2 .{ }^{[155]}$ As part of this work, X-band EPR spectroscopy was performed, illustrating a very broad axial signal that could be fitted to the following values: $g_{1}=4.300, g_{2}=2.095$ (Appendix Figure 4). In contrast, 9 shows notably different spectroscopic features due to its octahedral coordination environment (Figure 31). While a similar isomer shift $\left(\delta=0.09 \mathrm{~mm} \mathrm{~s}^{-1}\right)$ is found in the MB spectrum, the quadrupole splitting $\left(\Delta E_{\mathrm{Q}}=0.63 \mathrm{~mm} \mathrm{~s}^{-1}\right)$ is significantly decreased. This finding corresponds well to a spherically symmetric electron distribution around the iron center in 9 and is indicative of a low spin $S=1 / 2$ spin state rather than the intermediate $S=3 / 2$ spin state found in 8. Consequently, the X-band EPR spectrum of 9 shows a sharp cubic signal with a $g$-value of 2.037 . 

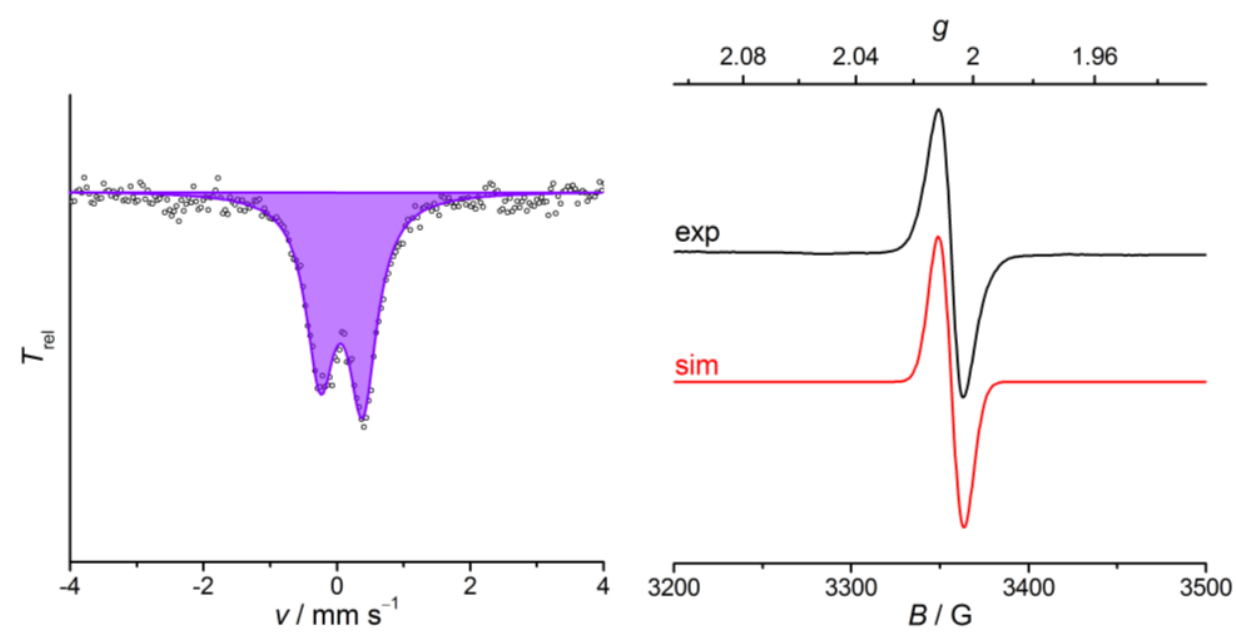

Figure 31. Zero-field ${ }^{57} \mathrm{Fe} \mathrm{MB}$ of 9 at $80 \mathrm{~K}$ (left, natural abundance ${ }^{57} \mathrm{Fe}, \quad \delta=0.09 \mathrm{~mm} \mathrm{~s}^{-1}$, $\Delta E_{\mathrm{Q}}=0.63 \mathrm{~mm} \mathrm{~s}^{-1}$ ) and X-band EPR spectrum of 9 in frozen MeCN solution at $143 \mathrm{~K}$ (black). The red line represents the simulation with $g=2.037$ and Gaussian line broadening of 8 G FWHT.

These results are consistent with the findings made for $\mu$-oxodiiron(III) compound $\mathbf{3}$. As described in chapter 5, 3 also has $5 \mathrm{C}$ iron centers in the $S=3 / 2$ spin state and coordination of a sixth ligand, namely $\mathrm{MeCN}$, is postulated to initiate cleavage of the $\mathrm{Fe}-\mathrm{O}-\mathrm{Fe}$ bond. Due to the energetically raised $d_{x^{2}-y^{2}}$ orbital in the tetracarbene coordinated iron centers studied within this work, an intermediate spin state for an iron(III) center directly correlates with single occupation of the $d_{z^{2}}$ orbital and destabilization of an octahedral coordination. In summary, the iron center in square pyramidal $\mathbf{8}$ is assigned to a $S=3 / 2$ spin state while such an intermediate spin state is unfavored in octahedrally coordinated $\mathbf{9}$, which is instead found in the low spin ground state.

According to the results reported by Iris Klawitter, $\mathbf{8}$ likely is of higher stability compared to $\mathbf{9}$. Although it was now possible to obtain single crystals of $\mathbf{9}$ for X-ray analysis along with MB and EPR studies, a full characterization including elemental analysis was not feasible. Several experiments aiming at clean material of $\mathbf{8}$ and $\mathbf{9}$ resulted in a mixture of both species and improvement of the synthetic strategies was thus desired. A modified method in generating 9 is provided by use of the radical cation thianthrenyl tetrafluoroborate instead of Magic Blue that does not provide the possibility to abstract a chloride ion. Unfortunately, crystallization of 9 was still very slow and only small amounts of crystalline material were obtained. The synthetic procedure for generation of chloridoiron(III) compound $\mathbf{8}$ could be improved as well. The iron(II) precursor $\mathbf{1}$ was oxidized with Magic Blue and $\left[{ }^{n} \mathrm{Bu}_{4} \mathrm{~N}\right] \mathrm{Cl}$ was added subsequently. This led to immediate formation of $\mathbf{8}$ indicated by a color change from purple to yellow and the corresponding crystals could be obtained within a shorter time and in a higher yield (83\%).

Finally, with these two iron(III) precursors in hand, the conversion with azide salts was studied; described in the following section. 


\subsubsection{Synthesis of Azidoiron(III) Species}

Both $\left[\left({ }^{\mathrm{NHC}} \mathrm{L}\right) \mathrm{FeCl}\right]^{2+}(\mathbf{8})$ and $\left[\left({ }^{\mathrm{NHC}} \mathrm{L}\right) \mathrm{Fe}(\mathrm{MeCN})_{2}\right]^{3+}(\mathbf{9})$ are conceivable precursors for the generation of azidoiron(III) species. Since $\mathbf{8}$ could be crystallized easier and in higher amounts than $\mathbf{9}$, initial studies have been performed on the chlorido compound $\mathbf{8}$.

Addition of $\left[{ }^{n} \mathrm{Bu}_{4} \mathrm{~N}\right] \mathrm{N}_{3}$ to an orange solution of $\mathbf{8}$ leads to an immediate color change to pink for both 1 eq. of azide salt or $\geq 2$ eq. Depending on the amount of $\mathrm{N}_{3}{ }^{-}$used, two different kinds of crystals were obtained after slow diffusion of $\mathrm{Et}_{2} \mathrm{O}$ into the cold reaction mixtures. The crystal structures for $\left[\left({ }^{\mathrm{NHC}} \mathrm{L}\right) \mathrm{Fe}(\mathrm{Cl})\left(\mathrm{N}_{3}\right)\right] \mathrm{OTf}(\mathbf{1 0})$ and $\left[\left({ }^{\mathrm{NHC}} \mathrm{L}\right) \mathrm{Fe}\left(\mathrm{N}_{3}\right)_{2}\right] \mathrm{Cl}(\mathbf{1 1})$ are shown in Figure 32, relevant structural parameters are given in Table 6. It can be concluded that the first equivalent of the azide coordinates at the free axial position in $\mathbf{8}$ while the second one substitutes the chlorido ligand.
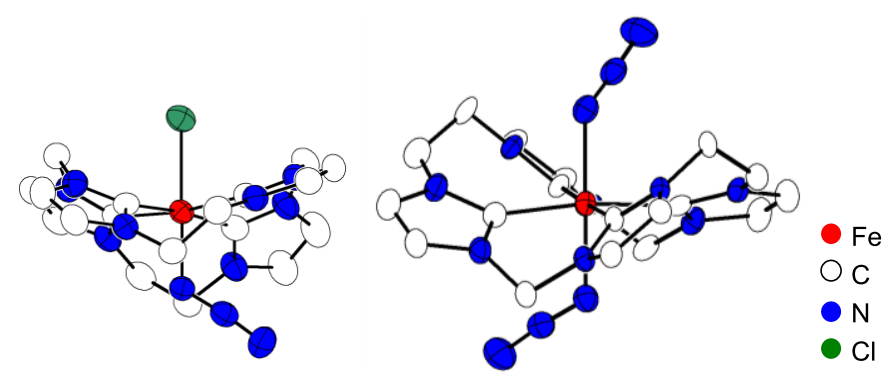

Figure 32. ORTEP plots of the molecular structures of the cations $\left.\left[\left({ }^{\mathrm{NHC}} \mathrm{L}\right) \mathrm{FeClN}\right]_{3}\right]^{+} \mathbf{1 0}$ (left) and $\left[\left({ }^{\mathrm{NHC}} \mathrm{L}\right) \mathrm{Fe}\left(\mathrm{N}_{3}\right)_{2}\right]^{+} \mathbf{1 1}$ (right) (50\% probability thermal ellipsoids); hydrogen atoms and anions are omitted for clarity, important bond lengths and angles are tabulated in Table 6 and chapter 13.

According to X-ray crystallography, both azide compounds $\mathbf{1 0}$ and $\mathbf{1 1}$ crystallize in the triclinic $P \overline{1}$ space group with an octahedrally coordinated iron center (Table 6). Due to the similarities between $\mathrm{Cl}^{-}$and $\mathrm{N}_{3}^{-}$ligands, the latter is also known as a pseudo-halide, the bond lengths and angles in $\mathbf{1 0}$ and $\mathbf{1 1}$ are quite similar. The main structural difference can be found for the $\mathrm{Fe}-\mathrm{N}_{3}$ bond length (10: $1.94 \AA$ A 11: $1.98 \AA$ ). Furthermore, these bonds are significantly shorter than the ones in their iron(II) analog 5, indicative of increased $\sigma$-donation of the anionic azido ligand into the $\mathrm{d}_{\mathrm{z}^{2}}$ orbital of the iron(III) center. Studying the spectroscopic features of both newly synthesized complexes with the help of Mössbauer, IR, and UV/vis spectroscopies, significant differences between $\mathbf{1 0}$ and 11 were observed (Figure 33). 

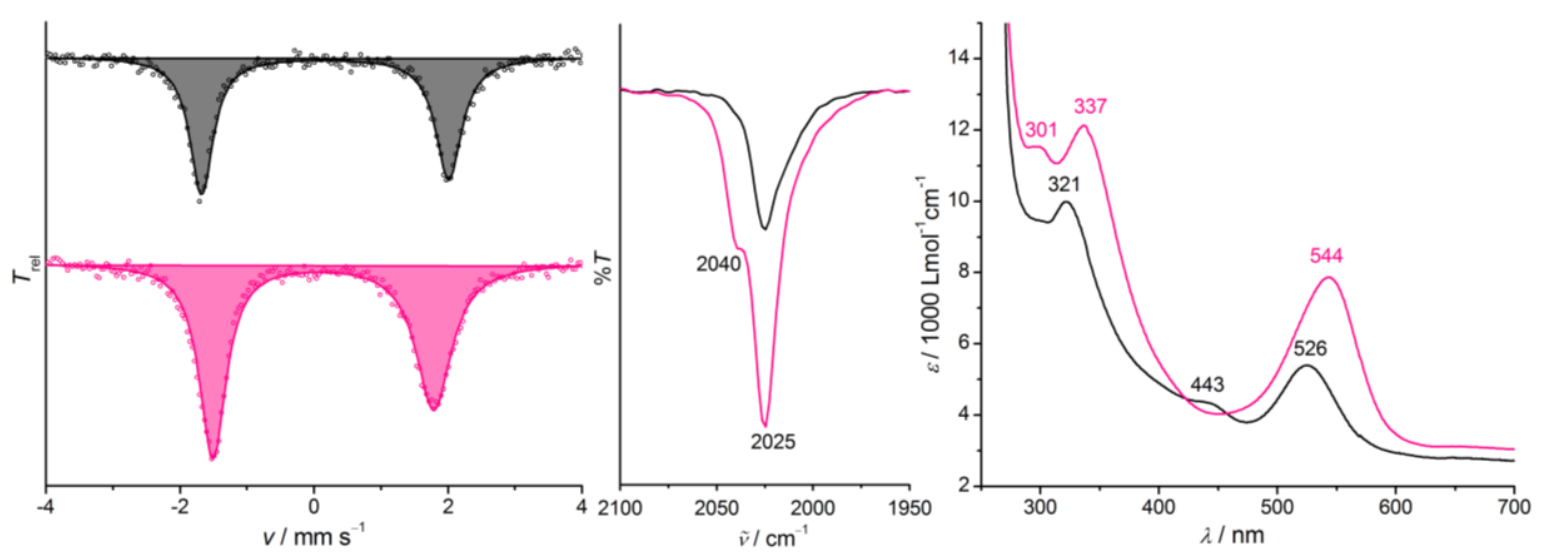

Figure 33. Zero-field ${ }^{57} \mathrm{Fe} \mathrm{MB}$ (left, natural abundance ${ }^{57} \mathrm{Fe}$ ) of $\mathbf{1 0}$ (top, black, $\delta=0.16 \mathrm{~mm} \mathrm{~s}^{-1}$, $\Delta E_{\mathrm{Q}}=3.71 \mathrm{~mm} \mathrm{~s}^{-1}$ ) and 11 (bottom, pink, $\delta=0.13 \mathrm{~mm} \mathrm{~s}^{-1}, \Delta E_{\mathrm{Q}}=3.32 \mathrm{~mm} \mathrm{~s}^{-1}$ ) at $80 \mathrm{~K}$ as well as parts of the corresponding IR (middle) and UV/vis absorption spectra (right) in MeCN solution at RT.

The MB doublet recorded for the chlorido-azido compound $\mathbf{1 0}$ (Figure 33, black) is characterized by an isomer shift $\left(\delta=0.16 \mathrm{~mm} \mathrm{~s}^{-1}\right)$ in the typical range of iron(III) species, and by a large quadrupole splitting $\left(\Delta E_{\mathrm{Q}}=3.71 \mathrm{~mm} \mathrm{~s}^{-1}\right)$. In agreement with increased symmetry at the iron center, the bis-azido species 11 shows a smaller quadrupole splitting $\left(\Delta E_{\mathrm{Q}}=3.32 \mathrm{~mm} \mathrm{~s}^{-1}\right.$, Figure 33, pink). Although $\mathrm{Cl}^{-}$and $\mathrm{N}_{3}^{-}$are very similar, the chlorido ligand forms bonds of higher ionic character corresponding to less $\sigma$-donation toward the iron center, which lowers the s-electron density. Accordingly, the isomer shift in $\mathbf{1 0}$ is slightly higher than what was found for $\mathbf{1 1}\left(\delta=0.13 \mathrm{~mm} \mathrm{~s}^{-1}\right)$. Furthermore, the bis-azido species generates an asymmetric quadrupole doublet in the $\mathrm{MB}$ spectrum, likely resulting from paramagnetic relaxation. ${ }^{[163]}$ Similar results have been reported for the $\left[\left({ }^{\mathrm{NHC}} \mathrm{L}\right) \mathrm{FeCl}\right]^{2+}$ precursor $\mathbf{8} .{ }^{[155]}$

Significant differences were also found in the IR spectra of $\mathbf{1 0}$ and $\mathbf{1 1}$ (Figure 33, middle). For instance, the intensity of the stretching frequency at $\tilde{v}=2025 \mathrm{~cm}^{-1}$ in $\mathbf{1 1}$, corresponding to the coordination of two azido ligands, is doubled compared to the signal for $\mathbf{1 0}$ in a solution of the same concentration. Compared to the bis-azidoiron(II) compound $\mathbf{5}$, this signal is found at slightly higher frequency consistent with decreased back-bonding of the iron(III) centers. While the main signal is found at the same position in both iron(III) species, an additional shoulder at $\tilde{v}=2040 \mathrm{~cm}^{-1}$ is found for the bis-azido compound. The IR stretches for $\mathbf{1 1}$ have also been computed using DFT calculations, providing further insight into the nature of the observed bands. For 11, two azide stretches were calculated at $\tilde{v}=2023$ and $2054 \mathrm{~cm}^{-1}$ in agreement with the experimental results (Figure 34 and DFT Figure 4). Both of these signals result mainly from the movement of the nitrogen atoms in the $\beta$ position in $\mathrm{Fe}-\mathrm{N}_{\alpha}-\mathrm{N}_{\beta}-\mathrm{N}_{\gamma}$. Based on the calculation, the signal at lower energy results from in-phase movement of the two $\mathrm{N}_{\beta}$ atoms in the two azido ligands while the out of phase movement is slightly higher in energy. 


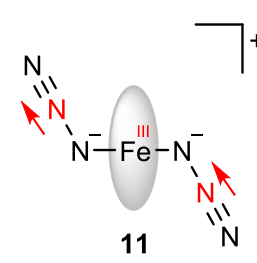

DFT: $2023 \mathrm{~cm}^{-1}$

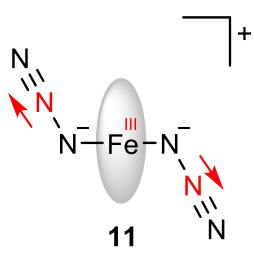

DFT: $2054 \mathrm{~cm}^{-1}$

Figure 34. DFT-calculated IR active stretching vibrations within the $\mathrm{N}_{3}$ moieties in 11, depicted is the inphase movement of $\mathrm{N}_{\beta}$ (left) and the corresponding out-of-phase movement (right). The arrows describe the movement of the atoms that are in the same color.

The main transitions in the UV/vis spectrum recorded for $\mathbf{1 0}\left(\lambda_{\max }=321\right.$ and $\left.526 \mathrm{~nm}\right)$ are shifted to lower energy in $11\left(\lambda_{\max }=337\right.$ and $\left.544 \mathrm{~nm}\right)$ where they also appear in higher intensity. The broad band around $530 \mathrm{~nm}$ for both species is assigned to a $\mathrm{N}_{3} \rightarrow$ Fe LMCT transition and found at an unusually high wavelength (low energy) compared to related octahedral $\mathrm{N}$-coordinated iron-azido complexes $\left(\lambda_{\max } \sim 470 \mathrm{~nm}\right) .^{[5,57,92]}$

Additionally, an EPR spectrum of $\mathbf{1 1}$ in frozen $\mathrm{MeCN}$ at $10 \mathrm{~K}$ was recorded. The observed broad signal could be simulated to afford $g$-values $g_{\mathrm{x}}=2.700, g_{\mathrm{y}}=1.990$, and $g_{\mathrm{z}}=1.676$ and corresponding line widths 380, 230, and $500 \mathrm{G}$ (Appendix Figure 4). The absence of the half-field signal around $g=4$ is perfectly in line with a low spin $S=1 / 2$ species in an octahedral coordination environment. Additionally, a ${ }^{1} \mathrm{H}-\mathrm{NMR}$ spectrum of the paramagnetic species was measured giving rise to shifted signals for the imidazole backbones $(\delta=21.50,8.94$, and $7.40 \mathrm{ppm})$ and the methylene $(\delta=-2.38 \mathrm{ppm})$ and ethylene bridges $(\delta=-5.72 \mathrm{ppm})$ when compared to diamagnetic iron species coordinated by the tetracarbene macrocycle (Appendix Figure 5). ${ }^{[153]}$

Most of these measurements were performed on powder material since crystallization was usually very slow and only small amounts of crystalline material could be obtained. Nevertheless, the synthesis of $\mathbf{1 1}$ using precursor compound $\mathbf{8}$ instead for $\mathbf{9}$ was preferred since initial studies on the bis-acetonitrile iron(III) compound revealed that the reaction proceeded not as cleanly.

In conclusion, crude $\left[\left({ }^{\mathrm{NHC}} \mathrm{L}\right) \mathrm{Fe}\left(\mathrm{N}_{3}\right)_{2}\right] \mathrm{Cl}$ (11) could be obtained in high yields and characterized via MB, EPR, UV/vis, IR and NMR spectroscopy. A schematic drawing illustrating the formation of the azidoiron(II) and -iron(III) compounds is given in Scheme 19. With these compounds in hand, irradiation experiments have been performed as described in the next section. 


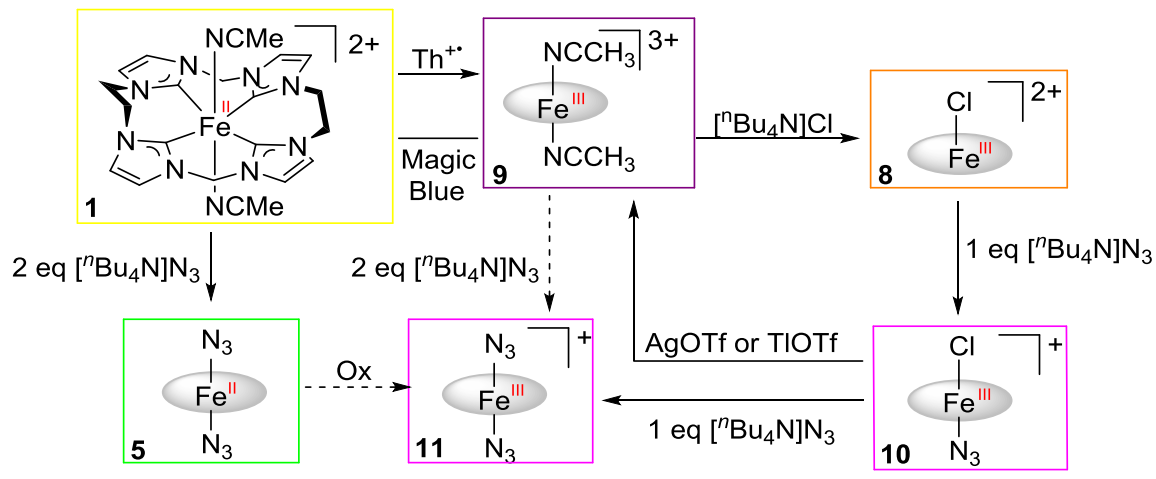

Scheme 19. Illustration of the synthetic strategy for generation of the bis(azido) species $\mathbf{5}$ and $\mathbf{1 1}$ described within this work. The colors of the boxes represent the actual colors of the compounds.

\subsubsection{Irradiation of $\left[\left({ }^{\mathrm{NHC}} \mathrm{L}\right) \mathrm{Fe}\left(\mathrm{N}_{3}\right)_{2}\right] \mathrm{Cl}(\mathbf{1 1})$}

Initial irradiation experiments were performed in $\mathrm{MeCN}$ solution at RT using a Mercury arc lamp that emits in a wide spectral range $(200-600 \mathrm{~nm}, 15 \mathrm{~W})$. Within five minutes, the pink color of 11 disappeared and a slight yellow solution was obtained. This solution showed absorption maxima in the UV/vis spectrum at $\lambda_{\max }=338$ and $290 \mathrm{~nm}$, while only the latter peak was stable in time. In a second experiment, a solution of $\mathbf{1 1}$ was cooled to $-40{ }^{\circ} \mathrm{C}$ before irradiation and a UV/vis spectrum of the resulting yellow solution was measured. However, only broadening and decrease of all bands and no characteristic maxima could be observed. Nevertheless, these experiments revealed that the bis-azido species is light-sensitive and that formation of the corresponding nitridoiron $(\mathrm{V})$ might be possible under appropriate conditions.

As described in the introduction (1.3.1), nitridoiron(V) species in 4-fold symmetry, as it would be the case for the desired tetracarbene coordinated species, are highly unstable and could only be generated upon matrix isolation so far. ${ }^{[57,92]}$ Consequently, the following irradiation studies were performed on frozen MeCN solutions using liquid nitrogen. In the labs of Jana Roithová in Prague, a mixture of $\mathbf{1 1}$ and $\mathrm{PBn}_{3}$ in a Young-NMR tube was irradiated with a blue laser $\left(\lambda_{\max }=445-\right.$ $450 \mathrm{~nm}, 1000-1200 \mathrm{~mW}$ ) for $\sim 6 \mathrm{~h}$. During that time, the pink color on the surface changed to yellow-green. After irradiation, the mixture was warmed up to RT and a ${ }^{31} \mathrm{P}-\mathrm{NMR}$ spectrum was recorded. In accord with the report by Smith et al. ${ }^{[97]}$ and the expected nitrogen atom transfer reactivity of high-valent nitridoiron species, formation of a phosphiniminato complex such as $\left({ }^{\mathrm{NHC}} \mathrm{L}\right) \mathrm{Fe}-\mathrm{N}=\mathrm{PBn}_{3}$ was desired but no oxidized phosphine was detected. This finding does not necessarily mean that $\mathrm{Fe}^{\mathrm{V}} \equiv \mathrm{N}$ was not formed but instead it indicates that the proposed nitrido compound would not be reactive toward the chosen phosphine. Furthermore, the resulting solution after melting of the reaction mixture was again pink, indicative of only negligible changes during 
the reaction progress or a reversible process such as release and subsequent rebonding of $\mathrm{N}_{3}{ }^{-}$ instead for release of $\mathrm{N}_{2}$.

Back in Göttingen, a frozen suspension of $\mathbf{1 1}$ in $\mathrm{MeCN}$ was irradiated in a MB cell by a diode (3x $\lambda_{\max }=530 \mathrm{~nm}, 900 \mathrm{~mW}$ ) for $\sim 24 \mathrm{~h}$. No color change was observed during that time indicating that no reaction had occurred which was subsequently confirmed by MB spectroscopy (13 K). Literature studies have shown that the wavelength of the laser is a crucial factor that determines which bond of the iron azide is cleaved. ${ }^{[5,94]}$ Accordingly, three pink suspensions of $\mathbf{1 1}$ in frozen MeCN were irradiated in EPR tubes with three different diodes at $\lambda_{\max }=365,450$, and $530 \mathrm{~nm}$. During $8 \mathrm{~h}$, no color change was observed for the samples irradiated with the $450 \mathrm{~nm}$ and the $530 \mathrm{~nm}$ diodes. A subsequent EPR measurement revealed a silent spectrum in agreement with the silent spectrum of $\mathbf{1 1}$ at 150-200 K, while a signal for $\mathbf{1 1}$ was only detected at significantly lower temperature $(10 \mathrm{~K})$. In the case of irradiation at $365 \mathrm{~nm}$, the color of the sample changed to brown during the reaction time. The corresponding product mixture showed a weak signal in the X-band EPR spectrum with $g=2.044,2.020,2.004$ (Figure 35, left). This signal does not resemble any signal known for the iron(III) species reported herein, nor does it correspond to any diamagnetic photooxidized iron(II) product. Thus, it could belong to the desired iron(V) $S=1 / 2$ system. Consequently, irradiation with the $365 \mathrm{~nm}$ diode was repeated in a sample cell suitable for subsequent MB measurements. However, irradiation for three days at $-196{ }^{\circ} \mathrm{C}$ revealed no color change of the sample and no change in the MB spectrum.
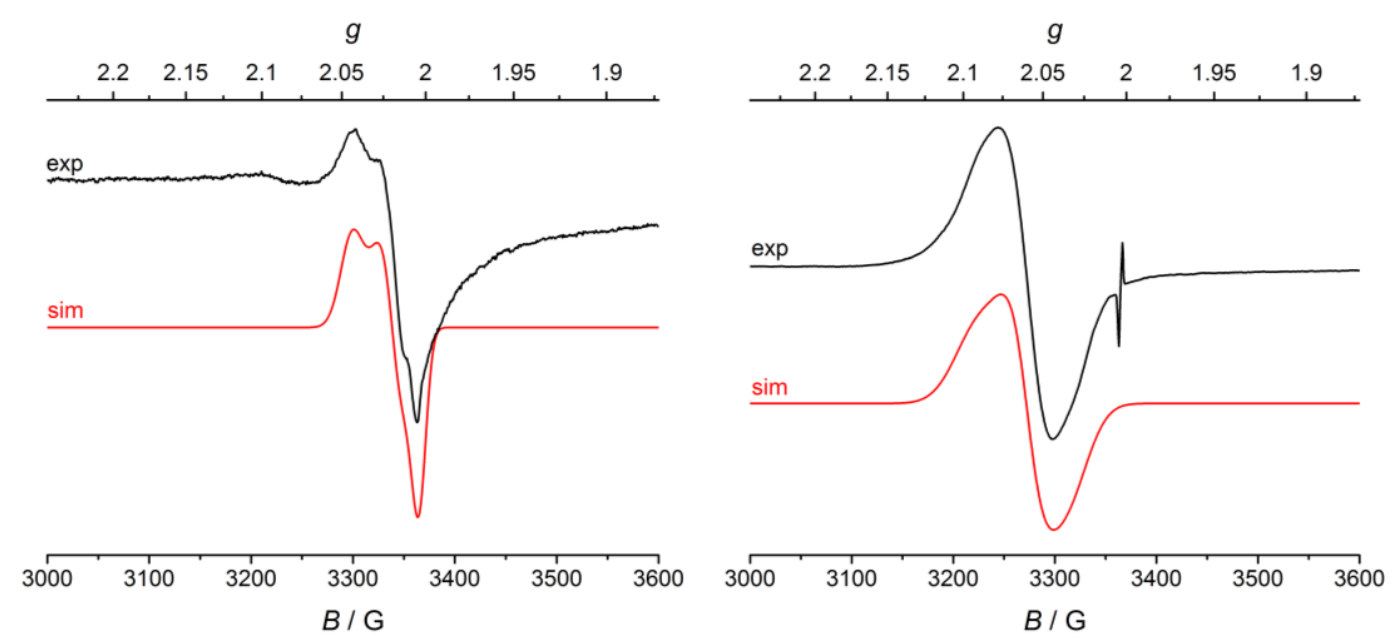

Figure 35. Experimental (black) and simulated (red) X-band EPR spectra of $\mathbf{1 1}$ after irradiation at $-196{ }^{\circ} \mathrm{C}$ (left) and $0{ }^{\circ} \mathrm{C}$ (black) with a $365 \mathrm{~nm}$-diode. The measurement was done in frozen MeCN solution at $160 \mathrm{~K}$. Simulation parameters for the left species: $g=2.0440,2.0199,2.0040$; $1 \mathrm{w}=13,12$, $8 \mathrm{G}$; right species: $g=2.092,2.060,2.034 ; \mathrm{lw}=27,18,24 \mathrm{G}$.

For comparison, one sample was also irradiated for $2 \mathrm{~min}$ in $\mathrm{MeCN}$ suspension at higher temperature $\left(\sim 0^{\circ} \mathrm{C}\right)$. Irradiation with the $450 \mathrm{~nm}$ and the $530 \mathrm{~nm}$ diode resulted in no immediate changes, while irradiation at $365 \mathrm{~nm}$ induced a fast color change from pink to orange and an 
increase in the solubility. The X-band EPR spectrum of the reaction mixture revealed a sharp band of higher intensity compared to irradiation in the frozen matrix; simulation afforded the $g$-values 2.092, 2.060, 2.034 (Figure 35, right). Furthermore, a very sharp, organic radical impurity at $g=2$ was observed.

In parallel, the MB sample that remained unchanged upon irradiation at $-196{ }^{\circ} \mathrm{C}$ was also irradiated in solution. The resulting MB spectrum was almost silent and showed only a very small signal in the IS region of about $0.27 \mathrm{~mm} \mathrm{~s}^{-1}$ clearly ruling out the clean formation of a high-valent iron compound. Thus, so far it was not possible to obtain MB spectra corresponding to the promising EPR signals. Furthermore, a suspension of 11 in MeCN was irradiated with the $365 \mathrm{~nm}$ diode in a NMR tube for $8 \mathrm{~h}$ at $-196{ }^{\circ} \mathrm{C}$ leading to a color change of the frozen sample from pink to dark brown. Before melting the sample, excess $\mathrm{PMe}_{3}$ was added and then the mixture was allowed to warm to RT. However, the resulting ${ }^{31} \mathrm{P}-\mathrm{NMR}$ spectrum did again not show any formation of a phosphinimin. Compared to tributylphosphine, the substrate that was used during initial studies, trimethylphosphine is of even higher reactivity and thus is expected to work as suitable trapping reagent for a putative nitridoiron $(\mathrm{V})$ species. Thus, based on this experiment, it is rather unlikely that $\left({ }^{\mathrm{NHC}} \mathrm{L}\right) \mathrm{Fe}^{\mathrm{IV}} \equiv \mathrm{N}$ was formed during the irradiation.

In conclusion, irradiation of $\mathbf{1 1}$ with a diode at $\lambda_{\max }=365 \mathrm{~nm}$ in an EPR tube led to promising EPR spectra for both irradiation in frozen $\mathrm{MeCN}$ matrix $\left(-196^{\circ} \mathrm{C}\right)$ and in solution $\left(\sim 0{ }^{\circ} \mathrm{C}\right)$. Based on the expected low stability of the desired nitridoiron $(\mathrm{V})$ species and the silent $\mathrm{MB}$ spectrum after irradiation in solution, irradiation in frozen matrix seems to be more encouraging. However, the intensity of the diode's light was too low to initiate any product formation when a suspension of $\mathbf{1 1}$ was irradiated in frozen $\mathrm{MeCN}$ in a Mössbauer cell. This cell is about $1 \mathrm{~cm}$ thick and the sample can only be irradiated from one side. Consequently, it may be necessary to irradiate with a high energy laser so that not only the surface of the sample is affected. Another idea for future experiments is to first oxidize $\mathbf{1 1}$ with a suitable oxidant and thus irradiate the resulting azidoiron(IV) species. Release of dinitrogen might then result in formation of a iron(VI) species that, according to ligand field theory, should be of relatively high stability. A problem in this regard might be the weak solubility of $\mathbf{1 1}$ that aggravates the measurement of an applicable cyclic voltammogram. Thus, it might be a problem to find a suitable oxidant without knowing if there is a promising oxidation wave in the cyclic voltammogram. 


\subsection{Reactivity of a $\mu$-Nitridodiiron(III) Compound}

\subsubsection{Characterization of 6}

As reported by Iris Klawitter, the conversion of 1 with 0.5 eq. $\left[{ }^{n} \mathrm{Bu}_{4} \mathrm{~N}\right] \mathrm{N}_{3}$ leads to the formation of the corresponding $\left.\left[{ }^{\mathrm{NHC}} \mathrm{L}\right) \mathrm{Fe}{ }^{\mathrm{III}}-\mathrm{N}-\mathrm{Fe}^{\mathrm{III}}\left({ }^{\mathrm{NHC}} \mathrm{L}\right)\right]^{3+}$ compound $\mathbf{6}$, likely via an $\mathrm{Fe}^{\mathrm{IV}} \equiv \mathrm{N}$ intermediate. ${ }^{[155]}$ The oxidation state was verified by MB spectroscopy $\left(\delta=-0.06 \mathrm{~mm} \mathrm{~s}^{-1}, \Delta E_{\mathrm{Q}}=1.27 \mathrm{~mm} \mathrm{~s}^{-1}\right)$ and SQUID measurements $\left(S_{\mathrm{T}}=0,-J>900, g=2.000\right)$. Furthermore, single crystals suitable for X-ray crystallography have been isolated after addition of $\mathrm{Et}_{2} \mathrm{O}$ to $\mathrm{DMF}$ solutions of the product but the obtained structures showed a strong disorder of the tetracarbene macrocycles; hence, crystals without such a disorder were desired.

To this end, diffusion of $\mathrm{Et}_{2} \mathrm{O}$ into a $\mathrm{MeCN}$ solution of $\mathbf{6}$ afforded non-disordered single crystals (69\% yield, Figure 36). The purity of the crystalline material was confirmed by elemental analysis. The highly air sensitive compound crystallizes in the triclinic crystal system with the space group $P \overline{1}$. The structure of 6 resembles the related $\mu$-oxo complex $3{ }^{[153]}$ The $\mathrm{Fe}-\mathrm{C}$ bonds (1.96-2.02 $\AA$ ) are very similar compared to 3 (1.97-2.04 $\AA$ ), whereas the symmetric Fe-N bond (1.69 $\AA$ ) is much shorter than the $\mathrm{Fe}-\mathrm{O}$ bond $(1.75 \AA)$. Overall, the $\mathrm{Fe}-\mathrm{N}$ distance is comparable to the previously described $\mu$-nitrido complexes, indicative of an appreciable $\mathrm{Fe}-\mathrm{N}$ double bond character in $\mathbf{6}^{[208,209]}$
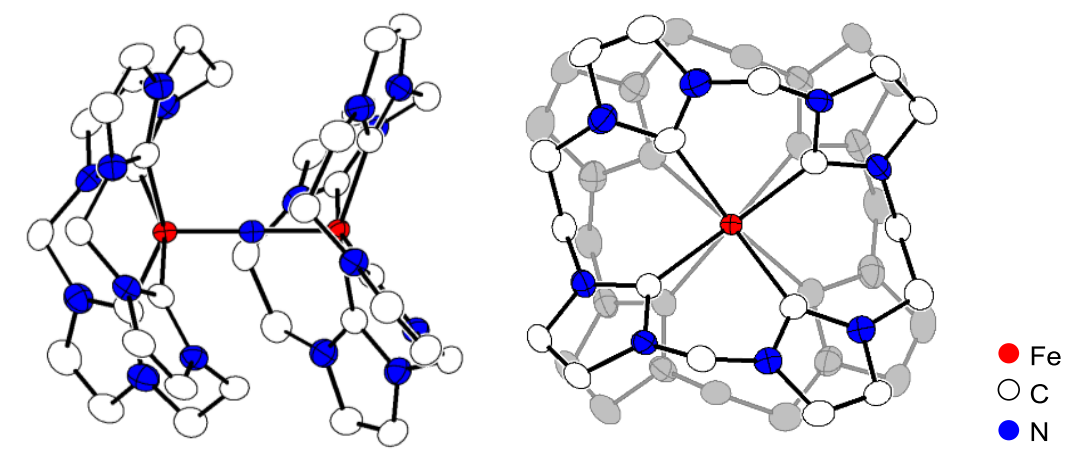

Figure 36. ORTEP plots of the molecular structure of the cation $\left[\left\{\left({ }^{\mathrm{NHC}} \mathrm{L}\right) \mathrm{Fe}\right\}_{2} \mathrm{~N}\right]^{3+} \mathbf{6}(50 \%$ probability thermal ellipsoids). $\mathrm{H}$ atoms, counter ions and solvent molecules were omitted for clarity. Right: View along the $\mathrm{Fe}-\mathrm{N}-\mathrm{Fe}$ axis $\left(80^{\circ}\right.$ twist of the ligand. Important bond lengths and angles are tabulated in Table 7. Further crystallographic details are given in Chapter 13. 
Table 7. Structural parameters, bond lengths $[\AA]$ and angles $\left[{ }^{\circ}\right]$ for complexes $\mathbf{6}\left[\left\{\left({ }^{\mathrm{NHC}} \mathrm{L}\right) \mathrm{Fe}^{\mathrm{III}}\right\}_{2} \mathrm{~N}\right]^{3+}, \mathbf{1 2}$ $\left[\left({ }^{\mathrm{NHC}} \mathrm{L}\right) \mathrm{Fe}{ }^{\mathrm{III}}-\mathrm{N}-\mathrm{Fe}^{\mathrm{IV}}\left({ }^{\mathrm{NHC}} \mathrm{L}\right)(\mathrm{NCMe})\right]^{3+}$, and $\mathbf{1 4}\left[\left\{\left(\mathrm{O}_{2} \mathrm{NO}\right)\left({ }^{\mathrm{NHC}} \mathrm{L}\right) \mathrm{Fe}^{\mathrm{IV}}\right\}_{2} \mathrm{~N}\right]^{3+}$ presented in section 6.3 .

\begin{tabular}{cccc}
\hline & $\mathbf{6}$ & $\mathbf{1 2}$ & $\mathbf{1 4}$ \\
\hline Space group & $P \overline{\mathbf{1}}$ & $P 2_{1} / \mathrm{n}$ & $I 4_{1} / \mathrm{acd}$ \\
coordination & $\mathrm{SPY}-5$ & $\mathrm{SPY}-5 /$ OC-6 & OC-6 \\
av. $\mathbf{d}\left(\mathbf{F e}-\mathbf{C}^{\mathbf{N H C}}\right)[\AA]$ & 1.99 & $1.99 / 2.01$ & 2.02 \\
$\mathbf{d}(\mathbf{F e}-\mathbf{N}-\mathbf{F e})[\AA \AA$ & $1.72 / 1.69$ & 1.68 \\
$\mathbf{d}(\mathbf{X}-\mathbf{F e})[\AA \AA$ & 1.69 & $-/ 2.09$ & 2.07 \\
$<(\mathbf{F e}-\mathbf{N}-\mathbf{F e})\left[{ }^{\circ}\right]$ & 178 & 179 & 180 \\
$<(\mathbf{X}-\mathbf{F e}-\mathbf{N})\left[^{\circ}\right]$ & - & $-/ 179$ & 179 \\
\hline
\end{tabular}

Since there is a strong antiferromagnetic coupling between the two iron centers, ${ }^{[155]}$ the $\mu$-nitridodiiron(III) compound $\mathbf{6}$ shows diamagnetic characteristics such as sharp signals in the ${ }^{1} \mathrm{H}-\mathrm{NMR}$ spectrum. While the analogous $\mu$-oxodiiron(III) compound $\mathbf{3}$ gives rise to only one set of signals in the ${ }^{1} \mathrm{H}-\mathrm{NMR}$ spectrum, a more complicated situation is observed for 6 (Appendix Figure 6). With the help of two-dimensional NMR spectroscopy, four different sets of signals could be assigned. All of these signal sets consist of the expected two signals for the imidazole backbone protons $(7.4-7.0 \mathrm{ppm})$, two doublets for the protons on the methylene bridges $(6.3-5.1 \mathrm{ppm})$ and one signal for the protons of the ethylene bridges (4.4-3.6 ppm). The part of the NMR spectrum corresponding to the protons of the methylene bridges is depicted in Figure 37, where the dark cyan peaks (a) represent the main isomer with signals at $6.09 \mathrm{ppm}$ and $5.23 \mathrm{ppm}$. The three other signal sets b-d are found with about half the intensity compared to the main signal set. According to a DOSY measurement, all proton signals belong to species with the same diffusion coefficient, thus all isomers should be similar N-bridged diiron species. Furthermore, a NOESY experiment is indicative of interaction between the ethylene bridges of the signal sets $\mathbf{c}$ (light cyan) and $\mathbf{d}$ (black). Consequently, they likely belong to one single species with unsymmetric carbene ligands. As depicted in Figure 36 (right), the two tetracarbene macrocycles are found to be twisted by about $80^{\circ}$ with respect to one another, thus no interaction of the ethylene bridges of the two rings is expected. Therefore, it is likely that the signal sets $\mathbf{c}$ and $\mathbf{d}$ correspond to an isomer without such a twist and the ethylene bridges close to each other. 


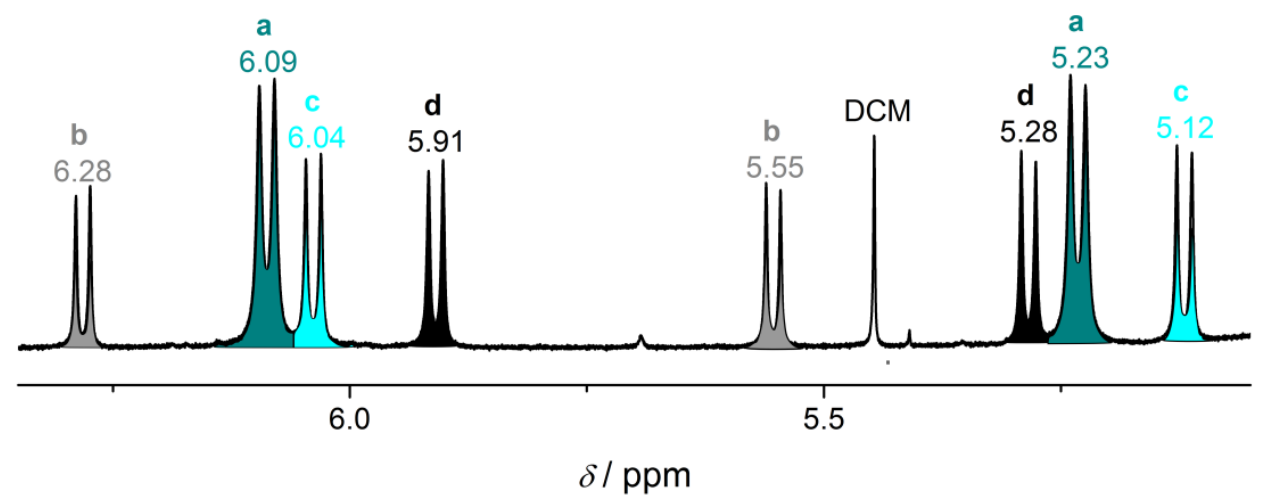

Figure 37. Part of the ${ }^{1} \mathrm{H}-\mathrm{NMR}$ spectrum of 6 illustrating the four isomers observed in $\mathrm{MeCN}-\mathrm{d}_{3}$.

Different batches of crystalline material gave identical NMR spectra as well as a sample of $\mathbf{6}$ that was dissolved in cold solvent and subsequently measured at lower temperature. In particular, only broadening of some of the signal sets was observed when cooling down or heating up the solution $\left(-35-70{ }^{\circ} \mathrm{C}\right)$. The obtained spectroscopic data strongly suggest that a solution of $\mu$-nitridodiiron(III) compound $\mathbf{6}$ consists of one symmetric isomer (a) and an unsymmetric isomer $(\mathbf{c}+\mathbf{d})$ in a 1:1 ratio as well as a minor isomer $(\mathbf{b})$. The nature of the minor species could not be clarified yet.

The spin state of the iron centers in the $\mu$-nitridodiiron(III) species $\mathbf{6}$ is not fully understood yet. DFT calculations predict negligible energy difference between the low spin and the intermediate spin state at the iron centers, only a high spin state can be ruled out. Thus, comparison of $\mathbf{6}$ with its oxo-bridged analog 3 reveals many similarities with respect to the molecular and electronic structure of both $5 \mathrm{C}$ iron centers. As described in chapter 5, the square pyramidal coordination of the iron center in $\mathbf{3}$ correlates with an intermediate spin state with a singly occupied $\mathrm{d}_{\mathrm{z}^{2}}$ orbital. Thus, it is rather likely that 6 also has two iron centers in the $S=3 / 2$ spin states.

\subsubsection{Cyclic Voltammetry on 6}

Detailed insight into the redox properties of $\mathbf{6}$ is provided by cyclic voltammetry measurements. As shown in Figure 38, five main electron transfer processes between $-3.3 \mathrm{~V}$ and $1.5 \mathrm{~V} v s$. the ferrocene couple can be observed. The three cathodic processes observed at $-2.10,-2.45$, and $-2.65 \mathrm{~V} v s$. $\mathrm{Fc} / \mathrm{Fc}^{+}$are not fully reversible. Compared to $3(-1.33$ and $-2.03 \mathrm{~V})$, these reduction processes occur at very low potentials. In particular, the exchange of an oxo bridge for a nitrido bridge leads to an appreciable shift of about $800 \mathrm{mV}$ for the first reduction. ${ }^{[155]}$

Furthermore, two anodic processes can be observed at very low potentials, consistent with the high reactivity toward dioxygen, which leads to the formation of $\mu$-oxo compound $\mathbf{3}$. A first oxidation 
takes place at $-0.59 \mathrm{~V}$, followed by a second oxidation at $0.19 \mathrm{~V} v s . \mathrm{Fc} / \mathrm{Fc}^{+}$. For comparison, the first oxidation of $\mathbf{3}$ occurs at significantly higher potential $\left(0.87 \mathrm{~V} v s . \mathrm{Fc} / \mathrm{Fc}^{+}\right)$in agreement with its stability in air. The shape of the first oxidation process (Figure 38, right) suggests a one-electron process leading to a mixed valent $\mathrm{Fe}^{\mathrm{III}}-\mathrm{N}-\mathrm{Fe}^{\mathrm{IV}}$ compound. As indicated by a Cotrell plot (Appendix Figure 7), this oxidation is electrochemically reversible. The second oxidation appears to be not fully reversible and was not detected by Iris Klawitter in her measurements on compound $\mathbf{6}$. Within her studies, only a broad second oxidation around $0.62 \mathrm{~V}$ was detected, while the first oxidiation appeared to be more prominent. ${ }^{[155]}$
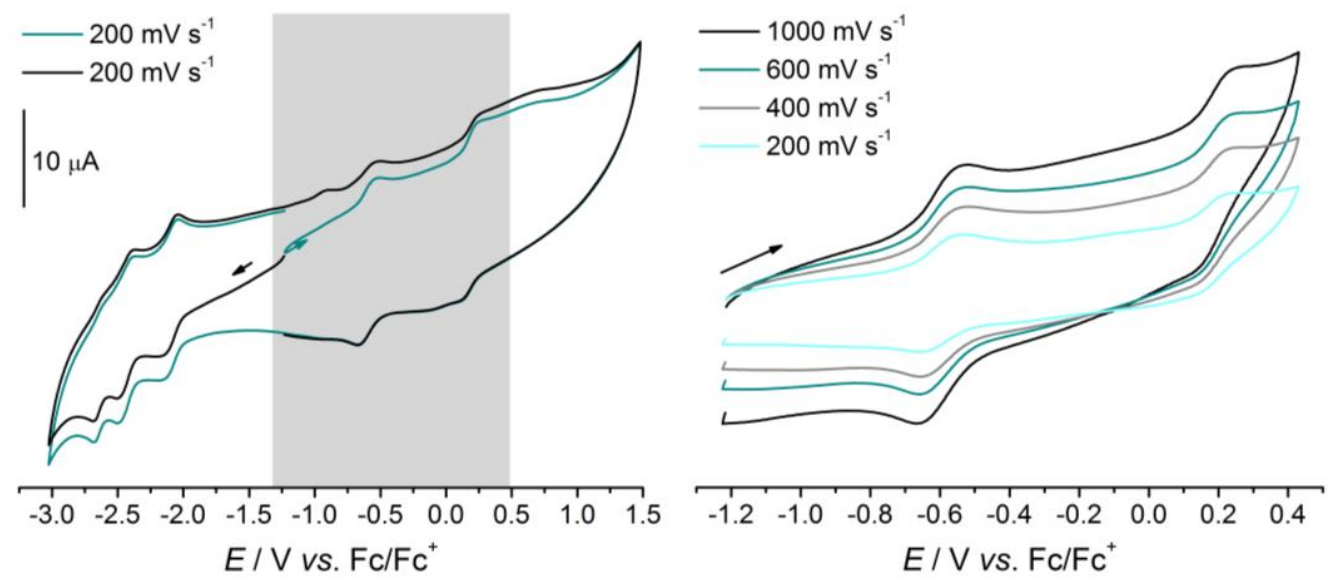

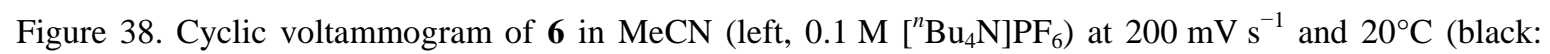
reduction first, cyan: oxidation first). The area shaded in grey was measured separately at various scan rates (right).

As introduced in the previous section, the iron centers in $\mathbf{6}$ are likely in the intermediate spin state. Thus, oxidation may correspond to the removal of the electron in the $\mathrm{d}_{\mathrm{z}^{2}}$ orbital. To overcome the resulting high positive charge at the iron(IV) center, interaction with $\sigma$-donating ligands is required. While the tetracarbene macrocycle may act as the perfect donor in the equatorial plane, the $\mathrm{N}^{3-}$ bridging ligand interacts with two iron centers along the $z$-axis. Nevertheless, the coordination mode probably switches from $5 \mathrm{C}$ to $6 \mathrm{C}$ upon oxidation, because of coordination of an additional solvent molecule trans to the nitrido bridge. Moreover, it is also likely that the availability of an anionic ligand instead for $\mathrm{MeCN}$ may further stabilize the oxidation state $+\mathrm{IV}$ and enables a fully reversible second oxidation. Thus, the cyclic voltammogram of $\mathbf{6}$ was measured in a comparative experiment in the presence of excess $\left[{ }^{n} \mathrm{Bu}_{4} \mathrm{~N}\right] \mathrm{NO}_{3}$ (Figure 39).

The first oxidative process is basically not affected by addition of nitrate salt while the second oxidation appears essentially different. The oxidative wave has a maximum at $0.21 \mathrm{~V}$ which is very similar to the oxidative wave in the original spectrum. However, the wave now appears significantly broadened and oxidative processes already start at about $-0.08 \mathrm{~V}$. Futhermore, the corresponding reduction wave is broadened as well and, even more important, shifted to lower potential. The maximum is now found at $-0.22 \mathrm{~V}$, thus the process becomes electrochemically less 
reversible but at the same time the redox potential of the entire oxidative process shifts from $0.19 \mathrm{~V}$ to $\sim 0.0 \mathrm{~V}$. Consequently, addition of nitrate anions is benefitial for the oxidation of $\mathbf{6}$ to the corresponding $\mu$-nitridoiron(IV) species. Chemical interpretation suggests that during the first oxidative process a mixed-valent $\mathrm{Fe}^{\mathrm{III}}-\mathrm{N}-\mathrm{Fe}^{\mathrm{IV}}$ species is formed that is subsequently further oxidized to $\mathrm{Fe}^{\mathrm{IV}}-\mathrm{N}-\mathrm{Fe}^{\mathrm{IV}}$ accompanied by coordination of nitrate. Compared to the non-nitrate coordinated $\mu$-nitridoiron(IV) compound that is formed in the normal $\mathrm{CV}$ of $\mathbf{6}$, the putative $\mathrm{O}_{3} \mathrm{~N}-\mathrm{Fe}^{\mathrm{IV}}-\mathrm{N}-\mathrm{Fe}^{\mathrm{IV}}-\mathrm{NO}_{3}$ species is more negatively charged which aggravates its reduction, in perfect agreement with the observed shift in the maximum potential of the reductive wave.

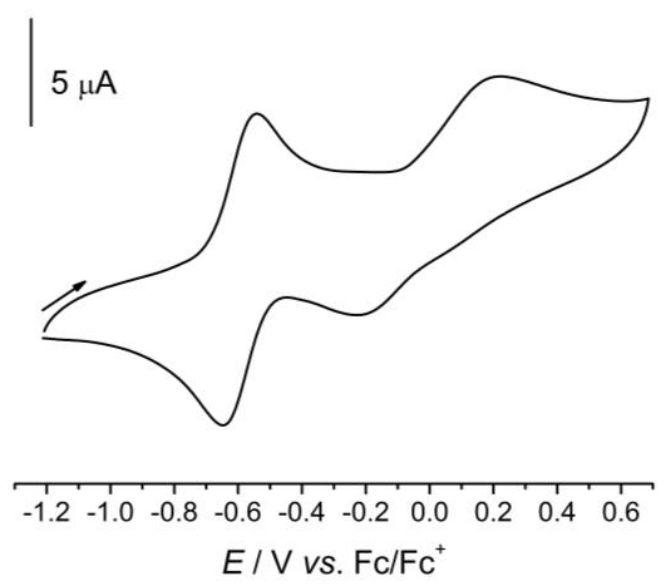

Figure 39. Cyclic voltammogram of $6(1.0 \mathrm{mM})$ after addition of $\sim 20$ eq. of $\left[{ }^{n} \mathrm{Bu}_{4} \mathrm{~N} \mathrm{NO}_{3}\right.$ in $\mathrm{MeCN}(0.1 \mathrm{M}$ $\left[{ }^{n} \mathrm{Bu}_{4} \mathrm{~N}\right] \mathrm{PF}_{6}$ ) at $50 \mathrm{mV} \mathrm{s}^{-1}$.

In conclusion, the observation of two oxidations at rather low potentials in the cyclic voltammogram of 6 provides motivation to study the corresponding chemical oxidation processes. Furthermore, a distinct influence of nitrate ions as potential axial coordinating ligands is observed electrochemically, thus it is of high interest to compare chemical oxidation processes with and without coordinating anions having available.

\subsubsection{Oxidation of 6 with Silver Salts}

Initial studies on the one-electron oxidation of $\mathbf{6}$ have been performed by Iris Klawitter, using the soft oxidizing agent 7,7,8,8-tetracyanoquionodimethan (TCNQ, $\mathrm{E}_{1 / 2}=-0.3 \mathrm{~V}$ vs. $\mathrm{Fc} / \mathrm{Fc}^{+}$in $\mathrm{MeCN})^{[207]}$ in $\mathrm{MeCN}$ at $-35^{\circ} \mathrm{C}$. Based on the $\mathrm{CV}$ shown in Figure 38, TCNQ is expected to only mediate the first oxidation to the corresponding mixed-valent $\mathrm{Fe}^{\mathrm{III}}-\mathrm{N}-\mathrm{Fe}^{\mathrm{IV}}$ species. The $\mathrm{MB}$ spectrum of the frozen reaction mixture showed a temperature independent quadrupole doublet at $\delta=-0.14 \mathrm{~mm} \mathrm{~s}^{-1}$ with $\Delta E_{\mathrm{Q}}=2.44 \mathrm{~mm} \mathrm{~s}^{-1}$. The significantly lower isomer shift compared to the non-oxidized compound $\mathbf{6}$ indicates a metal centered oxidation. In the case of mixed valent $\mu$-nitrido species, it is known that the nitrido bridge can enable fast electron exchange resulting in 
the observation of only one doublet in the MB spectrum. ${ }^{[210-212]}$ However, the isomer shift of the oxidized species is very similar to that of the oxoiron(IV) compound $\left[\left({ }^{\mathrm{NHC}} \mathrm{L}\right) \mathrm{Fe}=\mathrm{O}\right]^{2+} \mathbf{2}$ $\left(\delta=-0.13 \mathrm{~mm} \mathrm{~s}^{-1}\right){ }^{[153]}$ Thus, further evidence for a successful one-electron oxidation and isolation of the product compound was pursued.

As part of this work, 6 was oxidized with 1 eq. of the radical cation thianthrenyl tetrafluoroborate $\left(E_{1 / 2}=0.86 \mathrm{~V} \text { vs. } \mathrm{Fc} / \mathrm{Fc}^{+} \text {in } \mathrm{MeCN}\right)^{[207]}$ as well as with silver hexafluoroantimonate and silver triflate $\left(\mathrm{Ag}^{+}, E_{1 / 2}=0.0 \mathrm{~V} v s . \mathrm{Fc} / \mathrm{Fc}^{+}\right.$in $\mathrm{MeCN} .{ }^{[207]}$ In the case of silver oxidants, addition of $\mathrm{AgX}$ led to an immediate color change from the green-blue color of 6 to a brown suspension. Removal of elemental silver upon filtration via celite resulted in brown-red solutions. A subsequent EPR measurement on both oxidations (thianthrenyl and silver salt) revealed a signal at $g_{\mathrm{x}}=2.095$, $g_{\mathrm{y}}=2.085$, and $g_{\mathrm{z}}=2.013$ that can be assigned to the one-electron oxidized compound 12 (Figure 40 , left). This signal is suggestive of a low spin $S=1 / 2$ iron(III) center rather than an intermediate spin $S=3 / 2$ ion due to the absence of a half-field signal around $\mathrm{g} \sim 4$.
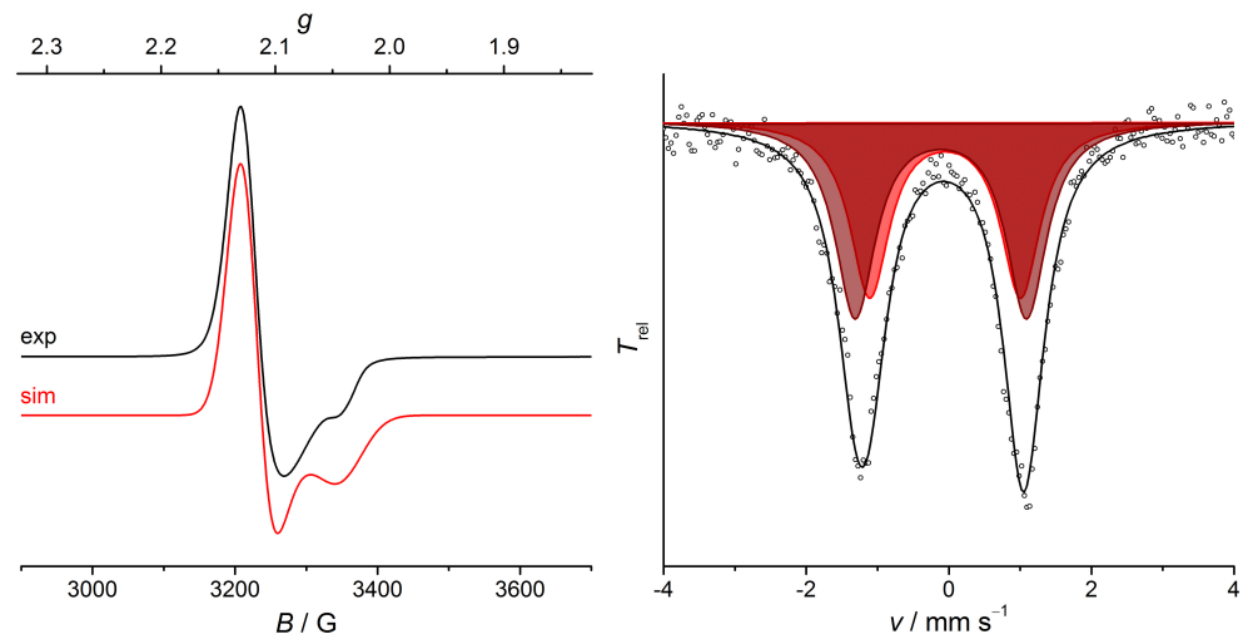

Figure 40. Left: X-band EPR spectrum (black, $163 \mathrm{~K}$ ) of 12, product of the oxidation of 6 with 1 eq. $\mathrm{Ag}\left(\mathrm{SbF}_{6}\right)$ and simulation (red) with the following $g$-values: $g_{\mathrm{x}}=2.095, g_{\mathrm{y}}=2.085$, and $g_{\mathrm{z}}=2.013$ (corresponding line widths 21, 30, $40 \mathrm{G}$ ). Right: Zero-field ${ }^{57} \mathrm{Fe} \mathrm{MB}$ spectrum of 12 at $7 \mathrm{~K}$ (natural abundance ${ }^{57} \mathrm{Fe}, \delta=-0.05 \mathrm{~mm} \mathrm{~s}^{-1}$ and $\Delta E_{\mathrm{Q}}=2.11 \mathrm{~mm} \mathrm{~s}^{-1} ; \delta=-0.11 \mathrm{~mm} \mathrm{~s}^{-1}$ and $\Delta E_{\mathrm{Q}}=2.40 \mathrm{~mm} \mathrm{~s}^{-1}$ ).

Furthermore, a MB spectrum of the corresponding brown powder was recorded (Figure 40, right). The observed signal was simulated as two relatively similar doublets in a ratio of $1: 1$ with the parameters $\delta=-0.05 \mathrm{~mm} \mathrm{~s}^{-1}$ and $\Delta E_{\mathrm{Q}}=2.11 \mathrm{~mm} \mathrm{~s}^{-1}$ as well as $\delta=-0.11 \mathrm{~mm} \mathrm{~s}^{-1}$ and $\Delta E_{\mathrm{Q}}=2.40 \mathrm{~mm} \mathrm{~s}^{-1}$. The former signal is assigned to the iron(III) center while the latter one corresponds to the iron(IV) side. The iron(III) center shows a similar IS value as found for the precursor complex $\mathbf{6}$, but the quadrupole splitting is significantly increased. This may be an indication for a change in the spin state from $S=3 / 2$ in $\mathbf{6}$ to $S=1 / 2$ in $\mathbf{1 2}$ or a result of the observed changes in the $\mathrm{Fe}-\mathrm{N}$ bond lengthes. The small difference in the IS of the iron(III) and the iron(IV) center can be explained with the longer average $\mathrm{Fe}^{\mathrm{IV}}-\mathrm{C}$ bond distance compared to the $\mathrm{Fe}^{\mathrm{III}}-\mathrm{C}$ 
distances as observed in the crystal structure (Figure 41, Table 7). The longer bonds suggest a weaker $\sigma$-donation of the carbenes to the iron(IV) center, consequently decreasing the s-electron density, in stark contrast to the increased s-electron density resulting from the metal-centered oxidation (less shielding by d-electrons). The spectrum was also simulated with only one doublet giving rise to an IS of $\delta=-0.08 \mathrm{~mm} \mathrm{~s}^{-1}$ and a QS of $\Delta E_{\mathrm{Q}}=2.27 \mathrm{~mm} \mathrm{~s}^{-1}$, however, the resulting fit was not as good as the one with two doublets. Futhermore, the obtained crystal structure largely suggests localization of the charge rather than the existence of two undistinguishable iron(3.5) centers.

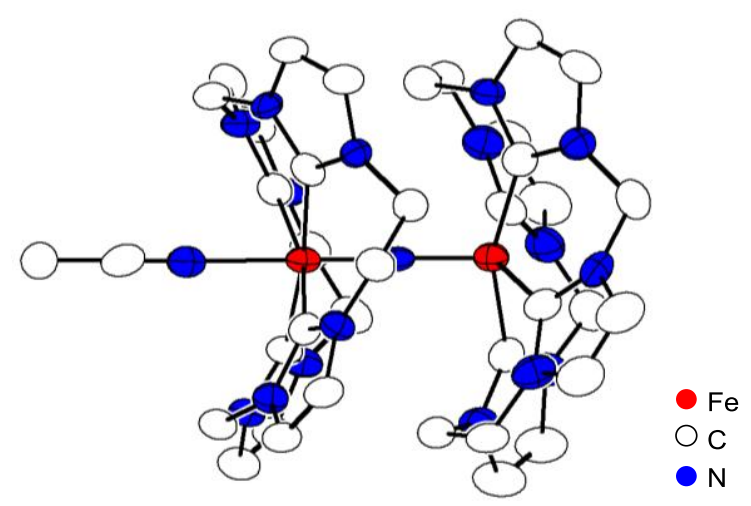

Figure 41. ORTEP plot of the molecular structure of $\left[\left({ }^{\mathrm{NHC}} \mathrm{L}\right) \mathrm{Fe}^{\mathrm{III}}-\mathrm{N}-\mathrm{Fe}^{\mathrm{IV}}\left({ }^{\mathrm{NHC}} \mathrm{L}\right)(\mathrm{MeCN})\right]^{4+} \mathbf{1 2}$ (left) (50\% probability thermal ellipsoids); hydrogen atoms and anions (OTf) are omitted for clarity, important bond lengths and angles are tabulated in Table 7.

Diffusion of $\mathrm{Et}_{2} \mathrm{O}$ into a brown solution of $\mathbf{1 2}$ in DMF at $\mathrm{RT}$ allowed for the isolation of red crystals suitable for X-ray crystallography. The molecular structure is depicted in Figure 41, characteristic bond lengths and angles are tabulated in Table 7. X-ray diffraction gave evidence for the formation of the mixed-valent species $\left[\left({ }^{\mathrm{NHC}} \mathrm{L}\right) \mathrm{Fe}-\mathrm{N}-\mathrm{Fe}\left({ }^{\mathrm{NHC}} \mathrm{L}\right)(\mathrm{MeCN})\right]^{4+} \mathbf{1 2}$, crystallizing in the monoclinic space group $P 21 /$ n. One iron center in $\mathbf{1 2}$ is coordinated in a square pyramidal fashion similar to what was found for the iron centers in the precursor complex $\mathbf{6}$. It shows a similar average $\mathrm{Fe}-\mathrm{C}$ bond length $(1.99 \AA)$, while the $\mathrm{Fe}-\mathrm{N}$ bond length is significantly longer $(1.71 \AA$ ). The second iron center in $\mathbf{1 2}$ is octahedrally coordinated with an additional MeCN molecule at the free axial position. This finding is in line with the considerations discussed in the previous sections: oxidation of the SPY-5 iron(III) center to iron(IV) induces a change of the coordination mode to OC-6. The average Fe-C (2.01 $\AA$ ) bond length of the iron(IV) center is longer than for the iron(III) center, while a shorter Fe-N bond is observed (1.69 $)$ ). Thus, the Fe-N-Fe bridge in the mixedvalent species is still linear $\left(179^{\circ}\right)$ but not symmetric anymore.

Unfortunately, the crystals were unstable in ambient conditions preventing full characterization of crystalline material. In hopes of increasing the stability of the corresponding mixed-valent species, 6 was also oxidized with 1 eq. of $\mathrm{AgNO}_{3}$. According to the results obtained in the cyclic voltammetry experiments, the nitrate ligand is expected to further stabilize the iron(IV) center and 
coordinate at the axial position replacing the $\mathrm{MeCN}$ molecule. The putative $\left[\left({ }^{\mathrm{NHC}} \mathrm{L}\right) \mathrm{Fe}-\mathrm{N}-\right.$ $\left.\mathrm{Fe}\left({ }^{\mathrm{NHC}} \mathrm{L}\right)\left(\mathrm{ONO}_{2}\right)\right]^{3+}$ compound $\mathbf{1 3}$ gave rise to a very similar signal in the EPR spectrum $\left(g_{\mathrm{x}}=2.094, g_{\mathrm{y}}=2.080\right.$, and $\left.g_{\mathrm{z}}=2.010\right)$ compared to 12 in agreement with the spin being located at a similar iron(III) center (Figure 42, left).

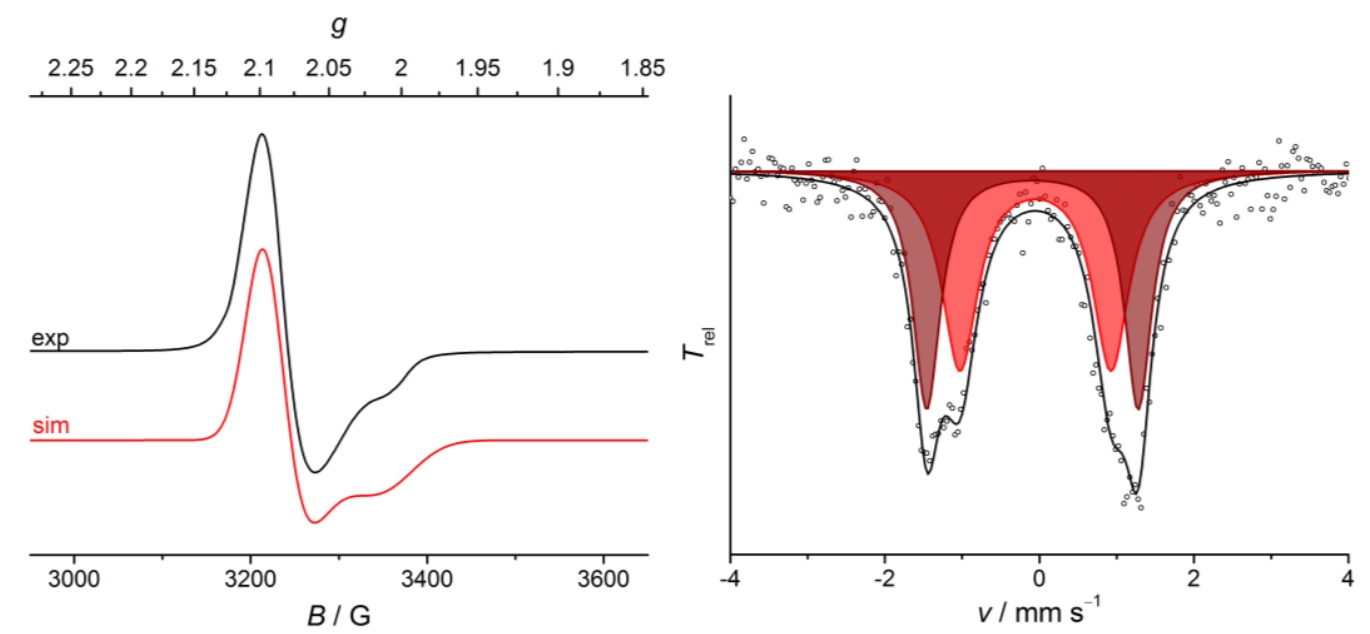

Figure 42. Left: X-band EPR spectrum (black, $162 \mathrm{~K}$ ) of 13, product of the oxidation of 6 with one equivalent of $\mathrm{AgNO}_{3}$ and simulation (red) with the following $g$-values: $g_{\mathrm{x}}=2.0935, g_{\mathrm{y}}=2.0802$, and $g_{\mathrm{z}}=2.0100$ (corresponding line widths 23, 30, $40 \mathrm{G}$ ). Right: Zero-field ${ }^{57} \mathrm{Fe} \mathrm{MB}$ spectrum of 13 at $7 \mathrm{~K}$ (natural abundance ${ }^{57} \mathrm{Fe}, \quad \delta=-0.06 \mathrm{~mm} \mathrm{~s}^{-1}$ and $\Delta E_{\mathrm{Q}}=1.96 \mathrm{~mm} \mathrm{~s}^{-1} ; \quad \delta=-0.10 \mathrm{~mm} \mathrm{~s}^{-1}$ and $\left.\Delta E_{\mathrm{Q}}=2.74 \mathrm{~mm} \mathrm{~s}^{-1}\right)$.

Furthermore, a MB spectrum (Figure 42, right) of the red solid 13 showed two temperature independent doublets in a $1: 1$ ratio. One doublet with $\delta=-0.06 \mathrm{~mm} \mathrm{~s}^{-1}$ and $\Delta E_{\mathrm{Q}}=1.96 \mathrm{~mm} \mathrm{~s}^{-1}$ can be assigned to the iron(III) center. The second doublet shows a lower IS $\left(\delta=-0.10 \mathrm{~mm} \mathrm{~s}^{-1}\right)$ and a larger QS $\left(\Delta E_{\mathrm{Q}}=2.74 \mathrm{~mm} \mathrm{~s}^{-1}\right)$ and is assigned to the iron(IV) center. In this case, a simulation with only one doublet does not represent the experimental data, which is indicative of a more pronounced charge localization in $\mathbf{1 3}$ when compared to $\mathbf{1 2}$. While the parameters for the iron(III) centers are very similar in $\mathbf{1 2}$ and 13, the QS of the iron(IV) center is significantly increased in 13. This is in line with a change in the symmetry as result of the coordination of the nitrite instead for-NCMe. However, it was not possible to crystallize this compound so far and, consequently, no direct evidence for the formation of $\left[\left({ }^{\mathrm{NHC}} \mathrm{L}\right) \mathrm{Fe}^{\mathrm{III}}-\mathrm{N}-\mathrm{Fe}^{\mathrm{IV}}\left({ }^{\mathrm{NHC}} \mathrm{L}\right)\left(\mathrm{ONO}_{2}\right)\right]^{4+} \mathbf{1 3}$ was obtained.

Interestingly, when 6 was reacted with $\geq 2$ eq. of $\mathrm{AgNO}_{3}$, a diamagnetic brown compound was obtained, showing only one doublet in the MB spectrum with $\delta=-0.16 \mathrm{~mm} \mathrm{~s}^{-1}$ and $\Delta E_{\mathrm{Q}}=3.12 \mathrm{~mm} \mathrm{~s}^{-1}$, indicative of two identical iron(IV) centers (Figure 43, left). Crystallization by diffusion of $\mathrm{Et}_{2} \mathrm{O}$ into a $\mathrm{MeCN}$ solution at $\mathrm{RT}$ led to the formation of brown crystals suitable for $\mathrm{X}$-ray diffraction analysis. The molecular structure of $\left[\left\{\left(\mathrm{O}_{2} \mathrm{NO}\right)\left({ }^{\mathrm{NHC}} \mathrm{L}\right) \mathrm{Fe}^{\mathrm{IV}}\right\}_{2} \mathrm{~N}\right]^{3+} \mathbf{1 4}$ is depicted in Figure 43 (right), important structural parameters are given in Table 7. 


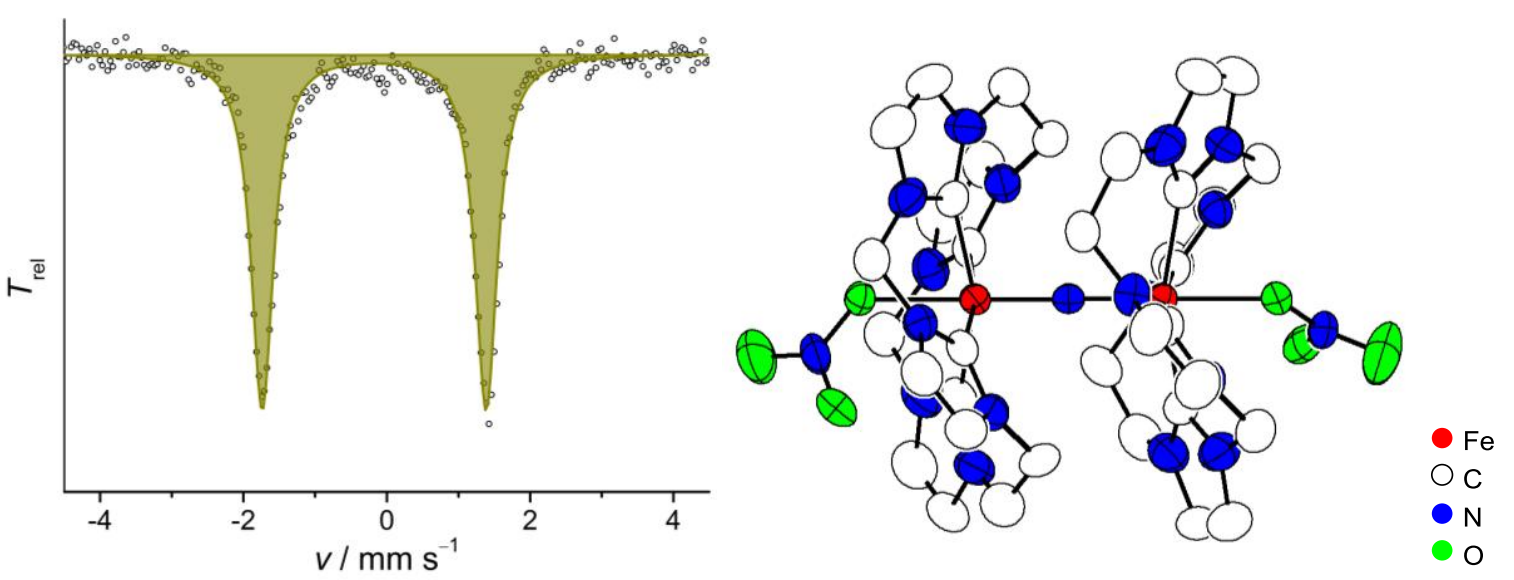

Figure 43. Left: Zero-field ${ }^{57} \mathrm{Fe} \mathrm{MB}$ spectrum of $\mathbf{1 4}$ at $80 \mathrm{~K}$ (natural abundance ${ }^{57} \mathrm{Fe}, \delta=-0.16 \mathrm{~mm} \mathrm{~s}^{-1}$, $\left.\Delta E_{\mathrm{Q}}=3.12 \mathrm{~mm} \mathrm{~s}^{-1}\right)$. Right: ORTEP plot of the molecular structure of $\left[\left\{\left(\mathrm{O}_{2} \mathrm{NO}\right)\left({ }^{\mathrm{NHC}} \mathrm{L}\right) \mathrm{Fe}^{\mathrm{IV}}\right\}^{2} \mathrm{~N}\right]^{3+} \mathbf{1 4}(50 \%$ probability thermal ellipsoids); hydrogen atoms and anions (OTf) are omitted for clarity, important bond lengths and angles are tabulated in Table 7.

Compound 14 crystallizes in the space group $I 4_{1} /$ acd with two iron centers in the oxidation state + IV. As expected, the iron centers are octahedrally coordinated by the tetracarbene ligand scaffold in the local equatorial plane with a nitrite group as well as a bridging nitrido ligand in the axial positions. As in the $\mu$-nitridoiron(III) system $\mathbf{6}$, the Fe-N-Fe moiety is symmetric (1.69 $\AA$ ) and almost perfectly linear $\left(179^{\circ}\right)$. While the $\mathrm{Fe}-\mathrm{N}$ bonds in $\mathbf{6}$ and $\mathbf{1 4}$ are similar, significantly longer $\mathrm{Fe}-\mathrm{C}$ bonds are found in the doubly oxidized compound (6: $1.99 \AA$ ․ 14: $2.02 \AA$ ).

Additionally, sharp NMR spectra typical for a diamagnetic species were recorded for 14. Similar to the results for the diiron(III) analog 6, four sets of signals are observed in the ${ }^{1} \mathrm{H}-\mathrm{NMR}$ spectrum but in this case, one signal set represents a major species and values for only this isomer are given in the experimental part. Compared to $\mathbf{6}$, the signals for the imidazole backbones and the methylene bridges in $\mathbf{1 4}$ are shifted downfield in the ${ }^{1} \mathrm{H}$-NMR spectrum. Most of the corresponding ${ }^{13} \mathrm{C}$ signals are found in a similar region, a significant difference was only observed for the carbene ${ }^{13} \mathrm{C}$ values (6: $187.5 \mathrm{ppm}, \mathbf{1 4}: 169.7 \mathrm{ppm})$.

Futhermore, UV/vis spectra were recorded for the $\mu$-nitridodiiron(III) compound $\mathbf{6}$, the mixedvalent species 12 and 13, and the high-valent diiron(IV) species 14; the obtained spectra are depicted in Figure 44 (left). As already reported by Iris Klawitter, complex 6 shows characteristic absorptions at $\lambda_{\max }=365,636$ and $785 \mathrm{~nm}$. Upon oxidation, the characteristic low energy bands in the visible region vanish while the transition at $\lambda_{\max }=365 \mathrm{~nm}\left(\mathbf{6}: \varepsilon \sim 25000 \mathrm{~L} \mathrm{~mol}^{-1} \mathrm{~cm}^{-1}\right)$ appears at significantly lower intensities; at $\varepsilon \sim 10000 \mathrm{~L} \mathrm{~mol}^{-1} \mathrm{~cm}^{-1}$ for $\mathbf{1 2}$ and $\mathbf{1 3}$, and at $\varepsilon \sim 3300 \mathrm{~L} \mathrm{~mol}^{-1} \mathrm{~cm}^{-1}$ for $\mathbf{1 4}$. Thus, following the reactions in the $\mathrm{UV} / \mathrm{vis}$ region gave rise to a decrease of the typical bands of $\mathbf{6}$ while no formation of characteristic new absorptions was observed. As expected based on the similar spectra of $\mathbf{1 2}$ and $\mathbf{1 3}$, it was not possible to differentiate 
the axial ligands in the UV/vis region. Still, the observed changes in the UV/vis spectra agree well with the observed color changes.
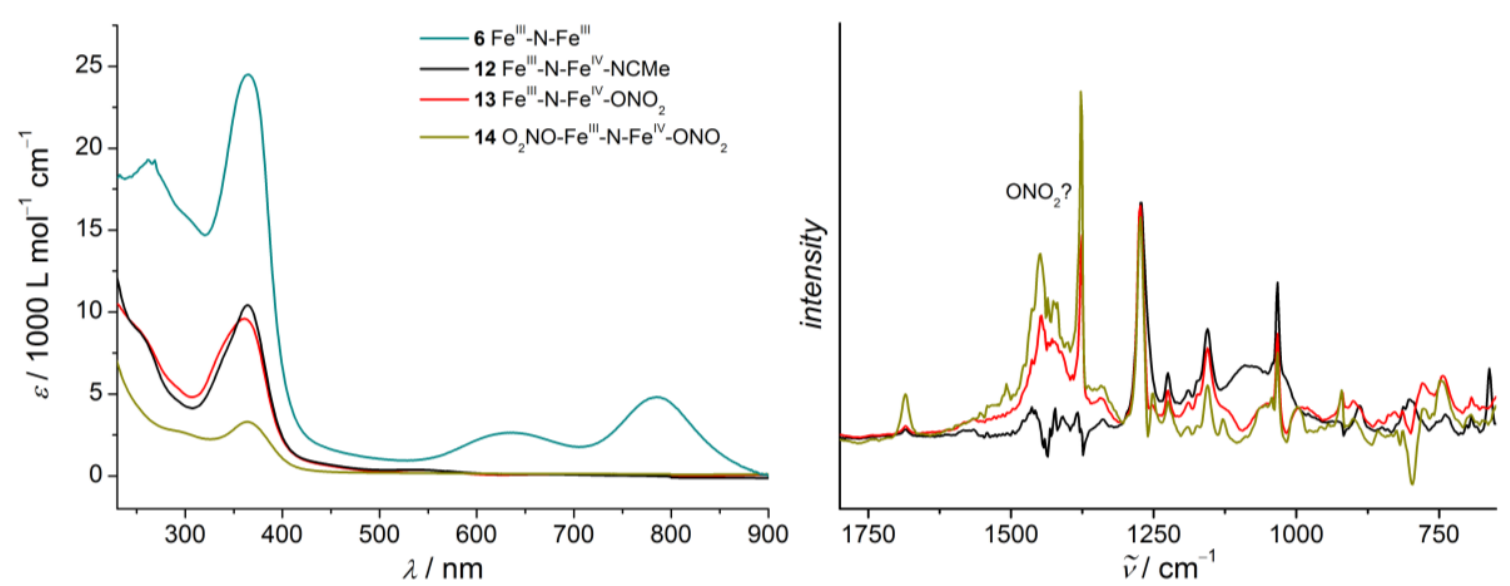

Figure 44. Comparative UV/vis (left) and Dial Path IR (right) measurements in MeCN solution of the $\mu$-nitridodiiron complexes discussed within this section.

Finally, comparative IR spectra were recorded. The Dial Path IR spectra of 12, 13 and 14 in $\mathrm{MeCN}$ solution show close similarities below $1300 \mathrm{~cm}^{-1}$ (Figure 44). In particular, all species give intense signals at $\tilde{v}=1033,1156$ and $1274 \mathrm{~cm}^{-1}$ which, consequently, likely correspond to vibrations within the tetracarbene ligand scaffold. Besides these similarities, the spectra of $\mathbf{1 3}$ and $\mathbf{1 4}$ show intense peaks in the range of $1300-1500 \mathrm{~cm}^{-1}$ that are not well pronounced in case of $\mathbf{1 2}$. In agreement with DFT calculations on 14 (DFT Figure 5), these signals are assigned to symmetric (left) and antisymmetric (right) vibrations within the $-\mathrm{ONO}_{2}$ moiety, as shown in Figure 45.

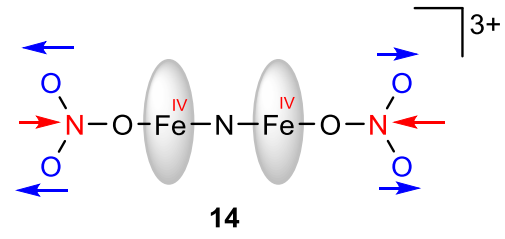

DFT: $1223+1227 \mathrm{~cm}^{-1}$

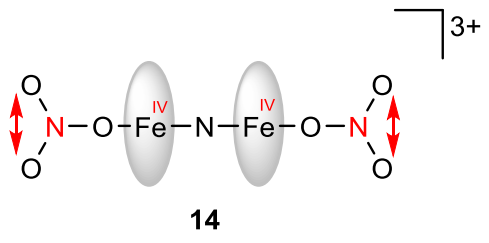

DFT: $2 \times 1528 \mathrm{~cm}^{-1}$

Figure 45. DFT-calculated IR active stretching vibrations within the $\mathrm{ONO}_{2}$ moiety, depicted is the in-phase movement of the two $\mathrm{ONO}_{2}$ groups, the second vibration close in energy corresponds to the out-of-phase movement. The arrows describe the movement of the atoms that are in the same color.

Furthermore, DFT predicts a $\mathrm{Fe}-\mathrm{N}-\mathrm{Fe}$ stretches of rather high intensity at $\tilde{v}=864$ and $945 \mathrm{~cm}^{-1}$. Since the spectra in solution do not allow for an unambiguous assignment of this stretch to an experimentally observed signal, solid state spectra have been recorded. Unfortunately, the solid state spectra give rise to only very slight differences among the series of $\mu$-nitrido species studies herein (Appendix Figure 8). Surprisingly, even the strong bands between $1500-1300 \mathrm{~cm}^{-1}$ for $\mathbf{1 3}$ and $\mathbf{1 4}$ are not observed in the solid spectra. For comparison, IR spectra of the starting material $\mathrm{AgNO}_{3}$ were recorded. In solution, four overlapping signals with maxima at $\tilde{v}=1303,1341,1381$ 
and $1417 \mathrm{~cm}^{-1}$ are observed. These signals are consistent with the findings made in solution spectra of 13 and 14 (maxima at around $\tilde{v} \sim 1376,1425,1448 \mathrm{~cm}^{-1}$ ) but at the same time the observed shifts clearly rule out the possibility that free $\mathrm{AgNO}_{3}$ was still present in the samples of $\mathbf{1 3}$ and $\mathbf{1 4}$. ATR measurements of solid $\mathrm{AgNO}_{3}$ reveal a broad and rather weak signal with a maximum at $\tilde{v} \sim 1295 \mathrm{~cm}^{-1}$ indicative of less prominent vibrations of the $-\mathrm{ONO}_{2}$ moiety in solid state. In order to provide a better understanding of the characteristics of these species in the IR region, the starting complex 6 was synthesized using $\left[{ }^{n} \mathrm{Bu}_{4} \mathrm{~N}\right]\left({ }^{15} \mathrm{~N}^{14} \mathrm{~N}_{2}\right)^{3-}$. Unfortunately, the obtained isotopically labeled product (theoretical ratio of ${ }^{14} \mathrm{~N} /{ }^{15} \mathrm{~N}$ : 1/1) gave rise to very similar IR spectra in both solution and solid state (Appendix Figure 9) indicative of the intense signals to be largely ligand based. Thus, it was so far not possible to assign the $\mathrm{Fe}-\mathrm{N}$ stretches.

In conclusion, the $\mu$-nitridodiiron(III) compound $\mathbf{6}$ could be sequentially oxidized twice using different silver oxidants. Oxidation of the iron(III) center to iron(IV) induces coordination of a sixth ligand at the free axial site and thus a change in the coordination environment, permitting the iron(IV) and the iron(III) ions to be distinguished in the MB spectrum. In the case of oxidation with $\mathrm{AgSbF}_{6}$, a MeCN solvent molecule is coordinated, while the use of $\mathrm{AgNO}_{3}$ presumably leads to coordination of $\mathrm{NO}_{3}{ }^{-}$. Conversion of 6 with $\geq 2$ eq. of $\mathrm{AgNO}_{3}$ leads to the formation of the doubly oxidized $\mu$-nitridodiiron(IV) compound $\mathbf{1 4}$, in accord with observations in the cyclic voltammetry measurements. Based on the cyclic voltammogram, the oxidizing potential of $\mathrm{Ag}^{+}$is sufficient to oxidize $\mathbf{1 3}$ to $\mathbf{1 4}$ in the presence of nitrate anions while it is too low to oxidize $\mathbf{1 2}$ when no coordinating anions are available. An overview about the MB parameters for the $\mu$-nitridodiiron complexes studied within this work is tabulated in Table 8 .

Table 8. Mössbauer parameters of the $\mu$-nitridodiiron complexes studied within this work.

\begin{tabular}{|c|c|c|}
\hline & $\delta\left[\mathrm{mm} \mathrm{s}^{-1}\right]$ & $\Delta E_{\mathrm{Q}}\left[\mathrm{mm} \mathrm{s}^{-1}\right]$ \\
\hline 6 & -0.06 & 1.27 \\
\hline+1 eq. $\operatorname{AgSbF}_{6}(12)$ & $-0.05 \&-0.11$ & $2.11 \& 2.40$ \\
\hline+1 eq. $\mathrm{AgNO}_{3}(13)$ & $-0.06 \&-0.10$ & $1.96 \& 2.74$ \\
\hline+2 eq. $\mathrm{AgNO}_{3}$ or + excess $\mathrm{NO} / \mathrm{O}_{2}(14)$ & -0.16 & 3.12 \\
\hline$+\operatorname{excess} N O_{(g)}$ & -0.16 & 2.69 \\
\hline+1 eq. $\mathrm{NOBF}_{4}(12)$ & $-0.05 \&-0.11$ & $2.01 \& 2.65$ \\
\hline+5 eq. $\mathrm{NOBF}_{4}(15)$ & -0.14 & 2.84 \\
\hline
\end{tabular}

Interestingly, the same $\mu$-nitridodiiron(IV) compound $\mathbf{1 4}$ was formed when $\mathbf{6}$ was reacted with a mixture of gaseous nitric oxide and dioxygen (see Table 8). Addition of oxygen to the nitric oxide gas phase leads to formation of a brown mixture of unstable ${ }^{\circ} \mathrm{NO}_{2}$ and $\mathrm{NO}_{3} \cdot{ }^{[213]}$ Presumably, the nitrogen trioxide radical oxidizes the iron(III) center in $\mathbf{6}$ and the resulting nitrate coordinates at the 
free axial position. When $\mathbf{6}$ was reacted with 'NO only, a compound with similar MB parameters as observed for 14 was generated (Figure $46, \delta=-0.16 \mathrm{~mm} \mathrm{~s}^{-1}$ and $\Delta E_{\mathrm{Q}}=2.69 \mathrm{~mm} \mathrm{~s}^{-1}$ ). Diffusion of $\mathrm{Et}_{2} \mathrm{O}$ into the corresponding brown $\mathrm{MeCN}$ solution resulted in co-crystallization of $\left[(\mathrm{ON})\left({ }^{\mathrm{NHC}} \mathrm{L}\right) \mathrm{Fe}^{\mathrm{IV}}-\mathrm{N}-\mathrm{Fe}^{\mathrm{IV}}\left({ }^{\mathrm{NHC}} \mathrm{L}\right)(\mathrm{NO})\right]^{3+}(17 \%)$ and $\left[(\mathrm{ONO})\left({ }^{\mathrm{NHC}} \mathrm{L}\right) \mathrm{Fe}^{\mathrm{IV}}-\mathrm{N}-\mathrm{Fe}^{\mathrm{IV}}\left({ }^{\mathrm{NHC}} \mathrm{L}\right)(\mathrm{ONO})\right]^{3+}$ (83\%). Impurities of ${ }^{\circ} \mathrm{NO}_{2}$ have been also observed during studies on the conversion of the iron(II) complex 1 with gaseous nitric oxide (Chapter 8). In the case of reaction of $\mathbf{6}$ with $\mathrm{NO}$, it remains unclear if the observed co-crystallization corresponds to the actual mixture of the two species in the whole sample or is just a result of the crystallization process. As mentioned, the MB spectrum gave rise to only one distinct signal; however, a mixture of two species with similar MB parameters in a ratio of 17:83 cannot be excluded (Appendix Figure 10).

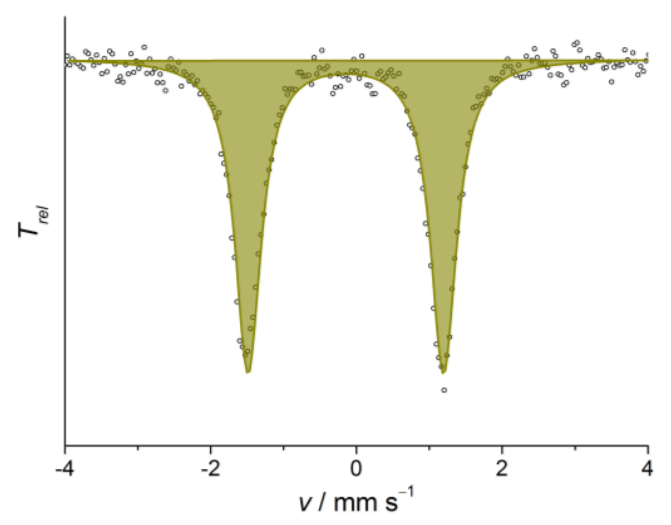

Figure 46. Zero-field ${ }^{57} \mathrm{Fe} \mathrm{MB}$ of the product obtained upon conversion of 6 with $\mathrm{NO}_{(\mathrm{g})}(80 \mathrm{~K}$, natural abundance ${ }^{57} \mathrm{Fe}, \delta=-0.16 \mathrm{~mm} \mathrm{~s}^{-1}$ and $\Delta E_{\mathrm{Q}}=2.69 \mathrm{~mm} \mathrm{~s}^{-1}$ ).

\subsubsection{Conversion of $\mathbf{6}$ with Nitrosonium Tetrafluoroborate}

Similar to the experiments with $\mathrm{AgNO}_{3}$, the strong oxidant nitrosonium tetrafluoroborate $\left(\mathrm{NOBF}_{4}\right.$, $E_{1 / 2}=0.87 \mathrm{~V} v s . \mathrm{Fc} / \mathrm{Fc}^{+}$in $\left.\mathrm{MeCN}\right)^{[207]}$ was also used for studies on 6. 1 eq. of $\mathrm{NO}^{+}$is expected to oxidize one iron center in $\mathbf{6}$ while it is reduced to NO. There are several possibilities regarding how the reaction proceeds further (Scheme 20). As shown in pathway (a), the NO radical could presumably oxidize the second iron ion and subsequently coordinate at one of the iron(IV) centers. Based on the results obtained so far, it is likely that either a NO-moiety or a solvent molecule (L) coordinates at the oxidized iron centers. Pathway (b) describes the direct coordination of the $\mathrm{NO}$ ligand at the iron(IV) center. However, this scenario is unlikely since an electron deficient $\{\mathrm{FeNO}\}^{5}$ species would be formed; a moiety that is so far not reported for iron nitrosyl compounds (discussed in chapter 8). Moreover, it is also possible that the ${ }^{-N O}$ coordinates on the iron(III) side resulting in a $\{\mathrm{FeNO}\}^{6}$ species (c). Additionally, the gaseous ${ }^{\circ} \mathrm{NO}$ may simply leave the reaction mixture, thus the solvent would coordinate at the oxidized iron center generating species $\mathbf{1 2}$ (d). Finally, the $\mathrm{NO}$ radical could also react faster with the remaining starting material 6 than with the 
mixed-valent intermediate. In the corresponding scenario (e), only 0.5 eq. $\mathrm{NO}^{+}$would be necessary for the full conversion of $\mathbf{6}$ into $\mathbf{1 2}$.

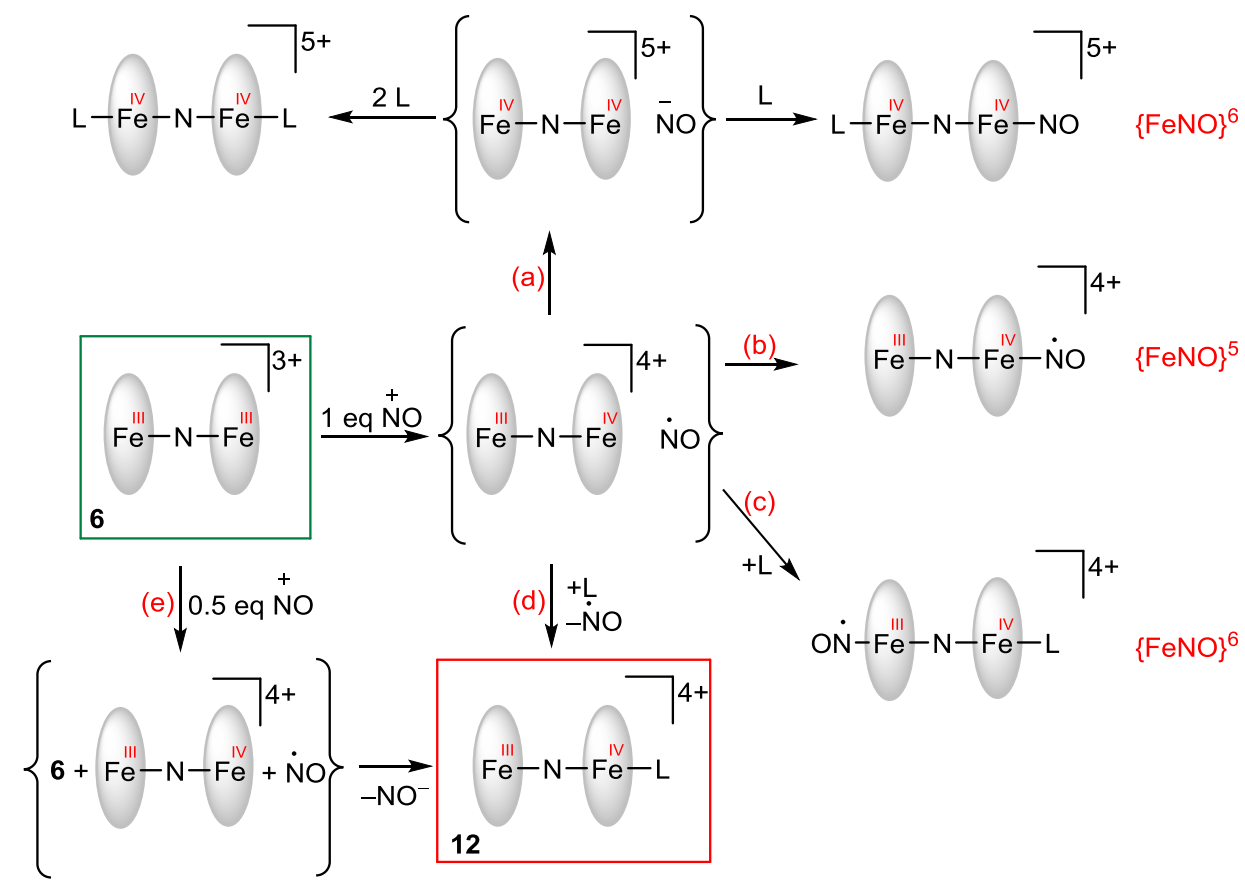

Scheme 20. Possible scenarios for the reaction of 6 with 1 eq. of $\mathrm{NOBF}_{4}$.
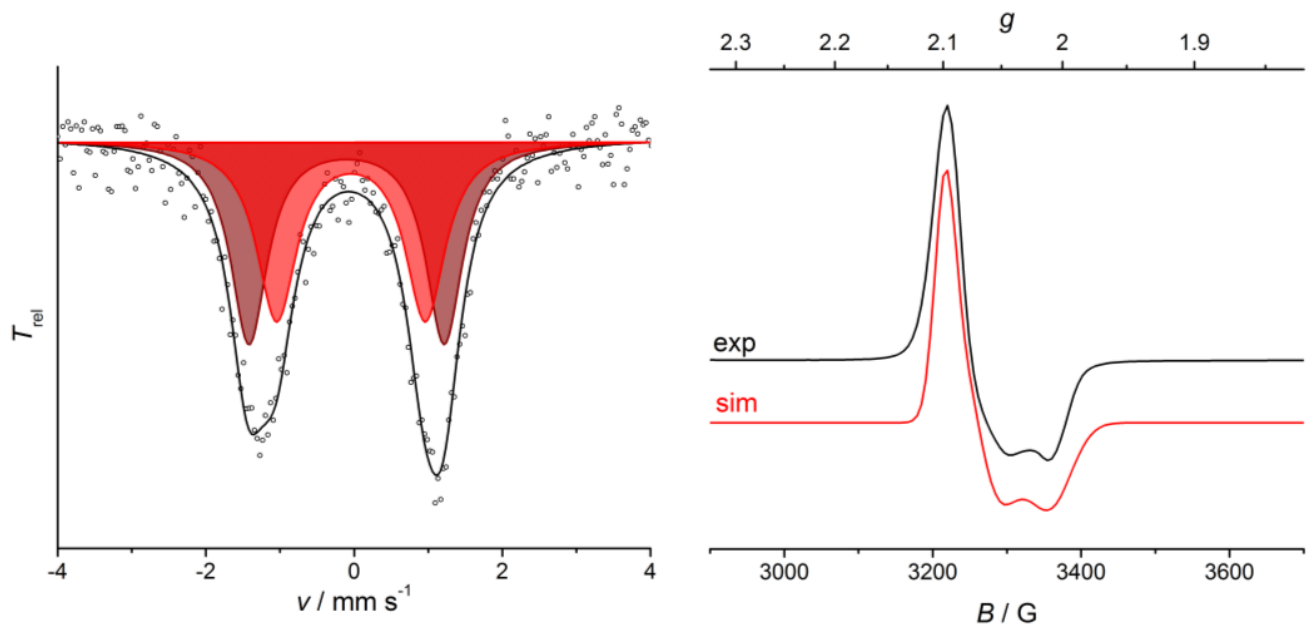

Figure 47. Left: Zero-field ${ }^{57} \mathrm{Fe} \mathrm{MB}$ spectrum after conversion of 6 with 1 eq. of $\mathrm{NOBF}_{4}$ at $7 \mathrm{~K}$ (natural abundance ${ }^{57} \mathrm{Fe}, \delta=-0.11 \mathrm{~mm} \mathrm{~s}^{-1}$ and $\Delta E_{\mathrm{Q}}=2.65 \mathrm{~mm} \mathrm{~s}^{-1} ; \delta=-0.05 \mathrm{~mm} \mathrm{~s}^{-1}$ and $\Delta E_{\mathrm{Q}}=2.01 \mathrm{~mm} \mathrm{~s}^{-1}$ ). Right: X-band EPR spectrum (black, $163 \mathrm{~K}$ ) of the product of conversion of 6 with 1 eq. of $\mathrm{NOBF}_{4}$ and simulation (red) with the following $g$-values: $g_{\mathrm{x}}=2.093, g_{\mathrm{y}}=2.063$, and $g_{\mathrm{z}}=2.004$ (corresponding line widths 15,28 , $30 \mathrm{G})$.

Addition of 1 eq. of $\mathrm{NOBF}_{4}$ to a MeCN solution of $\mathbf{6}$ induced an immediate color change from green-blue to red. The solvent was removed under vacuum and a MB spectrum of the resulting red solid was recorded. As depicted in Figure 47 (left), this spectrum showed a broad signal that can be simulated as two doublets with similar parameters as observed for the oxidations with $\mathrm{AgSbF}_{6}$ (Figure 40) and $\mathrm{AgNO}_{3}$ (Figure 42). One doublet shows a slightly lower IS and a larger QS 
$\left(\delta=-0.11 \mathrm{~mm} \mathrm{~s}^{-1}, \Delta E_{\mathrm{Q}}=2.65 \mathrm{~mm} \mathrm{~s}^{-1}\right)$ and likely corresponds to an iron(IV) center, while the other doublet is assigned to an iron(III) center $\left(\delta=-0.05 \mathrm{~mm} \mathrm{~s}^{-1}, \Delta E_{\mathrm{Q}}=2.01 \mathrm{~mm} \mathrm{~s}^{-1}\right)$. Based on these results, very similar species are formed in the experiments with 1 eq. of $\mathrm{AgSbF}_{6}, \mathrm{AgNO}_{3}$ and $\mathrm{NOBF}_{4}$. Consequently, pathway (a) resulting in a diiron(IV) species can be excluded. Furthermore, conversion with 0.5 eq. of $\mathrm{NOBF}_{4}$ gave rise to a mixture of the starting material 6 and the mixedvalent $\mathrm{Fe}^{\mathrm{III}}-\mathrm{N}-\mathrm{Fe}^{\mathrm{IV}}$ compound, as clarified by MB spectroscopy (Appendix Figure 11). This result allows excluding pathway (e) as well.

Futhermore, an EPR measurement after conversion of $\mathbf{6}$ with 1 eq. of $\mathrm{NO}^{+}$gave rise to the signal depicted in Figure 47 (right) that was simulated with $g$-values 2.093, 2.063 and 2.004. These values are very similar to the parameters for compounds 12 and 13 even though the shape of the spectrum slightly differs. The slight differences could be due to the presence of different counter anions $\left(\mathrm{OTf}^{-}, \mathrm{SbF}_{6}^{-}\right.$or $\left.\mathrm{BF}_{4}^{-}\right)$in the reaction mixtures.

To further clarify the mechanism, comparative IR spectra have been recorded. The spectra obtained after conversion of 6 with $0.5,1.0$ and 2.0 eq. of $\mathrm{NOBF}_{4}$ are shown in Figure 48. None of the spectra showed a signal in the expected area for a putative iron nitrosyl species $\left(\{\mathrm{FeNO}\}^{6}\right.$, $\tilde{v}=1800-2000 \mathrm{~cm}^{-1}$ ), ruling out the possibility of pathways (b) and (c). Furthermore, all the obtained IR spectra are essentially the same and resemble the spectrum recorded after conversion with $\mathrm{AgSbF}_{6}$. In particular, the most characteristic signals are again found at about $\tilde{v}=1272,1155$ and $1033 \mathrm{~cm}^{-1}$. In contrast to the products after conversion with $\mathrm{AgNO}_{3}$, no intense signals around $\tilde{v} \sim 1400 \mathrm{~cm}^{-1}$ are observed, in agreement with the assignement of these signals to vibrations within the $-\mathrm{ONO}_{2}$ ligand. Thus, pathway (d) is operative and the mixed-valent compound $\mathbf{1 2}$ is generated.
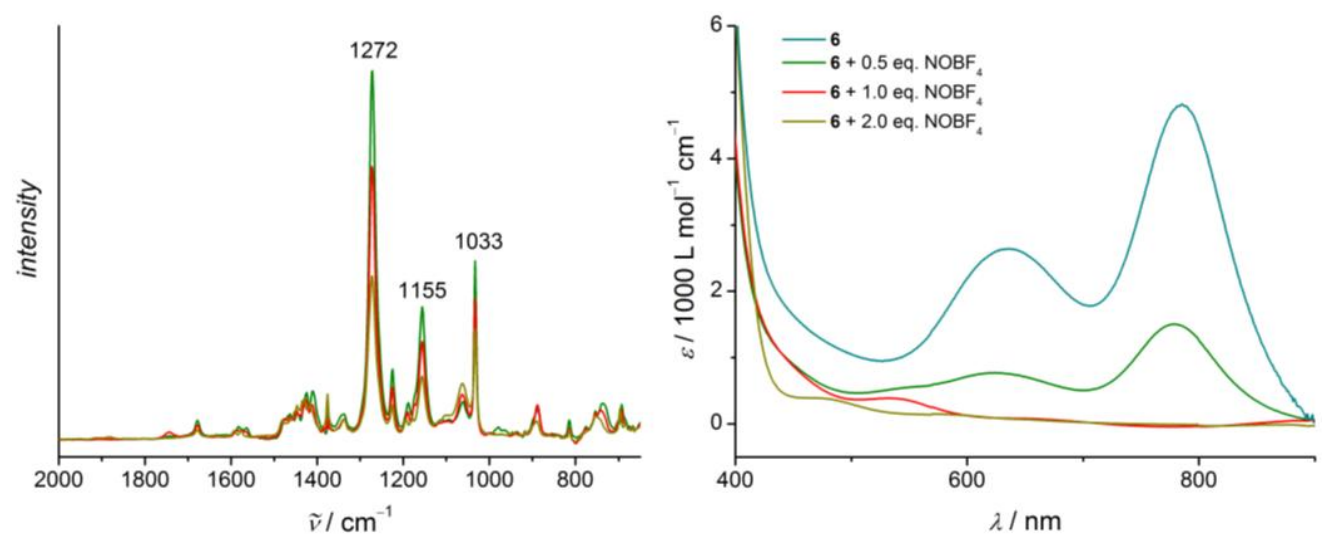

Figure 48. Dial Path IR and UV/vis spectra of the reaction mixtures obtained after conversion of 6 with 0.5 , 1.0 and 2.0 eq. of $\mathrm{NOBF}_{4}$ in $\mathrm{MeCN}$.

Moreover, UV/vis spectra were recorded. Similar to the results after conversion of 6 with silver oxidants, a decrease of the characteristic bands of 6 at $\lambda_{\max }=636$ and $785 \mathrm{~nm}$ is observed. This 
decrease is completed after addition of 1.0 eq. of $\mathrm{NOBF}_{4}$ and the resulting spectrum of the red solution with a small band at $\lambda_{\max }=535 \mathrm{~nm}$ is similar to the spectra of the mixed-valent species $\mathbf{1 2}$ and 13. In agreement with the MB studies described before, reaction of 6 with only 0.5 eq of $\mathrm{NO}^{+}$ leads to spectral features that agree well with the generation of a 1:1 mixture of the starting material and the mixed-valent product.

Conversion of 6 with 2 eq. of $\mathrm{NOBF}_{4}$ gave rise to a brown solution that features a UV/vis spectrum (Figure 48) similar to the one of $\mathbf{1 4}$ and a silent EPR spectrum. These observations agree well with the formation of a $\mu$-nitridodiiron(IV) species, which is in accordance with the high oxidizing potential of $\mathrm{NO}^{+}$. The brown solid obtained after conversion of 6 with 2 eq. of $\mathrm{NOBF}_{4}$ was analyzed by MB spectroscopy (Figure 49, left). The low IS of $\delta=-0.14 \mathrm{~mm} \mathrm{~s}^{-1}$ and the large quadrupole splitting of $\Delta E_{\mathrm{Q}}=2.84 \mathrm{~mm} \mathrm{~s}^{-1}$ are reminiscent of the spectra recorded after conversion with nitric oxide gas $\left(\delta=-0.16 \mathrm{~mm} \mathrm{~s}^{-1}, \Delta E_{\mathrm{Q}}=2.69 \mathrm{~mm} \mathrm{~s}^{-1}\right)$ and with excess of $\mathrm{AgNO}_{3}$ $\left(\delta=-0.16 \mathrm{~mm} \mathrm{~s}^{-1}, \Delta E_{\mathrm{Q}}=3.12 \mathrm{~mm} \mathrm{~s}^{-1}\right)$. These spectroscopic data are indicative of the formation of an $\mathrm{Fe}^{\mathrm{IV}}-\mathrm{N}-\mathrm{Fe}^{\mathrm{IV}}$ species. Based on the results obtained so far, it is very likely that the iron(IV) center is octahedrally coordinated and that, according to the IR spectrum, this ligand is not NO. Upon various crystallization attempts, it was possible to grow crystals suitable for X-ray diffraction by diffusion of $\mathrm{Et}_{2} \mathrm{O}$ into a $\mathrm{MeCN}$ solution of the crude product at RT (Figure 49, right). However, due to cocrystalliztion with small amounts of the mixed-valent species $\mathbf{1 2}$ the quality of the resulting data does not allow for the analysis of bond lengths and angles but does instead provide strong evidence for formation of $\left[(\mathrm{MeCN})\left({ }^{\mathrm{NHC}} \mathrm{L}\right) \mathrm{Fe}-\mathrm{N}-\mathrm{Fe}\left({ }^{\mathrm{NHC}} \mathrm{L}\right)(\mathrm{NCMe})\right]^{5+} \mathbf{1 5}$.
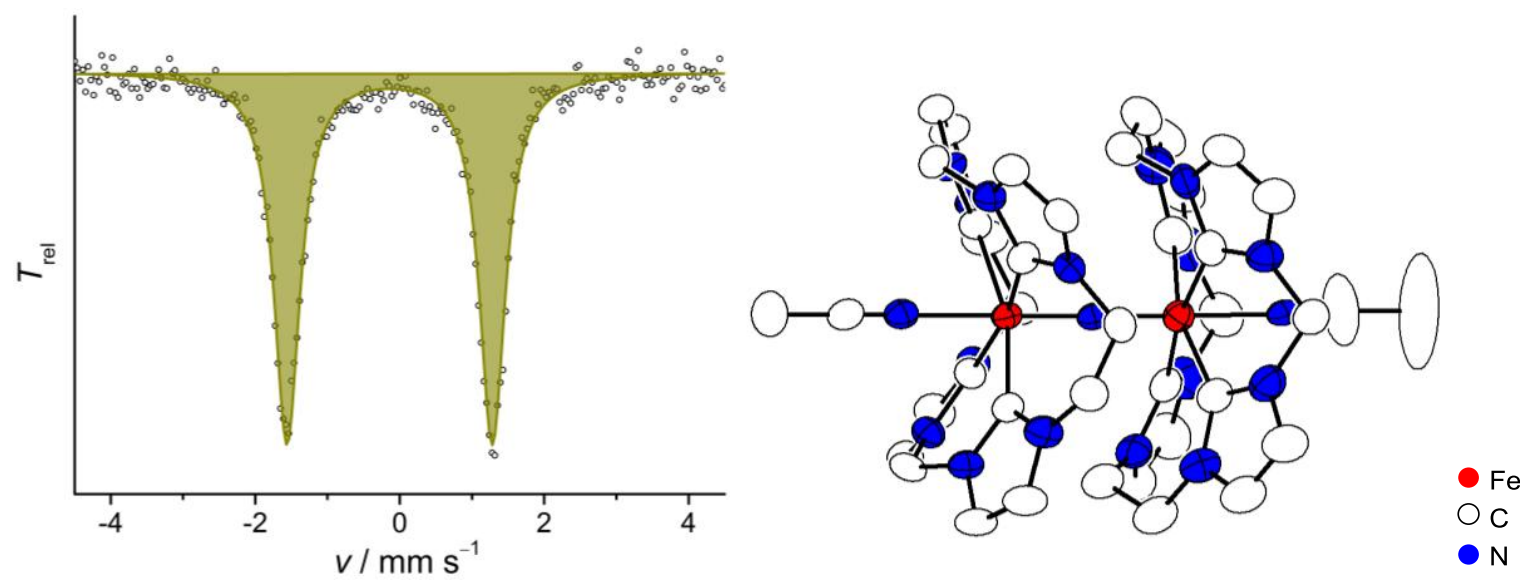

Figure 49. Left: Zero-field ${ }^{57} \mathrm{Fe} \mathrm{MB}$ spectrum after conversion of 6 with 2 eq. of $\mathrm{NOBF}_{4}$ at $80 \mathrm{~K}$ (natural abundance ${ }^{57} \mathrm{Fe}, \delta=-0.14 \mathrm{~mm} \mathrm{~s}^{-1}, \Delta E_{\mathrm{Q}}=2.84 \mathrm{~mm} \mathrm{~s}^{-1}$ ). Right: ORTEP plot of the molecular structure of $\left[\left\{(\mathrm{MeCN})\left({ }^{\mathrm{NHC}} \mathrm{L}\right) \mathrm{Fe}^{\mathrm{IV}}\right\}_{2} \mathrm{~N}\right]^{5+} \mathbf{1 5}(50 \%$ probability thermal ellipsoids); hydrogen atoms and anions (OTf) are omitted for clarity. The quality of the crystal structure does not allow a discussion of the observed bond lengths and angles.

In conclusion, conversion of 6 with 1 and 2 eq. of $\mathrm{NO}^{+}$allows for the generation of the mixedvalent $\mathrm{Fe}^{\mathrm{III}}-\mathrm{N}-\mathrm{Fe}^{\mathrm{IV}}$ compound 12 and the high-valent $\mathrm{Fe}^{\mathrm{IV}}-\mathrm{N}-\mathrm{Fe}^{\mathrm{IV}}$ species $\mathbf{1 5}$, respectively. The 
obtained spectroscopic data strongly speak for the coordination of $\mathrm{MeCN}$ at the iron(IV) sites in both complexes. Thus, $\mathrm{NO}^{+}$works as oxidizing reagent only. An overview on the reactivity of the $\mu$-nitridodiiron(III) complex 6 studied in this work is depicted in Scheme 21.

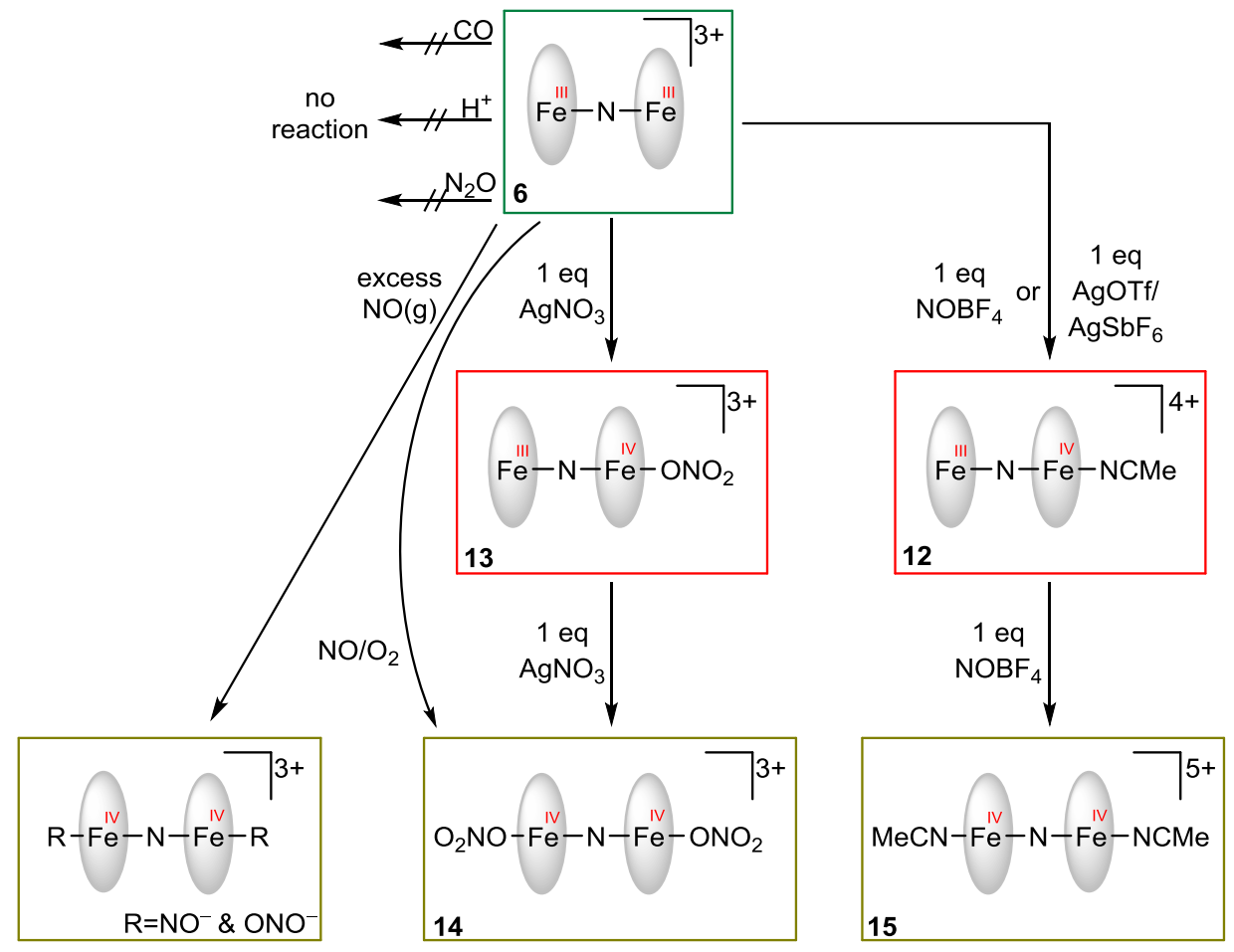

Scheme 21. Illustration of the reactivity of $\mathbf{6}$ studied within this work.

In addition to the discussed oxidative processes, 6 was also treated with acid (1,5-dimethylpyrdinium, $\left.\mathrm{p} K_{\mathrm{a}}=14.1\right)$, ${ }^{[214]} \mathrm{CO}$, or $\mathrm{N}_{2} \mathrm{O}$ in order to observe reactivity of the bridging nitrido ligand. In all these conversions, no reaction was observed. Thus, no conclusive data on the potential cleavage of the $\mathrm{Fe}-\mathrm{N}-\mathrm{Fe}$ bridge could be obtained, so far. According to literature reported studies on the reactivity of mixed-valent and high-valent $\mu$-nitrodo species, ${ }^{[105]}$ 12-15 might represent promising starting points for further reactivity studies. Besides cleavage of the nitrido bridge, exchange of the axial $\mathrm{MeCN}$ or ${ }^{-} \mathrm{ONO}_{2}$ ligands by an oxo group may yield high-valent $\mathrm{R}-$ $\mathrm{N}-\mathrm{Fe}=\mathrm{O}$ compounds as suitable catalysts for the oxidation of organic substrates. 


\section{Formation of Organometallic $\operatorname{Iron}(V)$ Species}

\subsection{Aim of this Project}

Beyond the synthesis of the first organometallic oxoiron(IV) complex 2 by Steffen Meyer, ${ }^{[153]}$ two new high-valent $\mu$-nitridodiiron(IV) compounds (14 and 15, $\left[\left\{(\mathrm{L})\left({ }^{\mathrm{NHC}} \mathrm{L}\right) \mathrm{Fe}\right\}_{2} \mathrm{~N}\right]^{4+}$, section 6.3) were presented as part of this work. Furthermore, first indications for the generation of a nitridoiron(V) compound upon irradiation of $\left.\mathbf{1 1}\left(\left[{ }^{\mathrm{NHC}} \mathrm{L}\right) \mathrm{Fe}\left(\mathrm{N}_{3}\right)_{2}\right]^{+}\right)$have been described in the previous chapter. While several bioinorganic iron(IV) model compounds have been presented so far, ${ }^{[7,52,58,60,69,215]}$ the number of spectroscopically characterized oxoiron(V) complexes is still limited. ${ }^{[56,81,216,217]}$ This provides further motivation to study possible strategies on the way to tetracarbene-coordinated iron $(\mathrm{V})$ compounds.

A possibility to generate a tetracarbene coordinated iron $(\mathrm{V})$ complex is one-electron oxidation of 2 at suitable conditions. Electrochemical studies show an irreversible oxidation of 2 at $E_{1 / 2} \sim 1.1 \mathrm{~V} v s$. $\mathrm{Fc} / \mathrm{Fc}^{+}$which is indicative of instability of the corresponding oxidized species. Thus, further stabilization of the corresponding oxidized species might be necessary. According to literature, binding of Lewis acids can enable isolation of highly unstable intermediates due to shifts in the redox potentials. ${ }^{[58,218]}$ An important example in this regard is the Lewis acid $\mathrm{Sc}^{3+}$ whose reactivity toward 2 was studied by Iris Klawitter. ${ }^{[155]}$ In the course of her work, it was observed that addition of $\mathrm{Sc}^{3+}$ mediates reduction of 2 to the iron(III) species $\left[\left({ }^{\mathrm{NHC}} \mathrm{L}\right) \mathrm{Fe}(\mathrm{MeCN})_{2}\right]^{3+}(\mathbf{9})$, ruling out the possibility to stabilize the desired oxoiron(V) compound. Thus, a strong oxidizing agent that simultaneously provides the possibility to stabilize the oxidized species is needed. A promising oxidant in this regard is $\mathrm{XeF}_{2}$, which is expected to both oxidize the iron center and transfer a fluorido ligand. The results on studies with $\mathrm{XeF}_{2}$ are presented in the following section.

Another strategy on the way to oxoiron(V) centers is protonation of the oxoiron(IV) center and subsequent $\mathrm{H}$-atom abstraction. However, according to UV/vis experiments conducted by Iris Klawitter, protonation of $\mathbf{2}$ leads again to formation of the bis-acetonitrile coordinated iron(III) complex 9.

Searching the literature for further oxoiron $(\mathrm{V})$ species, a report by Que and co-workers is of particular interest. ${ }^{[169]}$ Treatment of $\left[(\mathrm{TMC}) \mathrm{Fe}^{\mathrm{IV}}=\mathrm{O}(\mathrm{NCMe})\right]^{2+}$ with ${ }^{t} \mathrm{BuOOH}$ and base was proposed to mediate attack on the $\mathrm{MeCN}$ ligand and generation of a putative $\left[(\mathrm{TMC}) \mathrm{Fe}^{\mathrm{V}}=\mathrm{O}(\mathrm{NC}(\mathrm{O}) \mathrm{Me})\right]^{+}$compound. Due to the similar coordination sphere when compared to the TMC coordinated compound, $\mathbf{2}$ is a perfect candidate for studies on the proposed reactivity; the results are described in section 7.3. 


\subsection{Using $\mathrm{XeF}_{2}$ as Oxidizing Agent}

Xenon difluoride is a strong oxidizing and fluorination agent. In aqueous medium, its oxidizing power is based on equation (vii). ${ }^{[213]}$

$$
\mathrm{XeF}_{2}+2 \mathrm{H}^{+}+2 \mathrm{e}^{-} \rightarrow \mathrm{Xe}_{(\mathrm{g})}+2 \mathrm{HF} \quad E_{0}=+2.3 \mathrm{~V}
$$

For comparison, the standard potential of ferrocene in aqueous medium is $E_{0}=+0.4 \mathrm{~V}$. In nonaqueous solvents, in particular $\mathrm{MeCN}$, the value is expected to be similar but no distinct data are available. However, several studies have shown that it both oxidizes substrates and transfers fluorine. ${ }^{[219-222]}$ Consequently, oxidative fluorination of $\left[\left({ }^{\mathrm{NHC}} \mathrm{L}\right) \mathrm{Fe}^{\mathrm{IV}}=\mathrm{O}(\mathrm{MeCN})\right]^{2+} \boldsymbol{2}$ with $\mathrm{XeF}_{2}$ may result in the formation of $\left[\left({ }^{\mathrm{NHC}} \mathrm{L}\right) \mathrm{Fe}^{\mathrm{V}}=\mathrm{O}(\mathrm{F})\right]^{2+}$.

In a first experiment, a cold solution of 2 in $\mathrm{MeCN}\left(-35^{\circ} \mathrm{C}\right)$ was treated with an excess of $\mathrm{XeF}_{2}$, resulting in a brown reaction mixture. An EPR measurement of the crude reaction mixture showed a signal with $g$-values $2.29,2.05,1.93$ and a hyperfine coupling with one ${ }^{19} \mathrm{~F}$-atom $(A \sim 20 \mathrm{G})$ However, subsequent mass spectrometry experiments did not verify the formation of a high-valent tetracarbene-coordinated iron species and a MB measurement demonstrated the formation of a iron(III) high spin compound ( $\delta=0.49 \mathrm{~mm} \mathrm{~s}^{-1}, \Delta E_{\mathrm{Q}}=0.90 \mathrm{~mm} \mathrm{~s}^{-1}$ ), likely an iron(III)fluorido complex that is no longer coordinated by ${ }^{\mathrm{NHC}} \mathrm{L}$. Presumably, the chosen conditions were too harsh to enable the stabilization and isolation of a putative $\left[\left({ }^{\mathrm{NHC}} \mathrm{L}\right) \mathrm{Fe}^{\mathrm{V}}=\mathrm{O}(\mathrm{F})\right]^{2+}$ intermediate. Consequently, 2 was converted at low temperatures with 0.5 and 1 eq. of $\mathrm{XeF}_{2}$ only, resulting in a purple solution that shows the same UV/vis features than have been reported for iron(III) complex 9. ${ }^{[155]}$ Thus, this synthetic strategy does not provide promising results and was not studied in further detail.

Instead, the reactivity of the iron(II) complex $\left[\left({ }^{\mathrm{NHC}} \mathrm{L}\right) \mathrm{Fe}(\mathrm{MeCN})_{2}\right]^{2+}(\mathbf{1})$ toward $\mathrm{XeF}_{2}$ was examined. Treatment of a solution of $\mathbf{1}$ in $\mathrm{MeCN}$ at $-35^{\circ} \mathrm{C}$ with 1 eq. of $\mathrm{XeF}_{2}$ and subsequent addition of $\mathrm{Et}_{2} \mathrm{O}$ led to precipitation of a bright yellow solid. The obtained compound shows an IS of $\delta=0.11 \mathrm{~mm} \mathrm{~s}^{-1}$ and a QS of $\Delta E_{\mathrm{Q}}=4.15 \mathrm{~mm} \mathrm{~s}^{-1}$ in its $\mathrm{MB}$ spectrum (Figure 50, left). These values are similar to the parameters reported for the chloridoiron(III) compound $\mathbf{8}\left(\delta=0.11 \mathrm{~mm} \mathrm{~s}^{-1}\right.$, $\left.\Delta E_{\mathrm{Q}}=4.52 \mathrm{~mm} \mathrm{~s}^{-1}\right),{ }^{[155]}$ suggesting formation of a similar $\left[\left({ }^{\mathrm{NHC}} \mathrm{L}\right) \mathrm{Fe}^{\mathrm{III}} \mathrm{F}\right]^{2+}$ complex. So far, crystallization of this compound as well as assignment of signals detected in mass spectrometry experiments was not successful.

Addition of further excess of $\mathrm{XeF}_{2}$ resulted in a color change to brown and appearance of a second signal in the $\mathrm{MB}$ spectrum. The use of 5 eq. $\mathrm{XeF}_{2}$ gave rise to the $\mathrm{MB}$ spectrum depicted in Figure 50 (right) with only the new MB doublet at $\delta=-0.22 \mathrm{~mm} \mathrm{~s}^{-1}$ and $\Delta E_{\mathrm{Q}}=4.47 \mathrm{~mm} \mathrm{~s}^{-1}$. This isomer shift is significantly lower than what has been reported for the tetracarbene coordinated high-valent 
iron(IV) species $2\left(\delta=-0.13 \mathrm{~mm} \mathrm{~s}^{-1}\right)^{[153]}$ and $\mathbf{1 4}\left(\delta=-0.16 \mathrm{~mm} \mathrm{~s}^{-1}\right)$ and is in agreement with formation of an iron $(\mathrm{V})$ species. However, conversion of the yellow compound into the putative iron(V) system is accompanied by a decrease in MB intensity, indicative of simultaneous decomposition processes.
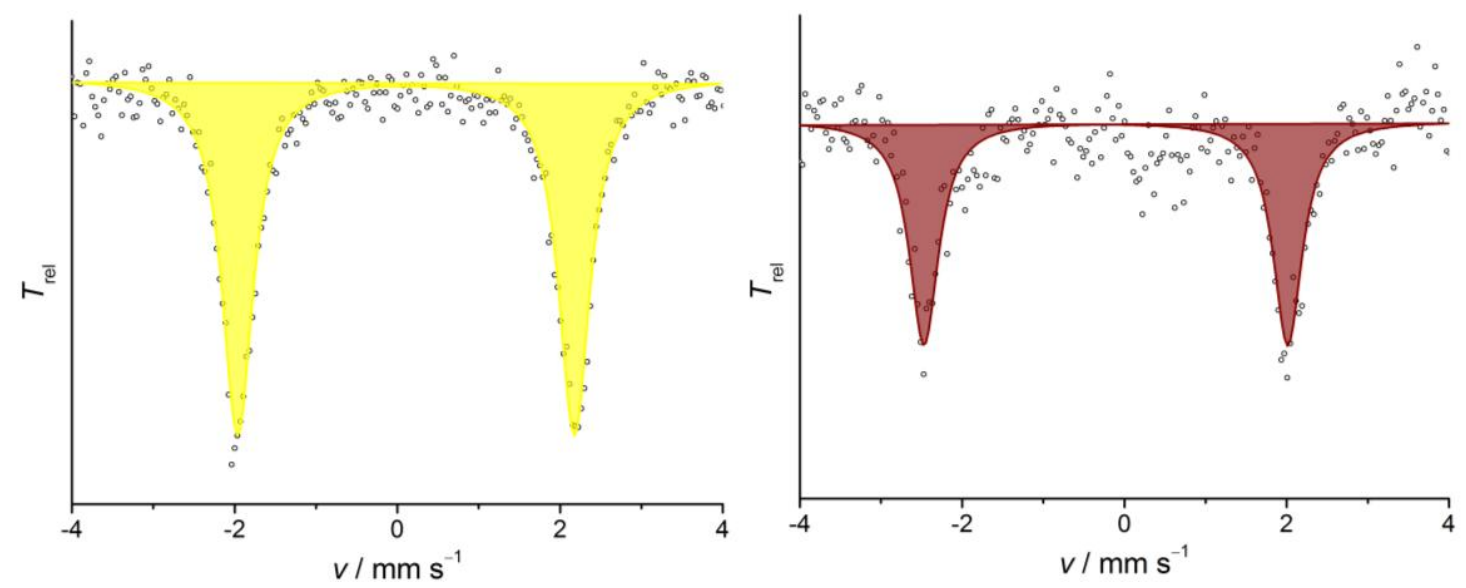

Figure 50. Zero-field ${ }^{57} \mathrm{Fe} \mathrm{MB}$ spectra after conversion of 1 with 1 eq. $\mathrm{XeF}_{2}$ (left, $\delta=0.11 \mathrm{~mm} \mathrm{~s}^{-1}$ and $\Delta E_{\mathrm{Q}}=4.15 \mathrm{~mm} \mathrm{~s}^{-1}$ ) and 5 eq. $\mathrm{XeF}_{2}$ (right, $\delta=-0.22 \mathrm{~mm} \mathrm{~s}^{-1}$ and $\left.\Delta E_{\mathrm{Q}}=4.47 \mathrm{~mm} \mathrm{~s}^{-1}\right)(80 \mathrm{~K}$, natural abundance ${ }^{57} \mathrm{Fe}$ ).
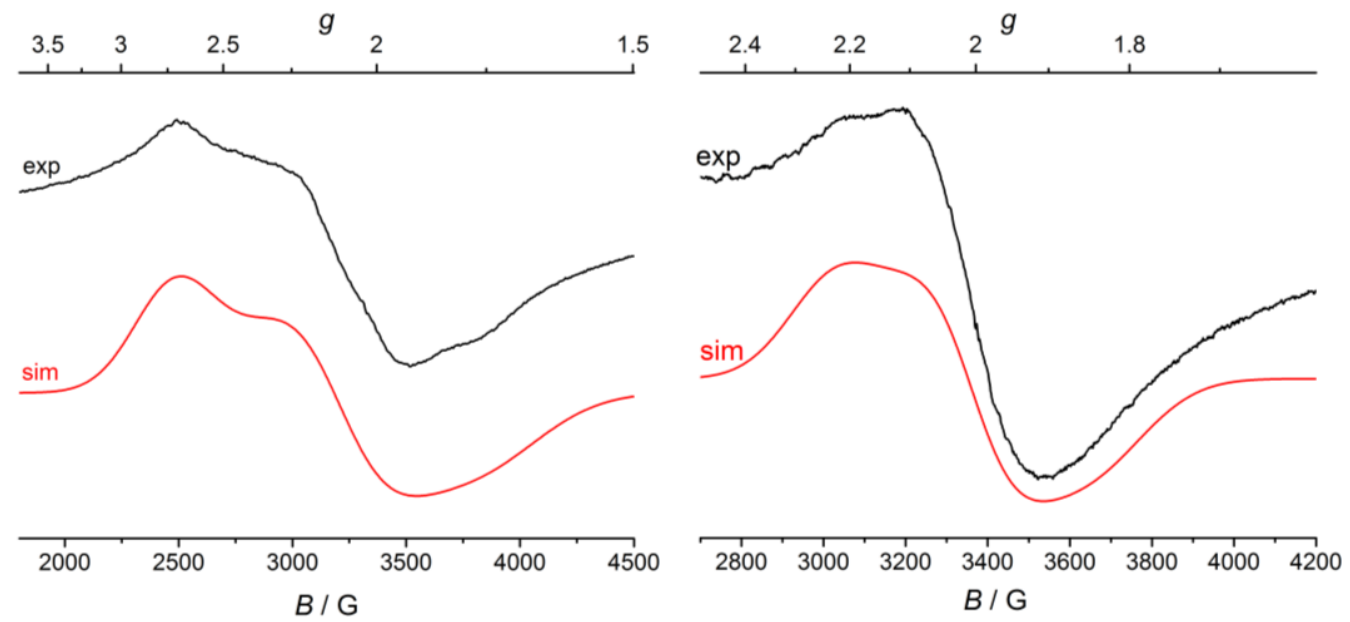

Figure 51. X-band EPR spectra after conversion of 1 with 1 eq. of $\mathrm{XeF}_{2}$ (left) and 5 eq. of $\mathrm{XeF}_{2}$ (right), experimental spectra are given in black, simulation in red. Left: $g(\mathrm{lw} / \mathrm{mT})=2.700(265), 2.095(240), 1.750$ (270), MeCN, 150 K. Right: $g(\mathrm{lw} / \mathrm{mT})=2.225$ (135), 2.005 (120), 1.840 (169), MeCN, $160 \mathrm{~K}$.

Both conversion with 1 eq. of the oxidizing agent and 5 eq. of $\mathrm{XeF}_{2}$ were repeated and EPR spectra of the reaction mixtures were recorded; the obtained spectra are shown in Figure 51. The broad signal obtained for the putative fluoridoiron(III) compound could be simulated with $g$-values 2.700 , 2.095 and 1.750. This differs from the spectral characteristics of the chloridoiron(III) complex 8 $(g=4.300,2.095,2.095$, Appendix Figure 4) and is consistent with a low spin ground state rather than intermediate spin. The weak signal after conversion with 5 eq. of $\mathrm{XeF}_{2}$ was simulated with the following values: $g(\mathrm{lw} / \mathrm{mT})=2.225(135), 2.005(120), 1.840(160)$. Even though the shape of 
the signals looks similar, the $g$-values are quite different indicative of the detection of different paramagnetic species. Unfortunately, no hyperfine coupling with ${ }^{19} \mathrm{~F}$ was observed in both spectra and thus, no conclusive evidence for formation of a $\left[\left({ }^{\mathrm{NHC}} \mathrm{L}\right) \mathrm{Fe}^{\mathrm{V}}(\mathrm{F})_{\mathrm{n}}\right]^{+(5-\mathrm{n})}$ species $(\mathrm{n}=1$ or 2$)$ was obtained.

Crystallization upon diffusion of $\mathrm{Et}_{2} \mathrm{O}$ into a reaction mixture in $\mathrm{MeCN}$ at $-35{ }^{\circ} \mathrm{C}$ was so far not successful. Furthermore, it was not possible to unambiguously assign the signals detected in the ESI mass spectrum. However, ESI-MS might not be a suitable technique in this case due to the expected instability of the iron(V) species. Finally, the conversion of both species with CHD was followed in the UV/vis region (Figure 52). The putative fluoridoiron(III) compound shows a strong absorption at $\lambda_{\max }=345 \mathrm{~nm}$ (Figure 52, left) that is similar to the main band in the iron(II) precursor $1\left(\lambda_{\max }=340 \mathrm{~nm}\right)$. Addition of CHD did not induce any changes in the spectral features. In contrast to this, the brown species obtained after conversion of 1 with 5 eq. of $\mathrm{XeF}_{2}$ shows a broad absorption throughout the spectral range between $300-700 \mathrm{~nm}$ that decreases in intensity after addition of CHD (Figure 52, right). However, this decrease is very slow and corresponds more likely to decomposition processes instead for a reaction with the substrate. Decomposition is in agreement with the expected high instability of a putative iron(V) species and the so far unsuccessful crystallization attempts. Furthermore, the lack of fast reactivity toward CHD does not directly rule out the existence of a high-valent iron species in this mixture since it is, up to now, not clear what kind of reactivity is expected for a compound with a sum formula of $\left[\left({ }^{\mathrm{NHC}} \mathrm{L}\right) \mathrm{Fe}^{\mathrm{V}}(\mathrm{F})_{\mathrm{n}}\right]^{+(5-n)}$. Literature reported models for hydrogenase enzymes have a $(\mathrm{L}) \mathrm{Fe}^{\mathrm{IV}}=\mathrm{O}(\mathrm{X})$ moiety that is able to mediate the activation of $\mathrm{C}-\mathrm{H}$ to $\mathrm{C}-\mathrm{X} \cdot{ }^{[223]}$ However, it is not clear if a similar reactivity can be observed without the oxo ligand that is responsible for the initial hydrogen atom abstraction.
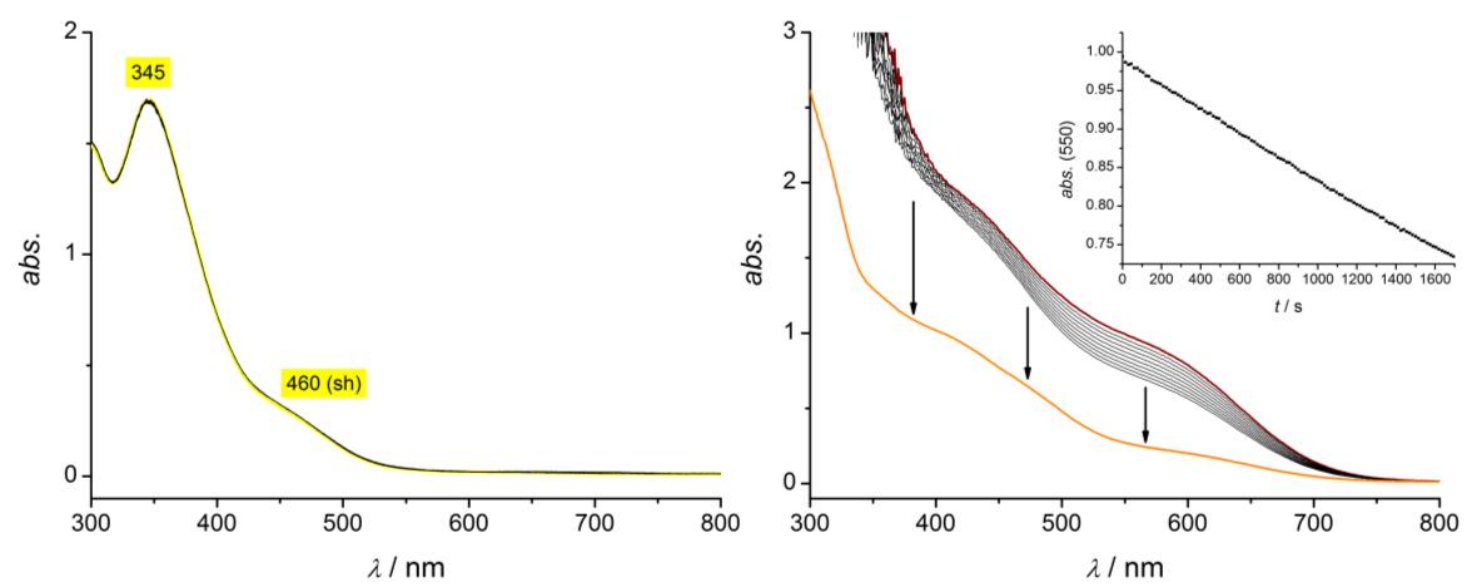

Figure 52. UV/vis spectra of the species obtained after conversion of 1 with 1 eq. of $\mathrm{XeF}_{2}$ (left, yellow line, $0.5 \mathrm{mM}$ ) and with 5 eq. of $\mathrm{XeF}_{2}$ (right, brown line, $1 \mathrm{mM}$ ) as well as the spectral changes upon addition of 10 eq. of CHD at $-40^{\circ} \mathrm{C}$. Left: Additon of CHD did not induce any changes and the species was stable at $-40{ }^{\circ} \mathrm{C}$. Right: Addition of substate was followed by a slow decrease of the absorbance throughout the whole spectal range, the inset shows the kinetic trace for the absorption at $550 \mathrm{~nm}$. 
In conclusion, the obtained data upon conversion of $\mathbf{1}$ with excess $\mathrm{XeF}_{2}$ are promising and indicative of the successful formation of a high-valent and EPR active species, thus an iron(V) compound. For future crystallization attempts it might be usuful to try lower temperatures in order to prevent decomposition processes. 


\subsection{Using ${ }^{t} \mathrm{BuOO}^{-}$as Oxidizing Agent}

In analogy to the procedure described by Que et al., a $1 \mathrm{mM}$ solution of in situ generated $\left[\left({ }^{\mathrm{NHC}} \mathrm{L}\right) \mathrm{Fe}=\mathrm{O}(\mathrm{NCMe})\right]^{2+} \mathbf{2}$ in $\mathrm{MeCN}$ at $-35{ }^{\circ} \mathrm{C}$ was treated with 5 eq. ${ }^{t} \mathrm{BuOOH}$ and 2 eq. $\mathrm{KO}^{t} \mathrm{Bu}$ (Scheme 22) and the reaction was followed in the UV/vis region (Figure 53, left). ${ }^{[169]}$

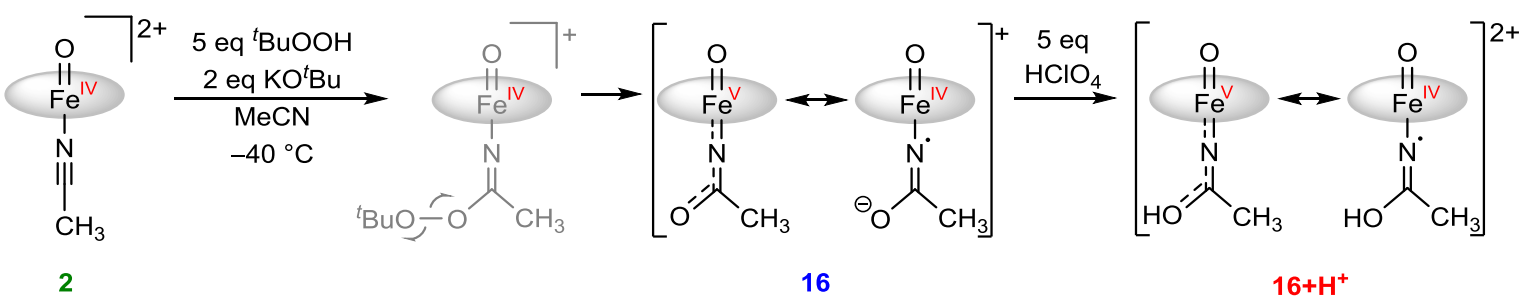

Scheme 22. Proposed steps upon treatment of the MeCN-coordinated $\mathrm{Fe}^{\mathrm{IV}}=\mathrm{O}$ species 2 with basic ${ }^{t} \mathrm{BuOOH}$ according to Que et $_{\text {al. }}{ }^{[169]}$
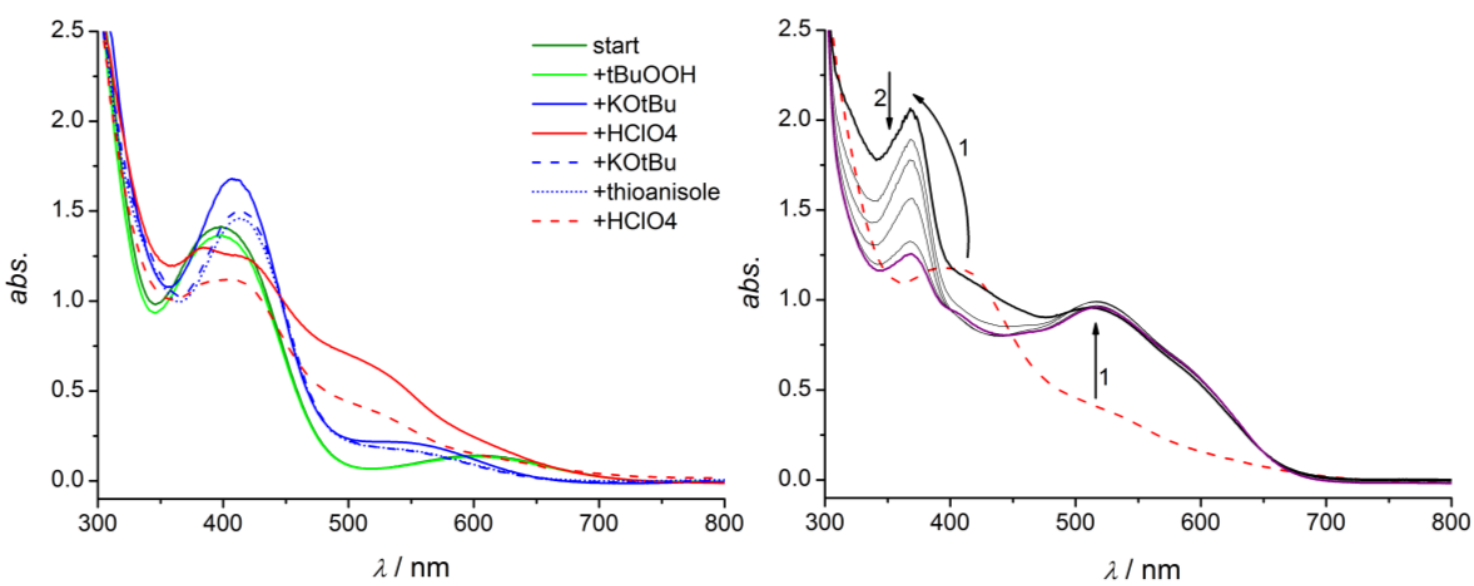

Figure 53. Formation of the putative iron(V) species $\mathbf{1 6}$ (blue) and $\mathbf{1 6}+\mathbf{H}^{+}$(red) followed in the UV/vis region (left, $\mathrm{MeCN},-40{ }^{\circ} \mathrm{C},[2]_{0}=1 \mathrm{mM}$ ) and subsequent reaction of $\mathbf{1 6}+\mathbf{H}^{+}$with 50 eq. $\mathrm{CHD}$ (right).

The spectrum of the starting material $\mathbf{2}$ is depicted in dark green and shows its typical absorptions at $\lambda_{\max }=400$ and $610 \mathrm{~nm}$. Addition of ${ }^{t} \mathrm{BuOOH}$ leads to a slight decrease of the intensity at $400 \mathrm{~nm}$, likely corresponding to dilution effects, while no further significant changes are observed. This result is in line with the mechanism proposed by Que and co-workers in which only the deprotonated tert-butyl hydroperoxide is reactive. Thus, subsequent addition of $\mathrm{KO}^{t} \mathrm{Bu}$ starts the reaction, clearly visible by a shift of the prominent absorbances to $\lambda_{\max }=410$ and $545 \mathrm{~nm}$ (blue spectrum). Following further the literature reported synthetic strategy, excess of aqueous $\mathrm{HClO}_{4}$ was added, resulting in a broad spectrum with maxima at $\lambda_{\max }=383,417$ and $520 \mathrm{~nm}$ (red). Subsequent treatment with excess base allowed for regeneration of the spectral features of the deprotonated species. However, the intensities could not be fully recovered (blue, dashed line) indicative of decomposition processes. When again 5 eq. of acid was added to the reaction mixture, the spectrum of $\mathbf{1 6}+\mathbf{H}^{+}$(red, dashed line) was partly recovered. 
The putative iron(V) species $\mathbf{1 6}$ is expected to show reactivity in OAT and $\mathrm{C}-\mathrm{H}-$-bond activation, thus thioanisole was added to the reaction mixture. However, no significant changes in absorbance were observed, similar to the weak activity in OAT observed for oxoiron(IV) species 2. Instead, addition of excess CHD to $\mathbf{1 6}+\mathbf{H}^{+}$(red dashed line) induced fast changes in the UV/vis region as depicted in Figure 53, right. Within a first process, the band at $\lambda_{\max }=417 \mathrm{~nm}$ decreases, while two new bands at $\lambda_{\max }=367$ and $520 \mathrm{~nm}$ are formed (bold black line). During a second process, the maximum at $\lambda_{\max }=367 \mathrm{~nm}$ decreases in intensity; the final solution had a purple color. The band at $\lambda_{\max }=367 \mathrm{~nm}$ was already reported as a typical characteristic for $\mu$-oxodiiron(III) compound $\mathbf{3}$ while the band at $\lambda_{\max }=520 \mathrm{~nm}$ is associated with the bis-acetonitrile coordinated iron(III) complex 9. These observations are in line with oxidation of CHD mediated by a high-valent oxoiron center that is reduced to iron(III) during this process.

Motivated by these promising results, the processes were studied in further detail. Therefore, $\mathrm{KO}^{t} \mathrm{Bu}$ was added in portions of 0.25 eq. and the changes were followed in the UV/vis region (Figure 54). The initial spectrum of a mixture of 1 with 2 eq. of ${ }^{t} \mathrm{BuOOH}$ is depicted in green. Addition of the first 0.25 eq. of base leads to a decrease of the absorption at $\lambda_{\max }=390 \mathrm{~nm}$ and a shift to about $\lambda_{\max }=395 \mathrm{~nm}$ while addition of further base up to 1 eq. results in the formation of a band at $\lambda_{\max }=405 \mathrm{~nm}$. The spectra obtained after addition of $0.25,0.5,0.75$ and 1.0 eq. of base give isosbestic points at $\lambda_{\mathrm{i}}=383 \mathrm{~nm}$ and $\lambda_{\mathrm{i}}=591 \mathrm{~nm}$. Addition of further amount of base (Figure 54 , right) results in further increase and shift of the characteristic absorption to $\lambda_{\max }=412 \mathrm{~nm}$ and the final spectrum depicted in blue that resembles well the spectrum obtained for $\mathbf{1 6}$ during the initial UV/vis studies. Furthermore, during titration of 1-3 eq. of base, two different isosbestic points at $\lambda_{\mathrm{i}}=391 \mathrm{~nm}$ and $\lambda_{\mathrm{i}}=457 \mathrm{~nm}$ were observed. According to these spectral data, addition of 2 with 1 eq. of $\mathrm{K}^{t} \mathrm{BuO}$ corresponds to a different process than treatment with excess of the base. During the following studies, both possibilities were examined.
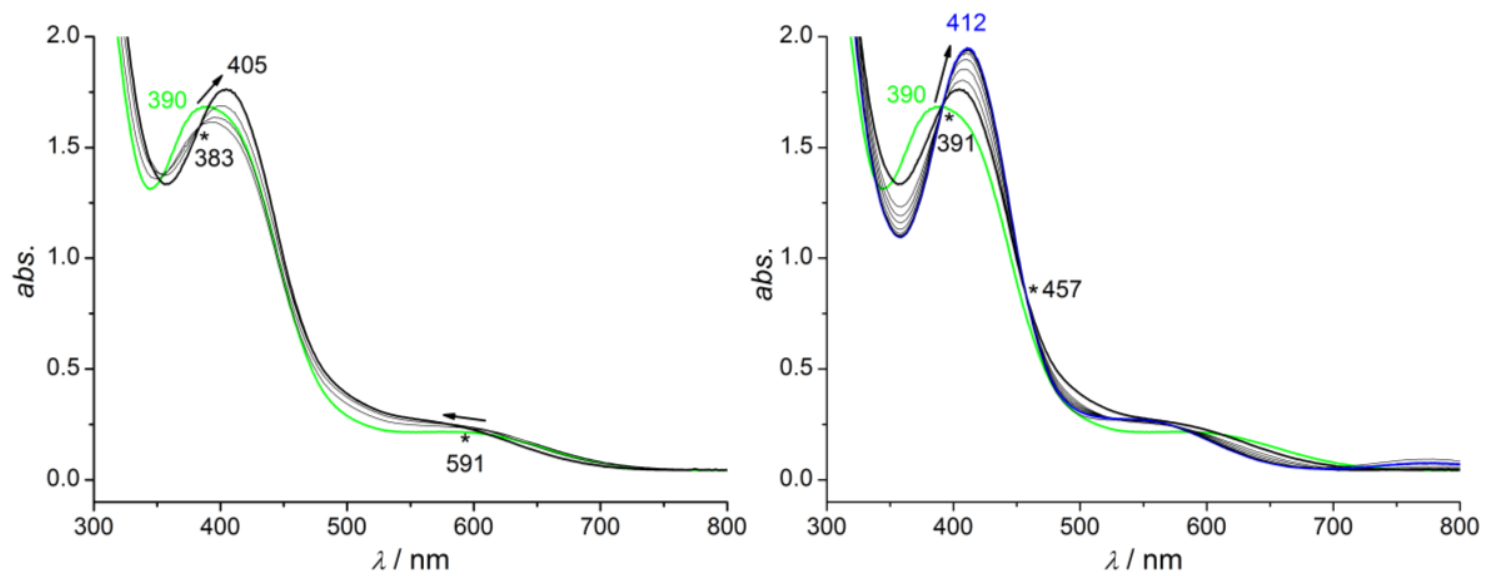

Figure 54. UV/vis spectral changes upon addition of $0-1$ eq. (left) and 1-3 eq. of $\mathrm{KO}^{t} \mathrm{Bu}$ in steps of 0.25 eq. to a mixture of $2(1 \mathrm{mM})$ and 2 eq. of ${ }^{t} \mathrm{BuOOH}$ in $\mathrm{MeCN}$ at $-40{ }^{\circ} \mathrm{C}$. 
Additionally, the second process (protonation of $\mathbf{1 6}$ to $\mathbf{1 6}+\mathbf{H}^{+}$) was investigated in further detail, as well. A reconsideration of the spectral characteristics obtained for the putative $\mathbf{1 6}+\mathbf{H}^{+}$species (red line in Figure 53) reveals that some of the bis-acetonitrile coordinated iron(III) complex 9 might be already present at this stage of the process. Thus, a much weaker acid compared to perchloric acid $\left(\mathrm{p} K_{\mathrm{A}}=-0.7 \text { in } \mathrm{MeCN}\right)^{[224]}$ was tested instead: 1,5-bis(dimethyl)-piperidinium ( $\mathrm{p} K_{\mathrm{a}} \geq 1$, value for piperidinium in $\mathrm{MeCN}) .{ }^{[225]}$ As a result of the studies with base that have been described before, the acid was added to both the mixture generated with 1 eq. of $\mathrm{KO}^{t} \mathrm{Bu}$ (Figure 55, left, bold black line) and the one with 3 eq. of $\mathrm{KO}^{t} \mathrm{Bu}$ (Figure 55, right, bold blue line). The less harsh conditions compared to conversion with $\mathrm{HClO}_{4}$ resulted in different spectral features and lack of any high intense absorption at $520 \mathrm{~nm}$ in both cases. These observations are consistent with a cleaner formation of the desired species $\mathbf{1 6}+\mathbf{H}^{+}$. In case of the titration depicted on the left side, stepwise addition of acid (process shown in black) leads to a small shift of the maximum at $\lambda_{\max }=404 \mathrm{~nm}$ to $\lambda_{\max }=402 \mathrm{~nm}$ accompanied by a slight decrease in intensity (final spectrum depicted in red). Subsequent addition of base (shown in red, final spectrum as dotted black line) allows for the recovery of the starting spectrum (bold black line).
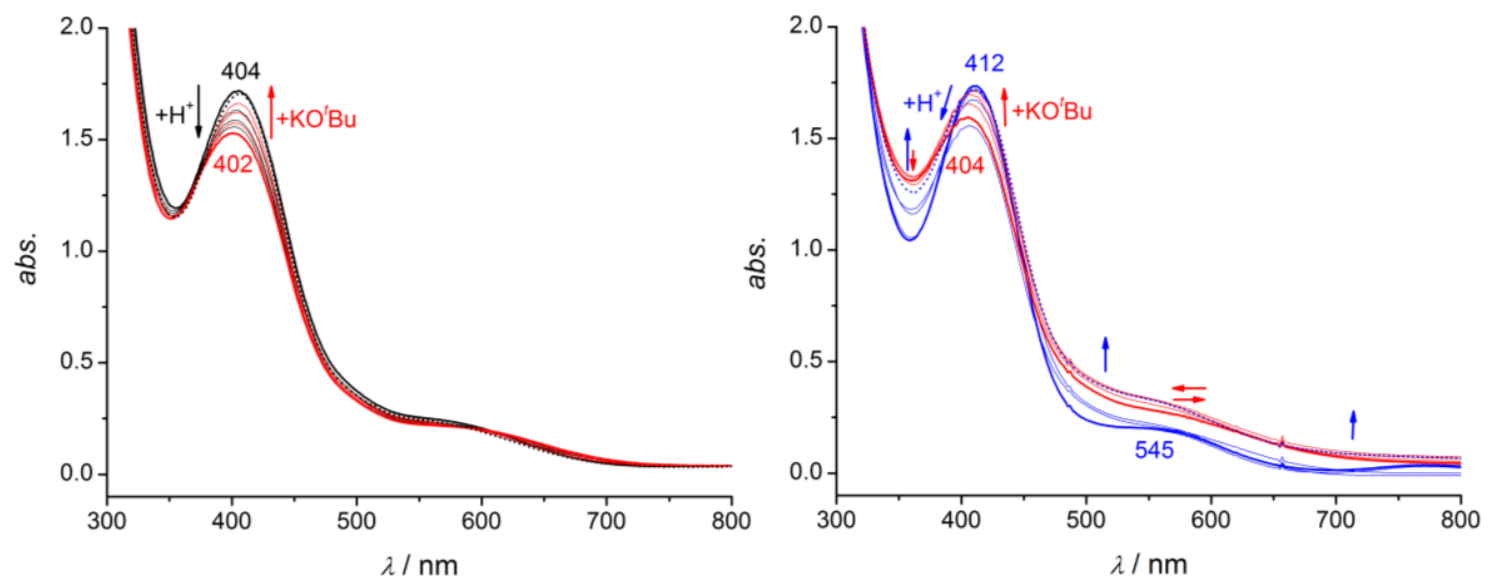

Figure 55. Left: UV/vis spectral changes upon addition of 1 eq. of 1,5-bis(dimethyl)-piperidinium in steps of 0.25 eq. to a mixture of $2(1 \mathrm{mM})$ with 2 eq. of ${ }^{t} \mathrm{BuOOH}$ and 1 eq. of $\mathrm{KO}^{t} \mathrm{Bu}$ (black line) in $\mathrm{MeCN}$ at $-40^{\circ} \mathrm{C}$. Addition of acid is shown in black while subsequent addition of base is given in red. The final spectrum (black, dotted line) represents recovery of the initial spectrum. Right: UV/vis spectral changes upon addition of up to 4 eq. of 1,5-bis(dimethyl)-piperidinium in steps of 1 eq. to a mixture of $2(1 \mathrm{mM})$ with 2 eq. of ${ }^{t} \mathrm{BuOOH}$ and 3 eq. of $\mathrm{KO}^{t} \mathrm{Bu}$ (blue line) in $\mathrm{MeCN}$ at $-40^{\circ} \mathrm{C}$. Addition of acid is shown in blue while subsequent addition of base is given in red. The final spectrum (blue, dotted line) represents incomplete recovery of the initial spectrum.

Compared to this, the titration shown on the right side in Figure 55 is less clean, indicative of concurrent decomposition processes. Starting with the very basic mixture ( 3 eq. $\mathrm{KO}^{t} \mathrm{Bu}$ ), a similar decrease and increase of the bands at $\lambda_{\max }=412 \mathrm{~nm}$ (blue) and $\lambda_{\max }=404 \mathrm{~nm}$ (red) was observed during the titration experiment. However, also the intensity of absorption around $370 \mathrm{~nm}$ and 
460-800 nm increases during addition of acid and does not decrease upon subsequent addition of base, thus the spectrum of the starting material (blue) is not fully recovered.

A test reaction with crystalline material of 2 instead for the in situ generated oxoiron(IV) starting material gave the same results, thus it seems to be not necessary to isolate $\mathbf{2}$ before use. Treatment of both $\mathbf{1 6}$ (basic solution, Figure 56, left) and $\mathbf{1 6}+\mathbf{H}^{+}$(acidic solution, Figure 56, right) with DHA leads to a fast reaction, indicative of high oxidative reactivity. Compared to the basic species 16, $\mathbf{1 6}+\mathrm{H}^{+}$was more reactive within these initial studies in line with a lower effective electron density at the proposed iron(V) / iron(IV) radical center. Both conversions lead to different final spectra that are not reminiscent of any known tetracarbene coordinated iron complexes.
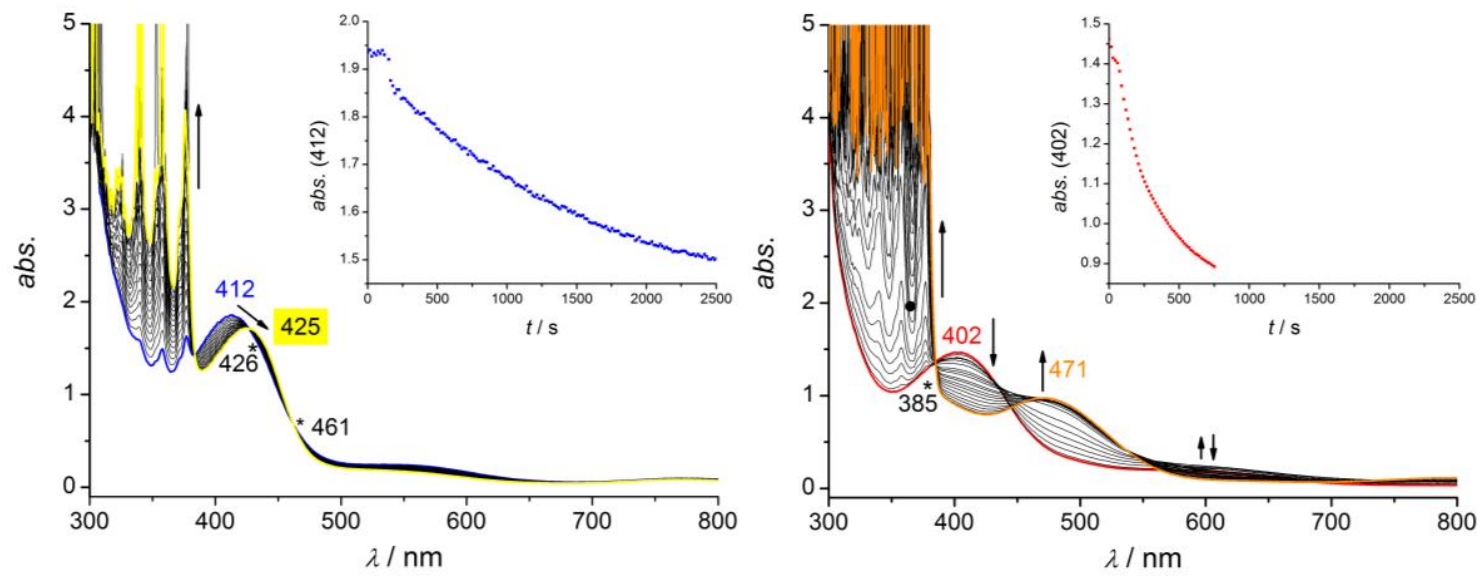

Figure 56. UV/vis spectral changes observed upon reaction of $\mathbf{1 6}$ (left) and $\mathbf{1 6}+\mathbf{H}^{+}$(right) with 10 eq. of DHA at $-40{ }^{\circ} \mathrm{C}$ in $\mathrm{MeCN}$. The insets show the kinetic traces for the spectral changes at 412 (left) and $402 \mathrm{~nm}$ (right) for these reactions.

In conclusion, the experimental data obtained in these UV/vis studies strongly suggest successful generation of new high-valent oxoiron compounds that are active in $\mathrm{C}-\mathrm{H}$ activation on DHA and CHD. Motivated by these promising initial studies, further characterization of intermediates $\mathbf{1 6}$ and $\mathbf{1 6}+\mathbf{H}^{+}$was attempted.

Treatment of a green solution of in situ generated $2(\sim 10-20 \mathrm{mM})$ with ${ }^{t} \mathrm{BuOOH}$ at $-35^{\circ} \mathrm{C}$ did not induce an immediate color change, in line with the UV/vis measurement. Subsequent addition of $\mathrm{KO}^{t} \mathrm{Bu}$ however was followed by a color change to red-brown. The MB spectrum of the crude reaction mixture is given in Figure 57, left. The obtained spectrum was simulated as MB doublet with a small IS of $\delta=-0.15 \mathrm{~mm} \mathrm{~s}^{-1}$ and a QS of $\Delta E_{\mathrm{Q}}=2.78 \mathrm{~mm} \mathrm{~s}^{-1}$. These values differ only slightly from the MB parameters obtained for oxoiron(IV) starting material 2 (frozen MeCN solution: $\left.\delta=-0.13 \mathrm{~mm} \mathrm{~s}^{-1}, \Delta E_{\mathrm{Q}}=3.08 \mathrm{~mm} \mathrm{~s}^{-1}\right) \cdot{ }^{[153]}$ The obtained IS value is in good agreement with the two isomeric structures of $\mathbf{1 6}$ (Scheme 22), in which iron centers in oxidation states +IV and $+\mathrm{V}$ are proposed. 
A second sample was generated in the same way but subsequently also treated with 1 eq. of 1,5-bis(dimethyl)-piperidinium in order to generate $\mathbf{1 6}+\mathbf{H}^{+}$. The resulting $\mathrm{MB}$ spectrum shows one broad unsymmetric signal (Figure 57, right). This can have two reasons: First, it is possible that nevertheless only one species is generated and the broadening effect is due to magnetic relaxation. The compound would then show an IS of $\delta=-0.10 \mathrm{~mm} \mathrm{~s}^{-1}$ and a QS of $\Delta E_{\mathrm{Q}}=2.77 \mathrm{~mm} \mathrm{~s}^{-1}$. Second, the sample might consist of two species in a 1:1 ratio with isomer shifts of $\delta=-0.14 \mathrm{~mm} \mathrm{~s}^{-1}$ and $\delta=-0.04 \mathrm{~mm} \mathrm{~s}^{-1}$ as well as quadrupole splittings of $\Delta E_{\mathrm{Q}}=2.93 \mathrm{~mm} \mathrm{~s}^{-1}$ and $\Delta E_{\mathrm{Q}}=2.50 \mathrm{~mm} \mathrm{~s}^{-1}$, respectively. The resulting simulation is of comparable quality as in scenario one. The parameters for the first species in scenario two are relatively similar to the values obtained for $\mathbf{1 6}$ what might be indicative of partly protonation only. Thus, the experiment was repeated with excess of acid but the recorded spectrum looked very similar, indicative of full protonation after 1 eq. of the acid already. The new compound $\mathbf{1 6}+\mathbf{H}^{+}$in both scenarios shows a higher IS value than 16. The $\mathrm{NC}(\mathrm{O}) \mathrm{Me}$ ligand in $\mathbf{1 6}$ has significantly higher anionic character than in $\mathbf{1 6}+\mathbf{H}^{\mathbf{+}}$. Consequently, it is expected, that the ligand forms a stronger, thus shorter, Fe-N bond in $\mathbf{1 6}$ than in $\mathbf{1 6}+\mathbf{H}^{+}$. The bond lengths around the iron center show direct influence on the IS. As described in section 1.6, shorter bonds correlate with compression of all orbitals resulting in increased s-electron density at the iron center and a lower isomer shift. Consequently, $\mathbf{1 6}+\mathbf{H}^{+}$with its longer $\mathrm{Fe}-\mathrm{N}$ bond should show a higher IS than $\mathbf{1 6}$ in agreement with the experimental result.
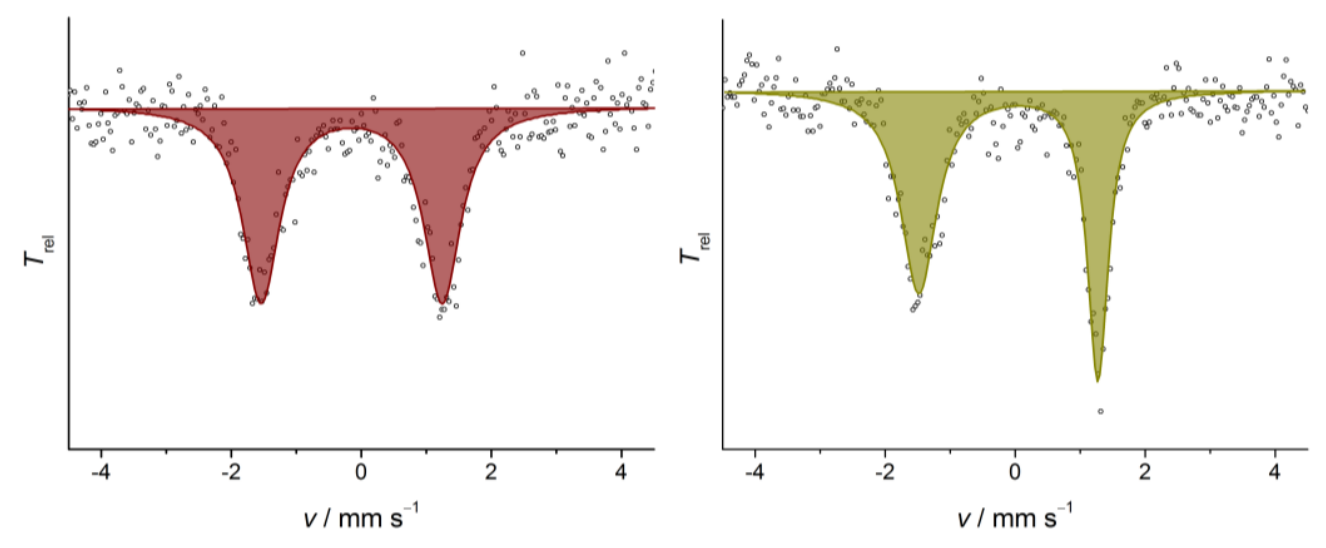

Figure 57. Zero-field ${ }^{57} \mathrm{Fe} \mathrm{MB}$ spectra after conversion of in situ generated 2 with 5 eq. ${ }^{t} \mathrm{BuOOH}$ and 2.5 eq. $\mathrm{KO} \mathrm{Bu}^{t}$ (left, $\delta=-0.15 \mathrm{~mm} \mathrm{~s}^{-1}$ and $\Delta E_{\mathrm{Q}}=2.78 \mathrm{~mm} \mathrm{~s}^{-1}$ ) as well as after subsequent addition of 1 eq. of 1,5-bis(dimethyl)-piperidinium (right, $\delta=-0.10 \mathrm{~mm} \mathrm{~s}^{-1}$ and $\Delta E_{\mathrm{Q}}=2.77 \mathrm{~mm} \mathrm{~s}^{-1}$ ) (MeCN, $7 \mathrm{~K}$, natural abundance $\left.{ }^{57} \mathrm{Fe}\right)$.

In this regard, it should be noted that the quality of the MB spectra in solution does not allow a statement on the purity of the generated species. In future experiments, it may be useful to synthesize ${ }^{57} \mathrm{Fe}$ labeled material resulting in a significantly increased intensity and, thus, a better signal-to-noise ratio. 
Since a lower concentration and volume of the sample is needed for EPR studies, it was possible to use crystalline material of $\mathbf{2}$. Addition of ${ }^{t} \mathrm{BuOOH}$ and base to a green solution of $\mathbf{2}$ resulted in a slight blue reaction mixture in this case; the corresponding EPR signal is depicted in Figure 58. A good simulation was achieved using $g$-values $g_{\mathrm{x}, \mathrm{y}, \mathrm{z}}=2.1980,2.0694,1.9920$ and a hyperfine coupling with one ${ }^{14} \mathrm{~N}$ at $g_{\mathrm{z}}\left(A_{\mathrm{z}}=12.6 \mathrm{G}\right)$. This result is in perfect agreement with the proposed structure of $\mathbf{1 6}$ and in line with the formation of a low spin $S=1 / 2$ system. Furthermore, the EPR spectrum resembles well the shape of the signal reported for the TMC analog $\left(g_{\mathrm{x}, \mathrm{y}, \mathrm{z}}=2.053,2.010\right.$, $1.971),{ }^{[169]}$ even though the $g$-values are slightly shifted and the signal for the TMC complex shows wider $g$ anisotropy.

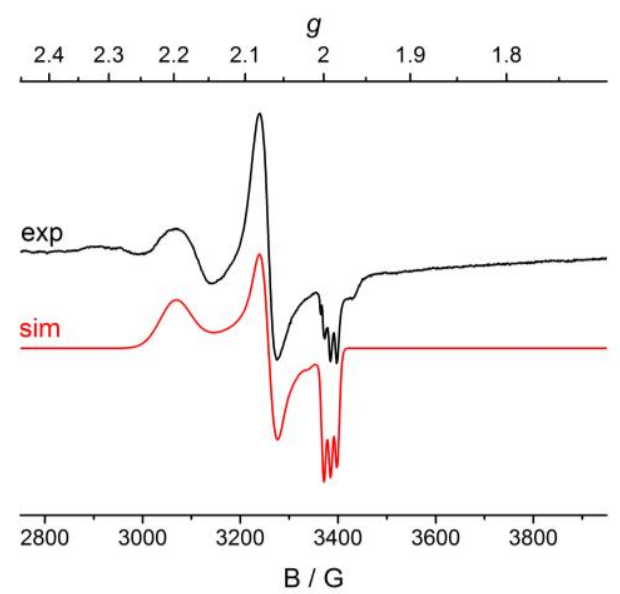

Figure 58. EPR spectrum after conversion of $\left[\left({ }^{\mathrm{NHC}} \mathrm{L}\right) \mathrm{Fe}^{\mathrm{IV}}=\mathrm{O}(\mathrm{NCMe})\right](2)$ with ${ }^{t} \mathrm{BuOOH}+\mathrm{KO}{ }^{t} \mathrm{Bu}$ (black), simulation (red) was performed using the following parameters: $g(\mathrm{lw} / \mathrm{G})=2.1980(38), 2.0694(16), 1.9920$ (5), $A_{\mathrm{z}}=12.6 \mathrm{G}$ for one ${ }^{14} \mathrm{~N}$.

When the blue solution was transferred back to a glove box after the measurement, the mixture turned brown. So far, it was not possible to reproduce the blue color obtained in this experiment. For comparison, the EPR experiment was repeated starting with in situ generated material. Even though the UV/vis experiments did not indicate differences in reactivity, the conversion of in situ generated material was not as clean as conversion of crystalline $\mathbf{2}$ and at least two different species were observed in the EPR measurements. Thus, further studies are needed in order to obtain conclusive data.

So far, crystallization attempts starting with the brownish reaction mixtures of both $\mathbf{1 6}$ and $\mathbf{1 6}+\mathbf{H}^{+}$ were not successful. Still, the obtained data strongly indicate a similar reactivity of the tetracarbene coordinated oxoiron(IV) complex 2 toward ${ }^{t} \mathrm{BuOO}^{-}$when compared to the TMC coordinated analog. As described within the previous chapters, the $\mathrm{Fe}=\mathrm{O}$ moiety in $\mathbf{2}$ shows similar properties (bond length, $\tilde{v}_{\mathrm{Fe}=\mathrm{O}}$ ) than nitrogen-coordinated model compounds since the strong $\sigma$-donating carbene donors mainly show influence on the unoccupied $d_{x^{2}-y^{2}}$ orbital. Thus, it would not surprise if the similar coordination sphere, in particular the coordination of an axial $\mathrm{MeCN}$ ligand, allows 
for a similar one-electron oxidation of 2 into a $\mathrm{O}=\mathrm{Fe}^{\mathrm{V}}(\mathrm{NC}(\mathrm{O}) \mathrm{Me}) \leftrightarrow \mathrm{O}=\mathrm{Fe}^{\mathrm{IV}}\left({ }^{\circ} \mathrm{NC}(\mathrm{O}) \mathrm{Me}\right)$ compound. 


\section{Macrocyclic Iron NHC Complexes with Nitric Oxides}

Major parts of this project have recently been published online and parts of this chapter have been adapted from this publication with permission from ACS. ${ }^{[22,23]}$

\subsection{Synthesis of Organometallic $\{\text { FeNO }\}^{n}$ Compounds}

Motivated by the high affinity of iron(II) species toward nitric oxide ${ }^{[28,114-116]}$ the reactivity of $\mathbf{1}$ toward NO was studied. According to the Enemark-Feltham notation, ${ }^{[113]}$ this conversion is expected to yield a mononitrosyl $\{\mathrm{FeNO}\}^{7}$ species (see section 1.4.1). Treatment of a yellow $\mathrm{MeCN}$ solution of the iron(II) tetracarbene $\left[\left({ }^{\mathrm{NHC}} \mathrm{L}\right) \mathrm{Fe}(\mathrm{MeCN})_{2}\right](\mathrm{OTf})_{2}(\mathbf{1})$ with either gaseous nitric oxide, or with one equivalent of trityl $S$-nitrosothiol leads to a fast color change to blue (Scheme 23). An ESI-MS spectrum of the reaction mixture shows signals at $217.1 \mathrm{~m} / \mathrm{z}$ and $583.1 \mathrm{~m} / \mathrm{z}$ which are assigned to $\left[\left({ }^{\mathrm{NHC}} \mathrm{L}\right) \mathrm{Fe}(\mathrm{NO})\right]^{2+}$ and $\left[\left({ }^{\mathrm{NHC}} \mathrm{L}\right) \mathrm{Fe}(\mathrm{NO})(\mathrm{OTf})\right]^{+}$(Appendix Figure 12) indicative of successful formation of the new iron nitrosyl complex 17. It was furthermore possible to characterize this species using a variety of spectroscopic methods including X-ray crystallography; the obtained data are discussed in section 8.2. ${ }^{[22]}$

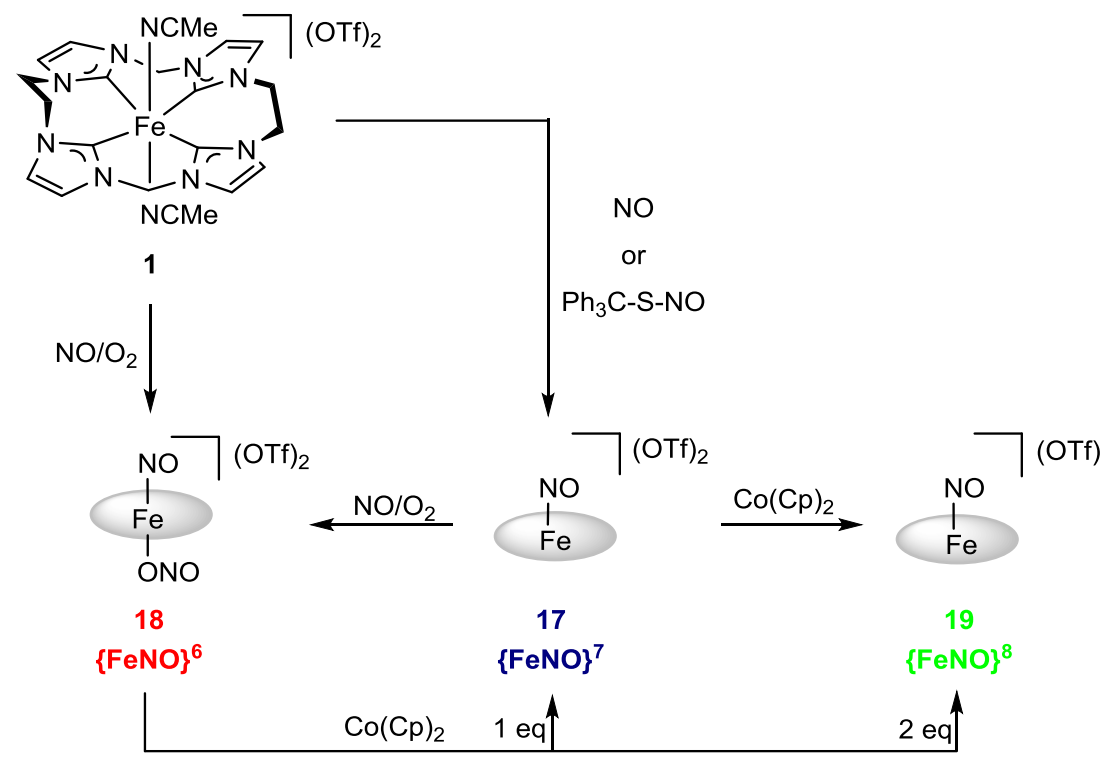

Scheme 23. Synthetic strategy for formation of the tetracarbene-coordinated $\{\mathrm{FeNO}\}^{6},\{\mathrm{FeNO}\}^{7}$ and $\{\mathrm{FeNO}\}^{8}$ species 18, 17 and 19. Starting with iron(II) precursor $\mathbf{1}$ it is possible to directly synthesize $\mathbf{1 8}$ and 17 which can be interconverted into each other. Furthermore, both species represent starting compounds for the generation of $19 .^{[22,23]}$

While solid material of $\{\mathrm{FeNO}\}^{7}$ compound $\mathbf{1 7}$ is stable under light and air for several days, a solution of $\mathbf{1 7}$ slowly turns yellow upon exposure to light because of cleavage of the Fe-NO bond. Depending on the experimental conditions, this photoactivated NO release leads to formation of the starting material $\mathbf{1}$ or, under air, of the corresponding $\mu$-oxodiiron(III) complex $\mathbf{3}$ as clarified by 
UV/vis measurements. ${ }^{[153]}$ Such light-sensitivity was observed for many iron nitrosyl complexes and was not studied in detail within this work. ${ }^{[226,227]}$

In the presence of small amounts of dioxygen ( $\geq 1$ eq.), compound $\left.\left[{ }^{\mathrm{NHC}} \mathrm{L}\right)\{\mathrm{FeNO}\}^{6}(\mathrm{ONO})\right](\mathrm{OTf})_{2}$ $\mathbf{1 8}$ containing an O-bound nitrito ligand trans to the NO was isolated as a side product when $\mathbf{1 7}$ was prepared from precursor complex 1 and an excess of ${ }^{\circ} O_{(\mathrm{g})}$ (see 8.2 for crystal structure and further spectroscopic data). Consequently, $\mathrm{Ph}_{3} \mathrm{C}-\mathrm{S}-\mathrm{NO}$ was the preferred reagent for the generation of pure 17. In 18, an oxidized $\{\mathrm{FeNO}\}^{6}$ moiety is found, providing motivation for the isolation of pure $\mathbf{1 8}$. Close examination of the reaction revealed that $\mathbf{1 8}$ is best synthesized in a twostep sequence by initially treating a MeCN solution of $\left[\left({ }^{\mathrm{NHC}} \mathrm{L}\right) \mathrm{Fe}^{\mathrm{II}}(\mathrm{MeCN})_{2}\right](\mathrm{OTf})_{2}$ with nitric oxide gas, which leads to a color change from yellow to blue indicative of the in situ formation of 17. In the second step, excess dioxygen is added to the nitric oxide atmosphere, causing the atmosphere to turn brownish immediately and the color of the solution to gradually change to yellow. As illustrated in Scheme 23, it is also possible to start from the isolated $\{\mathrm{FeNO}\}^{7}$ complex $\mathbf{1}^{[23]}$

In literature, different possibilities have been considered for the formation of a coordinated nitrite when reacting iron complexes with an excess of 'NO. In 1984, Yoshimura et al. reported that the reaction of nitrosyl porphyrinato iron(II) complexes with excess nitric oxide gas leads to a $\left[\mathrm{Fe}(\right.$ porph $\left.)(\mathrm{NO})\left(\mathrm{NO}_{2}\right)\right]$ species, which they explained by a disproportionation reaction between coordinated $\mathrm{NO}$ with three equivalents of free $\mathrm{NO}$ to form the coordinated ligands $(\mathrm{NO})\left(\mathrm{NO}_{2}\right)$ and free $\mathrm{N}_{2} \mathrm{O} \cdot{ }^{[228]}$ A similar mechanism was later found by Lippard et al. for an iron tropocoronand system. ${ }^{[133]}$ However, in the present case no evidence for such a mechanism was observed, because $\mathbf{1 7}$ is stable in the presence of excess $\mathrm{NO}$ when this is oxygen free. If instead dioxygen is added to the headspace of the reaction, conversion to $\{\mathrm{FeNO}\}^{6} \mathbf{1 8}$ accompanied by a color change from blue to yellow is initiated immediately. A possible scenario involving dioxygen could be the direct reaction of coordinated $\mathrm{NO}$ with $\mathrm{O}_{2}$ to give the iron nitrito complex, ${ }^{[229]}$ but this scenario is considered unlikely for two reasons. Firstly, the coordination mode of the resulting $\mathrm{NO}_{2}$ ligand would likely be N-bound nitro. Secondly, no $\mathrm{NO}_{2}$ ligand formation is observed upon treatment of $\mathbf{1 7}$ with $\mathrm{O}_{2}$ in the absence of nitric oxide gas. In fact, compound $\mathbf{1 7}$ is quite stable under dioxygen atmosphere. On the other hand, it is well established and exploited in large scale industrial processes that gaseous ' $\mathrm{NO}$ reacts with $\mathrm{O}_{2}$ to give nitrogen dioxide $\left(\mathrm{NO}_{2}\right.$ in equilibrium with its dimer $\left.\mathrm{N}_{2} \mathrm{O}_{4}\right){ }^{[213]} \mathrm{NO}_{2}$ can further react with additional $\mathrm{NO}$ to give unstable $\mathrm{N}_{2} \mathrm{O}_{3}{ }^{[23,230]}$ Consequently, it can be proposed that $\mathrm{NO}_{2}$ is formed in the headspace of the reaction and is then coordinating at the free axial position of the square pyramidal coordinated iron center in $\mathbf{1 7}$, accompanied by oxidation of the iron ion and formation of $\mathrm{NO}_{2}{ }^{-} \cdot{ }^{[23]}$

In order to obtain further support for this mechanistic idea, isotope labeling experiments using ${ }^{18} \mathrm{O}_{2}$ were performed. Non-labeled material of $\mathbf{1 8}$ detected by ESI-MS(+) shows a characteristic signal at 
$m / z=629.0$ that was assigned to $\left.\left[{ }^{\mathrm{NHC}} \mathrm{L}\right) \mathrm{Fe}(\mathrm{NO})(\mathrm{ONO})(\mathrm{OTf})\right]^{+}$(Appendix Figure 13). Upon using ${ }^{18} \mathrm{O}_{2}$, formation of the singly and doubly labeled compound $\mathbf{1 8}$ is observed in the mass spectrum. This is in agreement with formation of nitrogen dioxide $\left(\mathrm{NO}_{2}\right)$ in the gas phase that subsequently undergoes a redox reaction with the initially formed $\{\mathrm{FeNO}\}^{7}$ complex $\mathbf{1 7}$ to finally give the $\left(\mathrm{ONO}^{-}\right)\left\{\mathrm{FeNO}^{6}\right.$ product 18. The experiment did not show any significant amounts of labeled product 18 when only one equivalent of ${ }^{18} \mathrm{O}_{2}$ was used even though the reaction proceeded to completion. Presumably, the instability of $\mathrm{N}_{\mathrm{x}} \mathrm{O}_{\mathrm{y}}$ compounds allows for very fast exchange of the oxygen atoms and scrambling with the excess of $\mathrm{N}^{16} \mathrm{O}$. Depending on the amount of ${ }^{18} \mathrm{O}_{2}$, formation of both, partially labeled $\mathrm{NO}$ as well as $\mathrm{NO}_{2}$ and hence singly and doubly labeled compound 18 with ${ }^{18} \mathrm{O}$ enrichment was observed. The corresponding shifts in the IR spectra are discussed in section 8.2.3. ${ }^{[23]}$

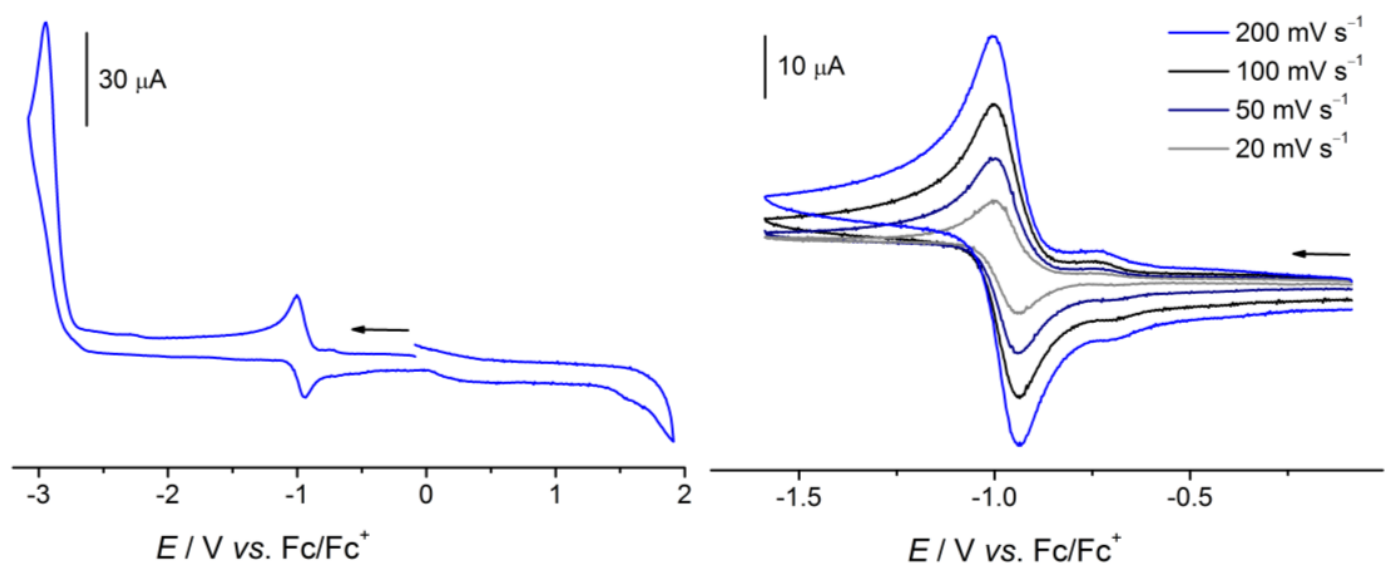

Figure 59. Left: Cyclic voltammogram of $17\left(1.5 \mathrm{mM}\right.$ in $\left.\left.\mathrm{MeCN} / 0.1 \mathrm{M}^{n}{ }^{n} \mathrm{Bu}_{4} \mathrm{~N}\right] \mathrm{PF}_{6}\right)$ at $\mathrm{RT}$ and $v=100 \mathrm{mV} \mathrm{s}^{-1}$; potentials given $v s$. $\mathrm{Fc} / \mathrm{Fc}^{+}$. Right: Reversible Reduction of $\mathbf{1 7}$ at $E_{1 / 2}=-0.98 \mathrm{~V}$ at various scan rates. ${ }^{[22]}$

Being interested in the redox properties of $\mathbf{1 7}$, a cyclic voltammogram of the $\{\mathrm{FeNO}\}^{7}$ compound was recorded. As shown in Figure 59, a reversible reduction of $\mathbf{1 7}$ at $E_{1 / 2}=-0.98 \mathrm{~V} v s$. the ferrocene couple was observed, providing motivation for the isolation of the corresponding $\{\mathrm{FeNO}\}^{8}$ species. To this end, a mixture of $\mathbf{1 7}$ and $\mathrm{Co}(\mathrm{Cp})_{2}$ in $\mathrm{MeCN}$ was reacted in the absence of light at $-35^{\circ} \mathrm{C}$ resulting in a color change of the solution from blue to green. Addition of $\mathrm{Et}_{2} \mathrm{O}$ gave a green precipitate of $\mathbf{1 9}$ that was washed with $\mathrm{Et}_{2} \mathrm{O}$ and hexane (47\% yield). Compared to other $\{\mathrm{FeNO}\}^{8}$ compounds, 19 is relatively stable even in solution and could be identified as the corresponding $\left.\left[{ }^{\mathrm{NHC}} \mathrm{L}\right)\{\mathrm{FeNO}\}^{8}\right](\mathrm{OTf})$ complex 19 based on a variety of spectroscopic methods as described within the next section. ${ }^{[22,23]}$ Alternative to cobaltocene, it is also possible to use sodium borohydride as the reducing agent. Especially in terms of in situ generation of the $\{\mathrm{FeNO}\}^{8}$ compound for reactivity studies, $\mathrm{NaBH}_{4}$ is of advantage since it does not absorb in the UV/vis region. 
Having in mind the successful isolation of $\mathbf{1 8}$, it is rather surprising that electrochemical experiments in $\mathrm{MeCN}$ solution did not show any oxidation of 17 up to $+1.5 \mathrm{~V}$ vs. $\mathrm{Fc} / \mathrm{Fc}^{+}$. In accordance with the results obtained on oxidation of the $\mu$-nitridodiiron(III) species $\mathbf{6}$, it is likely that formation of the $\{\mathrm{FeNO}\}^{6}$ species requires coordination of a second axial ligand. Formation of 18 is proposed to occur via reaction of $\{\mathrm{FeNO}\}^{7} \mathbf{1 7}$ with $\mathrm{NO}_{2}$ in a redox process to form $\left(\mathrm{ONO}^{-}\right)\left\{\mathrm{FeNO}^{6} \mathbf{1 8}\right.$. In agreement with this, treatment of $\mathbf{1 7}$ directly with sodium nitrite in the absence of a further oxidant did not lead to any reaction. To support the proposed mechanism, the cyclic voltammogram of $\mathbf{1 7}$ was recorded again in the presence of 1 eq. [ ${ }^{n} \mathrm{Bu}_{4} \mathrm{~N} \mathrm{NO}_{2}$ (Figure 60, left, red curve). In this case, a new electrochemically irreversible wave at $E_{1 / 2}=-0.51 \mathrm{~V}$ appeared. ${ }^{[23]}$ This wave is associated with anodic coordination and cathodic release of axial $\mathrm{NO}_{2}^{-}$ upon redox conversion between $\{\mathrm{FeNO}\}^{7}$ and $\{\mathrm{FeNO}\}^{6}$. The obtained cyclic voltammogram nicely resembles the one observed for clean 18 (Figure 60, right). Furthermore, the second reduction process equals the reduction observed for $\mathbf{1 7}$ to give 19. In agreement with the electrochemical findings, treatment of $(\mathrm{ONO})\{\mathrm{FeNO}\}^{6} \mathbf{1 8}$ with one equivalent of cobaltocene on a preparative scale leads to formation of the blue complex $\mathbf{1 7}$, and treatment of $\mathbf{1 8}$ with two equivalents of cobaltocene leads to the green complex 19, according to Scheme 23. Upon reduction, the more weakly bound trans nitrito ligand is rapidly released, giving the stable $\{\mathrm{FeNO}\}^{7}$ complex $\mathbf{1 7}$ that can be reversibly reduced in a subsequent process to the $\{\mathrm{FeNO}\}^{8}$ complex $19 .{ }^{[23]}$
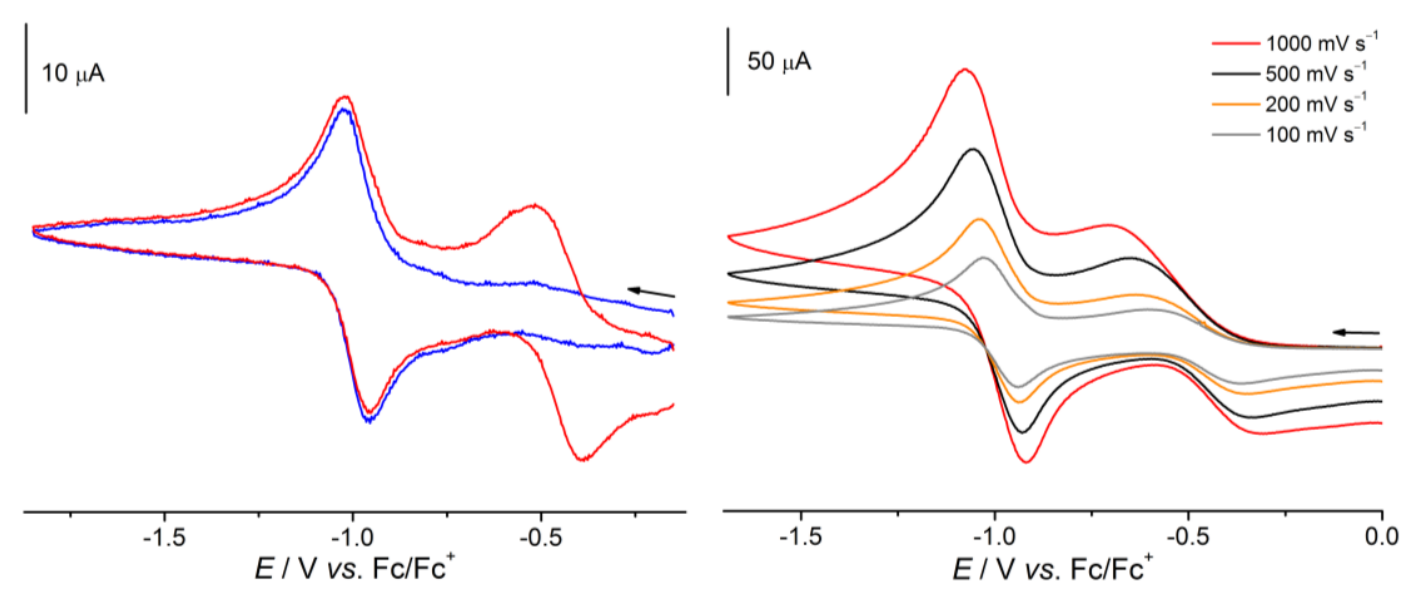

Figure 60. Left: Cyclic voltammograms of $\mathbf{1 7}$ prior to (blue) and after addition of 1 eq. $\left[{ }^{n} \mathrm{Bu}_{4} \mathrm{~N}^{2} \mathrm{NO}_{2}\right.$ (red) in

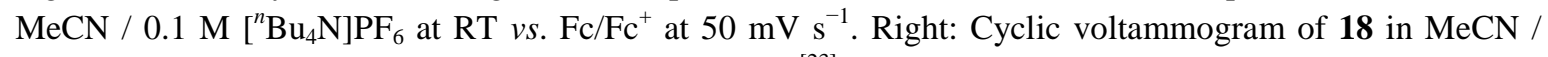
$0.1 \mathrm{M}\left[{ }^{n} \mathrm{Bu}_{4} \mathrm{~N}\right] \mathrm{PF}_{6}$ at $\mathrm{RT} v s . \mathrm{Fc} / \mathrm{Fc}^{+}$at various scan rates. ${ }^{[23]}$

In conclusion, a complete series of $\{\mathrm{FeNO}\}^{6-8}$ coordinated by a tetracarbene macrocycle was obtained and the species have been shown to reversibly interconvert into each other (electro)chemically. These compounds represent the first iron nitrosyl and nitroxyl systems coordinated by organometallic carbene donors. So far, Fe-C bonds in iron nitrosyls have been limited to $\mathrm{N}$-confused porphyrin systems with an $\left\{\mathrm{N}_{3} \mathrm{C}\right\}$ donor set. ${ }^{[132]}$ Parallel to this work, Kühn et al. synthesized a similar $\{\mathrm{FeNO}\}^{7}$ species coordinated by a smaller tetracarbene marcrocycle with 
methyl bridges only (Chapter 1, Scheme 10). ${ }^{[160]}$ Similar to $\mathbf{1 7}$, this compound did not show any oxidation in the cyclic voltammogram and only an irreversible reduction was found, thus no $\{\mathrm{FeNO}\}^{6-8}$ series was obtained. Having the series of $\mathbf{1 7 - 1 9}$ in hand, the species were characterized spectroscopically and theoretically, as presented within the next sections. 


\subsection{Characterization of a Complete Series of $\left\{\mathrm{FeNO}^{6},\{\mathrm{FeNO}\}^{7}\right.$ and $\{\mathrm{FeNO}\}^{8}$}

\subsubsection{Structural Characterization}

It was possible to obtain single crystals suitable for X-ray diffraction of all three $\{\mathrm{FeNO}\}^{n}$ compounds, what allowed for a comparison of their structural characteristics as will be presented within this section. The molecular structures of all species are shown in Figure 61, characteristic bond lengths and angles are depicted in Table 9. ${ }^{[22,23]}$
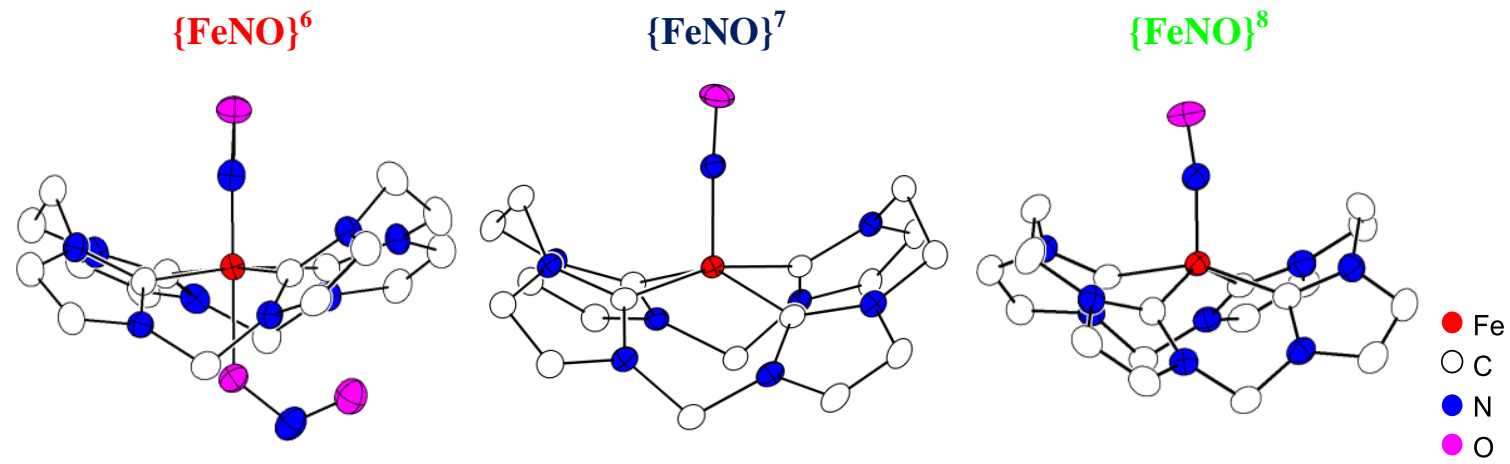

Figure 61. ORTEP plots of the molecular structures of the compounds $\left[\left({ }^{\mathrm{NHC}} \mathrm{L}\right)\{\mathrm{FeNO}\}^{6}(\mathrm{ONO})\right]^{2+} \mathbf{1 8}$ (left), $\left.\left[{ }^{\mathrm{NHC}} \mathrm{L}\right)\{\mathrm{FeNO}\}^{7}\right]^{2+} \mathbf{1 7}$ (middle), and $\left.\left[{ }^{\mathrm{NHC}} \mathrm{L}\right)\{\mathrm{FeNO}\}^{8}\right]^{+} \mathbf{1 9}$ (right) (50\% probability thermal ellipsoids); hydrogen atoms and anions (OTf) are omitted for clarity. ${ }^{[22,23]}$

Table 9. Selected structural parameters, bond lengths $[\AA]$ and angles $\left[^{\circ}\right]$ for the $\{\mathrm{FeNO}\}^{n}$ species presented herein, DFT calculated parameters in brackets. ${ }^{[22,23]}$

\begin{tabular}{|c|c|c|c|}
\hline & $\begin{array}{c}\text { FeNO }^{6} \\
18 \\
\end{array}$ & $\begin{array}{c}\text { FeNO }^{7} \\
17 \\
\end{array}$ & $\begin{array}{c}\text { FeNO }^{8} \\
19 \\
\end{array}$ \\
\hline space group & $P \overline{1}$ & $P \overline{1}$ & $P 2_{1 / \mathrm{C}}$ \\
\hline coordination & OC-6 & SPY -5 & SPY-5 \\
\hline Fe displacement from $\{\mathrm{C} 4\}$ plane $[\AA]$ & 0.12 & 0.41 & 0.56 \\
\hline$<(\mathbf{F e}-\mathbf{N}-\mathbf{O})\left[{ }^{\circ}\right]$ & $\begin{array}{c}176.6 \\
(175.3)\end{array}$ & $\begin{array}{c}176.9 \\
(179.5)\end{array}$ & $\begin{array}{c}169.1 \\
(174.8)\end{array}$ \\
\hline$<(\mathrm{ON}-\mathrm{Fe}-\mathrm{ONO})\left[^{\circ}\right]$ & $\begin{array}{c}175.6 \\
(174.0)\end{array}$ & - & - \\
\hline$d(\mathrm{~N}-\mathrm{O})[\AA]$ & $\begin{array}{c}1.162 \\
(1.159)\end{array}$ & $\begin{array}{c}1.166 \\
(1.168)\end{array}$ & $\begin{array}{c}1.207 \\
(1.190)\end{array}$ \\
\hline$d(\mathrm{Fe}-\mathrm{NO})[\AA]$ & $\begin{array}{c}1.625 \\
(1.635)\end{array}$ & $\begin{array}{c}1.670 \\
(1.661)\end{array}$ & $\begin{array}{c}1.660 \\
(1.655)\end{array}$ \\
\hline av. $d\left(\mathrm{Fe}-\mathrm{C}^{\mathrm{NHC}}\right)[\AA]$ & $\begin{array}{c}2.008 \\
(2.019)\end{array}$ & $\begin{array}{c}1.994 \\
(1.987)\end{array}$ & $\begin{array}{c}1.968 \\
(1.957)\end{array}$ \\
\hline
\end{tabular}

Blue crystals of 17 could be obtained in $54 \%$ yield by slow diffusion of $\mathrm{Et}_{2} \mathrm{O}$ into a $\mathrm{MeCN}$ solution of the crude $\{\mathrm{FeNO}\}^{7}$ compound at $-35^{\circ} \mathrm{C}$. Complex 17 crystallizes in the triclinic space group $P \overline{1}$. The iron center is found five-coordinated $(5 \mathrm{C})$ in roughly square pyramidal environment (SPY-5, $\tau=0.27)^{[231]}$ with the tetracarbene macrocycle in the equatorial positions and the NO 
ligand occupying the axial position. The iron atom is displaced by $0.41 \AA$ out of the basal $\left\{\mathrm{C}_{4}\right\}$ plane toward the NO. The macrocycle is ruffled similar to findings made for the other complexes presented within this work, and the $\mathrm{C}-\mathrm{Fe}-\mathrm{C}$ angles are relatively small $\left(164.3^{\circ}\right.$ and $\left.148.1^{\circ}\right)$. Importantly, $\mathbf{1 7}$ is a rare example of an $\{\mathrm{FeNO}\}^{7}$ complex that shows a quasi-linear $\mathrm{Fe}-\mathrm{N}-\mathrm{O}$ moiety $\left(176.9^{\circ}\right)$. A space-filling model (Chapter 13, X-ray Figure 11) confirms that linearity is not imposed by steric constraints, implicating that it originates from the electronic structure instead. The N-O bond length of $1.166 \AA$ is in the expected range (1.15-1.18 $\mathrm{A}$ ), whereas the Fe-NO bond in $17(1.670 \AA)$ is shorter than in most $5 \mathrm{C}\left\{\mathrm{FeNO}^{7}\right.$ complexes reported so far (1.73$1.78 \AA){ }^{[28,114,115,126]}$ The latter value is actually close to the $\mathrm{Fe}-\mathrm{NO}$ bond lengths of several $\{\mathrm{FeNO}\}^{6}$ complexes $\left(1.63-1.67 \AA\right.$ ) which are usually described as having some nitrosonium $\left(\mathrm{NO}^{+}\right)$ character. ${ }^{[11,22,23,28,116]}$

Green single crystals of $\mathbf{1 9}$ suitable for X-ray diffraction were obtained by slow diffusion of $\mathrm{Et}_{2} \mathrm{O}$ into a $\mathrm{MeCN}$ solution of the green reaction mixture at $-35^{\circ} \mathrm{C}$. As in compound $\mathbf{1 7}$, the iron center in the $\{\mathrm{FeNO}\}^{8}$ species is found in a roughly square pyramidal environment while the iron atom's displacement out of the $\left\{\mathrm{C}_{4}\right\}$ basal plane of the ${ }^{\mathrm{NHC}} \mathrm{L}$ scaffold is more pronounced in 19 (0.56 $\AA$ ). This observation directly correlates with reduced interaction of the iron center with the carbene ligand in the $\{\mathrm{FeNO}\}^{8}$ species compared to the corresponding $\{\mathrm{FeNO}\}^{7}$ compound. Increased displacement from the $\left\{\mathrm{C}_{4}\right\}$ plane could be due to stronger bonding with the NO as evidenced by the slightly shorter Fe-NO bond (1.669 ̊̊). Alternatively, increased repulsion by the carbene could push the iron center up, and the only redox-active orbital that would explain this is the $\mathrm{d}_{\mathrm{z}^{2}}$ with its strongly $\sigma$-antibonding torus. Linearity of the $\mathrm{Fe}-\mathrm{N}-\mathrm{O}$ moiety is decreased compared to $\{\mathrm{FeNO}\}^{7}$ 17 upon reduction to $\{\mathrm{FeNO}\}^{8} \mathbf{1 9}\left(169.1^{\circ}\right)$, in line with recently published crystallographic data by $\mathrm{Hu}$ and $\mathrm{Li}$ as well as DFT predictions discussed in section $1.4{ }^{[121,124,125]}$ It should be noted that in those previous examples the $\mathrm{Fe}-\mathrm{N}-\mathrm{O}$ moiety was already bent in the $\{\mathrm{FeNO}\}^{7}$ compound, ${ }^{[125]}$ in contrast to the situation in $\mathbf{1 7}$. The $\mathrm{N}-\mathrm{O}$ distance in $\mathbf{1 9}$ is slightly elongated while the $\mathrm{Fe}-\mathrm{NO}$ distance is shortened compared to 17. This trend is explained by addition of one further electron into the SOMO of the $\{\mathrm{FeNO}\}^{7}$ unit that has mainly $\mathrm{Fe}\left(\mathrm{d}_{\mathrm{z}^{2}}\right)$ character $(53 \%)$ and $\sigma^{*}$ character with respect to the NO moiety (see DFT, section 7.2.6). Intuitively one might assume that the addition of a further electron would lead to enhanced electronic repulsion between the $\mathrm{Fe}$ and the NO ligand and therefore to a longer Fe-NO bond. However, increased bending within this moiety reduces this repulsion. Conversely, the higher electron density at the Fe center enables better $\pi$-backbonding from $\mathrm{Fe}\left(\mathrm{d}_{\mathrm{xz}}, \mathrm{d}_{\mathrm{yz}}\right)$ into the $\pi^{*}(\mathrm{NO})$ orbital leading to a shorter $\mathrm{Fe}-\mathrm{NO}$ bond and a longer $\mathrm{N}-\mathrm{O}$ bond. $^{[23]}$

Yellow crystals of 18 could be obtained in $63 \%$ yield by diffusion of $\mathrm{Et}_{2} \mathrm{O}$ into the reaction mixture at RT. The $\{\mathrm{FeNO}\}^{6}$ compound proved to be stable in air for extended periods and was fully 
characterized. The Fe center in $\mathbf{1 8}$ (Figure 61) is coordinated octahedrally (OC-6) with the NO and O-nitrito ligands in the axial positions $\left(\angle \mathrm{ON}-\mathrm{Fe}-\mathrm{ONO}=175.6^{\circ}\right)$. In this case, the $\mathrm{Fe}$ atom is displaced only slightly out of the $\{\mathrm{C} 4\}$ basal plane by $0.12 \AA$ toward the nitric oxide. The $\mathrm{Fe}-\mathrm{N}-\mathrm{O}$ angle is very similar to the one in complex $\mathbf{1}\left(176.6^{\circ}\right)$ while both the $\mathrm{N}-\mathrm{O}(1.162 \AA)$ and the $\mathrm{Fe}-\mathrm{NO}(1.625 \AA)$ distances are slightly shorter. The distance between the $\mathrm{Fe}$ and the O-bound nitrito ligand ( $\mathrm{Fe}-\mathrm{ONO})$ is $1.98 \AA$ and therefore much longer than the distance between the Fe and the NO, indicating a much stronger bonding of the latter ligand. This is in agreement with release of the nitrite upon reduction of the $\{\mathrm{FeNO}\}^{6}$ compound. The $\mathrm{N}-\mathrm{O}$ bonds within the nitrito ligand are also much longer than the one in the NO ligand (FeO-NO $1.31 \AA$ and FeON-O $1.20 \AA$ ), as expected. ${ }^{[23]}$

Even though many reports on oxidized ferric nitrosyls $\{\mathrm{FeNO}\}^{6}$ are found in literature, ${ }^{[11,115]}$ few octahedral non-heme compounds have been reported. ${ }^{[129,138]}$ Interestingly, in complex $\mathbf{3}$ an O-bound nitrito ligand is found, contrasting the predominant N-nitrito (also: nitro) coordination. ${ }^{[115]}$ Some similar compounds with a nitrosyl and an N-nitrito group trans to each other and an equatorial macrocyclic ligand are found in the literature ${ }^{[133,228,232]}$ while only Wieghardt et al. reported a comparable O-nitrito $\{\mathrm{FeNO}\}^{6}$ complex. ${ }^{[23,138]}$

As described within the introduction, analysis of changes in properties upon both oxidation and reduction of $\{\mathrm{FeNO}\}^{7}$ species is rather limited since the related $\{\mathrm{FeNO}\}^{8}$ species are usually lacking due to their instability. ${ }^{[23]}$ Within this work, it was achieved to structurally characterize a complete series of $\{\mathrm{FeNO}\}^{6},\{\mathrm{FeNO}\}^{7}$ and $\{\mathrm{FeNO}\}^{8}$ complexes for the first time. Furthermore, the presented species are the first examples of fully organometallic iron nitrosyl and nitroxyl compounds. Their structures show characteristics that differ from both typical heme and non-heme models, in particular remarkable linear $\mathrm{Fe}-\mathrm{N}-\mathrm{O}$ moieties and short $\mathrm{Fe}-\mathrm{NO}$ bonds. The comparable $\{\mathrm{FeNO}\}^{7}$ species that was reported by Kühn et al. shows similar bond lengths and angles indicative of the special characteristics being a typical feature of tetracarbene coordinated $\{\mathrm{FeNO}\}$ species (Scheme 10). ${ }^{[160]}$ Consequently, it was necessary to obtain further spectroscopic data on compounds 17-19 in order to provide a comprehensive comparison with the model compounds previously reported in literature. ${ }^{[23]}$ As described within the introduction, the redox processes within $\{\mathrm{FeNO}\}^{6-8}$ species can occur ligand - meaning NO centered - or metal centered, thus providing motivation to identify the nature of reduction and oxidation of the $\{\mathrm{FeNO}\}^{7}$ species $\mathbf{1 7}$ herein. 


\subsubsection{Magnetic Properties of the Newly Synthesized FeNO Compounds}

As indicated by the Enemark-Feltham notation, the $\{\mathrm{FeNO}\}^{7}$ species $\mathbf{1 7}$ has an uneven number of electrons. Consequently, a SQUID magnetic susceptibility measurement was performed to specify the spin state of 17. As shown in Figure 62, the $\chi_{\mathrm{M}} T$ value of $0.39 \mathrm{~cm}^{3} \mathrm{~K} \mathrm{~mol}^{-1}$ is almost constant in the range from 10 to $295 \mathrm{~K}$ and is close to the spin-only value expected for an $S=1 / 2$ system $\left(0.375 \mathrm{~cm}^{3} \mathrm{~K} \mathrm{~mol}^{-1}\right) .^{[22]}$ Thus, $\mathbf{1 7}$ is another example for a low spin iron complex coordinated by the tetracarbene macrocycle ${ }^{\mathrm{NHC}} \mathrm{L}$. As shown within this work, this spin state is prevalent for this class of organometallic iron species while an intermediate spin state was only observed for the iron(III) species 3,6 and $\mathbf{8}$.

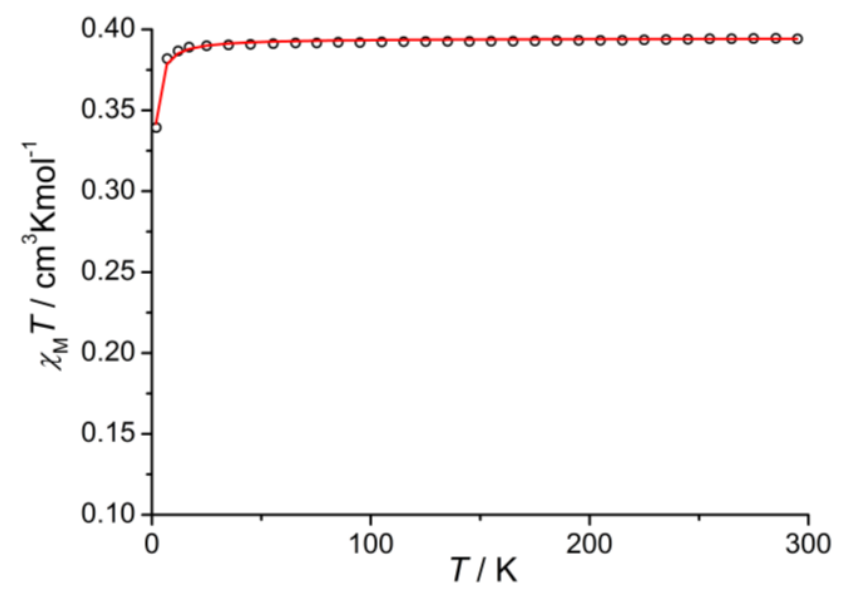

Figure 62. $\chi_{\mathrm{M}} T v s$. T of solid $\mathbf{1 7}$ (blue dots). The red line represents the simulation using $g=2.05$.
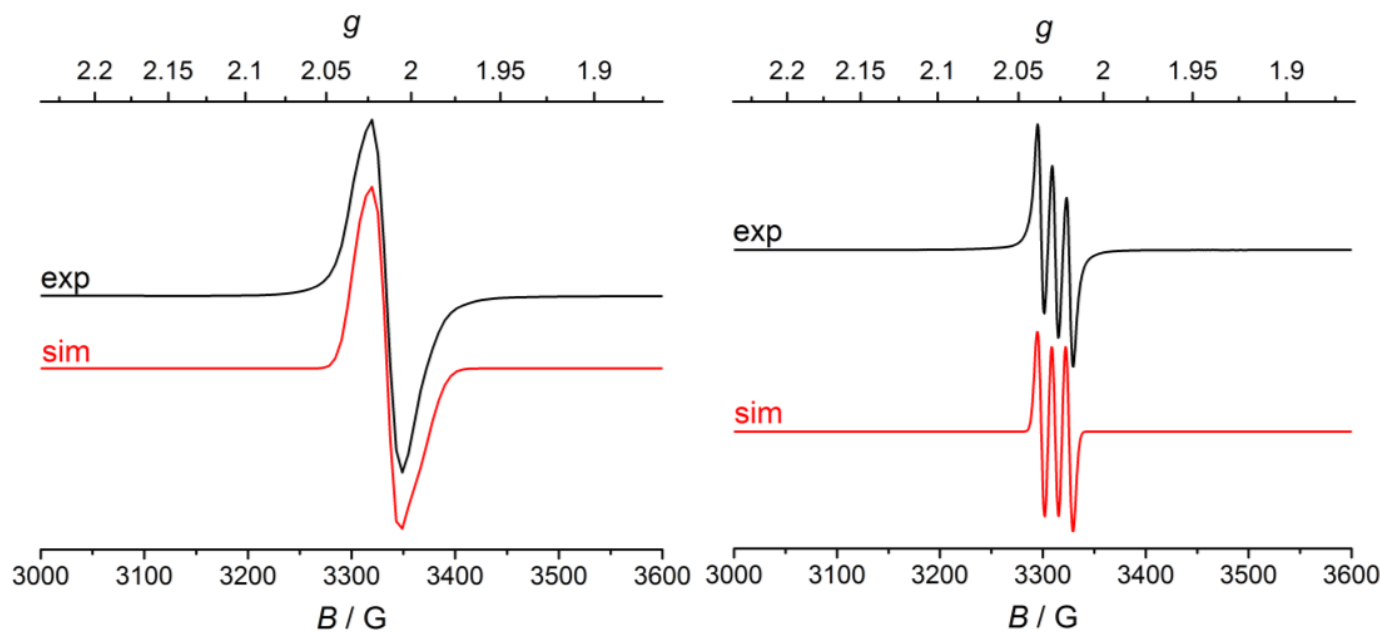

Figure 63. Left: X-Band EPR spectrum of $\left[\left({ }^{\mathrm{NHC}} \mathrm{L}\right)\{\mathrm{FeNO}\}^{7}\right](\mathrm{OTf})_{2} \mathbf{1 7}$ in frozen MeCN solution at $159 \mathrm{~K}$. The red line represents a simulation with the parameters $g=2.0290,2.0136,1.996$ and Gaussian line broadening of $1 \mathrm{mT}$ FWHM. Right: X-Band EPR spectrum of $\mathbf{1 7}$ in frozen MeCN solution at $220 \mathrm{~K}$. The red line represents a simulation with the parameters $g_{\text {iso }}=2.027$ and $A\left({ }^{14} \mathrm{~N}_{\mathrm{NO}}\right)=38 \mathrm{MHz}$ and Gaussian line broadening of 0.9 mT FWHM. 
Most reported low-spin $\{\mathrm{FeNO}\}^{7}$ compounds feature EPR spectra with $g$ values close to 2.0 and small $g$ anisotropy. X-Band EPR spectroscopy of a frozen MeCN solution of 19 at $159 \mathrm{~K}$ shows a rather broad and almost isotropic signal which can be simulated with the $g$ values 2.029,2.014, and $1.996\left(g_{\text {av }}=2.013\right.$; Figure 63, left $)$. However, at $220 \mathrm{~K}$, an isotropic triplet at $g_{\text {iso }}=2.027$ with resolved ${ }^{14} \mathrm{~N}(I=1)$ hyperfine coupling $A\left({ }^{14} \mathrm{~N}_{\mathrm{NO}}\right)=13.4 \mathrm{G}$ is observed for $\mathbf{1 9}$ (Figure 63, right). Similar temperature dependent broadening effects were reported for other paramagnetic iron nitrosyl complexes before, and a large anisotropy of the line width has been mentioned as a typical feature of square pyramidal $(S=1 / 2)\{\mathrm{FeNO}\}^{7}$ complexes with four equatorial N-donors. ${ }^{[22,138,233]}$

The $\{\mathrm{FeNO}\}^{8}$ compound 19 shows diamagnetic low spin character, what allows for a characterization via NMR spectroscopy (Appendix Figure 15). As typically observed for $\left[\left({ }^{\mathrm{NHC}} \mathrm{L}\right) \mathrm{Fe}(\mathrm{X})_{\mathrm{n}}\right]$ species $(\mathrm{n}=1$ or 2$)$ with only one or with two different axial ligands, the methylene $\left(\mathrm{CH}_{2}\right)$ linkers of the tetracarbene macrocycle show two doublets at $\delta=6.21$ and $5.78 \mathrm{ppm}$ $\left({ }^{2} J_{\mathrm{HH}}=13.5 \mathrm{~Hz}\right)$. Similar to compound 19, also the $\{\mathrm{FeNO}\}^{6}$ species 18 shows a diamagnetic NMR spectrum with a comparable signal set. Notably, one of the two doublets for the methylene linkers of the macrocycle $\left(\delta=6.18\right.$ and $\left.5.15 \mathrm{ppm} ;{ }^{2} J_{\mathrm{HH}}=13.5 \mathrm{~Hz}\right)$ is significantly shifted upfield compared to $\mathbf{1 9}$ and is thus assigned to the proton that directs to the side of the nitrito-O ligand. ${ }^{[23]}$

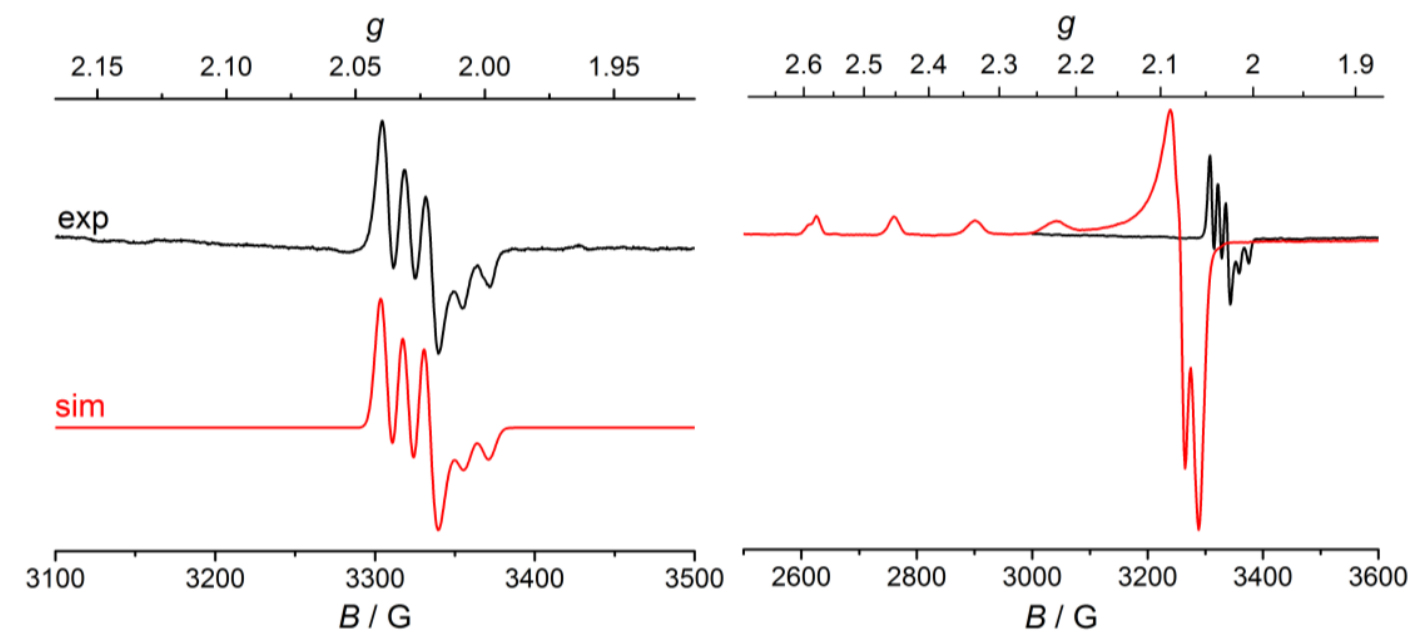

Figure 64. Left: X-band EPR spectrum of the $\{\mathrm{FeNO}\}^{6}$ species $\mathbf{1 8}$ in frozen MeCN solution at $159 \mathrm{~K}$. The red line represents a simulation with the parameters $g=2.0334,2.0334$, and 2.011 with $\mathrm{A}\left({ }^{14} \mathrm{~N}\right)=\left[\begin{array}{ll}38 & 38 \\ 43\end{array}\right]$ MHz. Right: X-Band EPR spectra of $18(10 \mathrm{mM})$ in frozen MeCN solution (black trace) and a reference spectrum of $\mathrm{CuSO}_{4}(1 \mathrm{mM})$ with $\mathrm{NaClO}_{4}(200 \mathrm{mM})$ in $\mathrm{HClO}_{4}(1 \mathrm{mM})$ in water (red trace) at $158 \mathrm{~K}$ and $10.0 \mathrm{~mW}$ each. Taking into account the differences in concentration, integration assigns the detected EPR active species in $\mathbf{1 8}$ to represent less than $5 \%$ of the sample.

While the X-band EPR spectrum of $\mathbf{1 9}$ is silent as expected, a signal was observed in the spectrum of 18 (Figure 64, left). With the help of a Cu-standard, by integration this signal was shown to corresponded to about $5 \%$ of the overall sample. A similar impurity of a paramagnetic species in solution has been often reported for $\{\mathrm{FeNO}\}^{6}$ complexes, and Wieghardt et al. assigned this signal to trace amounts of the corresponding $\{\mathrm{FeNO}\}^{7}$ compound. ${ }^{[234]}$ Thus, it can be concluded that the 
major species of a frozen MeCN solution of $\mathbf{1 8}$ is EPR silent in agreement with NMR signals in the diamagnetic region.

\subsubsection{IR and UV/vis Spectroscopy}

A very characteristic parameter for iron nitrosyl and nitroxyl compounds is the $\mathrm{N}-\mathrm{O}$ stretching frequency that directly correlates with the strength of this bond. The signals observed for the $\tilde{v}_{\mathrm{N}-\mathrm{O}}$ stretches of the $\{\mathrm{FeNO}\}^{6-8}$ species $\mathbf{1 7 - 1 9}$ in solid state are shown in Figure 65, left.
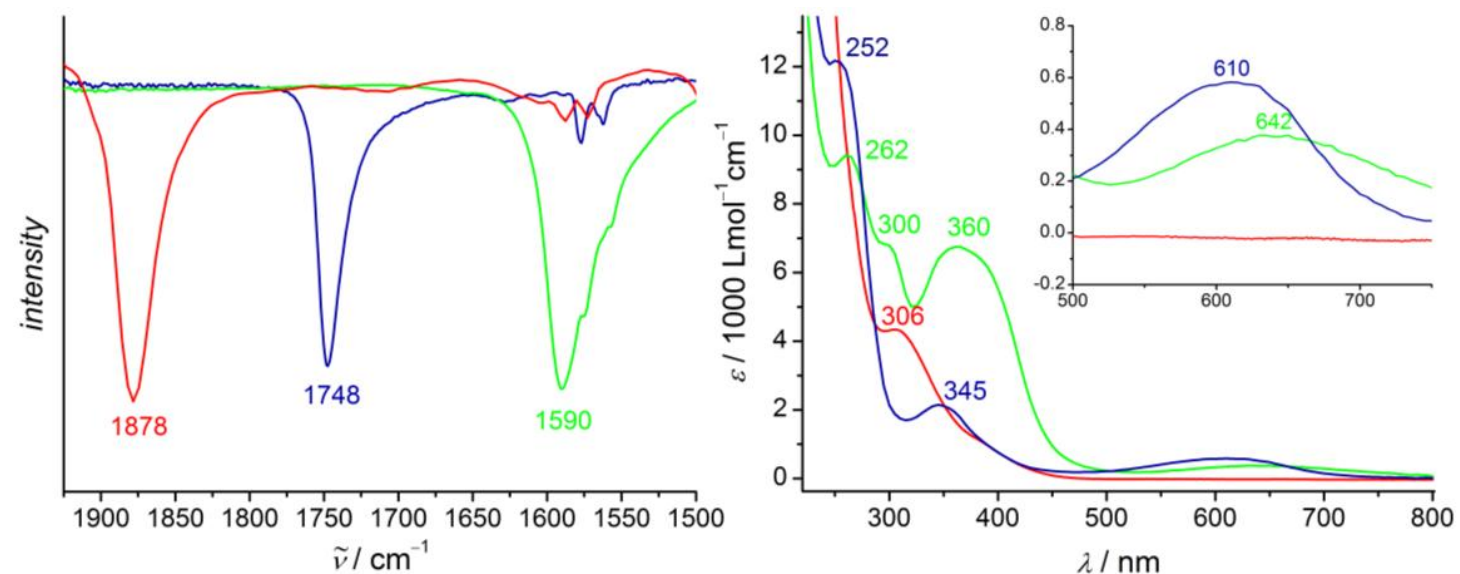

Figure 65. IR (left) and UV/vis spectra (right) of $\left[\left({ }^{\mathrm{NHC}} \mathrm{L}\right)\{\mathrm{FeNO}\}^{6}(\mathrm{ONO})\right]^{2+} \mathbf{1 8}$ (red), $\left.\left[{ }^{\mathrm{NHC}} \mathrm{L}\right)\{\mathrm{FeNO}\}^{7}\right]^{2+} \mathbf{1 7}$ (blue), and $\left[\left({ }^{\mathrm{NHC}} \mathrm{L}\right)\{\mathrm{FeNO}\}^{8}\right]^{+} \mathbf{1 9}$ (green). ${ }^{[23]}$

In case of the $\{\mathrm{FeNO}\}^{7}$ species $17, \tilde{v}_{\mathrm{N}-\mathrm{O}}$ is found at $1748 \mathrm{~cm}^{-1}$ for solid material and at $\tilde{v}=1740 \mathrm{~cm}^{-1}$ in MeCN solutions, which is at higher energy than for literature reported low spin $\{\mathrm{FeNO}\}^{7}$ complexes that usually have their $\tilde{v}_{\mathrm{N}-\mathrm{O}}$ stretches in the range of $1600-1700 \mathrm{~cm}^{-1}$. ${ }^{[1,28,124]}$ In general, the position of the $\tilde{v}_{\mathrm{N}-\mathrm{O}}$ band is sensitive to the oxidation and spin state of the iron center, and low spin $\{\mathrm{FeNO}\}^{7}$ species typically show lower $\mathrm{N}-\mathrm{O}$ stretching frequencies than high spin $\{\mathrm{FeNO}\}^{7}$ systems $\left(1700-1850 \mathrm{~cm}^{-1}\right) \cdot{ }^{[16]}$ On the other hand, linear $\mathrm{Fe}-\mathrm{N}-\mathrm{O}$ moieties have higher $\tilde{v}_{\mathrm{N}-\mathrm{O}}$ values than their bent analogues, in line with the relatively high frequency for the low spin system 17. ${ }^{[235,236]}$ Thus, the unusual linearity of the $\mathrm{Fe}-\mathrm{N}-\mathrm{O}$ group in the newly synthesized tetracarbene coordinated $\{\mathrm{FeNO}\}^{7}$ system leads to an unusually high value for $\tilde{v}_{\mathrm{N}-\mathrm{O}}$. This finding already suggests rather small electron density at the NO ligand and some $\mathrm{NO}^{+}$-character. ${ }^{[2]}$ For comparison, the $\{\mathrm{FeNO}\}^{7}$ species synthesized by Kühn et al. shows a slightly lower $\mathrm{N}-\mathrm{O}$ stretching frequency $\left(\tilde{v}=1729 \mathrm{~cm}^{-1}\right) \cdot{ }^{[160]}$

The $\tilde{v}_{\mathrm{N}-\mathrm{O}}$ stretching frequency for solid $\{\mathrm{FeNO}\}^{8} \mathbf{1 9}$ was found at $1590 \mathrm{~cm}^{-1}$ in the IR spectrum and at $1604 \mathrm{~cm}^{-1}$ for a MeCN solution, in line with the longer $\mathrm{N}-\mathrm{O}$ bond compared to the precursor complex 17. Again, this value is relatively high compared to other literature reported iron nitroxyl 
compounds and is in the range of high spin complexes rather than low spin species, due to the linearity of the $\mathrm{Fe}-\mathrm{N}-\mathrm{O}$ moiety. The shift of $158 \mathrm{~cm}^{-1}$ upon reduction from $\mathbf{1 7}$ to $\mathbf{1 9}$ can originate from either ligand or metal centered reduction. Addition of one electron into the $\pi^{*}(\mathrm{NO})$ orbital would directly weaken the $\mathrm{N}-\mathrm{O}$ bond, whereas reduction of $\mathrm{Fe}$ would lead to increased $\pi$ backbonding inducing kind of an indirect weakening of the $\mathrm{N}-\mathrm{O}$ bond. ${ }^{[23]}$ Compared to literaturereported shifts upon reduction from $\{\mathrm{FeNO}\}^{7}$ to $\{\mathrm{FeNO}\}^{8}$ species $\left(100-250 \mathrm{~cm}^{-1}\right),{ }^{[11,116,237]}$ the observed difference between $\mathbf{1 7}$ and $\mathbf{1 9}$ is rather moderate, suggesting a metal centered redox process. ${ }^{[23]}$ A similar moderate shift was found for the oxidation process going from $\{\mathrm{FeNO}\}^{7}$ to $\left\{\mathrm{FeNO}^{6}\right.$. In line with the shorter $\mathrm{N}-\mathrm{O}$ bond in the structure of $\mathbf{1 8}, \tilde{v}_{\mathrm{NO}}$ is shifted by $130 \mathrm{~cm}^{-1}$ to higher wavenumbers compared to the $\{\mathrm{FeNO}\}^{7}$ complex 17 (solid: $1886 \mathrm{~cm}^{-1}$; MeCN solution: $1882 \mathrm{~cm}^{-1}$ ). Unfortunately, the stretching frequencies within the O-nitrito moiety could not be assigned unambiguously even though labeling with ${ }^{18} \mathrm{O}$ and ${ }^{15} \mathrm{~N}$ was performed. For the latter, nitric oxide was generated by treatment of $\mathrm{Na}^{15} \mathrm{NO}_{2}$ with an aqueous solution of ascorbic acid. The stretching frequencies of the labeled compounds showed the expected shift of the $\tilde{v}_{\mathrm{N}-\mathrm{O}}$ stretch to lower energy by $31-38 \mathrm{~cm}^{-1}$. The obtained values are summarized in Table $10{ }^{[23]}$ Labeling with ${ }^{18} \mathrm{O}$ in case of the $\{\mathrm{FeNO}\}^{6}(\mathrm{ONO})$ species furthermore gave rise to a new signal at $1841 \mathrm{~cm}^{-1}$ (MeCN solution), that was assigned to the $\tilde{v}_{\mathrm{N}-\mathrm{O}}$ stretch in ${ }^{18} \mathrm{ON}-\mathrm{Fe}-\mathrm{ONO}$. However, the labeling experiments did not allow for an assignment of the Fe-NO stretching frequencies. Based on DFT calculations, the corresponding signals in the IR spectrum are expected to be in the region of $600-$ $700 \mathrm{~cm}^{-1}$. More importantly, the calculations predict very weak intensity of these signals $\left(\mathrm{T}^{* * 2}=1-5\right)$ which prevents their detection next to more intense vibrations within the tetracarbene ligand $\left(\mathrm{T}^{* * 2} \approx 90\right)$. ${ }^{[23]}$ Further information about the DFT calculations are given in section 7.2.6 and chapter 12 .

Table 10. Experimental and DFT calculated (in brackets) $\tilde{v}_{\mathrm{N}-\mathrm{O}}$ stretching frequencies for $\mathbf{1 8}, \mathbf{1 7}$ and $\mathbf{1 9} .^{[23]}$

\begin{tabular}{|c|c|c|c|}
\hline & $\begin{array}{c}\text { FeNO }^{6} \\
18 \\
\end{array}$ & $\begin{array}{c}\text { FeNO }^{7} \\
17 \\
\end{array}$ & $\begin{array}{c}\text { FeNO }\}^{8} \\
19 \\
\end{array}$ \\
\hline$\tilde{v}_{\mathrm{N}-\mathrm{o}}\left[\mathrm{cm}^{-1}\right]$ (solid) & $\begin{array}{c}1886 \\
(1887)\end{array}$ & $\begin{array}{c}1748 \\
(1796 / 1752)\end{array}$ & $\begin{array}{c}1590 \\
(1697 / 1601)\end{array}$ \\
\hline$\tilde{v}_{14 \mathrm{~N}-\mathrm{O}}\left[\mathrm{cm}^{-1}\right](\mathrm{MeCN})$ & 1882 & 1740 & 1604 \\
\hline$\tilde{v}_{15 \mathrm{~N}-\mathrm{o}}\left[\mathrm{cm}^{-1}\right](\mathrm{MeCN})$ & 1844 & 1708 & 1573 \\
\hline$\Delta \tilde{v}_{14 \mathrm{~N}-15 \mathrm{~N}}\left[\mathrm{~cm}^{-1}\right](\mathrm{MeCN})$ & 38 & 32 & 31 \\
\hline
\end{tabular}

The UV/vis/NIR spectrum of 17 (Figure 65, right and Figure 66) shows a prominent absorption around $\lambda_{\max }=345 \mathrm{~nm}(\varepsilon=2100)$ which is only slightly shifted in comparison with the characteristic $\mathrm{C}^{\mathrm{NHC}} \rightarrow \mathrm{Fe}$ LMCT band of the iron(II) precursor $1\left(\lambda_{\max }=339 \mathrm{~nm}, \varepsilon=9100\right)$. However, it is much weaker in intensity in accordance with increased displacement of the iron center out of the basal $\left\{\mathrm{C}_{4}\right\}$ plane that correlates with reduced interaction with the carbene ligand system. In addition, the 
$\{\mathrm{FeNO}\}^{7}$ species features a broad band at $\lambda_{\max }=615 \mathrm{~nm}$. With the help of DFT and natural transition orbital calculations, this band was assigned to a transition within the FeNO unit with largely $\mathrm{NO} \rightarrow \mathrm{Fe}$ LMCT character (Figure 66). In case of the reduced $\{\mathrm{FeNO}\}^{8}$ compound 19, the $\mathrm{C}^{\mathrm{NHC}} \rightarrow \mathrm{Fe}$ LMCT band at $\lambda_{\max }=360 \mathrm{~nm}(\varepsilon=6800$; Figure 65, right $)$ is more intense and red shifted compared to the one of $\mathbf{1 7}$, in line with shorter $\mathrm{Fe}-\mathrm{C}$ bonds. Moreover, the broad $\mathrm{NO} \rightarrow \mathrm{Fe}$ transition at $\lambda_{\max }=610 \mathrm{~nm}$ in $\{\mathrm{FeNO}\}^{7}$ is shifted to $\lambda_{\max }=642 \mathrm{~nm}$ in case of $\{\mathrm{FeNO}\}^{8} \mathbf{1 9}$ while the intensity is slightly decreased. In 19, this band represents the lowest energy HOMO $\rightarrow$ LUMO transition (see section 7.2.6) and, according to DFT, the shift to lower energies agrees well with a smaller HOMO-LUMO gap in 19 compared to 17. Interestingly, a vis transition within the $\{\mathrm{FeNO}\}$ moiety is completely missing for the $\{\mathrm{FeNO}\}^{6}$ species $\mathbf{1 8}$ which is lacking any characteristic transition beyond $450 \mathrm{~nm}$; only a broad band at $\lambda_{\max }=306 \mathrm{~nm}$ with a shoulder around $380 \mathrm{~nm}$ was found in the UV/vis spectrum of $\mathbf{1 8}$ (Figure 65). These transitions were assigned to be mainly $\mathrm{Fe}-\mathrm{ONO}$ and $\mathrm{Fe}-\mathrm{NO}$ related (DFT Figure 10). ${ }^{[22,23]}$

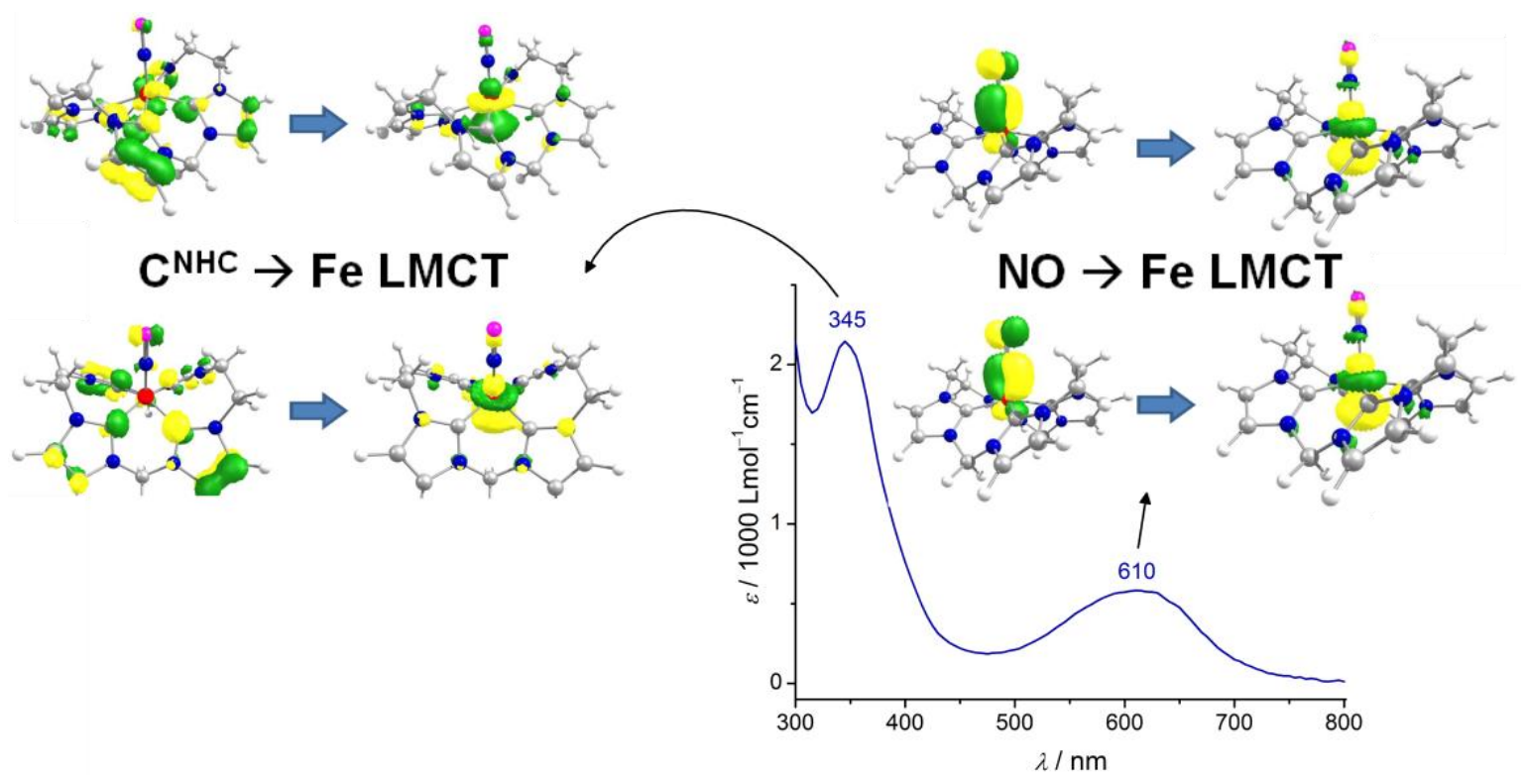

Figure 66. Natural transition orbitals of complex 17 for states $4 \& 5$ (right; band at $610 \mathrm{~nm}$, DFT: $500 \mathrm{~nm}$ ) and $16 \& 17$ (left; band at $345 \mathrm{~nm}$, DFT: $300 \mathrm{~nm}$ ) generated by Chemcraft (contour value 0.058) and calculated with B3LYP functional, RIJCOSX approximation def2-tzvp and def2-tzvp/j basis sets. The picture represents that the experimental band at $\lambda_{\max }=610 \mathrm{~nm}$ mainly originates from a LMCT within the $\{\mathrm{FeNO}\}$ unit while the band around $\lambda_{\max }=350 \mathrm{~nm}$ is best described as a LMCT with the tetracarbene ligand. ${ }^{\text {[22] }}$ 


\subsubsection{Mössbauer Spectroscopy}

In order to further characterize the electronic structures of 17-19 and the electron density at their iron nuclei, all species were analyzed by Mössbauer spectroscopy. As depicted in Figure 67, the compounds show sharp doublets in the solid state at $80 \mathrm{~K}$ that allow for a thorough interpretation of their isomer shifts and quadrupole splittings. ${ }^{[23]}$

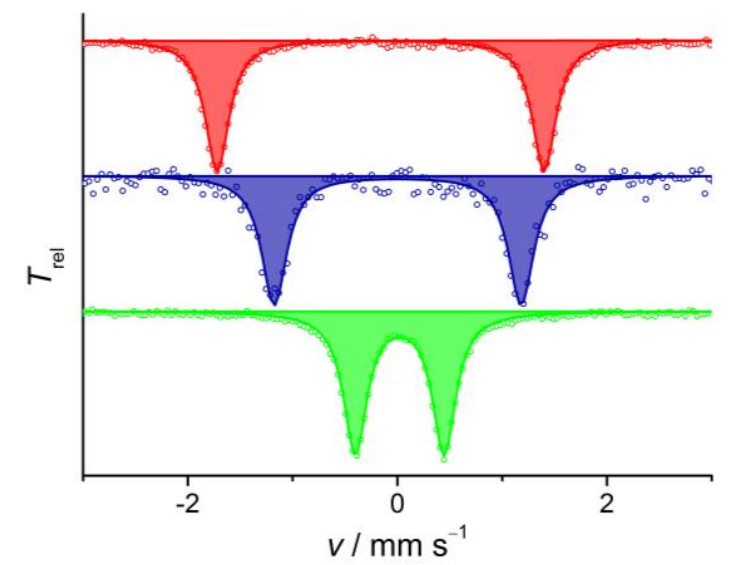

Figure 67. Zero-field ${ }^{57}$ Fe Mössbauer spectra of 17 (blue), 18 (red) and 19 (green) at $80 \mathrm{~K}$ (natural abundance $\left.{ }^{57} \mathrm{Fe}\right) .{ }^{[23]}$

Table 11. Experimental and DFT calculated (in brackets) MB parameters for 18, 17 and 19. ${ }^{[23]}$

\begin{tabular}{|c|c|c|c|}
\hline & $\begin{array}{c}\text { FeNO }^{6} \\
18\end{array}$ & $\begin{array}{c}\text { FeNO }^{7} \\
17\end{array}$ & $\begin{array}{c}\left\{\mathrm{FeNO}^{8}{ }^{8}\right. \\
19\end{array}$ \\
\hline$\delta\left[\mathrm{mm} \mathrm{s}^{-1}\right]$ & $\begin{array}{c}-0.16 \\
(-0.15)\end{array}$ & $\begin{array}{l}-0.01 \\
(0.05)\end{array}$ & $\begin{array}{c}0.02 \\
(-0.05)\end{array}$ \\
\hline$\Delta E_{\mathrm{Q}}\left[\mathrm{mm} \mathrm{s}^{-1}\right]$ & $\begin{array}{c}3.12 \\
(3.15)\end{array}$ & $\begin{array}{c}2.36 \\
(2.13)\end{array}$ & $\begin{array}{c}0.85 \\
(1.37)\end{array}$ \\
\hline
\end{tabular}

Complex 17 shows an isomer shift (IS) of $\delta=-0.01 \mathrm{~mm} \mathrm{~s}^{-1}$ and a rather large quadrupole splitting of $\Delta E_{Q}=2.36 \mathrm{~mm} \mathrm{~s}^{-1}$. Compared with the other tetracarbene coordinated iron complexes reported in this work and previously by Meyer ${ }^{[153,156]}$ and Klawitter, ${ }^{[155]}$ the IS is in the range typical for iron(III) species. However, in case of low spin $\{\mathrm{FeNO}\}^{n}$ compounds, the Mössbauer IS can generally not be used for a reliable assignment of oxidation states. As introduced in section 1.6, the isomer shift is a function of the s-electron density at the nucleus that depends both on the shielding by d-electrons but also on the interactions with surrounding ligands. For example, strong $\sigma$-donating ligands directly increase the s-electron density at the iron nucleus. Additionally, short $\mathrm{Fe}-\mathrm{L}$ bonds correspond to a compressed radial extension of the $4 \mathrm{~s}$ wave function and thus increased s-electron density at the iron nucleus, as well. In case of the NO ligand, the $\sigma$-donating ability drastically changes when going from $\mathrm{NO}^{+}$via ${ }^{\circ} \mathrm{NO}$ to $\mathrm{NO}^{-}{ }^{-[123]}$ In particular, the very strong $\sigma$-donating nitroxyl ligand in a hypothetical $\left\{\mathrm{Fe}^{\mathrm{III}} \mathrm{NO}^{-}\right\}^{7}$ species leads to increased s-electron density at the iron center resulting in a higher IS than expected for an iron(III) center. On the other 
hand, the $\mathrm{NO}^{+}$ligand in a low-valent $\left\{\mathrm{Fe}^{\mathrm{I}} \mathrm{NO}^{+}\right\}^{7}$ allows for strong $\mathrm{Fe} \rightarrow \mathrm{NO} \pi$-backbonding that lowers the shielding of s-electrons and results in an unusual low isomer shift. Consequently, the actual electron density at the iron nucleus in all possible oxidation states (I-III) is proposed to be similar, which prevents an unambiguous assignment of oxidation states in the highly covalent iron nitrosyl compounds based solely on the IS. While no direct conclusions can be drawn out of the IS in 17, the large QS nicely represents the oblate, and thus non-cubic, charge distribution around the iron center due to the strong $\sigma$-donating carbene ligands in the equatorial plane and due to the square pyramidal coordination. ${ }^{[22,23]}$

Even though the IS in the iron nitrosyl species $\mathbf{1 7}$ did not allow for an assignment of the oxidation state, the changes upon reduction and oxidation are of key importance. A Mössbauer spectrum of solid material of the reduced $\left.\left[{ }^{\mathrm{NHC}} \mathrm{L}\right)\{\mathrm{FeNO}\}^{8}\right]^{+}$species 19 at $80 \mathrm{~K}$ shows an almost identical IS of $\delta=0.01 \mathrm{~mm} \mathrm{~s}^{-1}$ compared to the one of $\left\{\mathrm{FeNO}^{7}\right.$ 17. Some literature reported $\{\mathrm{FeNO}\}^{8}$ systems have shown a drastic increase in the IS compared to their $\{\mathrm{FeNO}\}^{7}$ precursors, ${ }^{[139]}$ while negligible changes have been interpreted in terms of purely ligand-centered reductions. ${ }^{[138]}$ However, taken into account the observed structural changes when going from $\mathbf{1 7}$ to $\mathbf{1 9}$, the negligible changes observed within the tetracarbene coordinated species do not indicate a NO-centered reduction. The molecular structure of $\{\mathrm{FeNO}\}^{8} \mathbf{1 9}$ shows a slightly shorter $\mathrm{Fe}-\mathrm{N}$ bond and a markedly longer $\mathrm{N}-\mathrm{O}$ bond than what has been observed for 17. Moreover, the increased displacement of the iron center out of the $\{\mathrm{C} 4\}$ plane in $\mathbf{1 9}$ correlates with reduced interaction with the $\sigma$-donating macrocylce. Both findings are in line with a metal-centered reduction resulting in an iron center with less electron-accepting ability and enhanced electron donating ability. Consequently, two opposing effects are observed: On the one hand, a metal centered reduction (addition of a further electron into an iron d-orbital) leads to increased shielding of the $3 \mathrm{~s}$ and $4 \mathrm{~s}$ electrons, resulting in a higher IS. On the other hand, the higher electron density at the iron center results in increased $\pi$ backbonding to the NO-ligand and decreased $\sigma$-interaction with the carbenes, lowering the $\mathrm{d}$ electron density (and hence shielding of the s electrons) at the Fe core. In conclusion, the interplay of these contrary effects leads to the observed negligible changes in the IS upon reduction from $\mathbf{1 7}$ to 19. Still, it should be noted that a similar scenario could also be imagined for a ligand centered reduction. However, the interplay of all spectroscopic data with the results obtained upon DFT calculations (section 7.2.6) strongly suggests a metal centered reduction. In line with a metal centered reduction is also the marked change in the quadrupole splitting when going from $\mathbf{1 7}$ to $\mathbf{1 9}$ $\left(\Delta E_{Q}=0.87 \mathrm{~mm} \mathrm{~s}^{-1}\right)$. In particular, the QS in $\mathbf{1 9}$ is surprisingly small for a tetracarbene coordinated species, ${ }^{[22,23]}$ a similar small value was only found for $\left[\left({ }^{\mathrm{NHC}} \mathrm{L}\right) \mathrm{Fe}(\mathrm{MeCN})_{2}\right]^{3+}$ compound 9 $\left(\Delta E_{Q}=0.63 \mathrm{~mm} \mathrm{~s}^{-1}\right.$, Figure 31). In case of the ligand-centered reductions reported by Wieghardt $e t$ al., significantly smaller varieties have been observed for the QS values within their cyclam coordinated $\{\mathrm{FeNO}\}^{n}$ series. $^{[123,138]}$ 
The $\{\mathrm{FeNO}\}^{6}$ species 18 shows a very low IS at $\delta=-0.16 \mathrm{~mm} \mathrm{~s}^{-1}$, which can be explained with the changes in the $\mathrm{Fe}-\mathrm{C}^{\mathrm{NHC}}$ bond lengths. Even though this octahedral species is not completely analogous to the square pyramidal ones, the average $\mathrm{Fe}-\mathrm{C}^{\mathrm{NHC}}$ distances show significant variation among compounds 17-19 (Table 9). Specifically, there is a clear shortening of the $\mathrm{Fe}-\mathrm{C}^{\mathrm{NHC}}$ bonds upon going from the $\{\mathrm{FeNO}\}^{6}$ via $\{\mathrm{FeNO}\}^{7}$ to the $\{\mathrm{FeNO}\}^{8}$ complex, with a rather short average $\mathrm{Fe}-\mathrm{C}^{\mathrm{NHC}}$ distance of $1.968 \AA$ in $\{\mathrm{FeNO}\}^{8}$ 19. The long $\mathrm{Fe}-\mathrm{C}^{\mathrm{NHC}}$ bonds in $\{\mathrm{FeNO}\}^{6} \mathbf{1 8}$ are connected to weak interaction with the carbene ligands, perfectly in line with the small isomer shift. Interestingly, this value is even more negative than the one observed for the oxoiron(IV) complex $\left[\left({ }^{\mathrm{NHC}} \mathrm{L}\right) \mathrm{Fe}^{\mathrm{IV}}=\mathrm{O}\right]^{2+}\left(\delta=-0.13 \mathrm{~mm} \mathrm{~s}^{-1}\right)^{[153]}$ indicative of an oxidized iron center. This agrees with the mechanistic idea behind the formation of $\left[\left({ }^{\mathrm{NHC}} \mathrm{L}\right)\{\mathrm{FeNO}\}^{6}(\mathrm{ONO})\right]^{2+}$ in which $\mathbf{1 8}$ is expected to be generated by reaction of a $\mathrm{NO}_{2}$ radical with the $\{\mathrm{FeNO}\}^{7}$ species 17 . While the radical is clearly reduced to $\mathrm{NO}_{2}{ }^{-}$, the oxidation occurs largely metal centered. Moreover, $\mathbf{1 8}$ shows a very large quadrupole splitting $\Delta E_{\mathrm{Q}}=3.12 \mathrm{~mm} \mathrm{~s}^{-1}$. As already discussed, a longer $\mathrm{Fe}-\mathrm{C}$ bond is found in the molecular structure of $\mathbf{1 8}$ while at the same time the Fe center shows a smaller displacement from the $\{\mathrm{C} 4\}$ plane. This still allows for extensive charge donation into the $\mathrm{Fe} 4 \mathrm{~s}$ and $3 \mathrm{~d}_{x^{2}-y^{2}}$ orbitals, giving an oblate charge distribution around the Fe nucleus. ${ }^{[22,23]}$

\subsubsection{X-ray Emission Spectroscopy}

In collaboration with the group of Serena DeBeer, the complexes 17-19 were further characterized by $\mathrm{K} \beta \mathrm{X}$-ray emission spectroscopy (XES). Additionally, the spectrum for the iron(II) precursor 1 was recorded, which allowed to distinguish between NO- and tetracarbene-related features. Within this work, only the main results of the XES analysis are presented, a detailed description is given in a joint publication. ${ }^{[23]}$

$\mathrm{K} \beta \mathrm{X}$-ray emission spectra consist of a $\mathrm{K} \beta$ mainline region and a valence-to-core $(\mathrm{VtC})$ region. Signals within the mainline region correspond to $\mathrm{Fe} 3 \mathrm{p} \rightarrow 1 \mathrm{~s}$ transitions and are a sensitive probe of metal-centered spin density. ${ }^{[238]}$ As shown in Appendix Figure 16, no low-energy shoulders (K $\beta$, features) are observed in the mainlines of 17-19 which is consistent with a low spin state in all of the complexes. X-ray emission spectra are dominated by $\sigma$-interactions between the metal center and the coordinated ligands, since only these transitions are of appreciable intensity. ${ }^{[163,239]}$ The transitions of interest in terms of iron nitrosyl compounds are observed between the iron d-electrons and suitable NO orbitals. Due to the linear coordination mode for the NO ligand in compounds 17-19, optimal interaction with the iron center is expected for the $2 \mathrm{~s}_{\sigma^{*}}$ and the $2 \mathrm{p}_{\sigma} \mathrm{NO}$ orbitals. Both interactions are anticipated to be of relatively high energy within the valence-to-core $(\mathrm{VtC})$ region that is depicted in Figure 68, top. ${ }^{[23]}$ 


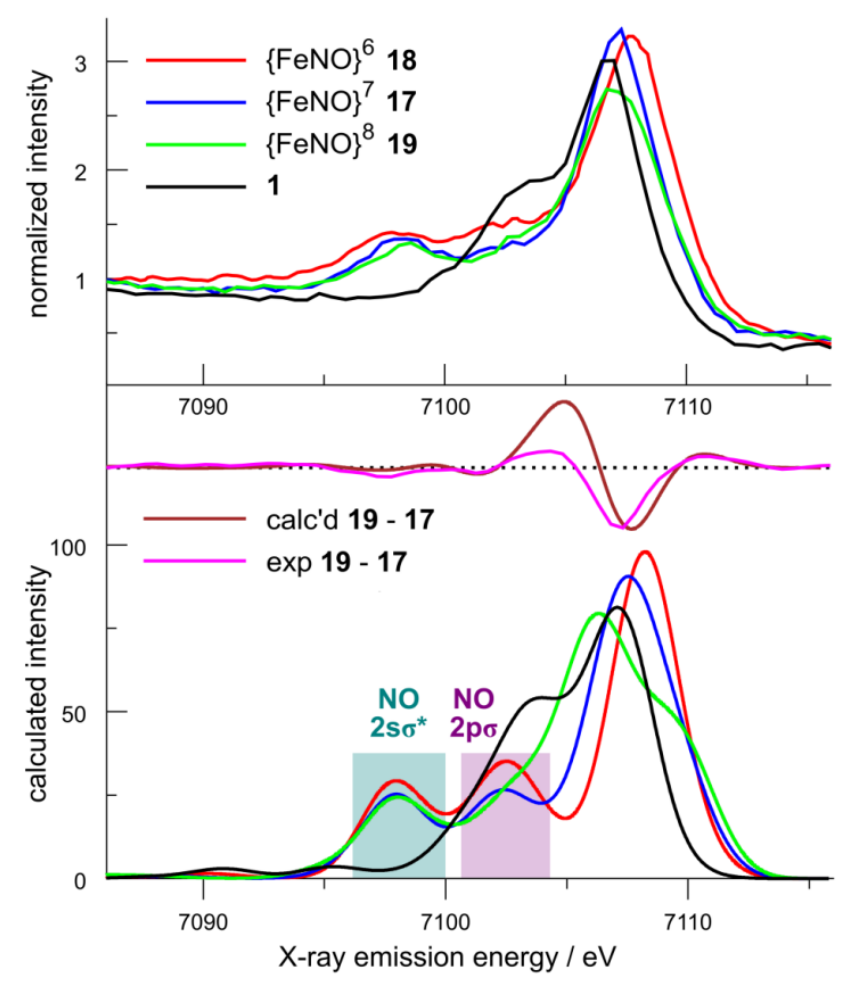

Figure 68. Top: Experimental (top) and DFT calculated (bottom) Fe K $\beta$ VtC XES spectra of 17-19 and the iron(II) precursor 1 . The $\mathrm{K} \beta$ " feature at $\sim 7098 \mathrm{eV}$ is assigned as transitions from $\mathrm{NO} 2 \mathrm{~s}_{\sigma^{*}}$, and the difference $\left(19\right.$ - 17) highlights the spectral changes upon reduction of the $\{\mathrm{FeNO}\}^{7}$ species. Reprinted with permission from ACS. ${ }^{[23]}$

The experimental spectra of the $\{\mathrm{FeNO}\}^{n}$ compounds 17-19 show an intense $\mathrm{K} \beta_{2,5}$ peak in the 7106-7108 eV region that can be attributed to the tetracarbene macrocycle for two reasons: Based on the strong $\sigma$-donating ability of the ligand, an intense signal in the $\mathrm{VtC}$ region is expected. Furthermore, a similar feature is also present in the spectrum of the NO-free iron(II) precursor 1. The spectrum of $\mathbf{1}$ additionally has a pronounced low-energy shoulder, likely from the lone pairs of the axial MeCN donors. The spectra of the three $\{\mathrm{FeNO}\}^{n}$ species exhibit a much weaker lowenergy $\mathrm{K} \beta_{2,5}$ shoulder, as well as a $\mathrm{K} \beta$ " peak at $\sim 7098 \mathrm{eV}$, which is absent in the precursor spectrum. These peaks are assigned to interactions with the NO ligand. According to a recent study by Liaw and co-workers, shifts in the energy between the NO $2 \mathrm{~s}_{\sigma^{*-}}$ and the $2 \mathrm{p}_{\sigma^{-}}$-derived features indicate changes in the oxidation state of the NO ligand. ${ }^{[240]}$ Among the series studied herein, only negligible changes in the energy difference between these features are observed indicative of metal centered redox processes. ${ }^{[23]}$

Furthermore, The VtC features of the $\{\mathrm{FeNO}\}^{7}$ species are found to be more intense than the one of reduced compound 19. This is in line with the increased bending of the $\mathrm{Fe}-\mathrm{N}-\mathrm{O}$ angle in the $\{\mathrm{FeNO}\}^{8}$ species and thus decreased $\sigma$-overlap between metal and ligand orbitals. Moreover, it agrees well with a metal-centered reduction since this is expected to decrease iron $\mathrm{p}-\mathrm{d}$ mixing that leads to weaker electric dipole transitions. ${ }^{[241]}$ The spectral features of the $\{\mathrm{FeNO}\}^{6}$ species $\mathbf{1 8}$ are 
highest in intensity due to the additional $\sigma$-donating O-nitrito ligand. Transitions arising from the ONO molecular orbitals are found in the same energy range and are thus experimentally indistinguishable. $^{[23]}$

Using a one-electron DFT approach, ${ }^{[242]}$ the VtC XES spectra for 17-19 were calculated computationally as it is shown in Figure 68, bottom. The calculated spectra agree very well with the experimentally obtained data, in particular also the differences in intensity between $\mathbf{1 7}$ and $\mathbf{1 9}$ show good agreements. A deconvolution and assignment of the calculated spectral features as well as a fragment analysis has been performed and is shown in Figure 69. ${ }^{[23]}$

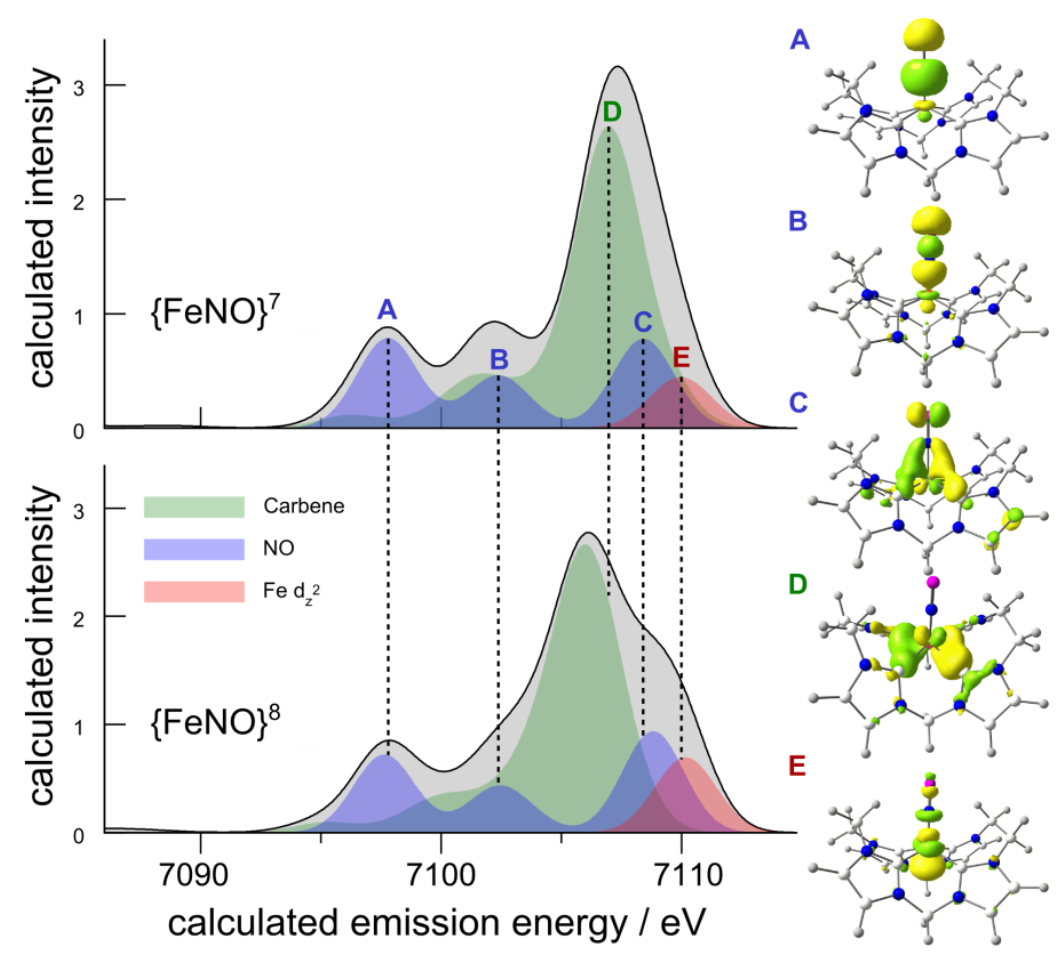

Figure 69. Left: Fragment analysis and spectral deconvolutions of the DFT-calculated VtC XES spectra of 17 (top) and 19 (bottom), showing the contributions to key spectral features. Right: Donor molecular orbitals of 17 corresponding to the most intense transitions at the indicated energies. Importantly, the approximate doubling of the highest-energy $\mathrm{d}_{\mathrm{z}^{2}}$ feature upon reduction is due to a second transition of the spin-down electron, indicating a metal-centered reduction. Reprinted with permission from ACS. ${ }^{[23]}$

As shown in Figure 69, the $\mathrm{K} \beta$ " and $\mathrm{K} \beta_{2,5}$ shoulders (A and $\mathbf{B}$ ) indeed correspond to the NO $2 \mathrm{~s}_{\sigma^{*}}$ and the $2 \mathrm{p}_{\sigma}$ molecular orbitals. As expected, the large $\mathrm{K} \beta_{2,5}$ peak in the $\sim 7106-7108 \mathrm{eV}$ region (D) is dominanted by iron-carbene interactions. However, its high energy shoulder at $\sim 7111 \mathrm{eV}$ (E) corresponds to a $\mathrm{Fe}\left(\mathrm{d}_{\mathrm{z}^{2}}\right) \rightarrow \mathrm{NO}$ interaction. Having a closer look at the experimental and calculated difference spectra, the $\{\mathrm{FeNO}\}^{8}$ species is more intense than the $\{\mathrm{FeNO}\}^{7}$ compound in this region. This increase in intensity upon reduction to $\mathbf{1 9}$ is in perfect agreement with higher electron density in the $\mathrm{Fe}\left(\mathrm{d}_{\mathrm{z}^{2}}\right)$ orbital as result of a metal-centered reduction. ${ }^{[23]}$ 
In conclusion, XES measurements provide strong evidence for a metal-centered reduction process. The $\mathrm{K} \beta$ " and $\mathrm{K} \beta_{2,5}$ features could be characterized as mainly $\mathrm{NO} 2 \mathrm{~s}_{\sigma^{*}}$ and $2 \mathrm{p}_{\sigma}$ in nature, respectively. Among the complete series, their energies remain largely the same which is indicative of a similar oxidation state of the NO ligands in all $\{\mathrm{FeNO}\}^{n}$ species. Furthermore, a shoulder at $\sim 7111 \mathrm{eV}$ that largely corresponds to an iron $\mathrm{d}_{\mathrm{z}^{2}}$-based transition is found to be increased in intensity in case of the reduced species. This finding also agrees well with higher electron density at the reduced iron center. To further investigate the electronic structures of the newly synthesized $\{\mathrm{FeNO}\}^{n}$ compounds, extensive DFT calculations have been performed. Their results are presented within the next section.

\subsubsection{Density Functional Calculations}

In order to provide an in depth understanding of the electronic structures of the $\{\mathrm{FeNO}\}^{n}$ compounds 17-19, unrestricted DFT calculations (ORCA, BP86 functional, RI approximation, def2-tzvp and def2-tzvp/j basis sets; see chapter 12 for details) have been performed. As shown in Table 9, the structure optimizations of all three complexes reproduce well the key structural parameters that have been determined crystallographically. ${ }^{[22,23]}$

Furthermore, the calculated Mössbauer parameters (Table 11) agree reasonably well with the experimentally obtained values, supporting the suitability of the DFT model. In particular, the DFT calculations are in good agreement with the negligible changes observed in the IS upon reduction from 17 to 19. Even though DFT slightly overestimates the experimentally observed trend, it gives strong support for dependency of the IS on the Fe-ligand bond lengths rather than the oxidation state. The minor discrepancies between the calculated and experimental MB parameters are within the expected error range (compare DFT Figure 14). ${ }^{[243]}$ In conclusion, experiment as well as DFT clearly evidence that the IS of $\{\mathrm{FeNO}\}^{7} \mathbf{1 7}$ and $\{\mathrm{FeNO}\}^{8} 19$ are essentially identical whereas the QS shows distinct changes upon reduction. ${ }^{[22,23]}$

Vibrational features in the IR region and UV/vis/NIR transitions could also be calculated with adequate agreement between theory and experiment (see chapter 12 for further details). Especially the high intensity of the $\mathrm{C}^{\mathrm{NHC}} \rightarrow \mathrm{Fe}$ LMCT transition at $360 \mathrm{~nm}$ for complex 19 and the lack of any $\mathrm{NO} \rightarrow \mathrm{Fe}$ transition for $\mathbf{1 8}$ are confirmed by the TD-DFT calculations. In case of the $\{\mathrm{FeNO}\}^{7}$ and $\{\mathrm{FeNO}\}^{8}$ species, the $\tilde{v}_{\mathrm{NO}}$ stretching frequencies resulting from the structural optimization overestimate the actual energy. Therefore, the calculation was optimized in order to obtain analytical frequencies in higher accordance with the experimentally obtained values (for details see chapter 12). ${ }^{[22,23]}$ 
The calculated MO schemes of the $\{\mathrm{FeNO}\}^{7}$ species $\mathbf{1 7}$ and $\{\mathrm{FeNO}\}^{8}$ compound $\mathbf{1 9}$ are depicted in Figure 70 (right). Comparison reveals that the orbital interactions within the valence electrons undergo only small changes upon reduction as indicated by the same energetic order of the molecular orbitals.

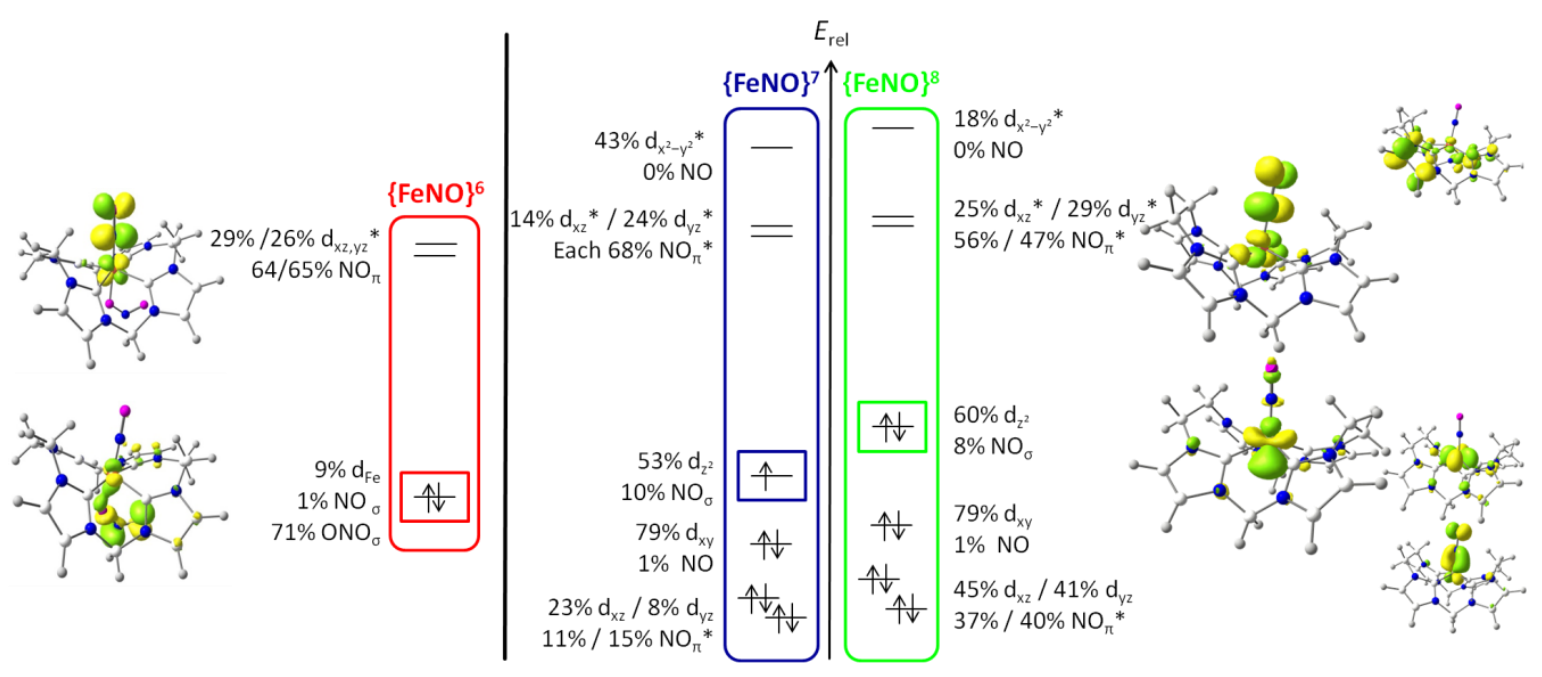

Figure 70. Left: DFT-calculated HOMO and LUMO of complex 18. Right: DFT-calculated MO scheme (BP86, def2-tzvp) according to $E_{\text {rel }}$ of $\{\mathrm{FeNO}\}^{7} \mathbf{1 7}$ (left) and $\{\mathrm{FeNO}\}^{8} \mathbf{1 9}$ (right). Values are given for the majority $(\alpha)$ spin orbitals with their dominating iron d-orbital contributions and DFT calculated molecular frontier orbitals of $\mathbf{1 9}$ are depicted on the right side (Counter value 0.06). Reprinted with permission from ACS. ${ }^{[23]}$

According to the MO scheme, the Fe-NO bond is based on two interactions. First, a $\sigma$-donation of the $\mathrm{NO}_{\sigma^{*}}$ orbitals into the $\mathrm{d}_{\mathrm{z}^{2}}$ of the iron center and, second, a weak $\pi$-backbonding of the occupied $\mathrm{Fe}\left(\mathrm{d}_{\mathrm{xz}} / \mathrm{d}_{\mathrm{yz}}\right)$ orbitals into the $\mathrm{NO}_{\pi^{*}}$. In detail, the $\sigma$-donation results in antibonding orbitals that show predominantly Fe character (17: 53\% Fe, 10\% NO, 19: 60\% Fe, $8 \%$ NO). In case of the $\{\mathrm{FeNO}\}^{7}$ species, this orbital is singly occupied and represents the SOMO. Furthermore, a plot of the calculated electron spin density of $\mathbf{1 7}$ reflects that the $\alpha$-spin is largely located at the central iron (DFT Figure 15). Upon reduction, a $\beta$-spin electron is added into this $\mathrm{NO}_{\sigma^{*}} \rightarrow \mathrm{d}_{\mathrm{z}^{2}}$ interaction, resulting in a HOMO with even higher iron character. This finding is in perfect agreement with a metal centered reduction and contrasts earlier reports on low spin $\{\mathrm{FeNO}\}^{7 / 8}$ species for which a SOMO/HOMO with mainly NO character and a largely ligand centered reduction was reported. ${ }^{[123,125]} \mathrm{A}$ detailed analysis of the nature of iron contribution in this $\sigma$-interaction provides a theoretical picture for the observed linearity of the $\{$ FeNO $\}$ moieties. DFT studies by Conradie and Ghosh have identified metal $\mathrm{d}_{\mathrm{z}^{2}} / \mathrm{p}_{\mathrm{z}}$ mixing, which is enhanced upon pyramidalization in squarepyramidal $\{\mathrm{FeNO}\}^{7}$ complexes, as a key factor that allows for linearity of the $\mathrm{Fe}-\mathrm{N}-\mathrm{O}$ unit because it minimizes repulsion between the $\mathrm{Fe}\left(\mathrm{d}_{\mathrm{z}^{2}}\right)$ orbital and the $\sigma$ lone pair of $\mathrm{NO} \cdot{ }^{[136,137]}$ The present experimental and DFT results on $\mathbf{1 7}$ and $\mathbf{1 9}$ are in accordance with that picture. As reflected by its shape, the SOMO of the $\{\mathrm{FeNO}\}^{7}$ species 17 indeed shows significant admixture of $\mathrm{Fe}\left(\mathrm{p}_{\mathrm{z}}\right)$ 
character ( $8 \%$ based on Mulliken, $13 \%$ based on Loewdin spin population analysis per MO, see DFT Table 6) which is likely related to the pronounced pyramidalization at Fe that results from the rather acute $\mathrm{C}-\mathrm{Fe}-\mathrm{C}$ angles. ${ }^{[22,23]}$

The $\mathrm{Fe}\left(\mathrm{d}_{\mathrm{xz}} / \mathrm{d}_{\mathrm{yz}}\right) \rightarrow \mathrm{NO}_{\pi^{*}} \pi$-backbonding interaction in $\{\mathrm{FeNO}\}^{7} \mathbf{1 7}$ has only slight NO character ( $\mathrm{d}_{\mathrm{xz}}$-interaction: $11 \% \mathrm{NO}, \mathrm{d}_{\mathrm{xz}}$-interaction: $15 \% \mathrm{NO}$ ), while the NO character for the same interaction in 19 is significantly increased (37\% and 40\%). This finding is in perfect agreement with largely enhanced backbonding ability of the reduced $\{\mathrm{FeNO}\}^{8}$ complex. The corresponding antibonding interaction of the $\mathrm{d}_{\mathrm{xz}}$ orbital with the $\mathrm{NO}_{\pi^{*}}$ orbital represents the LUMO in both $\{\mathrm{FeNO}\}^{n}$ compounds. The analogous interaction in the yz-plane is again very close in energy. When going from $\mathbf{1 7}$ to $\mathbf{1 9}$, the contrary effect than for the bonding interaction is observed: a very high NO character $(68 \%)$ in the $\{\mathrm{FeNO}\}^{7}$ species and a significantly lower NO character in the $\{\mathrm{FeNO}\}^{8}$ species ( $\mathrm{d}_{\mathrm{xz}}$-interaction: $56 \% \mathrm{NO}, \mathrm{d}_{\mathrm{xz}}$-interaction: $\left.47 \% \mathrm{NO}\right)$. Due to the extreme in-plane $\sigma$-donor strength of the tetracarbene macrocycle, the $\mathrm{Fe}\left(\mathrm{d}_{\mathrm{x}^{2}-\mathrm{y}^{2}}\right)$ orbital is markedly destabilized and raised high in energy. ${ }^{[22,23]}$

Taken together, the DFT results on the electronic structure of $\mathbf{1 7}$ and the reduced species $\mathbf{1 9}$ are in perfect agreement with a metal centered reduction. Furthermore, they strongly support the proposed enhanced backbonding character of the reduced iron center. According to the spin density calculations and the high iron character of the SOMO in the $\{\mathrm{FeNO}\}^{7}$ species, a considerable

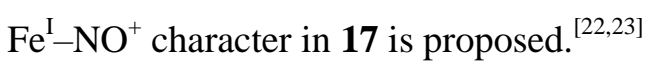

A complete MO scheme similar to the one for $\mathbf{1 7}$ and $\mathbf{1 9}$ could not be prepared for the oxidized $\{\mathrm{FeNO}\}^{6}$ species 18 since it was not possible to assign the calculated interactions to specific $\mathrm{Fe}(\mathrm{d})$ orbitals in a similar unambiguous manner. Nonetheless, the calculated energetic order of $\alpha$ and $\beta$ orbitals is shown in Chapter 12 (DFT Figure 17) while only the HOMO and LUMO of the $\{\mathrm{FeNO}\}^{6}$ species is shown in Figure 70 (left). Interestingly, the LUMO in $\{\mathrm{FeNO}\}^{6} \mathbf{1 8}$ largely resembles the ones observed for $\mathbf{1 7}$ and 19. This result is in agreement with a similar $\pi$-interaction between the iron center and the NO ligand in all three species. In contrast, the HOMO in $\mathbf{1 8}$ looks fundametally different. While it showed mainly iron character in the $\{\mathrm{FeNO}\}^{7}$ species, only minor contribution of $\mathrm{Fe}(\mathrm{d})$ orbitals $(9 \%)$ is found within the HOMO of the corresponding oxidized species. Instead, the HOMO represents a strong $\sigma$-bonding interaction with the O-bound nitrito ligand with largely ONO character (71\%). This observation nicely supports the mechanistic idea presented herein: The ${ }^{\mathrm{NO}} \mathrm{O}_{2}$ radical induces a largely metal centered oxidation when coordinating at the $\{\mathrm{FeNO}\}^{7}$ center by removal of the unpaired electron from the SOMO. Thus, the electron density is shifted to the nitrite $\left(\mathrm{ONO}^{-}\right)$ligand. Consequently, the iron $\mathrm{d}_{\mathrm{z}^{2}}$ orbital, which is left unoccupied after this removal, can no longer participate in a $\sigma$-bonding interaction between the iron center and the NO ligand. This finding can also give an explanation for the missing HOMO-LUMO 
transition in UV/vis/NIR compared to $\mathbf{1 7}$ and $\mathbf{1 9}$ since the character of the HOMO is completely changed upon oxidation. Furthermore, the DFT calculations reveal that a simple reduction of $\mathbf{1 8}$ is not favored, due to the antibonding character of the LUMO. Instead, addition of an electron is followed by a chemical reaction, in particular release of the nitrito ligand and regeneration of the square pyramidal coordinated $\{\mathrm{FeNO}\}^{7}$ species (EC mechanism) to lower the energy of the electron-accepting $\mathrm{d}_{\mathrm{z}^{2}}$ orbital. ${ }^{[22,23]}$

In conclusion, the DFT calculations provide strong evidence for metal centered redox processes within this series of tetracarbene coordinated low spin $\{\mathrm{FeNO}\}^{n}$ compounds. As presented within the introduction, studies on heme and non-heme iron-nitroxyl compounds reported in the literature prior to this work, led to the conclusion that metal centered reduction is a general feature of nonheme high spin species while low spin complexes show ligand centered reduction. ${ }^{[122]}$ The organometallic $\{\mathrm{FeNO}\}^{n}$ species presented within this work do not agree with this picture. The non-heme model compounds 17-19 are all low spin in nature and show metal centered redox processes, a combination that has not been reported in the literature, so far. Consequently, this result provides motivation to characterize the reactivity of the newly synthesized $\{\mathrm{FeNO}\}^{8}$ compound with a reduced iron center. 


\subsection{Reactivity Studies on a $\{\text { FeNO }\}^{8}$ Complex}

The protonation of $\{\mathrm{FeNO}\}^{8}$ species is of current interest in literature since the resulting $\{\mathrm{FeNHO}\}^{8}$ compounds are proposed intermediates in enzymatic processes. ${ }^{[34,35]}$ Furthermore, it is challenging to distinguish between $\mathrm{NO}^{-}$and its protonated form (NHO) in biological systems, and model compounds are thus highly desired. So far, studies on the protonation of $\{\mathrm{FeNO}\}^{8}$ compounds in most cases have led to highly unstable $\{\mathrm{FeNHO}\}^{8}$ intermediates that release $\mathrm{H}_{2}$ concomitant with formation of the corresponding $\{\mathrm{FeNO}\}^{7}$ species. ${ }^{[119-121,124]}$ Protonation of the $\mathrm{N}$-atom of the NO ligand in these systems is rationalized by ligand centered reduction processes and the basic nature of the resulting $\mathrm{NO}^{-}$ligand. As a consequence, protonation at the nitrogen atom in the newly synthesized $\left.\left[{ }^{\mathrm{NHC}} \mathrm{L}\right)\{\mathrm{FeNO}\}^{8}\right]^{+}$compound 19 is unlikely since high electron density is found at the iron center rather than the NO ligand. Accordingly, treatment of $\mathbf{1 9}$ with the weak acids 1,5-bis(dimethyl)-piperidinium $\left(\mathrm{p} K_{\mathrm{a}} \geq 19 \text {, value for piperidinium in } \mathrm{MeCN}\right)^{[225]}$ or 1,5-dimethylpyrdinium $\left(\mathrm{p} K_{\mathrm{a}}=14.1 \text { in } \mathrm{MeCN}\right)^{[214]}$ under strictly anaerobic conditions in a stoppedflow experiment or in a glove box led to no reaction. ${ }^{[23]}$ Thus, an important consequence of the metal centered redox processes in the $\{\mathrm{FeNO}\}^{n}$ species described herein for their reactivity is found, which contrasts the ligand centered reductions usually described in literature. ${ }^{[23,124,244]}$

Interestingly, when 1,5-bis(dimethyl)-piperidinium was added in small portions to a solution of $\mathbf{1 9}$ and the titration was followed in the UV/vis, clean formation of $\mathbf{1 7}$ was observed (Appendix Figure 22). Further investigation of this process revealed that trace amounts of dioxygen induced this reactivity. In particular, addition of oxygen to an acidic solution (1 eq. of 1,5-bis(dimethyl)piperidinium) of $\mathbf{1 9}$ induces the same reaction, indicated by an immediate color change from green to blue. When monitored in the UV/vis spectrometer, very fast decrease of the characteristic band of $\mathbf{1 9}$ at $\lambda_{\max }=360 \mathrm{~nm}$ was observed, leading to a spectrum that resembles the typical features of $\mathbf{1 7}$ (Figure 71, left). Subsequent IR measurements demonstrated full conversion to the oxidized $\{\mathrm{FeNO}\}^{7}$ species. When dioxygen was instead added to a pure solution of the $\{\mathrm{FeNO}\}^{8}$ species in $\mathrm{MeCN}$, a different reaction was observed (Figure 71, right). In the absence of $\mathrm{H}^{+}$, addition of $\mathrm{O}_{2}$ was followed by a significantly slower process with three different isosbestic points. According to IR, NMR, and ESI-MS studies, the resulting brown suspension contained only small amounts of the $\{\mathrm{FeNO}\}^{7}$ compound but mainly decomposition products of the type $\left({ }^{\mathrm{NHC}} \mathrm{LH}_{2}\right)\left(\mathrm{NH}_{\mathrm{y}} \mathrm{O}_{\mathrm{x}}\right)_{2}$ and $\mathrm{Fe}_{\mathrm{x}} \mathrm{O}_{\mathrm{y}}$. In particular, an ESI mass spectrum of the decomposed $\{\mathrm{FeNO}\}^{8}$ mixture shows signals corresponding to the protonated ligand system with $\mathrm{NO}$ and $\mathrm{NO}_{2}$ but no isotopic pattern typical for iron. According to NMR data, one main species is formed in which two of the carbenes are reprotonated ( $\mathrm{NCHN} 136.5 \mathrm{ppm}$ ) while the other two presumably bind to the $\mathrm{NO}_{\mathrm{x}}$ group indicated by a downfield shifted carbon signal at $152.1 \mathrm{ppm}$ (Appendix Figure $17 \mathrm{ff}$.). This proposal is in agreement with signals in the IR spectrum $\left(1665\right.$ and $\left.1702 \mathrm{~cm}^{-1}\right)$ in the range of organic nitrites. 
Furthermore, a lot of insoluble material is formed, assigned to not further characterized ironoxide. $^{[23]}$
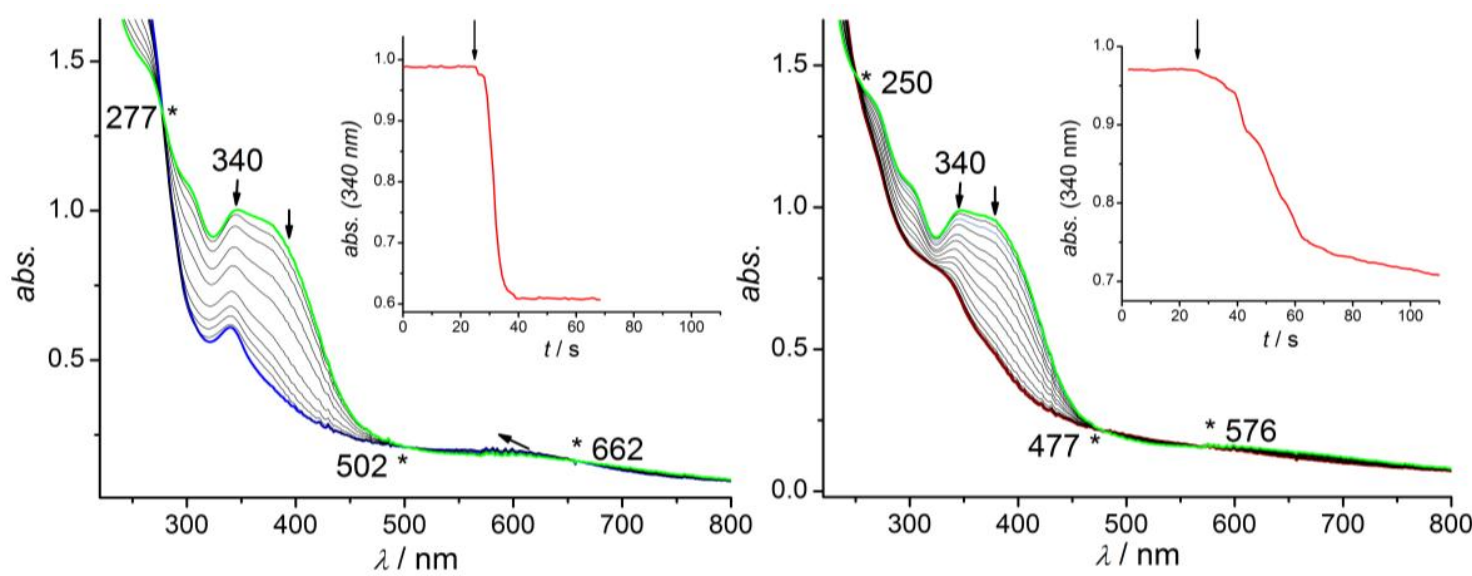

Figure 71. Left: UV/vis spectral changes during the reaction of a mixture of $19(0.14 \mathrm{mM}, \mathrm{MeCN})$ and 1 eq. of 1,5-bis(dimethyl)-piperidinium with excess of dioxygen at RT. Right: UV/vis spectral changes during the reaction of $19(0.14 \mathrm{mM}, \mathrm{MeCN})$ with excess of oxygen at $\mathrm{RT}$ in the absence of protons. The inset in both spectra shows the kinetic trace of the absorbance at $340 \mathrm{~nm}$; addition of dioxygen is marked with an arrow. ${ }^{[23]}$

In conclusion, oxidation of the $\{\mathrm{FeNO}\}^{8}$ species 19 with $\mathrm{O}_{2}$ in the presence of protons results in clean formation of $\{\mathrm{FeNO}\}^{7}$ precursor $\mathbf{1 7}$ and suppression of subsequent decomposition processes. Only very slowly, $\mathbf{1 7}$ is decomposing, most likely due to generated water. So far, the obtained data are indicative of the presence of a proton coupled electron transfer process (PCET) in which dioxygen oxidizes the $\{\mathrm{FeNO}\}^{8}$ compound and the resulting (su)peroxide species react further with protons instead for the iron compex. To further clarify the mechanism, it should be attempted to detect possible intermediates and products such as hydrogen peroxide or water in the reaction mixture. While it could be already clarified that 1 eq. of the acid is sufficient for full conversion, it is so far not clear how much dioxygen is needed. Still, it can be concluded that the $\{\mathrm{FeNO}\}^{8}$ compound can be reoxidized to the corresponding $\{\mathrm{FeNO}\}^{7}$ complex in a mechanism different to the stepwise fashion, namely initial protonation of the NO moiety followed by an intermolecular redox process, that has previously been described for heme and non-heme model systems. Taken together, the metal centered reduction when going from the $\{\mathrm{FeNO}\}^{7}$ species $\mathbf{1 7}$ to $\mathbf{1 9}$ shows direct influence on the reactivity of the resulting $\{\mathrm{FeNO}\}^{8}$ compound. $^{[23]}$ 


\section{Development of New Hybride Ligand Systems}

\subsection{Aim of this Project}

The organometallic iron compounds coordinated by the macrocyclic tetracarbene ligand reported prior to this work ${ }^{[153-156]}$ and as part of this work, ${ }^{[22,23,170]}$ provide fascinating features when compared to typical $\mathrm{N}$-coordinated model systems. For example, the unusually strong $\sigma$-donating ability of the equatorial ligand scaffold raises the $d_{x^{2}-y^{2}}$ orbital in energy resulting in a triplet-only mechanistic pathway for $\mathrm{C}-\mathrm{H}$ activation (compare chapters 3 and 4). As shown in the previous chapter, conversions within a series of low spin $\{\mathrm{FeNO}\}^{6-8}$ compounds occur largely metal centered in huge contrast to conclusions derived from the work on heme and non-heme model compounds with $\mathrm{N}$-donor ligands prior to this work. Thus, a better understanding of the influence of the carbene donors compared to nitrogen donor atoms is desired.

In this regard, it is aimed at developing new iron complexes coordinated by hybrid ligands providing both carbene and nitrogen donors. These hybrid ligand systems are expected to lead to properties intermediate between nitrogen-only and carbene-only coordinated iron centers. It would be of high interest to explore if, for example, a $2 \mathrm{~N} 2 \mathrm{C}$ donor system results in ligand centered redox processes within the corresponding $\{\mathrm{FeNO}\}$ species. A suitable starting point for this project is

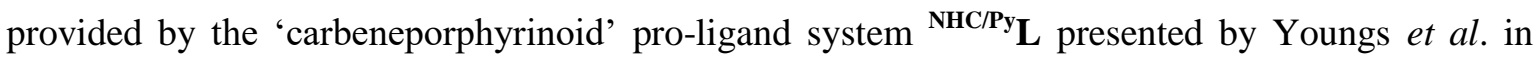
which two imidazolium rings are linked with two pyrrols via methylene bridges (Scheme 24). ${ }^{[25]}$ This macrocycle represents a perfect conjunction between the porphyrin system prevalent in nature and the organometallic tetracarbene ligand scaffold studied within this work. 


\subsection{A ‘Carbeneporphyrinoid’ Macrocycle for the Generation of Metal Complexes}

The hybrid ligand system ${ }^{\mathbf{N H C / P y}} \mathbf{L}$ could be successfully prepared according to literature described procedures $^{[245-248]}$ with an overall yield of $15 \%$ (chapter 11, Scheme 33). In order to allow for the four-fold coordination of the two carbene donors and the two nitrogen donors to the iron center, four protons need to be abstracted. Imidazolium groups $\left(\mathrm{Im}^{+} \mathrm{X}^{-}\right)$have been shown to interact with silver oxide yielding the corresponding silver carbene complexes as well as water and $\mathrm{AgX}$ without addition of an external base. ${ }^{[155,247,249,250]}$ In a subsequent transmetallation reaction, the obtained complexes can be transformed into their corresponding iron compounds. For that purpose, a $\mathrm{MeCN}$ solution of the imidazolium-pyrrole macrocycle was treated with 1.2 eq. of $\mathrm{AgO}_{2}$ at $55{ }^{\circ} \mathrm{C}$ in the absence of light and the reaction progress was followed by NMR measurements (Scheme 24). Full conversion was observed after two weeks. However, the signals detected in the resulting NMR spectrum are only slightly shifted compared to the spectrum recorded for 2,5-bis-(imidazolmethyl)pyrrole. Furthermore, an ESI-MS measurement of the product mixture showed signals corresponding to the decomposed macrocyclic ligand system coordinated to silver $(\mathrm{m} / \mathrm{z}=334$ : $\mathrm{C}_{12} \mathrm{H}_{12} \mathrm{~N}_{5} \mathrm{Ag}^{+}$and $\left.m / z=561:\left(\mathrm{C}_{12} \mathrm{H}_{13} \mathrm{~N}_{5}\right)_{2} \mathrm{Ag}^{+}\right)$while only very small signals could be assigned to the desired intact macrocyclic silver complex $\left(m / z=425.1: \mathrm{C}_{18} \mathrm{H}_{18} \mathrm{~N}_{6} \mathrm{Ag}^{+}\right)$. It can be concluded that this synthetic strategy leads to decomposition of the 'carbeneporphyrinoid' macrocycle ${ }^{{ }^{N H C / P y}} \mathbf{L}$ and does not provide the possibility of generating the intended bis-carbene-bis-pyrrole coordinated iron center. Thus, the reaction was not studied in further detail but instead alternative routes were examined.

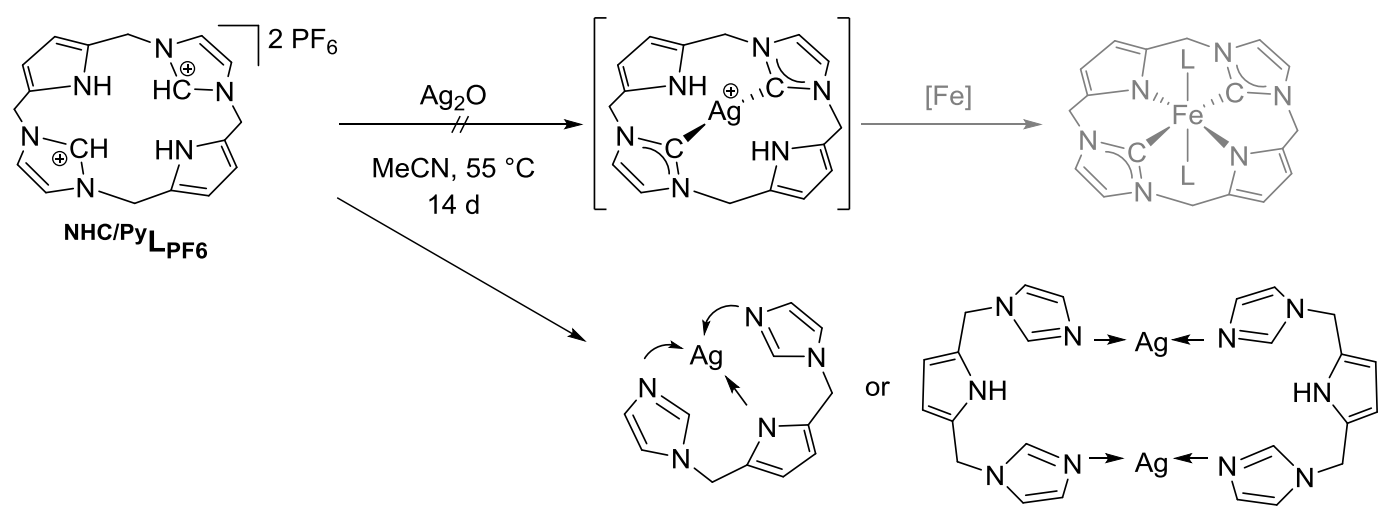

Scheme 24. Conversion of ${ }^{\mathrm{NHC} / \mathrm{Py}} \mathbf{L}$ with $\mathrm{Ag}_{2} \mathrm{O}$ resulting in decomposition of the macrocycle.

Subsequently, the generation of the desired iron complex was attempted via direct conversion of the ligand precursor with various bases and iron salts. While the use of $\left\{\mathrm{Fe}\left[\mathrm{N}(\mathrm{SiMe})_{2}\right]_{2}\right\}_{2}$ did lead to an inextricable mixture, promising results were obtained upon treatment of ${ }^{\mathrm{NHC} / \mathbf{P y}} \mathbf{L}$ with $\mathrm{KO}^{t} \mathrm{Bu}$ and different iron(II) salts $\left(\mathrm{FeBr}_{2}(\mathrm{THF})_{2},\left[\mathrm{Fe}(\mathrm{MeCN})_{6}\right]\left(\mathrm{BF}_{4}\right)_{2}, \mathrm{Fe}(\mathrm{OTf})_{2}\right.$; compare Scheme 25). Addition of a precooled solution of the base to a cold suspension of the ligand precursor in $\mathrm{MeCN}$ 
led to spontaneous formation of a bright yellow solution. After $15 \mathrm{~min}$, a solution of the iron salt in $\mathrm{MeCN}$ was added dropwise causing precipitation of a yellow solid. The precipitate turned out to be insoluble in agreement with the expected neutral charge of the desired complex but preventing any analysis in solution. Still, a MB spectrum at $80 \mathrm{~K}$ was recorded, indicative of the formation of a high spin iron(II) species $\left(\delta=1.01 \mathrm{~mm} \mathrm{~s}^{-1}, \Delta E_{\mathrm{Q}}=2.62 \mathrm{~mm} \mathrm{~s}^{-1}\right)$. This finding is in huge contrast to the vast number of low spin and the few examples of intermediate spin iron complexes coordinated by the tetracarbene macrocycle. If exposed to air, the yellow solid rapidly turns brown and the corresponding MB spectrum is typical for a low spin iron(III) complex $\left(\delta=0.46 \mathrm{~mm} \mathrm{~s}^{-1}\right.$, $\Delta E_{\mathrm{Q}}=0.74 \mathrm{~mm} \mathrm{~s}^{-1}$ ). A similar color change to brown was also observed when it was tried to dissolve the yellow compound in DMSO- $\mathrm{d}_{6}$ for NMR measurements or in various solvents for crystallization attempts.

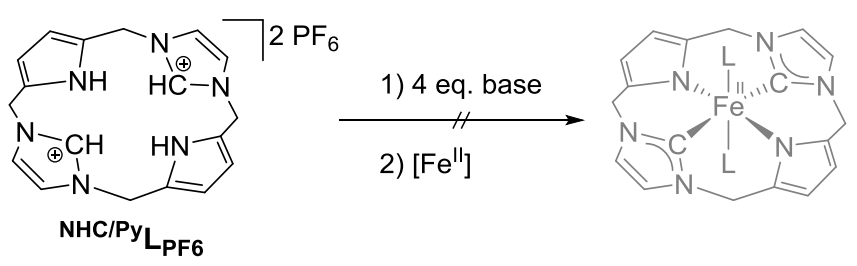

Scheme 25. Synthetic strategy for the preparation of iron complexes coordinated by ${ }^{\mathrm{NHC/Py}} \mathbf{L}$.

To circumvent the instability problems observed with the iron(II) product, the development of a suitable synthetic strategy by generating first the corresponding nickel(II) complex was attempted. Typically, nickel(II) ions in their $\mathrm{d}_{8}$ low spin state prefer square planar coordination and thus provide a suitable testing probe to discover if it is possible to obtain any metal complex with this ligand system. Therefore, a cold $\mathrm{MeCN}$ solution of ${ }^{\mathbf{N H C} /{ }^{\mathbf{y}}} \mathbf{L}$ was treated with $\mathrm{KO}^{t} \mathrm{Bu}$ before $\mathrm{NiBr}_{2}(\mathrm{dme})$ was added to the resulting yellow solution (Figure 72, left). The mixture was warmed to RT and stirred for two days leading to a red suspension. While both solution and solid showed rather similar NMR spectra, no isotopic pattern typical for nickel was found in mass spectrometry experiments of the solution. Crystallization of the solid upon diffusion of $\mathrm{Et}_{2} \mathrm{O}$ into a red DMSO solution gave rise to formation of complex 20 (Figure 72, right). As shown in the molecular structure, decomposition of the macrocyclic ligand was observed. However, the imidazolium groups do not coordinate at the nickel center but instead the released pyrrole ring couples with two $\mathrm{MeCN}$ molecules on each side leading to a $4 \mathrm{~N}$-coordination of the central nickel ion. Based on this crystal structure, it was possible to assign all the signals in the ${ }^{1} \mathrm{H}-\mathrm{NMR}$ spectrum of the crude product; thus, compound $\mathbf{2 0}$ found in the obtained crystal represents the major product species. 

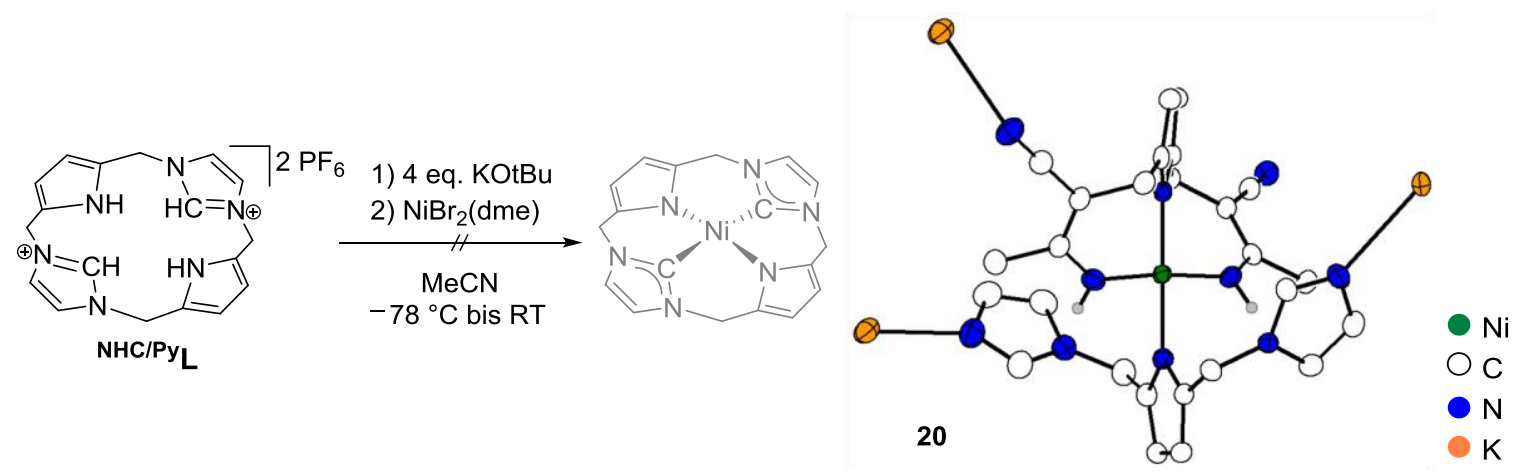

Figure 72. Left: Synthetic strategy for the generation of a $2 \mathrm{C} 2 \mathrm{~N}$-coordinated nickel(II) complex. Right: ORTEP plot of the molecular structure of the obtained product $\mathbf{2 0}$ (50\% probability thermal ellipsoids); hydrogen atoms except for $\mathrm{N} H$ are omitted for clarity.

In conclusion, decomposition of the ligand scaffold turned out to be a rather general pathway upon attempts to generate corresponding metal complexes from ${ }^{{ }^{\mathbf{H C}} \mathbf{P} \mathbf{P}} \mathbf{L}$. Two scenarios are conceivable: Firstly, the size of the macrocyclic ring could be too small for the chosen metal ions and coordination is only possible after breakage of the ligand. Secondly, already the deprotonation of

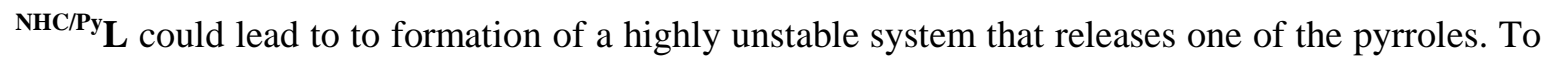
investigate the latter scenario, the hybrid ligand scaffold was treated with four equivalents of $\mathrm{KO}^{t} \mathrm{Bu}$ and a NMR spectrum of the reaction mixture was recorded. The spectrum consisted in four signals in a ratio of 1:1:1:2 that correspond to two different protons in the imidazol backbone $\left(\delta=7.04\right.$ and $6.79 \mathrm{ppm}, \int 2 \mathrm{H}$ each), one signal for the protons in the pyrrole backbone $(\delta=5.79 \mathrm{ppm}, \sqrt{2} \mathrm{H})$, and a signal for the protons of the methylene bridges $\left(\delta=4.92 \mathrm{ppm}, \int 4 \mathrm{H}\right)$. These spectral properties are in good agreement with successful abstraction of the protons at the pyrrole $\mathrm{NH}$ position as well as at the imidazolium $\mathrm{N}=\mathrm{CH}-\mathrm{N}$ position. However, the intact deprotonated macrocycle would give only three signals in the ratio 1:1:2 while the observed signal set is consistent with a three times deprotonated 2,5-bis-(imidazolmethyl)-pyrrole fragment. Thus, deprotonation with $\mathrm{KO}^{t} \mathrm{Bu}$ already leads to decomposition of the macrocycle. The same result was observed using $\mathrm{Cs}_{2} \mathrm{CO}_{3}$ or a $\mathrm{Fe}(\mathrm{OAc})_{2} /$ pyridine mixture as alternative basic systems.

Taken together, it was not possible to obtain a metal complex coordinated by the 'carbeneporphyrinoid' ligand scaffold ${ }^{\mathbf{N H C / P y}} \mathbf{L}$. The deprotonated macrocycle turned out to be highly unstable and showed decomposition to 2,5-bis-(imidazolmethyl)-pyrrole in all experimental conversions. 


\subsection{Modification of the Ligand Scaffold}

An idea to enable the successful synthesis of a $2 \mathrm{C} 2 \mathrm{~N}$-coordinated iron complex was to modify the solubility of the species by using additional groups in the pyrrole and imidazole backbones. Such alkyl- and aryl-groups furthermore enable adjustment of the acidity of the protons in the donor positions and presumably increase the stability of the deprotonated macrocycle. However, using a similar synthetic strategy as for the unmodified ${ }^{\mathbf{N H C} / \mathbf{P}_{\mathbf{y}}} \mathbf{L}$ system as well as changes regarding solvent, base, reaction time or temperature, it was not possible to obtain any positive results with 4,5-dimethyl- and 4,5-diphenyl-imidazole, as well as 3,4-diethylpyrrole.

The ring size, which is connected to the type of bridging units, also shows direct influence on the stability of macrocyclic systems. In particular, the tetracarbene ligand scaffold used in this work shows significant differences with respect to its stability and properties if compared to a smaller analogue bridged by methylene groups only (Chapter 1, Scheme 10). ${ }^{[160]}$ However, no 2,5bis(ethyl)-pyrroles with suitable leaving groups at the ethylene-substituents are found in the literature. Instead, the synthesis of a benzotriazole system with $-\mathrm{CH}_{2} \mathrm{CH}_{2} \mathrm{Br}$ groups at $\mathrm{N} 1$ and $\mathrm{N} 3$ is described by Gandelman et al. and provides a starting point for the generation of a bisbenzotriazole-bis-imidazole macrocycle (Scheme 26, left). ${ }^{[251]}$ 1,3-Di-(2'-bromoethyl)benzotriazolium bromide was successfully synthesized according to the literature described strategy and several attempts were made to replace the bromo leaving group by an imidazole. However, treatment of 1,3-di-(2'-bromoethyl)-benzotriazolium bromide with potassium imidazol1-ide under both atmospheric and inert conditions did not lead to any conversion to the desired product.

Triazole systems can be interpreted as nitrogen-derived analogues of N-heterocyclic carbenes. ${ }^{[251]}$ The nitrenium ion shows donating properties that differ from both the C-donor in a NHC and pyrrole-derived nitrogen donors. Thus, not only a macrocyclic ligand with benzotriazole in combination with imidazole but also in combination with the pyrrole was of interest. For that reason, excess of benzotriazole was deprotonated with $\mathrm{KOH}$ in DCM and 2,5-bis(trimethylaminomethyl)-pyrrole diiodide was added (Scheme 26, right). Removal of the solvent and washing of the crude product with ether, $\mathrm{DCM}, \mathrm{MeOH}$ and water allowed for the isolation of a clean 2,5-bis-(benzotriazolmethyl)-pyrrole system (21). However, it was not possible to obtain the closed macrocycle by conversion of $\mathbf{2 1}$ with another equivalent of 2,5-bis-(trimethylaminomethyl)pyrrole diiodide. Unsuccessful alkylation of 21 with $\mathrm{MeI}$ or $\mathrm{Bu}_{3} \mathrm{SnCl}$ furthermore revealed that the $\mathrm{N} 3$ position of the triazole group in $\mathbf{2 1}$ is highly unreactive, thus preventing the desired reactivity. 


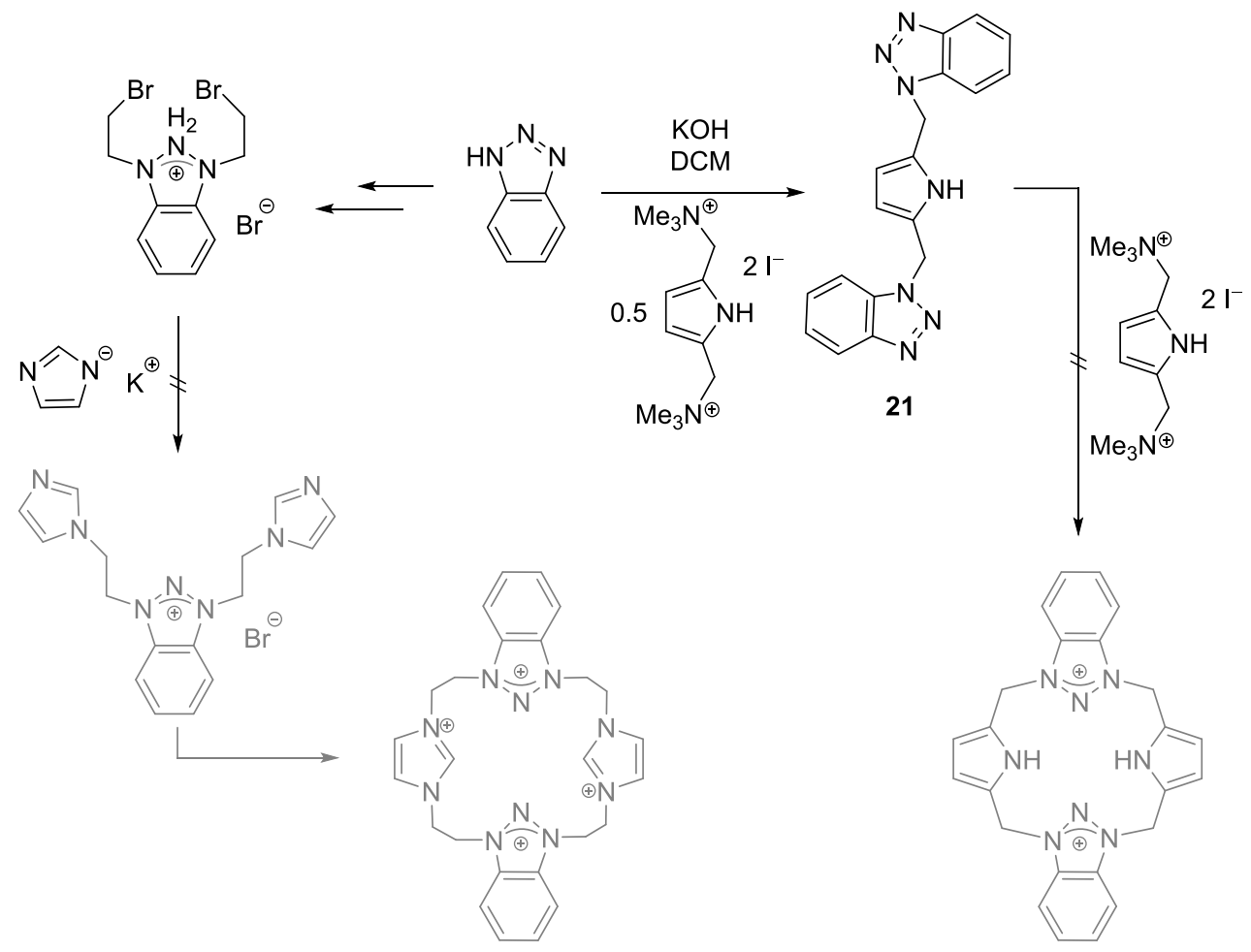

Scheme 26. Illustration of the attempts in the generation of benzotriazole derived macrocyclic ligand scaffolds.

Searching the literature for further possible ligand scaffolds, a carbazole derived pincer system first presented by Kunz and co-workers was of particular interest (Scheme 27, middle). ${ }^{[252]} 9 \mathrm{H}$ carbazole, also known as dibenzopyrrole, contains a pyrrole moiety that is rather protected by fusion with benzene rings. Furthermore, carbazole derived ligand systems likely show increased stability compared to simple pyrroles due to the modified bridging unit. A first attempt to close the macrocycle with 2,5-bis-(trimethylaminomethyl)-pyrrole diiodide was unsuccessful while ring closure with 2,6-bis-(bromomethyl)-pyridine according to Scheme 27 led to precipitation of the ligand scaffold 22, that was characterized by NMR and mass spectrometry.

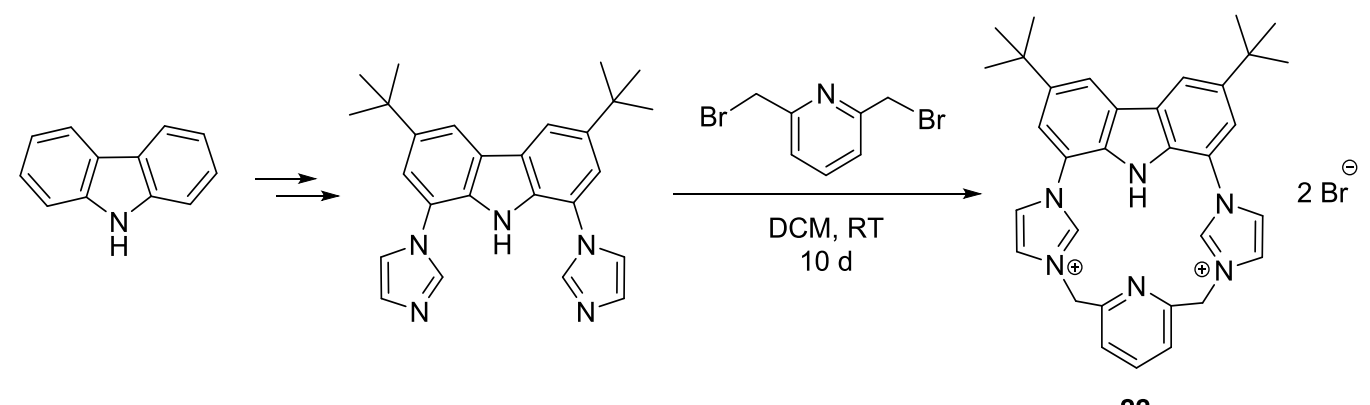

22

Scheme 27. Successful generation of the carbazole derived 2N2C macrocyclic ligand scaffold 22.

In conclusion, a new route for the synthesis of new hybrid ligand systems has been established. The synthesis of the new macrocyclic system 22 represents a suitable starting point for further projects, in particular attempts to generate corresponding iron complexes. Moreover, the synthetic procedure 
used for the successful ring closure can provide the basis for further experiments toward the preparation of other derivatives of the macrocycle, for example with imidazole- and pyrrole linkers instead for the pyridine in $\mathbf{2 2}$. 


\section{Summary}

Within this work, the characteristics and reactivity of several tetracarbene-coordinated iron complexes were studied and compared to literature-described N-coordinated model systems. Therefore, $\left[\left({ }^{\mathrm{NHC}} \mathrm{L}\right) \mathrm{Fe}^{\mathrm{II}}(\mathrm{MeCN})_{2}\right]^{2+} \mathbf{1}$ was used as starting material for the generation of bioinspired iron species such as $\left[\left({ }^{\mathrm{NHC}} \mathrm{L}\right) \mathrm{Fe}^{\mathrm{IV}}=\mathrm{O}(\mathrm{MeCN})\right]^{2+} \boldsymbol{2},\left[\left\{\left({ }^{\mathrm{NHC}} \mathrm{L}\right) \mathrm{Fe}^{\mathrm{III}}\right\}_{2} \mathrm{~N}\right]^{3+} \mathbf{6}$ and $\left[\left({ }^{\mathrm{NHC}} \mathrm{L}\right)\{\mathrm{FeNO}\}^{7}\right]^{2+} \mathbf{1 7}$.

In a first project, the properties of the organometallic oxoiron(IV) compound $\mathbf{2}$ could be further elucidated providing a thorough understanding of the effects of the carbene donors on the iron center. Helium tagging IRPD spectroscopy and ${ }^{18} \mathrm{O}$ labeling experiments allowed for the determination of the $\mathrm{Fe}=\mathrm{O}$ stretching frequency. The obtained value of $\tilde{v}=832 \pm 3 \mathrm{~cm}^{-1}$ is perfectly in line with data reported on other non-heme model systems, thus indicative of a similar $\mathrm{Fe}=\mathrm{O}$ core. This finding is rather surprising since the carbene donors are expected to have a significant influence on the iron center. Consequently, motivation for an intense study of the electronic properties of $\left[\left({ }^{\mathrm{NHC}} \mathrm{L}\right) \mathrm{Fe}^{\mathrm{IV}}=\mathrm{O}(\mathrm{MeCN})\right]^{2+}$ was provided. Using the interplay of UV/vis/MCD spectroscopy and DFT calculations, it was possible to unambiguously characterize the ligand field splitting in 2 . Due to the strong $\sigma$-donating ability of the carbene ligands in the equatorial positions, the empty $\mathrm{d}_{\mathrm{x}^{2}-\mathrm{y}^{2}}$ orbital is raised in energy resulting in the so far unprecedented orbital order $\left(d_{\mathrm{xy}}\right)^{2}\left(d_{\mathrm{xz}} / d_{\mathrm{yz}}\right)^{2}\left(d_{\mathrm{z}^{2}}\right)^{0}\left(d_{\mathrm{x}^{2}-\mathrm{y}^{2}}\right)^{0}$ (Scheme 28). Thus, the tetracarbene ligand shows mainly an influence on the empty $d_{x^{2}-y^{2}}$ orbital and hence on the order of the unoccupied $d_{x^{2}-y^{2}}$ and $d_{z^{2}}$ orbitals, while the occupied $d_{x z, y z, x y}$ orbitals remain unchanged. This is in perfect agreement with the similar properties of the $\mathrm{Fe}=\mathrm{O}$ core when compared to typical nitrogen-donor model systems with a $\left(d_{\mathrm{xy}}\right)^{2}\left(d_{\mathrm{xz}} / d_{\mathrm{yz}}\right)^{2}\left(d_{\mathrm{x}^{2}-\mathrm{y}^{2}}\right)^{0}\left(d_{\mathrm{z}^{2}}\right)^{0}$ orbital order. Futhermore a vibronic progression at about $12000 \mathrm{~cm}^{-1}$ corresponding to $v(\mathrm{Fe}=\mathrm{O} *)$ was detected that is very similar to the one observed for a TMC coordinated $\mathrm{Fe}^{\mathrm{IV}}=\mathrm{O}$ analog. Thus, it can be concluded that not only the stretching frequencies in both ground and excited state but also the $\sigma$ - and $\pi$-contributions to the $\mathrm{Fe}=\mathrm{O}$ bond are the same in both complexes despite the differences in the ligand system. Still, the energetic raise of the $d_{x^{2}-y^{2}}$ orbital leads to destabilization of the quintet state in $\mathrm{Fe}^{\mathrm{IV}}=\mathrm{O} 2$ and preference of the triplet ground state. Thus, it was of high interest to study the reactivity of $\mathbf{2}$ in order to clarify effects of this large triplet-quintet gap on the pathway of the reaction. Typically, the quintet excited state of non-heme model compounds is energetically close to the triplet ground state, allowing for two-state reactivity. In case of the organometallic model compound $\mathbf{2}$, significant differences are expected, providing motivation for further studies.

In order to gain insight into the mechanism of hydrogen atom abstraction (HAA) by 2 , the reactivity of this oxoiron(IV) complex in $\mathrm{C}-\mathrm{H}$ bond activation was studied experimentally and theoretically. It was possible to obtain kinetic parameters for the pseudo-first order oxidation 
reactions of 2 toward xanthene, DHA, CHD and fluorene. The determined $k_{2}$ values largely depend on the bond dissociation energy of the weakest $\mathrm{C}-\mathrm{H}$ bond of the substrates in line with HAA as the rate determining step. In comparative studies on DHA and DHA- $\mathrm{d}_{4}$, a high kinetic isotope effect $\left(k_{\mathrm{H}} / k_{\mathrm{D}}=32\right.$ at $\left.-40{ }^{\circ} \mathrm{C}\right)$ in the range of the value reported for taurine $\alpha-\mathrm{KG}$ dioxygenase was found. The first HAA step of this reaction was also analyzed by DFT calculations, providing information about the spin state of the most favorable transition state and the possible hydroxoiron(III) intermediates (Scheme 28). In perfect agreement with expectations, the large triplet-quintet gap rules out the possibility to react via the quintet pathway and the HAA process proceeds via the triplet pathway only. This is the first time that a 'single-state reactivity' model is presented and studied in detail both experimentally and theoretically. In literature, the quintet pathway is typically associated with higher reactivity than the triplet pathway due to lower activation barriers. Thus, it is quite fascinating that - in terms of reaction rates - the organometallic oxoiron(IV) compound 2 studied herein outperforms several $\mathrm{N}$-coordinated model systems with available quintet pathways, e.g. a tetramethylcyclam coordinated complex. Consequently, the results obtained during these studies on 2 give a perfect example for the complexity of the interplay of different factors that determine the reactivity of high-valent $\mathrm{Fe}=\mathrm{O}$ complexes.

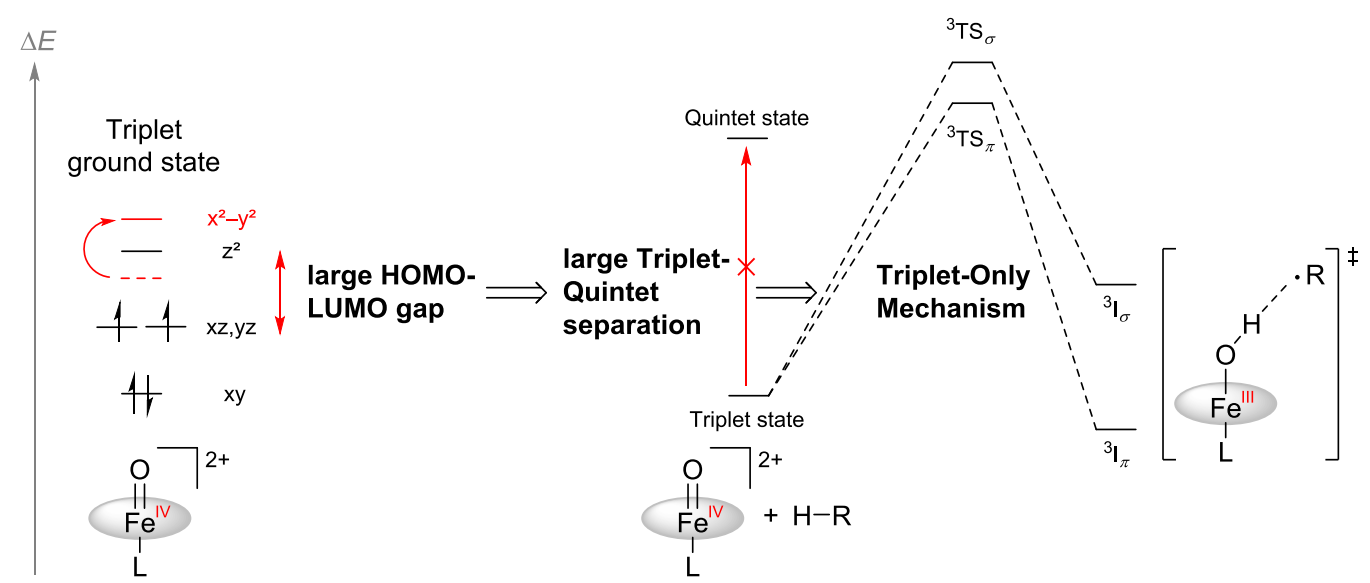

Scheme 28. Illustration of the mechanistic implication of the observed high energy of the $d_{x^{2}-y^{2}}$ orbital in 2 . The grey ellipse represents the strongly $\sigma$-donating macrocyclic tetracarbene ligand system ${ }^{\mathrm{NHC}} \mathrm{L}$ that lifts the $d_{x^{2}-y^{2}}$ orbital to higher energy.

Furthermore, in order to characterize the generated hydroxoiron(III) intermediate, EPR studies were performed. It was possible to detect both low spin $\left(d_{\mathrm{xy}}\right)^{2}\left(d_{\mathrm{xz}} / d_{\mathrm{yz}}\right)^{3}\left(d_{\mathrm{z}^{2}}\right)^{0}\left(d_{\mathrm{x}^{2}-\mathrm{y}^{2}}\right)^{0}$ and intermediate spin $\left(d_{\mathrm{xy}}\right)^{2}\left(d_{\mathrm{xz}} / d_{\mathrm{yz}}\right)^{2}\left(d_{\mathrm{z}^{2}}\right)^{1}\left(d_{\mathrm{x}^{2}-\mathrm{y}^{2}}\right)^{0}$ iron radicals corresponding to the ${ }^{3} \pi$ and ${ }^{3} \sigma$ pathways, respectively. According to comparative studies using crystalline material of $\mathbf{2}$ and in situ generated substance, it is rather likely that coordination of iodoarene is involved in the change of the spin state. Based on DFT results, the low spin state would be preferred in case of an octahedrally coordinated $\mathrm{Fe}^{\mathrm{III}}-\mathrm{OH}$ intermediate (Scheme 28) while square pyramidal coordination around the iron center is correlated 
to an intermediate spin state. However, these results are not fully understood so far and further studies such as ENDOR measurements are necessary.

Another species of interest within this work is the $\mu$-oxodiiron(III) complex 3 . In contrast to similar $\mathrm{N}$-coordinated $\mathrm{Fe}-\mathrm{O}-\mathrm{Fe}$ compounds, this system shows reactivity toward substrates with weak $\mathrm{C}-\mathrm{H}$ bonds and low oxidizing potentials. This unprecedented oxidizing activity was attributed to a donor induced disproportionation equilibrium into the corresponding iron(II) complex $\mathbf{1}$ and the oxoiron(IV) compound 2. The square pyramidal iron centers in $\mathbf{3}$ were determined to have intermediate spin ground states corresponding to the following orbital occupation: $\left(d_{\mathrm{xy}}\right)^{2}\left(d_{\mathrm{xz}} / d_{\mathrm{yz}}\right)^{2}\left(d_{\mathrm{z}^{2}}\right)^{1}\left(d_{\mathrm{x}^{2}-\mathrm{y}^{2}}\right)^{0}$. Thus, the axial anti-bonding $d_{\mathrm{z}^{2}}$ orbital is singly occupied and coordination of a second axial donor ligand trans to the bridging oxo unit is disadvantageous. It is proposed that coordination of $\mathrm{MeCN}$ or $\mathrm{PMe}_{3}$ in solution induces cleavage of the $\mathrm{Fe}-\mathrm{O}-\mathrm{Fe}$ bond and thus initiates the observed disproportionation. The resulting reactivity of $\mathbf{3}$ toward $\mathrm{PMe}_{3}$ and DHA was studied in comparison with the reactivity of free $\mathbf{2}$. As expected, $\mathbf{3}$ shows much lower kinetic rates than 2 , which is in line with the pre-equilibrium to lie mainly on the side of the $\mu$-oxo dimer. In case of the $\mathrm{C}-\mathrm{H}$ activation reaction on DHA, it was possible to determine the equilibrium constant by addition of subsequent amounts of the iron(II) precursor. The obtained very small value of $K_{\mathrm{eq}}=7.5 \pm 2.5 \cdot 10^{-8} \mathrm{~mol}^{-1}$ is perfectly in line with the long reaction time as well as data reported in literature for a similar equilibrium of a $\mu$-oxodiiron(IV) compound.

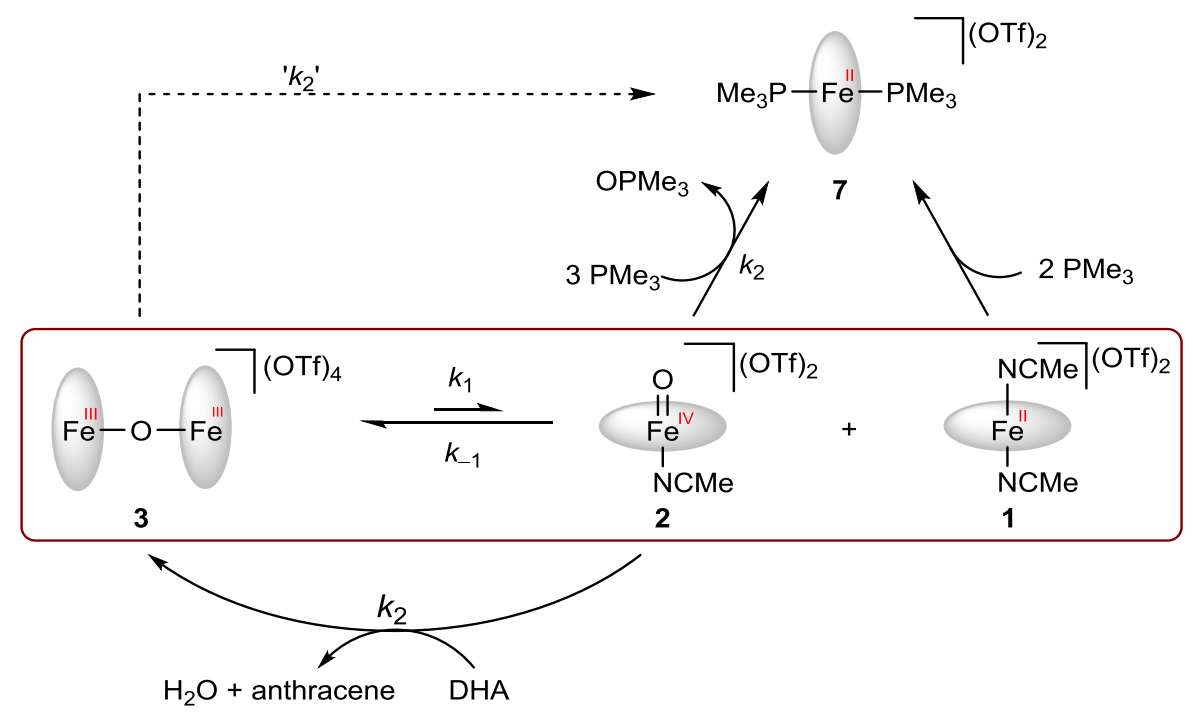

Scheme 29. Overview about the disproportionation equilibrium of the $\mu$-oxodiiron(III) system studied in this work (brown) and the resulting reactivity in OAT (top) and HAA (bottom).

Azidoiron(II) and -iron(III) compounds have been shown to be suitable precursors for high-valent nitridoiron(IV) and -iron(V) complexes, respectively. Studies prior to this work have revealed that irradiation of $\left[\left({ }^{\mathrm{NHC}} \mathrm{L}\right) \mathrm{Fe}^{\mathrm{II}}\left(\mathrm{N}_{3}\right)_{2}\right]$ did not initiate any reaction, in particular no $\mathrm{N}_{2}$ release and concomitant formation of $\left[\left({ }^{\mathrm{NHC}} \mathrm{L}\right) \mathrm{Fe}^{\mathrm{IV}} \equiv \mathrm{N}\left(\mathrm{N}_{3}\right)\right]$. Thus, it was now planned to generate the 
corresponding bis-(azido)iron(III) species 11. Comparative investigation of different synthetic strategies demonstrated that $\mathbf{1 1}$ is best prepared by first oxidizing the iron(II) precursor $\mathbf{1}$ with Magic Blue and subsequently treating the obtained $\mathrm{Fe}{ }^{\mathrm{III}}-\mathrm{Cl}$ compound 8 with 2 eq. of azide salt (Scheme 30). Conversion of 8 with only 1 eq. of $\left[{ }^{n} \mathrm{Bu}_{4} \mathrm{~N}\right] \mathrm{N}_{3}$ led to formation of the $\mathrm{Cl}-\mathrm{Fe}{ }^{\mathrm{IIII}}-\mathrm{N}_{3}$ species 10.

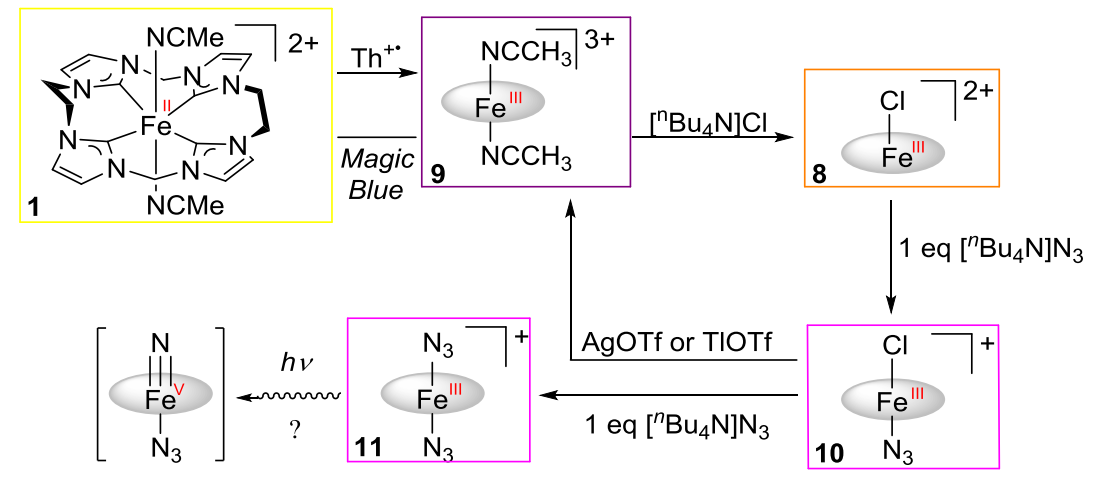

Scheme 30. Illustration of the synthetic strategy for generation of bis-(azido)iron(III) 11 explored within this work. The colors of the boxes represent the actual colors of the compounds.

It was rather surprising that oxidation of 1 with $\left[\mathrm{N}\left(\mathrm{C}_{6} \mathrm{H}_{4}-4-\mathrm{Br}\right)_{3}\right] \mathrm{SbCl}_{6}$ (Magic Blue) results in the isolation of chloridoiron(III) complex 8. Within this work, it was possible to identify the initial product of the oxidation, $\left[\left({ }^{\mathrm{NHC}} \mathrm{L}\right) \mathrm{Fe}^{\mathrm{III}}(\mathrm{MeCN})_{2}\right]\left(\mathrm{SbCl}_{6}\right)_{3}$ 9. During the crystallization process, this compound abstracts a chlorido ligand of the counter anion and thus, formation of $\mathbf{8}$ can be accelerated by addition of a chloride salt. All iron(II) and iron(III) compounds obtained in this project have been comprehensively analyzed and spectroscopic trends have been explored, adding up to the number of novel organometallic models for bioinorganic key structures that have been presented prior to this work.

Having in hand the newly synthesized $\left[\left({ }^{\mathrm{NHC}} \mathrm{L}\right) \mathrm{Fe}^{\mathrm{III}}\left(\mathrm{N}_{3}\right)_{2}\right] \mathrm{Cl}$ species, MeCN solutions of 11 were irradiated at different temperatures $\left(0{ }^{\circ} \mathrm{C},-40{ }^{\circ} \mathrm{C},-196{ }^{\circ} \mathrm{C}\right)$. According to literature reports, the desired four-fold nitridoiron(V) compound is expected to have low stability and comparable systems could be isolated in frozen matrix only. While irradiation with a diode at $365 \mathrm{~nm}$ led to a color change and promising EPR data, it was not possible to detect any conversion when the frozen sample was irradiated in a sample cell suitable for subsequent MB measurements. So far, no conclusive data on the successful generation of a high-valent nitrido species could be obtained, yet, the gained results provide motivation for future experiments with modified experimental setups and conditions.

Prior to this work, Iris Klawitter synthesized the organometallic compound $\left[\left\{\left({ }^{\mathrm{NHC}} \mathrm{L}\right) \mathrm{Fe}^{\mathrm{III}}\right\}_{2} \mathrm{~N}\right]^{3+}(\mathbf{6})$, an analog to $\mu$-oxodiiron(III) species 3. Comparison with literature has shown that this species is the first example of a nitrido bridged compound with both iron centers in oxidation state +III, while 
several mixed-valent $\mathrm{Fe}^{\mathrm{III}}-\mathrm{N}-\mathrm{Fe}^{\mathrm{IV}}$ and high-valent $\mathrm{Fe}^{\mathrm{IV}}-\mathrm{N}-\mathrm{Fe}^{\mathrm{IV}}$ species have been described before. Consequently, characterization of $\mathbf{6}$ was continued within this work including the examination of its electrochemical properties. In accordance with a reversible one-electron oxidation wave at low potential in the cyclic voltammogram, it was possible to isolate the first tetracarbene-coordinated mixed-valent $\mu$-nitridoiron systems 12 and 13 (Scheme 31). With the help of X-ray diffraction analysis, compound $\mathbf{1 2}$ could be described as $\left[\left({ }^{\mathrm{NHC}} \mathrm{L}\right) \mathrm{Fe}{ }^{\mathrm{III}}-\mathrm{N}-\right.$ $\left.\mathrm{Fe}^{\mathrm{IV}}\left({ }^{\mathrm{NHC}} \mathrm{L}\right)(\mathrm{NCMe})\right]^{4+}$. Thus, the coordination mode changes from square pyramidal as found for the iron(III) centers $(S=3 / 2)$ to octahedral for the iron(IV) center $(S=1)$. If $\mathrm{AgNO}_{3}$ was used as oxidizing agent instead of $\mathrm{AgSbF}_{6}$, the obtained spectroscopic data are indicative of coordination of the anionic nitrate ligand at the free axial position and thus formation of 13. Furthermore, the use of two equivalents $\mathrm{AgNO}_{3}$ enabled for the isolation of the corresponding compound $\left[\left\{\left(\mathrm{O}_{2} \mathrm{NO}\right)\left({ }^{\mathrm{NHC}} \mathrm{L}\right) \mathrm{Fe}^{\mathrm{IV}}\right\}_{2} \mathrm{~N}\right]^{3+}(\mathbf{1 4})$. Cyclic voltammetry measurements revealed that the second oxidation is facilitated in the presence of nitrate ions and thus becomes feasible with the weak oxidant silver(I). Moreover, conversion with the stronger oxidant nitrosonium tetrafluoroborate led to formation of 12 and $\mathbf{1 5}$ when using 1 eq. or 2 eq. of $\mathrm{NO}^{+}$, respectively, The spectroscopic data obtained so far give strong evidence for $\mathrm{NO}^{+}$acting as one-electron oxidant only and $\mathrm{MeCN}$ coordinating at the free axial position. The spectroscopic trends within these series of $\mu$-nitridoiron compounds were explored using the combination of X-ray, MB, EPR, NMR, IR and UV/vis spectroscopy.

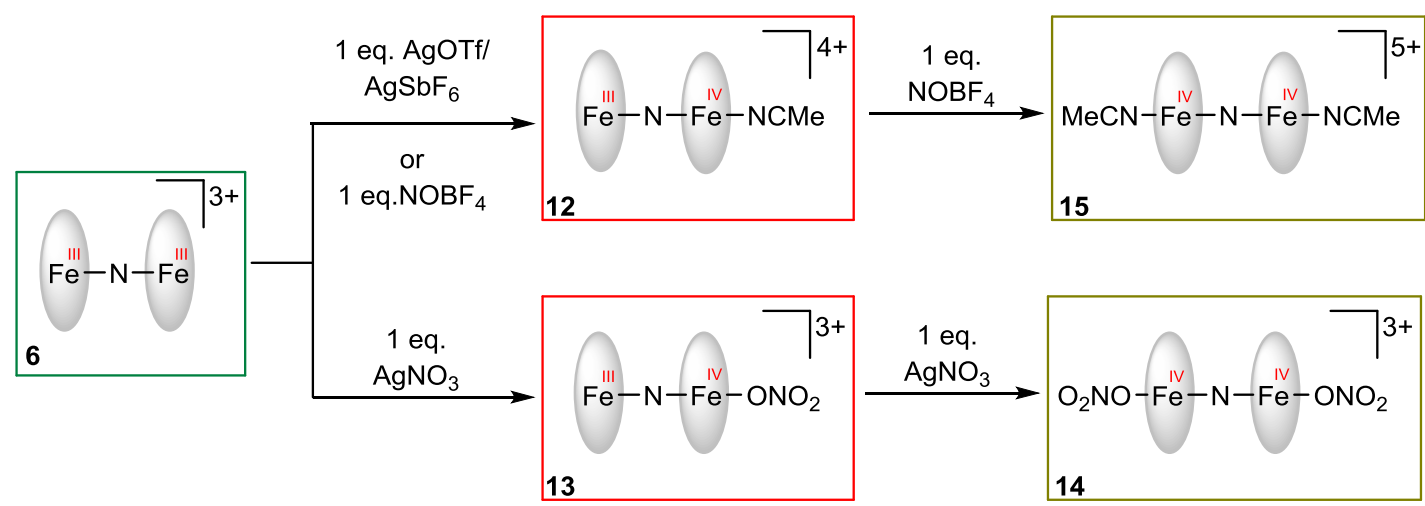

Scheme 31. Oxidation of $\mathbf{6}$ with different oxidants as studied in this work.

The newly synthesized $\mu$-nitridoiron(IV) compounds $\mathbf{1 4}$ and $\mathbf{1 5}$ are new examples for tetracarbenecoordinated high-valent iron systems and represent promising starting points for future reactivity studies. Furthermore, they turned out to be surprisingly stable at RT as well as atmospheric conditions, providing further motivation to study the generation of tetracarbene coordinated iron centers in the even higher oxidation state $+\mathrm{V}$. In this regard, two synthetic strategies revealed promising results: Firstly, conversion of $\left.\left[{ }^{\mathrm{NHC}} \mathrm{L}\right) \mathrm{Fe}^{\mathrm{II}}(\mathrm{MeCN})_{2}\right](\mathrm{OTf})_{2} \mathbf{1}$ with excess $\mathrm{XeF}_{2}$ gave rise to a brown species with a very low IS in the MB spectrum $\left(\delta=-0.22 \mathrm{~mm} \mathrm{~s}^{-1}\right)$ and a broad signal in the EPR spectrum. Secondly, treatment of $\left[\left({ }^{\mathrm{NHC}} \mathrm{L}\right) \mathrm{Fe}^{\mathrm{IV}}=\mathrm{O}(\mathrm{MeCN})\right](\mathrm{OTf})_{2} 2$ with deprotonated tert- 
butylperoxide monitored in the $\mathrm{UV} / \mathrm{vis}$ region is indicative of successful formation of a $\mathrm{O}=\mathrm{Fe}^{\mathrm{V}}(\mathrm{NC}(\mathrm{O}) \mathrm{Me}) \leftrightarrow \mathrm{O}=\mathrm{Fe}^{\mathrm{IV}}\left({ }^{\cdot} \mathrm{NC}(\mathrm{O}) \mathrm{Me}\right)$ compound in analogy to work on a $\mathrm{N}$-ligated $\mathrm{Fe}=\mathrm{O}$ system described in a recent publication by Que et al. Furthermore, the obtained MB and EPR data as well as initial reactivity studies are consistent with this picture. However, in both cases it was not yet possible to obtain crystalline material suitable for X-ray diffraction analysis.

Moreover the first fully organometallic $\{\mathrm{FeNO}\}^{7}$ compound (17) was generated upon conversion of 1 with nitric oxide (Scheme 32). Iron nitrosyl species are of relevance in numerous biosystems and especially their redox conversions are of high interest, i.e. due to their implication in the biogeochemical nitrogen cycle. Motivated by a reversible reduction wave in its cyclic voltammogram, the $\{\mathrm{FeNO}\}^{7}$ complex was reduced with cobaltocene which allowed for the isolation of the corresponding $\{\mathrm{FeNO}\}^{8}$ species (19). Although no oxidative process was observed in the $\mathrm{CV}$, a (ONO) $\{\mathrm{FeNO}\}^{6}$ compound (18) could be synthesized by treatment of $\mathbf{1 7}$ with in situ generated $\mathrm{NO}_{2}$. Thus, a complete series of mononitrosyl $\{\mathrm{FeNO}\}^{6-8}$ complexes was generated and their solid state structures were successfully characterized.

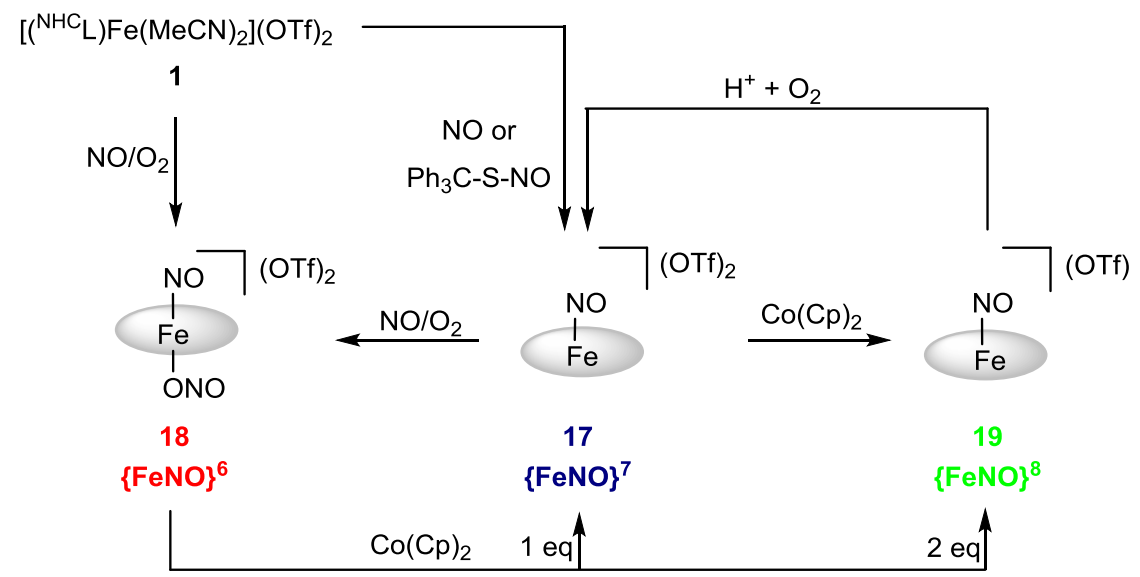

Scheme 32. Synthetic procedure for the generation of tetracarbene-coordinated $\{\mathrm{FeNO}\}^{6},\{\mathrm{FeNO}\}^{7}$ and $\{\mathrm{FeNO}\}^{8}$ compounds and the possible interconversions within this series.

Having these systems in hand, they have been characterized using the interplay of several spectroscopic and theoretical methods; in particular Mössbauer, EPR, SQUID, NMR, UV/vis/IR, XES measurements and DFT calculations. Analysis by this set of methods revealed novel properties that differ from both the well studied heme and non-heme iron nitrosyl compounds presented in literature so far. For example, the iron centers in all three compounds are in the low spin ground state and linear $\mathrm{Fe}-\mathrm{N}-\mathrm{O}$ angles are found; a combination that was unprecedented before this work. Even more interesting, the redox processes occur largely metal centered, in contrast to the former proposal that low spin $\{\mathrm{FeNO}\}^{7}$ species undergo NO-centered reductions while iron centered reductions are a primary feature of non-heme high spin iron nitrosyls. Practical relevance for the metal centered reduction of $\mathbf{1 7}$ was found in reactivity studies. While $\{\mathrm{FeNO}\}^{8}$ 
systems with a ligand reduced $\left\{\mathrm{Fe}^{\mathrm{II}} \mathrm{NO}^{-}\right\}$moiety are easily protonated to the corresponding, and mostly unstable, $\{\mathrm{FeNHO}\}^{8}$ compounds, the tetracarbene coordinated $\{\mathrm{FeNO}\}^{8}$ complex 19 is stable toward weak acids. Only in the presence of dioxygen, clean oxidation back to the $\{\mathrm{FeNO}\}^{7}$ compound is observed, likely via a PCET process. In conclusion, the first complete series of $\{\mathrm{FeNO}\}^{6},\{\mathrm{FeNO}\}^{7}$, and $\{\mathrm{FeNO}\}^{8}$ was presented as part of this work including the extensive analysis of their properties and comparison to literature reported heme and non-heme iron nitrosyls.

A last project of this work dealt with the development of new iron complexes coordinated by hybrid ligand systems with both carbene C- and N-donation. Such 2N2C ligand scaffolds are expected to allow for a better understanding of the different properties of the tetracarbenecoordinated iron complexes studied herein when compared to $\mathrm{N}$-coordinated systems prevalent in nature. Initial experiments used a promising bis-pyrrole-bis-imidazole macrocycle but it was not possible to obtain any iron complex coordinated by this ligand system. Consequently, the preparation of further suitable ligand scaffolds was attempted, finally resulting in the successful synthesis of a carbazole derived 2N2C macrocycle (22, Figure 73). This system represents a good starting point for future experiments regarding the generation of bioinspired iron compounds coordinated by nitrogen-carbene-hybrid ligands.

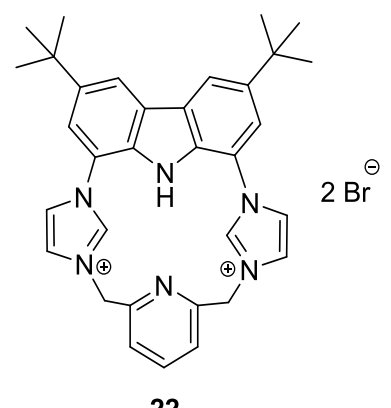

22

Figure 73. Chemical structure of the newly synthesized ligand scaffold 22. 


\section{Experimental Part}

\subsection{Materials and Methods}

\section{General Considerations}

If not stated differently, all reactions were carried out under an anaerobic and anhydrous atmosphere of dry argon using standard Schlenk techniques or in a glovebox (MBRAUN LabMaster) under dinitrogen atmosphere with less than 0.1 or $0.5 \mathrm{ppm} \mathrm{O}_{2}$ and $\mathrm{H}_{2} \mathrm{O}$. Solvents were dried and degassed by standard procedures before use.

\section{Chemicals}

Chemicals were purchased from commercial sources and used as received.

The following compounds were prepared according to literature: Tetraimidazolium ligand scaffold

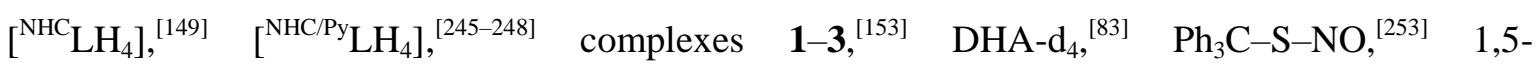
bis(dimethyl)piperidinium tetrafluoroborate ${ }^{[225]}$ and 1,5-dimethylpyrdinium triflate. ${ }^{[214,254]}$

Nitric oxide gas was purchased from Linde and passed through a column with Ascarite II and a cooling trap $\left(-78{ }^{\circ} \mathrm{C}\right)$ before being added to the reaction mixture. ${ }^{15} \mathrm{NO}$ gas was prepared in situ by treatment of $\mathrm{Na}^{15} \mathrm{NO}_{2}$ with an aqueous solution of ascorbic acid. ${ }^{[23]}$

\section{UV/vis/NIR Spectroscopy}

UV/vis/NIR spectra were collected with a Varian Cary 5000 instrument as well as a Varian Cary 60 $1 \mathrm{~cm}$ UV quartz cells. Spectra were analyzed by Cary Win UV software. UV-vis experiments for chapter 4 were performed using an Agilent $8453 \mathrm{UV} /$ vis spectrophotometer. The low temperature control was performed with a cryostat from Unisoku Scientific Instruments (Japan).

\section{Spectroscopy ${ }^{[170]}$}

MCD experiments were carried out at the MPI-CED with an Olis DSM17 CD spectrapolarimeter while the sample was placed in the Oxford cryostat Spectromag SM4000. The temperature ranged from $2 \mathrm{~K}$ to $80 \mathrm{~K}$ and the light energy was varied from 5000 to $30000 \mathrm{~cm}^{-1}$ (2000-333 nm). Global fits of MCD and absorption spectra of consistent temperature and field series with Gaussian lines were performed with an in-house program developed at the MPI-CEC (mcd_bf). Line positions were kept identical throughout the series, whereas line widths were allowed to vary as a function of temperature by $10-20 \%$; intensities have been released unconstrained except for pseudo- $A$ term signals. 


\section{Spectro-Elektrochemical Measurements and Cyclic Voltammetry ${ }^{[22,23]}$}

Electronic spectra during spectro-electrochemical measurements were recorded with the Cary 50 Bio Spectrophotometer supplied with the quartz dip probe $(1 \mathrm{~mm}$, Hellma Analytics). Spectra were analyzed by Cary Win UV software.

Cyclic voltammograms were measured using a Perkin Elemer 263 A potentiostat controlled by electrochemistry Powersuite software or a Gamry Reference 600 controlled by the Gamry Framework progamm. A three electrode arrangement with a glassy carbon working electrode, a Ag / $0.01 \mathrm{M} \mathrm{AgNO}_{3}$ reference electrode and a $\mathrm{Pt}$ wire counter electrode was utilized. All $\mathrm{CV}$ experiments were performed under an atmosphere of dry argon in $\mathrm{MeCN} / 0.1 \mathrm{mM}\left[{ }^{n} \mathrm{Bu}_{4} \mathrm{~N}\right] \mathrm{PF}_{6}$. The resulting data were converted to the $\mathrm{Fc} / \mathrm{Fc}^{+}$scale by using $\mathrm{Cp}_{2} \mathrm{Fe}$ or $\mathrm{Cp}_{2}{ }_{2} \mathrm{Fe}$, added after the experiment, as internal standard or according to published conversion constants. ${ }^{[255]}$ All electrochemical measurements were performed at room temperature.

\section{IR Spectroscopy ${ }^{[22,23]}$}

Solid state and liquid IR measurements were performed with a Cary 630 FTIR spectrometer with Dial Path Technology and analyzed by FTIR MicroLab software. IR Stopped Flow experiments were performed using a $\mathrm{T}_{\mathrm{g}} \mathrm{K}$ Scientific SF-61/VERTEX 70 IR. The stopped flow machine was controlled with Kineta Drive and the cell was cooled to $5{ }^{\circ} \mathrm{C}$ using a Cryostat. Data were recorded and analysed with the OPUS 7.0 program.

\section{Helium Tagging IR Spectroscopy ${ }^{[170]}$}

IRPD spectra were measured with the ISORI instrument in the lab of Jana Roithová in Prague. ${ }^{[170,174]}$ The electrospray ion source was kept at room temperature and soft ionization conditions were used. The $\left[\left({ }^{\mathrm{NHC}} \mathrm{L}\right) \mathrm{Fe}^{\mathrm{IV}} \mathrm{O}(\mathrm{NCMe})\right]^{2+}$ ions were mass selected $(\mathrm{m} / \mathrm{z}=230.5)$ by the first quadrupole and transferred with an octopole toward a cryo-cooled wire quadrupole ion trap operated at $3 \mathrm{~K}$ and $1 \mathrm{~Hz}$. The ions were introduced to the trap during the first $200 \mathrm{~ms}$ and trapped with a helium buffer gas pulse (130 ms long). About $15 \%$ of the trapped ions were transformed to the helium tagged complexes, $\left[\left({ }^{\mathrm{NHC}} \mathrm{L}\right) \mathrm{Fe}^{\mathrm{IV}} \mathrm{O}(\mathrm{NCMe})(\mathrm{He})\right]^{2+}(\mathrm{m} / z=232.5)$. After a $400 \mathrm{~ms}$ time delay, the ion cloud was irradiated by 8 photon pulses generated in an optical parametric oscillator/amplifier (OPO/OPA) operating at $10 \mathrm{~Hz}$ frequency. At $990 \mathrm{~ms}$, the exit electrode of the trap was opened, the ions were mass-analyzed by the second quadrupole, and their number $(N)$ was determined by a Daly type detector operated in ion-counting mode. In the following cycle the light from the OPO was blocked by a mechanical shutter, giving the number of unirradiated ions $\left(N_{0}\right)$. The IRPD spectra are constructed as the wavenumber dependence of $\left(1-N / N_{0}\right)$. The calibration was done using the absorption of methane and water; the positions of the bands are determined with accuracy better than $\pm 3 \mathrm{~cm}^{-1}$. 


\section{Mass Spectrometry}

ESI mass spectra were measured on a Thermo Finnigan Trace LCQ spectrometer or on an Bruker APEX IV (FTICR-MS). The GC measurement was performed on a Thermo Finnigan TRACE GC with EI and Varian GC Capillary Column. Cryo Mass spectrometry was recorded using a high resolution mass spectra (HR-MS) Bruker MicrOTOF-Q IITM instrument with cryospray ionization sources using a cryospray attachment and setting the temperature of the nebulizing and drying gas at $-40^{\circ} \mathrm{C}$

\section{CHN Analyses}

Elemental analyses were performed by the analytical laboratory of the Institute of Inorganic Chemistry at the Georg-August-University Göttingen using an Elementar Vario EL III instrument.

\section{NMR Spectroscopy}

${ }^{1} \mathrm{H},{ }^{13} \mathrm{C},{ }^{31} \mathrm{P}$ and ${ }^{15} \mathrm{~N}$ NMR spectra were recorded on Bruker Avance instruments with given $\mathrm{MHz}$ frequencies at $25{ }^{\circ} \mathrm{C}$ except otherwise mentioned. ${ }^{1} \mathrm{H}$ and ${ }^{13} \mathrm{C}$ chemical shifts $(\delta)$ are given in ppm and are referenced to the solvent residual signals according to Fulmer et al. ${ }^{[256]}$ The peaks are labeled according to their splitting patterns with s (singlet), $d$ (doublet), $t$ (triplet), or m (multiplet) and an additional $\mathrm{b}$ in case of broadened signals that prevent determination of coupling constants $(J$ $[\mathrm{Hz}])$.

\section{Mössbauer Spectroscopy}

Mössbauer (MB) spectra were recorded at $80 \mathrm{~K}$ with a ${ }^{57} \mathrm{Co}$ source in a Rh matrix using an alternating constant-acceleration WissEl Mössbauer spectrometer operated in the transmission mode and equipped with a Janis closed-cycle helium cryostat. Isomer shifts $(\delta)$ as well as the quadrupole splitting $\left(\Delta E_{\mathrm{Q}}\right)$ are given in $\mathrm{mm} \mathrm{s}^{-1}$ relative to iron metal at ambient temperature. Simulation of the experimental data was performed with the Mfit program. ${ }^{[257]}$

\section{Magnetic Susceptibility Measurements}

Temperature-dependent magnetic susceptibilities were measured by using a SQUID magnetometer (Quantum Design MPMS XL-5). The powdered sample was contained in a gel bucket and fixed in a non-magnetic sample holder. Each raw data file for the measured magnetic moment was corrected for the diamagnetic contribution of the gelatin capsule according to $M^{\mathrm{dia}}$ (capsule) $=\chi_{\mathrm{g}} \cdot m \cdot H$, with an experimentally obtained gram susceptibility of the gelatin capsule. The molar susceptibility data were corrected for the diamagnetic contribution according to $\chi_{\mathrm{M}}{ }^{\text {dia }}$ (sample) $=-0.5 \cdot M \cdot 10^{-6}$ $\mathrm{cm}^{3} \cdot \mathrm{mol}^{-1} \cdot{ }^{[258]}$ Intermolecular interactions were considered in a mean field approach by using a Weiss temperature $\Theta$. The Weiss temperature $\Theta$ (defined as $\Theta=z J_{\text {inter }} S(S+1) / 3 k$ ) relates to intermolecular interactions $z J_{\text {inter }}$, where $J_{\text {inter }}$ is the interaction parameter between two nearest 
neighbor magnetic centers, $k$ is the Boltzmann constant $\left(0.695 \mathrm{~cm}^{-1} \cdot \mathrm{K}^{-1}\right)$ and $z$ is the number of nearest neighbors $\left(\Theta=-0.3 \mathrm{~K}\right.$ corresponds to $\left.z J_{\text {inter }}=-0.834\right)$. Magnetic properties were simulated using the $j u l X$ program. ${ }^{[259]}$ Temperature-independent paramagnetism (TIP) was included according

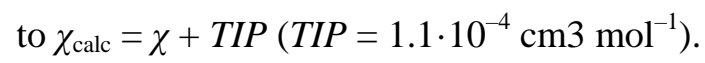

\section{$\mathbf{E P R}^{[22,23]}$}

X-band EPR spectra were measured with a Bruker E500 ELEXSYS spectrometer equipped with a standard cavity (ER4102ST, $9.45 \mathrm{GHz}$ ). The sample temperature was maintained constant with an Oxford Instruments Helium flow cryostat (ESP910) and an Oxford temperature controller (ITC-4). The microwave frequency was measured with the built-in frequency counter and the magnetic field was calibrated using an NMR field probe (Bruker ER035M). EPR spectra were simulated using $\mathrm{XSophe}^{[260]}$ or Easy-Spin. ${ }^{[261]}$

\section{$\mathbf{X E S}^{[23]}$}

X-ray emission spectra were collected at beamline C-1 at the Cornell High Energy Synchrotron Source (CHESS, 1, 17, and 19) and at beamline ID-26 at the European Synchrotron Radiation Facility (ESRF, 1 and 18). Samples were prepared by grinding neat solid into a fine powder using a mortar and pestle, which was sealed in a $1 \mathrm{~mm} \mathrm{Al}$ spacer using $38 \mu \mathrm{m}$ Kapton tape. In both cases, the spectrometers consisted of an array of five Ge(620) analyzer crystals arranged in a Rowland geometry of Johann type, as described previously. ${ }^{[163,262]}$ The spectrometer flight path was filled with helium to mitigate signal attenuation, and samples were maintained below $40 \mathrm{~K}$ using a displex (CHESS) or liquid helium flow (ESRF) cryostat. Incident X-ray energies were selected at CHESS and ESRF using a pair of molybdenum-carbide multilayers or a Si(111) double-crystal monochromator, respectively. Analyzed X-ray emission was detected using a spatially-resolved Pilatus CCD or APD. In all cases, samples were screened for radiation damage, and only spectra that exhibited no evidence of photochemistry were included. Both spectrometers were calibrated to the spectral features of $\mathrm{Fe}_{2} \mathrm{O}_{3},{ }^{[163]}$ and comparison of the iron(II) precursor spectra recorded on both beamlines confirmed the internal experimental consistency. ${ }^{[23]}$

\section{Irradiation Experiments}

Mercury arc lamb: 200 V, 20 Hz, 15 W, 200-600 nm

Diodes: Sahlmann Photochemical Solutions

3x530 nm, 900 mW, LED-Typ Luxeon LXML-PM01

3x450 nm, $2600 \mathrm{~mW}$, LED-Typ Luxeon LXML-PM01

$3 \times 365 \mathrm{~nm}, 3400 \mathrm{~mW}$, LED-Typ Nichia NC4U133A

Blue laser: 445-450 nm, 1000-1200 mW, high power blue laser module TTL DC 12V input 


\subsection{Synthetic Procedures}

\section{Sample Preparation for Helium Tagging IRPD Spectroscopy on $\left[\left({ }^{\mathrm{NHC}} \mathrm{L}\right) \mathrm{FeO}(\mathrm{MeCN})\right](\mathrm{OTf})_{2}$} $(2)^{[170]}$

$\left[\left({ }^{\mathrm{NHC}} \mathrm{L}\right) \mathrm{Fe}(\mathrm{MeCN})_{2}\right](\mathrm{OTf})_{2}(1.9 \mathrm{mg}, 2.4 \mu \mathrm{mol}, 1.0 \text { eq.) and 2-(tBuSO})_{2}-\mathrm{C}_{6} \mathrm{H}_{4}-\mathrm{IO}(3 \mathrm{mg}, 8.8 \mu \mathrm{mol}$, 3.7 eq.) were charged to a flask cooled by liquid nitrogen. Then, $\mathrm{MeCN}(3 \mathrm{~mL})$ was added and the mixture was slowly warmed to $-40{ }^{\circ} \mathrm{C}$ while stirring until all components were dissolved. The solution was transferred to a syringe operating at $-10{ }^{\circ} \mathrm{C}$ and introduced via a silica capillary to the mass spectrometer. ${ }^{18} \mathrm{O}$ labeled complex 2 was prepared in the same way with addition of $\mathrm{H}_{2}{ }^{18} \mathrm{O}$ $(8 \mu \mathrm{L})$ to the $\mathrm{MeCN}(3 \mathrm{~mL})$ solution.

\section{General Procedure for C-H Activity Experiments on $\left[\left({ }^{\mathrm{NHC}} \mathrm{L}\right) \mathrm{FeO}(\mathrm{MeCN})\right](\mathrm{OTf})_{2}(2)^{[179]}$}

A $1 \mathrm{mM}$ solution of $\left[\left({ }^{\mathrm{NHC}} \mathrm{L}\right) \mathrm{Fe}(\mathrm{MeCN})_{2}\right](\mathrm{OTf})_{2}(1.569 \mathrm{mg}, 2 \mu \mathrm{mol}, 1.0$ eq.) in $\mathrm{MeCN}(2 \mathrm{~mL})$ was cooled to $-40{ }^{\circ} \mathrm{C}$. A precooled and freshly prepared solution of 2-(tBuSO$)_{2}-\mathrm{C}_{6} \mathrm{H}_{4}-\mathrm{IO}(1.905 \mathrm{mg}$, $5.60 \mu \mathrm{mol}, 2.8$ eq. $)$ in $\mathrm{CH}_{2} \mathrm{Cl}_{2}(190.5 \mu \mathrm{L})$ was added and the cuvette was transferred out of the glove box and the UV/vis absorption measurement was started at $-40{ }^{\circ} \mathrm{C}$. The appropriate amount of substrate (10-160 eq.) was dissolved in $\mathrm{MeCN}$ (CHD) or $\mathrm{CH}_{2} \mathrm{Cl}_{2}$ (DHA, xanthene, fluorene) to give $100 \mu \mathrm{L}$ (because of solubility problems sometimes up to $200 \mu \mathrm{L}$ ) which were then added to the cuvette using a syringe. The subsequent increase of the characteristic absorption band of the iron(III)-hydroxo complex $\left(\lambda_{\max }=448 \mathrm{~nm}\right)$ was directly monitored and fitted to a single exponential function.

\section{Determination of the Yield in DHA Oxidation by $\left[\left({ }^{\mathrm{NHC}} \mathrm{L}\right) \mathrm{FeO}(\mathrm{MeCN})\right](\mathrm{OTf})_{2}(2)^{[179]}$}

Product analysis for the oxidation of DHA was performed by gas chromatography. Therefore, a $1 \mathrm{mM}$ solution of crystalline material of $\left.\left[{ }^{\mathrm{NHC}} \mathrm{L}\right) \mathrm{FeO}(\mathrm{MeCN})\right](\mathrm{OTf})_{2}(2.21 \mathrm{mg}, 2.91 \mu \mathrm{mol})$ in $\mathrm{MeCN}\left(2.91 \mathrm{~mL}\right.$ ) was reacted with 10 eq. of DHA (5.25 mg in $100 \mu \mathrm{L} \mathrm{CH}_{2} \mathrm{Cl}_{2}$ ) as described previously. The yield was determined after $3 \mathrm{~min}$, which corresponds to maximum absorption at $448 \mathrm{~nm}$, and after $1 \mathrm{~h}$. Prior to injection and to stop the reaction progress, the solution was filtered through silica and washed with $\mathrm{MeCN}$, ethyl acetate and $\mathrm{CH}_{2} \mathrm{Cl}_{2}$. Anthracene was the only observed product and its amount was compared to leftover amount of DHA since both substances show very similar intensities in GC. After $3 \mathrm{~min}, 0.93 \mu \mathrm{mol}$ anthracene was detected (64\% compared to the maximum theoretical yield), after $1 \mathrm{~h} 1.08 \mu \mathrm{mol}(74 \%)$.

\section{Sample Preparation for EPR Experiments on $\left[\left({ }^{\mathrm{NHC}} \mathrm{L}\right) \mathrm{FeO}(\mathrm{MeCN})\right](\mathrm{OTf})_{2}(2)$}

Experiments dealing with the detection of EPR-active hydroxoiron(III) intermediates during $\mathrm{C}-\mathrm{H}$ activation reactivity were performed starting with crystalline $\mathbf{2}$ or in situ generated material. When 
starting with crystalline substance, a concentrated and cold stock solution of $\left[\left({ }^{\mathrm{NHC}} \mathrm{L}\right) \mathrm{FeO}(\mathrm{MeCN})\right](\mathrm{OTf})_{2}$ was diluted with $\mathrm{MeCN}$ to generate $1000 \mu \mathrm{L}$ of a $3 \mathrm{mM}$ solution. The green solution was transferred to a $1.5 \mathrm{~mL}$ vial equipped with a small stirring bar and sealed with a septum. This mixture was transferred out of the glove box and kept at $-40{ }^{\circ} \mathrm{C}$ with a cooling bath. The appropriate amount of CHD (5-50 eq.) was dissolved in $\mathrm{MeCN}$ to give $100 \mu \mathrm{L}$ which were then added to the reaction vial using a syringe. At the desired reaction time (10-120 s), this mixture was transferred with a syringe to a dry and argon equipped EPR tube, properly sealed and frozen in liquid nitrogen. In analogy to this procedure, the reaction was performed in a similar way when starting with in situ generated material of $\mathbf{2}$. Therefore, the corresponding amount of $\left[\left({ }^{\mathrm{NHC}} \mathrm{L}\right) \mathrm{Fe}(\mathrm{MeCN})_{2}\right](\mathrm{OTf})_{2}$ in $\mathrm{MeCN}$ was treated with 2.8 eq. of 2-( $\left.{ }^{t} \mathrm{BuSO}_{2}\right)-\mathrm{C}_{6} \mathrm{H}_{4}-\mathrm{IO}$ in a minimum amount of DCM.

General Procedure for OAT from $\left[\left({ }^{\mathrm{NHC}} \mathrm{L}\right) \mathrm{FeO}(\mathrm{MeCN})\right](\mathrm{OTf})_{2}$ (2) toward $\mathrm{PMe}_{3}$ as Followed by UV/vis Spectroscopy

A $0.2 \mathrm{mM}$ solution of $\left[\left({ }^{\mathrm{NHC}} \mathrm{L}\right) \mathrm{Fe}(\mathrm{MeCN})_{2}\right](\mathrm{OTf})_{2}(0.31 \mathrm{mg}, 0.40 \mu \mathrm{mol})$ in $\mathrm{MeCN}(2 \mathrm{~mL})$ in a cuvette was cooled to $-40{ }^{\circ} \mathrm{C}$. A precooled and freshly prepared solution of 2-( $\left.{ }^{t} \mathrm{BuSO}_{2}\right)-\mathrm{C}_{6} \mathrm{H}_{4}-\mathrm{IO}$ (0.38 mg, $0.11 \mu \mathrm{mol}, 2.8$ eq.) in DCM $(100 \mu \mathrm{L})$ was added and the cuvette was transferred out of the glove box and the $\mathrm{UV} /$ vis absorption measurement was started at $0{ }^{\circ} \mathrm{C}$. The appropriate amount of $\mathrm{PMe}_{3}(100-400$ eq.) was dissolved in $\mathrm{MeCN}$ to give $100 \mu \mathrm{L}$ in a glove box which was then added to the cuvette using a syringe. The subsequent increase of the characteristic absorption band of bis-phosphinoiron(II) 7 (346 nm) was monitored and fitted to a single exponential function.

\section{General Procedure for UV/vis Studies on the Reactivity of $\left[\left\{\left({ }^{\mathrm{NHC}} \mathrm{L}\right) \mathrm{Fe}\right\}_{2} \mathrm{O}\right](\mathrm{OTf})_{4}(3)$ toward $\mathrm{PMe}_{3}$}

A $0.1 \mathrm{mM}$ solution of $\left.\left[\left\{{ }^{\mathrm{NHC}} \mathrm{L}\right) \mathrm{Fe}\right\}_{2} \mathrm{O}\right](\mathrm{OTf})_{4}(0.30 \mathrm{mg}, 0.20 \mu \mathrm{mol})$ in $\mathrm{MeCN}(2 \mathrm{~mL})$ in a cuvette was transferred out of the glove box and the UV/vis absorption measurement at $0{ }^{\circ} \mathrm{C}$ was started. All four experiments were started simultaneously from the same stock solution. The appropriate amount of $\mathrm{PMe}_{3}$ (60-240 eq.) was dissolved in a glove box in $\mathrm{MeCN}$ to give $100 \mu \mathrm{L}$ which was then added to the cuvette using a syringe. Due to the long reaction time, the cuvette was stored in the fridge $\left(0{ }^{\circ} \mathrm{C}\right)$ and the $\mathrm{UV} / \mathrm{vis}$ spectrum was measured about twice a day. The subsequent decrease of the characteristic absorption band of $\mu$-oxoiron(III) 3 (367 nm) was monitored and fitted to a linear function.

\section{Characterization of $\left[\left({ }^{\mathrm{NHC}} \mathrm{L}\right) \mathrm{Fe}\left(\mathrm{PMe}_{3}\right)_{2}\right](\mathrm{OTf})_{2}(7)$}

${ }^{1} \mathrm{H}-\mathrm{NMR}\left(300 \mathrm{MHz}, \mathrm{MeCN}-\mathrm{d}_{3}\right): \delta=0.69\left(\mathrm{~s}, 18 \mathrm{H} ; \mathrm{CH}_{3}\right), 3.98\left(\mathrm{~d},{ }^{2} \mathrm{~J}_{\mathrm{H}, \mathrm{H}}=14.7 \mathrm{~Hz}, 4 \mathrm{H} ; \mathrm{C}_{2} H_{4}\right), 4.41$ $\left(\mathrm{d},{ }^{2} J_{\mathrm{H}, \mathrm{H}}=14.7 \mathrm{~Hz}, 4 \mathrm{H} ; \mathrm{C}_{2} H_{4}\right), 5.43\left(\mathrm{~d},{ }^{2} J_{\mathrm{H}, \mathrm{H}}=13.1 \mathrm{~Hz}, 4 \mathrm{H} ; \mathrm{CH}_{2}\right), 6.15\left(\mathrm{~d},{ }^{2} J_{\mathrm{H}, \mathrm{H}}=13.3 \mathrm{~Hz}, 2 \mathrm{H}\right.$; $\left.\mathrm{CH}_{2}\right), 7.15\left(\mathrm{~d},{ }^{3} J_{\mathrm{H}, \mathrm{H}}=2.0 \mathrm{~Hz}, 4 \mathrm{H} ; \mathrm{CH}\right), 7.39(\mathrm{~s}, 4 \mathrm{H} ; \mathrm{CH}) ;{ }^{13} \mathrm{C} \mathrm{NMR}\left(75 \mathrm{MHz}, \mathrm{MeCN}-\mathrm{d}_{3}\right): \delta=21.61$ 
$\left(\mathrm{CH}_{3}\right), 55.16\left(\mathrm{C}_{2} \mathrm{H}_{4}\right), 62.47\left(\mathrm{CH}_{2}\right), 123.19(\mathrm{CH}), 128.38(\mathrm{CH}), 194.88(\mathrm{NCFe}) .{ }^{31} \mathrm{P}$ NMR $(121 \mathrm{MHz}$, $\left.\mathrm{MeCN}-\mathrm{d}_{3}\right): 22.7$.

(+)-ESI-MS: $m / z=202.1\left[\left({ }^{\mathrm{NHC}} \mathrm{L}\right) \mathrm{Fe}\right]^{2+}, 278.1\left[\left({ }^{\mathrm{NHC}} \mathrm{L}\right) \mathrm{Fe}\left(\mathrm{PMe}_{3}\right)_{2}\right]^{2+}, 553.1\left[\left({ }^{\mathrm{NHC}} \mathrm{L}\right) \mathrm{Fe}\right](\mathrm{OTf})^{+}, 705.1$ $\left[\left({ }^{\mathrm{NHC}} \mathrm{L}\right) \mathrm{Fe}\left(\mathrm{PMe}_{3}\right)_{2}\right](\mathrm{OTf})^{+}$.

\section{General Procedure for the Reaction of $\left[\left\{\left({ }^{\mathrm{NHC}} \mathrm{L}\right) \mathrm{Fe}\right\}_{2} \mathrm{O}\right](\mathrm{OTf})_{4}(3)$ with DHA Studied via NMR Spectroscopy}

$18 \mathrm{mg}$ of $\left[\left\{\left({ }^{\mathrm{NHC}} \mathrm{L}\right) \mathrm{Fe}\right\}_{2} \mathrm{O}\right](\mathrm{OTf})_{4}(12 \mu \mathrm{mol})$ were solved in $\mathrm{MeCN}_{-} \mathrm{d}_{3}(2 \mathrm{~mL})$ and transferred into 4 Young-NMR tubes in $500 \mu \mathrm{L}$ portions. Dihydroanthracene $(15.1 \mathrm{mg}, 84 \mu \mathrm{mol})$ was solved in $\mathrm{CDCl}_{3}(400 \mu \mathrm{L})$ and added to the 4 NMR tubes in $100 \mu \mathrm{L}$ portions each. Additional amount of $\left[\left({ }^{\mathrm{NHC}} \mathrm{L}\right) \mathrm{Fe}(\mathrm{MeCN})_{2}\right](\mathrm{OTf})_{2}$ was added (0-6.4 eq.) as solid material and the NMR tubes were transferred out of the glove box. Due to the long reaction time, the NMR tube was stored at RT and the spectrum was measured about every second day. Formation of anthracene was followed using the line fitting method in MestReNova.

\section{Synthesis of $\left[\left({ }^{\mathrm{NHC}} \mathrm{L}\right) \mathrm{Fe}\left(\mathrm{N}_{3}\right)_{2}\right](5)$}

The synthesis was slightly changed in comparison to the description by Iris Klawitter. ${ }^{[155]} \mathrm{A}$ solution of $\left[\left({ }^{\mathrm{NHC}} \mathrm{L}\right) \mathrm{Fe}(\mathrm{MeCN})_{2}\right](\mathrm{OTf})_{2}(30 \mathrm{mg}, 38 \mu \mathrm{mol}, 1.0$ eq.) in $\mathrm{DMF}(4 \mathrm{~mL})$ was precooled to $-35{ }^{\circ} \mathrm{C}$ and $\left[{ }^{n} \mathrm{Bu}_{4} \mathrm{~N}\right] \mathrm{N}_{3}(22 \mathrm{mg}, 76 \mu \mathrm{mol}, 2.0$ eq.) was added in one portion. Precipitation of a bright green solid was fulfilled by addition of $\mathrm{Et}_{2} \mathrm{O}(4 \mathrm{~mL})$. The solution was removed and the precipitate was washed with $\mathrm{Et}_{2} \mathrm{O}$ and THF (19 mg, $38 \mu \mathrm{mol}$, quant.). Diffusion of $\mathrm{Et}_{2} \mathrm{O}$ into a $\mathrm{MeCN}$ solution gave both green (5) and red $\left(\mathbf{5}_{\mathrm{MeCN}}\right)$ crystals suitable for X-ray diffraction.

EA: Calcd. [\%] for $\mathrm{C}_{18} \mathrm{H}_{20} \mathrm{~N}_{14} \mathrm{Fe}$ (5): C 44.28; H 4.13; N 40.16. Found: C 43.95, H 4.39, N 39.52.

Further analysis has already been performed by Iris Klawitter. ${ }^{[155]}$

\section{Synthesis of $\left[\left({ }^{\mathrm{NHC}} \mathrm{L}\right) \mathrm{Fe}(\mathrm{MeCN})_{2}\right](\mathrm{OTf})_{3}(9)$}

$\left[\left({ }^{\mathrm{NHC}} \mathrm{L}\right) \mathrm{Fe}(\mathrm{MeCN})_{2}\right](\mathrm{OTf})_{2}(10 \mathrm{mg}, 13 \mu \mathrm{mol}, 1.0$ eq. $)$ was dissolved in $\mathrm{MeCN}(2 \mathrm{~mL})$ and cooled to $-35^{\circ} \mathrm{C}$ in a glove box. $\left[\mathrm{N}\left(\mathrm{C}_{6} \mathrm{H}_{4}-4-\mathrm{Br}\right)_{3}\right] \mathrm{SbCl}_{6}(11 \mathrm{mg}, 13 \mu \mathrm{mol}, 1.0$ eq. $)$ was added, and the reactants were properly mixed for $2 \mathrm{~min}$ at $\mathrm{RT}$ while the mixture turned purple. Diffusion of $\mathrm{Et}_{2} \mathrm{O}$ into the reaction mixture at $-35^{\circ} \mathrm{C}$ led to formation of purple crystals suitable for X-ray diffraction. The crystalline product was washed with $\mathrm{Et}_{2} \mathrm{O}$ and dried under vacuum ( $6 \mathrm{mg}, 6.4 \mu \mathrm{mol}, 49 \%$ ). IR (solid, ATR): $\tilde{v}\left[\mathrm{~cm}^{-1}\right]=3113$ (w), 2290 (w), 1490 (w), 1252 (s), 1224 (s), 1138 (s), 1026 (s), $747(\mathrm{~s}), 698(\mathrm{~m}), 635(\mathrm{~s}), 571(\mathrm{~s}), 515(\mathrm{~s})$.

$\operatorname{MB}(80 \mathrm{~K}): \delta=0.09 \mathrm{~mm} \mathrm{~s}^{-1} ; \Delta E_{\mathrm{Q}}=0.63 \mathrm{~mm} \mathrm{~s}^{-1}$.

EPR $(143 \mathrm{~K}): g=2.037, \mathrm{lw}=8.0 \mathrm{G}$.

The UV/vis spectrum has been already reported by Iris Klawitter. ${ }^{[155]}$ 


\section{Synthesis of $\left[\left({ }^{\mathrm{NHC}} \mathrm{L}\right) \mathrm{FeCl}\right](\mathrm{X})_{2}(8), \mathrm{X}=\mathrm{OTf}$ or $\mathrm{SbCl}_{6}$}

The synthetic strategy reported by Iris Klawitter ${ }^{[155]}$ was varied in order to obtain clean crystals of $\mathbf{8}$ faster and in higher yield. [( $\left.\left.{ }^{\mathrm{NHC}} \mathrm{L}\right) \mathrm{Fe}(\mathrm{MeCN})_{2}\right](\mathrm{OTf})_{2}(14 \mathrm{mg}, 17.8 \mu \mathrm{mol}, 1.0$ eq. $)$ was dissolved in $\mathrm{MeCN}(1 \mathrm{~mL})$ at RT in a glove box. [N( $\left.\mathrm{C}_{6} \mathrm{H}_{4}-4-\mathrm{Br}_{3}\right)_{3} \mathrm{SbCl}_{6}(15 \mathrm{mg}, 18.4 \mu \mathrm{mol}, 1.0$ eq. $)$ was added and the mixture was allowed to react for $5 \mathrm{~min}$ at $-35^{\circ} \mathrm{C}$. $\left[{ }^{n} \mathrm{Bu} 4 \mathrm{~N}\right] \mathrm{Cl}(5.3 \mathrm{mg}, 19 \mu \mathrm{mol}, 1.1 \mathrm{eq}$. $)$ was added to the brown solution leading to a spontaneous color change to yellow. Diffusion of $\mathrm{Et}_{2} \mathrm{O}$ into the reaction mixture at $\mathrm{RT}$ led to formation of orange crystals that were washed with $\mathrm{Et}_{2} \mathrm{O}$ and dried under vacuum ( $11 \mathrm{mg}, 15 \mu \mathrm{mol}, 83 \%$, calculated for $\mathrm{X}=\mathrm{OTf}$ ).

IR (solid, ATR): $\tilde{v}\left[\mathrm{~cm}^{-1}\right]=3129(\mathrm{w}), 1480(\mathrm{~m}), 1247$ (s), 1221 (s), 1191 (m), 1148 (s), 1125 (s), $754(\mathrm{~s}), 681(\mathrm{~m}), 633(\mathrm{~s}), 573(\mathrm{~s}), 516(\mathrm{~s})$.

HRMS ((+)-ESI, MeOH): $m / z=588.0365$ (calcd. 588.0364 for $\left.\left[\mathrm{C}_{19} \mathrm{H}_{20} \mathrm{~N}_{8} \mathrm{ClF}_{3} \mathrm{FeO}_{3} \mathrm{~S}_{1}\right]^{+}\right) ; 219.5418$ (calcd. 219.5419 for $\left[\mathrm{C}_{18} \mathrm{H}_{20} \mathrm{~N}_{8} \mathrm{Cl}\right]^{2+}$ ).

EA: Calcd. [\%] for $\mathrm{C}_{20} \mathrm{H}_{20} \mathrm{~N}_{8} \mathrm{ClF}_{6} \mathrm{FeO}_{6} \mathrm{~S}_{2}$ : C 32.56; H 2.73; N 15.19; S 8.69. Found: C 32.51; H 2.71; N 15.15; S 8.47.

$\operatorname{EPR}(160 \mathrm{~K}): g_{\mathrm{x}}(\mathrm{lw}[\mathrm{G}])=4.300(600), g_{\mathrm{y}}=2.095(800), g_{\mathrm{z}}=2.095(300)$.

Further analysis has already been performed by Iris Klawitter. ${ }^{[155]}$

\section{Synthesis of $\left[\left({ }^{\mathrm{NHC}} \mathbf{L}\right) \mathrm{FeCl}\left(\mathrm{N}_{3}\right)\right](\mathrm{OTf})(\mathbf{1 0})$ and $\left[\left({ }^{\mathrm{NHC}} \mathbf{L}\right) \mathrm{Fe}\left(\mathrm{N}_{3}\right)_{2}\right](\mathrm{Cl})(11)$}

Generation of 11: [ $\left.\left({ }^{\mathrm{NHC}} \mathrm{L}\right) \mathrm{FeCl}\right](\mathrm{OTf})_{2}(15.0 \mathrm{mg}, 20 \mu \mathrm{mol}, 1.0$ eq. $)$ was dissolved in $\mathrm{MeCN}(2 \mathrm{~mL})$, $\left[{ }^{n} \mathrm{Bu}_{4} \mathrm{~N}\right] \mathrm{N}_{3}$ (12.6 mg, $44 \mu \mathrm{mol}, 2.2$ eq.) was added and the mixture was stored at $-35^{\circ} \mathrm{C}$ for $3 \mathrm{~h}$. During this time, the mixture changed its color from orange to pink. Precipitation with $\mathrm{Et}_{2} \mathrm{O}$ led to a pink crude product ( $8 \mathrm{mg}, 15 \mu \mathrm{mol}, 76 \%$ ). Diffusion of $\mathrm{Et}_{2} \mathrm{O}$ into a $\mathrm{MeCN}$ solution of the crude product at $-35{ }^{\circ} \mathrm{C}$ led to formation of single crystals suitable for X-ray diffraction.

10 was synthesized in an analog procedure using only 1 eq. of $\left[{ }^{n} \mathrm{Bu}_{4} \mathrm{~N}\right] \mathrm{N}_{3}$.

Spectroscopic data for 10:

IR (solution, MeCN, ATR): $\tilde{v}\left[\mathrm{~cm}^{-1}\right]=3170$ (w), 3141 (w), 2025 (m), 1685 (w), 1404 (w), 1272 (s), $1155(\mathrm{~m}), 1032(\mathrm{~m})$.

$\mathrm{UV} / \mathrm{vis}$ (solution, MeCN): $\lambda_{\max }[\mathrm{nm}]\left(\varepsilon_{\text {rel }}\left[\mathrm{L} \mathrm{mol}^{-1} \mathrm{~cm}^{-1}\right]\right)=321$ (10000), 443 (4400), 526 (5400).

MB: $\delta=0.16 \mathrm{~mm} \mathrm{~s}^{-1} ; \Delta E_{\mathrm{Q}}=3.71 \mathrm{~mm} \mathrm{~s}^{-1}$.

Spectroscopic data for 11:

${ }^{1} \mathrm{H}-\mathrm{NMR}\left(300 \mathrm{MHz}, \mathrm{MeCN}-\mathrm{d}_{3}\right.$ ): $\delta=21.50$ (b, 2H, CH), 8.94 (b, 4H, CH), 7.49 (b, 2H, CH), -2.38 (b, $4 \mathrm{H}, \mathrm{CH}_{2}$ ), -5.29 (bs, $\left.4 \mathrm{H}, \mathrm{C}_{2} \mathrm{H}_{4}\right),-6.15$ (bs, 4H, $\mathrm{C}_{2} \mathrm{H}_{4}$ ).

IR (solution, MeCN, ATR): $\tilde{v}\left[\mathrm{~cm}^{-1}\right]=3170$ (w), 3141 (w), 2400 (m), 2025 (s), 1685 (w), 1404 (w), $1272(\mathrm{~s}), 1155(\mathrm{~m}), 1032(\mathrm{~m})$.

UV/vis (solution, MeCN): $\lambda_{\max }[\mathrm{nm}]\left(\varepsilon_{\text {rel }}\left[\mathrm{L} \mathrm{mol}^{-1} \mathrm{~cm}^{-1}\right]\right)=301$ (11600), 337 (12200), 544 (7900).

$(+)$-ESI-MS (MeCN): $m / z=488.1\left[\left({ }^{\mathrm{NHC}} \mathrm{L}\right) \mathrm{Fe}\left(\mathrm{N}_{3}\right)_{2}\right]^{+}$.

MB: $\delta=0.14 \mathrm{~mm} \mathrm{~s}^{-1} ; \Delta E_{\mathrm{Q}}=3.41 \mathrm{~mm} \mathrm{~s}^{-1}$. 
EPR (10 K): $g(1 \mathrm{w}[\mathrm{G}])=2.700(380), 1.990(230), 1.676(500)$.

\section{Synthesis of $\left[\left\{\left({ }^{\mathrm{NHC}} \mathrm{L}\right) \mathrm{Fe}\right\}_{2} \mathrm{~N}\right](\mathrm{OTf})_{3}(6)$}

The synthesis was performed according to the description by Iris Klawitter. ${ }^{[155]}\left[\left({ }^{\mathrm{NHC}} \mathrm{L}\right) \mathrm{Fe}\right](\mathrm{OTf})_{2}$ (50 mg, $64.0 \mu \mathrm{mol}, 1.0$ Äq.) was dissolved in DMF (3 mL), [ ${ }^{n} \mathrm{Bu}_{4} \mathrm{~N} \mathrm{~N}_{3}(5.6 \mathrm{mg}, 32 \mu \mathrm{mol}, 0.5$ eq.) was added and the mixture was stirred at $\mathrm{RT}$ for at least $4 \mathrm{~d} \mathrm{Et}_{2} \mathrm{O}(10 \mathrm{~mL})$ was added to the resulting green-blue solution and the product was obtained as dark green powder $(56 \mathrm{mg}, 44 \mu \mathrm{mol}$, $69 \%$ ). Recrystallization by diffusion of $\mathrm{Et}_{2} \mathrm{O}$ into a $\mathrm{MeCN}$ solution of $\mathbf{6}$ yielded crystals suitable for $\mathrm{X}$-ray diffraction.

a: ${ }^{1} \mathrm{H}$ NMR ( $\left.800 \mathrm{MHz}, \mathrm{MeCN}-\mathrm{d}_{3}\right): \delta=7.37$ (s, 4H; $\mathrm{CH}_{\mathrm{NHC}-\mathrm{Me}}$ ), 7.07 (s, 4H; CH $H_{\mathrm{NHC}-\mathrm{Et}}$ ), 6.08 (d, $J=$ $\left.12.8 \mathrm{~Hz}, 2 \mathrm{H} ; \mathrm{CH}_{2}\right), 5.23\left(\mathrm{~d}, J=12.7 \mathrm{~Hz}, 2 \mathrm{H} ; \mathrm{CH}_{2}\right), 4.84\left(\mathrm{dd}, J=14.3,7.5 \mathrm{~Hz}, 4 \mathrm{H} ; \mathrm{C}_{2} H_{4}\right), 3.56(\mathrm{dd}$, $\left.J=14.3,7.4 \mathrm{~Hz}, 4 \mathrm{H} ; \mathrm{C}_{2} H_{4}\right) ;{ }^{13} \mathrm{C} \mathrm{NMR}\left(201 \mathrm{MHz}, \mathrm{MeCN}^{-} \mathrm{d}_{3}\right): \delta=187.5\left(C_{\mathrm{NHC}}\right), 123.3\left(\mathrm{CH}_{\mathrm{NHC}-\mathrm{Et}}\right)$, $122.5\left(\mathrm{CH}_{\mathrm{NHC}-\mathrm{Me}}\right), 62.8\left(\mathrm{CH}_{2}\right), 47.8\left(C_{2} \mathrm{H}_{4}\right) ;{ }^{14} \mathrm{~N}$ NMR $\left(51 \mathrm{MHz}, \mathrm{MeCN}-\mathrm{d}_{3}\right): \delta=-201.8\left(N_{\mathrm{Me}}\right)$, $-192.7\left(N_{\mathrm{Et}}\right)$.

b: ${ }^{1} \mathrm{H}$ NMR $\left(800 \mathrm{MHz}, \mathrm{MeCN}-\mathrm{d}_{3}\right): \delta=7.09$ (br, 4H; $\left.\mathrm{CH}_{\mathrm{NHC}-\mathrm{Me}}\right), 7.05$ (br, 4H; $\left.\mathrm{CH}_{\mathrm{NHC}-\mathrm{Et}}\right), 6.28$ (d, $J$ $\left.=12.1 \mathrm{~Hz}, 2 \mathrm{H} ; \mathrm{CH}_{2}\right), 5.54\left(\mathrm{~d}, \mathrm{~J}=12.1 \mathrm{~Hz}, 2 \mathrm{H} ; \mathrm{CH}_{2}\right), 4.42\left(\mathrm{br}, 4 \mathrm{H} ; \mathrm{C}_{2} \mathrm{H}_{4}\right), 4.33\left(\mathrm{br}, 4 \mathrm{H} ; \mathrm{C}_{2} \mathrm{H}_{4}\right) ;{ }^{13} \mathrm{C}$ NMR (201 MHz, MeCN-d 3$): \delta=123.9$ (br, $\left.C \mathrm{H}_{\mathrm{NHC}-\mathrm{Et}}\right), 121.7\left(\mathrm{CH}_{\mathrm{NHC}-\mathrm{Me}}\right), 62.5\left(\mathrm{CH}_{2}\right)$. It was not possible to assign the carbon signals of $C_{\mathrm{NHC}}$ and $\mathrm{C}_{2} \mathrm{H}_{4} \cdot{ }^{14} \mathrm{~N} \mathrm{NMR}\left(51 \mathrm{MHz}, \mathrm{MeCN}-\mathrm{d}_{3}\right.$ ): no assignment was possible.

c: ${ }^{1} \mathrm{H}$ NMR (800 MHz, MeCN-d 3 ): $\delta=7.32$ (s, 4H; $\mathrm{CH}_{\text {NHC-Me }}$ ), 7.00 (s, 4H; $\mathrm{CH}_{\text {NHC-Et }}$ ), 6.03 (d, $J=$ $\left.12.7 \mathrm{~Hz}, 2 \mathrm{H} ; \mathrm{CH}_{2}\right), 5.11$ (d, $\left.J=12.6 \mathrm{~Hz}, 2 \mathrm{H} ; \mathrm{CH}_{2}\right), 4.98\left(\mathrm{br}, 4 \mathrm{H} ; \mathrm{C}_{2} H_{4}\right), 3.83(\mathrm{td}, J=9.8,3.6 \mathrm{~Hz}$, $\left.4 \mathrm{H} ; \mathrm{C}_{2} H_{4}\right) ;{ }^{13} \mathrm{C}$ NMR $\left(201 \mathrm{MHz}, \mathrm{MeCN}-\mathrm{d}_{3}\right): \delta=186.2\left(\mathrm{br}, \mathrm{C}_{\mathrm{NHC}}\right), 123.2\left(\mathrm{CH}_{\mathrm{NHC}-\mathrm{Et}}\right), 122.4\left(\mathrm{CH}_{\mathrm{NHC}-}\right.$ Me), $62.6\left(\mathrm{CH}_{2}\right), 47.9\left(\mathrm{br}, \mathrm{C}_{2} \mathrm{H}_{4}\right) ;{ }^{14} \mathrm{~N}$ NMR (51 MHz, MeCN-d 3 ): $\delta=-202.4\left(N_{\mathrm{Me}}\right),-193.2\left(N_{\mathrm{Et}}\right)$.

d: ${ }^{1} \mathrm{H}$ NMR $\left(800 \mathrm{MHz}, \mathrm{MeCN}-\mathrm{d}_{3}\right): \delta=7.15$ (s, 4H; $\mathrm{CH}_{\text {NHC-Et }}$ ), 7.09 (s, 4H; $\mathrm{CH}_{\text {NHC-Me }}$ ), 5.90 (d, $J=$ $\left.12.3 \mathrm{~Hz}, 2 \mathrm{H} ; \mathrm{CH}_{2}\right), 5.27$ (d, $\left.J=12.2 \mathrm{~Hz}, 2 \mathrm{H} ; \mathrm{CH}_{2}\right), 4.43\left(\mathrm{~s}, 4 \mathrm{H} ; \mathrm{C}_{2} \mathrm{H}_{4}\right) ;{ }^{13} \mathrm{C}$ NMR $(201 \mathrm{MHz}$, MeCN-d $\left.{ }_{3}\right): \delta=123.9$ (br, $\left.C \mathrm{H}_{\mathrm{NHC}-\mathrm{Et}}\right), 121.6\left(\mathrm{CH}_{\mathrm{NHC}-\mathrm{Me}}\right), 62.3\left(\mathrm{CH}_{2}\right), 50.6$ (br, $\left.\mathrm{C}_{2} \mathrm{H}_{4}\right)$. It was not possible to assign the proton and carbon signals of $\mathrm{C}_{2} H_{4}$ and $C_{\mathrm{NHC}}$, respectively. ${ }^{14} \mathrm{~N}$ NMR (51 $\left.\mathrm{MHz}, \mathrm{MeCN}-\mathrm{d}_{3}\right): \delta=-193.3\left(N_{\mathrm{Me}}\right),-188.74\left(N_{\mathrm{Et}}\right)$.

DOSY: $f_{1}=7.9 \cdot 10^{-6}$ diffusion units for all signals.

IR (solid, ATR): $\tilde{v}\left(\mathrm{~cm}^{-1}\right)=3110(\mathrm{~m}), 1667$ (m), 1578 (w), 1561 (w), $1466(\mathrm{w}), 1418(\mathrm{~m}), 1404$ (m), 1336 (w), 1249 (s), 1220 (m), 1184 (m), 1145 (s), 1110 (w), 1089 (w), 1026 (s), 959 (m), 811 (m), $756(\mathrm{w}), 731(\mathrm{~m}), 718(\mathrm{~m}), 684(\mathrm{~m}), 635(\mathrm{~s}), 571(\mathrm{~m}), 516(\mathrm{~s})$.

EA: Calcd. (\%) for $\mathrm{C}_{39} \mathrm{H}_{40} \mathrm{~N}_{17} \mathrm{~F}_{9} \mathrm{Fe}_{2} \mathrm{O}_{9} \mathrm{~S}_{3}+1 \mathrm{CH}_{3} \mathrm{CN}+0.5 \mathrm{C}_{4} \mathrm{H}_{10} \mathrm{O}$ : C 38.32; H 3.59; N 18.71 . Found: C 38.43; H 3.89; N 18.82.

$\mathrm{MB}(80 \mathrm{~K}, \mathrm{MeCN}): \delta=-0.07 \mathrm{~mm} \mathrm{~s}^{-1} ; \Delta E_{\mathrm{Q}}=1.35 \mathrm{~mm} \mathrm{~s}^{-1}$.

Further analysis has already been performed by Iris Klawitter. ${ }^{[155]}$ 


\section{Synthesis of $\left[\left({ }^{\mathrm{NHC}} \mathrm{L}\right) \mathrm{Fe}^{\mathrm{III}}-\mathrm{N}-\mathrm{Fe}^{\mathrm{IV}}\left({ }^{\mathrm{NHC}} \mathrm{L}\right)(\mathrm{MeCN})\right](\mathrm{OTf})_{3}\left(\mathrm{SbF}_{6}\right)(12)$}

$\left[\left\{\left({ }^{\mathrm{NHC}} \mathrm{L}\right) \mathrm{Fe}\right\}_{2} \mathrm{~N}\right](\mathrm{OTf})_{2}(11.9 \mathrm{mg}, 9.4 \mu \mathrm{mol}, 1.0$ eq. $)$ was dissolved in $\mathrm{MeCN}(2 \mathrm{~mL})$, precooled to $-35{ }^{\circ} \mathrm{C}$ and $\mathrm{AgSbF}_{6}(3.2 \mathrm{mg}, 9.4 \mu \mathrm{mol}, 1.0$ eq.) was added. The mixture immediately turned redbrown and a black precipitate was formed. The suspension was kept at $-35{ }^{\circ} \mathrm{C}$ for $10 \mathrm{~min}$ and filtered through dry celite in a glove box. Precipitation with $\mathrm{Et}_{2} \mathrm{O}$ led to the reddish crude product that was washed with $\mathrm{Et}_{2} \mathrm{O}(3 \times 3 \mathrm{~mL})(10.5 \mathrm{mg}, 6.6 \mu \mathrm{mol}, 70 \%)$. Diffusion of $\mathrm{Et}_{2} \mathrm{O}$ into a DMF solution of the crude product at RT did lead to formation of single crystals suitable for X-ray diffraction.

IR (solid, ATR): $\tilde{v}\left(\mathrm{~cm}^{-1}\right)=3131(\mathrm{~m}), 1579(\mathrm{w}), 1566$ (w), $1463(\mathrm{w}), 1425(\mathrm{~m}), 1410(\mathrm{~m}), 1253(\mathrm{~s})$, 1225 (m), 1158 (m), 1028 (s), 892 (m), 814 (w), 735 (m), 689 (w), 659 (m), 636 (s), 574 (w), 518 (m).

IR (solution, MeCN, Dial Path IR): $\tilde{v}\left(\mathrm{~cm}^{-1}\right)=2254$ (m), 2250 (s), 2031 (w), 1384 (w), 1339 (w), 1272 (s), 1225 (w), 1156 (m), 1033 (m), 889 (w), 802 (w), 739 (w), 662 (m).

$\mathrm{UV} /$ vis (solution, MeCN): $\lambda_{\max }[\mathrm{nm}]\left(\varepsilon_{\text {rel }}\left[\mathrm{L} \mathrm{mol}^{-1} \mathrm{~cm}^{-1}\right]\right)=332(\mathrm{sh}), 360$ (10400), 540 (400).

$\mathrm{MB}(7 \mathrm{~K}): \delta=-0.05 \mathrm{~mm} \mathrm{~s}^{-1} ; \Delta E_{\mathrm{Q}}=2.11 \mathrm{~mm} \mathrm{~s}^{-1} \& \delta=-0.11 \mathrm{~mm} \mathrm{~s}^{-1} ; \Delta E_{\mathrm{Q}}=2.40 \mathrm{~mm} \mathrm{~s}^{-1}$. EPR: $g(\mathrm{lw}[\mathrm{G}])=2.095(21), 2.085(30), 2.013(40)$.

\section{Synthesis of $\left[\left({ }^{\mathrm{NHC}} \mathrm{L}\right) \mathrm{Fe}-\mathrm{N}-\mathrm{Fe}\left({ }^{\mathrm{NHC}} \mathrm{L}\right)\left(\mathrm{ONO}_{2}\right)\right]\left(\mathrm{OTf}_{3}(\mathbf{1 3})\right.$}

$\left[\left\{\left({ }^{\mathrm{NHC}} \mathrm{L}\right) \mathrm{Fe}\right\}_{2} \mathrm{~N}\right](\mathrm{OTf})_{2}(11.5 \mathrm{mg}, 9.1 \mu \mathrm{mol}, 1.0$ eq. $)$ was dissolved in $\mathrm{MeCN}(1 \mathrm{~mL})$, precooled to $-35{ }^{\circ} \mathrm{C}$ and $\mathrm{AgNO}_{3}(35 \mu \mathrm{L}$ of a $0.25 \mathrm{M}$ solution in $\mathrm{MeCN}, \sim 1.5 \mathrm{mg}, 9.1 \mu \mathrm{mol}, 1.0$ eq.) was added. The mixture immediately turned reddish brown and a black precipitate was formed. The suspension was kept at $-35{ }^{\circ} \mathrm{C}$ for $10 \mathrm{~min}$ and filtered through dry celite in a glove box. Precipitation with $\mathrm{Et}_{2} \mathrm{O}$ led to the reddish crude product that was washed with $\mathrm{Et}_{2} \mathrm{O}$ and dried under vacuum before using it for a MB measurement (10 mg, $7.5 \mu \mathrm{mol}, 83 \%$ ). Diffusion of $\mathrm{Et}_{2} \mathrm{O}$ into a $\mathrm{MeCN}$ or DMF solution of the crude product at $-35^{\circ} \mathrm{C}$ or RT did not lead to formation of single crystals suitable for X-ray diffraction, so far.

IR (solid, ATR): $\tilde{v}\left(\mathrm{~cm}^{-1}\right)=3113(\mathrm{w}), 1581(\mathrm{w}), 1565$ (w), $1423(\mathrm{~m}), 1409$ (m), 1342 (w), 1336 (w), 1248 (s), 1222 (m), 1187 (m), 1150 (s), 1026 (s), 889 (m), 813 (m), 729 (m), 689 (m), 634 (s), $572(\mathrm{w}), 515(\mathrm{~m})$.

IR (solution, MeCN, Dial Path IR): $\tilde{v}\left(\mathrm{~cm}^{-1}\right)=2298(\mathrm{w}), 2258(\mathrm{~m}), 2250(\mathrm{~s}), 2242(\mathrm{w}), 1685(\mathrm{w})$, 1566 (w), 1447 (m), 1428 (m), 1376 (s), 1343 (m), 1274 (s), 1225 (w), 1156 (m), 1033 (m), 920 (w), $900(\mathrm{w}), 743(\mathrm{~m}), 693(\mathrm{w})$.

$\mathrm{UV} / \mathrm{vis}$ (solution, MeCN): $\lambda_{\max }[\mathrm{nm}]\left(\varepsilon_{\text {rel }}\left[\mathrm{L} \mathrm{mol}^{-1} \mathrm{~cm}^{-1}\right]\right)=332$ (sh), 360 (9600), 540 (400).

$\mathrm{MB}(7 \mathrm{~K}): \delta=-0.06 \mathrm{~mm} \mathrm{~s}^{-1} ; \Delta E_{\mathrm{Q}}=1.96 \mathrm{~mm} \mathrm{~s}^{-1} \& \delta=-0.10 \mathrm{~mm} \mathrm{~s}^{-1} ; \Delta E_{\mathrm{Q}}=2.74 \mathrm{~mm} \mathrm{~s}^{-1}$. EPR: $g(\mathrm{lw}[\mathrm{G}])=2.0935(23), 2.0802(30), 2.0100$ (40). 


\section{Synthesis of $\left[\left\{\left({ }^{\mathrm{NHC}} \mathrm{L}\right) \mathrm{Fe}\left(\mathrm{ONO}_{2}\right)\right\}_{2} \mathrm{~N}\right](\mathrm{OTf})_{3}(14)$}

$\left[\left\{\left({ }^{\mathrm{NHC}} \mathrm{L}\right) \mathrm{Fe}\right\}_{2} \mathrm{~N}\right](\mathrm{OTf})_{2}(24.9 \mathrm{mg}, 19.6 \mu \mathrm{mol}, 1.0$ eq. $)$ was dissolved in $\mathrm{MeCN}(2 \mathrm{~mL})$, precooled to $-35^{\circ} \mathrm{C}$ and $\mathrm{AgNO}_{3}$ (7.0 mg, $41.2 \mu \mathrm{mol}, 2.1$ eq.) was added. The mixture immediately turned brown and a black precipitate was formed. The suspension was kept at $-35{ }^{\circ} \mathrm{C}$ for $10 \mathrm{~min}$ and filtered through dry celite in a glove box. Precipitation with $\mathrm{Et}_{2} \mathrm{O}$ led to formation of the brownish crude product that was washed with $\mathrm{Et}_{2} \mathrm{O}$ and dried under vacuum before using it for a $\mathrm{MB}$ measurement $(21 \mathrm{mg}, 15.0 \mu \mathrm{mol}, 77 \%)$. Diffusion of $\mathrm{Et}_{2} \mathrm{O}$ into a $\mathrm{MeCN}$ solution of the crude product at $-35{ }^{\circ} \mathrm{C}$ did lead to formation of single crystals suitable for X-ray diffraction.

${ }^{1} \mathrm{H}$ NMR (300 MHz, MeCN-d $\left.{ }_{3}\right): \delta=7.66\left(\mathrm{~d}, J=2.0 \mathrm{~Hz}, 4 \mathrm{H}\right.$; $\left.\mathrm{CH}_{\text {NHC-Me }}\right), 7.37(\mathrm{~d}, J=2.0 \mathrm{~Hz}, 4 \mathrm{H}$; $\left.\left.\mathrm{CH}_{\mathrm{NHC}-\mathrm{Et}}\right), 6.45\left(\mathrm{~d}, J=13.4 \mathrm{~Hz}, 2 \mathrm{H} ; \mathrm{CH}_{2}\right), 5.31\left(\mathrm{~d}, J=13.5 \mathrm{~Hz}, 2 \mathrm{H} ; \mathrm{CH}_{2}\right),\right), 4.52-4.31(\mathrm{~m} 4 \mathrm{H}$; $\left.\mathrm{C}_{2} \mathrm{H}_{4}\right), 4.18-3.93\left(\mathrm{~m}, 4 \mathrm{H} ; \mathrm{C}_{2} \mathrm{H}_{4}\right) ;{ }^{13} \mathrm{C} \mathrm{NMR}\left(75 \mathrm{MHz}, \mathrm{MeCN}-\mathrm{d}_{3}\right): \delta=169.7\left(C_{\mathrm{NHC}}\right), 125.9\left(\mathrm{CH}_{\mathrm{NHC}-}\right.$ Me), $125.7\left(\mathrm{CH}_{\mathrm{NHC}-\mathrm{Et}}\right), 63.2\left(\mathrm{CH}_{2}\right), 48.9\left(C_{2} \mathrm{H}_{4}\right) ;{ }^{14} \mathrm{~N}$ NMR (51 MHz, MeCN-d $\left.\mathrm{d}_{3}\right): \delta=-199.1\left(N_{\mathrm{Me}}\right)$, $-186.5\left(N_{\mathrm{Et}}\right)$. Values are given for the main isomer.

IR (solid, ATR): $\tilde{v}\left(\mathrm{~cm}^{-1}\right)=3119(\mathrm{w}), 1590$ (w), 1572 (w), 1475 (s), 1454 (m), 1426 (m), 1413 (m), 1336 (m), 1252 (s), 1222 (m), 1190 (w), 1150 (s), 1027 (s), 980 (m), 891 (w), 814 (m), 736 (m), $695(\mathrm{~m}), 682(\mathrm{~m}), 634(\mathrm{~s}), 610(\mathrm{w}), 572(\mathrm{~m}), 516(\mathrm{~m})$.

IR (solution, MeCN, Dial Path IR): $\tilde{v}\left(\mathrm{~cm}^{-1}\right)=2297$ (w), 2258 (m), 2250 (s), 1685 (w), 1449 (m), 1377 (s), 1273 (s), 1156 (m), 1033 (m), 748 (m).

$\mathrm{UV} / \mathrm{vis}$ (solution, MeCN): $\lambda_{\max }[\mathrm{nm}]\left(\varepsilon_{\text {rel }}\left[\mathrm{L} \mathrm{mol}^{-1} \mathrm{~cm}^{-1}\right]\right)=365$ (3300).

$\operatorname{MB}(80 \mathrm{~K}): \delta=-0.16 \mathrm{~mm} \mathrm{~s}^{-1} ; \Delta E_{\mathrm{Q}}=3.12 \mathrm{~mm} \mathrm{~s}^{-1}$.

\section{Conversion of 6 with $\mathrm{NOBF}_{4}$}

$\left[\left\{\left({ }^{\mathrm{NHC}} \mathrm{L}\right) \mathrm{Fe}\right\}_{2} \mathrm{~N}\right](\mathrm{OTf})_{2}(13.4 \mathrm{mg}, 10.6 \mu \mathrm{mol}, 1.0$ eq.) was dissolved in $\mathrm{MeCN}(1 \mathrm{~mL})$, precooled to $-35^{\circ} \mathrm{C}$ and $\mathrm{NOBF}_{4}(90 \mu \mathrm{L}$ of a $0.12 \mathrm{M}$ solution in $\mathrm{MeCN}, \sim 1.2 \mathrm{mg}, 10.6 \mu \mathrm{mol}, 1.0$ eq.) was added. The reaction mixture was kept at $-35{ }^{\circ} \mathrm{C}$ for $6 \mathrm{~h}$ while the color changed from green-blue to red. Precipitation with $\mathrm{Et}_{2} \mathrm{O}$ resulted in a red crude product that was washed with $\mathrm{Et}_{2} \mathrm{O}$ and dried under vacuum before using it for a $\mathrm{MB}$ measurement. Assuming that a solvent molecule is coordinating to one iron center, compound 12 is formed: $\left[\left({ }^{\mathrm{NHC}} \mathbf{L}\right) \mathbf{F e}-\mathbf{N}-\mathbf{F e}\left({ }^{\mathrm{NHC}} \mathbf{L}\right)(\mathrm{NCMe})\right](\mathbf{O T f})_{3}\left(\mathrm{BF}_{4}\right)(12 \mathrm{mg}$, $9.1 \mu \mathrm{mol}, 85 \%)$.

$\mathrm{UV} /$ vis (solution, MeCN): $\lambda_{\max }[\mathrm{nm}]\left(\varepsilon_{\mathrm{rel}}\left[\mathrm{L} \mathrm{mol}^{-1} \mathrm{~cm}^{-1}\right]\right)=332$ (sh), 363 (12400), 535 (390).

$\operatorname{MB}(7 \mathrm{~K}): \delta=-0.11 \mathrm{~mm} \mathrm{~s}^{-1}, \Delta E_{\mathrm{Q}}=2.65 \mathrm{~mm} \mathrm{~s}^{-1} \& \delta=-0.05 \mathrm{~mm} \mathrm{~s}^{-1}, \Delta E_{\mathrm{Q}}=2.01 \mathrm{~mm} \mathrm{~s}^{-1}$.

In analogy to this experiment, $6\left(10 \mathrm{mg}, 7.9 \mu \mathrm{mol}, 1.0\right.$ eq.) was also treated with excess $\mathrm{NOBF}_{4}$ (335 $\mu \mathrm{L}$ of a $0.12 \mathrm{M}$ solution in $\mathrm{MeCN}, \sim 4.6 \mathrm{mg}, 39.4 \mu \mathrm{mol}, 5.0$ eq.). Assuming that $\mathrm{MeCN}$ is coordinating to both iron centers, the following compound is formed: $\left[\left\{\left({ }^{\mathbf{N H C}} \mathbf{L}\right) \mathbf{F e}(\mathbf{N C M e})\right\}_{2} \mathbf{N}\right](\mathbf{O T f})_{3}\left(\mathbf{B F}_{4}\right)_{2}(\mathbf{1 5})\left(12 \mathrm{mg}, 7.9 \mu \mathrm{mol}\right.$, quant.). Diffusion of $\mathrm{Et}_{2} \mathrm{O}$ into a DMF solution of the crude product at RT did lead to formation of single crystals suitable for X-ray diffraction. 
${ }^{1} \mathrm{H}$ NMR (300 MHz, MeCN-d 3 ): $\delta=7.78\left(\mathrm{~d}, J=2.0 \mathrm{~Hz}, 4 \mathrm{H} ; \mathrm{CH}_{\mathrm{NHC}-\mathrm{Me}}\right), 7.42(\mathrm{~d}, J=2.0 \mathrm{~Hz}, 4 \mathrm{H}$; $\mathrm{CH}_{\text {NHC-Et }}$ ), $6.70\left(\mathrm{~d}, J=14.3 \mathrm{~Hz}, 2 \mathrm{H} ; \mathrm{CH}_{2}\right), 5.58\left(\mathrm{~d}, J=14.0 \mathrm{~Hz}, 2 \mathrm{H} ; \mathrm{CH}_{2}\right)$, ), 4.38-4.22 (m 4H; $\left.\mathrm{C}_{2} H_{4}\right), 4.11-3.99\left(\mathrm{~m}, 4 \mathrm{H} ; \mathrm{C}_{2} H_{4}\right), 1.96\left(\mathrm{~s}, 6 \mathrm{H} ; \mathrm{CH}_{3} \mathrm{CN}\right) ;{ }^{13} \mathrm{C} \mathrm{NMR}\left(75 \mathrm{MHz}, \mathrm{MeCN}-\mathrm{d}_{3}\right): \delta=163.7$ $\left(C_{\mathrm{NHC}}\right), 126.9\left(\mathrm{CH}_{\mathrm{NHC}-\mathrm{Me}}\right), 126.8\left(\mathrm{CH}_{\mathrm{NHC}-\mathrm{Et}}\right), 63.6\left(\mathrm{CH}_{2}\right), 49.0\left(C_{2} \mathrm{H}_{4}\right)$. Values are given for the main isomer.

IR (solid, ATR): $\tilde{v}\left(\mathrm{~cm}^{-1}\right)=3121(\mathrm{~m}), 1588(\mathrm{w}), 1577(\mathrm{w}), 1463(\mathrm{w}), 1428(\mathrm{~m}), 1411(\mathrm{~m}), 1335$ (w), 1251 (s), 1224 (m), 1152 (m), 1056 (w), 1026 (s), 888 (m), 814 (w), 736 (m), 694 (m), 684 (m), $636(\mathrm{~s}), 574(\mathrm{~m}), 516(\mathrm{~m})$.

IR (solution, MeCN, Dial Path IR): $\tilde{v}\left(\mathrm{~cm}^{-1}\right)=3175(\mathrm{~m}), 3134(\mathrm{~m}), 3003(\mathrm{~m}), 2946(\mathrm{~m}), 2318(\mathrm{w})$, 2296 (m), 1480 (w), 1428 (w), 1338 (w), 1272 (s), 1156 (m), 1063 (m), 1032 (s), 890 (w), 741 (w), $696(\mathrm{w})$.

UV/vis (solution, MeCN): $\lambda_{\max }[\mathrm{nm}]\left(\varepsilon_{\text {rel }}\left[\mathrm{Lmol}^{-1} \mathrm{~cm}^{-1}\right]\right)=292$ (9400), 365 (9800).

$\operatorname{MB}(7 \mathrm{~K}): \delta=-0.14 \mathrm{~mm} \mathrm{~s}^{-1}, \Delta E_{\mathrm{Q}}=2.84 \mathrm{~mm} \mathrm{~s}^{-1}$.

\section{Conversion of $\left[\left({ }^{\mathrm{NHC}} \mathrm{L}\right) \mathrm{Fe}(\mathrm{MeCN})_{2}\right](\mathrm{OTf})_{2}(1)$ with $\mathrm{XeF}_{2}$}

$\left[\left({ }^{\mathrm{NHC}} \mathrm{L}\right) \mathrm{Fe}(\mathrm{MeCN})_{2}\right](\mathrm{OTf})_{2}(27.7 \mathrm{mg}, 35.3 \mu \mathrm{mol}, 1$ eq. $)$ was dissolved in $\mathrm{MeCN}(2 \mathrm{~mL})$, cooled to $-35^{\circ} \mathrm{C}$ and $\mathrm{XeF}_{2}(6.0 \mathrm{mg}, 35.3 \mu \mathrm{mol}, 1$ eq.) was added in one portion. The mixture was kept at $-35^{\circ} \mathrm{C}$ for $16 \mathrm{~h}$ before $\mathrm{Et}_{2} \mathrm{O}(10 \mathrm{~mL})$ was added. After $24 \mathrm{~h}$, the solution was removed and the precipitate was washed twice with $\mathrm{Et}_{2} \mathrm{O}$. The crude product was obtained as yellow solid.

$\mathrm{UV} / \mathrm{vis}$ (solution, MeCN): $\lambda_{\max }[\mathrm{nm}]\left(\varepsilon\left[\mathrm{L} \mathrm{mol}^{-1} \mathrm{~cm}^{-1}\right]\right)=345$ (3400), 460 (sh, 590).

$\mathrm{MB}(80 \mathrm{~K}): \delta=0.11 \mathrm{~mm} \mathrm{~s}^{-1}, \Delta E_{\mathrm{Q}}=4.15 \mathrm{~mm} \mathrm{~s}^{-1}$

EPR (150 K): $g(\mathrm{lw}[\mathrm{G}])=2.700$ (265), 2.095 (240), 1.750 (270).

After a MB measurement, the putative $\left.\left[{ }^{\mathrm{NHC}} \mathrm{L}\right) \mathrm{Fe}(\mathrm{F})\right](\mathrm{OTf})_{2}$ compound $(20.5 \mathrm{mg}, 28.4 \mu \mathrm{mol})$ was treated with further $\mathrm{XeF}_{2}(18.5 \mathrm{mg}, 109 \mu \mathrm{mol}, 4$ eq. $)$ and kept at $-35{ }^{\circ} \mathrm{C}$ for $10 \mathrm{~min} . \mathrm{Et}_{2} \mathrm{O}(10 \mathrm{~mL})$ was added to the resulting brown reaction mixture. After $5 \mathrm{~h}$, the solution was removed and the obtained solid was used for a further MB measurement.

UV/vis (solution, MeCN): $\lambda_{\max }[\mathrm{nm}]\left(\varepsilon\left[\mathrm{L} \mathrm{mol}^{-1} \mathrm{~cm}^{-1}\right]\right)=430$ (broad, 1900), 570 (broad, 940).

$\mathrm{MB}(80 \mathrm{~K}): \delta=-0.22 \mathrm{~mm} \mathrm{~s}^{-1}, \Delta E_{\mathrm{Q}}=4.47 \mathrm{~mm} \mathrm{~s}^{-1}$

$\operatorname{EPR}(160 \mathrm{~K}): g(\mathrm{lw}[\mathrm{G}])=2.225$ (135), 2.005 (120), 1.840 (169).

\section{Conversion of $\left[\left({ }^{\mathrm{NHC}} \mathrm{L}\right) \mathrm{FeO}(\mathrm{MeCN})\right](\mathrm{OTf})_{2}(2)$ with ${ }^{t} \mathrm{BuOO}^{-}$}

$\left[\left({ }^{\mathrm{NHC}} \mathrm{L}\right) \mathrm{Fe}(\mathrm{MeCN})_{2}\right](\mathrm{OTf})_{2}(21.6 \mathrm{mg}, 27.5 \mu \mathrm{mol}, 1$ eq. $)$ was dissolved in $\mathrm{MeCN}(1 \mathrm{~mL})$, cooled to $-35^{\circ} \mathrm{C}$ and 2 - $\left(\mathrm{tBuSO}_{2}\right)-\mathrm{C}_{6} \mathrm{H}_{4}-\mathrm{IO}(26.2 \mathrm{mg}, 77.0 \mu \mathrm{mol}, 2.8 \mathrm{eq}$. $)$ was added in one portion. The resulting green solution was transferred into a new vial to remove residual iodosobenzene. Afterwards, the solution was treated first with ${ }^{t} \mathrm{BuOOH}(6.9 \mu \mathrm{L}, 5.5 \mathrm{M}$ in ditertbutylperoxide:water $3: 2,2 \mathrm{eq})$ and second with a solution of $\mathrm{KO}^{t} \mathrm{Bu}(3.0 \mathrm{mg}, 27.5 \mu \mathrm{mol}, 1 \mathrm{eq})$ in $\mathrm{MeOH}(82 \mu \mathrm{L})$. The resulting red-brown solution was used for in situ measurements of MB. The EPR sample was 
prepared in an analog way starting with crystalline material of $\mathbf{2}$. Crystallization of $\mathbf{1 6}$ by diffusion of $\mathrm{Et}_{2} \mathrm{O}$ into the $\mathrm{MeCN}$ reaction mixture at $-35^{\circ} \mathrm{C}$ was not successful, so far.

$\mathrm{UV} /$ vis (solution, $\mathrm{MeCN}): \lambda_{\max }[\mathrm{nm}]\left(\varepsilon\left[\mathrm{Lmol}^{-1} \mathrm{~cm}^{-1}\right]\right)=405$ (1750), 550 (sh, 300).

$\operatorname{MB}(\mathrm{MeCN}, 7 \mathrm{~K}): \delta=-0.15 \mathrm{~mm} \mathrm{~s}^{-1}, \Delta E_{\mathrm{Q}}=2.78 \mathrm{~mm} \mathrm{~s}^{-1}$

$\operatorname{EPR}(160 \mathrm{~K}): g(\mathrm{w}[\mathrm{G}])=2.1980(38), 2.0694(16), 1.9920(5), A_{\mathrm{z}}=12.6 \mathrm{G}$ for one ${ }^{14} \mathrm{~N}$

The protonated species $\mathbf{1 6 H}^{+}$was prepared in a similar way adding 2 eq. of ${ }^{t} \mathrm{BuOOH}$ and 1 eq. of $\mathrm{KO}^{t} \mathrm{Bu}$ as well as subsequent addition of 1,5-bis(dimethyl)-piperidinium (6.1 mg, $\left.27.5 \mu \mathrm{mol}, 1 \mathrm{eq}\right)$ in $\mathrm{MeCN}(100 \mu \mathrm{L})$.

$\mathrm{UV} / \mathrm{vis}$ (solution, $\mathrm{MeCN}): \lambda_{\max }[\mathrm{nm}]\left(\varepsilon\left[\mathrm{L} \mathrm{mol}^{-1} \mathrm{~cm}^{-1}\right]\right)=402$ (1550), 590 (250).

$\operatorname{MB}(\mathrm{MeCN}, 7 \mathrm{~K}): \delta=-0.10 \mathrm{~mm} \mathrm{~s}^{-1}, \Delta E_{\mathrm{Q}}=2.77 \mathrm{~mm} \mathrm{~s}^{-1}$

\section{Synthesis of $\left[\left({ }^{\mathrm{NHC}} \mathrm{L}\right)\{\mathrm{FeNO}\}^{7}\right](\mathrm{OTf})_{2}(17)^{[22]}$}

To a pre-cooled solution of $\left[\left({ }^{\mathrm{NHC}} \mathrm{L}\right) \mathrm{Fe}(\mathrm{MeCN})_{2}\right](\mathrm{OTf})_{2}(30.0 \mathrm{mg}, 0.038 \mathrm{mmol}, 1.00$ eq. $)$ in $\mathrm{MeCN}$ $(3 \mathrm{~mL})$ was added trityl $S$-nitrosothiol $(11.7 \mathrm{mg}, 0.038 \mathrm{mmol}, 1.00$ eq.). The reaction mixture was allowed to react over night at $-35{ }^{\circ} \mathrm{C} . \mathrm{Et}_{2} \mathrm{O}(7 \mathrm{~mL})$ was added to the resulting blue solution to precipitate a blue solid. The solution was removed using a syringe and the solid was washed twice with $\mathrm{Et}_{2} \mathrm{O}(2 \times 5 \mathrm{~mL})$. Blue single crystals suitable for X-ray diffraction were obtained by slow diffusion of $\mathrm{Et}_{2} \mathrm{O}$ into a solution of $\mathrm{MeCN}$ at $-35{ }^{\circ} \mathrm{C}$ (15.2 $\left.\mathrm{mg}, 0.021 \mathrm{mmol}, 54 \%\right)$.

IR (solid, ATR): $\tilde{v}\left[\mathrm{~cm}^{-1}\right]=3122(\mathrm{w}), 1747(\mathrm{~m}), 1489(\mathrm{w}), 1464(\mathrm{w}), 1410(\mathrm{w}), 1266(\mathrm{~s}), 1244$ (s), 1223 (s), 1190 (m), 1152 (s), 1104 (m), 1084 (w), 1027 (s), 1013 (w), 815 (w), 767 (m), 753 (m), $745(\mathrm{~m}), 695(\mathrm{~m}), 674(\mathrm{w}), 634(\mathrm{~s}), 574(\mathrm{~m}), 516(\mathrm{~s}), 482(\mathrm{w}), 448(\mathrm{w})$.

$\mathrm{UV} /$ vis (solution, MeCN): $\lambda_{\max }[\mathrm{nm}]\left(\varepsilon_{\text {rel }}\left[\mathrm{L} \mathrm{mol}^{-1} \mathrm{~cm}^{-1}\right]\right)=350(1.00), 615(0.30)$.

(+)-ESI-MS: $m / z=202.1\left[\left({ }^{\mathrm{NHC}} \mathrm{L}\right) \mathrm{Fe}\right]^{2+}, 217.1\left[\left({ }^{\mathrm{NHC}} \mathrm{L}\right) \mathrm{Fe}(\mathrm{NO})\right]^{2+}, 583.1\left[\left({ }^{\mathrm{NHC}} \mathrm{L}\right) \mathrm{Fe}(\mathrm{NO})(\mathrm{OTf})\right]^{+}$

EA: Calcd. [\%] for $\mathrm{C}_{20} \mathrm{H}_{20} \mathrm{~F}_{6} \mathrm{FeN}_{9} \mathrm{O}_{7} \mathrm{~S}_{2}$ : C 32.80; H 2.75; N 17.21. Found: C 32.82, H 2.72, N 17.41.

$\operatorname{MB}(80 \mathrm{~K}): \delta=-0.01 \mathrm{~mm} \mathrm{~s}^{-1}, \Delta E_{Q}=2.36 \mathrm{~mm} \mathrm{~s}^{-1}$

EPR: $159 \mathrm{~K}: g(\mathrm{lw}[\mathrm{mT}])=2.0290(1), 2.0136(1), 1.996(1)$.

$220 \mathrm{~K}: g(\mathrm{lw}[\mathrm{mT}])=2.027(0.9), A=38 \mathrm{MHz}$ for one ${ }^{14} \mathrm{~N}_{\mathrm{NO}}$

\section{Synthesis of $\left[\left({ }^{\mathrm{NHC}} \mathrm{L}\right)\{\mathrm{FeNO}\}^{8}\right](\mathrm{OTf})(19)^{[23]}$}

To a pre-cooled and blue solution of $\left[\left({ }^{\mathrm{NHC}} \mathrm{L}\right)\{\mathrm{FeNO}\}^{7}\right](\mathrm{OTf})_{2}(20.0 \mathrm{mg}, 0.027 \mathrm{mmol}, 1.00$ eq. $)$ in $\mathrm{MeCN}(2 \mathrm{~mL})$ was added cobaltocene $(5.7 \mathrm{mg}, 0.030 \mathrm{mmol}, 1.1 \mathrm{eq}$.). The reaction mixture was allowed to react for $3 \mathrm{~h}$ at $-35{ }^{\circ} \mathrm{C}$ under exclusion of light. $\mathrm{Et}_{2} \mathrm{O}(7 \mathrm{~mL})$ was added to the resulting green solution, causing precipitation of a green solid. The solution was removed via a syringe and the solid was washed with $\mathrm{Et}_{2} \mathrm{O}(5 \mathrm{~mL})$ and hexane $(5 \mathrm{~mL})$ and then dried under reduced pressure to give the crude product $(7.5 \mathrm{mg}, 0.013 \mathrm{mmol}, 47 \%)$. Green single crystals suitable for X-ray 
diffraction were obtained by slow diffusion of $\mathrm{Et}_{2} \mathrm{O}$ into a solution of crude $\left[\left({ }^{\mathrm{NHC}} \mathrm{L}\right)\{\mathrm{FeNO}\}^{8}\right](\mathrm{OTf})$ in $\mathrm{MeCN}$ at $-35^{\circ} \mathrm{C}$.

${ }^{1} \mathrm{H}$ NMR $\left(500 \mathrm{MHz}, \mathrm{MeCN}-\mathrm{d}_{3}\right): \delta=7.49$ (s, 4H; CH), 7.29 (s, 4H; CH), $6.21(\mathrm{~d}, J=13.3 \mathrm{~Hz}, 2 \mathrm{H}$, $\left.\mathrm{CH}_{2}\right), 5.78\left(\mathrm{~d}, J=13.6 \mathrm{~Hz}, 2 \mathrm{H} ; \mathrm{CH}_{2}\right), 4.71\left(\mathrm{t}, J=8.6 \mathrm{~Hz}, 4 \mathrm{H} ; \mathrm{C}_{2} H_{4}\right), 4.54-4.27(\mathrm{~m}, 4 \mathrm{H}$; $\left.\mathrm{C}_{2} H_{4}\right) \cdot{ }^{13} \mathrm{C}\left\{{ }^{1} \mathrm{H}\right\}$ NMR (126 MHz, MeCN-d 3$): \delta=195.85(C), 125.00(\mathrm{CH}), 123.17(\mathrm{CH}), 62.54$ $\left(\mathrm{CH}_{2}\right), 50.09\left(\mathrm{C}_{2} \mathrm{H}_{4}\right)$.

IR (solid, ATR): $\tilde{v}\left[\mathrm{~cm}^{-1}\right]=3127(\mathrm{w}), 1590(\mathrm{~s}), 1464(\mathrm{~m}), 1403(\mathrm{~m}), 1340(\mathrm{~m}), 1259(\mathrm{~s}), 1222(\mathrm{~m})$, $1209(\mathrm{~m}), 1153(\mathrm{~s}), 1029(\mathrm{~s}), 811(\mathrm{~m}), 718(\mathrm{~s}), 687(\mathrm{~s}), 671(\mathrm{~s}), 635(\mathrm{~s}), 572(\mathrm{~s}), 516(\mathrm{~s}), 462(\mathrm{~m})$.

$\mathrm{UV} / \mathrm{vis}$ (solution, MeCN): $\lambda_{\max }[\mathrm{nm}]\left(\varepsilon\left[\mathrm{L} \mathrm{mol}^{-1} \mathrm{~cm}^{-1}\right]\right)=300$ (6850), 360 (6800), 642 (410).

(+)-ESI-MS: $\left.m / z=202.1\left[\left({ }^{\mathrm{NHC}} \mathrm{L}\right) \mathrm{Fe}\right]^{2+}, 217.1\left[\left({ }^{\mathrm{NHC}} \mathrm{L}\right) \mathrm{Fe}(\mathrm{NO})\right]^{2+}, 434.1\left[{ }^{\mathrm{NHC}} \mathrm{L}\right) \mathrm{Fe}(\mathrm{NO})\right]^{+}, 583.1$ $\left[\left({ }^{\mathrm{NHC}} \mathrm{L}\right) \mathrm{Fe}(\mathrm{NO})(\mathrm{OTf})\right]^{+}$

$\mathrm{MB}(80 \mathrm{~K}): \delta=0.01 \mathrm{~mm} \mathrm{~s}^{-1}, \Delta E_{Q}=0.87 \mathrm{~mm} \mathrm{~s}^{-1}$.

\section{Synthesis of $\left[{ }^{\mathrm{NHC}} \mathrm{L}\{\mathrm{FeNO}\}^{6}(\mathrm{ONO})\right](\mathrm{OTf})_{2}(18)^{[23]}$}

A yellow solution of $\left[\left({ }^{\mathrm{NHC}} \mathrm{L}\right) \mathrm{Fe}(\mathrm{MeCN})_{2}\right](\mathrm{OTf})_{2}(25 \mathrm{mg}, 0,032 \mathrm{mmol})$ in $\mathrm{MeCN}(3 \mathrm{~mL})$ in a Schlenk flask wrapped with aluminum foil and closed with a septum was put under slight vacuum and connected to an NO gas source. The gas connection was opened and the solution turned blue within 5 min. Dry dioxygen was added using a syringe (1 eq. or excess). The gas phase immediately turned brown indicating the formation of oxidized nitric oxide gases such as $\mathrm{NO}_{2}$, and the solution turned yellow. Yellow single crystals suitable for X-ray diffraction were obtained by diffusion of $\mathrm{Et}_{2} \mathrm{O}$ into the reaction mixture at $\mathrm{RT}(15.0 \mathrm{mg}, 0.019 \mathrm{mmol}, 63 \%)$.

${ }^{1} \mathrm{H}$ NMR (300 MHz, MeCN-d $\left.{ }_{3}\right): \delta=7.61-7.35\left(\mathrm{~m}, 8 \mathrm{H} ; \mathrm{CH}_{\mathrm{NHC}}\right), 6.18\left(\mathrm{~d}, J=13.5 \mathrm{~Hz}, 2 \mathrm{H} ; \mathrm{CH}_{2}\right)$, $5.15\left(\mathrm{~d}, J=13.5 \mathrm{~Hz}, 2 \mathrm{H} ; \mathrm{CH}_{2}\right), 5.05-4.65\left(\mathrm{~m}, 8 \mathrm{H} ; \mathrm{C}_{2} H_{4}\right) .{ }^{13} \mathrm{C}\left\{{ }^{1} \mathrm{H}\right\} \mathrm{NMR}\left(75 \mathrm{MHz}, \mathrm{MeCN}-\mathrm{d}_{3}\right) \delta=$ $167.7\left(C_{\mathrm{NHC}}\right), 126.1\left(\mathrm{CH}_{\mathrm{NHC}}\right), 124.3\left(\mathrm{CH}_{\mathrm{NHC}}\right), 62.2\left(\mathrm{CH}_{2}\right), 49.9\left(C_{2} \mathrm{H}_{4}\right)$.

IR (solid, ATR): $\tilde{v}\left(\mathrm{~cm}^{-1}\right)=3127(\mathrm{~m}), 1877(\mathrm{~s}), 1459(\mathrm{~m}), 1346(\mathrm{~s}), 1257(\mathrm{~m}), 1222(\mathrm{~s}), 1193(\mathrm{~s}), 1140$ (m), $1028(\mathrm{~s}), 746(\mathrm{~s}), 696(\mathrm{~s}), 633(\mathrm{~m}), 570(\mathrm{~s}), 514(\mathrm{~s}), 444(\mathrm{~s})$.

$\mathrm{UV} /$ vis (solution, MeCN): $\lambda_{\max }[\mathrm{nm}]\left(\varepsilon\left[\mathrm{L} \mathrm{mol}^{-1} \mathrm{~cm}^{-1}\right]\right)=306$ (4300), 380 (sh, 1200).

(+)-ESI-MS: $m / z=202.1\left[\left({ }^{\mathrm{NHC}} \mathrm{L}\right) \mathrm{Fe}\right]^{2+}, 217.1\left[\left({ }^{\mathrm{NHC}} \mathrm{L}\right) \mathrm{Fe}(\mathrm{NO})\right]^{2+}, 583.1\left[\left({ }^{\mathrm{NHC}} \mathrm{L}\right) \mathrm{Fe}(\mathrm{NO})(\mathrm{OTf})\right]^{+}$, $629.0\left[\left({ }^{\mathrm{NHC}} \mathrm{L}\right) \mathrm{Fe}(\mathrm{NO})(\mathrm{ONO})(\mathrm{OTf})\right]^{+}$.

EA: Calcd. [\%] for $\mathrm{C}_{20} \mathrm{H}_{20} \mathrm{~F}_{6} \mathrm{FeN}_{10} \mathrm{O}_{9} \mathrm{~S}_{2}$ : C 30.86; H 2.59; N 17.99. Found: C 30.88, H 2.62, N 18.13.

$\operatorname{MB}(80 \mathrm{~K}): \delta=-0.16 \mathrm{~mm} \mathrm{~s}^{-1}, \Delta E_{\mathrm{Q}}=3.12 \mathrm{~mm} \mathrm{~s}^{-1}$. 


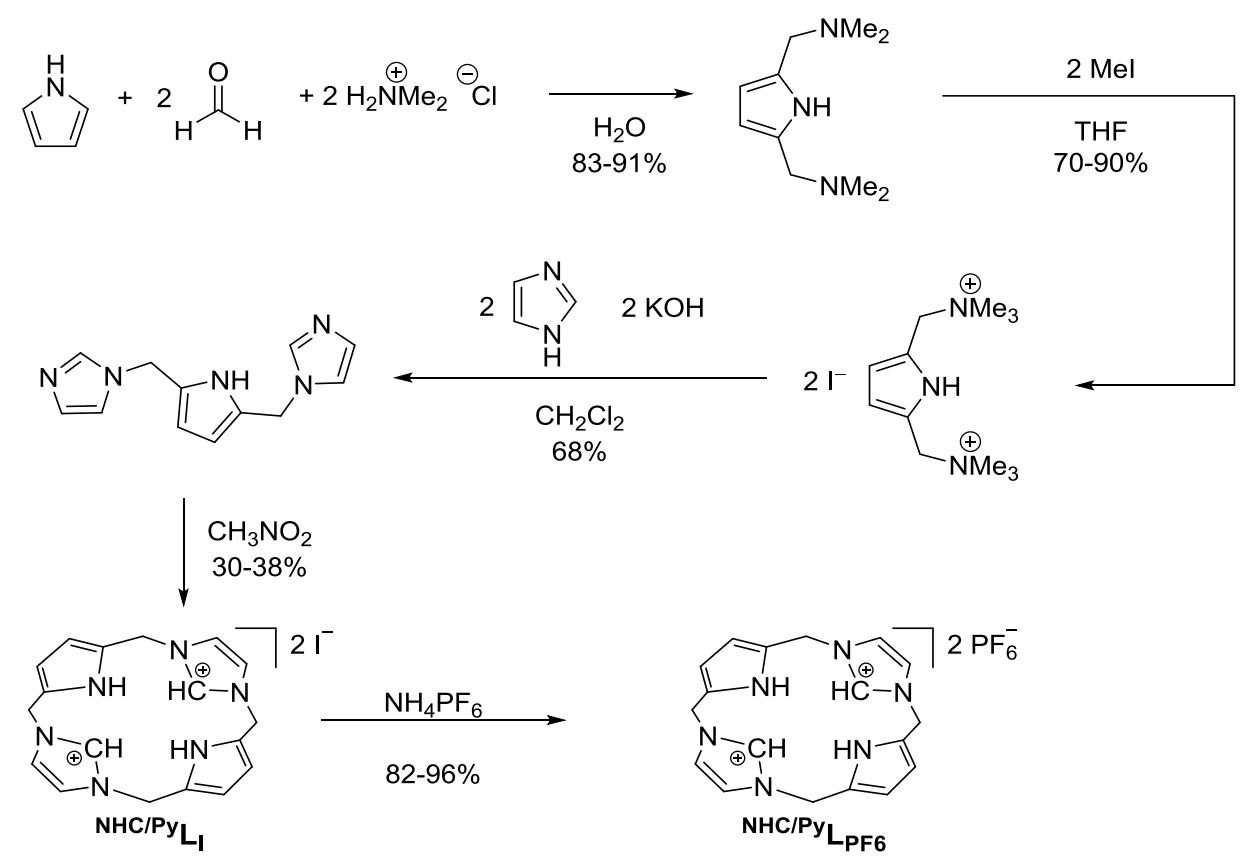

Scheme 33. Synthetic strategy for generation of the hybrid ligand system ${ }^{\mathrm{NHC} / \mathbf{P y}} \mathbf{L}$.

The hybrid ligand system ${ }^{\mathbf{N H C} / \mathbf{P y}} \mathbf{L}$ was prepared according to literature described procedures. ${ }^{[245-248]}$ The obtained yields are given in Scheme 33.

\section{Conversion of ${ }^{\mathrm{NHC} / \mathrm{Py}} \mathrm{L}$ with $\mathrm{AgO}_{2}$}

${ }^{\mathbf{N H C} / \mathbf{P y}} \mathbf{L}_{\mathbf{P F}}$ (400 mg, $0.66 \mathrm{mmol}, 1.0$ eq.) was dissolved in $\mathrm{MeCN}(60 \mathrm{~mL})$ at atmospheric conditions and the flask was wrapped in alumina foil. $\mathrm{AgO}_{2}(181 \mathrm{mg}, 0.79 \mathrm{mmol}, 1.2$ eq.) was added in one portion and the mixture was stirred at $55^{\circ} \mathrm{C}$ for 2 weeks. The reaction mixture was filtered through Celite and $\mathrm{Et}_{2} \mathrm{O}$ was added to the resulting brown solution, causing precipitation of a slight brown solid. The mixture was filtered and the solid was dried in vacuum $(90 \mathrm{mg}$, $0.19 \mathrm{mmol}, 29 \%)$.

${ }^{1} \mathrm{H}$ NMR (300 MHz, DMSO-d $\left.\mathrm{d}_{6}\right): \delta=11.21$ (s, $\left.1 \mathrm{H} ; \mathrm{NH}_{\mathrm{py}}\right), 7.88(\mathrm{bt}, 2 \mathrm{H} ; \mathrm{NCHN}$ im $), 7.41$ (bt, $2 \mathrm{H}$; $\mathrm{CH}_{\mathrm{im}}$ ), 7.05 (bt, 2H; $\left.\mathrm{CH}_{\mathrm{im}}\right), 6.10$ (d, J=2.4 Hz, 2H; $\left.\mathrm{CH}_{\mathrm{py}}\right), 5.17$ (s, $4 \mathrm{H} ; \mathrm{CH}_{2}$ ).

(+)-ESI-MS: $m / z=334\left[\mathrm{C}_{12} \mathrm{H}_{12} \mathrm{~N}_{5} \mathrm{Ag}\right]^{+}, 561\left[\left(\mathrm{C}_{12} \mathrm{H}_{13} \mathrm{~N}_{5}\right)_{2} \mathrm{Ag}\right]^{+}$.

\section{Synthesis of [ $\left.K_{2}\left\{L^{\prime}{ }^{\prime} N i\right\}\right](20)$}

${ }^{\mathbf{N H C} / \mathbf{P}^{\prime}} \mathbf{L}_{\mathbf{P F}}$ (100 mg, $0.16 \mathrm{mmol}, 1.0$ eq.) was dissolved in dry MeCN (50 mL) under atmosphere of dry argon and cooled to $-78^{\circ} \mathrm{C}$. In a second Schlenk flask, $\mathrm{KO}^{\mathrm{t}} \mathrm{Bu}(73.5 \mathrm{mg}, 0.66 \mathrm{mmol}, 4$ eq.) was dissolved in MeCN (50 mL) and the resulting solution was added slowly to the ${ }^{{ }^{\mathbf{N H C} / \mathbf{P}^{\mathbf{y}}} \mathbf{L}_{\mathbf{P F}}}$ solution using a metal canula. Within $1 \mathrm{~h}$, the reaction mixture was allowed to warm up to $-15^{\circ} \mathrm{C}$ resulting in a yellow solution. $\mathrm{NiBr}_{2}(\mathrm{dme})$ was added as a solid in one portion. The mixture was warmed up to RT and stirred for 2 days. The red suspension was filtered, yielding a slight red solid (36 mg, 
$0.05 \mathrm{mmol}, 30 \%)$. Crystallization upon diffusion of $\mathrm{Et}_{2} \mathrm{O}$ into a red solution of the crude product in DMSO led to crystals suitable for X-ray diffraction.

${ }^{1} \mathrm{H}$ NMR (300 MHz, DMSO-d ${ }_{6}$ ): $\delta=8.09$ (s, 2H), 7.66 (s, 2H), 6.88 (s, 2H), 6.45 (s, 2H), 5.69 (s, $4 \mathrm{H}), 5.02(\mathrm{~s}, 2 \mathrm{H}), 4.82(\mathrm{~s}, 2 \mathrm{H}), 1.32(\mathrm{~s}, 6 \mathrm{H})$.

\section{Synthesis of 2,5-Bis-(benzotriazolmethyl)-pyrrole (21)}

Benzotriazole (1.0 g, $8.4 \mathrm{mmol}$, 4 eq.) was dissolved in DCM $(20 \mathrm{~mL})$ under atmospheric conditions. The solution was cooled to $0{ }^{\circ} \mathrm{C}$ and $\mathrm{KOH}(0.47 \mathrm{~g}, 8.4 \mathrm{mmol}, 4 \mathrm{eq}$. $)$ was added, followed by addition of 2,5-bis-(trimethylaminomethyl)-pyrrole diiodide ( $0.98 \mathrm{~g}, 2.1 \mathrm{mmol}, 1 \mathrm{eq}$.). The reaction mixture was allowed to warm up to RT and stirred for two days. The solvent was removed under vacuum and the resulting solid was washed with $\mathrm{THF}, \mathrm{Et}_{2} \mathrm{O}, \mathrm{DCM}, \mathrm{MeOH}$, and $\mathrm{H}_{2} \mathrm{O}$. The product was dried under vacuum and obtained as off-white solid (160 mg, $0.48 \mathrm{mmol}$, $23 \%)$.

${ }^{1} \mathrm{H}$ NMR (300 MHz, DMSO-d 6 ): $\delta=11.51\left(\mathrm{~s}, 1 \mathrm{H} ; \mathrm{N}_{\mathrm{py}}\right), 8.00\left(\mathrm{~d}, J=8.1 \mathrm{~Hz}, 2 \mathrm{H} ; \mathrm{CH}_{\mathrm{benz}}\right), 7.66$ (d, $\left.J=8.1 \mathrm{~Hz}, 2 \mathrm{H} ; \mathrm{CH}_{\text {benz }}\right), 7.38\left(\mathrm{dt}, J=18.7,7.3 \mathrm{~Hz}, 4 \mathrm{H} ; \mathrm{CH}_{\text {benz }}\right), 6.11\left(\mathrm{~d}, J=2.9 \mathrm{~Hz}, 2 \mathrm{H} ; \mathrm{CH}_{\mathrm{py}}\right), 5.82$ $\left(\mathrm{s}, 4 \mathrm{H} ; \mathrm{CH}_{2}\right.$ ).

(+)-ESI-MS: $m / z=328.1[\mathrm{M}-\mathrm{H}]^{-}, 455.8[(\mathrm{M}) \mathrm{I}]^{-}$.

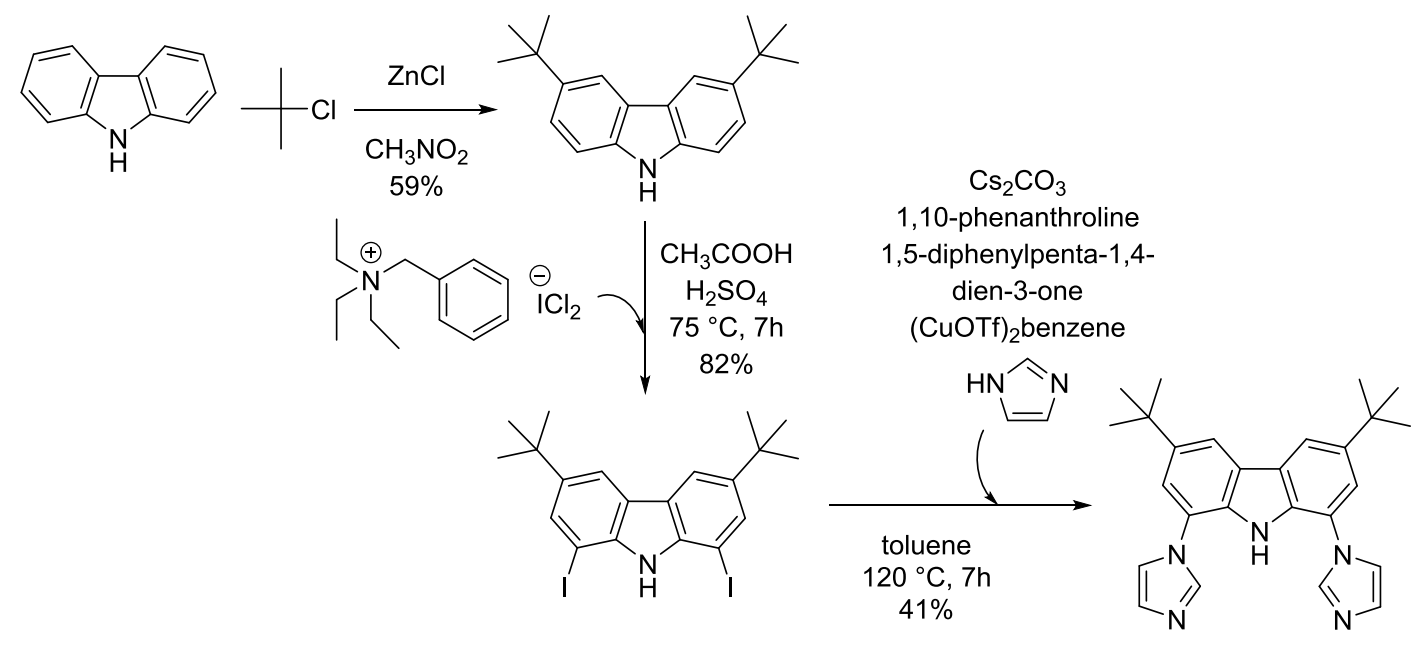

Scheme 34. Synthetic strategy for the generation of the 1,8-bis-(imidazol-1-yl)-carbazole. ${ }^{[263]}$

\section{Synthesis of the Carbazole-Ring 22}

The starting material 1,8-bis-(imidazol-1-yl)-carbazole was prepared according to literature described procedures (Scheme 34). ${ }^{[263]} 1,8$-bis-(imidazol-1-yl)-carbazole $(128.9 \mathrm{mg}, 0.31 \mathrm{mmol}$ ) and 2,6-bis-(bromomethyl)-pyridine $(83 \mathrm{mg}, 0.31 \mathrm{mmol})$ were dissolved in DCM $(6 \mathrm{~mL})$ and stirred at RT for 2 weeks. During this time, a white solid precipitates that was filtered off, washed with DCM and dryed under vacuum (95 mg, $0.18 \mathrm{mmol}, 59 \%)$. 
${ }^{1} \mathrm{H}$ NMR (300 MHz, DMSO-d6): $\delta=11.54$ (s, 1H; NH), $11.28(\mathrm{~s}, 1 \mathrm{H} ; \mathrm{NH}), 10.18(\mathrm{~s}, 2 \mathrm{H} ; \mathrm{NCHN})$, 10.04 (s, 2H; $\mathrm{NCHN}), 8.57$ (d, $\left.J=1.6 \mathrm{~Hz}, 2 \mathrm{H} ; \mathrm{CH}_{\text {carb }}\right), 8.52$ (d, $\left.J=1.7 \mathrm{~Hz}, 2 \mathrm{H} ; \mathrm{CH}_{\text {carb }}\right), 8.29$ (d, $J=$ $\left.1.8 \mathrm{~Hz}, 2 \mathrm{H} ; \mathrm{CH}_{\mathrm{im}}\right), 8.05$ (s, 2H; $\left.\mathrm{CH}_{\mathrm{im}}\right), 7.90\left(\mathrm{~d}, J=7.6 \mathrm{~Hz}, 2 \mathrm{H} ; \mathrm{CH}_{\mathrm{im}}\right), 7.81$ (d, $J=1.6 \mathrm{~Hz}, 2 \mathrm{H}$; $\left.\mathrm{CH}_{\text {carb }}\right), 7.74\left(\mathrm{~d}, J=1.7 \mathrm{~Hz}, 2 \mathrm{H} ; \mathrm{CH}_{\text {carb }}\right), 7.65$ (d, J=7.8 Hz, 2H; $\left.\mathrm{CH}_{\mathrm{im}}\right), 5.77$ (s, 4H; $\left.\mathrm{CH}_{2}\right), 5.61$ (s, $\left.4 \mathrm{H} ; \mathrm{CH}_{2}\right), 1.47$ (s, 18H; $\left.\mathrm{CH}_{3}\right), 1.46\left(\mathrm{~s}, 18 \mathrm{H} ; \mathrm{CH}_{3}\right)$.

(+)-ESI-MS: $m / z=258.1[\mathrm{M}]^{2+}, 515.3[\mathrm{M}-\mathrm{H}]^{+}, 595.2[\mathrm{MBr}]^{+}$. 


\section{DFT Calculations}

Geometry optimization calculations were performed with coordinates obtained from X-ray crystallographic structure determination as described in a recent publication. ${ }^{[23]}$ Unrestricted DFT calculations using the ORCA program (Ver. 3.0.1 or 3.0.2) were performed with BP86 respectively B3LYP functional, RI resp. RIJCOSX approximation, def2-tzvp and def2-tzvp/j basis sets ${ }^{[264]}$ Solvent effects were considered by invoking the conductor like screening model (COSMO) with $\mathrm{MeCN}$ as the solvent. 80 excited states were calculated; the maximum dimension of the expansion space in the Davidson procedure (MaxDim) was 800. The $\mathrm{p}_{z}$ characters as well as the amount of iron and nitrogen within the HOMO and LUMO result from 'Mulliken orbital population MO' analyses. Mössbauer parameters were computed via unrestricted DFT calculations with the B3LYP functional, def2-tzvp basis set + enlarged CP(PPP) basis set for the Fe atom, dispersion correction D3ZERO, COSMO (MeCN). Mössbauer isomer shifts were obtained using the correlation formula and parameters reported by Neese et al. ${ }^{[243]}$

DFT Table 1. Coordinates of the energy-optimized structure of $\left[\left({ }^{\mathrm{NHC}} \mathrm{L}\right) \mathrm{Fe}\left(\mathrm{N}_{3}\right)_{2}\right] \mathbf{5}$.

\begin{tabular}{|c|c|c|c|}
\hline $\mathrm{Fe}$ & 0.006216000 & -0.111663000 & -0.047572000 \\
\hline $\mathrm{C}$ & -0.139833000 & 1.851536000 & 0.067823000 \\
\hline $\mathrm{C}$ & -1.961765000 & -0.274280000 & 0.082128000 \\
\hline $\mathrm{C}$ & 0.243893000 & -2.027402000 & -0.116008000 \\
\hline $\mathrm{C}$ & 1.928149000 & -0.073840000 & -0.133121000 \\
\hline $\mathrm{N}$ & -0.127809000 & -0.015803000 & -2.126232000 \\
\hline $\mathrm{N}$ & 0.092411000 & -0.186743000 & 2.028654000 \\
\hline $\mathrm{N}$ & 0.567565000 & 2.939900000 & -0.414361000 \\
\hline $\mathrm{N}$ & -1.169388000 & 2.466774000 & 0.753284000 \\
\hline $\mathrm{N}$ & -2.726350000 & 0.650677000 & 0.763745000 \\
\hline $\mathrm{N}$ & -2.931751000 & -1.144033000 & -0.392133000 \\
\hline $\mathrm{N}$ & -0.379591000 & -2.999156000 & -0.858788000 \\
\hline $\mathrm{N}$ & 1.233332000 & -2.735978000 & 0.534082000 \\
\hline $\mathrm{N}$ & 2.777208000 & -0.940353000 & 0.524895000 \\
\hline $\mathrm{N}$ & 2.792363000 & 0.686656000 & -0.879077000 \\
\hline $\mathrm{N}$ & -0.961845000 & 0.667489000 & -2.677986000 \\
\hline $\mathrm{N}$ & 0.769622000 & 0.576394000 & 2.678693000 \\
\hline $\mathrm{C}$ & -0.012832000 & 4.148749000 & -0.034042000 \\
\hline $\mathrm{C}$ & 1.871832000 & 2.987781000 & -1.088909000 \\
\hline $\mathrm{C}$ & -1.109365000 & 3.850780000 & 0.703019000 \\
\hline $\mathrm{C}$ & -2.139952000 & 1.728000000 & 1.534631000 \\
\hline $\mathrm{C}$ & -4.084550000 & 0.378591000 & 0.722525000 \\
\hline $\mathrm{C}$ & -4.214206000 & -0.755438000 & -0.007104000 \\
\hline $\mathrm{C}$ & -2.790755000 & -2.430638000 & -1.087229000 \\
\hline $\mathrm{C}$ & -1.466986000 & -2.701654000 & -1.780441000 \\
\hline $\mathrm{C}$ & 0.200664000 & -4.252193000 & -0.679557000 \\
\hline $\mathrm{C}$ & 1.228891000 & -4.084499000 & 0.194518000 \\
\hline $\mathrm{C}$ & 2.253488000 & -2.048086000 & 1.310706000 \\
\hline $\mathrm{C}$ & 4.110073000 & -0.726829000 & 0.189202000 \\
\hline $\mathrm{C}$ & 4.119125000 & 0.309171000 & -0.691602000 \\
\hline $\mathrm{C}$ & 2.327826000 & 1.721070000 & -1.793296000 \\
\hline $\mathrm{N}$ & -1.744538000 & 1.306417000 & -3.263822000 \\
\hline $\mathrm{N}$ & 1.401377000 & 1.284413000 & 3.362313000 \\
\hline
\end{tabular}




\begin{tabular}{llll}
\hline $\mathrm{H}$ & 0.402601000 & 5.105573000 & -0.326025000 \\
$\mathrm{H}$ & 2.629709000 & 3.290879000 & -0.346969000 \\
$\mathrm{H}$ & 1.804666000 & 3.786649000 & -1.843947000 \\
$\mathrm{H}$ & -1.842039000 & 4.491111000 & 1.179392000 \\
$\mathrm{H}$ & -1.649255000 & 1.302268000 & 2.420804000 \\
$\mathrm{H}$ & -2.940020000 & 2.416009000 & 1.834213000 \\
$\mathrm{H}$ & -4.828150000 & 1.011286000 & 1.192393000 \\
$\mathrm{H}$ & -5.097788000 & -1.310630000 & -0.298420000 \\
$\mathrm{H}$ & -3.580003000 & -2.462366000 & -1.854703000 \\
$\mathrm{H}$ & -1.603375000 & -3.579169000 & -2.428159000 \\
$\mathrm{H}$ & -0.158762000 & -5.140579000 & -1.185386000 \\
$\mathrm{H}$ & 1.934398000 & -4.797351000 & 0.604718000 \\
$\mathrm{H}$ & 1.801700000 & -1.666329000 & 2.234198000 \\
$\mathrm{H}$ & 3.068887000 & -2.747074000 & 1.531617000 \\
$\mathrm{H}$ & 4.923265000 & -1.306025000 & 0.610054000 \\
$\mathrm{H}$ & 4.944051000 & 0.802955000 & -1.191459000 \\
$\mathrm{H}$ & 3.165709000 & 1.984217000 & -2.453565000 \\
$\mathrm{H}$ & 1.515013000 & 1.285908000 & -2.394163000 \\
$\mathrm{H}$ & -2.998893000 & -3.236407000 & -0.362875000 \\
$\mathrm{H}$ & -1.154193000 & -1.843451000 & -2.394174000 \\
\hline
\end{tabular}

DFT Table 2. Coordinates of the energy-optimized structure of $\left[\left({ }^{\mathrm{NHC}} \mathrm{L}\right) \mathrm{Fe}\left(\mathrm{N}_{3}\right)_{2}\right]^{+} \mathbf{1 1}$.

\begin{tabular}{|c|c|c|c|}
\hline $\mathrm{Fe}$ & -0.020363000 & -0.017853000 & 1.986096000 \\
\hline $\mathrm{N}$ & -0.039073000 & -0.040617000 & 3.961197000 \\
\hline $\mathrm{N}$ & 0.506169000 & -0.914064000 & 4.613641000 \\
\hline $\mathrm{N}$ & 0.093737000 & -0.018773000 & 0.015047000 \\
\hline $\mathrm{N}$ & 0.465515000 & 0.903566000 & -0.685046000 \\
\hline $\mathrm{C}$ & 1.949894000 & -0.023482000 & 2.100613000 \\
\hline $\mathrm{C}$ & 0.088888000 & -2.019883000 & 1.844516000 \\
\hline $\mathrm{C}$ & -2.014359000 & -0.016325000 & 2.014957000 \\
\hline $\mathrm{C}$ & -0.144351000 & 1.989153000 & 1.920372000 \\
\hline $\mathrm{N}$ & 1.007718000 & -1.699753000 & 5.303710000 \\
\hline $\mathrm{N}$ & 0.813542000 & 1.732821000 & -1.418961000 \\
\hline $\mathrm{N}$ & 2.793183000 & 0.717549000 & 2.872842000 \\
\hline $\mathrm{N}$ & 2.776469000 & -0.901758000 & 1.466602000 \\
\hline $\mathrm{N}$ & 1.157027000 & -2.637698000 & 1.233506000 \\
\hline $\mathrm{N}$ & -0.689932000 & -3.084997000 & 2.230532000 \\
\hline $\mathrm{N}$ & -2.896994000 & -0.781466000 & 2.716615000 \\
\hline $\mathrm{N}$ & -2.812728000 & 0.884613000 & 1.376981000 \\
\hline $\mathrm{N}$ & -1.185642000 & 2.625084000 & 1.276135000 \\
\hline $\mathrm{N}$ & 0.622766000 & 3.046098000 & 2.351327000 \\
\hline $\mathrm{C}$ & 4.115048000 & 0.311683000 & 2.715222000 \\
\hline $\mathrm{C}$ & 2.353238000 & 1.803712000 & 3.746515000 \\
\hline $\mathrm{C}$ & 4.106329000 & -0.717705000 & 1.826679000 \\
\hline $\mathrm{C}$ & 2.251727000 & -1.928603000 & 0.585562000 \\
\hline $\mathrm{C}$ & 1.047431000 & -4.019659000 & 1.234906000 \\
\hline $\mathrm{C}$ & -0.118311000 & -4.301930000 & 1.863479000 \\
\hline $\mathrm{C}$ & -2.037363000 & -3.118589000 & 2.826921000 \\
\hline $\mathrm{C}$ & -2.510122000 & -1.889664000 & 3.584408000 \\
\hline $\mathrm{C}$ & -4.209326000 & -0.365828000 & 2.515834000 \\
\hline $\mathrm{C}$ & -4.158022000 & 0.695002000 & 1.666822000 \\
\hline $\mathrm{C}$ & -2.252851000 & 1.941893000 & 0.558741000 \\
\hline $\mathrm{C}$ & -1.069882000 & 4.006765000 & 1.305805000 \\
\hline $\mathrm{C}$ & 0.070799000 & 4.271762000 & 1.984444000 \\
\hline $\mathrm{C}$ & 1.950816000 & 3.056340000 & 2.989144000 \\
\hline $\mathrm{H}$ & 4.935570000 & 0.775414000 & 3.249259000 \\
\hline $\mathrm{H}$ & 3.195833000 & 2.056697000 & 4.402125000 \\
\hline $\mathrm{H}$ & 1.519100000 & 1.442894000 & 4.363149000 \\
\hline $\mathrm{H}$ & 4.914158000 & -1.320326000 & 1.429697000 \\
\hline
\end{tabular}




\begin{tabular}{llll}
\hline $\mathrm{H}$ & 3.048731000 & -2.645504000 & 0.361017000 \\
$\mathrm{H}$ & 1.879200000 & -1.465991000 & -0.337393000 \\
$\mathrm{H}$ & 1.799021000 & -4.671445000 & 0.806302000 \\
$\mathrm{H}$ & -0.584925000 & -5.252541000 & 2.091593000 \\
$\mathrm{H}$ & -2.037742000 & -3.960613000 & 3.534115000 \\
$\mathrm{H}$ & -2.756648000 & -3.356588000 & 2.027579000 \\
$\mathrm{H}$ & -3.402304000 & -2.181465000 & 4.153407000 \\
$\mathrm{H}$ & -1.760374000 & -1.530780000 & 4.296414000 \\
$\mathrm{H}$ & -5.054069000 & -0.847676000 & 2.993126000 \\
$\mathrm{H}$ & -4.944920000 & 1.313111000 & 1.251861000 \\
$\mathrm{H}$ & -1.869767000 & 1.519875000 & -0.378503000 \\
$\mathrm{H}$ & -3.039328000 & 2.670053000 & 0.332925000 \\
$\mathrm{H}$ & -1.796424000 & 4.669153000 & 0.851245000 \\
$\mathrm{H}$ & 0.536958000 & 5.215621000 & 2.239763000 \\
$\mathrm{H}$ & 1.941429000 & 3.891195000 & 3.704845000 \\
$\mathrm{H}$ & 2.700634000 & 3.289679000 & 2.217249000 \\
\hline
\end{tabular}

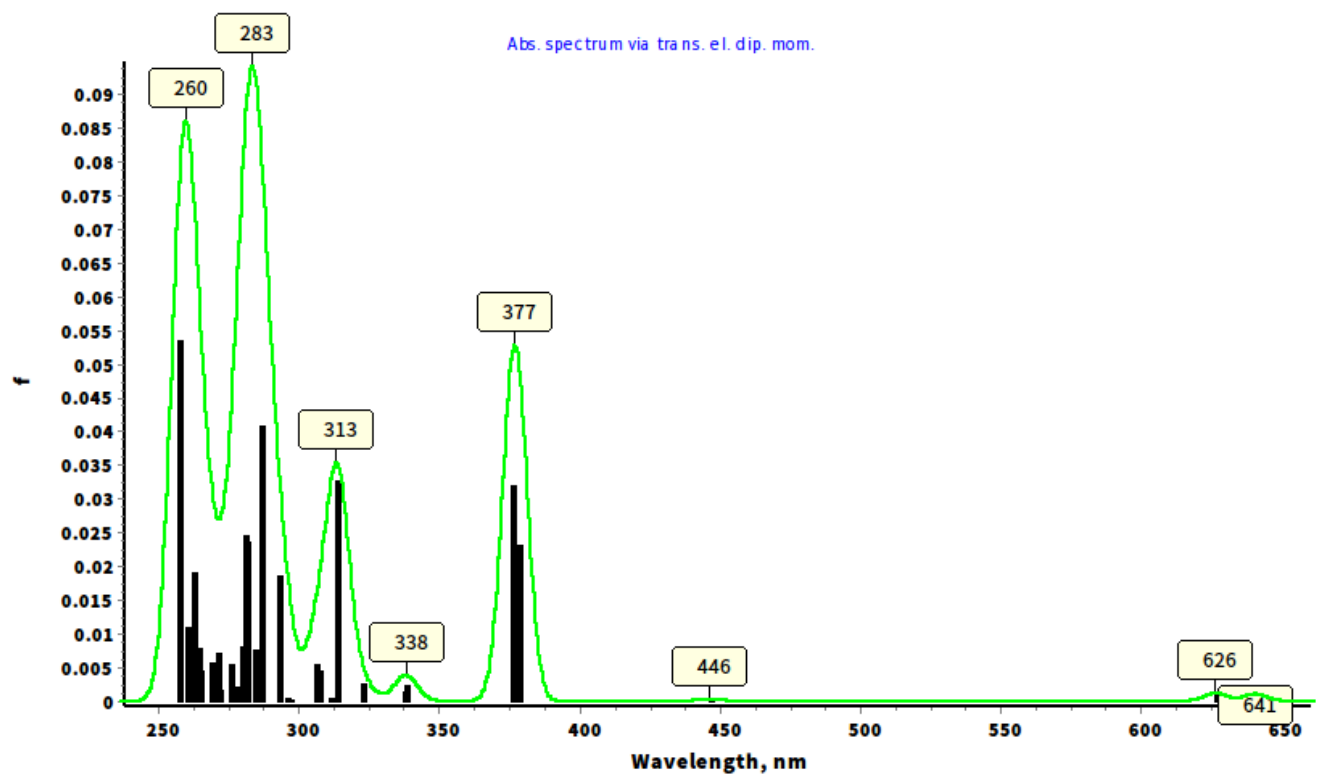

DFT Figure 1. Calculated absorption spectrum of $\left.\left[{ }^{\mathrm{NHC}} \mathrm{L}\right) \mathrm{Fe}\left(\mathrm{N}_{3}\right)_{2}\right] \mathbf{5}$ generated by Chemcraft and calculated with B3LYP functional, COSMO MeCN, RIJCOSX approximation def2-tzvp and def2-tzvp/j basis sets. 


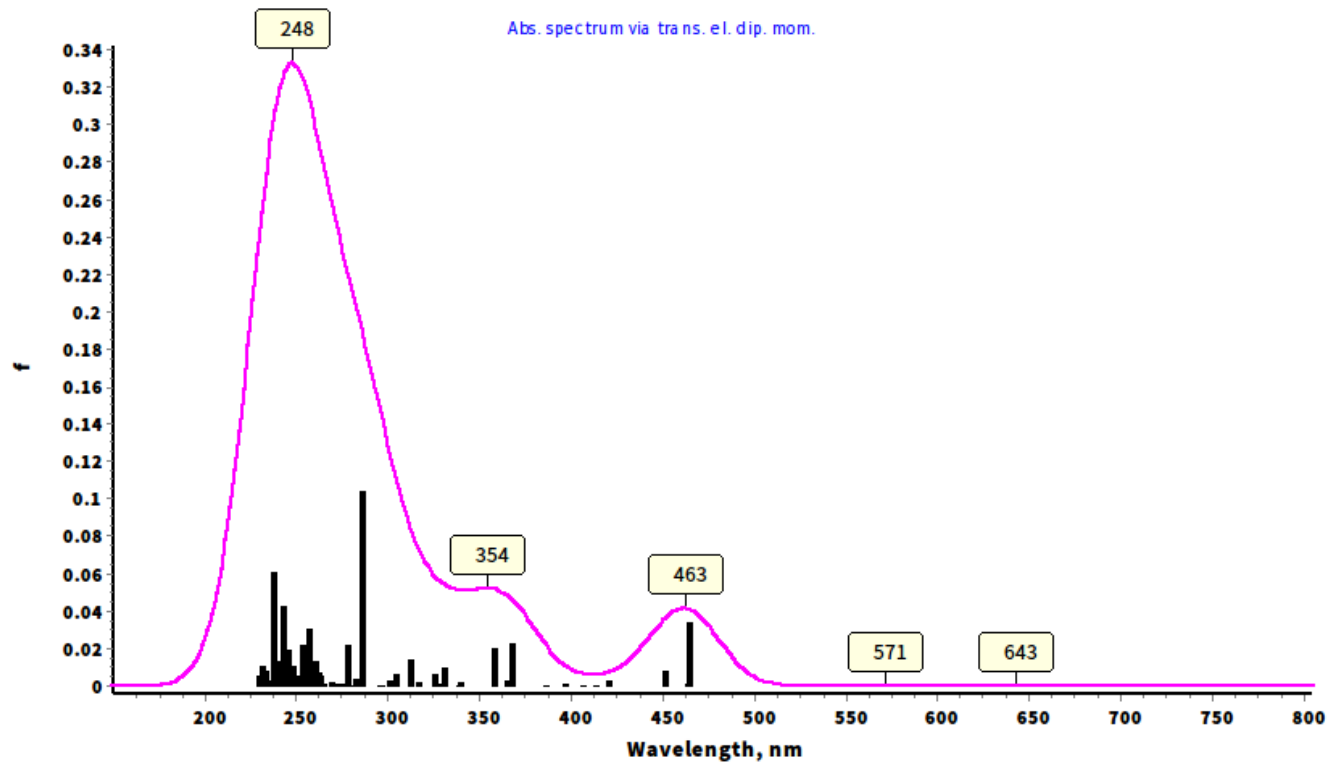

DFT Figure 2. Calculated absorption spectrum of complex $\left.\left[{ }^{\mathrm{NHC}} \mathrm{L}\right) \mathrm{Fe}\left(\mathrm{N}_{3}\right)_{2}\right]^{+} \mathbf{1 1}$ generated by Chemcraft and calculated with B3LYP functional, COSMO MeCN, RIJCOSX approximation def2-tzvp and def2-tzvp/j basis sets.

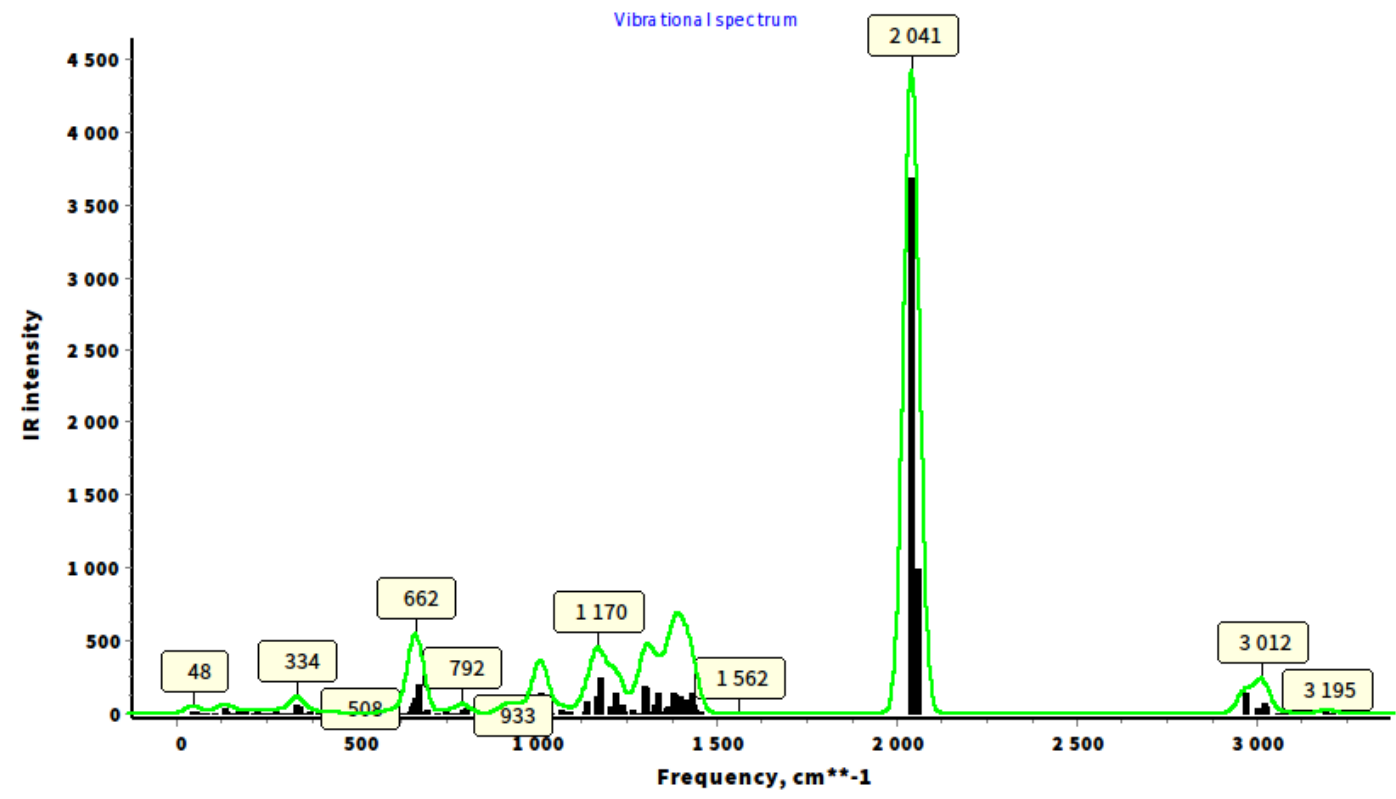

DFT Figure 3. Calculated infrared spectrum spectrum of $\left[\left({ }^{\mathrm{NHC}} \mathrm{L}\right) \mathrm{Fe}\left(\mathrm{N}_{3}\right)_{2}\right] \mathbf{5}$ generated by Chemcraft and calculated with BP86 functional, COSMO MeCN, RI approximation def2-tzvp and def2-tzvp/j basis sets. The large signal at $2041 \mathrm{~cm}^{-1}$ represents the calculated the azide peak. 


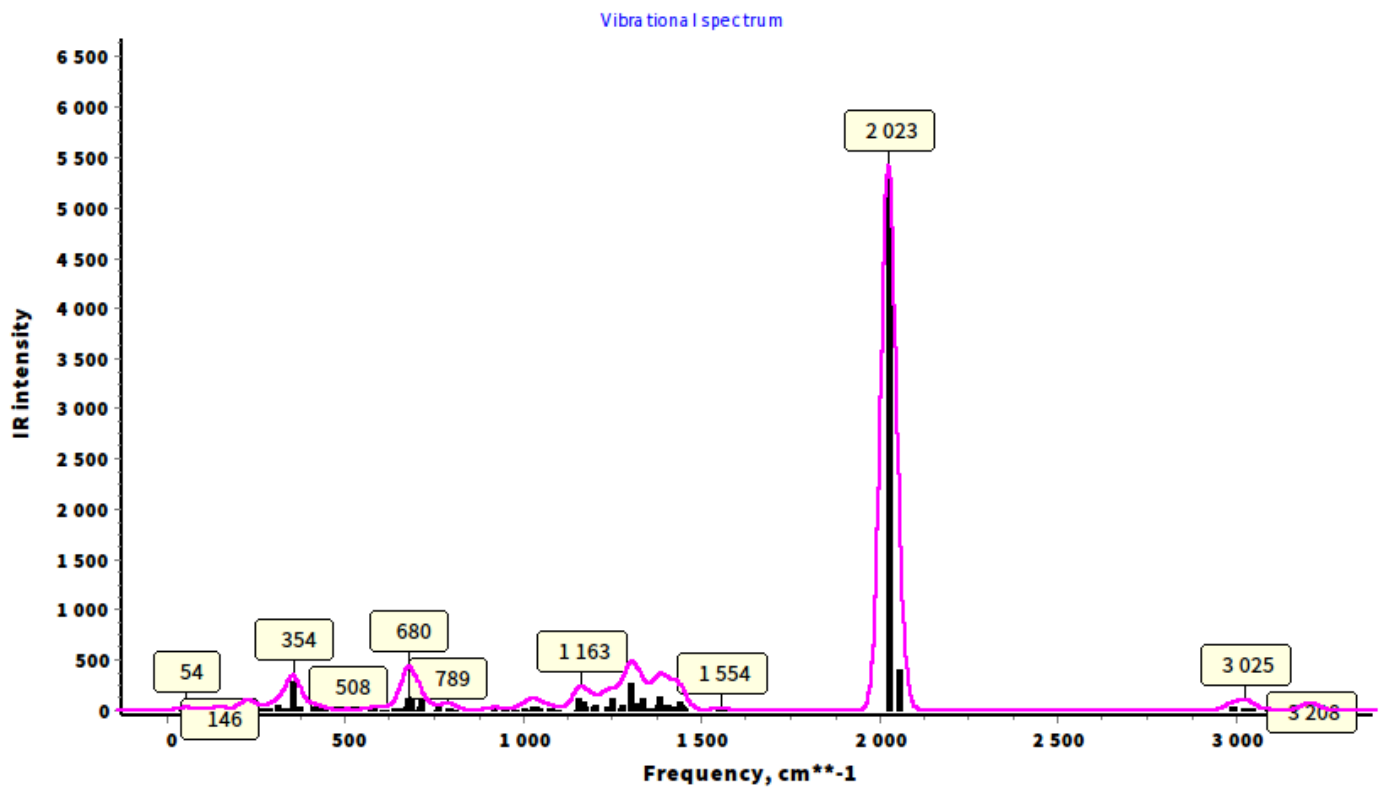

DFT Figure 4. Calculated infrared spectrum of complex $\left.\left[{ }^{\mathrm{NHC}} \mathrm{L}\right) \mathrm{Fe}\left(\mathrm{N}_{3}\right)_{2}\right]^{+} \mathbf{1 1}$ generated by Chemcraft and calculated with BP86 functional, COSMO MeCN, RI approximation def2-tzvp and def2-tzvp/j basis sets. The large signal at $2023 \mathrm{~cm}^{-1}$ represents the calculated the azide peak.

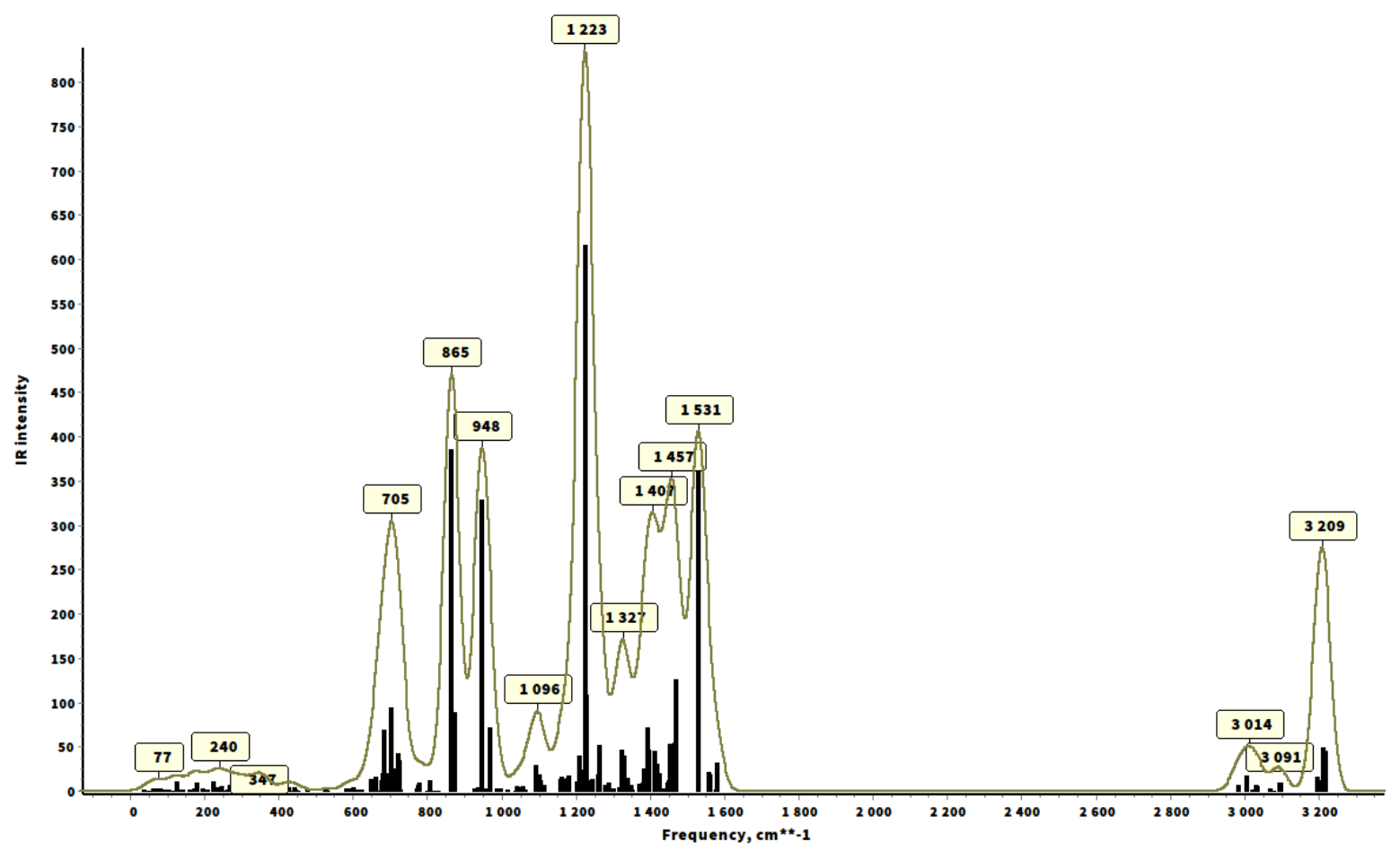

DFT Figure 5. Calculated infrared spectrum of complex $\left[\left\{\left(\mathrm{O}_{2} \mathrm{NO}\right)\left({ }^{\mathrm{NHC}} \mathrm{L}\right) \mathrm{Fe}\right\}(\mathrm{N})\right]^{3+} \mathbf{1 4}$ generated by Chemcraft and calculated with BP86 functional, UKS d3bj approximation def2-tzvp and def2-tzvp/j basis sets. Stretches within the $\mathrm{ONO}_{2}$ moiety are calculated at 1223,1227 and $1528 \mathrm{~cm}^{-1}$; the Fe-N stretch is predicted to be at relatively high intensity at 864 and $945 \mathrm{~cm}^{-1}$. 
DFT Table 3. Coordinates of the energy-optimized structure of $\left[\left({ }^{\mathrm{NHC}} \mathrm{L}\right)\{\mathrm{FeNO}\}^{6}(\mathrm{ONO})\right]^{2+} \mathbf{1 8}$.

\begin{tabular}{|c|c|c|c|}
\hline $\mathrm{Fe}$ & 0.109433000 & 0.011100000 & 0.010767000 \\
\hline $\mathrm{N}$ & 1.737487000 & -0.016851000 & -0.141278000 \\
\hline $\mathrm{O}$ & 2.880061000 & -0.098155000 & -0.319704000 \\
\hline $\mathrm{O}$ & -1.896232000 & -0.023888000 & -0.000584000 \\
\hline $\mathrm{C}$ & -0.153681000 & 1.978542000 & 0.214504000 \\
\hline $\mathrm{C}$ & 0.014378000 & 0.011620000 & 1.998910000 \\
\hline $\mathrm{C}$ & 0.006527000 & -2.023265000 & -0.084118000 \\
\hline $\mathrm{C}$ & -0.190010000 & 0.153167000 & -2.013246000 \\
\hline $\mathrm{N}$ & -2.689596000 & -0.824362000 & 0.697545000 \\
\hline $\mathrm{N}$ & 0.415413000 & 3.003044000 & -0.471694000 \\
\hline $\mathrm{N}$ & -0.919017000 & 2.590682000 & 1.159650000 \\
\hline $\mathrm{N}$ & -0.744764000 & 0.847133000 & 2.761736000 \\
\hline $\mathrm{N}$ & 0.728879000 & -0.712555000 & 2.897028000 \\
\hline $\mathrm{N}$ & 0.542418000 & -3.035756000 & 0.660788000 \\
\hline $\mathrm{N}$ & -0.557371000 & -2.663487000 & -1.150333000 \\
\hline $\mathrm{N}$ & -0.788238000 & -0.850133000 & -2.721116000 \\
\hline $\mathrm{N}$ & 0.117031000 & 1.082181000 & -2.972025000 \\
\hline $\mathrm{O}$ & -2.204311000 & -1.634661000 & 1.446882000 \\
\hline $\mathrm{C}$ & -0.004768000 & 4.235290000 & 0.020755000 \\
\hline $\mathrm{C}$ & 1.357863000 & 2.836370000 & -1.576752000 \\
\hline $\mathrm{C}$ & -0.846677000 & 3.975399000 & 1.054903000 \\
\hline $\mathrm{C}$ & -1.626090000 & 1.870229000 & 2.211101000 \\
\hline $\mathrm{C}$ & -0.531330000 & 0.627476000 & 4.118900000 \\
\hline $\mathrm{C}$ & 0.397555000 & -0.359480000 & 4.203022000 \\
\hline $\mathrm{C}$ & 1.703269000 & -1.748701000 & 2.552489000 \\
\hline $\mathrm{C}$ & 1.076941000 & -3.032819000 & 2.036674000 \\
\hline $\mathrm{C}$ & 0.294444000 & -4.273556000 & 0.068433000 \\
\hline $\mathrm{C}$ & -0.399158000 & -4.042182000 & -1.070620000 \\
\hline $\mathrm{C}$ & -1.407039000 & -2.030971000 & -2.144056000 \\
\hline $\mathrm{C}$ & -0.871878000 & -0.558986000 & -4.074555000 \\
\hline $\mathrm{C}$ & -0.300549000 & 0.658228000 & -4.231349000 \\
\hline $\mathrm{C}$ & 0.698235000 & 2.438274000 & -2.882383000 \\
\hline $\mathrm{H}$ & 0.337451000 & 5.176961000 & -0.392627000 \\
\hline $\mathrm{H}$ & 2.146899000 & 2.136690000 & -1.283484000 \\
\hline $\mathrm{H}$ & 1.843884000 & 3.806778000 & -1.741446000 \\
\hline $\mathrm{H}$ & -1.388320000 & 4.643505000 & 1.714508000 \\
\hline $\mathrm{H}$ & -1.882989000 & 2.575772000 & 3.008066000 \\
\hline $\mathrm{H}$ & -2.544057000 & 1.420421000 & 1.812353000 \\
\hline $\mathrm{H}$ & -1.047937000 & 1.183913000 & 4.892286000 \\
\hline $\mathrm{H}$ & 0.855997000 & -0.825878000 & 5.067294000 \\
\hline $\mathrm{H}$ & 2.241027000 & -1.999461000 & 3.475653000 \\
\hline $\mathrm{H}$ & 2.448657000 & -1.343871000 & 1.858237000 \\
\hline $\mathrm{H}$ & 1.846665000 & -3.816570000 & 2.070041000 \\
\hline $\mathrm{H}$ & 0.260091000 & -3.338047000 & 2.708860000 \\
\hline $\mathrm{H}$ & 0.633109000 & -5.202727000 & 0.511924000 \\
\hline $\mathrm{H}$ & -0.783670000 & -4.722726000 & -1.821362000 \\
\hline $\mathrm{H}$ & -2.372369000 & -1.758191000 & -1.694561000 \\
\hline $\mathrm{H}$ & -1.581108000 & -2.751634000 & -2.950192000 \\
\hline $\mathrm{H}$ & -1.321709000 & -1.232246000 & -4.795119000 \\
\hline $\mathrm{H}$ & -0.148625000 & 1.257775000 & -5.121777000 \\
\hline $\mathrm{H}$ & -0.102008000 & 3.154044000 & -3.123644000 \\
\hline $\mathrm{H}$ & 1.457228000 & 2.510174000 & -3.674771000 \\
\hline
\end{tabular}


DFT Table 4. Coordinates of the energy-optimized structure of $\left[\left({ }^{\mathrm{NHC}} \mathrm{L}\right)\{\mathrm{FeNO}\}^{7}\right]^{2+} \mathbf{1 7}$.

\begin{tabular}{|c|c|c|c|}
\hline $\mathrm{Fe}$ & -0.026286000 & -0.024655000 & 0.027791000 \\
\hline $\mathrm{C}$ & 1.892565000 & -0.033282000 & -0.389352000 \\
\hline $\mathrm{C}$ & -0.039439000 & 1.915983000 & -0.499799000 \\
\hline $\mathrm{C}$ & -1.967822000 & -0.014244000 & -0.261948000 \\
\hline $\mathrm{C}$ & -0.046676000 & -1.961269000 & -0.516798000 \\
\hline $\mathrm{N}$ & 0.031615000 & -0.027052000 & 1.687687000 \\
\hline $\mathrm{N}$ & 2.865043000 & -0.812492000 & 0.157752000 \\
\hline $\mathrm{N}$ & 2.560023000 & 0.768363000 & -1.261650000 \\
\hline $\mathrm{N}$ & 0.884354000 & 2.474293000 & -1.351001000 \\
\hline $\mathrm{N}$ & -0.821386000 & 2.984998000 & -0.141734000 \\
\hline $\mathrm{N}$ & -2.903045000 & 0.760924000 & 0.351986000 \\
\hline $\mathrm{N}$ & -2.690026000 & -0.808373000 & -1.096683000 \\
\hline $\mathrm{N}$ & -1.023781000 & -2.512655000 & -1.311043000 \\
\hline $\mathrm{N}$ & 0.756557000 & -3.033005000 & -0.218929000 \\
\hline $\mathrm{O}$ & 0.070416000 & -0.019540000 & 2.855394000 \\
\hline $\mathrm{C}$ & 4.115980000 & -0.501585000 & -0.366312000 \\
\hline $\mathrm{C}$ & 2.588745000 & -1.849421000 & 1.150216000 \\
\hline $\mathrm{C}$ & 3.9256860000 & 0.498332000 & -1.268826000 \\
\hline $\mathrm{C}$ & 1.869154000 & 1.718974000 & -2.113838000 \\
\hline $\mathrm{C}$ & 0.687594000 & 3.836751000 & -1.517443000 \\
\hline $\mathrm{C}$ & -0.383554000 & 4.157249000 & -0.750698000 \\
\hline $\mathrm{C}$ & -2.027231000 & 3.072943000 & 0.713026000 \\
\hline $\mathrm{C}$ & -2.563475000 & 1.790486000 & 1.332243000 \\
\hline $\mathrm{C}$ & -4.184957000 & 0.455846000 & -0.095396000 \\
\hline $\mathrm{C}$ & -4.052966000 & -0.536599000 & -1.016255000 \\
\hline $\mathrm{C}$ & -2.056588000 & -1.751162000 & -2.000550000 \\
\hline $\mathrm{C}$ & -0.838431000 & -3.873521000 & -1.501438000 \\
\hline $\mathrm{C}$ & 0.280034000 & -4.200216000 & -0.808323000 \\
\hline $\mathrm{C}$ & 2.014420000 & -3.127452000 & 0.556575000 \\
\hline $\mathrm{H}$ & 5.025347000 & -0.996154000 & -0.045132000 \\
\hline $\mathrm{H}$ & 1.940726000 & -1.435259000 & 1.927421000 \\
\hline $\mathrm{H}$ & 3.536820000 & -2.117914000 & 1.634331000 \\
\hline $\mathrm{H}$ & 4.630381000 & 1.031563000 & -1.896465000 \\
\hline $\mathrm{H}$ & 1.373215000 & 1.187040000 & -2.939689000 \\
\hline $\mathrm{H}$ & 2.598893000 & 2.420168000 & -2.534058000 \\
\hline $\mathrm{H}$ & 1.312693000 & 4.450955000 & -2.155544000 \\
\hline $\mathrm{H}$ & -0.864020000 & 5.113619000 & -0.578271000 \\
\hline $\mathrm{H}$ & -2.809708000 & 3.535479000 & 0.093033000 \\
\hline $\mathrm{H}$ & -1.803821000 & 3.769786000 & 1.534739000 \\
\hline $\mathrm{H}$ & -1.868711000 & 1.369923000 & 2.064527000 \\
\hline $\mathrm{H}$ & -3.478864000 & 2.055775000 & 1.877433000 \\
\hline $\mathrm{H}$ & -5.071717000 & 0.948514000 & 0.286309000 \\
\hline $\mathrm{H}$ & -4.796423000 & -1.063786000 & -1.602813000 \\
\hline $\mathrm{H}$ & -1.616531000 & -1.211913000 & -2.853013000 \\
\hline $\mathrm{H}$ & -2.812630000 & -2.448895000 & -2.377341000 \\
\hline $\mathrm{H}$ & -1.503607000 & -4.482766000 & -2.102642000 \\
\hline $\mathrm{H}$ & 0.770192000 & -5.158022000 & -0.675230000 \\
\hline $\mathrm{H}$ & 2.755666000 & -3.585331000 & -0.115297000 \\
\hline $\mathrm{H}$ & 1.842750000 & -3.830141000 & 1.385829000 \\
\hline
\end{tabular}


DFT Table 5. Coordinates of the energy-optimized structure of $\left[\left({ }^{\mathrm{NHC}} \mathrm{L}\right)\{\mathrm{FeNO}\}^{8}\right]^{+} \mathbf{1 9}$.

\begin{tabular}{|c|c|c|c|}
\hline $\mathrm{Fe}$ & 0.018142000 & -0.004533000 & 0.013002000 \\
\hline $\mathrm{N}$ & -0.126935000 & -0.136388000 & -1.630635000 \\
\hline $\mathrm{O}$ & -0.303704000 & -0.308930000 & -2.794857000 \\
\hline $\mathrm{C}$ & -1.829635000 & 0.050303000 & 0.573451000 \\
\hline $\mathrm{C}$ & 0.086520000 & -1.827524000 & 0.753773000 \\
\hline $\mathrm{C}$ & 1.928827000 & -0.011499000 & 0.350322000 \\
\hline $\mathrm{C}$ & 0.034357000 & 1.894441000 & 0.593066000 \\
\hline $\mathrm{N}$ & -2.839380000 & 0.807988000 & 0.038638000 \\
\hline $\mathrm{N}$ & -2.462143000 & -0.667677000 & 1.551098000 \\
\hline $\mathrm{N}$ & -0.787953000 & -2.340724000 & 1.694292000 \\
\hline $\mathrm{N}$ & 0.844464000 & -2.940055000 & 0.425986000 \\
\hline $\mathrm{N}$ & 2.873660000 & -0.808874000 & -0.239868000 \\
\hline $\mathrm{N}$ & 2.666241000 & 0.784512000 & 1.182321000 \\
\hline $\mathrm{N}$ & 1.015450000 & 2.476734000 & 1.374112000 \\
\hline $\mathrm{N}$ & -0.753779000 & 2.982591000 & 0.259369000 \\
\hline $\mathrm{C}$ & -4.054310000 & 0.572837000 & 0.677692000 \\
\hline $\mathrm{C}$ & -2.597628000 & 1.761459000 & -1.033014000 \\
\hline $\mathrm{C}$ & -3.818517000 & -0.362636000 & 1.635968000 \\
\hline $\mathrm{C}$ & -1.692514000 & -1.501155000 & 2.449072000 \\
\hline $\mathrm{C}$ & -0.589432000 & -3.693749000 & 1.937066000 \\
\hline $\mathrm{C}$ & 0.438270000 & -4.068105000 & 1.139145000 \\
\hline $\mathrm{C}$ & 1.955994000 & -3.104631000 & -0.528026000 \\
\hline $\mathrm{C}$ & 2.510871000 & -1.847784000 & -1.190735000 \\
\hline $\mathrm{C}$ & 4.155030000 & -0.517667000 & 0.221315000 \\
\hline $\mathrm{C}$ & 4.026783000 & 0.492498000 & 1.122787000 \\
\hline $\mathrm{C}$ & 1.998684000 & 1.692264000 & 2.089761000 \\
\hline $\mathrm{C}$ & 0.852875000 & 3.848553000 & 1.514070000 \\
\hline $\mathrm{C}$ & -0.261290000 & 4.164432000 & 0.812055000 \\
\hline $\mathrm{C}$ & -1.984696000 & 3.069532000 & -0.546891000 \\
\hline $\mathrm{H}$ & 1.796354000 & -1.426486000 & -1.904507000 \\
\hline $\mathrm{H}$ & -1.962430000 & 1.283545000 & -1.784625000 \\
\hline $\mathrm{H}$ & -4.973841000 & 1.066204000 & 0.387111000 \\
\hline $\mathrm{H}$ & -3.558235000 & 2.003530000 & -1.508005000 \\
\hline $\mathrm{H}$ & -4.486842000 & -0.834054000 & 2.346654000 \\
\hline $\mathrm{H}$ & -1.109645000 & -0.853763000 & 3.128990000 \\
\hline $\mathrm{H}$ & -2.369456000 & -2.134778000 & 3.033825000 \\
\hline $\mathrm{H}$ & -1.183085000 & -4.260732000 & 2.644331000 \\
\hline $\mathrm{H}$ & 0.908473000 & -5.035261000 & 1.006128000 \\
\hline $\mathrm{H}$ & 1.628150000 & -3.788391000 & -1.327468000 \\
\hline $\mathrm{H}$ & 2.765237000 & -3.608033000 & 0.023886000 \\
\hline $\mathrm{H}$ & 3.410777000 & -2.140961000 & -1.748609000 \\
\hline $\mathrm{H}$ & 5.038085000 & -1.030583000 & -0.140391000 \\
\hline $\mathrm{H}$ & 4.771608000 & 1.022471000 & 1.704143000 \\
\hline $\mathrm{H}$ & 1.491751000 & 1.104365000 & 2.875092000 \\
\hline $\mathrm{H}$ & 2.733986000 & 2.364098000 & 2.547999000 \\
\hline $\mathrm{H}$ & 1.530076000 & 4.469216000 & 2.088649000 \\
\hline $\mathrm{H}$ & -0.740104000 & 5.122370000 & 0.646854000 \\
\hline $\mathrm{H}$ & -1.775765000 & 3.692543000 & -1.431677000 \\
\hline $\mathrm{H}$ & -2.722046000 & 3.609212000 & 0.068104000 \\
\hline
\end{tabular}




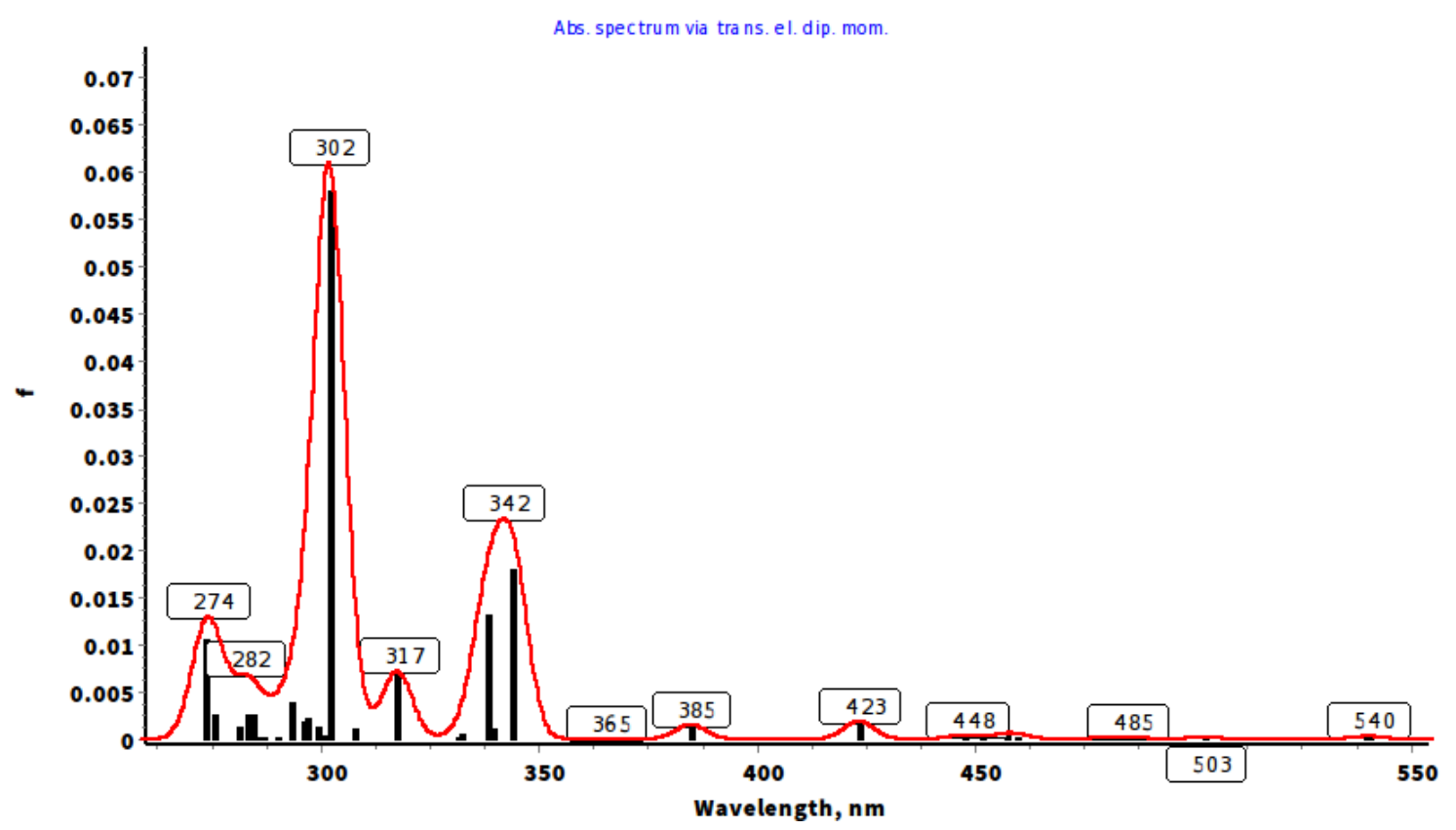

DFT Figure 6. Calculated absorption spectrum of $\left[\left({ }^{\mathrm{NHC}} \mathrm{L}\right)\{\mathrm{FeNO}\}^{6}(\mathrm{ONO})\right]^{2+} \mathbf{1 8}$ generated by Chemcraft and calculated with B3LYP functional, COSMO MeCN, RIJCOSX approximation def2-tzvp and def2-tzvp/j basis sets.

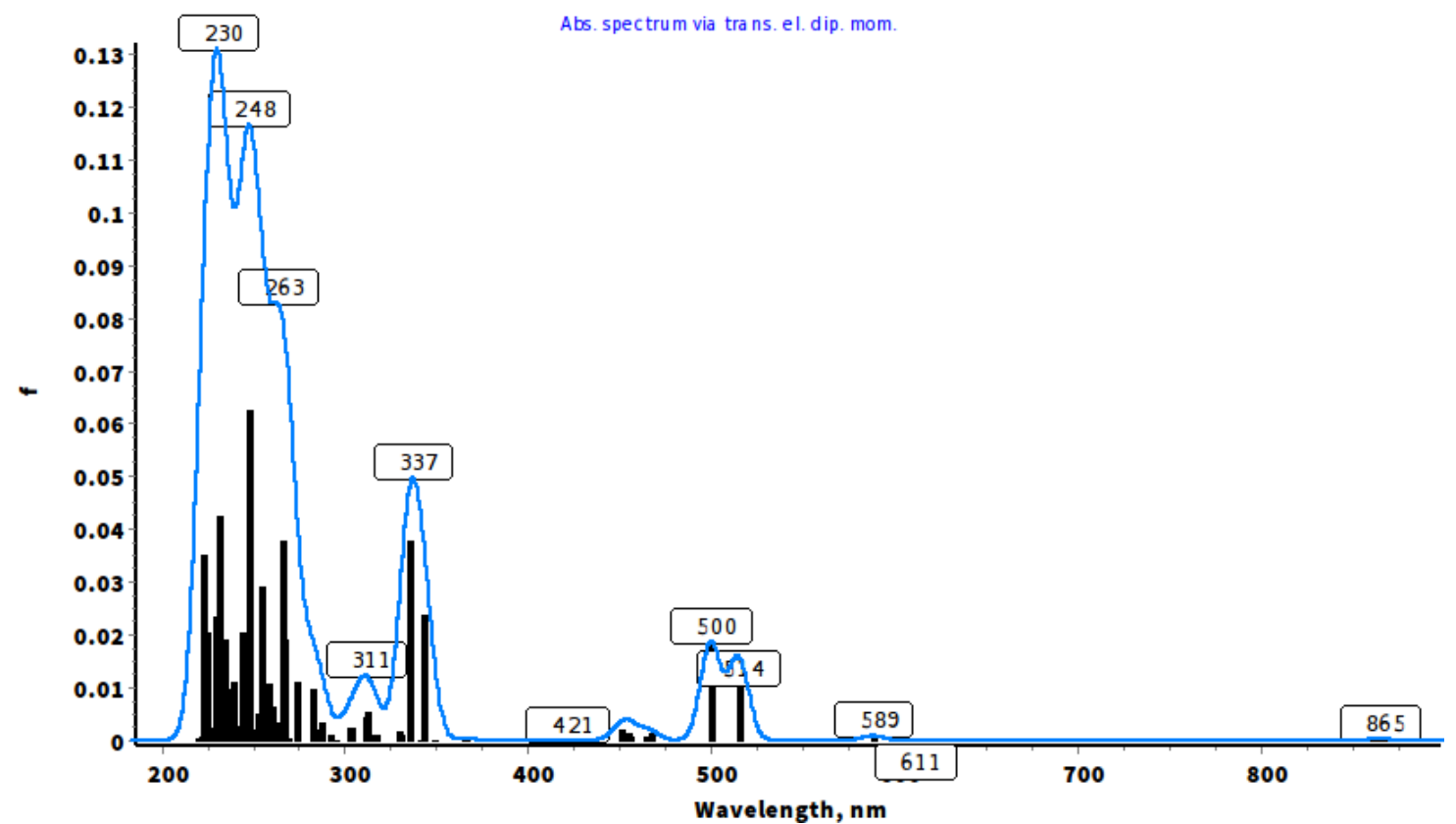

DFT Figure 7. Calculated absorption spectrum of $\left[\left({ }^{\mathrm{NHC}} \mathrm{L}\right)\{\mathrm{FeNO}\}^{7}\right]^{2+} \mathbf{1 7}$ generated by Chemcraft and calculated with B3LYP functional, COSMO MeCN, RIJCOSX approximation def2-tzvp and def2-tzvp/j basis sets. 


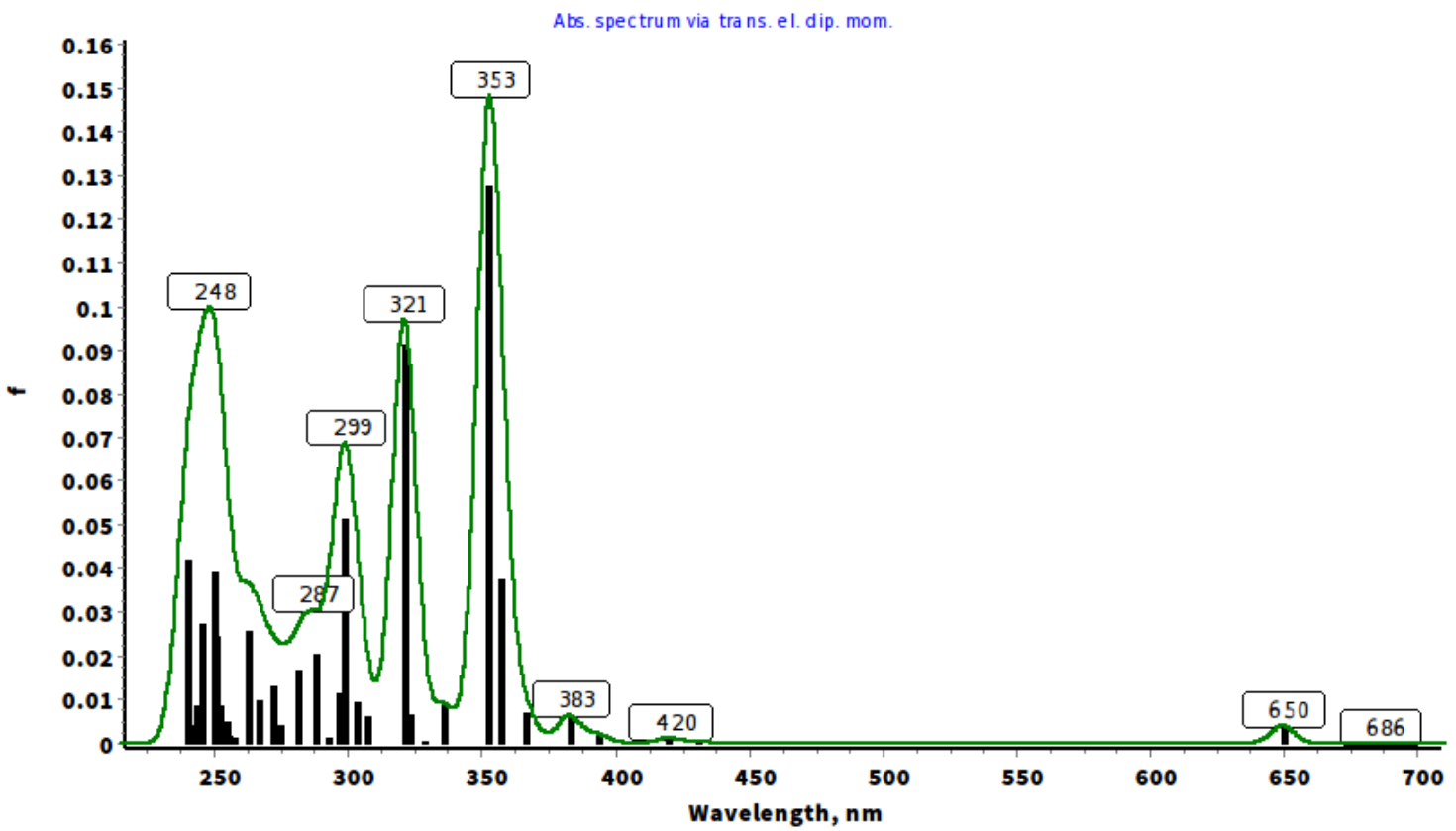

DFT Figure 8. Calculated absorption spectrum of $\left[\left({ }^{\mathrm{NHC}} \mathrm{L}\right)\{\mathrm{FeNO}\}^{8}\right]^{+} \mathbf{1 9}$ generated by Chemcraft and calculated with B3LYP functional, COSMO MeCN, RIJCOSX approximation def2-tzvp and def2-tzvp/j basis sets.

$450 \mathrm{~nm}$

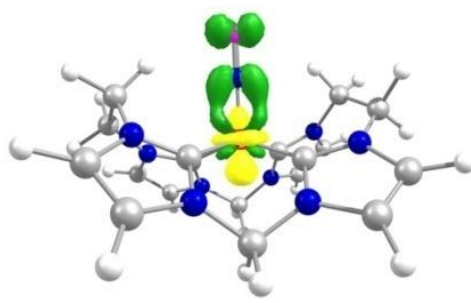

$515 \mathrm{~nm}$

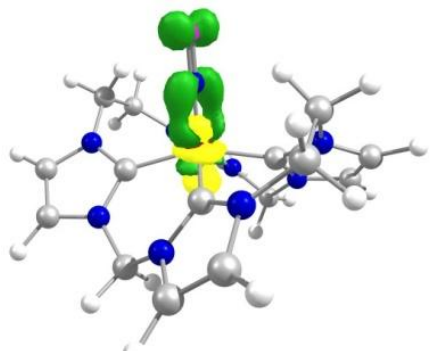

$335 \mathrm{~nm}$

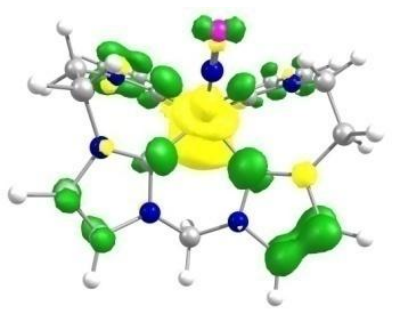

$343 \mathrm{~nm}$

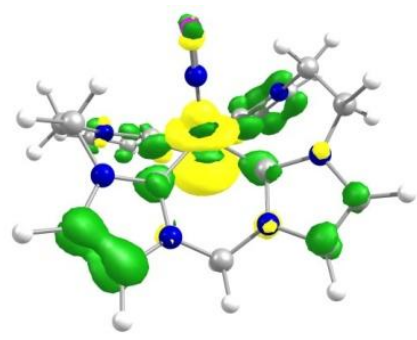

DFT Figure 9. Difference density orbitals of complex $\left[\left({ }^{\mathrm{NHC}} \mathrm{L}\right)\{\mathrm{FeNO}\}^{7}\right]^{2+} \mathbf{1 7}$ for spectroscopic transitions at $499.5 \mathrm{~nm} \& 514.8 \mathrm{~nm}$ (left; contour value 0.01 ) and $335.1 \mathrm{~nm} \& 343.1 \mathrm{~nm}$ (right; contour value 0.003) generated by Chemcraft and calculated with BP86 functional, RI approximation def2-tzvp and def2-tzvp/j basis stes. The orbitals represent that the experimentally observed band at around $615 \mathrm{~nm}$ results from a $\mathrm{Fe}-$ NO-CT and the band around $350 \mathrm{~nm}$ results from a mainly $\mathrm{Fe}-\left({ }^{\mathrm{NHC}} \mathrm{L}\right)-\mathrm{CT}$ but it cannot be assigned if these are ML- or LM-CT transitions. For this assignment NTOs are used (see Figure 66). 
$\{\mathrm{FeNO}\}^{8}$ :

$350 \mathrm{~nm}$

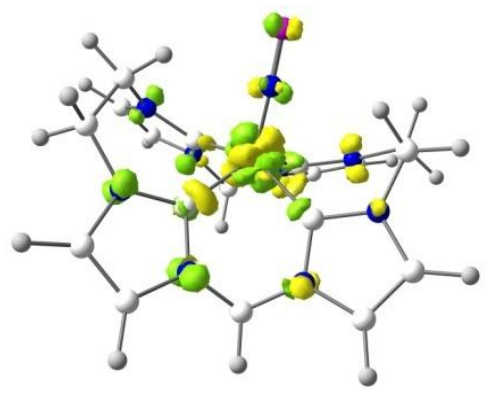

$652 \mathrm{~nm}$

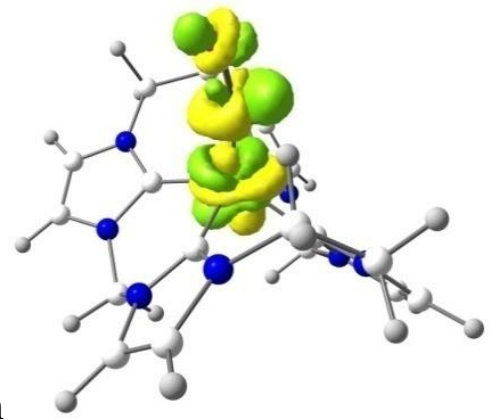

$\{\mathrm{FeNO}\}^{6}$ :

$302 \mathrm{~nm}$

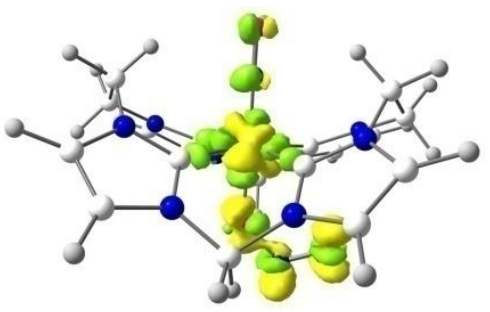

$344 \mathrm{~nm}$

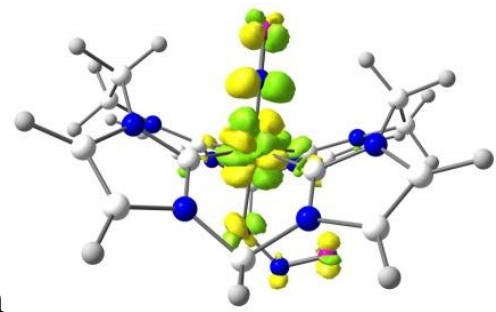

DFT Figure 10. Calculated difference density orbitals of $\left.\left[{ }^{\mathrm{NHC}} \mathrm{L}\right)\{\mathrm{FeNO}\}^{8}\right]^{+} \mathbf{1 9}$ (top) and $\left[\left({ }^{\mathrm{NHC}} \mathrm{L}\right)\{\mathrm{FeNO}\}^{6}(\mathrm{ONO})\right]^{2+} \mathbf{1 8}$ (bottom) generated by Chemcraft and calculated with B3LYP functional, RIJCOSX approximation def2-tzvp and def2-tzvp/j basis sets. In case of compound 19, the dd-transition around $350 \mathrm{~nm}$ shows interaction between the iron center and the tetracarbene ligand while the broad band around $652 \mathrm{~nm}$ is assigned to the lowest energy HOMO-LUMO transition. The main transitions in compound $\mathbf{1 8}$ are mainly $\mathrm{ONO}-\mathrm{Fe}(302 \mathrm{~nm})$ and $\mathrm{ON}-\mathrm{Fe}(344 \mathrm{~nm})$ based, respectively.

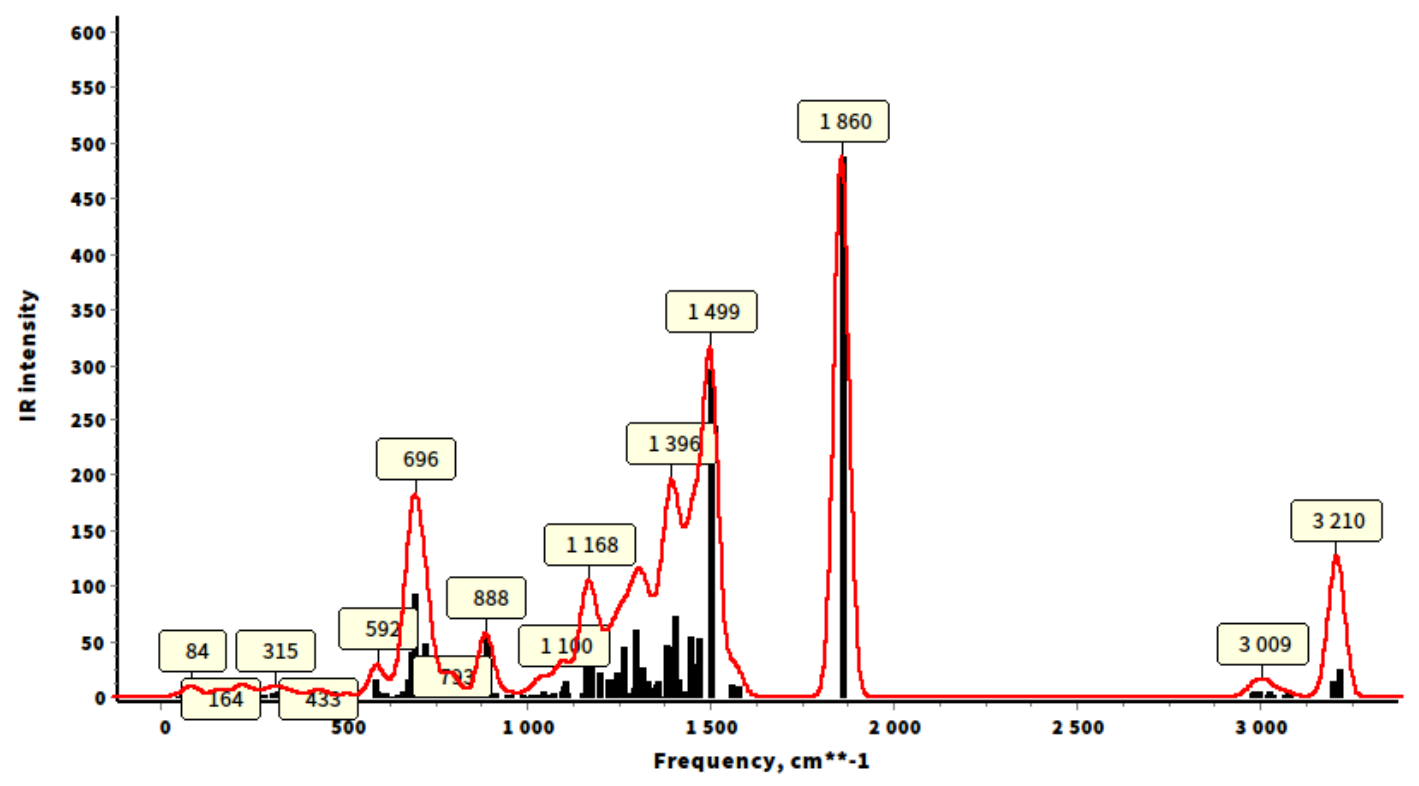

DFT Figure 11. Calculated infrared spectrum of $\left.\left[{ }^{\mathrm{NHC}} \mathrm{L}\right)\{\mathrm{FeNO}\}^{6}(\mathrm{ONO})\right]^{2+} \mathbf{1 8}$ generated by Chemcraft and calculated with BP86 functional, RI approximation def2-tzvp and def2-tzvp/j basis sets. While the intensity for $\tilde{v}_{\mathrm{N}-\mathrm{O}}=1859 \mathrm{~cm}^{-1}$ is predicted to be very high, $\tilde{v}_{\mathrm{Fe}-\mathrm{NO}}=663 \mathrm{~cm}^{-1}$ is of very low intensity and next to more intense ligand transitions. 


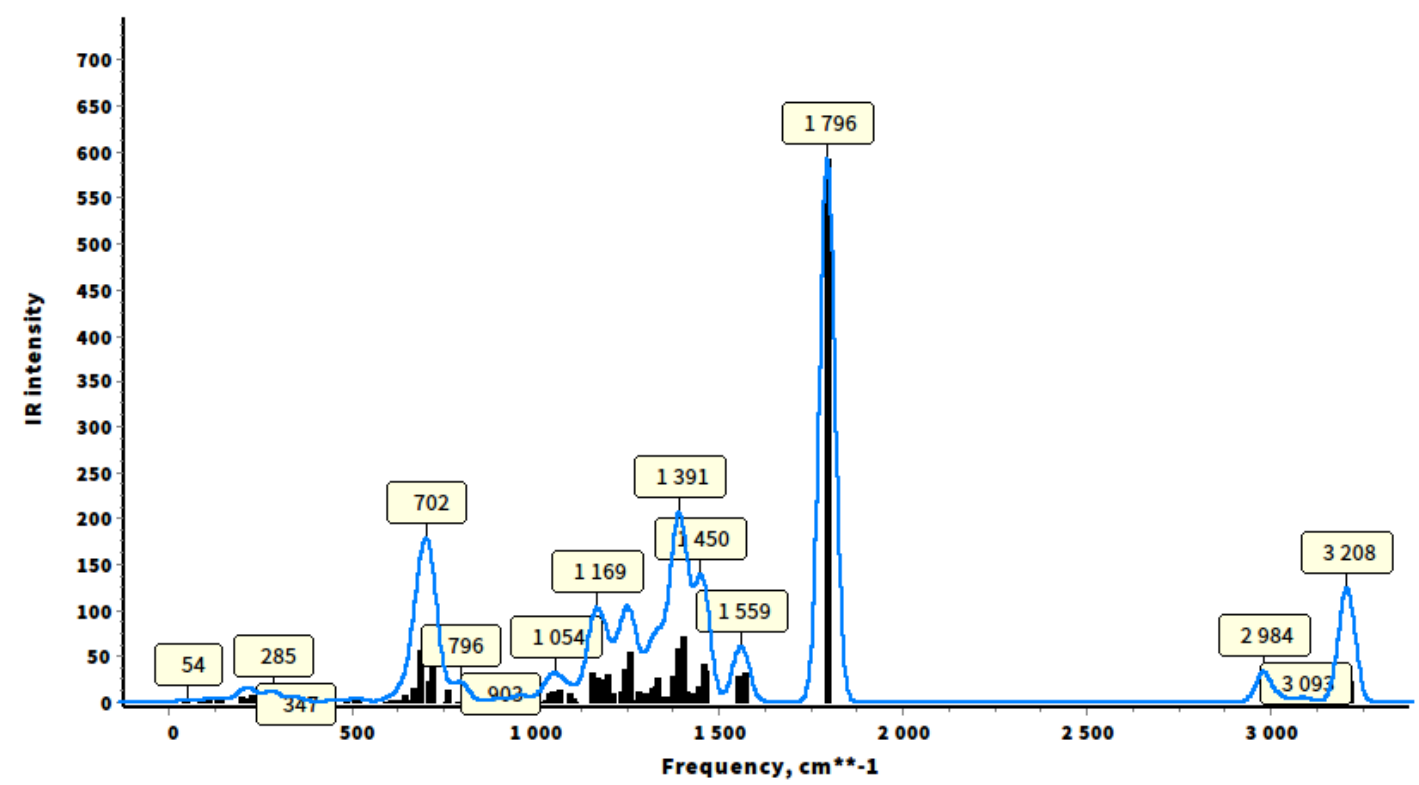

DFT Figure 12. Calculated infrared spectrum of $\left[\left({ }^{\mathrm{NHC}} \mathrm{L}\right)\{\mathrm{FeNO}\}^{7}\right]^{2+} \mathbf{1 7}$ (including spectroscopic transitions) generated by Chemcraft and calculated with BP86 functional, RI approximation def2-tzvp and def2-tzvp/j basis stes. While the intensity for $\tilde{v}_{\mathrm{N}-\mathrm{O}}=1796 \mathrm{~cm}^{-1}$ is predicted to be very high, $\tilde{v}_{\mathrm{Fe}-\mathrm{NO}}=626 \mathrm{~cm}^{-1}$ is of very low intensity and next to more intense ligand transitions.

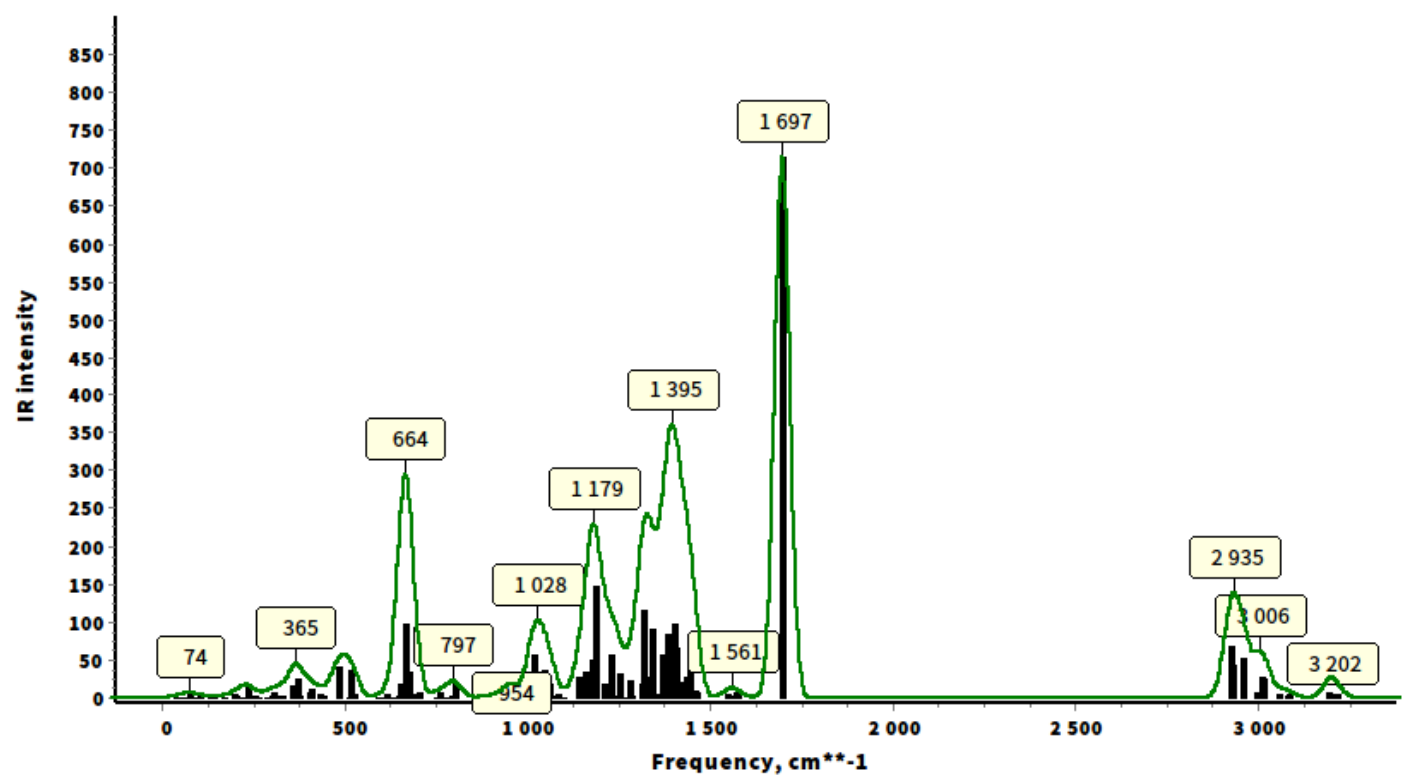

DFT Figure 13. Calculated infrared spectrum of $\left[\left({ }^{\mathrm{NHC}} \mathrm{L}\right)\{\mathrm{FeNO}\}^{8}\right]^{+} \mathbf{1 9}$ generated by Chemcraft and calculated with BP86 functional, RI approximation def2-tzvp and def2-tzvp/j basis sets. While the intensity for $\tilde{v}_{\mathrm{N}-\mathrm{O}}=1697 \mathrm{~cm}^{-1}$ is predicted to be very high, $\tilde{v}_{\mathrm{Fe}-\mathrm{NO}}=635 \mathrm{~cm}^{-1}$ is of very low intensity and next to more intense ligand transitions.

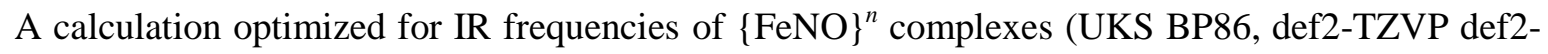
TZVP/J DecontractAux D3BJ ZORA Grid5 NoFinalGrid SlowConv TightSCF RI) led to the following values: 17: $\tilde{v}_{\mathrm{N}-\mathrm{O}}=1752 \mathrm{~cm}^{-1}$, 19: $\tilde{v}_{\mathrm{N}-\mathrm{O}}=1601 \mathrm{~cm}^{-1}$ 


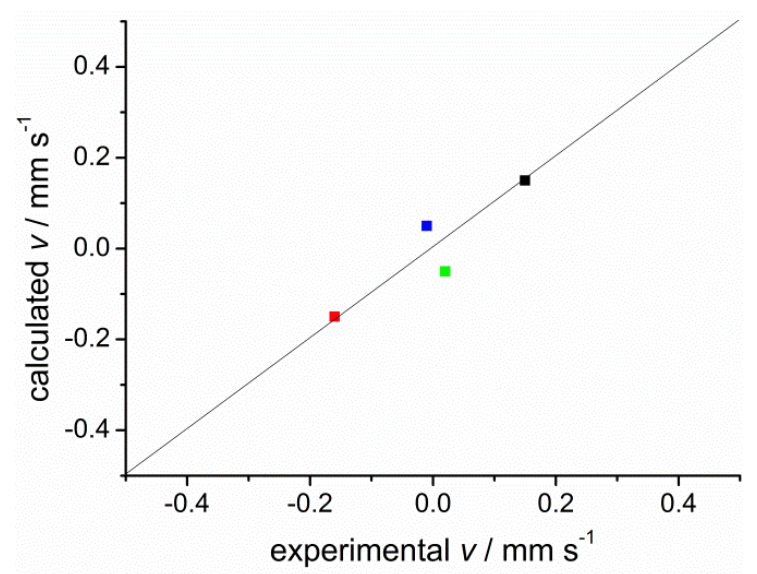

DFT Figure 14. Plot of the DFT-calculated Mössbauer IS vs. the experimental values for $\{\mathrm{FeNO}\}^{6} \mathbf{1 8}$ (red), $\{\mathrm{FeNO}\}^{7} 17$ (blue), $\{\mathrm{FeNO}\}^{8} 19$ (green) and the related tetracarbene $\left({ }^{\mathrm{NHC}} \mathrm{L}\right.$ ) coordinated $\mathrm{Fe}^{\mathrm{III}}-\mathrm{S}-\mathrm{S}-\mathrm{Fe}^{\mathrm{III}}$ species $\mathbf{3}_{\mathrm{SS}}$ (black). ${ }^{[156]}$ The straight line represents perfect agreement of calculation and experiment.

DFT Table 6. Calculated orbital populations per MO for $\left.\left[{ }^{\mathrm{NHC}} \mathrm{L}\right)\{\mathrm{FeNO}\}^{7}\right]^{2+} \mathbf{1 7}$. According to these data the $\mathrm{Fe}\left(\mathrm{p}_{\mathrm{z}}\right)$ character is $8 \%$ for Mulliken and $13 \%$ for Loewdin orbital populations.

\begin{tabular}{ccc}
\hline & $\begin{array}{c}\text { MULLIKEN ORBITAL } \\
\text { POPULATIONS PER MO }\end{array}$ & $\begin{array}{c}\text { LOEWDIN ORBITAL } \\
\text { POPULATIONS PER MO }\end{array}$ \\
\hline Number of orbital & 111 & 111 \\
Energy & -0.39963 & -0.39963 \\
Spin up population & 1.00000 & 1.00000 \\
0Fe 5s & 1.1 & 0.2 \\
0Fe 6s & -0.6 & 0.1 \\
0Fe 2pz & 0.5 & 0.7 \\
0Fe 2py & 0.0 & 0.0 \\
0Fe 3pz & - & 0.3 \\
OFe 1dz2 & 25.8 & 24.6 \\
0Fe 1dxz & 0.1 & 0.1 \\
OFe 1dyz & 0.0 & 0.0 \\
0Fe 1dx2y2 & 0.2 & 0.2 \\
0Fe 1dxy & 1.6 & 1.5 \\
0Fe 2dz2 & 14.4 & 15.7 \\
0Fe 2dxz & 0.0 & 0.1 \\
0Fe 2dyz & 0.0 & 0.0 \\
0Fe 2dx2y2 & 0.1 & 0.2 \\
0Fe 2dxy & 1.0 & 1.1 \\
0Fe 3dz2 & 4.7 & 2.9 \\
0Fe 3dxz & 0.0 & 0.0 \\
0Fe 3dyz & -0.0 & 0.0 \\
0Fe 3dxy & 0.4 & 0.3 \\
0Fe 4pz & 3.5 & 5.5 \\
0Fe 4px & 0.0 & 0.0 \\
0Fe 4py & 0.0 & 0.0 \\
0Fe 4dz2 & 0.3 & 0.1 \\
0Fe 4dxz & 0.0 & 0.0 \\
0Fe 4dyz & 0.0 & 0.0 \\
0Fe 4dxy & -0.1 & 0.0 \\
\hline
\end{tabular}




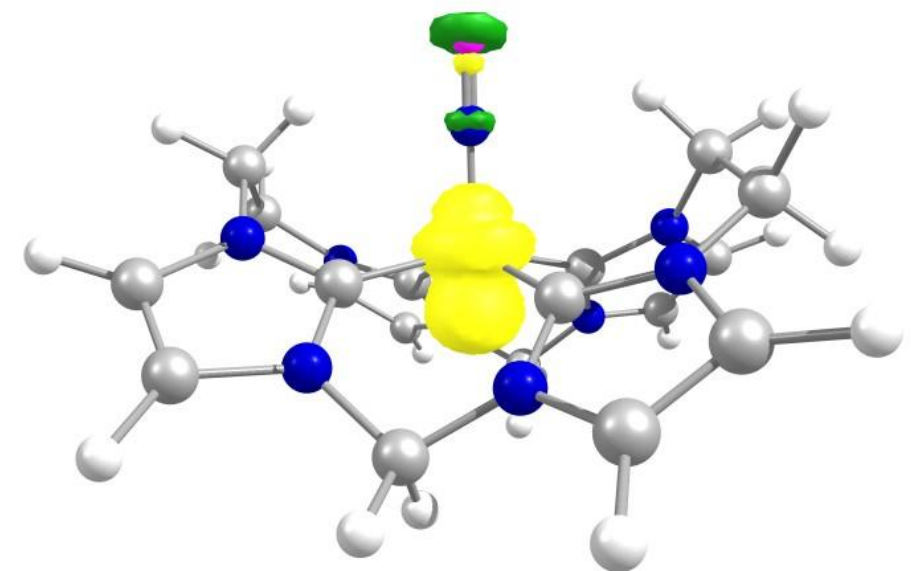

DFT Figure 15. Plot of the calculated electron spin density of $\left.\left[{ }^{\mathrm{NHC}} \mathrm{L}\right)\{\mathrm{FeNO}\}^{7}\right]^{2+} \mathbf{1 7}$ (contour value: 0.05). The picture reflects that the $\alpha$-spin is largely located at the iron center.

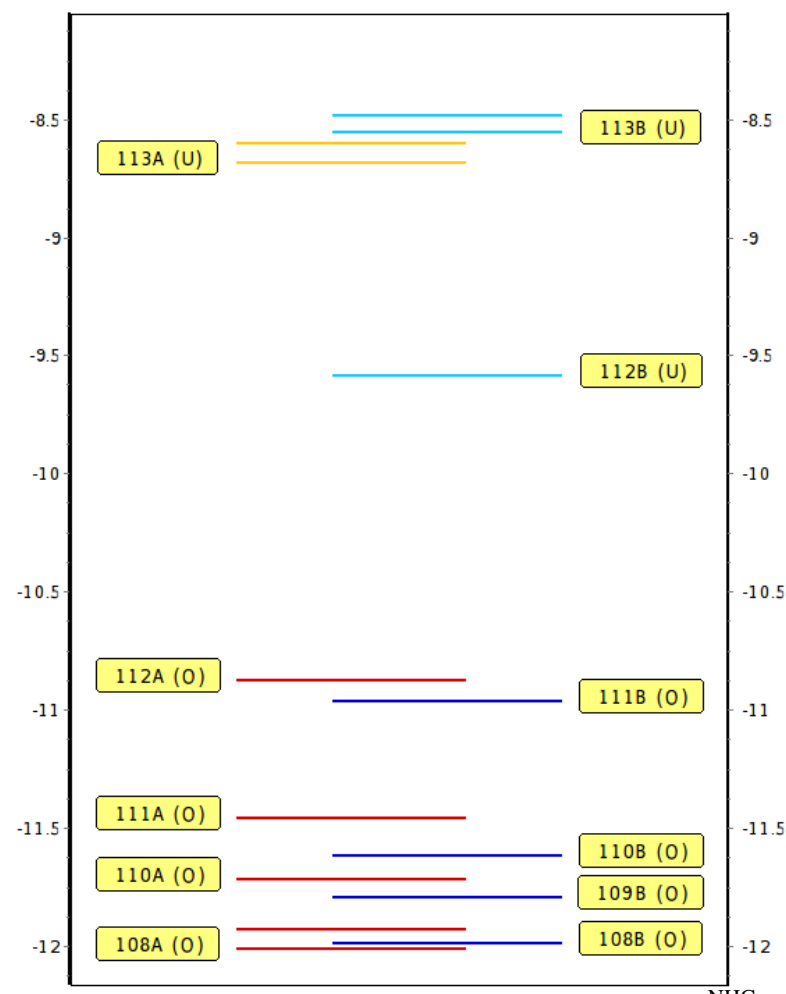

DFT Figure 16. Energetic order of $\alpha$ (left) and $\beta$ (right) frontier orbitals of $\left[\left({ }^{\mathrm{NHC}} \mathrm{L}\right)\{\mathrm{FeNO}\}^{7}\right]^{2+} \mathbf{1 7}$ genergated by Chemcraft and calculated with BP86 functional, RI approximation def2-tzvp and def2-tzvp/j basis stes. 


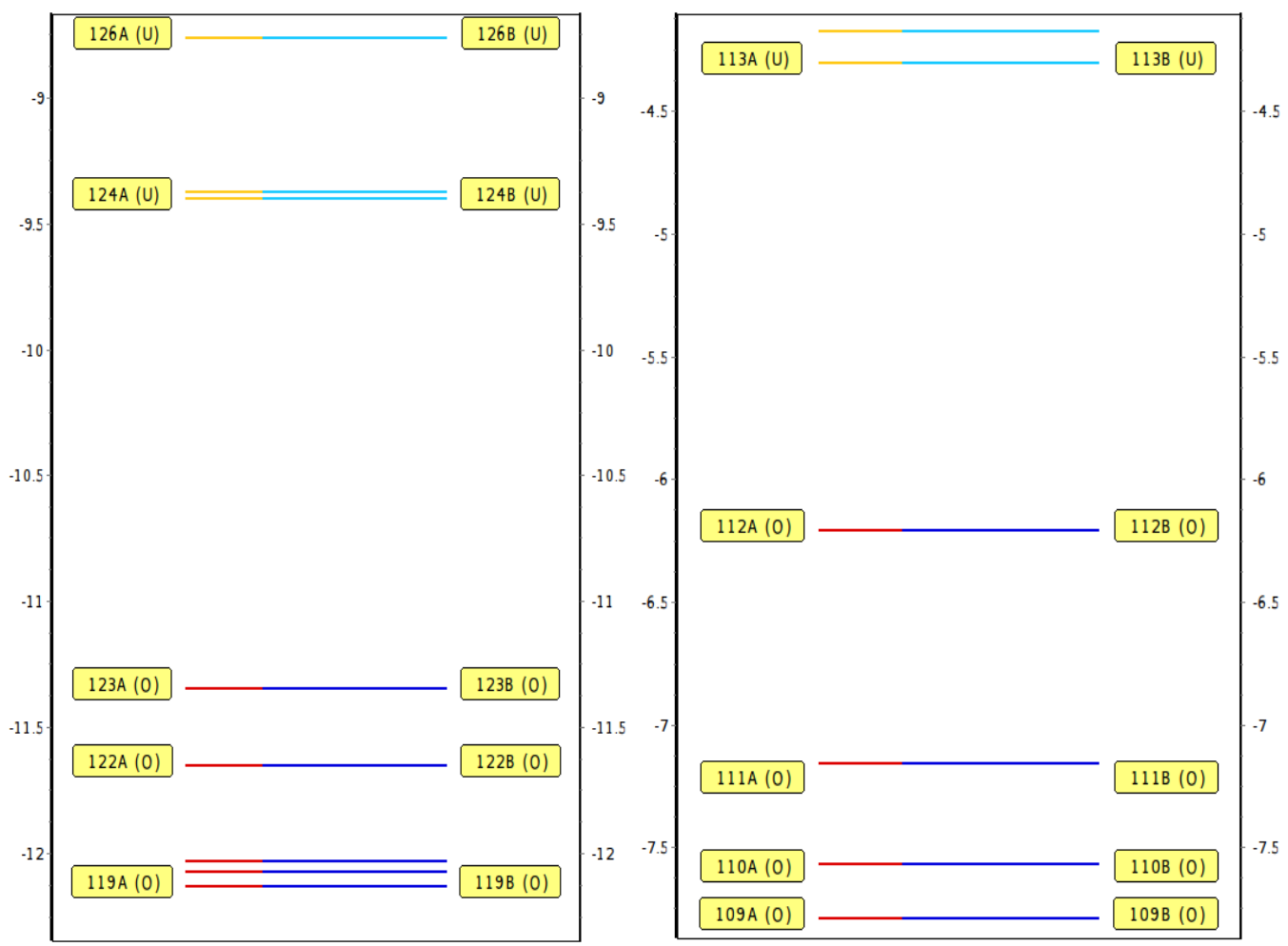

DFT Figure 17. Energetic order of $\alpha$ (left) and $\beta$ (right) frontier orbitals of $\left[\left({ }^{\mathrm{NHC}} \mathrm{L}\right)\{\mathrm{FeNO}\}^{6}(\mathrm{ONO})\right]^{2+} \mathbf{1 8}$ (left) and $\left[\left({ }^{\mathrm{NHC}} \mathrm{L}\right)\{\mathrm{FeNO}\}^{8}\right]^{+} \mathbf{1 9}$ (right) generated by Chemcraft and calculated with BP86 functional, RI approximation def2-tzvp and def2-tzvp/j basis sets. 


\section{Crystallography}

X-ray data were collected on a STOE IPDS II diffractometer with an area detector (graphite monochromated Mo- $\mathrm{K}_{\alpha}$ radiation, $\lambda=0.71073 \AA$ ) by use of $\omega$ scans at $-140{ }^{\circ} \mathrm{C}$. The structures were solved with SHELXT or SHELXS and refined on $F^{2}$ using all reflections with SHELXL2013/2014. ${ }^{[265]}$ Most non-hydrogen atoms were refined anisotropically (disordered MeCN in 10 was refined isotropically). Hydrogen atoms were placed in calculated positions and assigned to an isotropic displacement parameter of $1.2 / 1.5 U_{\text {eq }}(\mathrm{C})$ or $1.5 U_{\text {eq }}(\mathrm{O})$. Face-indexed absorption corrections were performed by the program X-RED. ${ }^{[266]}$

X-ray Table 1. Crystal data and refinement details for 7.

\begin{tabular}{ll}
\hline compound & $\left.\mathbf{7}\left[{ }^{\mathrm{NHC}} \mathbf{L}\right) \mathbf{F e}\left(\mathbf{P M e}_{3}\right)_{2}\right](\mathbf{O T f})_{2}$ \\
\hline empirical formula & $\mathrm{C}_{30} \mathrm{H}_{44} \mathrm{~F}_{6} \mathrm{FeN}_{10} \mathrm{O}_{6} \mathrm{P}_{2} \mathrm{~S}_{2}$ \\
formula weight & 936.66 \\
$T[\mathrm{~K}]$ & $133(2)$ \\
crystal size $\left[\mathrm{mm}^{3}\right]$ & $0.500 \times 0.490 \times 0.190$ \\
crystal system & monoclinic \\
space group & $P 2_{1} / n$ \\
$a\left[\AA{ }^{\circ}\right]$ & $12.8023(7)$ \\
$b[\AA]$ & $12.5818(9)$ \\
$c[\AA]$ & $13.6364(8)$ \\
$\alpha\left[^{\circ}\right]$ & 90 \\
$\beta\left[^{\circ}\right]$ & $114.737(4)$ \\
$\gamma\left[^{\circ}\right]$ & 90 \\
$V\left[\AA^{3}\right]$ & $1994.9(2)$ \\
$Z$ & 2 \\
$V\left[\mathrm{~g} / \mathrm{cm}^{3}\right]$ & 1.559 \\
$F(000)$ & 968 \\
$\mu\left[\mathrm{mm}{ }^{-1}\right]$ & 0.646 \\
$T_{\text {min }} / T_{\text {max }}$ & $0.7435 / 0.8620$ \\
$\theta$-range $\left[{ }^{\circ}\right]$ & $1.833-26.758$ \\
$h k l$-range & $\pm 16, \pm 15, \pm 17$ \\
measured refl. & 27321 \\
unique refl. $\left[R_{\text {int }}\right]$ & $4218[0.0239]$ \\
observed refl. $(I>2 \sigma(I))$ & 3909 \\
data / restraints / param. & $4218 / 0 / 263$ \\
goodness-of-fit $\left(F^{2}\right)$ & 1.017 \\
$R 1, w R 2(I>2 \sigma(I))$ & $0.0240,0.0619$ \\
$R 1, w R 2$ (all data) & $0.0268,0.0631$ \\
resid. el. dens. [e/ $\left./ \AA^{3}\right]$ & $-0.305 / 0.322$ \\
\hline
\end{tabular}




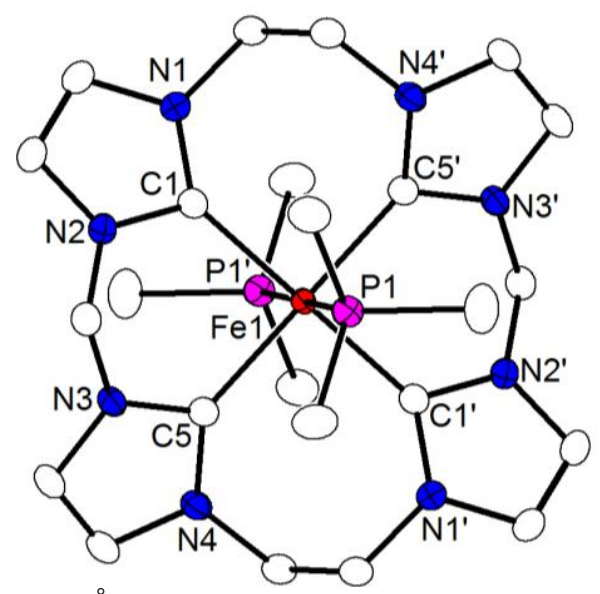

X-ray Figure 1. Selected bond lengths [Å] and angles [ $\left.{ }^{\circ}\right]$ for 7: Fe1-C5 2.0206(12), Fe1-C5' 2.0206(13), Fe1C1' 2.0231(13), Fe1-C1 2.0231(13), Fe1-P1 2.2612(4), Fe1-P1' 2.2613(4); C5-Fe1-C5' 180.0, C5-Fe1-C1' 90.95(5), C5'-Fe1-C1' 89.05(5), C5-Fe1-C1 89.05(5), C5'-Fe1-C1 90.95(5), C1'-Fe1-C1 180.00(5), C5-Fe1P1 93.87(4), C5'-Fe1-P1 86.13(4), C1'-Fe1-P1 85.40(4), C1-Fe1-P1 94.61(4), C5-Fe1-P1' 86.13(4), C5'-Fe1P1' 93.87(4), C1'-Fe1-P1' 94.60(4), C1-Fe1-P1' 85.39(4), P1-Fe1-P1' 180.0, C12-P1-Fe1 114.83(5), C11-P1Fe1 119.41(5), C10-P1-Fe1 119.27(5), N2-C1-Fe1 123.54(9), N1-C1-Fe1 134.37(9), N3-C5-Fe1 123.57(9), N4-C5-Fe1 134.35(10). Symmetry transformation used to generate equivalent atoms: (') 1-x, 1-y, 1-z.

X-ray Table 2. Crystal data and refinement details for $\mathbf{5}, \mathbf{5}_{\mathrm{MeCN}}$ and $\mathbf{9}$.

\begin{tabular}{|c|c|c|c|}
\hline compound & $\begin{array}{l}5 \\
{\left[\left({ }^{N H C} \mathbf{L}\right) \mathrm{Fe}\left(\mathrm{N}_{3}\right)_{2}\right]}\end{array}$ & $\begin{array}{l}5_{\mathrm{MeCN}} \\
{\left[\left(^{\mathrm{NHC}} \mathrm{L}\right) \mathrm{Fe}\left(\mathrm{N}_{3}\right)(\mathrm{MeCN})\right]\left(\mathrm{N}_{3}\right)}\end{array}$ & $\begin{array}{l}9 \\
{\left[\left({ }^{\mathrm{NHC}} \mathrm{L}\right) \mathrm{Fe}(\mathrm{MeCN})_{2}\right]\left(\mathrm{SbCl}_{6}\right)_{3}}\end{array}$ \\
\hline empirical formula & $\mathrm{C}_{18} \mathrm{H}_{20} \mathrm{FeN}_{14}$ & $\mathrm{C}_{22} \mathrm{H}_{26} \mathrm{FeN}_{16}$ & $\mathrm{C}_{24} \mathrm{H}_{29} \mathrm{Cl}_{18} \mathrm{FeN}_{11} \mathrm{Sb}_{3}$ \\
\hline formula weight & 488.33 & 570.44 & 1530.78 \\
\hline crystal size $\left[\mathrm{mm}^{3}\right]$ & $0.23 \times 0.17 \times 0.14$ & $0.29 \times 0.21 \times 0.14$ & $0.42 \times 0.26 \times 0.23$ \\
\hline crystal system & monoclinic & monoclinic & monoclinic \\
\hline space group & $P 2_{1} / n$ & $P 2_{1} / c$ & $C 2 / c$ \\
\hline$a[\AA]$ & $8.6391(7)$ & $16.3331(10)$ & $35.6040(14)$ \\
\hline$b[\AA]$ & $8.3117(5)$ & $8.1828(3)$ & $15.0289(4)$ \\
\hline$c[\AA]$ & $27.637(2)$ & $18.9734(12)$ & $23.3149(8)$ \\
\hline$\alpha\left[^{\circ}\right]$ & 90 & 90 & 90 \\
\hline$\beta\left[{ }^{\circ}\right]$ & $94.875(7)$ & $101.638(5)$ & $101.549(3)$ \\
\hline$\gamma\left[{ }^{\circ}\right]$ & 90 & 90 & 90 \\
\hline$V\left[\AA^{3}\right]$ & 1977.3(3) & $2483.7(2)$ & $12223.0(7)$ \\
\hline$Z$ & 4 & 4 & 8 \\
\hline$\rho\left[\mathrm{g} / \mathrm{cm}^{3}\right]$ & 1.640 & 1.526 & 1.664 \\
\hline$F(000)$ & 1008 & 1184 & 5880 \\
\hline$\mu\left[\mathrm{mm}^{-1}\right]$ & 0.806 & 0.656 & 2.360 \\
\hline$T_{\min } / T_{\max }$ & $0.7867 / 0.9119$ & $0.7236 / 0.9020$ & $0.4443 / 0.6414$ \\
\hline$\theta$-range $\left[{ }^{\circ}\right]$ & $1.479-25.711$ & $1.273-26.031$ & $1.475-25.682$ \\
\hline$h k l$-range & $-10-9, \pm 10, \pm 33$ & $\pm 19,-8-10, \pm 23$ & $\pm 43, \pm 18,-28-26$ \\
\hline measured refl. & 21958 & 28994 & 57100 \\
\hline unique refl. $\left[R_{\mathrm{int}}\right]$ & $3745[0.0842]$ & 28994 & 11535 [0.0398] \\
\hline observed refl. $(I>2 \sigma(I))$ & 2579 & 17226 & 10149 \\
\hline data / restraints / param. & 3745 / $546 / 587$ & 28994 / 0 / 355 & 11535 / 147 / 581 \\
\hline
\end{tabular}




\begin{tabular}{llll}
\hline goodness-of-fit $\left(F^{2}\right)$ & 1.088 & 0.926 & 1.094 \\
$R 1, w R 2(I>2 \sigma(I))$ & $0.0593,0.1236$ & $0.0651,0.1419$ & $0.0353,0.0810$ \\
$R 1, w R 2$ (all data) & $0.0978,0.1352$ & $0.1164,0.1600$ & $0.0426,0.0835$ \\
resid. el. dens. $\left[\mathrm{e} / \AA^{3}\right]$ & $-0.274 / 0.655$ & $-0.498 / 0.778$ & $-0.529 / 1.058$ \\
\hline
\end{tabular}

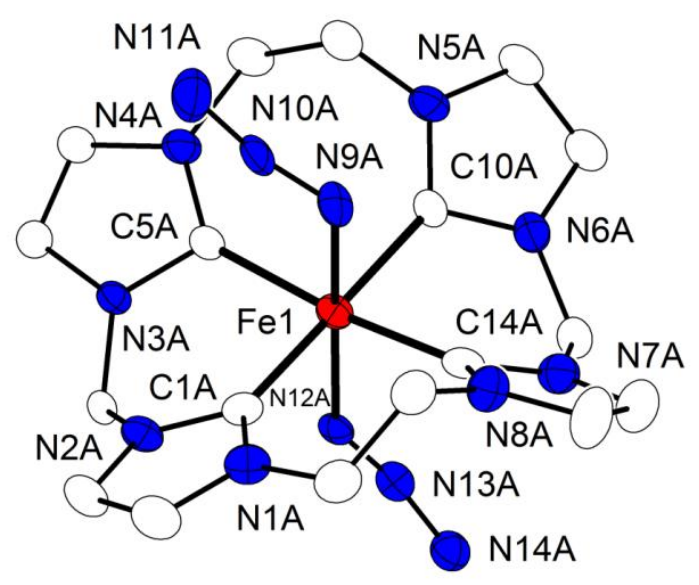

X-ray Figure 2. Plot of the molecular structure of 5 (30\% probability thermal ellipsoids; hydrogen atoms and disorder omitted for clarity). Selected bond lengths $[\AA]$ and angles $\left[{ }^{\circ}\right]$ : Fe1-C1A 1.960(10), Fe1-C5A 2.045(10), Fe1-C10A 2.017(9), Fe1-C14A 1.924(9), Fe1-N9A 2.13(3), Fe1-N12A 2.09(3); C14A-Fe1C1A 95.3(5), C14A-Fe1-C10A 83.9(5), C1A-Fe1-C10A 177.7(6), C14A-Fe1-C5A 173.8(5), C1A-Fe1C5A 90.5(5), C10A-Fe1-C5A 90.2(5), C14A-Fe1-N12A 91.7(9), C1A-Fe1-N12A 84.1(8), C10A-Fe1N12A 98.2(7), C5A-Fe1-N12A 90.7(10), C14A-Fe1-N9A 84.9(8), C1A-Fe1-N9A 91.9(11), C10A-Fe1N9A 85.9(11), C5A-Fe1-N9A 93.2(9), N12A-Fe1-N9A 174.4(12).

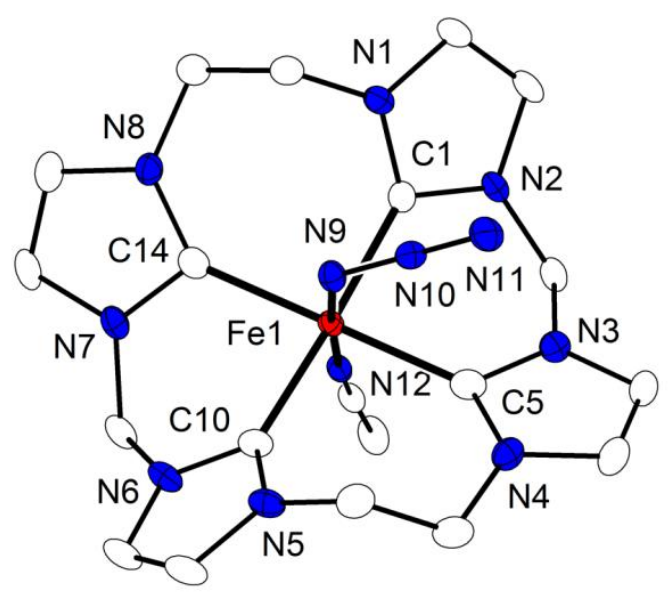

X-ray Figure 3. Plot of the molecular structure of the cationic part of $\mathbf{5}_{\mathrm{MeCN}}$ (30\% probability thermal ellipsoids; hydrogen atoms and solvent omitted for clarity). Selected bond lengths $[\AA]$ and angles $\left[{ }^{\circ}\right]$ : $\mathrm{Fe} 1-\mathrm{C} 1$ 1.957(6), Fe1-C5 1.998(6), Fe1-C10 1.969(6), Fe1-C14 2.021(6), Fe1-N9 2.056(5), Fe1-N12 1.919(5); N12-Fe1-C1 92.9(2), N12-Fe1-C10 94.3(2), C1-Fe1-C10 172.6(2), N12-Fe1-C5 88.0(2), C1-Fe1-C5 86.9(2), C10-Fe1-C5 94.6(3), N12-Fe1-C14 87.9(2), C1-Fe1-C14 92.4(2), C10-Fe1-C14 86.6(2), C5Fe1-C14 175.8(2), N12-Fe1-N9 176.21(18), C1-Fe1-N9 85.7(2), C10-Fe1-N9 87.1(2), C5-Fe1-N9 88.4(2), C14-Fe1-N9 95.6(2). 


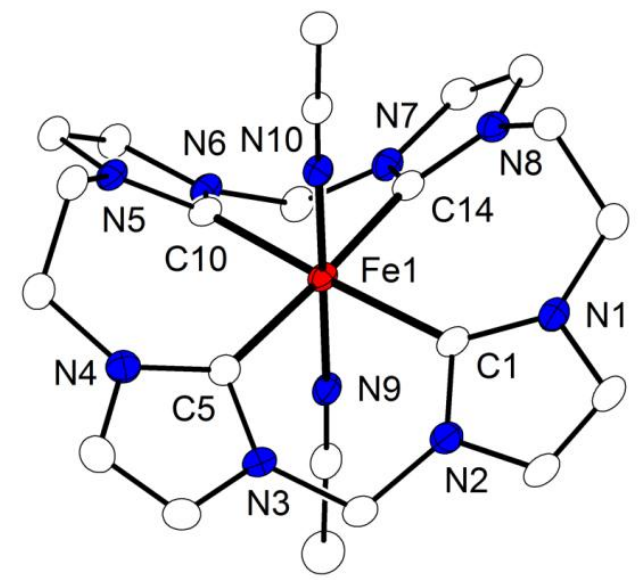

X-ray Figure 4. Plot of the molecular structure of the cationic part of $\mathbf{9}$ (30\% probability thermal ellipsoids; hydrogen atoms and solvent omitted for clarity). Selected bond lengths [ $[\AA]$ and angles [ $\left.{ }^{\circ}\right]$ : $\mathrm{Fe} 1-\mathrm{C} 12.053(4)$, Fe1-C5 2.044(4), Fe1-C10 1.995(4), Fe1-C14 1.986(4), Fe1-N9 1.934(3), Fe1-N10 1.923(3); N10-Fe1-N9 177.22(13), N10-Fe1-C14 90.99(13), N9-Fe1-C14 91.43(14), N10-Fe1-C10 92.09(13), N9-Fe1-C10 89.57(13), C14-Fe1-C10 82.60(15), N10-Fe1-C5 91.06(13), N9-Fe1-C5 86.63(13), C14-Fe1-C5 174.94(15), C10-Fe1-C5 92.70(15), N10-Fe1-C1 90.63(13), N9-Fe1-C1 87.92(13), C14-Fe1-C1 92.27(15), C10-Fe1-C1 174.23(15), C5-Fe1-C1 92.34(15).

X-ray Table 3. Crystal data and refinement details for 10, 11 and $\mathbf{6 .}$

\begin{tabular}{|c|c|c|c|}
\hline compound & $\begin{array}{l}10 \\
{\left[\left({ }^{\mathrm{NHC}} \mathrm{L}\right) \mathrm{FeN}\right.} \\
\end{array}$ & $\begin{array}{l}11 \\
{\left[\left({ }^{\mathrm{NHC}} \mathbf{L}\right) \mathrm{Fe}\left(\mathrm{N}_{3}\right)_{2}\right] \mathrm{Cl}}\end{array}$ & $\begin{array}{l}6 \\
{\left[\left\{\left({ }^{\mathrm{NHC}} \mathrm{L}\right) \mathrm{Fe}\right\}_{2} \mathrm{~N}\right]}\end{array}$ \\
\hline empirical formula & $\mathrm{C}_{22} \mathrm{H}_{24.50} \mathrm{ClF}_{3} \mathrm{FeN}_{12.50} \mathrm{O}_{3} \mathrm{~S}$ & $\mathrm{C}_{20} \mathrm{H}_{28} \mathrm{ClFeN}_{14} \mathrm{O}_{2}$ & $\mathrm{C}_{39} \mathrm{H}_{40} \mathrm{~F}_{9} \mathrm{Fe}_{2} \mathrm{~N}_{17} \mathrm{O}_{9} \mathrm{~S}_{3}$ \\
\hline formula weight & 692.40 & 587.86 & 1269.76 \\
\hline crystal size $\left[\mathrm{mm}^{3}\right]$ & $0.33 \times 0.31 \times 0.28$ & $0.50 \times 0.18 \times 0.16$ & $0.50 \times 0.46 \times 0.44$ \\
\hline crystal system & triclinic & triclinic & triclinic \\
\hline space group & $P \overline{1}$ & $P \overline{1}$ & $P \overline{1}$ \\
\hline$a[\AA]$ & $8.1960(8)$ & $9.6585(4)$ & $11.6348(5)$ \\
\hline$b[\AA]$ & $13.2376(13)$ & $11.1419(5)$ & $14.0648(7)$ \\
\hline$c[\AA]$ & $14.0594(16)$ & $12.6453(5)$ & $15.1177(7)$ \\
\hline$\alpha\left[^{\circ}\right]$ & $102.627(8)$ & $76.513(3)$ & $86.790(4)$ \\
\hline$\beta\left[{ }^{\circ}\right]$ & $103.731(9)$ & $72.612(3)$ & $86.774(4)$ \\
\hline$\gamma\left[{ }^{\circ}\right]$ & $96.053(8)$ & $89.394(3)$ & $81.295(4)$ \\
\hline$V\left[\AA^{3}\right]$ & $1425.9(3)$ & $1260.25(10)$ & $2438.7(2)$ \\
\hline$Z$ & 2 & 2 & 2 \\
\hline$\rho\left[\mathrm{g} / \mathrm{cm}^{3}\right]$ & 1.613 & 1.549 & 1.729 \\
\hline$F(000)$ & 708 & 610 & 1292 \\
\hline$\mu\left[\mathrm{mm}^{-1}\right]$ & 0.767 & 0.755 & 0.831 \\
\hline$T_{\min } / T_{\max }$ & $0.7957 / 0.9091$ & $0.7011 / 0.8706$ & $0.5886 / 0.8040$ \\
\hline$\theta$-range $\left[{ }^{\circ}\right]$ & $1.541-25.752$ & $1.739-26.731$ & $1.773-26.805$ \\
\hline$h k l$-range & $-10-9,-16-15, \pm 17$ & $\pm 12, \pm 14, \pm 15$ & $\pm 14, \pm 17, \pm 19$ \\
\hline measured refl. & 15687 & 17869 & 35363 \\
\hline unique refl. $\left[R_{\mathrm{int}}\right]$ & $5319[0.0734]$ & $5335[0.0394]$ & $10328[0.0496]$ \\
\hline observed refl. $(I>2 \sigma(I))$ & 4064 & 4805 & 8866 \\
\hline data / restraints / param. & $5319 / 59 / 456$ & 5335 / 550 / 591 & $10328 / 0 / 712$ \\
\hline
\end{tabular}




\begin{tabular}{llll}
\hline goodness-of-fit $\left(F^{2}\right)$ & 1.038 & 1.244 & 1.034 \\
$R 1, w R 2(I>2 \sigma(I))$ & $0.0659,0.1682$ & $0.0540,0.1159$ & $0.0433,0.1073$ \\
$R 1, w R 2$ (all data) & $0.0872,0.1805$ & $0.0611,0.1182$ & $0.0529,0.1118$ \\
resid. el. dens. $\left[\mathrm{e} / \AA^{3}\right]$ & $-0.493 / 0.920$ & $-0.606 / 0.358$ & $-0.389 / 1.109$ \\
\hline
\end{tabular}

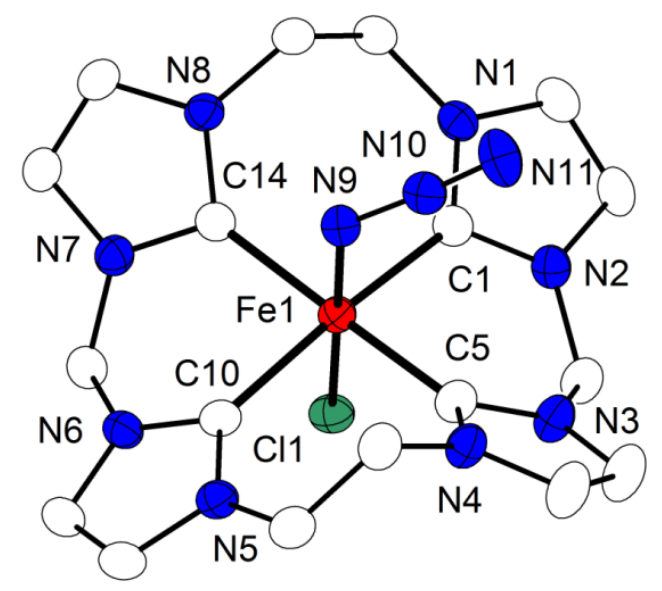

X-ray Figure 5. Plot of the molecular structure of the cationic part of $\mathbf{1 0}$ (30\% probability thermal ellipsoids; hydrogen atoms and solvent omitted for clarity). Selected bond lengths $[\AA]$ and angles $\left[{ }^{\circ}\right]$ : Fe1-C1 2.021(4), Fe1-C5 1.970(4), Fe1-C10 2.049(4), Fe1-C14 1.973(4), Fe1-N9 1.942(3), Fe1-Cl1 2.3355(12); N9-Fe1-C5 88.20(16), N9-Fe1-C14 86.01(16), C5-Fe1-C14 173.96(16), N9-Fe1-C1 93.00(15), C5-Fe1-C1 86.78(17), C14-Fe1-C1 95.23(17), N9-Fe1-C10 95.01(15), C5-Fe1-C10 93.03(18), C14-Fe1-C10 85.77(17), C1Fe1-C10 171.98(16), N9-Fe1-Cl1 177.22(11), C5-Fe1-Cl1 93.86(13), C14-Fe1-Cl1 91.99(12), C1-Fe1Cl1 85.25(12), C10-Fe1-C11 86.77(12)

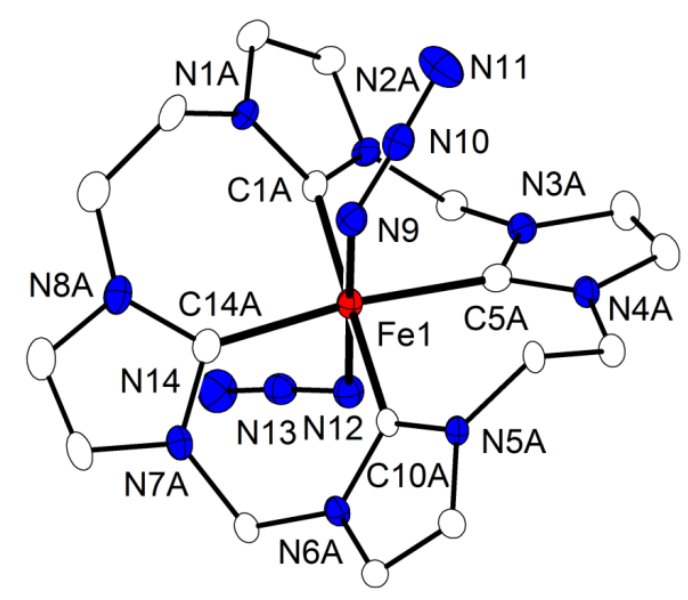

X-ray Figure 6. Plot of the molecular structure of the cationic part of $\mathbf{1 1}$ (30\% probability thermal ellipsoids; hydrogen atoms, disorder and solvent omitted for clarity). Selected bond lengths $[\AA]$ and angles $\left[^{\circ}\right]$ : Fe1C1A 1.945(11), Fe1-C5A 2.043(8), Fe1-C10A 2.024(10), Fe1-C14A 2.013(6), Fe1-N9 1.981(3), Fe1-N12 1.988(3); C1A-Fe1-N9 89.8(6), C1A-Fe1-N12 91.9(6), N9-Fe1-N12 178.31(13), C1A-Fe1-C14A 94.7(4), N9-Fe1-C14A 93.0(2), N12-Fe1-C14A 86.8(2), C1A-Fe1-C10A 178.1(7), N9-Fe1-C10A 88.4(5), N12Fe1-C10A 90.0(5), C14A-Fe1-C10A 86.0(3), C1A-Fe1-C5A 88.4(4), N9-Fe1-C5A 94.5(2), N12-Fe1C5A 85.6(2), C14A-Fe1-C5A 171.9(3), C10A-Fe1-C5A 91.1(4). 


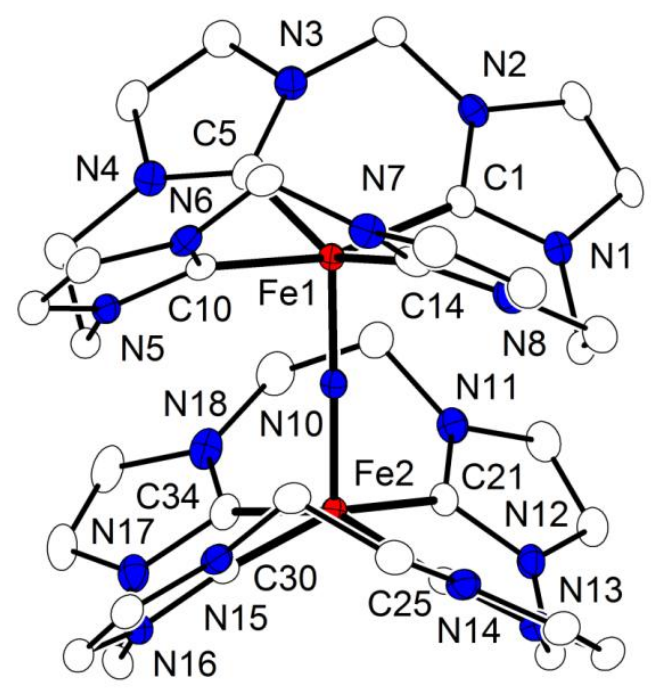

X-ray Figure 7. Plot of the molecular structure of the cationic part of $\mathbf{6}$ (30\% probability thermal ellipsoids; hydrogen atoms omitted for clarity). Selected bond lengths $[\AA]$ and angles $\left[^{\circ}\right]$ : Fe1-C1 1.962(2), Fe1-C5 2.016(3), Fe1-C10 1.957(2), Fe1-C14 2.006(3), Fe1-N10 1.690(2), Fe2-C21 1.963(2), Fe2-C25 2.024(3), Fe2-C30 1.960(2), Fe2-C34 2.015(3), Fe2-N10 1.689(2); N10-Fe1-C10 97.69(10), N10-Fe1-C1 100.12(10), C10-Fe1-C1 162.19(10), N10-Fe1-C14 104.60(10), C10-Fe1-C14 87.70(11), C1-Fe1-C14 87.58(11), N10-Fe1-C5 106.58(10), C10-Fe1-C5 87.58(10), C1-Fe1-C5 87.60(10), C14-Fe1-C5 148.82(11), N10-Fe2-C30 99.56(10), N10-Fe2-C21 98.78(10), C30-Fe2-C21 161.66(11), N10-Fe2-C34 103.45(10), C30-Fe2-C34 87.83(11), C21-Fe2-C34 87.96(10), N10-Fe2-C25 103.35(10), C30-Fe2-C25 88.04(10), C21-Fe2-C25 87.71(11), C34-Fe2-C25 153.19(11), Fe2-N10-Fe1 178.49(13)

X-ray Table 4. Crystal data and refinement details for $\mathbf{1 2}$ and $\mathbf{1 4 .}$

\begin{tabular}{lll}
\hline compound & $\begin{array}{l}\mathbf{1 2} \\
{\left[\left({ }^{\mathrm{NHC}} \mathbf{L}\right) \mathbf{F e N F e}\left({ }^{\mathrm{NHC}} \mathbf{L}\right)(\mathbf{N C M e})\right](\mathbf{O T f})_{3.7}\left(\mathbf{S b F}_{\mathbf{6}}\right)_{0.3}}\end{array}$ & $\begin{array}{l}\mathbf{1 4} \\
{\left[\left\{\left(\mathbf{O}_{2} \mathbf{N O}\right)\left({ }^{\mathrm{NHC}} \mathbf{L}\right) \mathbf{F e}\right\}_{2} \mathbf{N}\right]}\end{array}$ \\
\hline empirical formula & $\mathrm{C}_{43.70} \mathrm{H}_{46} \mathrm{~F}_{12.91} \mathrm{Fe}_{2} \mathrm{~N}_{19} \mathrm{O}_{11.09} \mathrm{~S}_{3.70} \mathrm{Sb}_{0.30}$ & $\mathrm{C}_{43} \mathrm{H}_{46} \mathrm{~F}_{9} \mathrm{Fe}_{2} \mathrm{~N}_{21} \mathrm{O}_{15} \mathrm{~S}_{3}$ \\
formula weight & 1527.09 & 1475.89 \\
$T[\mathrm{~K}]$ & $133(2)$ & $133(2)$ \\
crystal size $\left[\mathrm{mm}^{3}\right]$ & $0.460 \times 0.140 \times 0.080$ & $0.500 \times 0.280 \times 0.260$ \\
crystal system & monoclinic & tetragonal \\
space group & $P{ }_{1} / n$ & $I 4{ }_{1} / a c d$ \\
$a[\AA]$ & $21.8373(9)$ & $29.7738(6)$ \\
$b[\AA]$ & $15.8109(4)$ & $29.7738(6)$ \\
$c[\AA]$ & $22.4223(8)$ & $26.8238(5)$ \\
$\alpha\left[{ }^{\circ}\right]$ & 90 & 90 \\
$\beta\left[{ }^{\circ}\right]$ & $116.568(3)$ & 90 \\
$\gamma\left[{ }^{\circ}\right]$ & 90 & 90 \\
$V\left[\AA^{3}\right]$ & $6924.2(4)$ & $23778.7(11)$ \\
$Z$ & 4 & 16 \\
$\rho\left[\mathrm{g} / \mathrm{cm}^{3}\right]$ & 1.465 & 1.649 \\
$F(000)$ & 3091 & 12032 \\
$\mu\left[\mathrm{mm}^{-1}\right]$ & 0.744 & 0.704 \\
\hline
\end{tabular}




\begin{tabular}{lll}
\hline$T_{\min } / T_{\max }$ & $0.7420 / 0.9409$ & $0.7762 / 0.8885$ \\
$\theta$-range $\left[^{\circ}\right]$ & $1.640-25.888$ & $1.707-25.651$ \\
$h k l$-range & $\pm 26, \pm 19, \pm 27$ & $\pm 36,-36-35, \pm 32$ \\
measured refl. & 52972 & 71903 \\
unique refl. $\left[R_{\text {int }}\right]$ & $13134[0.1095]$ & $5630[0.0565]$ \\
observed refl. $(I>2 \sigma(I))$ & 7029 & 4402 \\
data / restraints / param. & $13134 / 338 / 968$ & $5630 / 222 / 567$ \\
goodness-of-fit $\left(F^{2}\right)$ & 1.068 & 1.094 \\
$R 1, w R 2(I>2 \sigma(I))$ & $0.0917,0.1776$ & $0.0758,0.2008$ \\
$R 1, w R 2$ (all data) & $0.1812,0.2275$ & $0.0992,0.2173$ \\
resid. el. dens. [e/ $\left.\AA^{3}\right]$ & $-0.534 / 0.614$ & $-0.394 / 0.899$ \\
\hline
\end{tabular}

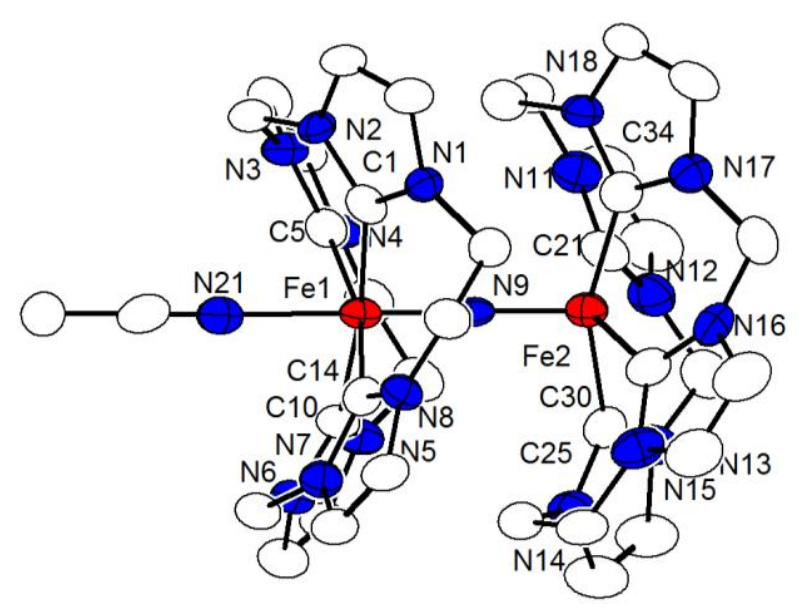

X-ray Figure 8. Plot of the molecular structure of the cationic part of $\mathbf{1 2}$ (30\% probability thermal ellipsoids; hydrogen atoms omitted for clarity). Selected bond lengths $[\AA]$ and angles $\left[{ }^{\circ}\right]$ :Fe1-N9 1.685(7), Fe2-N9 1.713(7), Fe1-C1 1.971(8), Fe1-C5 2.055(9), Fe1-C10 1.985(8), Fe1-C14 2.040(7), Fe1-N21 2.092(8), Fe2C21 2.014(9), Fe2-C34 1.964(8), Fe2-C25 1.965(9), Fe2-C30 2.014(9); N9-Fe1-C1 92.6(3), N9-Fe1-C10 94.3(3), C1-Fe1-C10 173.0(3), N9-Fe1-C14 97.1(3), C1-Fe1-C14 91.7(3), C10-Fe1-C14 88.0(3), N9-Fe1-C5 96.3(3), C1-Fe1-C5 87.1(3), C10-Fe1-C5 91.6(3), C14-Fe1-C5 166.6(3), N9-Fe1-N21 178.5(3), C1-Fe1-N21 88.5(3), C10-Fe1-N21 84.5(3), C14-Fe1-N21 83.8(3), C5-Fe1-N21 82.8(3), N9-Fe2-C34 101.8(3), N9-Fe2C25 101.4(3), C34-Fe2-C25 156.8(3), N9-Fe2-C30 107.3(3), C34-Fe2-C30 87.2(3), C25-Fe2-C30 85.1(3), N9-Fe2-C21 107.4(3), C34-Fe2-C21 85.8(3), C25-Fe2-C21 88.1(4), C30-Fe2-C21 145.3(4), Fe1-N9-Fe2 179.1(4), C41-N21-Fe1 172.7(7). 


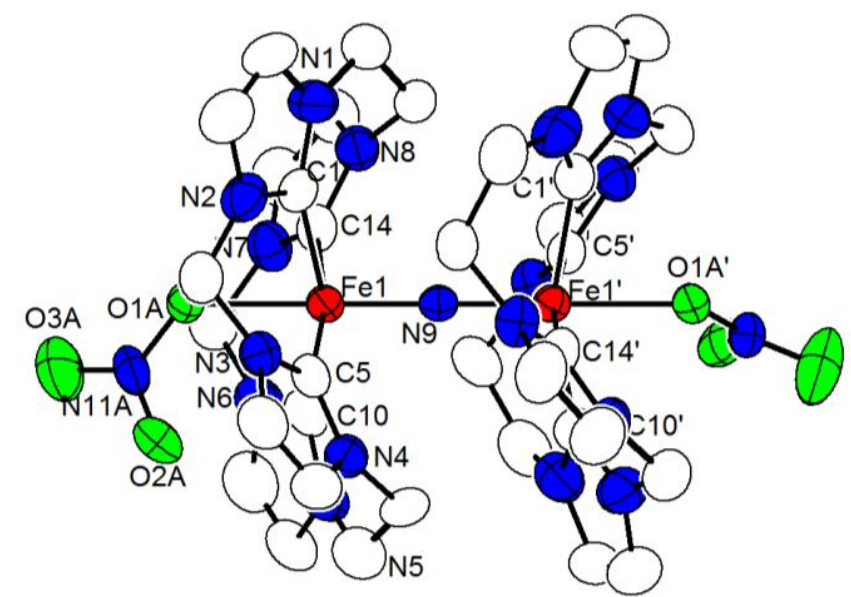

X-ray Figure 9. Plot of the molecular structure of the cationic part of $\mathbf{1 4}$ (30\% probability thermal ellipsoids; hydrogen atoms omitted for clarity). Selected bond lengths $[\AA]$ and angles $\left[{ }^{\circ}\right]$ : Fe1-N9 1.6837(6), N9-Fe1' 1.6838(6), Fe1-C1 2.061(5), Fe1-C5 1.988(5), Fe1-C10 2.024(5), Fe1-C14 1.989(5), Fe1-O1A 2.069(5), Fe1O1B 2.080(16); N9-Fe1-C1 99.9(2), C5-Fe1-C1 88.3(2), N9-Fe1-C5 94.55(13), N9-Fe1-C14 95.10(15), C5Fe1-C14 170.2(2), N9-Fe1-C10 99.5(2), C5-Fe1-C10 92.0(2), C14-Fe1-C10 88.1(2), C14-Fe1-C1 88.4(2), C10-Fe1-C1 160.6(2), N9-Fe1-O1A 179.0(3), C5-Fe1-O1A 86.3(3), C14-Fe1-O1A 84.1(3), C10-Fe1-O1A 81.1(3), C1-Fe1-O1A 79.5(3), N9-Fe1-O1B 179.0(13), C5-Fe1-O1B 86.4(13), C14-Fe1-O1B 83.9(13), C10Fe1-O1B 80.4(12), C1-Fe1-O1B 80.2(12), Fe1-N9-Fe1' 179.5(3), N11A-O1A-Fe1 127.0(6), N11B-O1B-Fe1 129(2). Symmetry transformation used to generate equivalent atoms: (') $x, 1-y, 3 / 2-z$.

X-ray Table 5. Crystal data and refinement details for $\{\mathrm{FeNO}\}^{6} \mathbf{1 8},\{\mathrm{FeNO}\}^{7} \mathbf{1 7}$ and $\{\mathrm{FeNO}\}^{8} \mathbf{1 9}$.

\begin{tabular}{|c|c|c|c|}
\hline compound & $\begin{array}{l}18 \\
{\left[\left({ }^{\mathrm{NHC}} \mathrm{L}\right)\{\mathrm{FeNO}\}^{6}(\mathrm{ONO})\right](\mathrm{OTf})_{2}}\end{array}$ & $\begin{array}{l}17 \\
\left.\left[{ }^{\mathrm{NHC}} \mathrm{L}\right)\{\mathrm{FeNO}\}^{7}\right](\mathrm{OTf})_{2}\end{array}$ & $\begin{array}{l}19 \\
{\left[\left({ }^{\mathrm{NHC}} \mathrm{L}\right)\{\mathrm{FeNO}\}^{8}\right](\mathrm{OTf})}\end{array}$ \\
\hline empirical formula & $\mathrm{C}_{20} \mathrm{H}_{20} \mathrm{~F}_{6} \mathrm{FeN}_{10} \mathrm{O}_{9} \mathrm{~S}_{2}$ & $\mathrm{C}_{20} \mathrm{H}_{20} \mathrm{~F}_{6} \mathrm{Fe} \mathrm{N} \mathrm{N}_{9} \mathrm{O}_{7} \mathrm{~S}_{2}$ & $\mathrm{C}_{19} \mathrm{H}_{20} \mathrm{~F}_{3} \mathrm{FeN}_{9} \mathrm{O}_{4} \mathrm{~S}$ \\
\hline formula weight & 778.43 & 732.42 & 583.35 \\
\hline crystal size $\left[\mathrm{mm}^{3}\right]$ & $0.21 \times 0.17 \times 0.06$ & $0.230 \times 0.210 \times 0.110$ & $0.48 \times 0.32 \times 0.21$ \\
\hline crystal system & triclinic & triclinic & monoclinic \\
\hline space group & $P \overline{1}$ & $P \overline{1}$ & $P 2_{1} / c$ \\
\hline$a[\AA]$ & 10.1194(9) & $10.506(2)$ & $12.6972(6)$ \\
\hline$b[\AA]$ & $11.5579(10)$ & $12.075(2)$ & $12.3023(4)$ \\
\hline$c[\AA]$ & $13.5781(11)$ & $12.948(3)$ & $15.4558(8)$ \\
\hline$\alpha\left[^{\circ}\right]$ & $74.854(7)$ & $66.52(3)$ & 90 \\
\hline$\beta\left[^{\circ}\right]$ & $86.990(7)$ & $88.96(3)$ & $113.068(4)$ \\
\hline$\gamma\left[^{\circ}\right]$ & $67.327(7)$ & $67.42(3)$ & 90 \\
\hline$V\left[\AA^{3}\right]$ & $1412.4(2)$ & $1373.3(6)$ & 2221.23(18) \\
\hline$Z$ & 2 & 2 & 4 \\
\hline$\rho\left[\mathrm{g} / \mathrm{cm}^{3}\right]$ & 1.830 & 1.771 & 1.744 \\
\hline$F(000)$ & 788 & 742 & 1192 \\
\hline$\mu\left[\mathrm{mm}^{-1}\right]$ & 0.792 & 0.802 & 0.849 \\
\hline$T_{\min } / T_{\max }$ & $0.6975 / 0.8894$ & $0.8099 / 0.9511$ & $0.6665 / 0.8201$ \\
\hline$\theta$-range $\left[{ }^{\circ}\right]$ & $1.556-26.794$ & $1.737-26.790$ & $1.743-26.799$ \\
\hline$h k l$-range & $\pm 12,-13,-14, \pm 17$ & $\begin{array}{l}-13,-13,-15,-15,-16, \\
-16\end{array}$ & $-16,-15, \pm 15, \pm 19$ \\
\hline measured refl. & 17760 & 14515 & 29419 \\
\hline
\end{tabular}




\begin{tabular}{llll}
\hline $\begin{array}{l}\left.\text { unique refl. [R } R_{\text {int }}\right] \\
\text { observed refl. }(I>\end{array}$ & $5991[0.0729]$ & $5817[0.0564]$ & 4713 [0.0611] \\
$\begin{array}{l}2 \sigma(I)) \\
\text { data / restraints / }\end{array}$ & 4286 & 4027 & 4083 \\
$\begin{array}{l}\text { param. } \\
\text { goodness-of-fit }\left(F^{2}\right)\end{array}$ & 1.056 & $5817 / 0 / 406$ & $4713 / 0 / 334$ \\
$R 1, w R 2(I>2 \sigma(I))$ & $0.0592,0.1007$ & 0.971 & 1.047 \\
$R 1, w R 2$ (all data) & $0.0973,0.1106$ & $0.0514,0.0994$ & $0.0386,0.0988$ \\
resid. el. dens. & $-0.561 / 0.673$ & $0.0870,0.1093$ & $0.0468,0.1025$ \\
{$\left[\mathrm{e} / \AA^{3}\right]$} & $-0.345 / 0.831$ & $-0.443 / 0.849$ \\
\hline
\end{tabular}

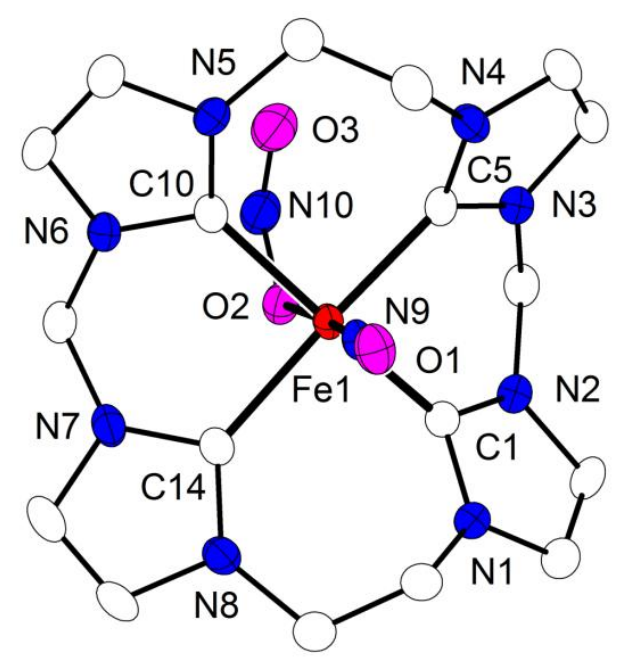

X-ray Figure 10. Plot (50\% probability thermal ellipsoids) of the molecular structure of the cationic part of $\left[\left({ }^{\mathrm{NHC}} \mathrm{L}\right)\{\mathrm{FeNO}\}^{6}(\mathrm{ONO})\right](\mathrm{OTf})_{2} \mathbf{1 8}$ (hydrogen atoms omitted for clarity). Selected bond lengths $[\AA]$ and angles [ ${ }^{\circ}$ ]: Fe1-N9 1.625(4), Fe1-O2 1.979(3), Fe1-C1 1.984(4), Fe1-C5 1.988(4), Fe1-C10 2.012(4), Fe1C14 2.047(4), N9-O1 1.162(4), N10-O3 1.201(5), N10-O2 1.305(4); N9-Fe1-O2 175.62(13), N9-Fe1-C1 97.07(16), O2-Fe1-C1 85.24(14), N9-Fe1-C5 93.90(15), O2-Fe1-C5 90.05(13), C1-Fe1-C5 83.53(15), N9-Fe1-C10 90.74(16), O2-Fe1-C10 87.11(14), C1-Fe1-C10 171.84(15), C5-Fe1-C10 93.62(15), N9Fe1-C14 92.62(15), O2-Fe1-C14 83.60(13), C1-Fe1-C14 91.26(15), C5-Fe1-C14 172.12(17), C10-Fe1C14 90.73(15), O1-N9-Fe1 176.6(3), N10-O2-Fe1 127.7(2), O2-N10-O3 118.0(4). 

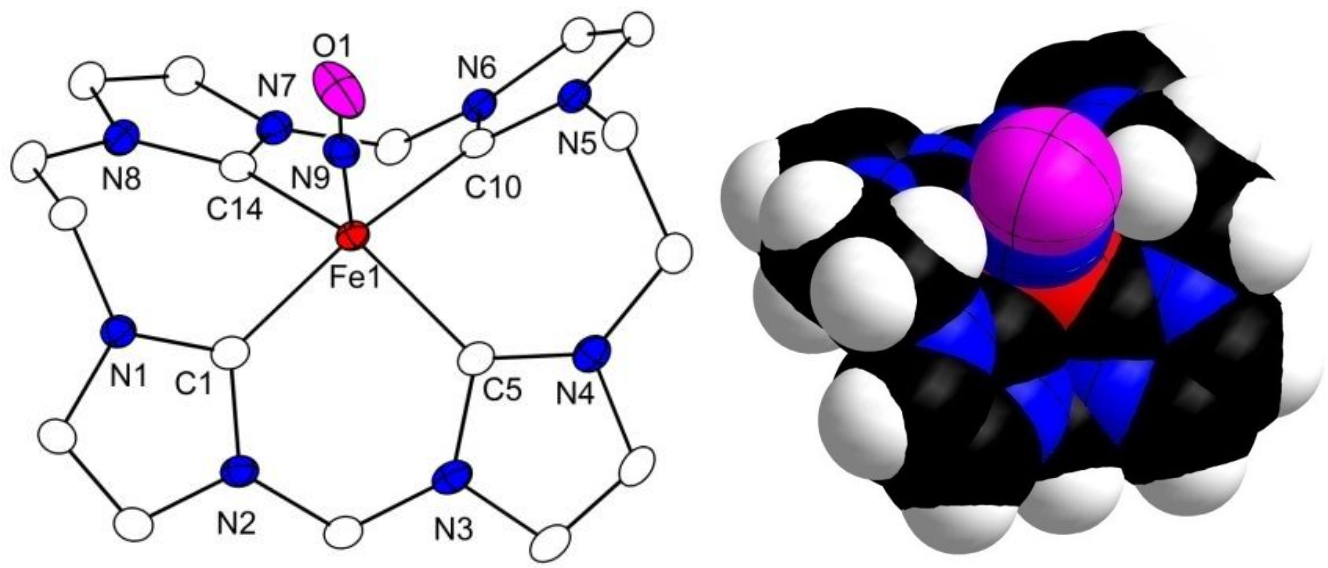

X-ray Figure 11. Molecular structure of the cation of $\left.\left[{ }^{\mathrm{NHC}} \mathrm{L}\right)\{\mathrm{FeNO}\}^{7}\right](\mathrm{OTf})_{2} \mathbf{1 7}$ (30\% displacement ellipsoids), determined by single crystal X-ray diffraction. Hydrogen atoms were omitted for clarity. Selected bond lengths $[\AA ̊ \Omega]$ and angles $\left[^{\circ}\right]$ :Fe1-C1 1.963(3), Fe1-C5 2.014(3), Fe1-C10 1.964(3), Fe1-C14 2.033(3), Fe1-N9 1.670(3), N9-O1 1.166(4), N9-Fe1-C1 98.75(14), N9-Fe1-C5 105.87(14), N9-Fe1-C10 96.88(14), N9-Fe1-C14 106.02(15), C1-Fe1-C5 88.13(14), C10-Fe1-C5 88.61(13), C1-Fe1-C14 87.94(14), C10-Fe1C14 86.74(14), C1-Fe1-C10 164.33(14), C5-Fe1-C14 148.10(14), O1-N9-Fe1 176.9(3)

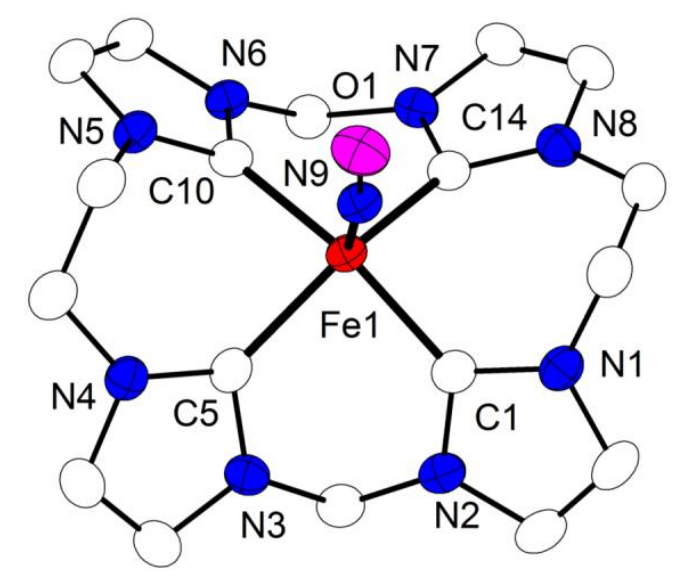

X-ray Figure 12. Plot (50\% probability thermal ellipsoids) of the molecular structure of the cationic part of $\left[\left({ }^{\mathrm{NHC}} \mathrm{L}\right)\{\mathrm{FeNO}\}^{8}\right](\mathrm{OTf}) \mathbf{1 9}$ (hydrogen atoms omitted for clarity). Selected distances $[\AA]$ and angles $\left[{ }^{\circ}\right]$ : $\mathrm{Fe} 1-$ N9 1.660(2), Fe1-C10 1.928(2), Fe1-C1 1.952(2), Fe1-C14 1.991(2), Fe1-C5 2.001(2), N9-O1 ; N9-Fe1C10 98.94(9), N9-Fe1-C1 107.21(10), C10-Fe1-C1 153.84(9), N9-Fe1-C14 107.53(9), C10-Fe1-C14 87.30(9), C1-Fe1-C14 84.28(9), N9-Fe1-C5 111.79(9), C10-Fe1-C5 84.50(9), C1-Fe1-C5 86.41(10), C14-Fe1-C5 140.61(9), O1-N9-Fe1 169.13(18).

X-ray Table 6. Crystal data and refinement details for $\mathbf{2 0 .}$

\begin{tabular}{ll}
\hline compound & $\mathbf{2 0}$ \\
& {$\left[\mathbf{K}_{\mathbf{2}}\{\mathbf{L L}, \mathbf{N i}\}\right]$} \\
\hline empirical formula & $\mathrm{C}_{32} \mathrm{H}_{40} \mathrm{~K}_{2} \mathrm{~N}_{12} \mathrm{NiO}_{2}$ \\
formula weight & 761.67 \\
$T[\mathrm{~K}]$ & $133(2)$ \\
crystal size $\left[\mathrm{mm}^{3}\right]$ & $0.500 \times 0.050 \times 0.030$ \\
crystal system & triclinic \\
space group & $P \overline{1}$ \\
$a[\AA]$ & $8.3987(4)$ \\
$b[\AA]$ & $10.9621(5)$ \\
$c[\AA]$ & $20.7837(10)$ \\
$\alpha\left[{ }^{\circ}\right]$ & $96.385(4)$ \\
\hline
\end{tabular}




\begin{tabular}{ll}
\hline$\beta\left[^{\circ}\right]$ & $93.696(4)$ \\
$\gamma\left[^{\circ}\right]$ & $108.374(4)$ \\
$V\left[\AA^{3}\right]$ & $1794.61(15)$ \\
$Z$ & 2 \\
$\rho\left[\mathrm{g} / \mathrm{cm}^{3}\right]$ & 1.410 \\
$F(000)$ & 796 \\
$\mu\left[\mathrm{mm}^{-1}\right]$ & 0.821 \\
$T_{\min } / T_{\max }$ & $0.8198 / 0.9665$ \\
$\theta$-range $\left[{ }^{\circ}\right]$ & $1.977-26.747$ \\
$h k l$-range & $-10-9, \pm 13, \pm 26$ \\
measured refl. & 22777 \\
unique refl. $\left[R_{\text {int }}\right]$ & $7610[0.0846]$ \\
observed refl. $(I>2 \sigma(I))$ & 5274 \\
data / restraints / param. & $7610 / 0 / 454$ \\
goodness-of-fit $\left(F^{2}\right)$ & 0.937 \\
$R 1, w R 2(I>2 \sigma(I))$ & $0.0460,0.0829$ \\
$R 1, w R 2$ (all data) & $0.0802,0.0914$ \\
resid. el. dens. $\left[\mathrm{e} / \AA^{3}\right]$ & $-0.431 / 0.428$ \\
\hline
\end{tabular}

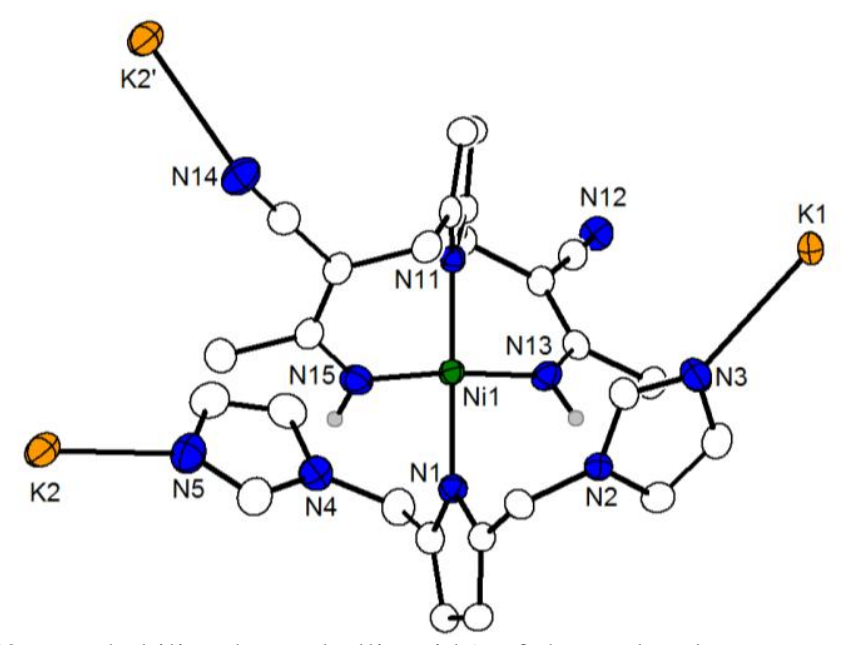

X-ray Figure 13. Plot (50\% probability thermal ellipsoids) of the molecular structure of the cationic part of 20 (hydrogen atoms except for $\mathrm{NH}$ omitted for clarity). Selected bond lengths $[\AA \AA]$ and angles $\left[^{\circ}\right]$ : Ni1-N1 1.934(2), Ni1-N11 1.890(2), Ni1-N15 1.896(3), Ni1-N13 1.904(2), Ni1-K1' 3.8053(7); N11-Ni1-N15 93.70(10), N11-Ni1-N13 92.26(10), N15-Ni1-N13 173.98(11), N11-Ni1-N1 178.39(10), N15-Ni1-N1 86.88(11), N13-Ni1-N1 87.18(10), N11-Ni1-K1' 49.99(6), N15-Ni1-K1' 130.07(8), N13-Ni1-K1' 55.18(8), N1-Ni1-K1' 128.63(7), Ni1-N11-K1' 100.81(8), Ni1-N13-K1' 94.92(9). Symmetry transformation used to generate equivalent atoms: (') 2-x, 1-y, $-z$. 


\section{Appendix}

\section{Further Spectroscopic Data}
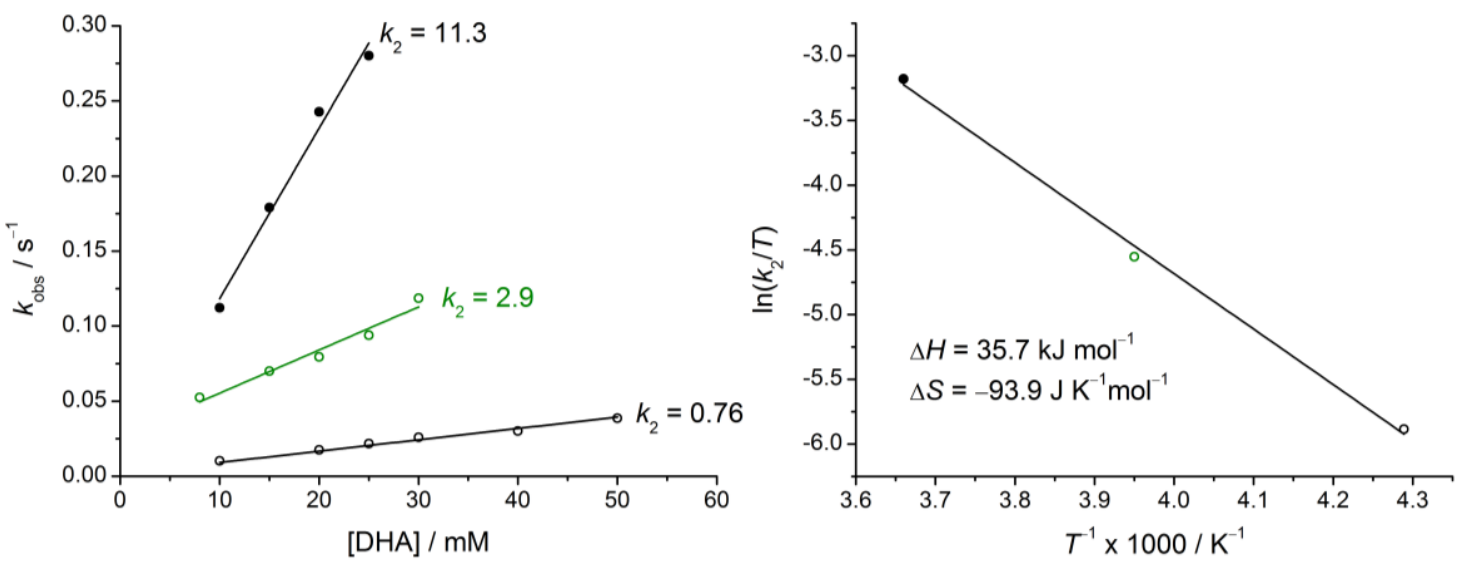

Appendix Figure 1. Plot of the observed reaction rates $k_{\mathrm{obs}}$ of $2(1 \mathrm{mM}) v s$. concentration of DHA at $-40{ }^{\circ} \mathrm{C}$, $-20{ }^{\circ} \mathrm{C}$ and $0{ }^{\circ} \mathrm{C}$ in $\mathrm{MeCN}$ (left) and the resulting Eyring plot (right). The $k_{2}$ values are given in $\mathrm{M}^{-1} \mathrm{~s}^{-1}$.
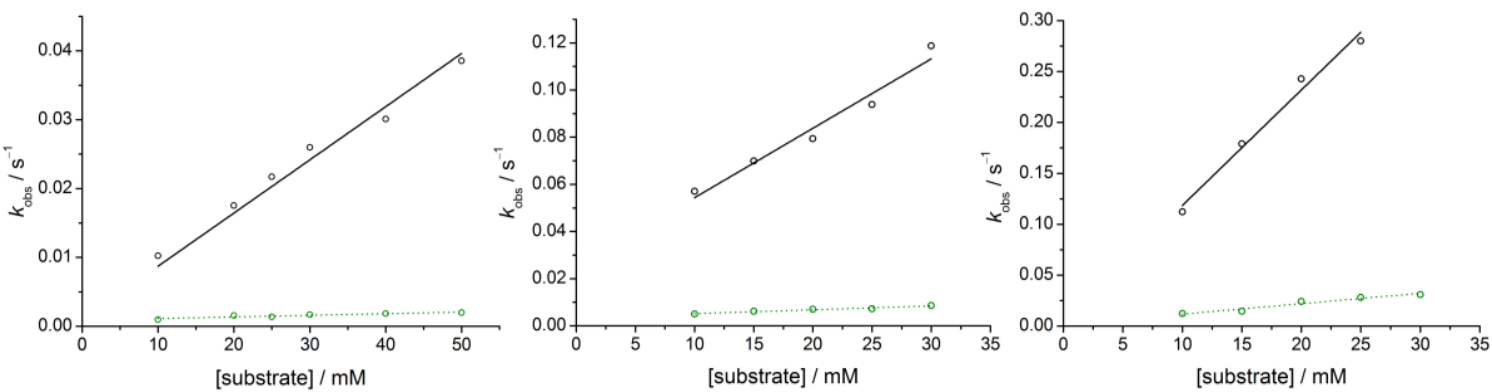

Appendix Figure 2. Plot of the observed reaction rates $\left(k_{\mathrm{obs}}\right)$ of $2(1 \mathrm{mM})$ vs. concentration of DHA (black, solid line) and DHA- $d_{4}$ (green, dashed line) at $-40{ }^{\circ} \mathrm{C}$ (left), $-20{ }^{\circ} \mathrm{C}$ (middle) and $0{ }^{\circ} \mathrm{C}$ (right) in MeCN. The different slopes lead to KIEs of 32, 18 and 11.
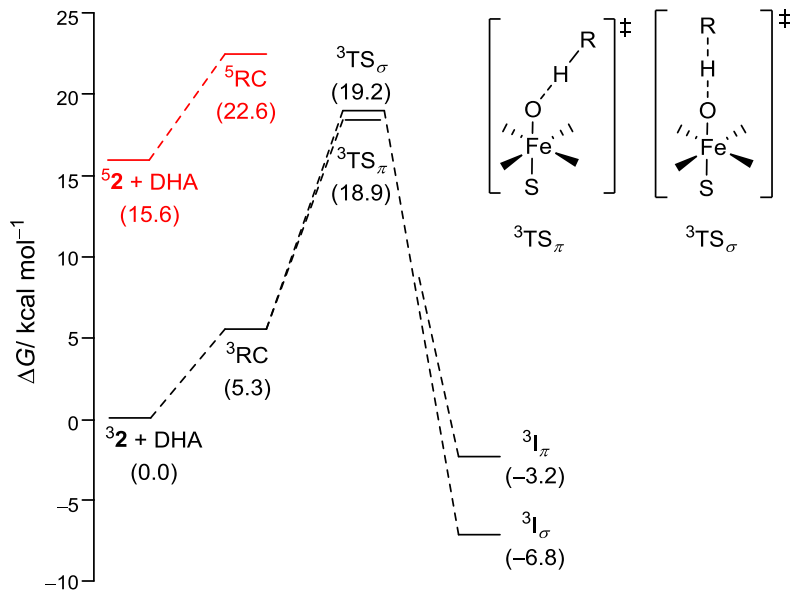

Appendix Figure 3. Reaction free energy profile $\left(\Delta G\right.$ in $\left.\mathrm{kcal} \mathrm{mol}^{-1}\right)$ for the first $\mathrm{H}$-atom abstraction of DHA by 2 . 

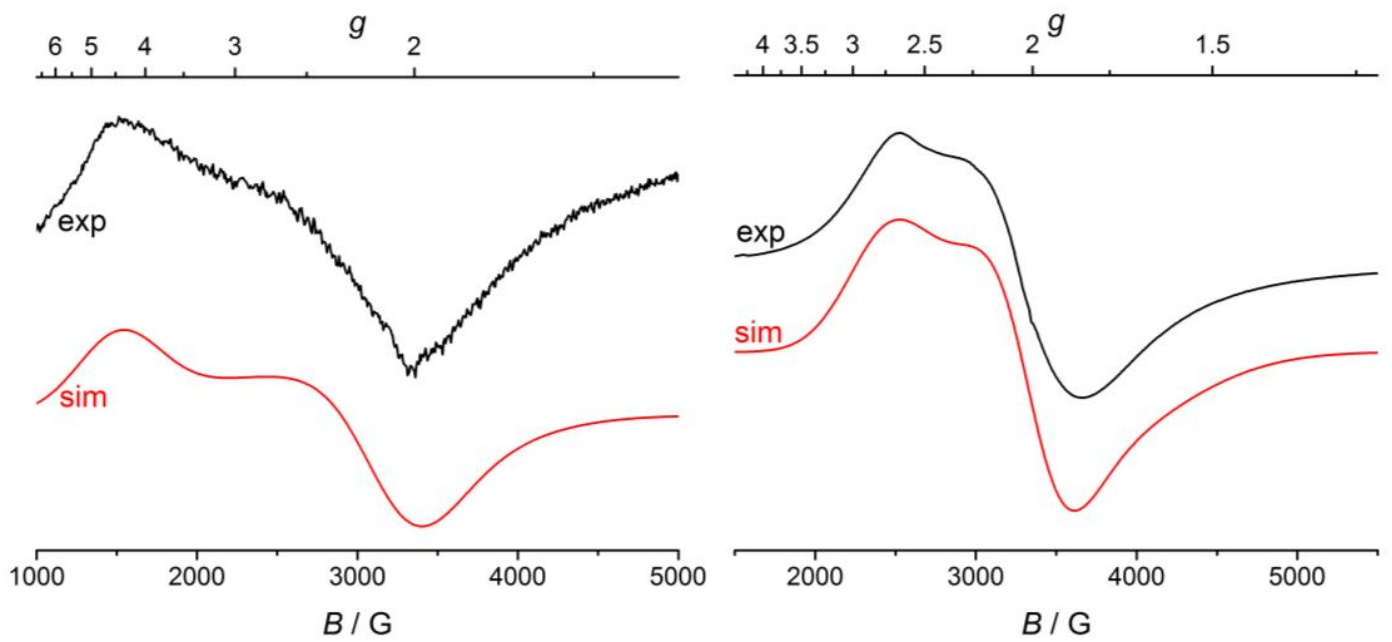

Appendix Figure 4. EPR spectrum of $\left[\left({ }^{\mathrm{NHC}} \mathrm{L}\right) \mathrm{FeCl}\right](\mathrm{X})_{2} \mathbf{8}\left(\mathrm{X}=\mathrm{OTf}\right.$ or $\mathrm{SbCl}_{6}$, frozen $\mathrm{MeCN}, 160 \mathrm{~K}, g(\mathrm{lw}$ $[\mathrm{G}])=4.300$ (600), 2.095 (800), $2.095(300)$ ) and $\left.\left[{ }^{\mathrm{NHC}} \mathrm{L}\right) \mathrm{Fe}\left(\mathrm{N}_{3}\right)_{2}\right](\mathrm{OTf}) 11$ (frozen $\mathrm{MeCN}, 10 \mathrm{~K}, g$ (lw [G]) $=2.700(380), 1.990(230), 1.676(500))$.

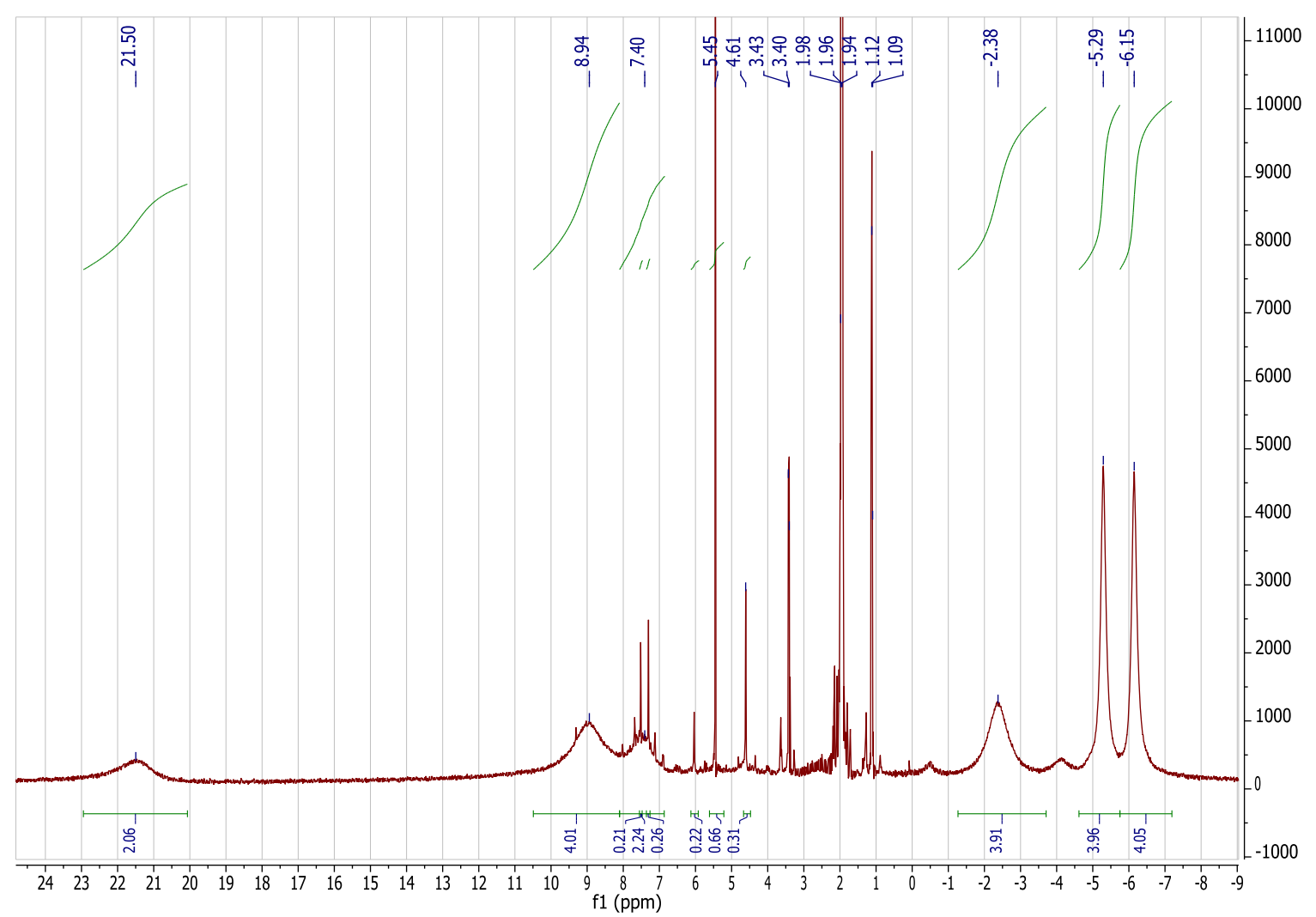

Appendix Figure 5. ${ }^{1} \mathrm{H}-\mathrm{NMR}$ spectrum of $\mathbf{1 1}$ in $\mathrm{MeCN}-\mathrm{d}_{3}$ wth a paramagnetic impurity of about $5 \%$ of $\mathbf{1}$. 


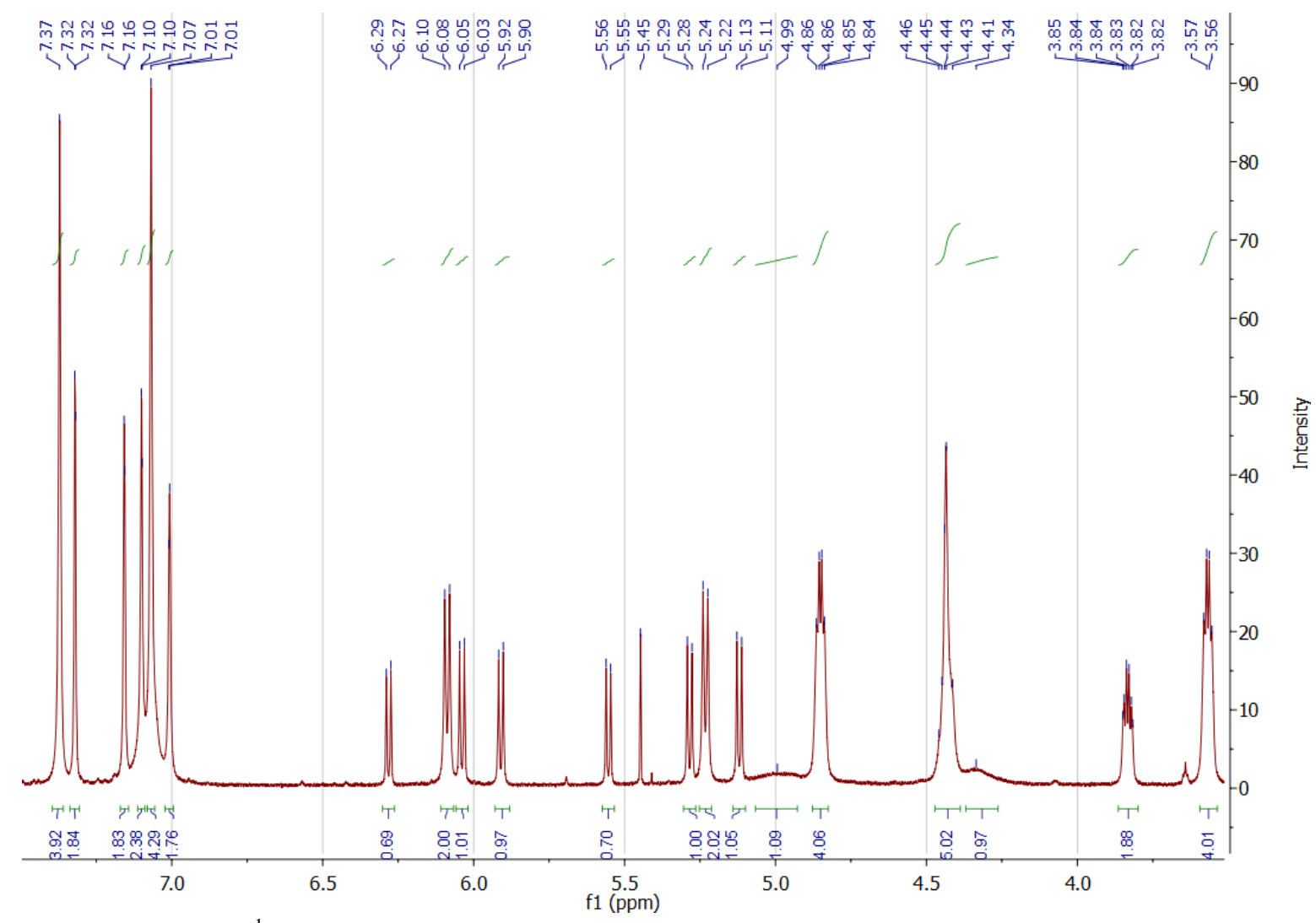

Appendix Figure 6. ${ }^{1} \mathrm{H}-\mathrm{NMR}$ spectrum of 6 in $\mathrm{MeCN}-\mathrm{d}_{3}$.

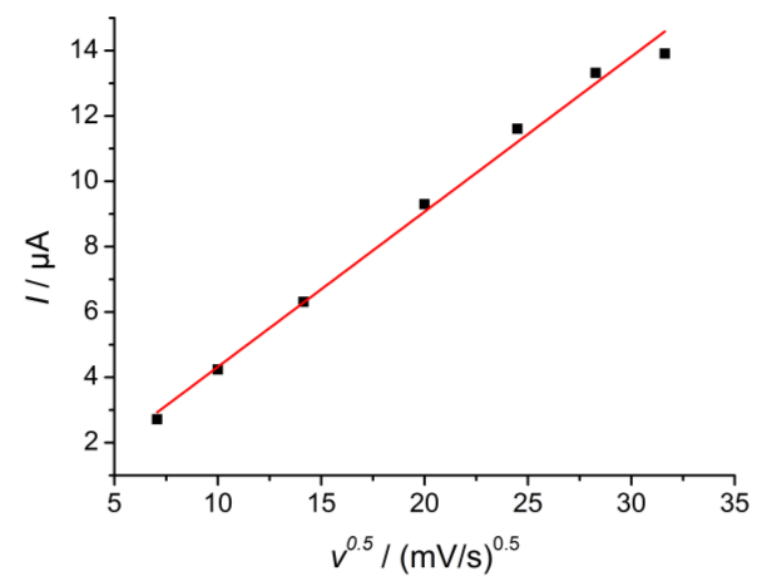

Appendix Figure 7. Plot of the recorded current vs. scan rate according to the Cotrell equation for the first oxidation of $\mathbf{6}$ in the cyclic voltammogram. The good linear fit (Pearson $\mathrm{R}=0.965$ and variation of the slope $=0.01782$ ) are indicative of a reversible process. 


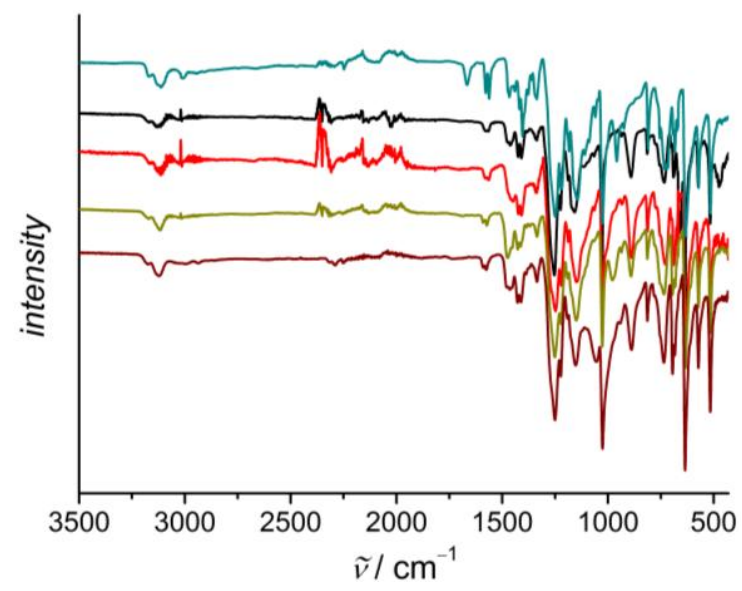

Appendix Figure 8. Solid state ATR measurements of the $\mu$-nitridodiiron complexes discussed in section 6 . Dark cyan: 6, black: 12, red: 13, dark yellow: 14, brown: 15 .
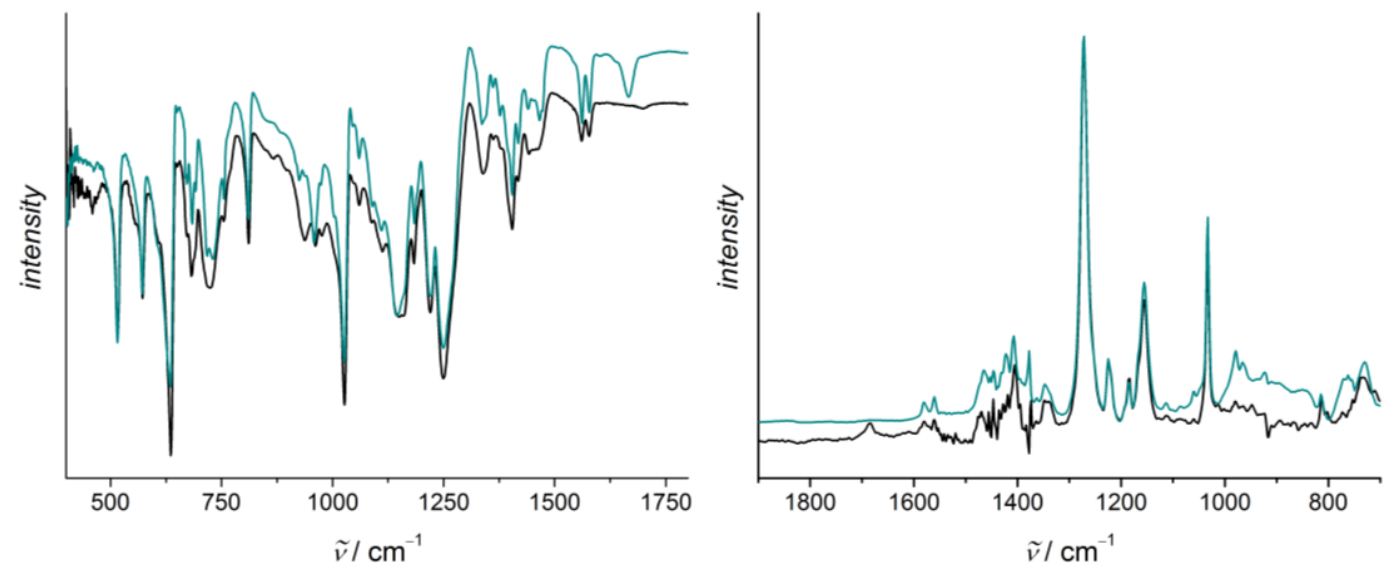

Appendix Figure 9. Solid state ATR (left) and Dial-Path solution IR (right, MeCN) spectra of the $\mu$-nitridodiiron complex 6 with natural isotopes distribution (dark cyan) and after labeling with ${ }^{15} \mathrm{~N}$ (black).
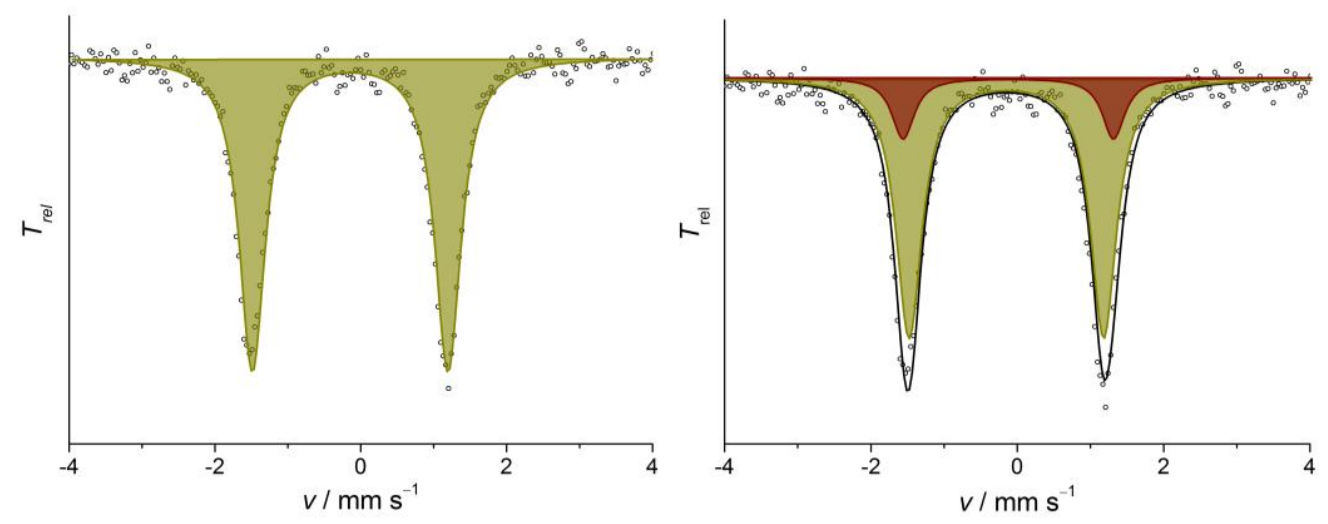

Appendix Figure 10. Zero-field ${ }^{57} \mathrm{Fe} \mathrm{MB}$ of the product obtained upon conversion of 6 with $\mathrm{NO}_{(\mathrm{g})}(80 \mathrm{~K}$, natural abundance ${ }^{57} \mathrm{Fe}$ ) and its simulation with only one doublet (left) and with two similar doublets in a 17:83 ratio (right). The parameters for the minor species were fixed to the values obtained after conversion of $\mathbf{6}$ with excess $\mathrm{NOBF}_{4}$. Both fits are of similar quality, thus a minor impurity of a similar species cannot be excluded. 


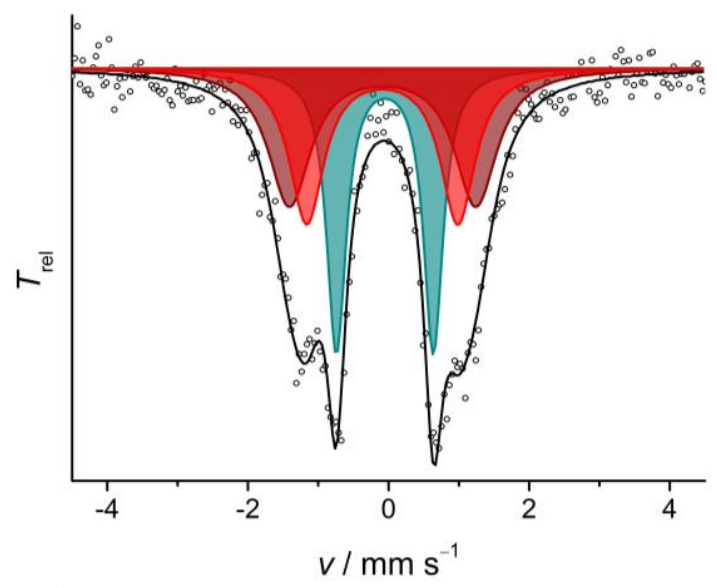

Appendix Figure 11. Zero-field ${ }^{57} \mathrm{Fe}$ MB spectrum of the product obtained upon conversion of 6 with 0.5 eq. $\mathrm{NOBF}_{4}\left(7 \mathrm{~K}\right.$, natural abundance ${ }^{57} \mathrm{Fe}$ ). The data correspond to a mixture of the starting material 6 (cyan, $\left.\delta=-0.04 \mathrm{~mm} \mathrm{~s}^{-1}, \Delta E_{\mathrm{Q}}=1.38 \mathrm{~mm} \mathrm{~s}^{-1}\right)$ and the mixed valent species $\mathbf{1 2}(\mathrm{red} / \mathrm{brown})$.

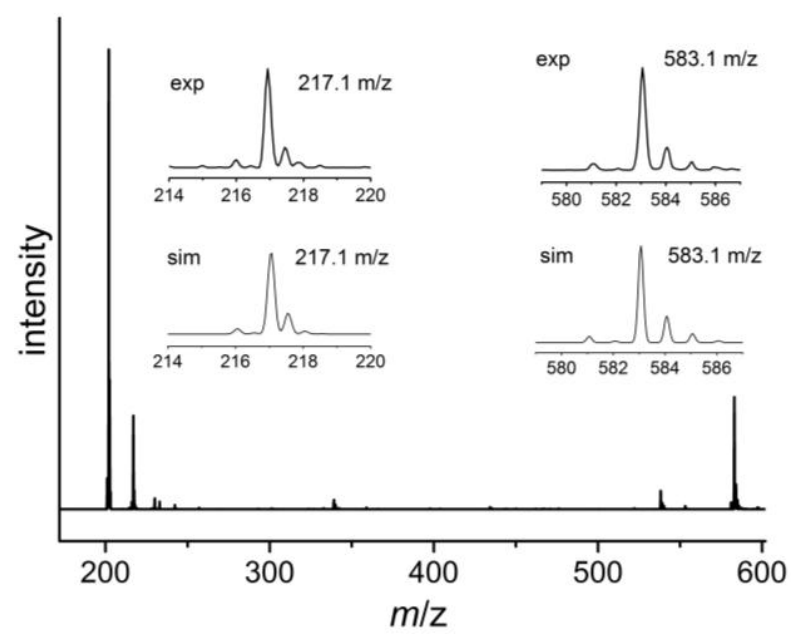

Appendix Figure 12. ESI(+)-MS of $\mathbf{1 7}$ in MeCN. The peak at $m / z=202.1$ can be assigned to $\left[\left({ }^{\mathrm{NHC}} \mathrm{L}\right) \mathrm{Fe}\right]^{2+}$, the peak at $m / z=217.1$ to $\left.\left[{ }^{\mathrm{NHC}} \mathrm{L}\right) \mathrm{Fe}(\mathrm{NO})\right]^{2+}$ and the peak at $m / z=583.1$ to $\left.\left[{ }^{\mathrm{NHC}} \mathrm{L}\right) \mathrm{Fe}(\mathrm{NO})(\mathrm{OTf})\right]^{+}$. The insets show the isotopic patterns for the peaks at $\mathrm{m} / z=217.1$ (left) and $\mathrm{m} / \mathrm{z}=583.1$ (right); experiment (top) and corresponding simulated pattern (bottom). 

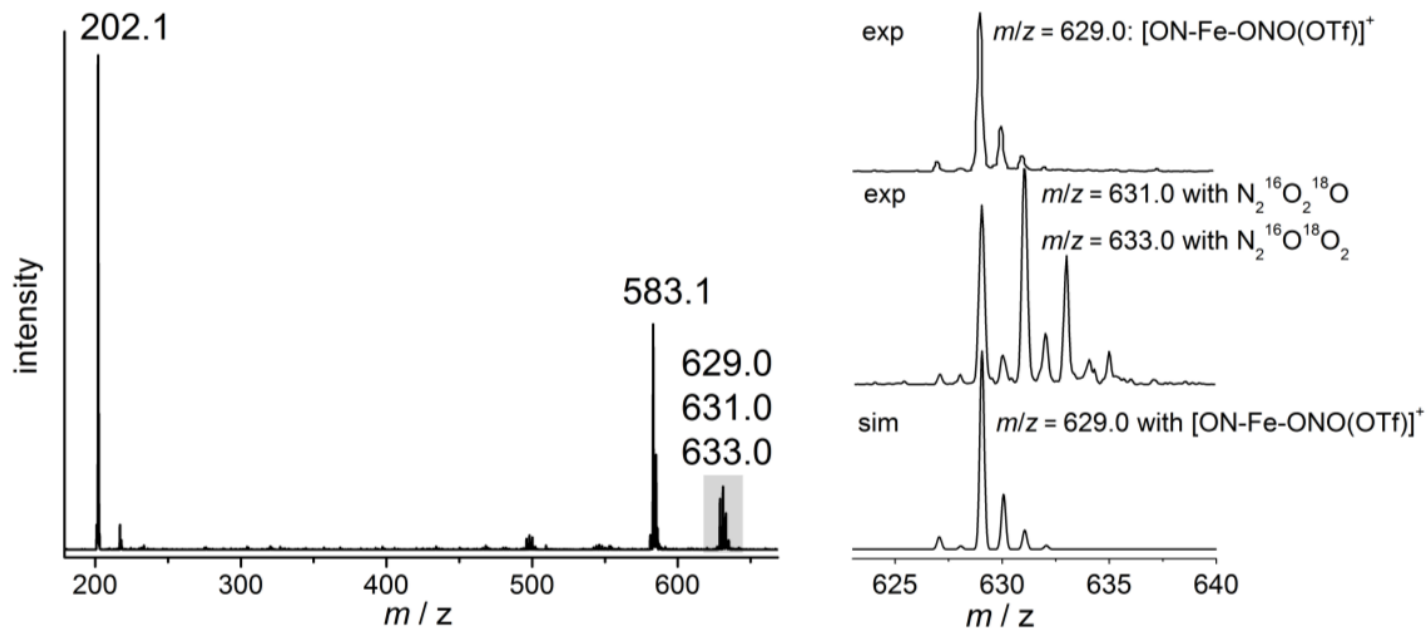

Appendix Figure 13. ESI(+)-MS of $\mathbf{1 8}$ in MeCN. The peak at $m / z=202.1$ can be assigned to $\left.\left[{ }^{\mathrm{NHC}} \mathrm{L}\right) \mathrm{Fe}\right]^{2+}$, the peak at $m / z=217.1$ to $\left.\left[{ }^{\mathrm{NHC}} \mathrm{L}\right) \mathrm{Fe}(\mathrm{NO})\right]^{2+}$ and the peak at $\mathrm{m} / z=583.1$ to $\left.\left[{ }^{\mathrm{NHC}} \mathrm{L}\right) \mathrm{Fe}(\mathrm{NO})(\mathrm{OTf})\right]^{+}$. Right: Isotopic pattern for the peak at $m / z=629.0$ assigned to $\left[\left({ }^{\mathrm{NHC}} \mathrm{L}\right) \mathrm{Fe}(\mathrm{NO})(\mathrm{ONO})(\mathrm{OTf})\right]^{+}$from the experiments using ${ }^{16} \mathrm{O}_{2}$ (top) and ${ }^{18} \mathrm{O}_{2}$ (middle), and the simulation for the ${ }^{16} \mathrm{O}$ isotopomer (bottom).

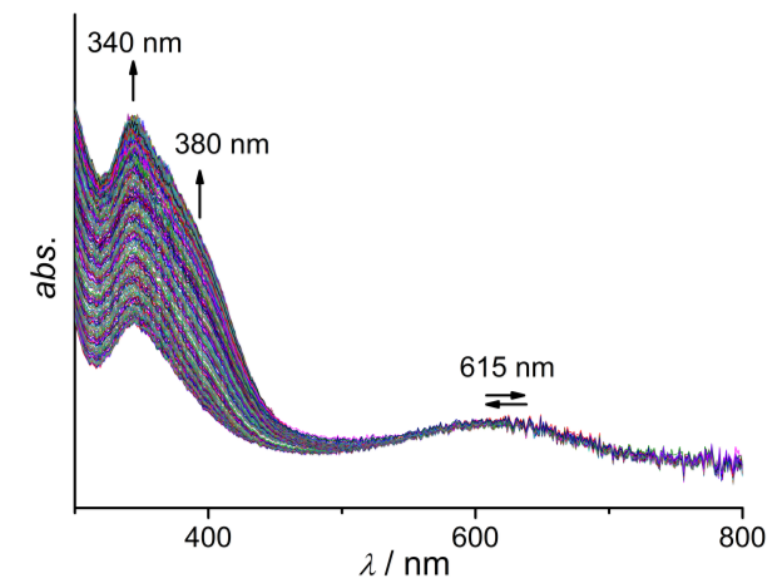

Appendix Figure 14. Electrochemical reduction of $17(c=0.13 \mathrm{mM})$ in $\mathrm{MeCN} / 0.1 \mathrm{M}\left[{ }^{n} \mathrm{Bu}_{4} \mathrm{~N}\right] \mathrm{PF}_{6}$ at RT at a potential of $-1.0 \mathrm{~V}$. The course of reduction was followed by UV/vis spectroscopy. The arrows represent the change in intensity of each band. 

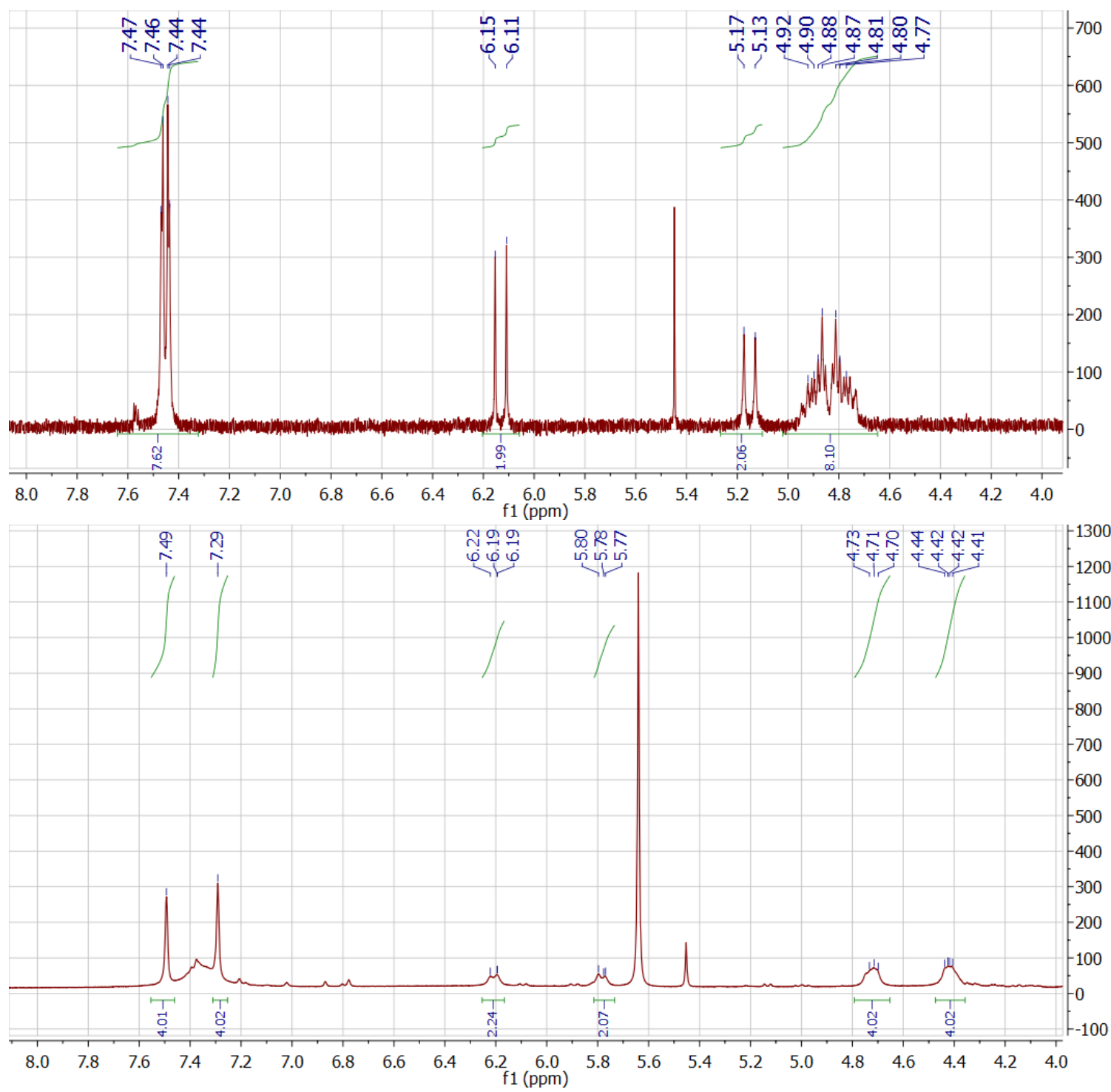

Appendix Figure 15. Top. ${ }^{1} \mathrm{H}$ NMR spectrum of $\{\mathrm{FeNO}\}^{6}$ 18. Bottom: ${ }^{1} \mathrm{H}$ NMR spectrum of $\{\mathrm{FeNO}\}^{8} \mathbf{1 9}$. The concentration of the sample could not be increased due to limited solubility. The signal at $5.63 \mathrm{ppm}$ is assigned to residual cobaltocene ( 0.7 eq.). 


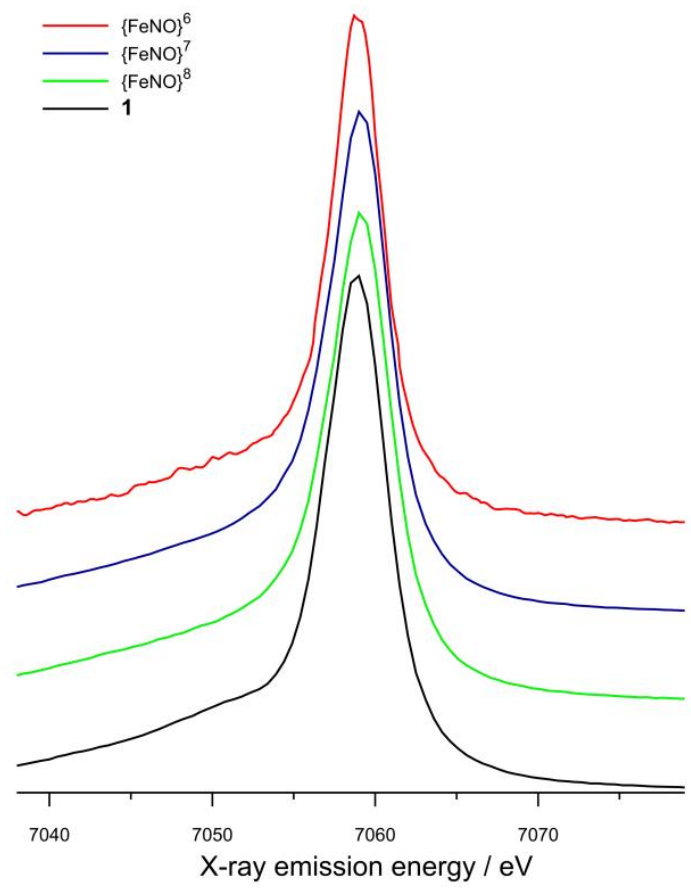

Appendix Figure 16. Fe $\mathrm{K} \beta$ mainline XES spectra of 17-19 and the iron(II) precursor $\left.\left[{ }^{\mathrm{NHC}} \mathrm{L}\right) \mathrm{Fe}(\mathrm{MeCN})_{2}\right](\mathrm{OTf})_{2} \mathbf{1}$. The spectra of $\mathbf{1 7}-\mathbf{1 9}$ do not exhibit any low-energy $\mathrm{K} \beta$ ' shoulder, indicative of low spin iron in all three complexes.

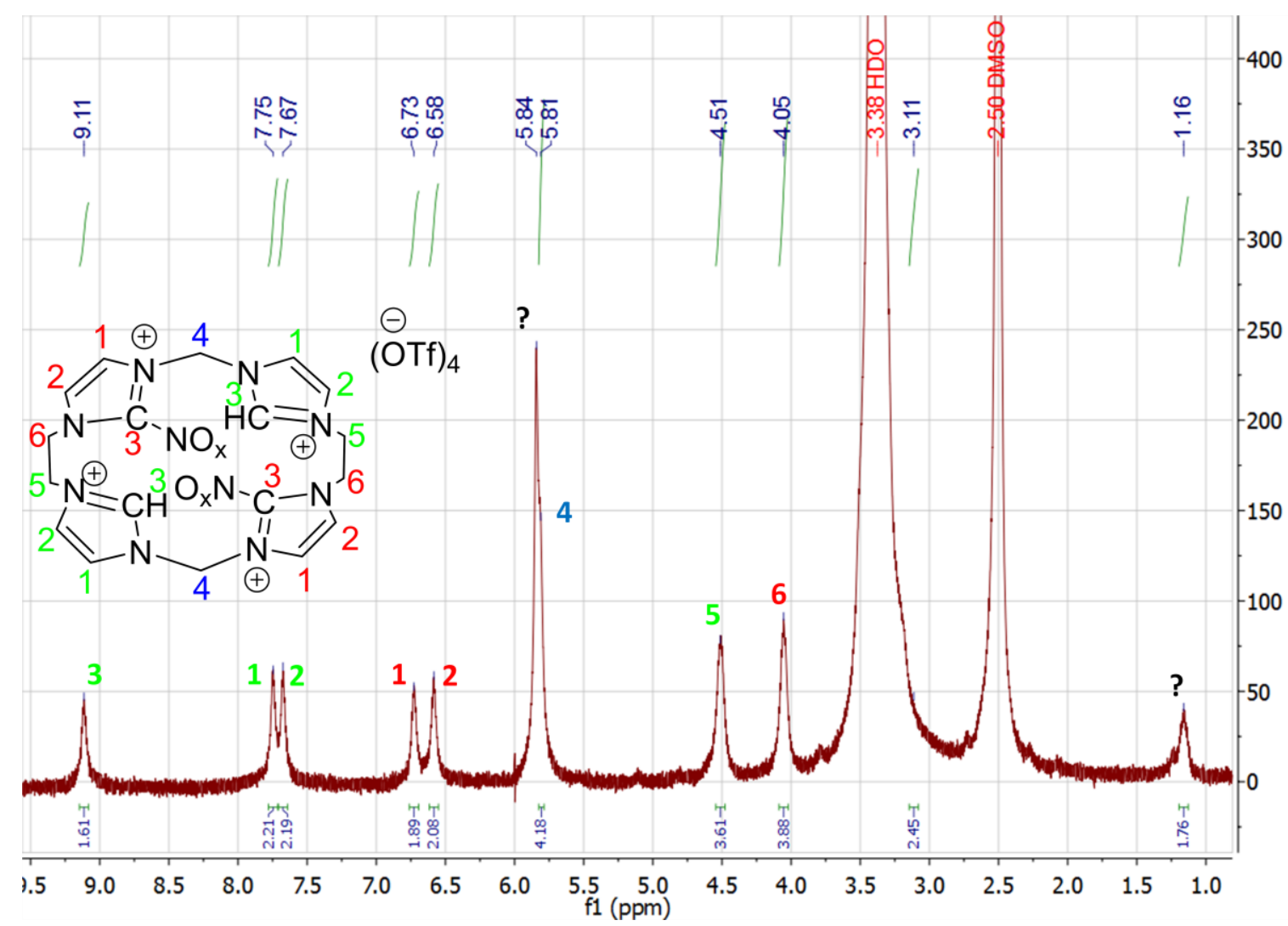

Appendix Figure 17. ${ }^{1} \mathrm{H}-\mathrm{NMR}$ spectrum of a decomposed $\{\mathrm{FeNO}\}^{8}$ mixture. 


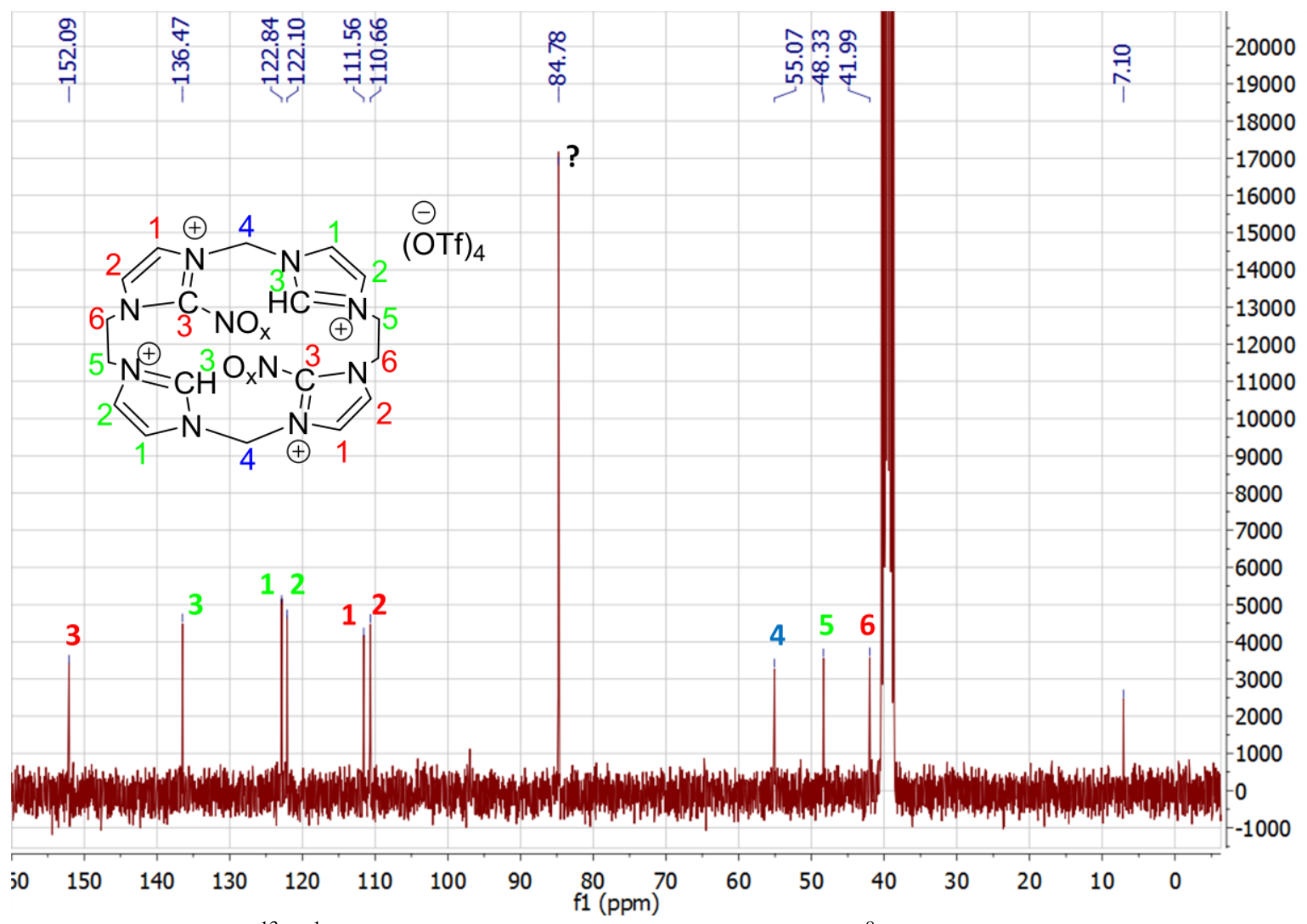

Appendix Figure $18 .{ }^{13} \mathrm{C}\left\{{ }^{1} \mathrm{H}\right\}$-NMR spectrum of a decomposed $\{\mathrm{FeNO}\}^{8}$ mixture.

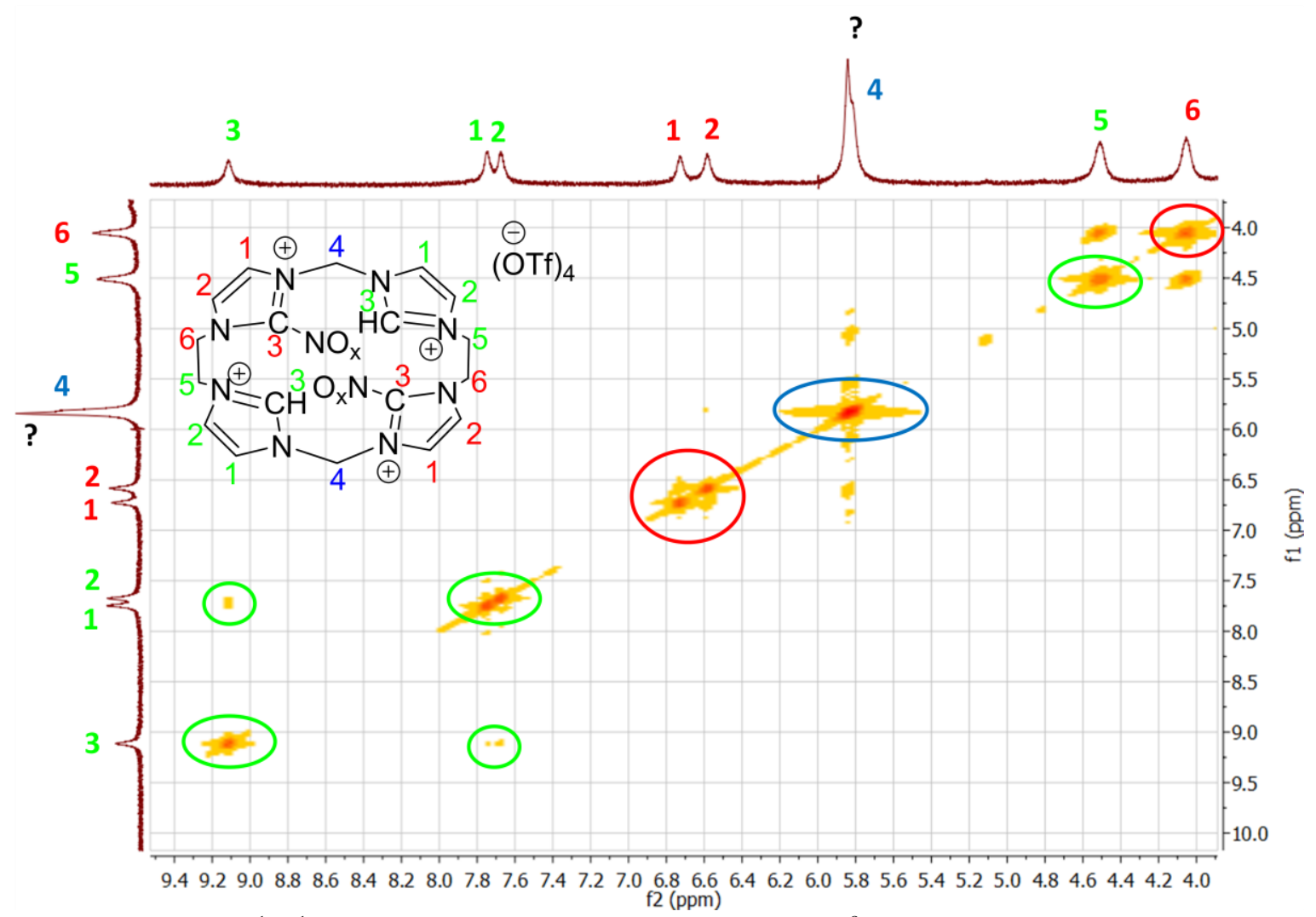

Appendix Figure 19. ${ }^{1} \mathrm{H}-{ }^{1} \mathrm{H}-\mathrm{COSY}$ spectrum of a decomposed $\{\mathrm{FeNO}\}^{8}$ mixture. 


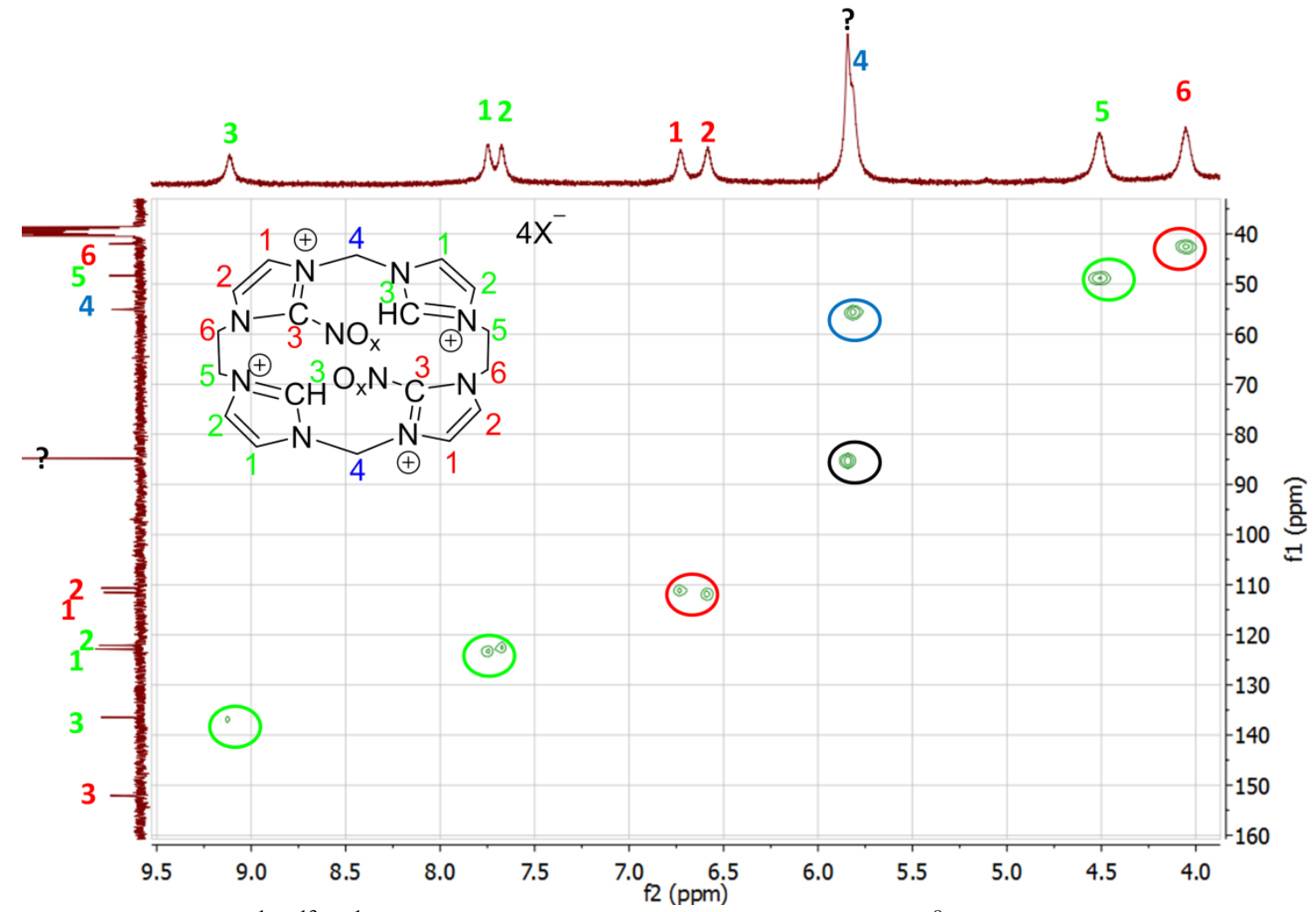

Appendix Figure 20. ${ }^{1} \mathrm{H}-{ }^{13} \mathrm{C}\left\{{ }^{1} \mathrm{H}\right\}$-HSQC spectrum of a decomposed $\{\mathrm{FeNO}\}^{8}$ mixture.

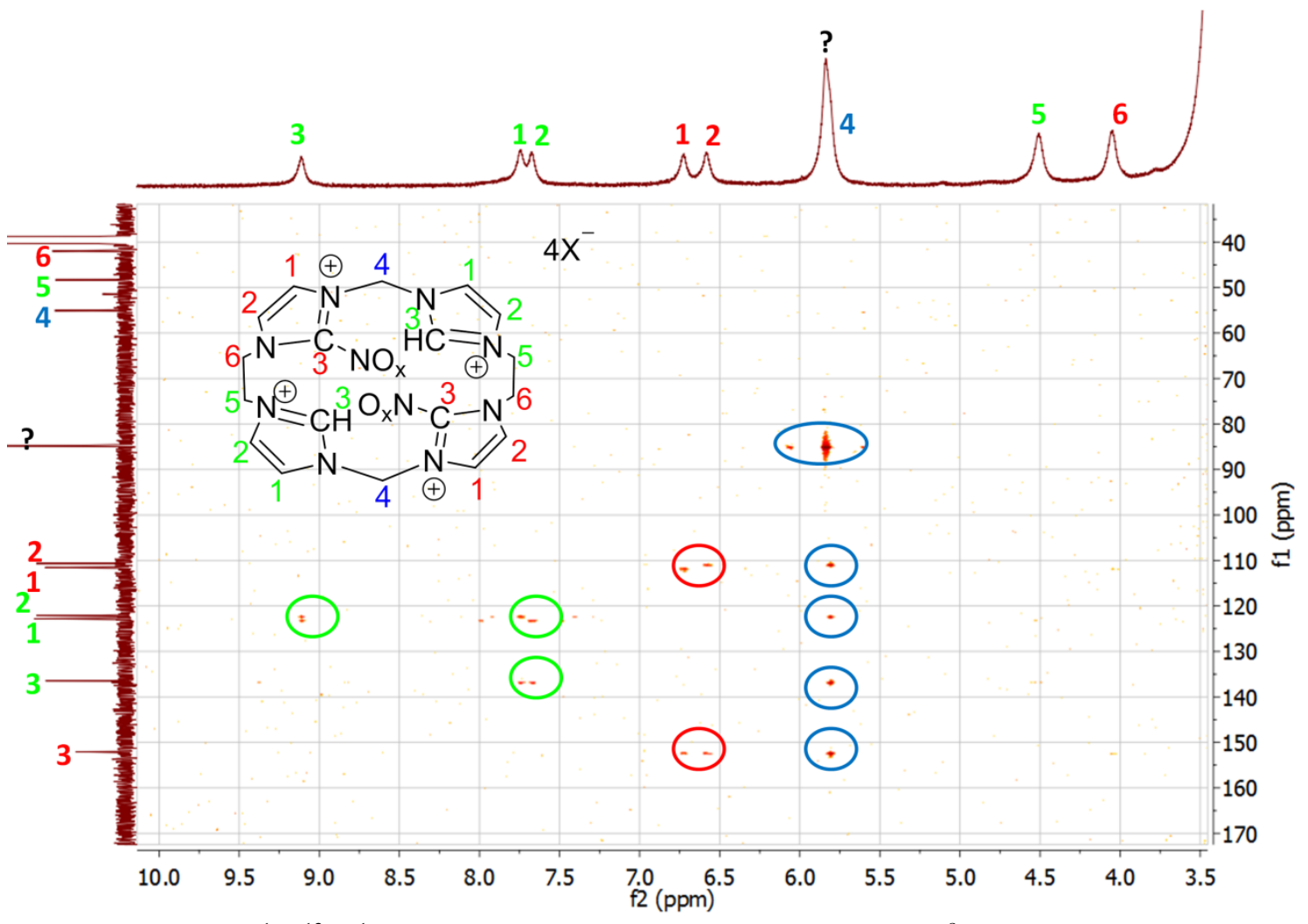

Appendix Figure 21. ${ }^{1} \mathrm{H}-{ }^{13} \mathrm{C}\left\{{ }^{1} \mathrm{H}\right\}-\mathrm{HMBC}$ spectrum of a decomposed $\{\mathrm{FeNO}\}{ }^{8}$ mixture. 


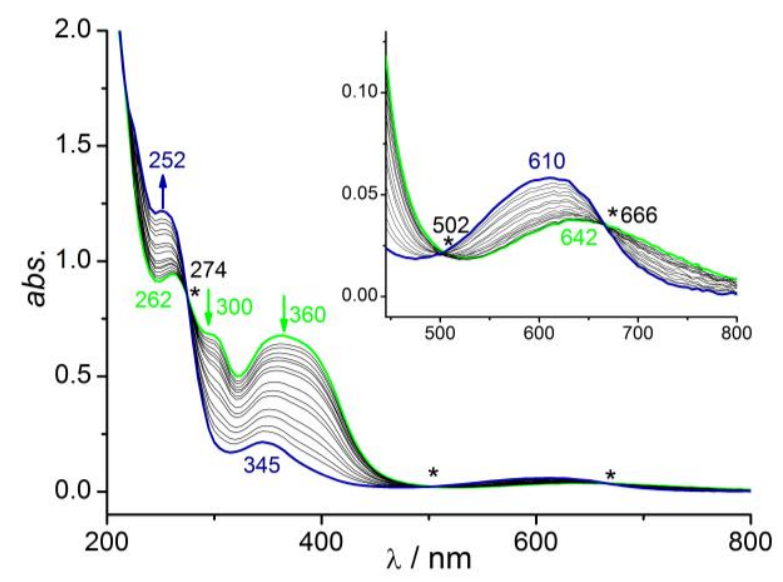

Appendix Figure 22. UV/vis/NIR changes during reaction of 19 (green) $(0.1 \mathrm{mM}$ in $\mathrm{MeCN}$ ) with 1,5-bis(dimethyl)-piperidinium. Full conversion to $\mathbf{1 7}$ (blue) was observed after addition of 8 eq. of acid. However, further studies have shown that slight amount of dioxygen led to the observed reaction. 


\section{References}

[1] P. A. Frey, G. H. Reed, ACS Chem. Biol. 2012, 7, 1477-1481.

[2] J. F. Berry, E. Bill, E. Bothe, S. D. George, B. Mienert, F. Neese, K. Wieghardt, Science 2006, 312, 1937-1941.

[3] H. Beinert, R. H. Holm, E. Münck, Science 1997, 277, 653-659.

[4] J. G. Riess, Chem. Rev. 2001, 101, 2797-2920.

[5] J. Torres-Alacan, O. Krahe, A. C. Filippou, F. Neese, D. Schwarzer, P. Vöhringer, Chem. Eur. J. 2012, 18, 3043-3055.

[6] Z. Liu, P. Hu, J. Am. Chem. Soc. 2002, 124, 5175-5182.

[7] J. Hohenberger, K. Ray, K. Meyer, Nat. Commun. 2012, 3, 720.

[8] N. Aliaga-Alcalde, S. DeBeer George, B. Mienert, E. Bill, K. Wieghardt, F. Neese, Angew. Chem. Int. Ed. 2005, 44, 2908-2912.

[9] K. M. Lancaster, M. Roemelt, P. Ettenhuber, Y. Hu, M. W. Ribbe, F. Neese, U. Bergmann, S. DeBeer, Science 2011, 334, 974-977.

[10] B. M. Hoffman, D. R. Dean, L. C. Seefeldt, Acc. Chem. Res. 2009, 42, 609-619.

[11] L. E. Goodrich, F. Paulat, V. K. K. Praneeth, N. Lehnert, Inorg. Chem. 2010, 49, 62936316.

[12] A. Decker, E. I. Solomon, Curr. Opin. Chem. Biol. 2005, 9, 152-163.

[13] W. Nam, Acc. Chem. Res. 2007, 40, 522-531.

[14] J. Rittle, M. T. Green, Science 2010, 330, 933-937.

[15] A. B. McQuarters, M. W. Wolf, A. P. Hunt, N. Lehnert, Angew. Chem. Int. Ed. 2014, 53, $4750-4752$.

[16] S. Chakrabarty, R. N. Austin, D. Deng, J. T. Groves, J. D. Lipscomb, J. Am. Chem. Soc. 2007, 129, 3514-3515.

[17] J. C. Price, E. W. Barr, B. Tirupati, J. M. Bollinger, C. Krebs, Biochemistry 2003, 42, $7497-$ 7508.

[18] C. Krebs, D. Galonić Fujimori, C. T. Walsh, J. M. Bollinger, Acc. Chem. Res. 2007, 40, 484-492.

[19] J. D. Lipscomb, L. Que Jr., J. Biol. Inorg. Chem. 1998, 3, 331-336.

[20] R. Banerjee, Y. Proshlyakov, J. D. Lipscomb, D. A. Proshlyakov, Nature 2015, 518, 431- 
434.

[21] G. B. Richter-Addo, P. Legdzins, Metal Nitrosyls, Oxford University Press, New York, 1992.

[22] C. Kupper, A. Schober, S. Demeshko, M. Bergner, F. Meyer, Inorg. Chem. 2015, 54, $3096-$ 3098. ACS Articles on Request 10.1021/acs.inorgchem.5b00301

[23] C. Kupper, J. A. Rees, S. Dechert, S. DeBeer, F. Meyer, J. Am. Chem. Soc. 2016, 138, 7888-7898. ACS Articles on Request 10.1021/jacs.6b00584

[24] J. L. E. Ignarro, Nitric Oxide, Biology and Pathobiology, Academic Press, San Diego, CA, 2000.

[25] L. Zweier, H. Li, A. Samouilov, X. Liu, Nitric Oxide 2010, 22, 83.

[26] D. L. Garbem, D. G. Lowell, D. L. Garbers, D. G. Lowell, Biol. Chem. 1995, 269, 3074130744 .

[27] D. J. Stuehr, Annu. Rev. Pharmacol. Toxicol. 1997, 37, 339-359.

[28] F. Roncaroli, M. Videla, L. D. Slep, J. A. Olabe, Coord. Chem. Rev. 2007, 251, 1903-1930.

[29] S. J. Ferguson, Curr. Opin. Chem. Biol. 1998, 2, 182-193.

[30] W. Zumft, J. Inorg. Biochem. 2005, 99, 194-215.

[31] A. L. Speelman, N. Lehnert, Angew. Chem. Int. Ed. 2013, 52, 12283-12287.

[32] B. A. Averill, Chem. Rev. 1996, 96, 2951-2964.

[33] P. Girsch, S. de Vries, Biochim. Biophys. Acta - Bioenerg. 1997, 1318, 202-216.

[34] A. Daiber, H. Shoun, V. Ullrich, J. Inorg. Biochem. 2005, 99, 185-193.

[35] N. Lehnert, V. K. K. Praneeth, F. Paulat, J. Comput. Chem. 2006, 27, 1338-1351.

[36] T. Hayashi, J. D. Caranto, D. A. Wampler, D. M. Kurtz, P. Moënne-Loccoz, Biochemistry 2010, 49, 7040-7049.

[37] D. M. Kurtz, Jr., Dalton Trans. 2007, 4115.

[38] D. E. Canfield, A. N. Glazer, P. G. Falkowski, Science 2010, 330, 192-196.

[39] Q. Cheng, J. Integr. Plant Biol. 2008, 50, 786-798.

[40] B. Thamdrup, Annu. Rev. Ecol. Evol. Syst. 2012, 43, 407-428.

[41] T. A. Betley, J. C. Peters, J. Am. Chem. Soc. 2004, 126, 6252-6254.

[42] B. K. Burgess, D. J. Lowe, Chem. Rev. 1996, 96, 2983-3012.

[43] J. Chatt, J. R. Dilworth, R. L. Richards, Nature 1970, 227, 419-419. 
[44] J. Chatt, A. J. Pearman, R. L. Richards, J. Chem. Soc. Dalt. Trans. 1977, 1852.

[45] M. P. Hendrich, W. Gunderson, R. K. Behan, M. T. Green, M. P. Mehn, T. A. Betley, C. C. Lu, J. C. Peters, Proc. Natl. Acad. Sci. 2006, 103, 17107-17112.

[46] M. P. Mehn, J. C. Peters, J. Inorg. Biochem. 2006, 100, 634-643.

[47] E. W. Svastits, J. H. Dawson, R. Breslow, S. H. Gellman, J. Am. Chem. Soc. 1985, 107, $6427-6428$.

[48] J. T. Groves, R. C. Haushalter, M. Nakamura, T. E. Nemo, B. J. Evans, J. Am. Chem. Soc. 1981, 103, 2884-2886.

[49] J. T. Groves, J. Inorg. Biochem. 2006, 100, 434-447.

[50] H. Fujii, Coord. Chem. Rev. 2002, 226, 51-60.

[51] L. Que Jr., Acc. Chem. Res. 2007, 40, 493-500.

[52] A. R. McDonald, L. Que Jr., Coord. Chem. Rev. 2013, 257, 414-428.

[53] J.-U. Rohde, J.-H. In, M. H. Lim, W. W. Brennessel, M. R. Bukowski, A. Stubna, E. Münck, W. Nam, L. Que Jr., Science 2003, 299, 1037-1039.

[54] J. England, Y. Guo, E. R. Farquhar, V. G. Young Jr., E. Münck, L. Que Jr., J. Am. Chem. Soc. 2010, 132, 8635-8644.

[55] J. England, M. Martinho, E. R. Farquhar, J. R. Frisch, E. L. Bominaar, E. Münck, L. Que, Angew. Chem. Int. Ed. 2009, 48, 3622-3626.

[56] F. T. de Oliveira, A. Chanda, D. Banerjee, X. Shan, S. Mondal, L. Que Jr., E. L. Bominaar, E. Münck, T. J. Collins, Science 2007, 315, 835-838.

[57] C. A. Grapperhaus, B. Mienert, E. Bill, T. Weyhermüller, K. Wieghardt, Inorg. Chem. 2000, 39, 5306-5317.

[58] W. Nam, Y. Lee, S. Fukuzumi, Acc. Chem. Res. 2014, 47, 1146-1154.

[59] W. N. Oloo, L. Que Jr., Acc. Chem. Res. 2015, 48, 2612-2621.

[60] W. Nam, Acc. Chem. Res. 2015, 48, 2415-2423.

[61] I. Prat, J. S. Mathieson, M. Güell, X. Ribas, J. M. Luis, L. Cronin, M. Costas, Nat. Chem. 2011, 3, 788-793.

[62] S. Rana, A. Dey, D. Maiti, Chem. Commun. 2015, 51, 14469-14472.

[63] K. Cho, X. Wu, Y. Lee, Y. H. Kwon, S. Shaik, W. Nam, J. Am. Chem. Soc. 2012, 134, 20222-20225.

[64] A. Company, I. Prat, J. R. Frisch, D. R. Mas-Ballesté, M. Güell, G. Juhász, X. Ribas, D. E. 
Münck, J. M. Luis, L. Que Jr., M. Costas, Chem. Eur. J. 2011, 17, 1622-1634.

[65] J. Kaizer, E. J. Klinker, N. Y. Oh, J.-U. Rohde, W. J. Song, A. Stubna, J. Kim, E. Münck, W. Nam, L. Que Jr., J. Am. Chem. Soc. 2004, 126, 472-473.

[66] J. J. Warren, T. A. Tronic, J. M. Mayer, Chem. Rev. 2010, 110, 6961-7001.

[67] J. J. Scepaniak, C. G. Margarit, J. N. Harvey, J. M. Smith, Inorg. Chem. 2011, 50, 95089517.

[68] R. H. Holm, Chem. Rev. 1987, 87, 1401-1449.

[69] D. Wang, K. Ray, M. J. Collins, E. R. Farquhar, J. R. Frisch, L. Gómez, T. A. Jackson, M. Kerscher, A. Waleska, P. Comba, M. Costas, L. Que Jr., Chem. Sci. 2013, 4, $282-291$.

[70] P. Comba, S. Fukuzumi, H. Kotani, S. Wunderlich, Angew. Chem. Int. Ed. 2010, 49, 26222625.

[71] M. S. Seo, J.-H. In, S. O. Kim, N. Y. Oh, J. Hong, J. Kim, L. Que Jr., W. Nam, Angew. Chem. Int. Ed. 2004, 43, 2417-2420.

[72] M. Puri, A. Company, G. Sabenya, M. Costas, L. Que, Inorg. Chem. 2016, 55, 5818-5827.

[73] S. P. de Visser, Y.-M. Lee, W. Nam, Eur. J. Inorg. Chem. 2008, 1027-1030.

[74] C. V. Sastri, K. Oh, Y. J. Lee, M. S. Seo, W. Shin, W. Nam, Angew. Chem. Int. Ed. 2006, 45, 3992-3995.

[75] D. Janardanan, Y. Wang, P. Schyman, L. Que, S. Shaik, Angew. Chem. Int. Ed. 2010, 49, $3342-3345$.

[76] D. C. Lacy, R. Gupta, K. L. Stone, J. Greaves, J. W. Ziller, M. P. Hendrich, A. S. Borovik, J. Am. Chem. Soc. 2010, 132, 12188-12190.

[77] A. N. Biswas, M. Puri, K. K. Meier, W. N. Oloo, G. T. Rohde, E. L. Bominaar, E. Münck, L. Que, J. Am. Chem. Soc. 2015, 137, 2428-2431.

[78] M. S. Seo, N. H. Kim, K.-B. Cho, J. E. So, S. K. Park, M. Clémancey, R. Garcia-Serres, J.M. Latour, S. Shaik, W. Nam, Chem. Sci. 2011, 2, 1039.

[79] S. H. Bae, M. S. Seo, Y.-M. Lee, K.-B. Cho, W.-S. Kim, W. Nam, Angew. Chem. Int. Ed. 2016, 55, 8027-8031.

[80] H. Hirao, L. Que Jr., W. Nam, S. Shaik, Chem. Eur. J. 2008, 14, 1740-1756.

[81] S. Kundu, J. V. K. Thompson, A. D. Ryabov, T. J. Collins, J. Am. Chem. Soc. 2011, 133, 18546-18549.

[82] C. Geng, S. Ye, F. Neese, Dalton Trans. 2014, 43, 6079-86.

[83] C. V. Sastri, J. Lee, K. Oh, Y. J. Lee, J. Lee, T. A. Jackson, K. Ray, H. Hirao, W. Shin, J. A. 
Halfen, J. Kim, L. Que Jr., S. Shaik, W. Nam, Proc. Natl. Acad. Sci. 2007, 104, 1918119186.

[84] D. Mandal, R. Ramanan, D. Usharani, D. Janardanan, B. Wang, S. Shaik, J. Am. Chem. Soc. 2015, 137, 722-733.

[85] P. R. Schreiner, H. P. Reisenauer, D. Ley, D. Gerbig, C.-H. Wu, W. D. Allen, Science 2011, $332,1300-1303$.

[86] D. Ley, D. Gerbig, P. R. Schreiner, Org. Biomol. Chem. 2012, 10, 3781-3790.

[87] S. Hong, Y. M. Lee, K. Bin Cho, K. Sundaravel, J. Cho, M. J. Kim, W. Shin, W. Nam, J. Am. Chem. Soc. 2011, 133, 11876-11879.

[88] S. Hong, H. So, H. Yoon, K. Cho, Y. Lee, S. Fukuzumi, W. Nam, Dalton Trans. 2013, 42, $7842-7845$.

[89] Y. Hitomi, K. Arakawa, M. Kodera, Chem. Eur. J. 2013, 19, 14697-14701.

[90] X. Sun, C. Geng, R. Huo, U. Ryde, Y. Bu, J. Li, J. Phys. Chem. B 2014, 118, 1493-1500.

[91] W. D. Wagner, K. Nakamoto, J. Am. Chem. Soc. 1989, 111, 1590-1598.

[92] K. Meyer, E. Bill, B. Mienert, T. Weyhermüller, K. Wieghardt, J. Am. Chem. Soc. 1999, $121,4859-4876$.

[93] T. Petrenko, S. DeBeer George, N. Aliaga-Alcalde, E. Bill, B. Mienert, Y. Xiao, Y. Guo, W. Sturhahn, S. P. Cramer, K. Wieghardt, F. Neese, J. Am. Chem. Soc. 2007, 129, 1105311060.

[94] J. Torres-Alacan, U. Das, A. C. Filippou, P. Vöhringer, Angew. Chem. Int. Ed. 2013, 52, $12833-12837$.

[95] C. Vogel, F. W. Heinemann, J. Sutter, C. Anthon, K. Meyer, Angew. Chem. Int. Ed. 2008, 47, 2681-2684.

[96] J. J. Scepaniak, C. S. Vogel, M. M. Khusniyarov, F. W. Heinemann, K. Meyer, J. M. Smith, Science 2011, 331, 1049-1052.

[97] J. J. Scepaniak, M. D. Fulton, R. P. Bontchev, E. N. Duesler, M. L. Kirk, J. M. Smith, J. Am. Chem. Soc. 2008, 130, 10515-10517.

[98] W. Lee, R. A. Juarez, J. J. Scepaniak, S. B. Muñoz, D. A. Dickie, H. Wang, J. M. Smith, Inorg. Chem. 2014, 53, 8425-8430.

[99] J. J. Scepaniak, J. A. Young, R. P. Bontchev, J. M. Smith, Angew. Chem. Int. Ed. 2009, 48, $3158-3160$.

[100] J. J. Scepaniak, R. P. Bontchev, D. L. Johnson, J. M. Smith, Angew. Chem. Int. Ed. 2011, 
$50,6630-6633$.

[101] W. R. Scheidt, D. A. Summerville, I. A. Cohen, J. Am. Chem. Soc. 1976, 98, 6623-6628.

[102] A. Ghosh, E. Tangen, E. Gonzalez, L. Que Jr., Angew. Chem. Int. Ed. 2004, 43, 834-838.

[103] M. Li, M. Shang, N. Ehlinger, C. E. Schulz, W. R. Scheidt, Inorg. Chem. 2000, 39, 580583.

[104] T. Jüstel, T. Weyhermüller, K. Wieghardt, E. Bill, M. Lengen, A. X. Trautwein, P. Hildebrandt, Angew. Chem. Int. Ed. 1995, 34, 669-672.

[105] C. Colomban, E. V. Kudrik, D. V. Tyurin, F. Albrieux, S. E. Nefedov, P. Afanasiev, A. B. Sorokin, Dalton Trans. 2015, 44, 2240-2251.

[106] S. D. Brown, M. P. Mehn, J. C. Peters, J. Am. Chem. Soc. 2005, 127, 13146-13147.

[107] A. B. Sorokin, E. V. Kudrik, D. Bouchu, Chem. Commun. 2008, 2562.

[108] E. V. Kudrik, P. Afanasiev, L. X. Alvarez, P. Dubourdeaux, M. Clémancey, J.-M. Latour, G. Blondin, D. Bouchu, F. Albrieux, S. E. Nefedov, A. B. Sorokin, Nat. Chem. 2012, 4, 1024-1029.

[109] E. V. Kudrik, A. B. Sorokin, Chem. Eur. J. 2008, 14, 7123-7126.

[110] Ü. İşci, P. Afanasiev, J.-M. M. Millet, E. V. Kudrik, V. Ahsen, A. B. Sorokin, Dalton Trans. 2009, 7410-7420.

[111] L. X. Alvarez, E. V. Kudrik, A. B. Sorokin, Chem. Eur. J. 2011, 17, 9298-9301.

[112] C. Colomban, E. V. Kudrik, P. Afanasiev, A. B. Sorokin, J. Am. Chem. Soc. 2014, 136, $11321-11330$.

[113] J. H. Enemark, R. D. Feltham, Coord. Chem. Rev. 1974, 13, 339-406.

[114] I. M. Lorković, P. C. Ford, Inorg. Chem. 2000, 39, 632-633.

[115] G. R. A. Wyllie, W. R. Scheidt, Chem. Rev. 2002, 102, 1067-1090.

[116] T. C. Berto, A. L. Speelman, S. Zheng, N. Lehnert, Coord. Chem. Rev. 2013, 257, 244-259.

[117] C. A. Brown, M. A. Pavlosky, T. E. Westre, Y. Zhang, B. Hedman, K. O. Hodgson, E. I. Solomon, J. Am. Chem. Soc. 1995, 117, 715-732.

[118] D. Lancon, K. M. Kadish, J. Am. Chem. Soc. 1983, 105, 5610-5617.

[119] I. K. Choi, Y. Liu, D. Feng, K. J. Paeng, M. D. Ryan, Inorg. Chem. 1991, 30, 1832-1839.

[120] Z. Wei, M. D. Ryan, Inorg. Chem. 2010, 49, 6948-6954.

[121] J. Pellegrino, S. E. Bari, D. E. Bikiel, F. Doctorovich, J. Am. Chem. Soc. 2010, 132, 989995. 
[122] A. L. Speelman, N. Lehnert, Acc. Chem. Res. 2014, 47, 1106-1116.

[123] R. García Serres, C. A. Grapperhaus, E. Bothe, E. Bill, T. Weyhermüller, F. Neese, K. Wieghardt, J. Am. Chem. Soc. 2004, 126, 5138-5153.

[124] L. E. Goodrich, S. Roy, E. E. Alp, J. Zhao, M. Y. Hu, N. Lehnert, Inorg. Chem. 2013, 52, 7766-7780.

[125] B. Hu, J. Li, Angew. Chem. Int. Ed. 2015, 54, 10579-10582.

[126] N. Lehnert, T. C. Berto, M. G. I. Galinato, L. E. Goodrich, The Handbook of Porphyrin Science, World Scientific, Singapore, 2011.

[127] A. Earnshaw, E. A. King, L. F. Larkworthy, J. Chem. Soc. A 1969, 2459-2463.

[128] K. J. Haller, P. L. Johnson, R. D. Feltham, J. H. Enemark, J. R. Ferraro, L. J. Basile, Inorg. Chim. Acta 1979, 33, 119-130.

[129] K. D. Hodges, R. G. Wollmann, S. L. Kessel, D. N. Hendrickson, D. G. Van Derveer, E. K. Barefield, J. Am. Chem. Soc. 1979, 101, 906-917.

[130] M. Radoń, E. Broclawik, K. Pierloot, J. Phys. Chem. B 2010, 114, 1518-1528.

[131] J. Conradie, A. Ghosh, J. Inorg. Biochem. 2006, 100, 2069-2073.

[132] J. Kopf, J. Schmidt, Zeitschrift für Naturforsch. B 1977, 32b, 275-280.

[133] K. J. Franz, S. J. Lippard, J. Am. Chem. Soc. 1999, 121, 10504-10512.

[134] M. Ray, A. P. Golombek, M. P. Hendrich, G. P. a. Yap, L. M. Liable-Sands, L. Rheingold, Arnold, A. S. Borovik, Inorg. Chem. 1999, 38, 3110-3115.

[135] J. Conradie, D. A. Quarless, H.-F. Hsu, T. C. Harrop, S. J. Lippard, S. A. Koch, A. Ghosh, J. Am. Chem. Soc. 2007, 129, 10446-10456.

[136] J. Conradie, A. Ghosh, Inorg. Chem. 2011, 50, 4223-4225.

[137] J. Conradie, K. H. Hopmann, A. Ghosh, J. Phys. Chem. B 2010, 114, 8517-8524.

[138] C. Hauser, T. Glaser, E. Bill, T. Weyhermüller, K. Wieghardt, J. Am. Chem. Soc. 2000, 122, $4352-4365$.

[139] A. K. Patra, K. S. Dube, B. C. Sanders, G. C. Papaefthymiou, J. Conradie, A. Ghosh, T. C. Harrop, Chem. Sci. 2012, 3, 364-369.

[140] Z. J. Tonzetich, F. Héroguel, L. H. Do, S. J. Lippard, Inorg. Chem. 2011, 50, 1570-1579.

[141] Z. Ouyang, Y. Meng, J. Cheng, J. Xiao, S. Gao, L. Deng, Organometallics 2016, 35, 13611367.

[142] K. Riener, S. Haslinger, A. Raba, M. P. Högerl, M. Cokoja, W. A. Herrmann, F. E. Kühn, 
Chem. Rev. 2014, 114, 5215-5272.

[143] M. J. Ingleson, R. A. Layfield, Chem. Commun. 2012, 48, 3579.

[144] M. Scholl, S. Ding, C. W. Lee, R. H. Grubbs, Org. Lett. 1999, 1, 953-956.

[145] M. M. Rogers, S. S. Stahl, in N-Heterocyclic Carbenes Transit. Met. Catal., Springer Berlin Heidelberg, 2006, pp. 21-46.

[146] K. R. Jain, W. A. Herrmann, F. E. Kühn, Curr. Org. Chem. 2008, 12, 1468-1478.

[147] W. Ching, C. Chuang, C. Wu, C. Peng, C. Hung, J. Am. Chem. Soc. 2009, 131, 7952-7953.

[148] W.-M. Ching, C.-H. Hung, Chem. Commun. 2012, 48, 4989.

[149] H. M. Bass, S. A. Cramer, J. L. Price, D. M. Jenkins, Organometallics 2010, 29, 32353238.

[150] S. A. Cramer, D. M. Jenkins, J. Am. Chem. Soc. 2011, 133, 19342-19345.

[151] S. A. Cramer, R. Hernández Sánchez, D. F. Brakhage, D. M. Jenkins, Chem. Commun. 2014, 50, 13967-13970.

[152] Z. Lu, S. A. Cramer, D. M. Jenkins, Chem. Sci. 2012, 3, 3081.

[153] S. Meyer, I. Klawitter, S. Demeshko, E. Bill, F. Meyer, Angew. Chem. Int. Ed. 2013, 52, 901-905.

[154] S. Meyer, Bioinspired Organometallic Analogues of Iron-Sites in Metalloproteins, GeorgAugust Universität Göttingen, 2013.

[155] I. Klawitter, Makrozyklische NHC-Liganden Zur Stabilisierung Hochvalenter Metallionen, Georg-August Universität Göttingen, 2015.

[156] S. Meyer, O. Krahe, C. Kupper, I. Klawitter, S. Demeshko, E. Bill, F. Neese, F. Meyer, Inorg. Chem. 2015, 54, 9770-9776.

[157] H. M. Bass, S. A. Cramer, A. S. McCullough, K. J. Bernstein, C. R. Murdock, D. M. Jenkins, Organometallics 2013, 32, 2160-2167.

[158] S. A. Cramer, F. L. Sturgill, P. P. Chandrachud, D. M. Jenkins, Dalton Trans. 2014, 43, 7687.

[159] R. McKie, J. A. Murphy, S. R. Park, M. D. Spicer, S. Zhou, Angew. Chem. Int. Ed. 2007, $46,6525-6528$.

[160] M. R. Anneser, S. Haslinger, A. Pöthig, M. Cokoja, J.-M. M. Basset, F. E. Kühn, Inorg. Chem. 2015, 54, 3797-3804

[161] M. R. Anneser, S. Haslinger, A. Pöthig, M. Cokoja, V. D’Elia, M. P. Högerl, J.-M. Basset, F. E. Kühn, Dalton Trans. 2016, 45, 6449-6455. 
[162] I. Klawitter, M. R. Anneser, S. Dechert, S. Meyer, S. Demeshko, S. Haslinger, A. Pöthig, F. E. Kühn, F. Meyer, Organometallics 2015, 34, 2819-2825.

[163] P. Gütlich, E. Bill, A. X. Trautwein, Mössbauer Spectroscopy and Transition Metal Chemistry, Springer, Heidelberg, 2011.

[164] R. L. Mössbauer, Zeitschrift für Phys. 1958, 151, 124-143.

[165] S. Ye, E. Bill, F. Neese, Inorg. Chem. 2016, 55, 3468-3474.

[166] Z. J. Tonzetich, L. H. Do, S. J. Lippard, J. Am. Chem. Soc. 2009, 131, 7964-7965.

[167] B. M. Lindley, A. Swidan, E. B. Lobkovsky, P. T. Wolczanski, M. Adelhardt, J. Sutter, K. Meyer, Chem. Sci. 2015, 6, 4730-4736.

[168] Y. Lee, N. P. Mankad, J. C. Peters, Nat. Chem. 2010, 2, 558-565.

[169] K. M. Van Heuvelen, A. T. Fiedler, X. Shan, R. F. De Hont, K. K. Meier, E. L. Bominaar, E. Münck, L. Que, Proc. Natl. Acad. Sci. 2012, 109, 11933-11938.

[170] S. Ye, C. Kupper, S. Meyer, E. Andris, R. Navrátil, O. Krahe, B. Mondal, M. Atanasov, E. Bill, J. Roithová, F. Meyer, F. Neese, J. Am. Chem. Soc. 2016, 138, 14312-14325. ACS Articles on Request 10.1021/jacs.6b07708

[171] E. J. Klinker, J. Kaizer, W. W. Brennessel, N. L. Woodrum, C. J. Cramer, L. Que Jr., Angew. Chem. Int. Ed. 2005, 44, 3690-3694.

[172] A. Thibon, J. England, M. Martinho, V. G. Young, J. R. Frisch, R. Guillot, J.-J. Girerd, E. Münck, L. Que Jr., F. Banse, Angew. Chem. Int. Ed. 2008, 47, 7064-7067.

[173] D. Macikenas, E. Skrzypczak-Jankun, J. D. Protasiewicz, J. Am. Chem. Soc. 1999, 121, 7164-7165.

[174] J. Roithová, A. Gray, E. Andris, J. Jašík, D. Gerlich, Acc. Chem. Res. 2016, 49, 223-230.

[175] F. Neese, E. I. Solomon, Inorg. Chem. 1999, 38, 1847-1865.

[176] S. Ye, G. Xue, I. Krivokapic, T. Petrenko, E. Bill, L. Que Jr., F. Neese, Chem. Sci. 2015, 6, 2909-2921.

[177] A. Decker, J.-U. Rohde, E. J. Klinker, S. D. Wong, L. Que Jr., E. I. Solomon, J. Am. Chem. Soc. 2007, 129, 15983-15996.

[178] A. Ghosh, F. Tiago de Oliveira, T. Yano, T. Nishioka, E. S. Beach, I. Kinoshita, E. Münck, A. D. Ryabov, C. P. Horwitz, T. J. Collins, J. Am. Chem. Soc. 2005, 127, 2505-2513.

[179] C. Kupper, B. Mondal, J. Serrano-Plana, I. Klawitter, F. Neese, M. Costas, S. Ye, F. Meyer, J. Am. Chem. Soc. 2017, DOI 10.1021/jacs.7b03255.

[180] S. Hong, B. Wang, M. S. Seo, Y. Lee, M. J. Kim, H. R. Kim, T. Ogura, R. Garcia-Serres, 
M. Clémancey, J. Latour, W. Nam, Angew. Chem. Int. Ed. 2014, 53, 6388-6392.

[181] T. H. Parsell, M.-Y. Yang, A. S. Borovik, J. Am. Chem. Soc. 2009, 131, 2762-2763.

[182] O. Planas, M. Clémancey, J.-M. Latour, A. Company, M. Costas, Chem. Commun. 2014, $50,10887$.

[183] J. C. Price, E. W. Barr, T. E. Glass, C. Krebs, J. M. Bollinger, J. Am. Chem. Soc. 2003, 125, 13008-13009.

[184] J. C. Nesheim, J. D. Lipscomb, Biochemistry 1996, 35, 10240-10247.

[185] A. N. Biswas, M. Puri, K. K. Meier, W. N. Oloo, G. T. Rohde, E. L. Bominaar, E. Münck, L. Que, J. Am. Chem. Soc. 2015, 137, 2428-2431.

[186] D. Mandal, S. Shaik, J. Am. Chem. Soc. 2016, 138, 2094-2097.

[187] S. T. Kleespies, W. N. Oloo, A. Mukherjee, L. Que, Inorg. Chem. 2015, 54, 5053-5064.

[188] F. G. Cantú Reinhard, S. P. de Visser, Chem. Eur. J. 2017, 23, 2935-2944.

[189] K. S. Murray, Coord. Chem. Rev. 1974, 12, 1-35.

[190] D. M. J. Kurtz, Chem. Rev. 1990, 90, 585-606.

[191] D.-H. Chin, A. L. Balch, G. N. La Mar, J. Am. Chem. Soc. 1980, 102, 1446-1448.

[192] D.-H. Chin, G. N. La Mar, A. L. Balch, J. Am. Chem. Soc. 1980, 102, 4344-4350.

[193] T. J. Collins, Acc. Chem. Res. 2002, 35, 782-790.

[194] A. Kejriwal, P. Bandyopadhyay, A. N. Biswas, Dalton Trans. 2015, 44, 17261-17267.

[195] M. Kodera, M. Itoh, K. Kano, T. Funabiki, M. Reglier, Angew. Chem. Int. Ed. 2005, 44, 7104-7106.

[196] S. Taktak, S. V. Kryatov, T. E. Haas, E. V. Rybak-Akimova, J. Mol. Catal. A Chem. 2006, $259,24-34$.

[197] É. Balogh-Hergovich, G. Speier, M. Réglier, M. Giorgi, E. Kuzmann, A. Vértes, Eur. J. Inorg. Chem. 2003, 1735-1740.

[198] P. Payra, S. Hung, W. H. Kwok, D. Johnston, J. Gallucci, M. K. Chan, Inorg. Chem. 2001, 40, 4036-4039.

[199] R. Horikawa, C. Fujimoto, R. Yazaki, T. Ohshima, Chem. Eur. J. 2016, 22, 12278-12281.

[200] J.-U. Rohde, L. Que Jr., Angew. Chem. Int. Ed. 2005, 44, 2255-2258.

[201] M. R. Bukowski, K. D. Koehntop, A. Stubna, E. L. Bominaar, J. A. Halfen, E. Münck, W. Nam, L. Que Jr., Science 2005, 310, 1000-1002.

[202] D.-H. Chin, G. N. La Mar, A. L. Balch, J. Am. Chem. Soc. 1980, 102, 5945-5947. 
[203] K. K. Singh, M. K. Tiwari, B. B. Dhar, K. Vanka, S. Sen Gupta, Inorg. Chem. 2015, 54, $6112-6121$.

[204] C. Ercolani, M. Gardini, G. Pennesi, G. Rossi, J. Chem. Soc. Chem. Commun. 1983, 10, $549-550$.

[205] M. K. Ellison, H. Nasri, Y.-M. Xia, J. Marchon, C. E. Schulz, P. G. Debrunner, W. R. Scheidt, Inorg. Chem. 1997, 36, 4804-4811.

[206] R. S. Czernuszewicz, W.-D. Wagner, G. B. Ray, K. Nakamoto, J. Mol. Struct. 1991, 242, 99-117.

[207] N. G. Connelly, W. E. Geiger, Chem. Rev. 1996, 96, 877-910.

[208] S. K. Dutta, U. Beckmann, E. Bill, T. Weyhermüller, K. Wieghardt, Inorg. Chem. 2000, 39, $3355-3364$.

[209] T. Jüstel, M. Müller, T. Weyhermüller, C. Kressl, E. Bill, P. Hildebrandt, M. Lengen, M. Grodzicki, A. X. Trautwein, B. Nuber, K. Wieghardt, Chem. Eur. J. 1999, 5, 793-810.

[210] D. A. Summerville, I. A. Cohen, J. Am. Chem. Soc. 1976, 98, 1747-1752.

[211] C. Ercolani, S. Hewage, R. Heucher, G. Rossi, Inorg. Chem. 1993, 32, 2975-2977.

[212] D. R. English, D. N. Hendrickson, K. S. Suslick, Inorg. Chem. 1983, 22, 367-368.

[213] A. F. Hollemann, E. Wiberg, N. Wiberg, Lehrbuch Der Anorganischem Chemie, Walter De Gruyter, Berlin, New York, New York, 2007.

[214] I. Kaljurand, A. Kütt, L. Sooväli, T. Rodima, V. Mäemets, I. Leito, I. A. Koppel, J. Org. Chem. 2005, 70, 1019-1028.

[215] K. Ray, F. F. Pfaff, B. Wang, W. Nam, J. Am. Chem. Soc. 2014, 136, 13942-13958.

[216] E. Kwon, K.-B. Cho, S. Hong, W. Nam, Chem. Commun. 2014, 50, 5572.

[217] J. Serrano-Plana, W. N. Oloo, L. Acosta-Rueda, K. K. Meier, B. Verdejo, E. García-España, M. G. Basallote, E. Münck, L. Que, A. Company, M. Costas, J. Am. Chem. Soc. 2015, 137, 15833-15842.

[218] J. Chen, Y.-M. Lee, K. M. Davis, X. Wu, M. S. Seo, K.-B. Cho, H. Yoon, Y. J. Park, S. Fukuzumi, Y. N. Pushkar, W. Nam, J. Am. Chem. Soc. 2013, 135, 6388-6391.

[219] C. A. Ramsden, R. G. Smith, J. Am. Chem. Soc. 1998, 120, 6842-6843.

[220] A. M. Forster, A. J. Downs, Polyhedron 1985, 4, 1625-1635.

[221] M. Tramšek, B. Žemva, Acta Chim. Slov. 2006, 53, 105-116.

[222] X. Ou, A. F. Janzen, J. Fluor. Chem. 2000, 101, 279-283. 
[223] S. Chatterjee, T. K. Paine, Angew. Chem. Int. Ed. 2016, 55, 7717-7722.

[224] A. Kütt, T. Rodima, J. Saame, E. Raamat, V. Mäemets, I. Kaljurand, I. A. Koppel, R. Y. Garlyauskayte, Y. L. Yagupolskii, L. M. Yagupolskii, E. Bernhardt, H. Willner, I. Leito, J. Org. Chem. 2011, 76, 391-395.

[225] E.-I. Rõõm, A. Kütt, I. Kaljurand, I. Koppel, I. Leito, I. A. Koppel, M. Mishima, K. Goto, Y. Miyahara, Chem. Eur. J. 2007, 13, 7631-7643.

[226] P. G. Wang, M. Xian, X. Tang, X. Wu, Z. Wen, T. Cai, A. J. Janczuk, Chem. Rev. 2002, 102, 1091-1134.

[227] U. Schatzschneider, Eur. J. Inorg. Chem. 2010, 1451-1467.

[228] T. Yoshimura, Inorg. Chim. Acta 1984, 83, 17-21.

[229] L. Cheng, M. A. Khan, G. B. Richter-Addo, D. R. Powell, Chem. Commun. 2000, 991, 2301-2302.

[230] M. A. Rhine, B. C. Sanders, A. K. Patra, T. C. Harrop, Inorg. Chem. 2015, 54, 9351-9366.

[231] A. W. Addison, T. N. Rao, J. Reedijk, J. van Rijn, G. C. Verschoor, J. Chem. Soc., Dalt. Trans. 1984, 1349-1356.

[232] B. Weber, I. Käpplinger, H. Görls, E.-G. Jäger, Eur. J. Inorg. Chem. 2005, 2794-2811.

[233] M.-C. Tsai, F.-T. Tsai, T.-T. Lu, M.-L. Tsai, Y.-C. Wei, I.-J. Hsu, J.-F. Lee, W.-F. Liaw, Inorg. Chem. 2009, 48, 9579-9591.

[234] K. Pohl, K. Wieghardt, B. Nuber, J. Weiss, J. Chem. Soc. Dalt. Trans. 1987, 187-192.

[235] B. Weber, H. Görls, M. Rudolph, E.-G. Jäger, Inorg. Chim. Acta 2002, 337, 247-265.

[236] J. Li, A. Banerjee, P. L. Pawlak, W. W. Brennessel, F. A. Chavez, Inorg. Chem. 2014, 53, 5414-5416.

[237] N. Lehnert, W. R. Scheidt, M. W. Wolf, Structure and Bonding in Heme-Nitrosyl Complexes and Implications for Biology, Springer-Verlag, Berlin Heidelberg, 2013.

[238] C. J. Pollock, M. U. Delgado-Jaime, M. Atanasov, F. Neese, S. DeBeer, J. Am. Chem. Soc. 2014, 136, 9453-9463.

[239] C. J. Pollock, S. DeBeer, Acc. Chem. Res. 2015, 48, 2967-2975.

[240] T.-T. Lu, T.-C. Weng, W.-F. Liaw, Angew. Chem. Int. Ed. 2014, 53, 11562-11566.

[241] T. E. Westre, P. Kennepohl, J. G. DeWitt, B. Hedman, K. O. Hodgson, E. I. Solomon, J. Am. Chem. Soc. 1997, 119, 6297-6314.

[242] N. Lee, T. Petrenko, U. Bergmann, F. Neese, S. DeBeer, J. Am. Chem. Soc. 2010, 132, 9715-9727. 
[243] M. Römelt, S. Ye, F. Neese, Inorg. Chem. 2009, 48, 784-785.

[244] A. P. Hunt, N. Lehnert, Acc. Chem. Res. 2015, 48, 2117-2125.

[245] R. S. Simons, J. C. Garrison, W. G. Kofron, C. A. Tessier, W. J. Youngs, Tetrahedron Lett. 2002, 43, 3423-3425.

[246] G. B. Bachman, L. Heisey, J. Am. Chem. Soc. 1946, 68, 2496-2499.

[247] A. Melaiye, Z. Sun, K. Hindi, A. Milsted, D. Ely, D. H. Reneker, C. A. Tessier, W. J. Youngs, J. Am. Chem. Soc. 2005, 127, 2285-2291.

[248] I. T. Kim, R. L. Elsenbaumer, Tetrahedron Lett. 1998, 39, 1087-1090.

[249] J. M. Smith, J. R. Long, Inorg. Chem. 2010, 49, 11223-11230.

[250] Z. Xi, X. Zhang, W. Chen, S. Fu, D. Wang, Organometallics 2007, 26, 6636-6642.

[251] Y. Tulchinsky, M. A. Iron, M. Botoshansky, M. Gandelman, Nat. Chem. 2011, 3, 525-531.

[252] M. Moser, B. Wucher, D. Kunz, F. Rominger, Organometallics 2007, 26, 1024-1030.

[253] T. C. Harrop, Z. J. Tonzetich, E. Reisner, S. J. Lippard, J. Am. Chem. Soc. 2008, 130, $15602-15610$.

[254] J. J. Curley, R. G. Bergman, T. D. Tilley, Dalton Trans. 2012, 41, 192-200.

[255] V. V Pavlishchuk, A. W. Addison, Inorg. Chim. Acta 2000, 298, 97-102.

[256] G. R. Fulmer, A. J. M. Miller, N. H. Sherden, H. E. Gottlieb, A. Nudelman, B. M. Stoltz, J. E. Bercaw, K. I. Goldberg, Organometallics 2010, 29, 2176-2179.

[257] E. Bill, Mfit, Max-Planck Institute For Chemical Energy Conversion, Mülheim/Ruhr, Germany, n.d.

[258] O. Kahn, Molecular Magnetism, VCH Publishers Inc., New York, 1993.

[259] E. Bill, julX, Max-Planck Institute For Chemical Energy Conversion, Mülheim/Ruhr, Germany, Germany, n.d.

[260] G. R. Hanson, K. E. Gates, C. J. Noble, M. Griffin, A. Mitchell, S. Benson, J. Inorg. Biochem. 2004, 98, 903-916.

[261] S. Stoll, A. Schweiger, J. Magn. Reson. 2006, 178, 42-55.

[262] P. Glatzel, U. Bergmann, Coord. Chem. Rev. 2005, 249, 65-95.

[263] D. C. Marelius, E. H. Darrow, C. E. Moore, J. A. Golen, A. L. Rheingold, D. B. Grotjahn, Chem. Eur. J. 2015, 21, 10988-10992.

[264] a) http://libint.valeyev.net; b) H - Kr: A. Schaefer, H. Horn and R. Ahlrichs, J. Chem. Phys. 1992, 97, 2571; c) F. Weigend, R. Ahlrichs, Phys. Chem. Chem. Phys. 2005, 7, 3297; d) 
https://orcaforum.cec.mpg.de/.

[265] G. M. Sheldrick, Acta Crystallogr. Sect. A Found. Crystallogr. 2008, 64, 112-122.

[266] X-RED, STOE \& CIE GmbH: Darmstadt (Germany), 2002. 


\section{List of Abbreviations}

\begin{tabular}{|c|c|}
\hline $5 \mathrm{C} / 6 \mathrm{C}$ & five/six coordinated \\
\hline $\mathrm{CHD}$ & 1,4-cyclohexadiene \\
\hline COSY & correlation spectroscopy \\
\hline $\mathrm{Cp} / \mathrm{Cp}^{*}$ & cyclopentadienyl / pentamethylcyclopentadienyl \\
\hline CT & charge transfer \\
\hline $\mathrm{CV}$ & cyclic voltammetry \\
\hline cy-ac & cyclam-acetate \\
\hline DCM & dichloromethane, $\mathrm{CH}_{2} \mathrm{Cl}_{2}$ \\
\hline DHA & 1,4-dihydroanthracene \\
\hline DFT & density functional theory \\
\hline $\mathrm{DMF}$ & $\mathrm{N}, \mathrm{N}$-dimethylformamide \\
\hline DMSO & dimethylsulfoxide \\
\hline DOSY & diffusion ordered spectroscopy \\
\hline EA & elemental analysis \\
\hline EPR & electron paramagnetic resonance \\
\hline ESI & electrospray ionization \\
\hline et al. & et alia \\
\hline ET & electron transfer \\
\hline $\mathrm{Fc} / \mathrm{Fc}^{+}$ & ferrocene/ferrocenium \\
\hline GC-FID & gas chromatography - flame inonization detector \\
\hline HAA & hydrogen atom abstraction \\
\hline HS/IS/LS & high spin / intermediate spin / low spin \\
\hline $\mathrm{IS}, \delta$ & isomer shift \\
\hline KIE & kinetic isotope effect \\
\hline LUMO & lowest unoccupated molecule orbital \\
\hline $\mathrm{m}$ & medium (IR) \\
\hline$m / z$ & mass per charge \\
\hline MB & Mössbauer \\
\hline $\mathrm{Me}_{3} \mathrm{NTB}$ & tris $((1-m e t h y l-1 H-b e n z o[d]$ imidazole-2-yl)methyl)amine \\
\hline $\mathrm{MeCN}$ & acetonitrile, $\mathrm{CH}_{3} \mathrm{CN}$ \\
\hline $\mathrm{MeOH}$ & methanole \\
\hline MS & mass spectrometry \\
\hline n.b. & non-bonding \\
\hline $\mathrm{NHC}$ & N-heterocyclic carbene \\
\hline NMR & nuclear magnetic resonance \\
\hline
\end{tabular}




\begin{tabular}{|c|c|}
\hline OAT & oxygen atom transfer \\
\hline OTf & trifluoromethanesulfonate \\
\hline ppm & parts per million \\
\hline PT & proton transfer \\
\hline${ }^{\mathrm{Me}, \mathrm{H}}$ Pytacn & 1-(2'-pyridylmethyl)-4,7-dimethyl-1,4,7-triazacyclo-nonane \\
\hline $\mathrm{QS}, \Delta E_{\mathrm{Q}}$ & quadrupole splitting \\
\hline rRaman & resonance Raman \\
\hline RT & room temperature, $21^{\circ} \mathrm{C}$ \\
\hline $\mathrm{s}$ & singlet (NMR), strong (IR) \\
\hline$S$ & spin \\
\hline Salen & $N, N$ '-ethylenebis(salicylimine) \\
\hline sPhIO & soluble iodosobenzene (2-(tert-butylsulfonyl)iodosobenzene) \\
\hline SR-TPA & tris(3,5-dimethyl-4-methoxypyridyl-2-methyl)amine \\
\hline SOMO & singly occupied molecule orbital \\
\hline SQUID & superconducting quantum interference device \\
\hline TACN & 1,4,7-triazacyclononane \\
\hline TAML & tetraamido macrocyclic ligand \\
\hline TCNQ & 7,7,8,8-tetracyanochinodimethane \\
\hline THF & tetrahydrofuran \\
\hline TIMEN $^{\text {mes }}$ & tris-carbene system \\
\hline TMC & tetramethylcyclam \\
\hline $\mathrm{TMG}_{3}$ tren & 1,1,1-tris $\left\{2-\left[N^{2}-(1,1,3,3\right.\right.$-tetramethylguanidino $\left.)\right]$ ethyl $\}$ amine \\
\hline TMP & meso-tetramesityl porphinato anion \\
\hline TQA & tris(quinolin-2-ylmethyl)amine \\
\hline TPP & tetraphenylporphyrin \\
\hline $\mathrm{UV} / \mathrm{vis} / \mathrm{IR}$ & ultraviolet/visible/infrared \\
\hline$v s$. & versus \\
\hline $\mathrm{w}$ & weak (IR) \\
\hline XAS/XES & X-ray absorption spectroscopy / X-ray emission spectroscopy \\
\hline
\end{tabular}




\section{Structures of Ligands and Complexes}
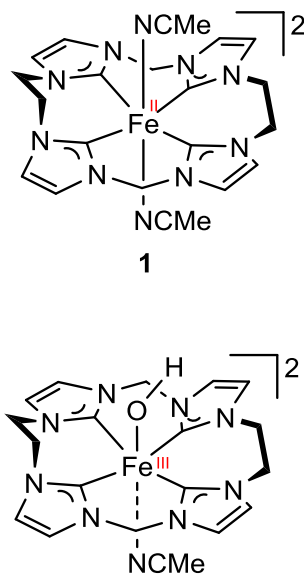

4

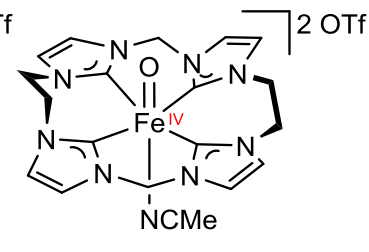

2

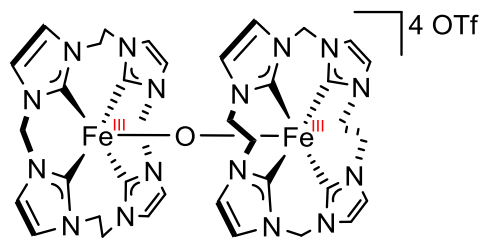

3

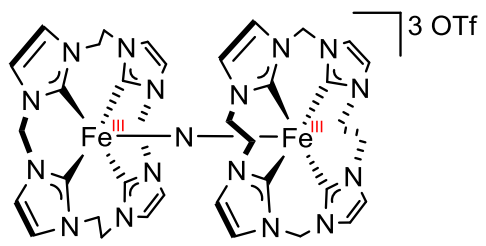

6

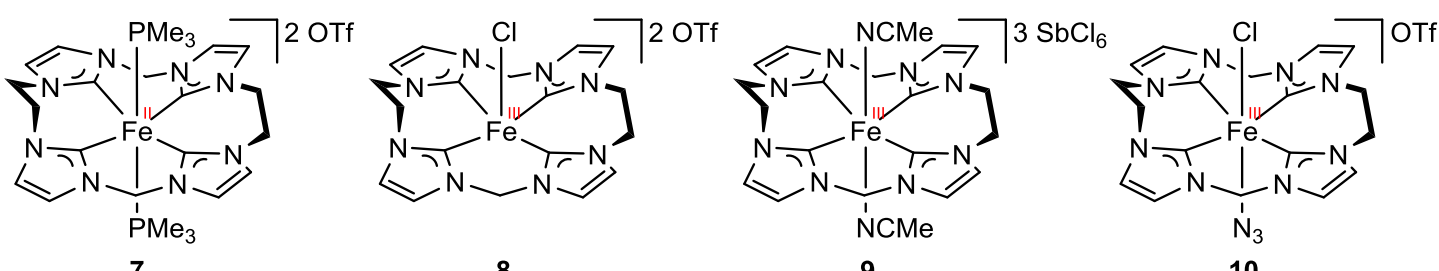

7

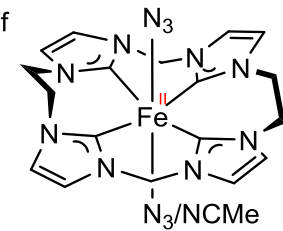

$5+5_{\mathrm{MeCN}}$

10

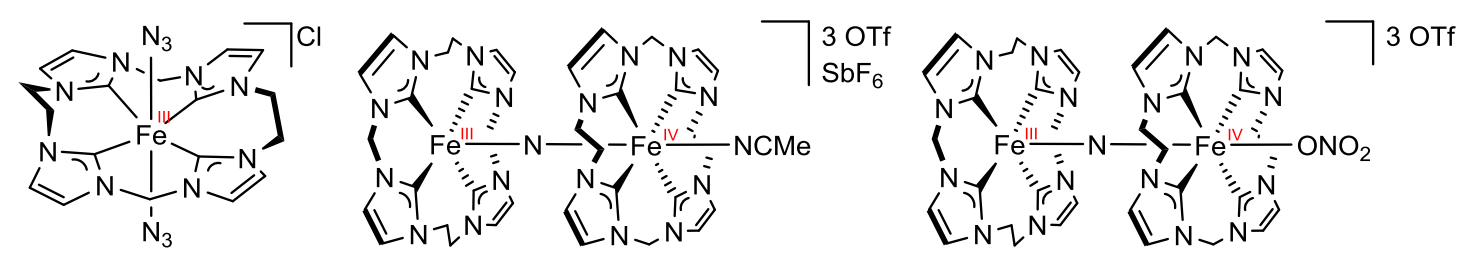

11

12

13

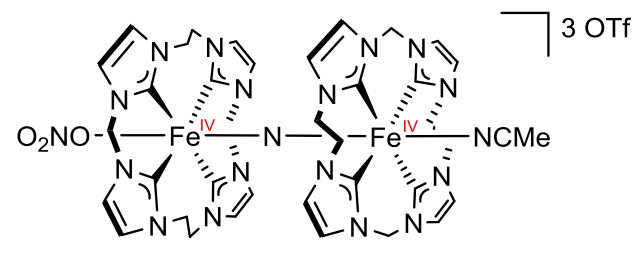

14

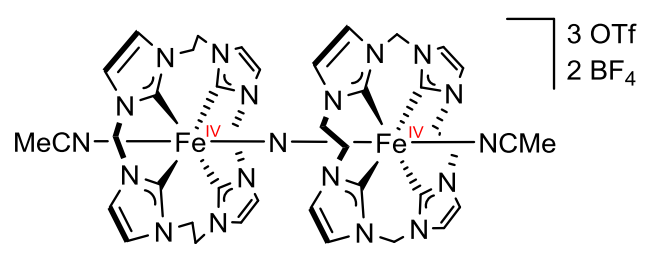

15 


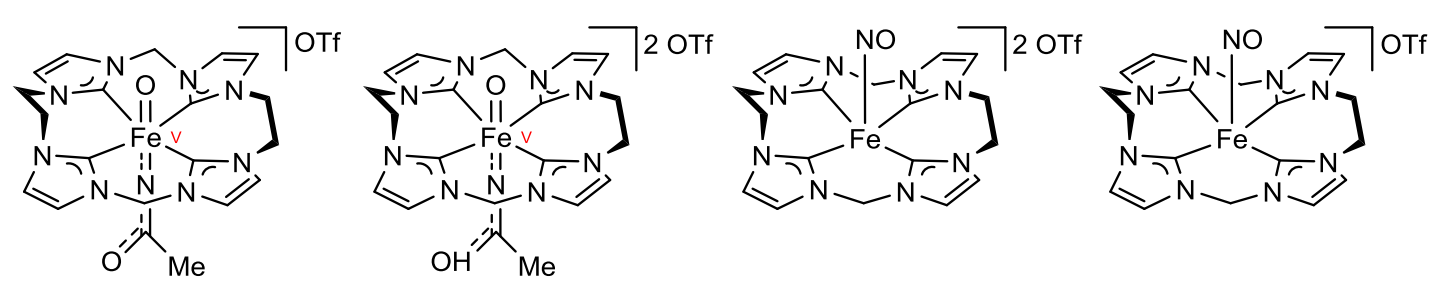

16

$16+\mathrm{H}^{+}$

17

19

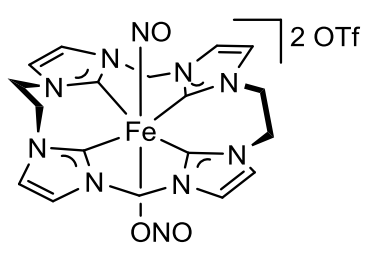

18

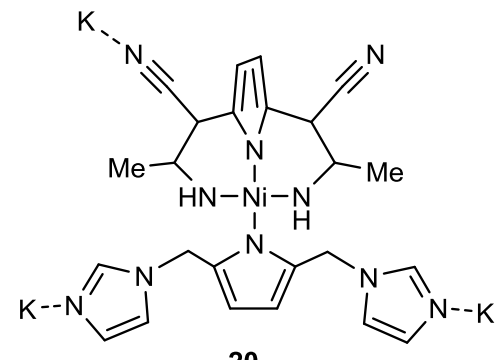

20

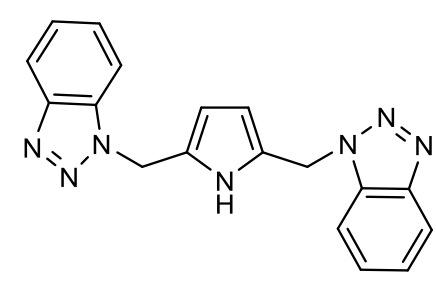

21<smiles></smiles>

22 


\section{List of Scientific Contributions}

Publications from this Work

- C. Kupper, A. Schober, S. Demeshko, M. Bergner, F. Meyer „An Exclusively Organometallic $\{\mathrm{FeNO}\}^{7}$ Complex with Tetracarbene Ligation and a Linear FeNO Unit" Inorg. Chem. 2015, 54, 3096-3098.

- S. Meyer, O. Krahe, C. Kupper, I. Klawitter, S. Demeshko, E. Bill, F. Neese, F. Meyer, "A trans-1,2 End-On Disulfide-Bridged Iron-Tetracarbene Dimer and Its Electronic Structure" Inorg. Chem. 2015, 54, 9770-9776.

- C. Kupper, J. A. Rees, S. Dechert, S. DeBeer, F. Meyer „Complete Series of $\{\mathrm{FeNO}\}^{8}$, $\{\mathrm{FeNO}\}^{7}$, and $\{\mathrm{FeNO}\}^{6}$ Complexes Stabilized by a Tetracarbene Macrocycle” J. Am. Chem. Soc. 2016, 138, 7888-7898.

- S. Ye, C. Kupper, S. Meyer, E. Andris, R. Navrátil, O. Krahe, B. Mondal, M. Atanasov, E. Bill, J. Roithová, F. Meyer, F. Neese "Magnetic Circular Dichroism Evidence for an Unusual Electronic Structure of a Tetracarbene-Oxoiron(IV) Complex" J. Am. Chem. Soc. 2016, 138, 14312-14325.

- C. Kupper, B. Mondal, J. Serrano-Plana, I. Klawitter, F. Neese, M. Costas, S. Ye, F. Meyer „Nonclassical Single-State Reactivity of an Oxo-Iron(IV) Complex Confined to Triplet Pathways" J. Am. Chem. Soc. 2017, DOI: 10.1021/jacs.7b03255.

Other Publications

- C. Kupper, S. Molitor, V. H. Gessner „Structure, Bonding, and Reactivity of RoomTemperature-Stable Lithium Chloride Carbenoids" Organometallics 2014, 33, 347-353.

- S. Molitor, K.-S. Feichtner, C. Kupper, and V. H. Gessner „Substitution Effects on the Formation of T-Shaped Palladium Carbene and Thioketone Complexes from $\mathrm{Li} / \mathrm{Cl}$ Carbenoids" Chem. - A Eur. J. 2014, 20, 10752-10762.

- K. Junold, F. M. Mück, C. Kupper, J. A. Baus, C. Burschka, R. Tacke „Activation of Sulfur Dioxide by Bis[N,N'-diisopropylbenz-amidinato(-)]silicon(II): Synthesis of Neutral SixCoordinate Silicon(IV) Complexes with Chelating O,O'-Sulfito or O,O'-Dithionito Ligands“ Chem. - A Eur. J. 2014, 20, 12781-12785.

- N. Laskowski, K. Junold, C. Kupper, J. A. Baus, C. Burschka, R. Tacke „Bis[N,N'-diisopropylbenzamidinato(-)]silicon(II): Cycloaddition Reactions with Organic 1,3-Dienes and 1,2-Diketones" Organometallics 2014, 33, 6141-6148.

- M. Bergner, S. Dechert, S. Demeshko, C. Kupper, J. M. Mayer, F. Meyer „Structural and Functional MitoNEET Model Shows Proton Coupled Electron Transfer" J. Am. Chem. Soc. 2017, 139, 701-707. 
Oral Presentations at Conferences

- C. Kupper, A. Rees, S. Dechert, S. DeBeer, F. Meyer "Complete series of $\{\mathrm{FeNO}\}^{6}$, $\left\{\mathrm{FeNO}^{7}\right.$ and $\{\mathrm{FeNO}\}^{8}$ complexes stabilized by a tetracarbene macrocycle" 12. Koordinationschemietreffen Kiel, Germany, February 2016.

Posters at Conferences and International Workshops

- C. Kupper, S. Meyer, I. Klawitter, S. Demeshko, E. Bill, F. Meyer "Iron(II)- and Oxoiron(IV)-Tetracarbenes: Reactivity towards Small Molecules and more" Workshop of the International Research Training Group GRK 1422 "Metal Sites in Biomolecules: Structures, Regulation and Mechanisms” Lund, Sweden, Mai 2014.

- A. Brinkmeier, C. Kupper, J. Odrobina „Energy Related Activation of Small Molecules and Catalysis in the Meyer Group" Kick-Off Meeting for the "International Center for Advanced Studies of Energy Conversion” Göttingen, Germany, November 2015.

- C. Kupper, B. Mondal, S. Ye, M. Costas, E. Bill, F. Meyer "Reactivity and Spectroscopy of an Oxoiron(IV)-Tetracarbene" Final Symposium of the International Research Training Group GRK 1422 "Metal Sites in Biomolecules: Structures, Regulation and Mechanisms", Göttingen, Germany, August 2015 and Girona Seminar: "Predictive Catalysis: TransitionMetal Reactivity by Design" Girona, Spain, April 2016.

- C. Kupper, J. A. Rees, S. Dechert, S. DeBeer, F. Meyer "Complete Series of $\{\mathrm{FeNO}\}^{8}$, $\{\mathrm{FeNO}\}^{7}$ and $\{\mathrm{FeNO}\}^{6}$ Complexes Stabilized by a Tetracarbene Macrocycle" 13th European Biological Inorganic Chemistry Conference Budapest, Hungary, August 2016. 

